RECEIVEDDOEMC/23075-5031

NOV 211995

Vol. III

\title{
OSTI
}

\section{Measurement and Modeling of Advanced Coal Conversion Processes \\ Volume III}

\section{User's Manual}

FBED-1: Fixed Bed Coal Combustion and

Gasification Model with a Generalized

Coal Devolatilization Submodel (FG-DVC)

M. Usman Ghani

Michael L. Hobbs

David G. Hamblen
L. Douglas Smoot

Yuxin Zhao

Predrag T. Radulovic

August 1993

Work Performed Under Contract No.: DE-AC21-86MC23075

For

U.S. Department of Energy

Office of Fossil Energy

Morgantown Energy Technology Center

Morgantown, West Virginia

By

Advanced Fuel Research, Inc.

East Hartford, Connecticut

and

Brigham Young University

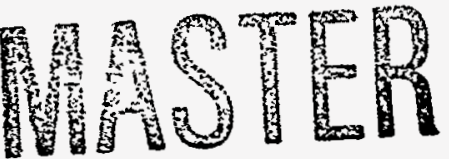

Provo, Utah 


\section{DISCLAIMER}

This report was prepared as an account of work sponsored by an agency of the United States Government. Neither the United States Government nor any agency thereof, nor any of their employees, makes any warranty, express or implied, or assumes any legal liability or responsibility for the accuracy, completeness, or usefulness of any information, apparatus, product, or process disclosed, or represents that its use would not infringe privately owned rights. Reference herein to any specific commercial product, process, or service by trade name, trademark, manufacturer, or otherwise does not necessarily constitute or imply its endorsement, recommendation, or favoring by the United States Government or any agency thereof. The views and opinions of authors expressed herein do not necessarily state or reflect those of the United States Government or any agency thereof.

This report has been reproduced directly from the best available copy.

Available to DOE and DOE contractors from the Office of Scientific and Technical Information, 175 Oak Ridge Turnpike, Oak Ridge, TN 37831; prices available at (615) 576-8401.

Available to the public from the National Technical Information Service, U.S. Department of Commerce, 5285 Port Royal Road, Springfield, VA 22161; phone orders accepted at (703) 487-4650. 


\title{
Measurement and Modeling of Advanced Coal Conversion Processes \\ Volume III
}

\author{
User's Manual \\ FBED-1: Fixed Bed Coal Combustion and \\ Gasification Model with a Generalized \\ Coal Devolatilization Submodel (FG-DVC)
}

M. Usman Ghani

L. Douglas Smoot

Michael L. Hobbs

Yuxin Zhao

David G. Hamblen

Predrag T. Radulovic

Work Performed Under Contract No.: DE-AC21-86MC23075

For

U.S. Department of Energy

Office of Fossil Energy

Morgantown Energy Technology Center

P.O. Box 880

Morgantown, West Virginia 26507-0880

By

Advanced Fuel Research, Inc.

87 Church Street

East Hartford, Connecticut 06108

and

Brigham Young University

Provo, Utah 84602 
Abstract

\section{Abstract}

A generalized, one-dimensional, heterogeneous, steady-state, fixed-bed model for gasification and combustion of coal is presented. The model, referred to as FBED-1, is a design and analysis tool that can be used to simulate a variety of fixed or moving bed gasification, combustion, and devolatilization processes. The model considers separate gas and solid temperatures, axially variable solid and gas flow rates, variable bed void fraction, coal drying, devolatilization based on chemical functional group composition, depolymerization, vaporization and crosslinking, oxidation and gasification of char, and partial equilibrium in the gas phase. The conservation equations and boundary conditions are formulated for gas and solid overall continuity, gas and solid energy equations, and gas and solid species or elemental continuity equations. Plug flow is assumed in both the solid and the gas phase with variable axial velocities. Gas phase pressure drop is calculated with the Ergun equation for packed beds. Large coal particle devolatilization is allowed to occur simultaneously with char oxidation and gasification. A generalized, coal devolatilization submodel, FG-DVC, is an important part of the model. Shell progressive or ash segregation, shrinking core char submodel describes oxidation and gasification. Turbulence is not treated formally in the slowly moving bed with low gas velocity, but is included implicitly through model correlations such as the effective heat transfer coefficient. A split, back-and-forth iteration and a Livermore solver for ordinary differential equations, LSODE, are used to solve a highly non-linear, stiff system of differential governing equations. Model formulation and solution method are presented, along with user and implementation guides and a sample problem. 


\section{Preface}

To expand the utilization of coal, it is necessary to reduce the technical and economic risks inherent in using a feed stock which is highly variable and which sometimes exhibits unexpected and unwanted behavior. Reducing these risks can be best achieved by developing technology for predicting coal's behavior in various processes. One such process is combustion and gasification of coal in fixed or slowly moving beds. Combustion and gasification of coal in fixed beds or slowly moving beds is of great commercial interest, since these systems can be conveniently integrated into combined cycle processes. In addition, these systems are reliable, require minimal pretreatment of feed coal, offer high thermal efficiencies, and generate easily disposable wastes. This manual presents a onedimensional, steady-state, fixed-bed model FBED-1, for simulating combustion and gasification of coal in fixed or slowly moving beds. The FBED-1 model incorporates a functional group - depolymerization, vaporization, and crosslinking devolatilization submodel, FG-DVC.

The manual is divided in two parts. The first part presents model formulation and solution method for the system of governing equations. The second part presents user's and implementation guides and a sample problem for the code. As the sample problem, a simulation for an atmospheric, air-blown, dryash Wellman-Galusha gasifier fired with Jetson bituminous coal is presented.

The complex nature of the physical and chemical processes being modeled results in a computer code that is sensitive to input parameters. Several input parameters, needed for FBED-1 simulations, are not known with certainty. The manual provides guidelines for selection of such parameters. Users are cautioned that an arbitrary set of input parameters will not necessarily produce a converged solution. A thorough understanding of the model equations and the numerical solution technique will give the user a better understanding of how to select appropriate input parameters and to adjust numerical parameters to obtain a converged solution. FBED-1 is thus considered a "user specialist" code. 


\section{Acknowledgments}

The development of this code and user's manual was sponsored by The U.S. Department of Energy, Morgantown Energy Technology Center (Contract Number DE-AC21-86MC23075), under subcontract from Advanced Fuel Research, Inc. with cost sharing by the Advanced Combustion Engineering Research Center (ACERC) at Brigham Young University. Funds for this center are provided by the U.S. National Science Foundation, the State of Utah, the U.S. Department of Energy, industrial participants, Brigham Young University and the University of Utah. Mr. Justin L. Beeson, Dr. Richard A. Johnson, and Dr. Norman T. Holcomb served as the DOE contracting Officer's Technical Representatives (COTRs). The financial support of these institutions, as well as the technical guidance of the DOE COTRs, are gratefully acknowledged.

At BYU, aside from the BYU authors of this manual, other contributors to the development of this code were Drs. B. Scott Brewster and Sung-Chul Yi. At AFR, aside from the AFR authors of this manual, major contributors to the development of the FG-DVC code were Drs. Peter R. Solomon and Michael A. Serio. Other contributors at AFR were Dr. Zhen Zhong Yu, Ms. Sylvie Charpenay, and Dr. Phillip E. Best. Professor Don E. Norton, of BYU Department of English, reviewed and edited the manual. The contributions of these individuals are greatly appreciated.

Finally, sincere appreciation is expressed to Ms. Nicole Banks and Ms. Melissa Wright for carefully typing and coordinating this document; to Mr. Mack R. Lund, ACERC computer system manger, for computer support; and to Mr. Michael R. King, ACERC manager, for administrative support. 


\section{Table of Contents}

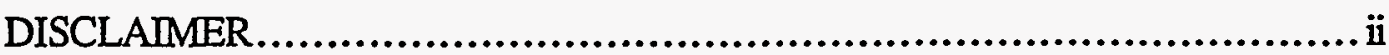

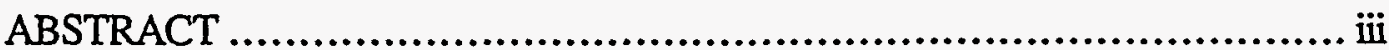

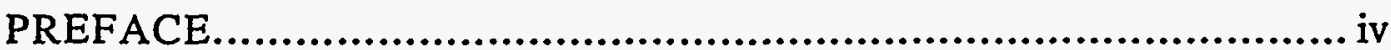

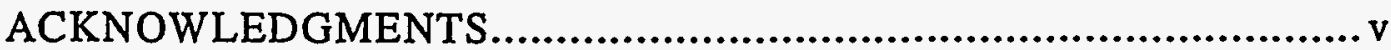

TABLE OF CONTENTS............................................................ vi

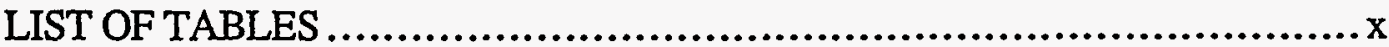

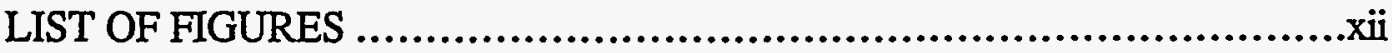

PART ONE: MODEL FORMULATION AND SOLUTION

\section{CHAPTER}

1 INTRODUCTION ...........................................

Code Description .............................................1-4

Code Development ............................................1-5

Limitations ......................................................1-6

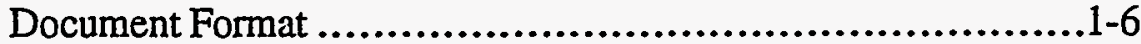

2 ZERO-DIMENSIONAL SUBMODEL ..........................2-1

Foundations and Assumptions.....................................2-2

Mass and Energy Balance ...........................................2-3

Gas Phase Chemistry ....................................................2-8

Devolatilization .....................................................2-9

Ultimate Yields and Compositions ................................2 2-10

Oxidation and Gasification ......................................2-11

Freeboard..................................................... 2-11

Summary ............................................... 2-12

3 ONE-DIMENSIONAL FIXED-BED MODEL ....................3-1

Foundations and Assumptions....................................... 
Conservation Equations …......................................3-5

Overall Gas and Solid Continuity Equations ...................3-8

Gas and Solid Energy Equations...................................3-8

Gas Phase Elemental Continuity Equations.......................3-12

Overall and Elemental Continuity Equations for Tar...........3-12

Solid Species Continuity Equations ............................3-13

Pressure Drop..................................................... 3-13

Gas Phase Chemistry .......................................... 3-14

Heat and Mass Transport....................................... 3-14

Transport and Thermodynamic Properties.....................3-15

Heat and Mass Transfer Correlations..............................3-17

Drying ......................................................3-22

Devolatilization ............................................ 3-22

Oxidation and Gasification .....................................3-23

Summary of Model Equations, Model Parameters and Options 3-27

4 FG-DVC DEVOLATILIZATION SUBMODEL ...................4-1

FG-DVC Model Basis .................................... 4-1

Depolymerization-Vaporization-Crosslinking (DVC)

Formulation ...............................................4-4

Functional Group (FG) Model Formulation...................4-7

Percolation Theory...................................................4-9

FG-DVC Solution Technique...................................4-11

FG Submodel ...............................................4-11

Distributed Rate Submodel (the f's) .......................... 4-13

DVC Submodel ...........................................4-15

Calculation of $\mathrm{F}_{\mathrm{j}}(\mathrm{p}, \mathrm{q})$ and $\mathrm{F}(\mathrm{p}, \mathrm{q}) \ldots \ldots \ldots \ldots \ldots \ldots \ldots \ldots \ldots . .18$

Summary of Active Differential Equations.........................4-21

5 SOLUTION METHOD ........................................5-1

Computational Algorithm..............................................5-2

PART TWO: CODE USER'S INSTRUCTIONS

6 USER'S GUIDE ...............................................

Description of Subroutines.....................................6-2

Program Input ............................................6-7

Main Input Data File, FB1IN.....................................6-7

Thermodynamic Data File, FBITHM ......................6-13 
FG-DVC Input Data File ...............................6-17

Input Data File, EXPT.DAT......................................6-18

Program Output....................................................6-18

Main Output File, FB1OUT ............................. 6-20

Output Files, OUTA through OUTJ.........................6-33

Other Output Files .......................................... 6-35

Graphics.....................................................6-36

Code Operation ................................................6-36

Setting the Logical Variables ...............................6-39

Iteration Procedure............................................6-41

7 IMPLEMENTATION GUIDE.....................................

Compiling, Linking, and Executing FBED-1 Code...............7-1

FORTRAN and Machine Incompatibilities.........................7-9

8 SAMPLE PROBLEM................................................

Atmospheric Pressure, Air-blown Gasification........................8-1

NOMENCLATURE

REFERENCES

APPENDICES
Nomenclature-1

References-1

A Adjustment of Functional Group Composition ........... A-1

B FG-SET Devolatilization Submodel.......................... B-1

FG-SET Submodel Basis............................. B-1

Arrhenius Rate Constants............................ B-5

Potential Tar-Forming Fraction ...................... B-5

C Prediction of effluent compositions for fixed-bed coal gasifiers........................................................

D Modeling Fixed-Bed Coal Gasifiers ..................... D-1

E Network models of coal thermal decomposition............ E-1 
F General Model of Coal Devolatilization...................... F-1

G A Characterization Method and Model for Predicing Coal Conversion Behaviour.....................................G-1

$\mathrm{H} \quad$ FBED-1 Subroutines.........................................

I Code Variables ....................................... I-1

J FBED-1 Graphics ....................................J-1

Compilation, Execution and Viewing/Printing of the Program PLOT1 ...................................

USER'S FORUM (Comment reporting form).................................. Forum-1

FBED-1 Code Installation Instructions : 


\section{List of Tables}

2.1 Partial molar heat capacity of slag components used in KoppNeumann rule .................................................................

3.1 Primary assumptions for the one-dimensional, fixed-bed model

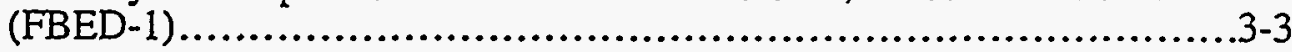

3.2 Conservation equations for FBED -1 ....................................

3.3 Enthalpy exchange associated with the oxidation reaction.................. 3-11

3.4 Physical property equations used in the one-dimensional fixed-bed

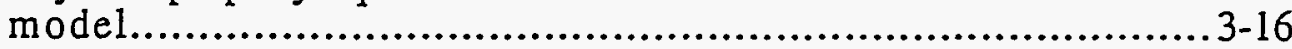

3.5 Heat and mass transport correlations used in FBED-1 ................... 3-18

3.6 Oxidation and gasification kinetic rate constants......................... 3-25

3.7 Summary of the one-dimensional fixed-bed model equations................. 3-28

3.8 Summary of the one-dimensional fixed-bed model parameters and options ................................................................. $3-30$

3.9 Guidelines for the selection of some critical model parameters ............ 3-32

6.1 Main data file, FBIIN.........................................................6-7

6.2 Thermodynamic input data file, FB1THM ...........................6-15

6.3 Sample input file for FG-DVC devolatilization submodel .................6-19

6.4 Sample data file EXPT.DAT ......................................... 6-18

6.5 Main output file, FB1OUT .......................................... 6-20 
6.6 Contents of output files OUTA through OUTJ 6-33

7.1 Listing of the make file MAKEFBED.....................................7-6

A.1 FG-DVC input data for Argonne Premium coal \#1 (Zap Lignite, Beulah, N.D.)

A.2 FG-DVC input data for Argonne Premium coal \#2 (WyodakAnderson, Wyoming)

A.3 FG-DVC input data for Argonne Premium coal \#3 (Illinois \#6). A-6

A.4 FG-DVC input data for Argonne Premium coal \#4 (Utah Blind Canyon)

A.5 FG-DVC input data for Argonne Premium coal \#5 (Upkana-Stokton, WV)

A.6 FG-DVC input data for Argonne Premium coal \#6 (Pittsburgh \#8)

A.7 FG-DVC input data for Argonne Premium coal \#7 (Upper Freeport,

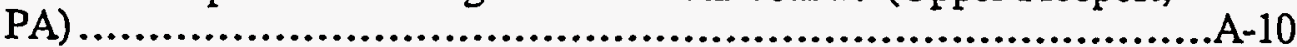

A.8 FG-DVC input data for Argonne Premium coal \#8 (Pocahontes \#3, VA)

B.1 Devolatilization kinetic parameters, functional group compositions, potential tar-forming fractions and ultimate analysis

J.1 Input file plot1.dim for the program plot1 $\mathrm{J}-2$

J.2 Input data file plot2.dim for the program plot2 $\mathrm{J}-3$ 


\section{List of Figures}

1.1 Typical atmospheric-pressure, fixed-bed gasifier, (WellmanGalusha), with input data for Jetson simulation (Thimsen et al., 1984)

2.1 Control volume for A) 1-zone total equilibrium submodel, B) 1-zone partial equilibrium submodel, and C) 2-zone partial equilibrium submodel.

3.1 Schematic of a coal particle with FG-SET devolatilization model based on chemical functional groups (Solomon et al., 1988).

3.2 Energy exchange due to the transport of mass between solid and gas phase due to oxidation

4.1 Bethe lattice for two- $\sigma$ model with $\sigma_{1}=\sigma_{2}=1$. (a) With most double bands representing the crosslinks not yet formed to represent the starting coal, the lattice is like one- $\sigma$ model with $\sigma=1$, linear chains. (b) Fully linked case $(p=q=1)$ is like one- $\sigma$ model with $\sigma=3$

5.1A Computational algorithm for the FBED-1 model

5.1B Details of computational algorithm for the zero-dimensional submodel, FBED0D.

5.1C Details of computational algorithm for the downward integration pass

5.1D Details of computational algorithm for the upward integration pass.........5-7

6.1 Structure of the main program FBED1D .............................6-4

6.2 Structure of the subprogram FBED0D.................................6-5 
6.3 Plot generated by the program plotl showing the predicted axial profiles.

6.4 Plot generated by the program plot 2 showing the comparison of the predicted and experimental data.

8.1 Predicted axial A) temperature, B) pressure, C) major species composition, D) minor species composition, E) volumetric char consumption rate, F) burnout and overall particle and unreacted core diameter in the atmospheric pressure, air-blown Wellman-Galusha gasifier fired with Jetson bituminous coal

8.2 Comparison of FBED-1 predictions with experimental data for the gasification of the Jetson bituminous coal in the atmospheric, airblown, dry-ash Wellman-Galusha gasifier.

B.1 Progress of thermal decomposition according to the functional group model: A) functional group composition of coal, B) initial state of decomposition, C) later stage of decomposition, D) completion of decomposition 


\section{PART ONE: MODEL FORMULATION AND SOLUTION}

\section{CHAPTER 1}

\section{INTRODUCTION}

Fixed-bed systems are simple, reliable, have high thermal efficiency, and require minimal pretreatment of feed coal. Combustion and gasification of coal in fixed or slowly moving beds of packed coal particles are of substantial commercial interest. Eighty-nine percent of the coal gasified in the world is gasified by the fixed-bed, ten percent by the entrained-bed, and only one percent by the fluidized bed process. Fixed beds can be operated at high pressure, providing opportunity for increased power generation efficiency through combined cycle processes. Fixed beds are also a popular choice for mild gasification since, by their countercurrent nature, the liquids can be quickly removed before being altered by substantial reaction. Fixed bed gasification and combustion processes are classified according to the flow pattern of coal and gas as countercurrent, cocurrent, and crosscurrent processes. The countercurrent process is the most common fixed bed combustion and gasification process.

Figure 1.1 shows a schematic of a large-scale, atmospheric pressure, airblown, dry-bottom, fixed-bed gasifier. Coal is fed to the top of the gasifier and moves downward under gravity, countercurrent to the rising gas stream. The dry ash is removed at the bottom of the gasifier. The feed gas is commonly composed of air or oxygen and steam. Excess steam is supplied to the gasifier to control the ash temperature. Figure 1.1 also shows the reactor divided into four overlapping zones: drying, devolatilization, gasification and combustion. As the coal slowly 


\section{Sampling Point For Offtake Gas}

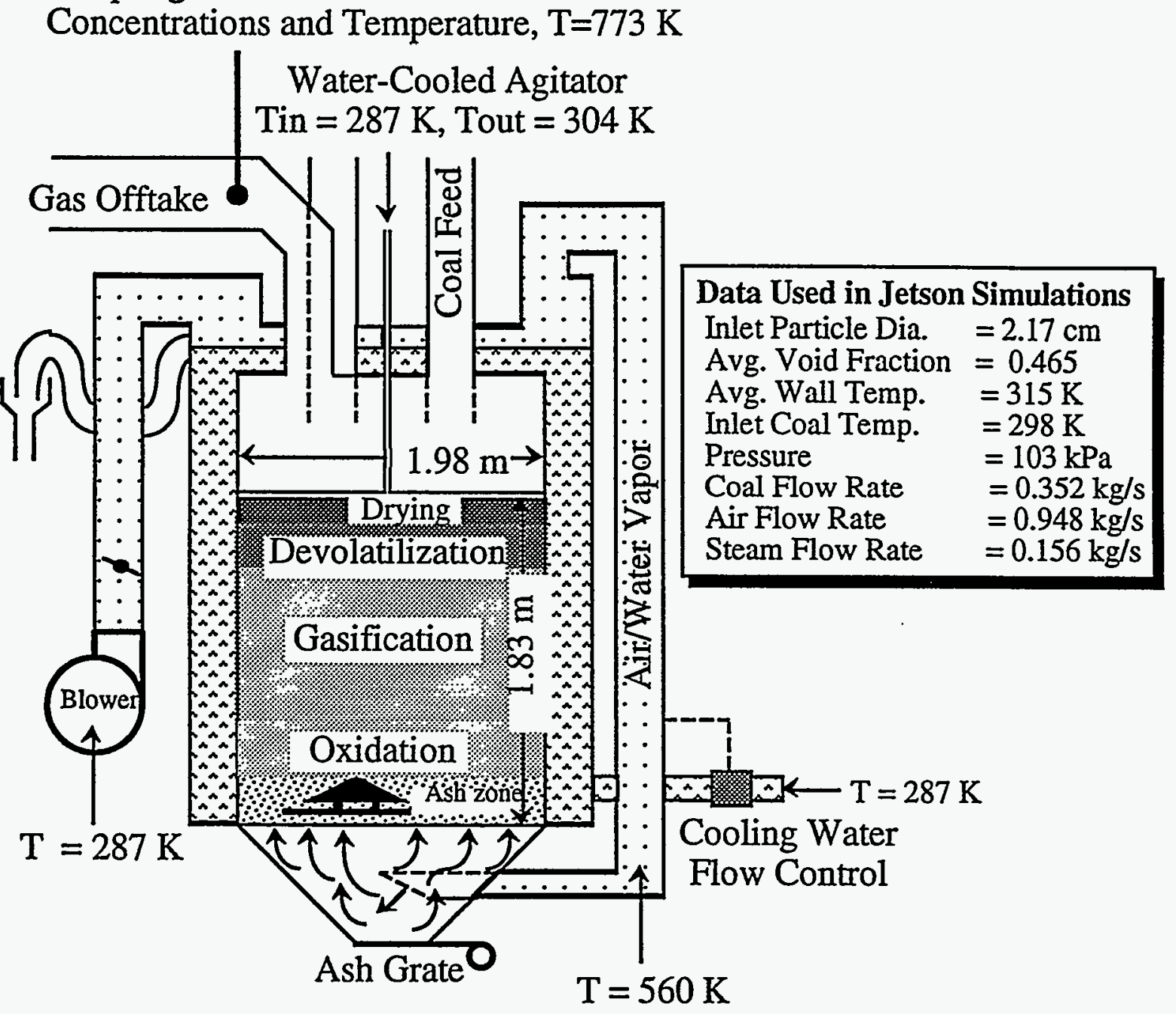

Figure 1.1. Typical atmospheric-pressure, fixed-bed gasifier (Wellman-Galusha), with input data for Jetson simulation (Thimsen et al., 1984).

descends, the hot gases produced in the gasification and combustion zones exchange energy with the colder solid. Water and subseqquently volatile matter are released when the solid reaches sufficiently high temperatures. After drying and 
devolatilization, the char enters the gasification zone where carbon reacts with steam, carbon dioxide and hydrogen. Endothermic reactions in this section produce carbon monoxide and hydrogen. The slightly exothermic reaction of hydrogen with carbon produces methane. Differentiation between the "gasification zone" and "combustion zone" is based on the presence or absence of free oxygen. Combustion and gasification reactions occur simultaneously in the "combustion zone." The combustible gases such as carbon monoxide and hydrogen may react with oxygen. The exothermic combustion reactions provide the necessary energy for the endothermic gasification reactions and drying. Blast gas, which is composed of steam and oxidant (air or oxygen), is preheated by the hot ash. Part of the process steam is produced by a water jacket surrounding the gasification chamber.

Solid residence times in the drying, gasification and oxidation zones may be on the order of several hours. Residence time in the ash layer may be even higher depending on the thickness of this zone. Gas residence times are on the order of seconds. Solid and gas temperature gradients are highest in the devolatilization and oxidation zones. In the fixed bed gasifiers, as opposed to the entrained and the fluidized bed gasifiers, the devolatilization products are released in the region of relatively low temperature and lack of free oxygen. Thus, tars, oils, and heavier hydrocarbon gases are neither cracked nor oxidized, but added to the product gas. This may be a disadvantage but it may also be used to advantage as in the mild gasification process.

The fixed bed systems have been the focus of significant modeling efforts (Desai and Wen, 1978; Yoon et al., 1978; Amundson and Arri, 1978; Thorness and Kang, 1986; Bhattacharya et al, 1986). Most of these models make simplifying assumptions such as equal gas and solid temperatures, plug flow, constant bed porosity, instantaneous devolatilization and simplified gas phase chemistry. This manual presents a one-dimensional fixed-bed model, FBED-1, in which most of these assumptions are relaxed. The major advances achieved by the FBED-1 model are: (1) A coal general devolatilization submodel which is based on a rigorous functional group, depolymerization, vaporization, and crosslinking submodel (FGDVC) proposed by Solomon et al., (1988); (2) Generalized gas chemistry to predict the composition of gas phase; (3) Finite, overlapping, drying, devolatilization, 
Introduction

gasification and oxidation zones; (4) Axially variable bed porosity and velocities to properly predict the pressure profile; (5) Ash layer effects; (6) A split back-andforth shooting method to satisfy the conditions at both boundaries. Furthermore, the FBED-1 model is the first fixed-bed combustion and gasification model which has been extensively validated by comparisons with measured temperature and pressure profiles.

\section{Code Description}

This manual presents the details of an advanced, comprehensive, onedimensional computer code, referred to as FBED-1 (Fixed-BED, 1-dimensional), which describes drying, devolatilization, combustion, and gasification of large coal particles in countercurrent flow. FBED-1 can be used to simulate dry-ash gasifiers, such as the atmospheric pressure Wellman-Galusha gasifier, the medium pressure, METC gasifier, and the high pressure Lurgi gasifier. FBED-1 is a one-dimensional steady-state model that simulates the major conversion processes during coal gasification, namely drying, devolatilization, oxidation and gasification of char with an ash layer, and tar decomposition in the gas phase. It considers separate gas and solid temperatures, axially variable solid and gas velocities, and variable bed void fraction. Coal devolatilization is based on the functional group, depolymerization, vaporization and crosslinking (FG-DVC) submodel (Solomon et al., 1988). A simpler devolatilization submodel, FG-SET, based on functional group composition (Solomon et al., 1988) and tar evolution using a semiempirical correlation (Ko et al., 1988), is also provided as an option. The gas composition is determined by considering either total or partial equilibrium in the gas phase. The input data include reactor dimensions, operating conditions, inlet solid and gas temperatures, pressure, composition, flow rates and wall temperature. The additional input data needed for the devolatilization submodel include the initial functional group composition, and kinetic parameters. For the FG-DVC submodel, bond population and kinetics, cross-link efficiency for each functional group, and probabilities for tar mass distribution are also needed. The predicted quantities include axial solid and gas temperatures, gas phase species concentration, pressure, char conversion, particle size and number density, gas and solid phase flow rates, 
Chapter 1

residence times, heating rates, velocities, film, ash and chemical resistances, gas phase transport properties, and wall heat loss.

The FBED-1 code is based on a set of 191 coupled, first order ordinary differential equations. These equations simulate the chemical and physical processes taking place in both the gas and the solid phase during the coal conversion in a fixed-bed. The system of differential equations is composed mainly of the overall continuity and energy equations for the gas and solid phases, the solid and gas species and elemental continuity equations, and equations for the functional groups composition in the gas, tar and char. In the FBED-1 model, plug flow is assumed for both solid and gas phase; gas phase pressure drop is determined with Ergun's equation (Ergun, 1952). In addition, an attempt has been made to formulate a general reaction scheme that can accommodate results of future measurements and improved kinetic parameters.

The model presented in this manual applies to countercurrent fixed-bed processes for large particles $(>1 \mathrm{~mm})$, where solid heating rates are small $(<10 \mathrm{~K} / \mathrm{s})$ and gas heating rates are moderate $\left(<10^{4} \mathrm{~K} / \mathrm{s}\right)$. This model is not applicable without modification to cocurrent fixed-bed, entrained-flow or fluidized-bed processes.

\section{Code Development}

The formulation and the numerical solution of FBED-1 are based on a previous model, MBED-1, developed by Hobbs et al., (1992). FBED-1 provides the following improvements over MBED-1: 1) improved predictions of product gas composition and temperature; 2) improved prediction of tar flow rate; 3 ) integration of the devolatilization submodel, FG-DVC; 4) modifications in the iteration scheme to satisfy the gas phase boundary conditions at the bottom of the gasifier; 5) improved modularity, code structure and user friendliness; and 6) improved graphics output. To evaluate and validate FBED-1, several practical cases have been simulated and comparisons have been made with the available experimental data. The appropriate use of this comprehensive code requires a thorough 
Introduction

understanding of its foundations and operation. A person with a strong technical background may require several months to understand and effectively use the code. Results can be obtained faster with the guidance of more experienced users.

\section{Limitations}

The assumptions employed in the development of FBED-1 are discussed in detail in Chapters 2 and 3. The input data also require several model parameters which may be difficult to determine from the available experimental data. These parameters include the bed void fraction distribution, the adjustable factor for reacting solid-to-gas heat transfer, and the effective ash diffusivity. Guidelines for specifying these parameters are provided in Chapter 3 . The kinetic parameters for devolatilization are assumed to be a function of coal rank. However, the kinetic parameters for the oxidation and gasification reactions are provided by the user and may be either rank dependent or rank independent. The kinetic parameters used herein were obtained from small particle data and may not apply to large particles. However, due to the dominant mass transport effects in large particles, these kinetic parameters are expected to give reasonable predictions.

\section{Document Format}

This manual is organized in two parts. Part One documents the theory (Chapters 2-4) and the solution method for the governing equations (Chapter 4-5). An understanding of the theoretical foundations of the code is essential for its correct application, and potential users should study this material carefully. Part Two describes the use of the code (Chapter 6), gives suggestions regarding its implementation on different computer systems (Chapter 7), and presents a sample simulation of the atmospheric pressure Wellman-Galusha gasifier (Chapter 8). 


\section{CHAPTER 2}

\section{ZERO-DIMENSIONAL SUBMODEL}

The core of the fixed-bed model, FBED-1, is a set of 191 coupled, first order ordinary differential equations. These equations simulate the chemical and physical processes taking place in both the gas and the solid phase during the coal conversion in a fixed-bed. Due to the countercurrent flows of gas and solids, these equations constitute a split boundary value problem. The input conditions for the solid phase are known at the top of the gasifier, whereas the input conditions for the gas phase are known at the bottom of gasifier. This system of equations can be converted to an initial value problem and integrated from the top to the bottom of the gasifier, provided the initial estimates for the gas phase quantities are made available at the top of the gasifier. In order to provide these estimates, a zero-dimensional submodel, FBED-0, is proposed and described in this chapter.

FBED-0 is a two-zone, well mixed, partial equilibrium model. Its primary use is to provide estimates for the product gas enthalpy, composition and species flow rates, as well as the product tar composition and flow rate. The zerodimensional submodel, FBED-0, considers drying and devolatilization on one side, and gasification and oxidation on the other to take place in separate zones. It uses an advanced devolatilization submodel, FG-DVC, which is based on the chemical structure of the coal. It provides an option to use a simpler devolatilization submodel, FG-SET, and a provision to consider tar recycle. FBED-0 can also be used as a stand-alone model to predict the temperature and composition of the effluent gas and tar streams. 
Zero-Dimensional Submodel

\section{Foundations and Assumptions}

The basic assumption of the zero-dimensional submodel, FBED-0, is that oxidation and gasification on one hand, and drying and devolatilization on the other occur at different temperatures in separate zones. The rationale behind this assumption is that oxidation and gasification of char take place at relatively high temperatures, as compared to the devolatilization and drying of coal. This temperature difference provides a natural division of the process into two zones -- a higher temperature oxidation and gasification zone, and a lower temperature drying and devolatilization zone. Higher temperatures in the oxidation and gasification region favor total chemical and thermal equilibrium. Lower temperatures in the devolatilization zone may favor partial chemical equilibrium while maintaining thermal equilibrium. In the subsequent discussions, these two zones are referred to as "equilibrium zone" and "drying and devolatilization zone."

In FBED-0, the equilibrium, and the drying and devolatilization zones are assigned different temperatures, $T_{\text {equilibrium }}$ and $T_{\text {exit }}$, respectively. All gases are assumed to be in thermal equilibrium in both zones. The gases produced in the equilibrium zone are assumed to be in chemical equilibrium in this zone, but can be considered to be either reactive or nonreactive in the drying and devolatilization zone. The volatile gases and the water vapor produced in the drying and devolatilization zone can also be assumed to be either reactive or nonreactive. Coal is assumed to be composed of various chemical functional groups (Solomon et al., 1988), which evolve to form light gases and tar. The input data for the zerodimensional model include the gasifier dimensions, operational variables such as pressure, coal and gas flow rates and temperatures, ultimate and proximate analysis of coal, composition of the feed gas, water jacket temperature, wall heat loss or overall heat transfer coefficient, and coal burnout. When the recycled tar is considered, its fraction must also be specified. The computations are based on an overall mass and energy balance. The output of FBED-0 includes the temperatures and compositions of the product gas, product tar and residual solid streams. 
Chapter 2

\section{Mass and Energy Balance}

The two-zone, zero-dimensional submodel has evolved from simpler onezone total equilibrium and partial equilibrium submodels. In the one-zone submodels, the complete conversion of coal is assumed to take place at a uniform temperature. The gases, however, may be taken to be in total or partial chemical equilibrium. The control volumes for these three submodels are shown in Figure 2.1. The one-zone submodels shown in Figures 2.1.A and 2.1.B are included for comparison only and show the evolution of the two-zone submodel. The mass and energy balances around any control volume shown in Figure 2.1 are given by

$$
\begin{gathered}
\dot{m}_{s, \text { in }}+\dot{m}_{g, \text { in }}-\dot{m}_{s, \text { out }}-\dot{m}_{g, \text { out }}=0 \\
\dot{m}_{s, \text { in }} h_{s, \text { in }}+\dot{m}_{g, \text { in }} h_{g, \text { in }}-\dot{m}_{s, \text { out }} h_{s, \text { out }}-\dot{m}_{g, \text { out }} h_{g, \text { out }}-Q=0
\end{gathered}
$$

where $\dot{m}(\mathrm{~kg} / \mathrm{s}), h(\mathrm{~J} / \mathrm{kg})$, and $Q$ (watts) refer to mass flow rate, total enthalpy, and heat loss, respectively. The subscripts $s, g$, in and out refer to solid, gas, control volume inlet, and control volume outlet, respectively. The total equilibrium submodel shown in Figure 2.1.A assumes complete reaction of the dry, ash-free coal by either devolatilization, gasification or oxidation. This submodel, however, does not differentiate among mechanisms for these chemical processes as do the one- and two-zone partial equilibrium submodels. The only difference between the one- and two-zone partial equilibrium submodels is the treatment of drying and devolatilization processes. The one-zone submodel assumes that drying and devolatilization occur in the same zone as oxidation and gasification. This assumption is relaxed in the two-zone partial equilibrium submodel.

The total enthalpy of a substance is computed from the enthalpy of formation and the sensible enthalpy:

$$
h=h_{f}^{o}+h^{s}
$$




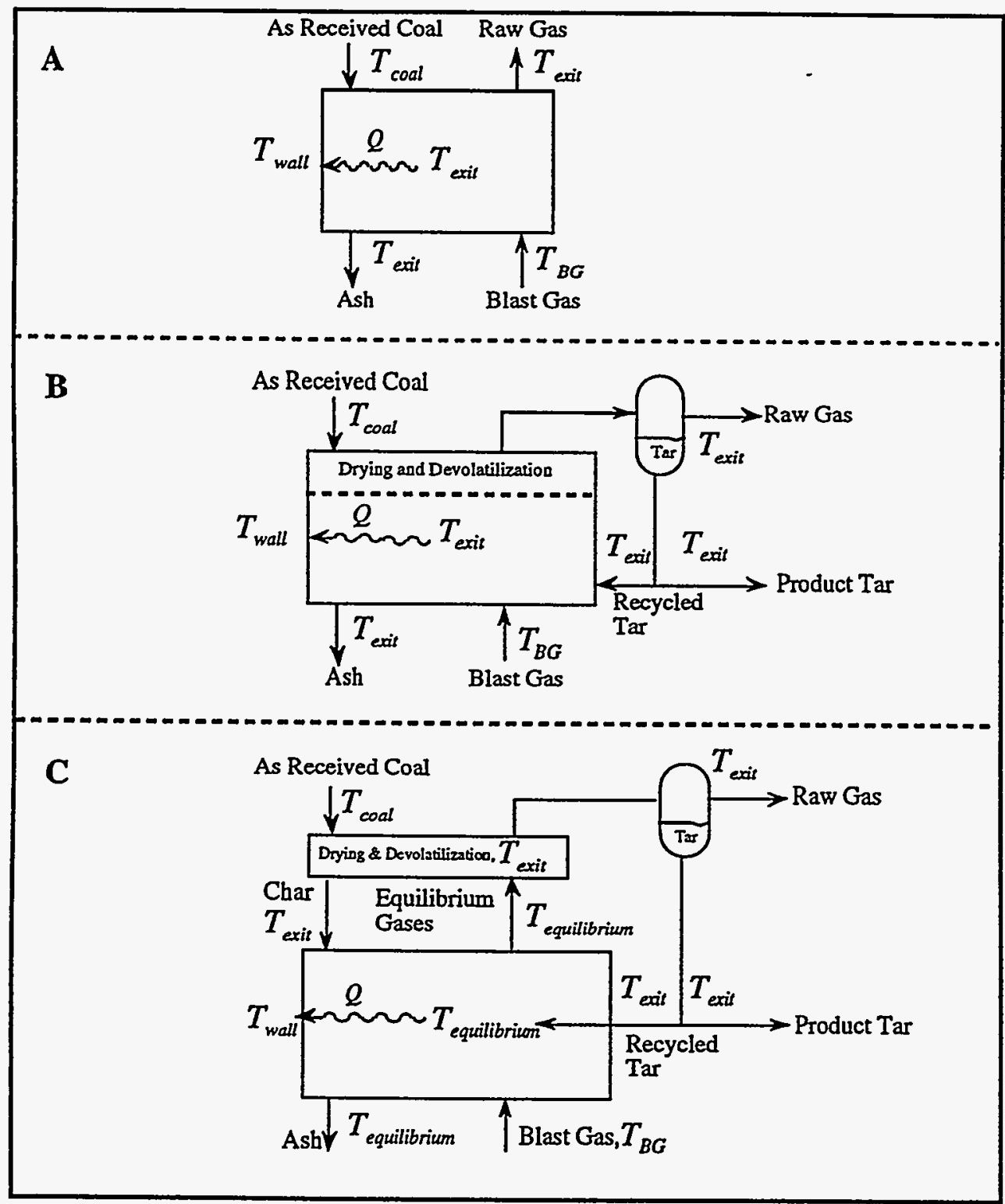

Figure 2.1. Control volume for A) 1-zone total equilibrium submodel, B) 1-zone partial equilibrium submodel, and C) 2-zone partial equilibrium submodel. 
where the superscripts $o$ and $s$ refer to the reference temperature $(298.15 \mathrm{~K})$ and sensible enthalpy from the reference temperature to the stream temperature.

The heat of formation of the coal can be obtained from its higher heating value, $H H V(J / k g)$, which is the negative of the heat of reaction (i.e., $\left.H H V=-\Delta h_{\text {reaction }}\right)$. The higher heating value of coal can be measured or estimated from the ultimate analysis using the Dulong's formula (Perry and Chilton, 1973). Ash effects are included via the experimentally determined HHV. The heat of formation of the coal, $h_{f, c}^{o}$, is then determined as the sum of the heats of formation of the products from the following reaction:

$$
C_{n} \mathrm{H}_{m} \mathrm{O}_{h} S_{j} N_{k}+\left(n+\frac{m}{4}-\frac{h}{2}+j\right) O_{2} \rightarrow n \mathrm{CO}_{2}+\frac{m}{2} \mathrm{H}_{2} \mathrm{O}+\frac{k}{2} \mathrm{~N}_{2}+j \mathrm{SO}_{2}
$$

and the higher heating value of the coal:

$$
h_{f, c}^{o}=\sum_{i=1}^{4} v_{i} h_{f, i}^{o}+H H V
$$

where $i$ represents the formation of $\mathrm{CO}_{2}, \mathrm{H}_{2} \mathrm{O}(l), \mathrm{N}_{2}$, and $\mathrm{SO}_{2}$, respectively and $v_{i}$ represents the stoichiometric coefficient for the $i$-th product given in Equation 2.4. Since both terms in Equation 2.5 are of similar magnitude and of limited accuracy, error may result in determining the heat of formation of coal, which is a relatively small number, when computed as the difference of these two numbers (Johnson, 1981).

The sensible enthalpy for the coal or char, $h_{c}^{S}(J / k g)$, can be determined using the Merrick's (1983) correlation evaluated at $298.15 \mathrm{~K}$ and the coal temperature:

$$
h_{c}^{s}=\left(\frac{R}{a}\right)\left[380 g_{o}\left(\frac{380}{T}\right)+3600 g_{o}\left(\frac{1800}{T}\right)\right]
$$




$$
g_{0}(z)=\frac{1}{\exp (z)-1}
$$

where $R(8314.4 \mathrm{~J} / \mathrm{kmol} \cdot K)$ is the universal gas constant, $T(K)$ is solid temperature, 380 and $1800(K)$ are characteristic Einstein temperatures, and $z$ is a dummy variable representing the ratio of the Einstein temperature and $T$. The mean atomic weight, $a$, is defined (Merrick, 1983) as

$$
\frac{l}{a}=\sum_{i=1}^{5} \frac{\Omega_{i}}{\mu_{i}}
$$

where $\mu_{\mathrm{i}}$ and $\Omega_{\mathrm{i}}$ represent the atomic weights and dry-ash free mass fraction of carbon, hydrogen, oxygen, nitrogen and sulfur respectively.

The specific heat of the ash, $C p_{a}(J / k g \cdot K)$, can be determined from Kirov's correlation (Merrick, 1983), given by:

$$
C p_{a}=754+0.586 t
$$

where $t\left({ }^{\circ} \mathrm{C}\right)$ is the ash temperature. The Kopp-Neumann rule (Mills and Rhine, 1989) can also be used to obtain reliable estimates of the ash heat capacity, provided various constituents of the ash are known:

$$
C_{p_{a}}=1000\left(\frac{\sum_{i=1}^{n} x_{i} C_{p_{i}}}{\bar{M}_{w}}\right)
$$

where $x_{i}, C_{p_{i}}(\mathrm{~J} / \mathrm{mol} \cdot K)$, and $\bar{M}_{w}$ represent the mole fraction of the $i$-th ash constituent, partial molar heat capacity of the pure $i$-th component, and the average molecular weight of the ash or slag. The temperature dependence of ash heat capacity, $C_{p}$, is expressed as $C_{p}=a+b T-c T^{-2}$. The values of the coefficients $a, b$, and $c$ for ash constituents used in the present formulation are given in Table 2.1. Equation 2.10 can 
Chapter 2

Table 2.1. Partial molar heat capacity of slag components used in Kopp-Neumann rule. $\$$

\begin{tabular}{|c|c|c|c|c|c|}
\hline Ash & $M_{w}$ & \multicolumn{3}{|c|}{$C_{p_{i}}(J / m o l K)=a+b T-C T^{-2}($ solid $)$} & $\overline{C_{p} \text { (liquid) }}$ \\
\hline Component & $\mathrm{kg} / \mathrm{kmol}$ & $a$ & $b$ & $c$ & $J /$ molK \\
\hline $\mathrm{SiO}_{2}$ & 60.09 & 53.591 & $1.8715 \times 10^{-2}$ & $1.2644 \times 10^{6}$ & 87.0 \\
\hline $\mathrm{CaO}$ & 56.08 & 41.868 & $2.0264 \times 10^{-2}$ & $4.5217 \times 10^{5}$ & 80.8 \\
\hline $\mathrm{Al}_{2} \mathrm{O}_{3}$ & 101.96 & 92.445 & $3.7560 \times 10^{-2}$ & $2.1876 \times 10^{6}$ & 146.4 \\
\hline $\mathrm{MgO}$ & 40.31 & 45.469 & $5.0116 \times 10^{-3}$ & $8.7379 \times 10^{5}$ & 90.4 \\
\hline $\mathrm{K}_{2} \mathrm{O}^{\ddagger}$ & 94.2 & 65.700 & $2.2600 \times 10^{-2}$ & 0.0 & 74.0 \\
\hline $\mathrm{Na}_{2} \mathrm{O}$ & 61.98 & 65.700 & $2.2600 \times 10^{-2}$ & 0.0 & 92.0 \\
\hline $\mathrm{TiO}_{2}$ & 79.9 & 49.446 & $3.1568 \times 10^{-2}$ & $1.7543 \times 10^{5}$ & 111.7 \\
\hline $\mathrm{MnO}$ & 70.94 & 31.108 & $4.3459 \times 10^{-2}$ & $5.5156 \times 10^{-5}$ & 79.9 \\
\hline $\mathrm{FeO}$ & 71.85 & 52.84 & $6.2470 \times 10^{-3}$ & $3.1903 \times 10^{5}$ & 76.6 \\
\hline $\mathrm{Fe}_{2} \mathrm{O}_{3}$ & 159.7 & 103.50 & $6.7156 \times 10^{-2}$ & $1.7727 \times 10^{6}$ & 191.2 \\
\hline$F e^{\frac{7}{f}}$ & 55.85 & 12.72 & $3.1710 \times 10^{-2}$ & $-2.5100 \times 10^{5}$ & 43.9 \\
\hline $\mathrm{P}_{2} \mathrm{O}_{5} \frac{7}{5}$ & 141.91 & 182.5 & $4.6400 \times 10^{-2}$ & $4.5440 \times 10^{6}$ & 242.7 \\
\hline $\mathrm{CaF}_{2}$ & 78.08 & 59.83 & $3.045 \times 10^{-2}$ & $-1.9600 \times 10^{5}$ & 96.2 \\
\hline $\mathrm{SO}_{3}^{\frac{7}{6}}$ & 80.06 & 70.2 & $9.774 \times 10^{-2}$ & 0.0 & 175.7 \\
\hline
\end{tabular}

§ All solid heat capacity coefficients obtained from Perry's Handbook (1973, page 3-119) except as noted. All liquid heat capacity values are obtained from Mills and Rhine (1989).

₹ Solid heat capacity coefficients obtained from Mills and Rhine correcting $\mathrm{b}$ and $\mathrm{c}$ by $10^{-3}$ and $10^{5}$, respectively.

also be used to determine the heat capacity of the liquid slag. In that case, melting is assumed to occur at the measured ash fluid temperature, and the associated enthalpy of fusion is taken to be $230 \mathrm{~J} / \mathrm{g}$ following Mills and Rhine (1989). The predicted temperatures of the solid phase were found to be insensitive to the enthalpy of fusion; therefore a single value for enthalpy of fusion is used for coals of all ranks. 
The total enthalpies of the feed gases are found with the polynomial expressions obtained by the regression analysis of the thermochemical data given in the JANAF tables (Stull and Prophet, 1971).

The final term in Equation 2.2 represents the heat loss through the reactor wall, $Q$ (watts). This quantity can be estimated provided the jacket steam flow rate is known. Otherwise, the heat loss through the wall can be calculated using the following expression, which requires that the overall heat transfer coefficient, $U$ (watts $/ m^{2} K$ ), be known:

$$
Q=U A\left(T_{e}-T_{w}\right)
$$

where $A, T_{e}$, and $T_{w}$ represent the surface area of steam jacket, the equilibrium zone temperature, and the wall temperature, respectively. The values of the overall heat transfer coefficient, $U$, are usually in the range from 50 to 200 watts $/ m^{2} K$, which represent the forced convection regime.

\section{Gas Phase Chemistry}

Most of the currently available fixed-bed models compute the gas phase composition by assuming the major gaseous species $\left(\mathrm{CO}, \mathrm{CO}_{2}, \mathrm{H}_{2}\right.$, and $\left.\mathrm{H}_{2} \mathrm{O}\right)$ to be in equilibrium, following the water-gas-shift reaction. Furthermore, these models do not account for the minor species in the equilibrium calculations. In FBED-0, all gaseous species are fully taken into account, and the gas composition can be determined based on either total or partial equilibrium in the gas phase. A mixture is said to be in partial equilibrium if at least one of its constituent species is considered to be out of chemical equilibrium. The "out of equilibrium" species may be chemically frozen or undergo changes following certain reactions with finite rate kinetics.

In FBED-0, minor species such as higher hydrocarbons $\left(\mathrm{C}_{2} \mathrm{H}_{2}, \mathrm{C}_{2} \mathrm{H}_{4}\right.$, $\mathrm{C}_{2} \mathrm{H}_{6}, \mathrm{C}_{3} \mathrm{H}_{6}, \mathrm{C}_{3} \mathrm{H}_{8}, \mathrm{C}_{4} \mathrm{H}_{10}$, etc.), and nitrogenous species ( $\mathrm{HCN}, \mathrm{NO}$, and $\mathrm{NH}_{3}$ ) are fully treated. The gas phase in the equilibrium zone is assumed to be in total 
chemical and thermal equilibrium. The composition and temperature are determined, using a generalized chemical equilibrium code, CREE (Pratt, 1979), by minimizing the Gibbs free energy, given the total static enthalpy, pressure and elemental composition of the gas phase. In the drying and devolatilization zone, thermal equilibrium is assumed, but the gas phase composition is determined using one of the following two options: 1) total chemical equilibrium is assumed for the gas phase; 2) the gases generated in the oxidation and gasification zone as well as the devolatilized gases and moisture released in the drying and devolatilization zone are assumed to be nonreactive. In both of these options, the tar evolved from the coal is taken to be in thermal equilibrium but kept out of chemical equilibrium. In countercurrent, fixed-bed gasifiers, the assumptions regarding nonreacting species may be justified due to the low temperature in the drying and devolatilization zone, which prevents further reactions of the volatile gases and moisture with the gases produced in the equilibrium zone.

\section{Devolatilization}

During the devolatilization process, as much as $40-60$ percent of the dry-ash free mass of feed coal is evolved as light gases and tar. A precise treatment of devolatilization is, therefore, essential to predict accurately the yield of volatiles and the composition of residual char and volatiles. In FBED-0, two devolatilization submodels FG-DVC and FG-SET are provided. In both submodels, char is assumed to be composed of various functional groups, which evolve to yield both light gases and tar. The main difference between these two models is in the treatment of tar evolution from the char matrix. The FG-DVC submodel computes the tar evolution from the char, based on the coal structure. The simpler FG-SET model uses a semiempirical correlation to determine the potential tar forming fraction, $\mathrm{x}^{0}$, and tar evolution is assumed to follow a first order decay of $\mathrm{x}^{0}$. It also provides an option to allow for user specified tar-forming fraction. The functional group composition is only available for a select group of standard coals, such as Argonne Premium Coals. In order to use the available data, the functional group composition of the standard coal, which is closest to the feed coal, must be adjusted to match the ultimate composition of the feed coal. The guidelines to adjust the 
functional group composition are presented in Appendix A. The FG-DVC submodel is presented and discussed in Chapter 4; the simpler FG-SET submodel is presented in Appendix B.

\section{Ultimate Yields and Compositions}

In the zero-dimensional submodel, the ultimate char, tar, and gas yields and composition are determined by integrating the set of differential equations, from time zero to infinity (taken as a suitably large value). After the integration, the ultimate yields, $\omega_{c}^{\infty}, \omega_{t}^{\infty}$, and $\omega_{g}^{\infty}$, are readily obtained using the following expressions:

$$
\begin{gathered}
\omega_{t}^{\infty}=\sum_{i=1}^{n f g} W_{T a r, i}^{\infty} \\
\omega_{g}^{\infty}=\sum_{i=1}^{n f g} W_{g a s, i}^{\infty} \\
\omega_{c}^{\infty}=\sum_{i=1}^{n f g} y_{i}^{\infty}
\end{gathered}
$$

The ultimate volatiles yield, $\omega_{v}^{\infty}$, is by definition sum of the tar and the gas ultimate yields, $\omega_{t}^{\infty}$ and $\omega_{g}^{\infty}$, and thus from Eqns. 2.12 and 2.13:

$$
\omega_{v}^{\infty}=\sum_{i=1}^{n f g}\left(W_{g a s, i}^{\infty}+W_{T a r, i}^{\infty}\right)
$$

The compositions of the devolatilized tar, gas and residual char are then determined by the following expressions: 


$$
\begin{aligned}
& \chi_{g a s, j}=\sum_{i=1}^{n f g} W_{g a s, i}^{\infty} \chi_{F G, i, j} \\
& \chi_{T a r, j}=\sum_{i=1}^{n f g} W_{T a r, i}^{\infty} \chi_{F G, i, j} \\
& \chi_{c h a r, j}=\sum_{i=1}^{n f g} y_{i}^{\infty} \chi_{F G, i, j}
\end{aligned}
$$

where $\chi_{F G, i, j}$ is the mass fraction of the $j$-th element in the $i$-th functional group.

\section{Oxidation and Gasification}

Oxidation and gasification in FBED-0 are treated by specifying the total burnout and accounting for devolatilization. The elemental mass from the residual char (i.e., after devolatilization) is released to the gas phase, and the composition and temperature are then determined assuming total equilibrium. The sensitivity of temperature and composition to specified burnout is discussed further by Hobbs et al., (1992b). The paper by Hobbs et al., (1992b) is included in this manual as Appendix C. If no estimate is available for burnout, a value of unity (i.e., $100 \%$ burnout) is recommended. Long solid residence times and high oxygen concentrations and temperatures in the equilibrium zone justify this assumption for fixed-beds.

\section{Freeboard}

The freeboard is the void space directly above the coal bed. The two-zone submodel predicts the temperature at the top of the coal bed, which is greater than 
the temperature at the sampling point due to heat loss in the water-cooled freeboard. The freeboard temperature, $T_{f b}(\mathrm{~K})$, can be determined by doing an energy balance around the freeboard zone and accounting for heat loss in this section, $Q_{f b}$ (watts), as follows:

$$
T_{f b}=T_{e x i t}+\frac{Q_{f b}}{\dot{m}_{g a s} C p_{g a s}}
$$

where $T_{\text {exit }}(\mathrm{K}), \dot{m}_{g a s}(\mathrm{~kg} / \mathrm{s})$, and $C p_{\text {gas }}(\mathrm{J} / \mathrm{kgK})$ are the exit temperature, exit gas mass flow rate, and exit gas heat capacity, respectively. The gas heat capacity is computed at the freeboard temperature and requires an iterative procedure. The freeboard temperature is the temperature usually reported in the literature. For the air-blown, Wellman-Galusha gasifier, the temperature drop from the bed top to the sampling point is on the order of $10-20 \mathrm{~K}$. Quantitative agreement between measured exit temperature and predicted exit temperature is not expected, since the zero-dimensional assumption gives an average temperature in the drying and devolatilization zone that is significantly higher than the exit temperature. However qualitative comparisons are possible and have been discussed by Hobbs et al., (1992b).

\section{Summary}

A zero-dimensional submodel, FBED-0, has been developed and discussed. The motivation for developing the two-zone fixed-bed submodel was to provide an initial estimate of effluent compositions and temperatures for the one-dimensional, fixed-bed model, which is discussed in detail in chapter 3. This two-zone, partial equilibrium submodel uses an advanced devolatilization submodel and a partial equilibrium gas phase submodel to predict effluent temperature and composition. Effluent composition is determined for all the major species and a number of minor species. Tar recycle is taken into account. The key assumption for the two-zone submodel is that oxidation and gasification occur at relatively high temperature compared to the colder drying and devolatilization zone. The temperature difference provides a natural division of the process into two zones. High temperature in the 
oxidation and gasification zone favors total equilibrium in the gas phase. Lower temperature in the drying and devolatilization zone favors partial equilibrium. The limitations to the two-zone submodel are a need to specify burnout and uncertainty in wall heat loss estimates. The solution method to solve the equations for FBED-0 is discussed in Chapter 5. 


\section{CHAPTER 3}

\section{ONE-DIMENSIONAL FIXED-BED MODEL}

The zero-dimensional submodel described in Chapter 2 can be used to estimate the temperature and composition of the product gas and the product tar. However, in order to design a gasifier or analyze the performance of an existing gasifier, additional information, such as temperature and composition profiles in the gasifier bed, is needed. Information such as peak solid temperature may also be needed to design a specific type of gasifier, e.g., dry-ash gasifier or a slagging gasifier. In order to predict these desired quantities, a one-dimensional fixed-bed model, FBED-1, is presented and discussed in this chapter. FBED-1 can predict axial solid and gas temperatures, species concentrations, pressure, char consumption rate, particle size and number density, gas and solid flow rates, residence times, heating rates, velocities, and wall heat loss. These predictive capabilities of FBED-1 make it a potentially important tool for design and analysis of coal gasification units. FBED-1 is a one-dimensional, steady-state model that considers separate gas and solid temperatures, axially variable solid and gas velocities, variable bed void fraction, devolatilization based on FG-DVC submodel, coal drying, oxidation and gasification of char with an ash layer, and gas composition based on either total or partial equilibrium in the gas phase. Input data include reactor dimensions, operating conditions, inlet solid and gas temperatures, pressure, concentrations, flow rates and wall temperature. The output data, in addition to the quantities listed above, include auxiliary quantities such as film, diffusion and chemical resistances, gas phase transport properties, and heat and mass transfer coefficients. The FBED-1 model is described by a system of 191 coupled, ordinary differential equations. These equations are integrated using the LSODE (Livermore Solver for Ordinary Differential Equations) package. The zerodimensional submodel presented in Chapter 2 is used to provide the initial effluent 
One-Dimensional Fixed-Bed Model

gas properties needed to perform the integration of the system of equations for the one-dimensional model.

\section{Foundations and Assumptions}

The conservation equations for mass and energy form the foundation of the FBED-1 model. The gas and solid phase equations are coupled through the source terms. These source terms account for the release of mass from the solid phase to the gas phase, and energy exchange between the two phases. The source terms in the continuity and energy equations are described by various physical and chemical submodels. Plug flow is assumed in both the solid and the gas phase with variable axial velocities. Gas phase pressure drop is calculated with the Ergun equation for packed beds (Ergun, 1952). An effective heat transfer coefficient is used for heat loss to the wall, including both stagnant and dynamic contributions, as well as conduction and diffusive radiation. Drying and devolatilization are allowed to occur simultaneously with char oxidation and gasification. Oxidation and gasification of char are described by either shell progressive or a shrinking core ash segregation submodel. Chemical equilibrium is assumed to calculate gas concentrations and temperature. Turbulence is not treated formally in the slowly moving bed with low gas velocities, but is included implicitly through correlations such as for the effective heat transfer coefficient. Primary assumptions employed in the development of the one-dimensional fixed-bed model (FBED-1) are listed in Table 3.1 .

The basis and justifications of these assumptions are now discussed. The one-dimensional assumption can be justified by following the development of Yoon et al., (1978). The fixed-bed reactor may consist of an adiabatic core and a boundary layer. The boundary layer thickness, $\ell(m)$, can be calculated from the following equation (Carslaw and Jaeger, 1959):

$$
\ell=\sqrt{\frac{k_{e} \tau}{\rho C_{p}}}
$$


Chapter 3

Table 3.1 Primary assumptions for the one-dimensional, fixed-bed model (FBED-1)

1. The moving-bed is one-dimensional.

2. Interdiffusion (diffusional transport of kinetic energy and enthalpy) is small compared to chemical reactions.

3. Both particles and gases are treated as a continuum.

4. Pressure is uniform at control surfaces surrounding particles.

5. Viscous heating is negligible.

6. Aerodynamic drag is negligible.

7. Conduction, radiation and convection to the wall are combined in an effective bulk heat transfer term.

8. Work performed by moving particles is negligible.

9. Work due to body forces is small compared to chemical reaction terms.

10. Work associated with particle volume changes is small.

11. Soret and Dufour effects are negligible.

12. Axial diffusion is negligible.

13. The ideal gas law is valid.

14. PV-work is negligible.

15. Viscous dissipation is negligible.

16. Turbulence effects are implicitly included in the effective heat transfer correlation.

17. The solid phase internal energy is equal to the solid phase enthalpy.

18. Particles are isothermal.

19. Feed coal is represented by a single type, and particles are assumed to be of uniform size.

where $k_{e}, \tau, \rho$, and $C p$ represent the effective radial thermal conductivity $(w / m K)$, residence time of the coal in the gasifier $(s)$, apparent density of the coal $\left(\mathrm{kg} / \mathrm{m}^{3}\right)$, and heat capacity of the coal $(\mathrm{J} / \mathrm{kg} \mathrm{K})$, respectively. Hobbs (1990) estimated the boundary layer thickness in a Wellman-Galusha gasifier to be approximately $0.07 \mathrm{~m}$, which when compared to the reactor diameter $(2 \mathrm{~m})$, justifies the one-dimensional assumption. However, two-dimensional models may be necessary to investigate wall effects or details near the grate.

The ideal gas assumption is justified because of relatively low pressure operation and high temperatures. The compressibility factor is essentially unity for conditions applying to both the Lurgi and Wellman-Galusha gasifiers; this justifies the use of the ideal gas law in the fixed-bed model. 
One-Dimensional Fixed-Bed Model

Yu (1981) discusses the steady-state operation of a fixed-bed gasifier at partial load. As the coal feed rate is reduced, the relative importance of convection to dispersion is lessened. Yu's calculation with and without axial dispersion indicates that when the coal feed rate is less than ten percent of full load, axial dispersion of heat is important and should be considered. However, mass dispersion is small for all conditions and can be neglected. Axial dispersion at low coal flow rates affects the predicted temperature profile by moving the profile axially and decreasing the temperature peak slightly. Young and Finlayson (1976) give the following criteria for neglecting axial diffusion:

$$
\begin{gathered}
\frac{d_{p} M_{w}}{P e_{m}^{a} \rho_{g} u_{z}} \sum_{i} \sum_{j} r_{j} \alpha_{i j} \ll 1 \\
\frac{d_{p}}{P e_{h}^{a}\left(H_{g}-H_{s}\right)} \sum_{j}\left(-\Delta H_{j}\right) r_{j} \ll 1
\end{gathered}
$$

where $d_{p}, M_{w}, P e_{a}^{m}, P e_{h}^{a}, \rho_{g}, u_{z}, r_{j}, \alpha_{i j}, H_{g}, H_{s}$, and $\Delta H_{j}$ represent the particle diameter, particle molecular weight, axial mass Peclet number, axial thermal Peclet number, gas density, gas velocity, reaction rate, stoichiometric coefficient for the $j$-th reaction, convective heat flux of gas and solid, and the $j$-th reaction enthalpy, respectively. Denn et al., (1982) give the magnitude of the expressions in Equations 3.2 and 3.3 as 0.0001 for fixed-bed reactors operating under full load. Denn et al., (1982) also show that axial thermal dispersion is equal to axial thermal convection at a flow rate of $1 \%$ of the normal load. Denn further states that axial mass dispersion is not important even at $1 \%$ of normal load. However, axial dispersion of heat is significant when the coal feed rate is less than $10 \%$ of full load (Yu, 1981). Negligible axial diffusion also implies negligible Dufour and Soret effects.

The importance of pressure-volume work (PV-work) can be shown by comparing the term $v_{z} \frac{\partial P}{\partial z}$ with its counterpart $\rho_{g} C p_{g} v_{z} \frac{\partial T}{\partial z}$ (Khanna and Seinfeld, 1987): 


$$
\frac{v_{z} \frac{\partial P}{\partial z}}{\rho_{g} C p_{g} v_{z} \frac{\partial T}{\partial z}}=\frac{\Delta P}{\rho_{g} C p_{g} \Delta T} \ll 1
$$

PV-work can be neglected if Equation (3.4) is satisfied. For a dry-ash Lurgi gasifier at typical operating conditions, the quantity $\frac{\Delta P}{\rho_{g} C p_{g} \Delta T}$ is approximately 0.0001, which justifies neglecting PV-work (Hobbs, 1990).

In FBED-1, multidimensional effects such as viscous dissipation and turbulence are included implicitly via correlations such as for the heat transfer and mass transfer coefficients.

\section{Conservation Equations}

The conservation equations for the one-dimensional, fixed-bed model are listed in Table 3.2. These equations are classified as gas and solid overall continuity, gas and solid energy equations, and gas and solid species or elemental continuity equations. The constitutive relations for solid flow have been only proposed recently, and no solution for these equations has been attempted (Gray and Stiles, 1988). Thus, only differential equations for continuity and energy are treated in the model described herein. Table 3.2 shows only the conservation equations for gas and solid phase. Remaining equations are related to the devolatilization and tar cracking submodels and will be discussed later.

The derivation of the two phase conservation equations can be found in Crowe and Smoot (1979). The conservation equations in Table 3.2 show reaction source terms that represent drying, devolatilization, char oxidation and gasification. These chemical and physical processes are shown in Figure 3.1, which depicts a conceptual coal particle divided into various functional groups, including moisture and ash. Drying is assumed to be diffusion-limited, and devolatilization is 
One-Dimensional Fixed-Bed Model

Table 3.2 Conservation Equations for FBED-1

Overall Gas Continuity

Overall Solid Continuity

Gas Phase Energy

Solid Phase Energy

Gas Phase Species Continuity

Gas Phase Elemental Continuity

Overall Tar Continuity

Tar Elemental Continuity

$$
\frac{d W_{g}}{d z}=A \sum_{i=1}^{6} r_{i}
$$

$$
\frac{d W_{s} h_{s}}{d z}=A\left(-Q_{s g}-Q_{s w}-\sum_{i=1}^{6} r_{i} h_{g, i}\right)
$$

$$
\frac{d W_{g, j}}{d z}=A \sum_{i=1}^{6} r_{i, j}^{g a s}
$$

$$
\frac{d W_{t a r}}{d z}=A r_{d}^{\operatorname{tar}}
$$

$$
\frac{d W_{\text {tar }, j}}{d z}=A r_{d, j}^{t a r}
$$

\section{Moisture Continuity}

$$
\frac{d W_{\text {moisture }}}{d z}=-A r_{\text {drying }}
$$

1. Equations 39-164 describe the FG-DVC devolatilization submodel presented in Chapter 4.

2. Equations $165-191$ describe the lower bound of the distribution function for the gas phase tar cracking reactions and follow the FG-DVC formulation.

3. Equations 5-26 are solved only when the gas pbase is not considered to be in chemical equilibrium.

4. $i=1-6$, represents drying, devolatilization, $\mathrm{CO}_{2}, \mathrm{H}_{2}, \mathrm{H}_{2} \mathrm{O}$ gasification, and oxidation reactions respectively.

5. $j=1-5$ represents elements $\mathrm{C}, \mathrm{H}, \mathrm{O}, \mathrm{N}$, and $\mathrm{S}$ respectively.

6 . $l=1-22$ represents 22 gaseous species considered in FBED-1. 


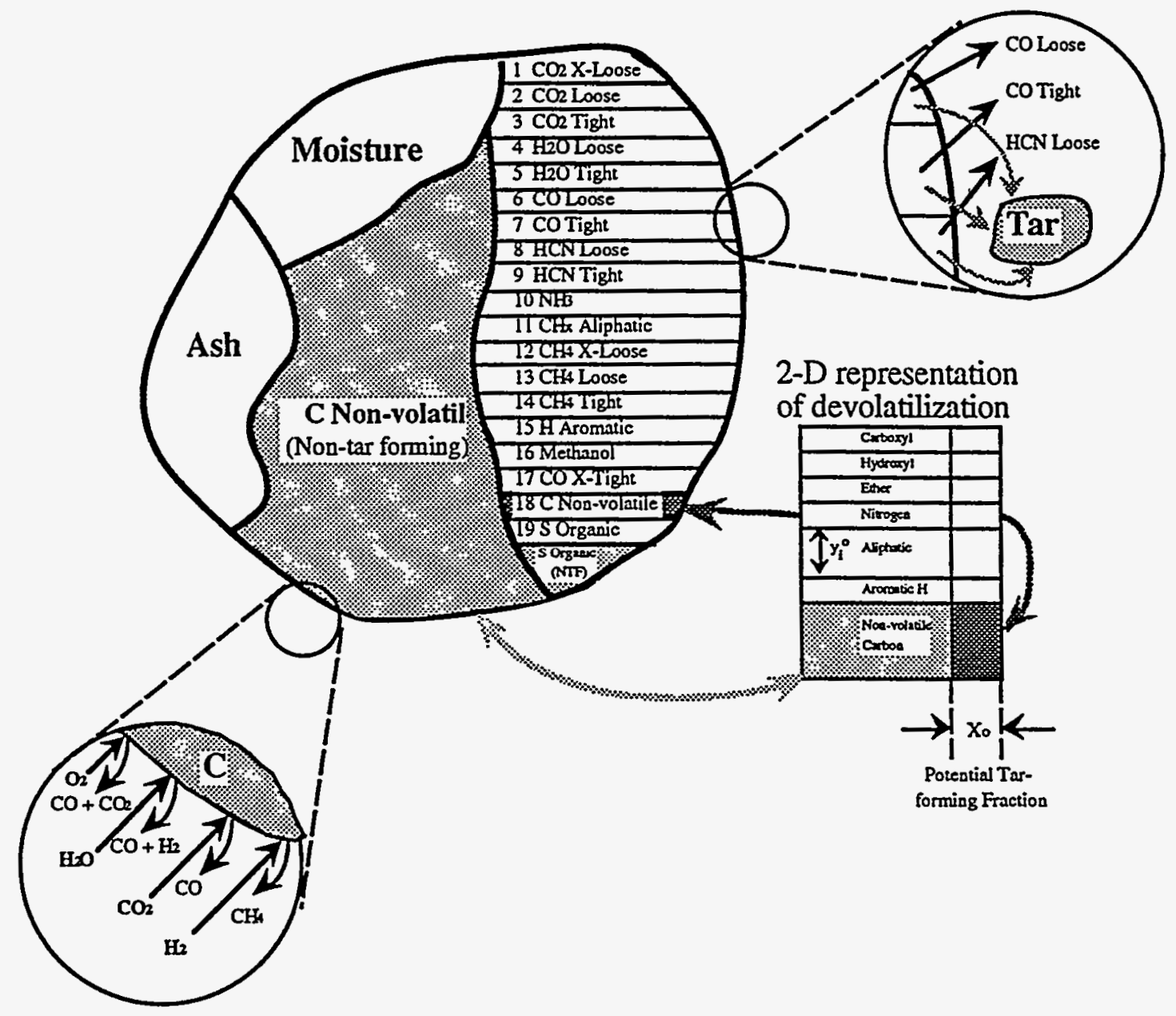

Figure 3.1. Schematic of a coal particle with FG-SET devolatilization model based on chemical functional groups (Solomon et al., 1988).

described by assuming that the organic portion of the coal particle is composed of various functional groups such as carboxyl, hydroxyl, ether, nitrogen, etc. A functional group model (FG model) has been used to describe the evolution of light gases from the char, and the evolution of tar is based on DVC submodel (Solomon and Hamblen, 1985; Serio et al., 1987; and Solomon et al., 1988). The coal 
One-Dimensional Fixed-Bed Model

dependent kinetics is used for the FG-DVC submodel, which is discussed in Chapter 4. A simpler devolatilization submodel, FG-SET, also provided as an option, is discussed in Appendix B.

Oxidation and gasification reactions act on all functional groups. As shown in Figure 3.1, three gasification agents are considered: steam, carbon dioxide, and hydrogen. The volatile functional groups can competitively evolve as either light gases or tar. In FBED-1, tar and char are treated as single species which have a variable composition, depending on the location in the reactor.

Gas temperature is determined by assuming all gas species to be in thermal equilibrium. Gas phase composition is determined by Gibbs free energy minimization. Solid temperature is determined from the solid enthalpy and the elemental composition of the char. All gas phase transport properties (conductivity, viscosity, diffusivity, etc.) are considered to be functions of both temperature and composition.

\section{Overall Gas and Solid Continuity Equations}

The overall gas and solid species continuity equations are given by Equations 1 and 2 in Table 3.2. The gas and solid flow rates are represented by $W_{g}$ $(\mathrm{kg} / \mathrm{s})$ and $W_{s}(\mathrm{~kg} / \mathrm{s})$, respectively. The axial distance and cross sectional areas are represented by $z(m)$ and $A\left(m^{2}\right)$, respectively. The volumetric reaction rate is represented by $r_{i}\left(\mathrm{~kg} / \mathrm{m}^{3} \mathrm{~s}\right)$, where $i$ depicts the different processes and heterogeneous reactions, such as drying, devolatilization, char gasification, and char oxidation. Summation of Equations 1 and 2 in Table 3.2 results in the overall continuity equation, with a zero source term that represents conservation of mass. Reaction rates are written as the volumetric rate of mass addition to the gas phase; thus a positive rate signifies a source of mass for the gas phase and a sink for the particle phase. The submodels used to determine these source terms are discussed later in this chapter.

\section{Gas and Solid Energy Equations}

The gas and solid energy equations are given by Equations 3 and 4 in Table 3.2. The total gas and solid enthalpies are represented by $h_{g}(J / k g)$ and $h_{s}(J / k g)$. 
The energy exchange between solid and gas is represented by $Q_{s g}\left(w / m^{3}\right)$. The heat losses to the wall from the gas and solid phases are represented by $Q_{g w}\left(w / m^{3}\right)$ and $Q_{s w}\left(w / m^{3}\right)$, respectively. Calculation of $Q_{s g}, Q_{g w}$, and $Q_{s w}$ will be discussed later. The last term in the energy equations represents the energy exchange due to the coal decomposition processes i.e., drying, devolatilization, and heterogeneous reactions of char with $\mathrm{CO}_{2}, \mathrm{H}_{2}, \mathrm{H}_{2} \mathrm{O}$ and $\mathrm{O}_{2}$. A discussion of these equations is presented in later sections.

Heat of Vaporization - The heat of vaporization accounts for the energy exchange due to drying of the feed coal. This term is calculated as

$$
h_{\text {total }}^{\text {vap }}=h_{f}^{o}+h_{\text {vap }}\left(T_{s}\right)+h_{s}\left(T_{s}\right)
$$

where $h_{f}^{o}$ is heat of formation of $\mathrm{H}_{2} \mathrm{O}, h_{\text {vap }}\left(T_{s}\right)$ is the heat of vaporization of water at the solid temperature, and $h_{s}\left(T_{s}\right)$ is the sensible heat from the reference temperature to the solid temperature.

Heat of Devolatilization - The energy exchange due to devolatilization is computed following the model proposed by Merrick (1983). The Merrick's model, which considers 9 species, including tar, was extended to accommodate 27 functional groups of the FG-DVC devolatilization submodel. The resulting equations are

$$
\frac{d h_{\text {devel }}}{d t}=\sum_{k=1}^{n f g+1} \frac{\partial q}{\partial m_{k}} \frac{d m_{k}}{d t} \quad(W / k g)
$$

where

$$
\frac{\partial q}{\partial m_{k}}=\sum_{j=l}^{5} A_{j k}\left(\lambda_{j}-\Phi_{j}+\frac{f(\bar{T})}{\mu_{i}}\right)-h_{k}
$$




$$
\begin{gathered}
\frac{d m_{k}}{d t}=r_{d, k}^{g a s} \quad(k=1, \ldots, n f g) \\
\frac{d m_{n f g+1}}{d t}=\sum_{k=1}^{n f g} r_{d, k}^{t a r}
\end{gathered}
$$

Heat of Reactions - The heat of reaction for the gasification and oxidation reactions of char can be calculated by performing an energy balance around the particle/gas interface. The location within the particle and the temperature at which the reaction occurs must be assumed. The energy exchange at the solid-to-gas interface due to transport of mass between the two phases during heterogeneous oxidation is shown in Figure 3.2. Solid conduction and solid-to-gas energy exchange are not shown. Since the products of heterogeneous reactions leave at the solid temperature, the energy exchange due to these reactions can be viewed as a nonisothermal heat of reaction. The enthalpy terms shown in Figure 3.2 are total enthalpies at the indicated temperature. Furthermore, the reaction rates are based on the given species and stoichiometry of the given reaction. A sample calculation of the enthalpy associated with the term $r_{i} h_{g, i}$ for the oxidation reaction is given in Table 3.3. The heats of reactions for the gasification reactions can be calculated in a similar manner.

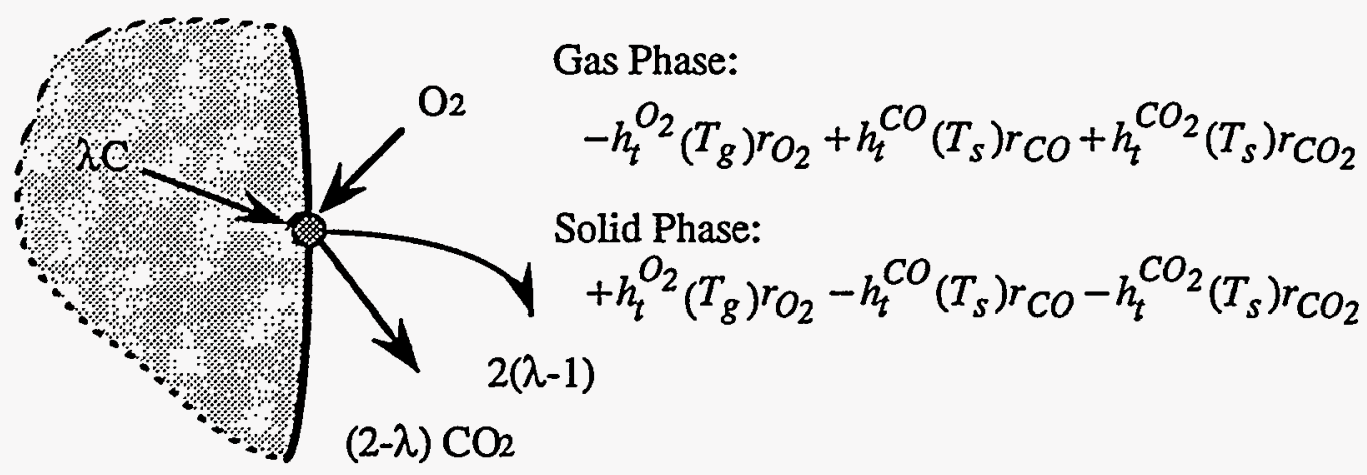

Figure 3.2. Energy exchange due to the transport of mass between solid and gas phase due to oxidation. 
Table 3.3 Enthalpy exchange associated with the oxidation reaction.

Oxidation Reaction:

$$
\lambda \mathrm{C}+\mathrm{O}_{2} \rightarrow 2(\lambda-1) \mathrm{CO}+(2-\lambda) \mathrm{CO}_{2}
$$

Formation Enthalpies:§

$$
\begin{aligned}
& h_{f}^{C O}, \frac{J}{k g(\text { carbon })}=-9.203 \times 10^{6} \frac{2(\lambda-1)}{\lambda} \\
& h_{f}^{C O_{2}}, \frac{J}{k g(\text { carbon })}=-3.276 \times 10^{7} \frac{(2-\lambda)}{\lambda} \\
& h_{f}^{O_{2}}, \frac{J}{k g(\text { carbon })}=0.000
\end{aligned}
$$

Sensible Enthalpies:‡

$$
\begin{aligned}
& h_{s}^{C O}\left(T_{s}\right), \frac{J}{k g(\text { carbon })}= \\
& 83.25\left[\frac{2(\lambda-1)}{\lambda}\right]\left[28.41\left(T_{s}-298\right)+0.0041\left(T_{s}^{2}-298^{2}\right)+4.6 \times 10^{4}\left(\frac{1}{T_{s}}-\frac{1}{298}\right)\right] \\
& h_{s}^{C O_{2}}\left(T_{s}\right), \frac{J}{k g(\text { carbon })}= \\
& 83.25 \frac{2-\lambda}{\lambda}\left[44.22\left(T_{s}-298\right)+0.00879\left(T_{s}^{2}-298^{2}\right)+8.62 \times 10^{5}\left(\frac{1}{T_{s}}-\frac{1}{298}\right)\right] \\
& h_{s}^{O_{2}}\left(T_{g}\right), \frac{J}{k g(\text { carbon })}= \\
& \frac{83.25}{\lambda}\left[29.96\left(T_{g}-298\right)+0.00418\left(T_{g}^{2}-298^{2}\right)+1.67 \times 10^{5}\left(\frac{1}{T_{g}}-\frac{1}{298}\right)\right]
\end{aligned}
$$


One-Dimensional Fixed-Bed Model

Table 3.3 Enthalpy exchange associated with the oxidation reaction (continued).

Enthalpy Exchange due to oxidation:

$r_{i} h_{g, i}, \frac{J}{m^{3} s}=r_{O_{2}}\left[\left(h_{f}^{C O}+h_{s}^{C O}\right)+\left(h_{f}^{C O_{2}}+h_{s}^{C O_{2}}\right)-\left(h_{f}^{O_{2}}+h_{s}^{O_{2}}\right)\right], \quad i=$ oxidation

$\S$ Formation enthalpies are from Laidler and Meiser (1982, page 66). Units for the heat of formation are on carbon basis. For example, the heat of formation for $\mathrm{CO}$ can be calculated as follows:

$$
-9.203 \times 10^{6} \frac{2(\lambda-I)}{\lambda}=\left(-110.54 \frac{K J}{\operatorname{molCO}}\right)\left(\frac{1000 J}{K J}\right)\left[\frac{2(\lambda-1) \text { molCO}}{\lambda \text { molC }}\right]\left(\frac{\text { molC }}{12.01115 g C}\right)\left(\frac{1000 g C}{k g C}\right)
$$

¥ Sensible enthalpies are taken from Laidler and Meiser (1982, page 63) by integration of gas heat capacity.

\section{Gas Phase Elemental Continuity Equations}

The mass rate of change of each reactive element in the gas phase is given by Equations 27-31 in Table 3.2. These elements include $\mathrm{C}, \mathrm{H}, \mathrm{O}, \mathrm{N}$, and $\mathrm{S}$. Elements related to inert species such as argon do not take part in any reaction, thus eliminating the need for a differential equation. The term $r_{i, j}$ represent the volumetric evolution rate of the $j-$ th element contributed by the $i$-th reaction. These reactions include drying, devolatilization, and heterogeneous oxidation and gasification reactions of char; these are discussed later in this chapter. The gas phase elemental continuity equations include the contribution of tar.

\section{Overall and Elemental Continuity Equations for Tar}

Tar, which is part of the gas phase, is treated as a pseudospecies in FBED-1. The use of the advanced devolatilization submodel makes tracking of tar throughout the reactor bed possible. Tar can be either assumed to be nonreactive in the gas phase, which would classify the one-dimensional model as a partial equilibrium model, or assumed to react to completion in the gas phase. The evolution of tar is based on either FG-DVC or FG-SET submodel; these are presented in Chapter 4 and Appendix B respectively. The overall tar continuity is 
Chapter 3

given by Equation 32; its elemental continuity is given by Equations 33-37 of Table 3.2 .

\section{Solid Species Continuity Equations}

The solid particle is represented as being composed of moisture, ash, and organic functional groups, as depicted in Figure 3.1. Ash is considered inert and does not require a differential equation. Moisture is released during the drying of the coal particles; the equations governing the drying process have been discussed previously. The composition of the char is updated from the instantaneous functional group composition of the char. It should be noted that the functional groups are depleted by devolatilization and gasification, as well as by oxidation reactions.

\section{Pressure Drop}

The pressure drop in the reactor is calculated from the Ergun's equation (Ergun, 1952):

$$
\begin{gathered}
-\Delta P_{t}=\frac{f L G^{2}}{g \rho_{g} d_{p}} \\
f=\frac{1-\varepsilon}{\varepsilon^{3}}\left(1.75+150 \frac{1-\varepsilon}{R e}\right) \text { for } \frac{R E}{1-\varepsilon}<500
\end{gathered}
$$

where $P_{\imath}, g, \rho_{g}, d_{p}, L, G$, and $f$ represent total pressure $\left(\mathrm{kg}\right.$ force $\left./ \mathrm{m}^{2}\right)$, acceleration due to gravity $\left(9.81 \mathrm{~m} / \mathrm{s}^{2}\right)$, gas density $\left(\mathrm{kg} / \mathrm{m}^{3}\right)$, particle diameter $(\mathrm{m})$, reactor length $(\mathrm{m})$, superficial gas mass flux $\left(\mathrm{kg} / \mathrm{m}^{2} \mathrm{~s}\right)$, and friction factor, respectively. Ergun's equation is used to determine pressure drop, since Reynolds numbers are typically less than 500 in fixed-bed coal gasifiers. Although the pressure drop in a fixed-bed reactor is relatively small, it is sensitive to the bed void fraction, $\varepsilon$, as 
One-Dimensional Fixed-Bed Model

shown in Equations 3.10 and 3.11, and can be used to determine if the bed void fraction distribution is appropriate.

\section{Gas Phase Chemistry}

The composition and temperature of the gas phase are determined by total or partial equilibrium submodels. In the total equilibrium submodel, all gaseous species, including tar, are assumed to be in chemical and thermal equilibrium. For the partial equilibrium option, tar is held out of chemical equilibrium. In this case, the remaining gaseous species are taken to be in equilibrium. However, an option is provided to prevent the gases from reacting to equilibrium below a user specified temperature. This option is mainly exercised during drying and devolatilization when the gas phase temperature is low. Gas phase kinetics would be required to hold certain gas species out of equilibrium in the high temperature region. For all these options, all species, including tar, are considered to be in thermal equilibrium. Equations 5-26 (Table 3.2) are solved only when the gas phase is assumed to be out of chemical equilibrium. Otherwise, the gas phase species concentrations are computed from the equilibrium submodel.

\section{Heat and Mass Transport}

Mass and heat transfer processes in fixed-bed gasifiers are affected by complex solid flow and chemical reactions. Coarsely crushed coal settles while undergoing heating, drying, devolatilization, gasification and combustion. Coal particles change in diameter, shape and porosity. Nonideal behavior may result from coal bridges, gas bubbles and channels. Variable bed void fraction may also change heat and mass transport properties. Steep temperature gradients, typical to fixed-bed gasification and oxidation of coal, make the use of correlations for solidto-gas heat transfer coefficients uncertain, since these are typically obtained under ideal conditions. Mass transfer occurs by diffusion and convection. The modes of heat transfer are conduction, convection and radiation for both the gas and solid phases. 


\section{Transport and Thermodynamic Properties}

Several physical properties for the gas and particle phases are required to obtain mass and heat transfer coefficients needed to solve the system of differential equation set given in Table 3.2. Chapman-Enskog theory has been used to calculate multicomponent gas mixture viscosity and diffusivity (Bird et al., 1960). The equations used to determine the gas species and mixture conductivity, viscosity, and diffusivity are given in Table 3.4. The particle is assumed to swell linearly with the extent of devolatilization as given by Equation 9 of Table 3.4. The particle diameter and number density are discussed later in this chapter.

Euken's formula is used to calculate the conductivity of individual gaseous species, as given by Equation 1, Table 3.4. The multicomponent gas viscosity is calculated from Equation 2, as given in Table 3.4. The gas species viscosity given by Equation 3 of Table 3.4 is based on the viscosity of a pure monatomic gas. The Stockmayer collision diameter, $\sigma$, and the collision integral for viscosity which describes the deviation from rigid sphere behavior, $\Omega_{\mathrm{m}}$, are computed from the relations of Neufield with Brokaw's correction (1969). The interaction parameter is obtained from Equation 5 of Table 3.4.

The effective mass diffusivity for a single gaseous species $i$ through the gas mixture is given by Equation 8 of Table 3.4. This formulation assumes that each species in the mixture moves with the same velocity. The species mass diffusivities are calculated using the Chapman-Enskog formula given by Equation 6 of Table 3.4. The collision integral for diffusion, $\Omega_{\mathrm{d}}$, is a dimensionless function of the temperature and of the intermolecular potential field for species $i$ and $k$. This function can be approximated by the Lennard-Jones function (Bird et al., 1960). This requires a suitable estimate of the Lennard-Jones parameters $\sigma_{i k}$ and $\varepsilon_{i k}$ from the individual species parameters $\sigma_{\mathbf{i}}$ and $\varepsilon_{\mathbf{i}}$. These interspecies parameters are computed as $\sigma_{i k}=0.5\left(\sigma_{i}+\sigma_{k}\right)$ and $\varepsilon_{i k}=\sqrt{\varepsilon_{i} \varepsilon_{k}}$. Furthermore, the diffusivity of gaseous tar is assumed to be $0.1 \mathrm{~cm}^{2} / \mathrm{s}$ at standard temperature and pressure following Suuberg et al., (1979). The pressure and temperature dependencies for 
Table 3.4 Physical property equations used in the one-dimensional fixed-bed model. $\$$

Gas species conductivity

$$
k_{i}=\left(C p_{i}+\frac{5 R}{4 M w_{i}}\right) \mu_{i}
$$

Gas mixture conductivity

$$
k_{g}=\sum_{i=1}^{n} \frac{x_{i} k_{i}}{\sum_{j=l}^{n} x_{j} \Phi_{i j}}
$$

Gas species viscosity

$$
\mu_{i}=2.6693 \times 10^{-5} \frac{\sqrt{M w_{i} T_{g}}}{\sigma_{i}^{2} \Omega_{m}^{2}}
$$

Gas mixture viscosity

$$
\mu_{g}=\sum_{i=1}^{n} \frac{x_{i} \mu_{i}}{\sum_{j=1}^{n} x_{j} \Phi_{i j}}
$$

Interaction parameter

$$
\begin{aligned}
\Phi_{i j} & =\frac{1}{\sqrt{8}}\left(1+\frac{M w_{i}}{M w_{j}}\right)^{-\frac{1}{2}}\left[1+\left(\frac{\mu_{i}}{\mu_{j}}\right)^{\frac{1}{2}}\left(\frac{M w_{j}}{M w_{i}}\right)^{\frac{1}{4}}\right]^{2} \\
D_{i j} & =0.0018583 \frac{\sqrt{T_{g}^{3}\left(\frac{1}{M w_{i}}+\frac{1}{M w_{j}}\right)}}{P \sigma_{i j}^{2} \Omega_{d, i j}}
\end{aligned}
$$

Species diffusivity

Tar diffusivity

$$
D_{\text {tar }}=0.1\left(\frac{T}{273}\right)^{1.5}\left(\frac{I}{P}\right)
$$

Effective species diffusivity

$$
\begin{aligned}
& D_{i m}=\frac{I-x_{i}}{\sum_{j \neq i} \frac{x_{j}}{D_{i j}}} \\
& d=d^{o}\left[I+\gamma\left(\frac{v^{\infty}-v}{v^{\infty}}\right)\right]
\end{aligned}
$$

$$
A_{p}=\pi d^{2}
$$

PThe physical property equations for all gases except tar are from Bird et al., (1960). The tar diffusivity is calculated following Suuberg et al. (1979). The particle diameter is assumed to swell during devolatilization; and the particle surface area is dependent on the instantaneous particle diameter. 
the tar diffusivity are assumed to be the same as predicted by Chapman-Enskog theory, as shown by Equation 7 of Table 3.4.

The thermodynamic properties of gaseous species are determined from the widely used polynomial expression (Kee et al., 1991) obtained from the regression analysis of the thermochemical data from JANAF tables (Stull and Prophet, 1971). These expressions for the dimensionless heat capacity, enthalpy, and entropy are given below:

$$
\begin{gathered}
\frac{C_{p}}{R}=a_{1}+a_{2} T+a_{3} T^{2}+a_{4} T^{3}+a_{5} T^{4} \\
\frac{H^{o}}{R T}=a_{1}+\frac{a_{2}}{2} T+\frac{a_{3}}{3} T^{2}+\frac{a_{4}}{4} T^{3}+\frac{a_{5}}{5} T^{4}+\frac{a_{6}}{T} \\
\frac{S^{o}}{R}=a_{1} \ln T+a_{2} T+\frac{a_{3}}{2} T^{2}+\frac{a_{4}}{3} T^{3}+\frac{a_{5}}{4} T^{4}+a_{7}
\end{gathered}
$$

where $\mathrm{T}=$ gas temperature $(K)$.

The enthalpies and heat capacities of char and ash are determined as discussed in chapter 2 and summarized below. Dulong's formula (Perry and Chilton, 1973) is used for the heating value of the char. The correlations proposed by Merrick (1983) for char enthalpy and heat capacity are used for computing the enthalpy of char. Finally, either Kirov (Merrick, 1983) or Kopp-Neumann (Mills and Rhine, 1989) relation is used to calculate the heat capacity of ash.

\section{Heat and Mass Transfer Correlations}

The correlations used for the calculation of the heat and mass transport coefficients used in the one-dimensional model are presented in Table 3.5. The 
Table 3.5 Heat and mass transport correlations used in FBED-1.

Bed-to-wall heat transfer coefficient $\quad h_{w}=\frac{2.44 k_{r}^{0}}{D^{4 / 3}}+\frac{0.033 k_{g} \operatorname{Pr} \operatorname{Re}}{d_{p}}$

Gas-to-wall heat transfer coefficient $\quad h_{w}^{g}=\frac{k_{r g}}{k_{r g}+k_{r s}} h_{w}$

Solid-to-wall heat transfer coefficient $\quad h_{w}^{s}=\frac{k_{r s}}{k_{r g}+k_{r s}} h_{w}$

Static effective radial conductivity $k_{r}^{o}=k_{g} \varepsilon\left(1+\frac{\beta d_{p} h_{r v}}{k_{g}}\right)+\frac{\beta k_{g}(I-\varepsilon)}{\left(\frac{1}{\phi}+\frac{h_{r s} d_{p}}{k_{g}}\right)^{-1}+\frac{2}{3 \kappa}}$

Gas effective radial conductivity $k_{r g}=k_{g}\left\{\varepsilon\left(1+\frac{\beta d_{p} h_{r v}}{k_{g}}\right)+\operatorname{Pr} \operatorname{Re}\left[\frac{0.14}{1+46\left(\frac{d_{p}}{D}\right)^{2}}\right]\right\}$

Solid effective radial conductivity $\quad k_{r s}=\frac{\beta k_{g}(1-\varepsilon)}{\left(\frac{1}{\phi}+\frac{h_{r s} d_{p}}{k_{g}}\right)^{-1}+\frac{2}{3 \kappa}}$

Solid conductivity

$$
k_{s}=\left(\frac{\rho_{s}^{t}}{4511}\right)^{3.5} \sqrt{T_{s}}
$$

Void-to-void radiation coefficient

$$
h_{\tau v}=\frac{2.27 \times 10^{-7} T_{g}^{3}}{1+\frac{\varepsilon}{2(1-\varepsilon)}\left(\frac{1-\varepsilon^{\prime}}{\varepsilon^{\prime}}\right)}
$$

Solid radiation coefficient

$$
h_{r s}=2.27 \times 10^{-7}\left(\frac{\varepsilon^{\prime}}{2-\varepsilon^{\prime}}\right) T_{s}^{3}
$$


Table 3.5 Heat and mass transport correlations used in FBED-1 (continued).

Packing parameter $\phi= \begin{cases}\phi_{2}+\left(\phi_{1}-\phi_{2}\right) \frac{\varepsilon-0.260}{0.476-0.260} & \text { if } \varepsilon_{1} \geq \varepsilon \geq \varepsilon_{2} \\ \phi_{1} & \text { if } \varepsilon>\varepsilon_{1}=0.476 \\ \phi_{2} & \text { if } \varepsilon>\varepsilon_{2}=0.260\end{cases}$

Loose packing parameter (for $\varepsilon_{1}$ )

$$
\phi_{1}=\frac{0.3525\left(\frac{\kappa-1}{\kappa}\right)^{2}}{\ln [\kappa-0.5431(\kappa-1)]-\frac{0.4569(\kappa-1)}{\kappa}}-\frac{2}{3 \kappa}
$$

Dense packing parameter (for $\varepsilon_{2}$ )

$$
\phi_{2}=\frac{0.07217\left(\frac{\kappa-1}{\kappa}\right)^{2}}{\ln [\kappa-0.9250(\kappa-1)]-\frac{0.07498(\kappa-1)}{\kappa}}-\frac{2}{3 \kappa}
$$

Solid-to-gas heat transfer coefficient $\quad h_{s g}=\frac{2.06 C p_{g} G}{\varepsilon} R e^{-0.575} \operatorname{Pr}^{-2 / 3}$

Mass transfer coefficient

$$
k_{m}=\frac{2.06 G}{\varepsilon \rho_{g}} R e^{-0.575} S c^{-2 / 3}
$$

Reynolds number

$$
\operatorname{Re}=d_{p} G / \mu_{g}=\rho_{g} d_{p} v_{g} / \mu_{g}
$$

Prandtl number

$$
\operatorname{Pr}=C p_{g} \mu_{g} / k_{g}
$$

Schmidt number

$$
S c_{i}=\mu_{g} / \rho_{g} D_{i m}
$$

Conductivity ratio

$$
\kappa=k_{s} / k_{g}
$$


One-Dimensional Fixed-Bed Model

Table 3.5 Heat and mass transport correlations used in FBED-1 (continued).

\begin{tabular}{lr}
\hline Bed void fraction & \multicolumn{1}{c}{$\varepsilon=$ void volume/bed volume } \\
Emissivity & $\varepsilon^{\prime}=0.85$ \\
\hline \multicolumn{1}{c}{ Units for Table 3.5} & $\mathrm{~J} / \mathrm{s} \mathrm{m}^{2} \mathrm{~K}$ \\
$h_{r s}, h_{r v}, h_{s g}, h_{w}, h_{w}^{g}$, and $h_{w}^{s}$ & $\mathrm{~J} / \mathrm{s} \mathrm{mK}$ \\
$k_{g}, k_{r g}, k_{r s}, k_{r}^{o}$, and $k_{s}$ & $\mathrm{~m} / \mathrm{s}$ \\
$k_{m}$ & $\mathrm{~m}$
\end{tabular}

References for Table $\mathbf{3 . 5}$

Equations (1)-(6), (8,9)

Equations (7)

Equations (10)-(12)

Equations (13)-(14)

Equations (15)-(19)

Equation (20)
Froment and Bischoff (1979)

Merrick (1983)

Kunii and Smith (1960)

Gupta and Thodos (1963)

Definitions

Estimated for coal

effective axial and radial conductivities have been correlated by Yagi et al., (1960) and Bischoff (1962), and by Froment and Bischoff (1979), respectively. Both the axial and radial effective conductivities take into account molecular as well as turbulent contributions. The effective radial conductivities of the gas and solid phases also account for radiation and have been correlated by DeWasch and Froment (1971).

No direct experimental data are available on the gas and the solid phase contributions to the bed-to-wall heat transfer. Therefore, the effective bed-to-wall heat transfer coefficient as well as the gas and solid phase contributions are determined by the correlations suggested by DeWasch and Froment (1971). The heat transfer to the wall is treated following Yagi and Wakao (1959) and Yagi and 
Kunii (1960). The reader is referred to Rohsenow et. al., (1985) for additional information.

The volumetric heat transfer rate from solid-to-gas, $Q_{s g}\left(\mathrm{~J} / \mathrm{m}^{3} \mathrm{~s}\right)$, is computed as

$$
Q_{s g}=\zeta h_{s g} \pi d_{p}^{2} \eta_{p}\left(T_{s}-T_{g}\right)
$$

where $\zeta, h_{s g}\left(\mathrm{~J} / \mathrm{sm}^{2} \mathrm{~K}\right)$, and $\eta_{p}\left(1 / \mathrm{m}^{3}\right)$ represent deviation from nonreactive solid-to-gas heat transfer (ranges from 0.02 to 1.0), the nonreactive solid-to-gas heat transfer coefficient (Equation 13, Table 3.5), and the particle number density, respectively. The volumetric wall heat loss from the solid and gas phase, $Q_{g w}$ and $Q_{s w}\left(\mathrm{~J} / \mathrm{m}^{3} \mathrm{~s}\right)$, can be calculated as follows:

$$
\begin{gathered}
Q_{g w}=\frac{4 h_{w}^{g}}{D}\left(T_{g}-T_{\text {wall }}\right) \\
Q_{s w}=\frac{4 h_{w}^{s}}{D}\left(T_{s}-T_{\text {wall }}\right)
\end{gathered}
$$

where $h_{w}^{g}, h_{w}^{s}, T_{g}, T_{s}, T_{w a l l}$, and $D$ represent the gas-to-wall heat transfer coefficient, solid-to-wall heat transfer coefficient, gas temperature, solid temperature, wall temperature and reactor diameter, respectively. The wall temperature can be varied from the bottom to the top of the reactor.

The mass transport coefficient given in Table 3.5 is used to calculate the film resistance and the effective particle resistance to mass transport. 


\section{Drying}

The chemical submodels are composed of coal drying, devolatilization, char oxidation and gasification, and gas phase chemistry. The drying of coal is based on the diffusion-limited vaporization of moisture from the coal particle, as described by Smoot and Smith (1979):

$$
r_{w}=k_{w m}\left(\rho_{w p}-\rho_{w g}\right)
$$

where $k_{w m}, \rho_{w p}$ and $\rho_{w g}$ represent the moisture mass transfer coefficient, surface moisture concentration, and bulk water concentrations, respectively. Blowing or transpiration effects influence the rates by less than $5 \%$ for large particles at typical operating conditions in fixed-bed coal gasifiers and therefore are assumed negligible (Hobbs et al., 1992a, see Appendix D).

\section{Devolatilization}

In FBED-1 model, devolatilization is based on either the FG-DVC submodel, or the simpler FG-SET submodel. These submodels provide the rates of evolution of light gases, tar, and char. These rates are per unit mass of initial char and can then be converted to the overall coal devolatilization rates using the following expression:

$$
r_{i}= \pm \rho_{s m}^{o}\left(1-\varepsilon^{o}\right)\left(1-\Omega_{a s h}^{o}-\Omega_{m o i s t u r e}^{o}\right) \frac{d \omega_{i,(\text { char }, \text { tar, or gas })}}{d t}
$$

where $\rho_{s m}^{o}$ is the measured apparent density of the feed coal, $\varepsilon^{o}$ is the bed void fraction at the gasifier top, $\Omega_{a s h}^{o}$ and $\Omega_{\text {moisture }}^{o}$ are the proximate ash and moisture 
fractions of the feed coal, and $\omega_{i}$, (char, tar, or gas) is the weight fraction of the $i$-th functional group in the char, tar, or gas.

\section{Oxidation and Gasification}

The two most common char oxidation submodels used in the currently available fixed-bed models are the Shell Progressive model (SP model) and the Ash Segregation model (AS model). The difference between the two models is in the treatment of the ash layer. In the SP model, the ash remains intact and the oxidant is required to diffuse through the film boundary layer and the ash layer. In the AS model, ash is assumed to crumble slowly and fall away from the char particle; the oxidant is required to diffuse through the film boundary layer only. Both these submodels assume global reactions and depend on the external surface area of the particle.

The SP or AS submodel is used to obtain the rates of oxidation and gasification reactions. The rate equation for a single particle is given by Hobbs et al., (1992a):

$$
r_{i}^{0, g}=\frac{A_{p} v_{s} M w_{p} C_{i g}}{\frac{1}{k_{r} \zeta}+\frac{1}{k_{m}}+\frac{1}{k_{e f f}}}
$$

where the resistances in the denominator represent surface reaction, molecular diffusion through the gaseous film, and diffusion through the ash layer. Equation 3.20 neglects the effects of diffusion-induced convective transport and assumes that the reactions are first order in oxidizer concentration. It also explicitly neglects pore diffusion, in which case, these effects are implicitly lumped into $k_{\text {eff. }}$. The diffusional resistance through the ash layer is set to zero if the AS model is used. The quantities $A_{p}, v_{s}, M_{p}, C_{i g}, k_{r}, \zeta, k_{m}$, and $k_{\text {eff }}$ represent the external surface area of the particle, the stoichiometric coefficient to identify the number of moles of product gas per mole of oxidant, char molecular weight, molar concentration of 
One-Dimensional Fixed-Bed Model

oxidizer or gasification agent in the bulk gas phase, Arrhenius chemical reaction rate constant, particle area factor to account for internal surface burning, bulk mass transfer coefficient, and ash layer mass transfer coefficient, respectively. Oxidation and gasification kinetic rate constants are given in Table 3.6. Baxter (1987) used nonlinear analysis to correlate the Arrhenius parameters listed in Table 3.6. The results of the original investigators obtained by linear analysis are also listed.

Oxidation and gasification kinetics data for large particle are limited and not readily available in the open literature. Essenhigh (1981) listed various publications regarding fixed-bed experimental methods used in obtaining kinetic data. Unfortunately, the experimental programs for which data were available primarily involved small particles (Essenhigh, 1981). Although the kinetic parameters given in Table 3.6 were derived from small particle experimentation, they are assumed to be applicable to the oxidation and gasification of large particles.

It is pointed out that several studies were done on large spherical carbon particles (Froberg, 1967; Kurylko, 1969) and might not be directly applicable to coal. However, mass transport may dominate the oxidation and gasification processes for large coal particles, thereby justifying the use of the available kinetic data. Hobbs (1990) reported an order of magnitude analysis showing the film resistance to be ten orders of magnitude higher than the chemical resistance for the oxidation reaction. It is, therefore, concluded that shrinking core models using effective internal diffusion may be adequate for comprehensive fixed-bed modeling.

The last resistance in the denominator of Equation 3.20 can be determined using an effective mass transfer coefficient (Thorsness and Kang, 1984):

$$
\frac{1}{k_{e f f}}=\frac{(1-F) d_{p}}{2 D_{e f f}}
$$

where $F, d_{p}$, and $D_{\text {eff }}$ represent the fraction of original carbon, particle diameter, and effective diffusivity, respectively. Walker et al., (1959) and Laurendeau (1978) discuss methods for calculating effective diffusivities. Park and Edgar (1987) show the effect of a developing ash layer on the burning rate of a sample core of coal. The core burning rate can be predicted by using an effective 
Chapter 3

Table 3.6 Oxidation and gasification kinetic rate constants (after Hedman et al., 1987).

\begin{tabular}{lllll}
\hline \hline$k=A T \cdot \exp \left(\frac{-E}{R T}\right), \frac{m}{s}$ & & & \\
\hline Reaction & $A\left(\frac{m}{s K}\right)$ & $\frac{E}{R}(K)$ & $\begin{array}{c}\text { Source of } \\
\text { Correlation }\end{array}$ & Source of Data \\
\hline & & & & \\
$C+0.50_{2} \rightarrow C O$ & & & & \\
All ranks & & & & \\
HVBA & $2.30 \times 10^{0}$ & $1.11 \times 10^{4}$ & Baxter (1987) & Field et al., (1967) \\
HVBC & $1.03 \times 10^{0}$ & $9.01 \times 10^{3}$ & Baxter (1987) & Goetz et al., (1982) \\
SUBC & $5.00 \times 10^{-1}$ & $6.31 \times 10^{3}$ & Baxter (1987) & Goetz et al., (1982) \\
Lignite A & $1.04 \times 10^{1}$ & $1.12 \times 10^{4}$ & Baxter (1987) & Goetz et al., (1982) \\
HVBA & $1.22 \times 10^{0}$ & $1.03 \times 10^{4}$ & Nsakala et al., (1985) & Nsakala et al., (1985) \\
HVBC & $2.26 \times 10^{0}$ & $1.02 \times 10^{4}$ & Goetz et al., (1982) & Goetz et al., (1982) \\
SUBC & $2.05 \times 10^{0}$ & $8.63 \times 10^{3}$ & Goetz et al., (1982) & Goetz et al., (1982) \\
\hline
\end{tabular}

$\mathrm{C}+\mathrm{CO}_{2} \rightarrow 2 \mathrm{CO}$

\begin{tabular}{lllll} 
All ranks $^{\mathrm{a}}$ & $5.89 \times 10^{2}$ & $2.68 \times 10^{4}$ & Hobbs et al., (1992a) & Goetz et al., (1982) \\
HVBA & $1.16 \times 10^{3}$ & $3.12 \times 10^{4}$ & Baxter (1987) & Goetz et al., (1982) \\
HVBC & $4.89 \times 10^{3}$ & $3.13 \times 10^{4}$ & Baxter (1987) & Goetz et al., (1982) \\
SUBC & $6.19 \times 10^{3}$ & $2.89 \times 10^{4}$ & Baxter (1987) & Goetz et al., (1982) \\
Lignite A & $3.42 \times 10^{0}$ & $1.56 \times 10^{4}$ & Baxter (1987) & Goetz et al., (1982) \\
HVBA & $9.51 \times 10^{1}$ & $2.70 \times 10^{4}$ & Goetz et al., (1982) & Goetz et al., (1982) \\
HVBC & $8.86 \times 10^{2}$ & $2.84 \times 10^{4}$ & Goetz et al., (1982) & Goetz et al., (1982) \\
SUBC & $7.11 \times 10^{1}$ & $2.14 \times 10^{4}$ & Goetz et al., (1982) & Goetz et al., (1982) \\
Lignite A & $4.51 \times 10^{1}$ & $1.99 \times 10^{4}$ & Goetz et al., (1982) & Goetz et al., (1982) \\
\hline
\end{tabular}

a Base case parameters used in sensitivity analysis.

b Baxter's (1987) rate constants were obtained by nonlinear analysis of Fields's et al., (1967) and Goetz' et al., (1982) data.

c Hobb's et al., (1992a) rate constants for all ranks were obtained by averaging Baxter's (1987) rate constants for four coal ranks.

diffusivity based on the molecular diffusivity multiplied by a constant, i.e., $D_{\text {eff }}=\phi D_{m}$. The constant, $\phi$, is based on the porosity of the developing ash layer. 
Thorsness and Kang (1985) have used 0.35 for $\phi$. Wang and Wen (1972) measured the porosity of a fire clay ash which varied from 0.4 to 0.8 . Laurendeau (1978) shows that $\phi$ can be estimated by the ash porosity divided by the tortuosity squared, which is estimated to be 2.0. Using Wang and Wen's values for the ash porosity $(0.4$ to 0.8$), \phi$ should range between 0.2 and 0.4 . However, lower values of ash porosity $(0.06-0.60)$ were determined for ash originating from the Lurgi and Wellman-Galusha gasifiers, indicating a lower range of 0.03 to 0.3 for the constant $\phi$ for the fixed-bed gasifiers.

The single particle model can be related to the bed by use of the particle number density and unreacted core particle surface area. Assuming spherical particles, Hobbs et al., (1992a) presented the expressions for the particle diameter, unreacted core diameter and particle number density for the SP submodel. These expressions are given by

$$
\begin{gathered}
d_{p}=\left[\left(1-\Omega_{a s h}^{o}\right) d_{u}^{3}+\Omega_{a s h}^{o} d_{o}^{3}\right]^{1 / 3} \\
d_{u}=F^{1 / 3} d_{o} \\
\eta_{p}=\frac{\sigma(1-\varepsilon)}{\pi d_{p}^{3}}
\end{gathered}
$$

where the subscripts $o$ and $u$ represent initial and unreacted core, respectively.

The heterogeneous oxidation of carbon produces both $\mathrm{CO}$ and $\mathrm{CO}_{2}$ as primary products as shown by the following reaction:

$$
\lambda \mathrm{C}+\mathrm{O}_{2} \rightarrow 2(\lambda-1) \mathrm{CO}+(2-\lambda) \mathrm{CO}_{2}, \quad 1 \leq \lambda \leq 2
$$

Carbon monoxide may be favored at higher temperatures if $\mathrm{CO}$ is formed at carbon edges and $\mathrm{CO}_{2}$ is formed at inorganic sites. Lower temperatures may favor $\mathrm{CO}_{2}$ 
due to catalytic activity. The $\mathrm{CO} / \mathrm{CO}_{2}$ ratio has been correlated by Laurendeau (1978):

$$
\frac{C O}{\mathrm{CO}_{2}}=A \exp \left(-\frac{E}{R T}\right)=\frac{2(\lambda-1)}{(2-\lambda)}
$$

where $A=10^{2.5}, E \approx 6-9 \mathrm{kcal} / \mathrm{mol}$ for low pressures and $A=10^{3.5}$, $E \approx 12-9 \mathrm{kcal} / \mathrm{mol}$ at high pressures. The stoichiometric coefficient for Equation 3.25 can be determined as a function of temperature from Equation 3.26:

$$
\lambda=\frac{2\left[A \exp \left(-\frac{E}{R T}\right)+1\right]}{A \exp \left(-\frac{E}{R T}\right)+2}
$$

The values for $\lambda$ at $500,1000,1500$, and $2000 \mathrm{~K}$ are $1.08,1.78,1.93$, and 1.96 using Equation 3.27 with the low pressure parameters and 1.00, 1.06, 1.47, and 1.76 using the high pressure parameters. This indicates that $\mathrm{CO}$ is favored at higher temperatures.

\section{Summary of Model Equations, Model Parameters and Options}

A summary of the one-dimensional fixed-bed model equations is given in Table 3.7. The system of ordinary differential equations listed in Table 3.2 is solved simultaneously from the top to the bottom of the reactor with LSODE (Livermore Solver for Ordinary Differential Equations, Hindmarsh, 1983). The equations for drying, devolatilization, combustion and gasification are described by Equations $3.18,3.19$, and 3.20. The volumetric heat transfer rate from solid-togas, solid-to-wall, and gas-to-wall are described by Equations 3.15, 3.16 and 3.17. 
One-Dimensional Fixed-Bed Model

Table.3.7 Summary of the one-dimensional fixed-bed model equations.

\begin{tabular}{lc}
\hline Basic Equations and Boundary Conditions & Reference \\
\hline Overall gas species continuity & Table 3.2 \\
Overall solid species continuity & Table 3.2 \\
Gas phase energy & Table 3.2 \\
Solid phase energy & Table 3.2 \\
Gas species continuity & Table 3.2 \\
Solid species continuity & Table 3.2 \\
Gas phase elemental & Table 3.2 \\
Gas phase tar species continuity & Table 3.2 \\
Gas phase tar elemental continuity & Table 3.2 \\
& \\
Auxiliary Equations & Reference \\
\hline Total enthalpies & Eq. (2.3) \\
Enthalpies of formation & Eq. (2.5) and Table 3.3 \\
Sensible enthalpies & Eqs. (2.6) and Table 3.3 \\
Enthalpy exchange due to reaction & Table 3.3 \\
Ash heat capacity & Eq. (2.9, 2.10) and Table 2.1 \\
Pressure drop & Eq. (3.11) \\
Gas conductivity, viscosity, and diffusivity & Table 3.4 \\
Particle diameter and surface area & Table 3.4, Eq. (3.22) \\
Particle number density & Eq. (3.24) \\
Volumetric solid-to-gas heat transfer rate & Eq. (3.15) \\
Volumetric wall heat loss from solid & Eq. (3.16) \\
Volumetric wall heat loss from gas & Eq. (3.17) \\
Gas-to-wall heat transfer coefficient & Table 3.5 \\
Solid-to-wall heat transfer coefficient & Table 3.5 \\
Effective gas and solid conductivities & Table 3.5 \\
Solid conductivity & Table 3.5 \\
Diffusive radiation coefficients & Table 3.5 \\
Solid-to-gas heat transfer coefficient & Table 3.5 \\
Mass transfer coefficient & Table 3.5 \\
Reynolds, Prandtl, and Schmidt numbers & Table 3.5 \\
Drying rate & Eq. (3.18) \\
Devolatilization rates & Eq. (3.19) \\
Oxidation and gasification rates & Eq. (3.20) \\
\hline & \\
& \\
&
\end{tabular}


The heat and mass transfer correlations are described in detail in Table 3.5. Elemental reaction rates can be determined from reaction stoichiometry. The tar reaction rates can be determined from Equation 3.19.

Table 3.8 provides a summary of the parameters and options for the onedimensional fixed-bed model. Parameters that are difficult to obtain and are highly uncertain are the solid-to-gas heat transfer coefficient, effective diffusivity, and bed void fraction. Most of these parameters can be determined independently, as shown in Table 3.9. However, if experimental values are unavailable, Table 3.9 can be used as a guide to select the optimum value of bed void fraction, $\varepsilon$, solid-togas heat transfer correction factor, $\zeta$, and the ash porosity, $\phi$, which can be used to determine the effective diffusivity. The solid-to-gas heat transfer correction factor ranges between 0.02 and 1.0 and is discussed by Hobbs et al., (1992a). Bed void fraction for the feed coal can be determined experimentally. However, the bed void fraction is not constant throughout the reactor. Values at the top and bottom of the reactor can be specified in the one-dimensional model. The bed void fraction is assumed to vary linearly from the specified values at the reactor top and bottom.

The choice of either the SP or AS char model options is difficult to determine a priori. The SP model option seems to give temperature profiles closer to experimental results (Hobbs et al., 1992a). Other model options include the gas phase tar reaction equilibrium option and volatiles mass transport option. Also, the combustion product distribution can be considered a model option. However, this option is not listed in Table 3.8, since the distribution is assumed to be sufficiently correlated to experimental data. 
Table 3.8 Summary of the one-dimensional fixed-bed model parameters and options.

PARAMETER TYPICAL VALUE

DESCRIPTION

\section{Devolatilization}

$x^{0}$

$k_{i}^{o}, 1 / s$

0.16 (Table B.1)

Potential tar-forming fraction

$\bar{E}_{i} / R, K$

$5.0 \times 10^{12}\left(\mathrm{CO}_{2}\right.$ extra loose, Table A.6)

$\sigma_{i} / R, K$

$20,850\left(\mathrm{CO}_{2}\right.$ extra loose, Table A.6)

$3600\left(\mathrm{CO}_{2}\right.$ extra loose, Table A.6)

$\chi_{i}$

$k_{x}^{o}, 1 / s$

$2048\left(\mathrm{CO}_{2}\right.$ extra loose, Table A.6)

27,500 (Tar, Table B.1)

$\bar{E}_{x} / R, K$

$0.86 \times 10^{15}$ (Tar, Table B.1)

\section{Oxidation/Gasification}

$k_{\mathrm{CO}_{2}}^{\mathrm{o}}, 1 / \mathrm{s}$

589 (Table 3.6)

$E_{\mathrm{CO}_{2}} / R, K \quad 26,800$ (Table 3.6)

$k_{H_{2}}^{o}, 1 / s$

0.589

$E_{H_{2}} / R, K \quad 26,800$

$k_{H_{2} \mathrm{O}}^{o}, 1 / s \quad 589$

$E_{\mathrm{H}_{2} \mathrm{O}} / \mathrm{R}, \mathrm{K} \quad 26,800$

$k_{\mathrm{O}_{2}}^{\mathrm{o}}, 1 / \mathrm{s}$

2.3 (Table 3.6)

$E_{\mathrm{O}_{2}} / R, K$

11,100 (Table 3.6)

Flow, Heat and Mass Transport

$\varepsilon^{\prime} \quad 0.85$ (Table 3.5)

$\varepsilon_{\text {top }} \quad 0.33$ (Thimson et al., 1984)

$\varepsilon_{\text {bottom }} \quad 0.6$ (Thimson et al., 1984)

$\zeta \quad 0.05$ (Lowry, 1963)

$\phi / \tau \quad 0.5$ (Laurendeau, 1978)
Functional group frequency factor

Functional group activation energy

Standard deviation for distribution function

Cross-link efficiency

Tar frequency factor

Tar activation energy

Frequency factor for $\mathrm{CO}_{2}$ gasification reaction

Activation energy for $\mathrm{CO}_{2}$ gasification reaction

Frequency factor for $\mathrm{CO}_{2}$ gasification reaction

Activation energy for $\mathrm{CO}_{2}$ gasification reaction

Frequency factor for $\mathrm{CO}_{2}$ gasification reaction

Activation energy for $\mathrm{CO}_{2}$ gasification reaction

Frequency factor for $\mathrm{CO}_{2}$ gasification reaction

Activation energy for $\mathrm{CO}_{2}$ gasification reaction

Coal emissivity

Bed void and bed top

Bed void at bed bottom

Reactive/nonreactive heat transfer coeff.

Ash porosity divided by tortuosity squared 
Table 3.8 Summary of the one-dimensional fixed-bed model parameters and options. (continued)

Operating Parameters

Prox. \&

ultimate comp.

$d_{p}, \mathrm{~cm}$

$D, m$

2.2 (Thimson et al., 1984)

Proximate and ultimate analysis

$L, m$

$T_{\text {coal }}, K$

$T_{B G}, K$

$T_{\text {wall }} K$

298

560

325 (Thimsen et al., 1984)

$\dot{m}_{c}, k g / s$

0.35 (Thimsen et al., 1984)

Particle diameter

Reactor diameter

Reactor length

Feed coal temperature

Feed gas temperature

Wall temperature

$\dot{m}_{o}, \mathrm{~kg} / \mathrm{s} \quad 0.95$ (Thimsen et al., 1984)

Coal mass flow rate

$\dot{m}_{s}, k g / s$

0.16

Oxidizer mass flow rate

Steam mass flow rate

Model Options: AS or SP char submodel, gas phase tar reaction equilibrium option, and volatiles mass transport option for FG-SET submodel. 
One-Dimensional Fixed-Bed Model

Table 3.9 Guidelines for the selection of some critical model parameter.

Parameter

Independent Calculation

Bed void fraction at gasifier top, $\varepsilon_{c}$, and gasifier bottom, $\varepsilon_{a}$

Solid-to-gas heat transfer correction factor, $\zeta$.

Effective diffusivity parameter, $\phi=\frac{\phi_{a}}{\tau^{2}}=\frac{\phi_{a}}{2}$

SP or AS model option
Void fractions can be measured or estimated from coal and ash properties. Typical values may range between 0.2 and 0.8 depending on particle distribution, sphericity and particle roughness\$.

$\zeta$ ranges from 0.02 to 1.0 based on reacting solid-to-gas heat transfer (Hobbs et al., 1992a). A value of 0.05 is recommended if no other data are available.

$\phi$ can be measured or estimated from the ash porosity, $\phi_{a}$. Ash porosities range from 0.06 to 0.60 for ash obtained from fixedbed gasifiers (Hobbs, 1990). An acceptable range for $\phi$ might be 0.02 to 0.5 . A value of 0.5 is recommended if no other data are available.

The SP model option is recommended if no other data are available as discussed by Hobbs et al., (1992a).
Selection Procedure

Bed void fraction is selected to match measured pressure profile. Initially, $\varepsilon_{c}$ and $\varepsilon_{a}$ are adjusted in equal increments; however, the top or bottom void fractions are adjusted independently if the measured profile indicates a larger or smaller void fraction.

$\zeta$ is selected to match the location of the maximum measured temperature.

$\phi$ can be selected to match the location of the maximum measured temperature. $\phi$ is usually adjusted after $\zeta$ is chosen. In other words, if adjusting $\zeta$ is insufficient to adjust the location of the maximum temperature, adjust $\phi$.

The AS model option can be chosen if a discontinuity is observed in the measured pressure profile, or if the oxidation zone is very small with a corresponding steep temperature gradient, or if burnout is high simultaneously with a large drying zone as is common for lignites with high moisture contents.

\footnotetext{
$\S$ MeFayed (1984) discusses bed void fraction. For spherical particles, bed void fraction for close random packing ranges from 0.359 to 0.375 ; for hexagonal close packing, bed void fraction is 0.26 . The effect of sphericity is to increase bed void fraction. For example, for a sphere with a sphericity of 1.0 , it is 0.4 ; for a cube with sphericity 0.8 , it is 0.5 ; and for a particle with sphericity of 0.2 it is 0.85 .
} 


\section{CHAPTER 4}

\section{FG-DVC DEVOLATILIZATION $S U B M O D E L$}

\section{FG-DVC Model Basis}

FG-DVC is a general model for coal pyrolysis that predicts the decomposition of coal into tar, char, and gas, given the ambient pressure and the temperature history of an isothermal coal particle. The model predicts the amount, functional group composition, elemental composition, and molecular weight distribution of tar and char and the amount and composition of the gas. The model is described in more detail in Appendices E, F, and G.

The letters FG stand for Functional Group, and DVC for Depolymerization, Vaporization and Crosslinking. The FG model considers certain functional groups in the coal which decompose to form the light gas species. At the same time, the DVC model describes the overall depolymerization of the macromolecular network which combines bridge breaking and crosslinking to produce fragments of the coal macromolecule. These fragments are then subjected to transport behavior, specifically the vaporization of the lightest fragments to form tar. The tar fragmentation process provides a second mechanism for the removal of functional groups from the coal. The model, whose parameters are determined in the laboratory at moderate temperatures and one atmosphere, can then be used to 
FG-DVC Devolatilization Submodel

extrapolate away from the laboratory conditions to predict pyrolysis and combustion in high temperature reactions, or liquefaction at high pressure.

The model for coal thermal decomposition has six basic concepts.

- Functional Groups (decompose to produce light gases),

- Macromolecular Network (decomposes to produce tar and metaplast),

- Network Coordination Number (possible number of attachments per cluster),

- Bridge Breaking (limited by hydrogen availability),

- Crosslinking (related to gas evolution),

- Mass Transport of Tar (evaporation of light network fragments into light gases).

The first concept is that light gases are formed by the decomposition of certain functional groups in the coal. For example, methyl groups can lead to the formation of methane, carboxyl groups can lead to the formation of $\mathrm{CO}_{2}$, etc. The second concept is that coal consists of a macromolecular network. This network is made up of fused aromatic ring clusters (which are described by their molecular weight) linked by bridges, some of which are relatively weak. There are some unattached parts of the network which can be extracted. Sometimes, there is also a second component of high polymethylene content. When heated, this network decomposes to produce smaller fragments. The lightest of the fragments evaporate to produce tar; the heavier fragments form the metaplast. These heavier molecules are the primary liquid fragments in liquefaction or the fragments that make coal fluid.

The third concept is that one of the most important properties of the network is its coordination number. The coordination number describes the geometry of the network, and specifies how many possible attachments there are per aromatic ring cluster. For example, a linear polymer chain has a coordination number of 2 , because each fused aromatic ring has two possible attachments to link it in the chain. On the other hand, a square "fish net" has a coordination number of 4 , because there are four possible attachments at each ring cluster. The coordination number controls the molecular weight distribution of the network fragments at a 
given extent of decomposition. The extent of decomposition is specified by the probability that the possible attachments are made. For example, for $20 \%$ of broken bridges, a linear chain is totally fragmented, while a "fish net" will have some holes but is almost totally connected. In describing the network, a crosslink is defined to occur at a branch point where there are more than two attachments on a ring cluster. The coordination number is thus related to the crosslink density. With no possible crosslinks, the coordination number is two. With increasing crosslink density, the coordination number increases.

The second important property of the network is the fraction of possible attachments, $p$, which are actually present. During thermal decomposition, this fraction is determined by the rates of bridge breaking. The factors that control how many of the weak links can break are the rate constant and the amount of hydrogen that can be donated from the coal to stabilize the free radicals which form when the links break.

A competitive process with the bond breaking is the retrogressive process of crosslinking. Crosslinking reactions appear to be related to the evolution of certain gases. Specifically, for low rank coals, crosslinking at low temperature (prior to bridge breaking) seems to be related to the evolution of carbon dioxide. For coals of all rank, a higher temperature crosslinking event (following bridge breaking) seems to be related to the evolution of methane. At high temperatures, the evolution of hydrogen should be related to crosslinking, but this is not currently done.

The final concept is that the tar evolution is controlled by mass transport. Bridge breaking and crosslinking produce a set of fragments with a molecular weight distribution. The lightest fragments can leave the coal melt by evaporation into the light gas species. The heavier fragments remain, forming the metaplast that controls the coal's fluidity.

In the following sections, the descriptions of DVC and FG submodels are given. More details can be found in Appendices E, F, and G. 
FG-DVC Devolatilization Submodel

\section{Depolymerization-Vaporization-Crosslinking (DVC) Formulation}

The DVC model predicts the yield and molecular weight distribution of the tar, and the yield and molecular weight distribution, extract yield, and the crosslink density of the char. The theory describes the combined effects of: 1) depolymerization and hydrogen consumption; 2) cross-linking; and 3) transport. These processes, described below, are incorporated into a computer code that employs a percolation theory method for performing the statistical analysis (Grant et al., 1989; Fisher and Essam, 1961).

Process 1. Depolymerization and Hydrogen Consumption. Bond cleavage in coal is likely to be very complicated, including homolytic cleavage, ipso substitution, and hydrogen-transfer-induced bond-scission reactions for a variety of bond types. However, it has been observed that tar evolution is consistent with a narrow distribution of activation energies; this allows consideration in the model of a single representative bridge (while acknowledging that other types may be present). Also, the rate for tar formation from coal, $k_{t a r}$ is in good agreement with the rate determined for the breaking of ethylene bridges between naphthalene rings, $k_{B}$. This kinetic rate, $k_{B}$, employs an activation energy that is in agreement with resonance-stabilization calculations and an overall rate that agrees with previous measurements on model compounds. In view of these observations, a single type of bond (ethylene) undergoing homolytic cleavage is assumed for coal as a simple approximation of a complex behavior.

Bond cleavage is accompanied by the consumption of donatable hydrogens, $\mathrm{H}(\mathrm{al})$, to cap free radicals, along with corresponding carbon-carbon double bond formation at the donor site. For simplicity, the DVC model assumes that all the coal's donatable hydrogens, whether in bridges or in hydroaromatic rings, are in bridges, i.e., $\mathrm{H}(\mathrm{al})=(2 / 28) W_{B}$, where $\mathrm{H}(\mathrm{al})$ is the weight fraction of donatable hydrogen and $W_{B}$ is the weight fraction of labile bridges. This approximation will produce some error in tar yield, since a broken bond in a hydroaromatic ring will not be as effective as a broken bond in a bridge in fragmenting the coal. But this effect will be compensated for, since $\mathrm{H}(\mathrm{al})$ is a parameter that is determined for each coal from a selected pyrolysis experiment. $\mathrm{H}$ (al) could, in principle, be determined by FT-IR or NMR, but not with sufficient accuracy. 
The equation describing the disappearance of labile bridges in the char, $W_{B}$ (char), due to bridge breaking and hydrogen donation is

$$
\frac{d W_{B}}{d t}=-2 k_{B} W_{B}
$$

or

$$
\frac{d p_{L}}{d t}=-2 k_{B} p_{L}
$$

where $p_{L}$ is the fraction of occupied bridges. The value for $k_{B}$ is the same as for tar formation, $k_{t a r}$. The rate of decrease of labile bridges is twice the rate of bridge breaking, since for each broken bridge, an additional labile bridge is converted to a nonlabile bridge with the donation of hydrogen. By assuming that all the donatable hydrogen is in the labile bridges, the consumption of labile bridges and donatable hydrogen occurs simultaneously. The redistribution of hydrogen creates source and loss terms, $d W_{i}(D V C) / d t$, in the equations for the char functional groups $W_{i}($ char $)$.

Equation 4.1 describes only the loss due to bridge breaking and hydrogen donation. The loss of labile bridges due to evolution with the tar is computed by using the transport equations (Eq. 4.6) discussed below.

Process 2. Crosslinking. Crosslinking reactions are important in describing the rank and heating rate dependence of the tar molecular weight distributions and yields. A correlation between gas evolution and crosslinking permits the rates for crosslinking and the number of crosslink sites to be related to rates and yields for gas evolution. The model assumes the following expression for the rate of increase of the fraction of crosslinks in the coal, $q$ :

$$
\frac{d q}{d t} \alpha \sum_{\mathrm{CH}_{4}, \mathrm{CO}_{2}}\left[\frac{d W_{i}(\text { gas })}{d t}\right] \cdot\left[\frac{\# \text { crosslinks formed }}{\text { unit of gas } i}\right]
$$


where the rates, $d W_{i} / d t$, of evolution per gram of coal of $\mathrm{CO}_{2}$ and $\mathrm{CH}_{4}$ are calculated in the FG submodel.

We caution that the assumed reactions must be a gross simplification of a very complicated set of chemical reactions. This is especially true for the crosslinks occurring during methane formation, during which time extensive bond breaking and crosslinking accompany tar formation.

Process 3. Transport. The external transport of tar components from the particle surface to the bulk gas by vaporization and diffusion through a gas boundary layer is described by simply assuming a constant pressure drop from the interior to the exterior of the coal particle. This assumption is plausible for a swelling coal.

In the DVC model, we assume that the tars achieve their equilibrium vapor pressure, $P_{j}$, in the evolving gases, which can be computed with a few assumptions. The rate of transport, per gram of coal $d n_{j} / d t$, for tar component $j$ is proportional to the volume of gases evolved, $d V / d t$. That is,

$$
\frac{d n_{j}}{d t}=P_{j} \chi_{j}\left(\frac{d V}{d t}\right)\left(\frac{1}{R T}\right)
$$

where $\chi_{j}$ is the mole fraction of tar species $j$ in the metaplast (using Raoult's Law). The volume flow rate of gases is given by the ideal gas law: proportional to the molar gas flow rate and the temperature, and inversely proportional to the pressure within the particle, $P_{0}+\Delta P$, where $P_{0}$ is the ambient pressure and $\Delta P$ is the average pressure difference between the surface and the particle's interior. That is,

$$
\frac{d V}{d t}=\sum_{i}\left(\frac{d n_{i}}{d t}\right)_{\text {gas \& light tar }}\left(\frac{R T}{P_{0}+\Delta P}\right)
$$

where $\Sigma_{i}\left(d n_{i} / d t\right)_{\text {gas } \& \text { light tar }}$ is the rate of production per gram of coal of gas components $i$ summed over all gas and light tar species. For gas molecules, $d n_{i} / d t$ is taken as the rate of production given by the FG model. For tar molecules, $d n_{i} / d t$ 
Chapter 4

is computed using Eqs. 4.4 and 4.5 simultaneously. Summing Eq. 4.4 over the light tar species, adding to it the sum over gas species, and substituting into Eq. 4.5 , we obtain an expression for $d V / d t$ in terms of the gas evolution rates and the tar vapor concentrations from Raoult's Law, which leads to

$$
\frac{d n_{j}}{d t}=P_{j} \chi_{j} \sum_{F G \text { gases only }}\left(\frac{d n_{i}}{d t}\right)\left(\frac{1}{P_{0}+\Delta P-\sum_{\text {light tars }} P_{j} \chi_{j}}\right)
$$

$\Delta P$ is used as an adjustable parameter that varies with the coal and experimental conditions.

While $\Delta P=0$ appears to be a good approximation for fluid coals at one atmosphere or more, $\Delta P>0$ is expected for some coals and situations. $\Delta P$ is proportional to the coal's viscosity and so will become important for less fluid coals. $\Delta P$ is also important when $P_{O}$ is small, for large particles, and when the heating rates are very high. For nonfluid coals, $\Delta P$ is very large due to the limited gas transport ability of these coals, while the highly fluid coals have a small $\Delta P$. We have found that reasonable results can be obtained with $\Delta P$ equal $10 \mathrm{~atm}$ for nonfluid coals and $0.2 \mathrm{~atm}$ for fluid coals. The quantities needed to evaluate Eq. 4.6 are discussed under Solution Technique later in this chapter.

\section{Functional Group (FG) Model Formulation}

The Functional Group (FG) model permits the detailed prediction of the composition of volatile species (gas yield, tar yield and tar functional group and elemental composition) and of char (elemental and functional group composition). It employs coal dependent rates for the decomposition of individual assumed functional groups in the coal and char to produce gas species. The ultimate yields of each gas species are related to the coal's functional group composition. Tar evolution is a parallel process that competes for all the functional groups in the coal. There are three additional processes in the FG submodel.

Process 4. Gas Formation. The evolution of each gas species is assumed to be a first order reaction, 


$$
\frac{d W_{i}(\text { gas })}{d t}=k_{i}\left[W_{i}(\text { char })+W_{i}(\text { tar }) \cdot\left[\begin{array}{l}
0 \\
1
\end{array}\right]\right]
$$

where, $d W_{i}(g a s) / d t$ is the rate of evolution of species $i$ into the gas phase, $k_{i}$ is a distributed rate for species $i$, and $W_{i}($ char $), W_{i}$ (tar) are the functional group sources remaining in the char and tar. The $[0,1]$ distinguish between the cases where tar does or does not continue to pyrolyze.

We employ the concept of the distributed rate where $k_{i}$ is given by an Arrhenius expression $k_{i}(E)=k_{i} \exp \left[-\left(E \pm \sigma_{i}\right) / R T\right]$, where $\pm \sigma_{i}$ indicates that a Gaussian distribution is employed to describe the source pools, $d W_{i}(E)$, as a function of the activation energies $E$ about the initial average activation energy $E_{i}$.

$$
d W_{i}(E)=\frac{W_{i}^{0}}{\sqrt{2 \pi \sigma_{i}}} \exp \left(\frac{-\left(E-E_{i}\right)^{2}}{2 \sigma_{i}^{2}}\right) d E
$$

where $\sigma_{i}$ is the standard deviation of the Gaussian distribution.

Process 5. Tar Formation. The tar composition is tracked by summing the functional group contributions evolved with the tar. The rate of evolution of each contribution is

$$
\frac{d W_{i}(\text { tar })}{d t}=\left(\frac{d W(\text { tar })}{d t}\right)_{D V C} \cdot\left(\frac{W_{i}(\text { char })}{\sum_{i} W_{i}(\text { char })}\right)-k_{i} \cdot W_{i}(\text { tar }) \cdot\left[\begin{array}{l}
0 \\
1
\end{array}\right]
$$

where $d W_{i}(t a r) / d t$ is the rate of evolution of each functional group component with the tar; $[d W(t a r) / d t]_{D V C}$, the rate of tar production from the char, is discussed later in this chapter under DVC submodel Solution Technique.

Process 6. Char Formation. The change in the $i$-th char pool, $W_{i}$ (char), is computed by summing the losses to the gas and tar, and the redistributions determined in the DVC submodel, 


$$
\frac{d W_{i}(\text { char })}{d t}=-\left(\frac{d W_{i}(\text { gas })}{d t}\right)-\left(\frac{d W(\text { tar })}{d t}\right)+\left(\frac{d W_{i}(D V C)}{d t}\right)
$$

\section{Percolation Theory}

The coal macromolecular network can be represented using several methods. This model uses the percolation theory, which predicts analytically the probability that a bond will be occupied, given the coordination number and the starting bond occupation probability (Appendix E).

The DVC model predicts, and experiments confirm, that there are more than one kind of bond bridges and crosslinks which have different coordination numbers, and independent probabilities of being broken. To take advantage of the benefits of percolation theory, we have extended percolation theory on a Bethe lattice (one with no loops) to use two independent subnetworks, as illustrated in Fig. 4.1. In the figure, double lines represent one of the bond types, while single lines represent the other. As can be seen by comparing Fig. 4.1a and 4.2b, this lattice has the desired feature of modeling a transition from chain-like structures (a) to fishnet structures (b). The mathematics of this two-bond percolation theory follows closely that of the standard theory. The mathematics are presented in Appendix E.

Four parameters describe the network: $p, q, \sigma_{1}$ and $\sigma_{2} . p$ and $q$ are the occupation probabilities of bridges and crosslinks, respectively, while $\sigma_{l}+I$ and $\sigma_{2}+1$ are the coordination numbers for bridge linkage and crosslink linkage, respectively. $\sigma_{1}$ and $\sigma_{2}$ are model constants. $p_{L}$ is the number fraction of occupied labile bridges; the evolution in time is given by Eq. 4.2. Similarly, $q$ is the number fraction of crosslinks, computed using Eq. 4.3. $p$ and $q$, are the state variables computed at each time step, and are set equal to the input parameters $p_{o}, q_{0}$, at time equal $0 . p_{o}, q_{o}$ vary with each coal. 

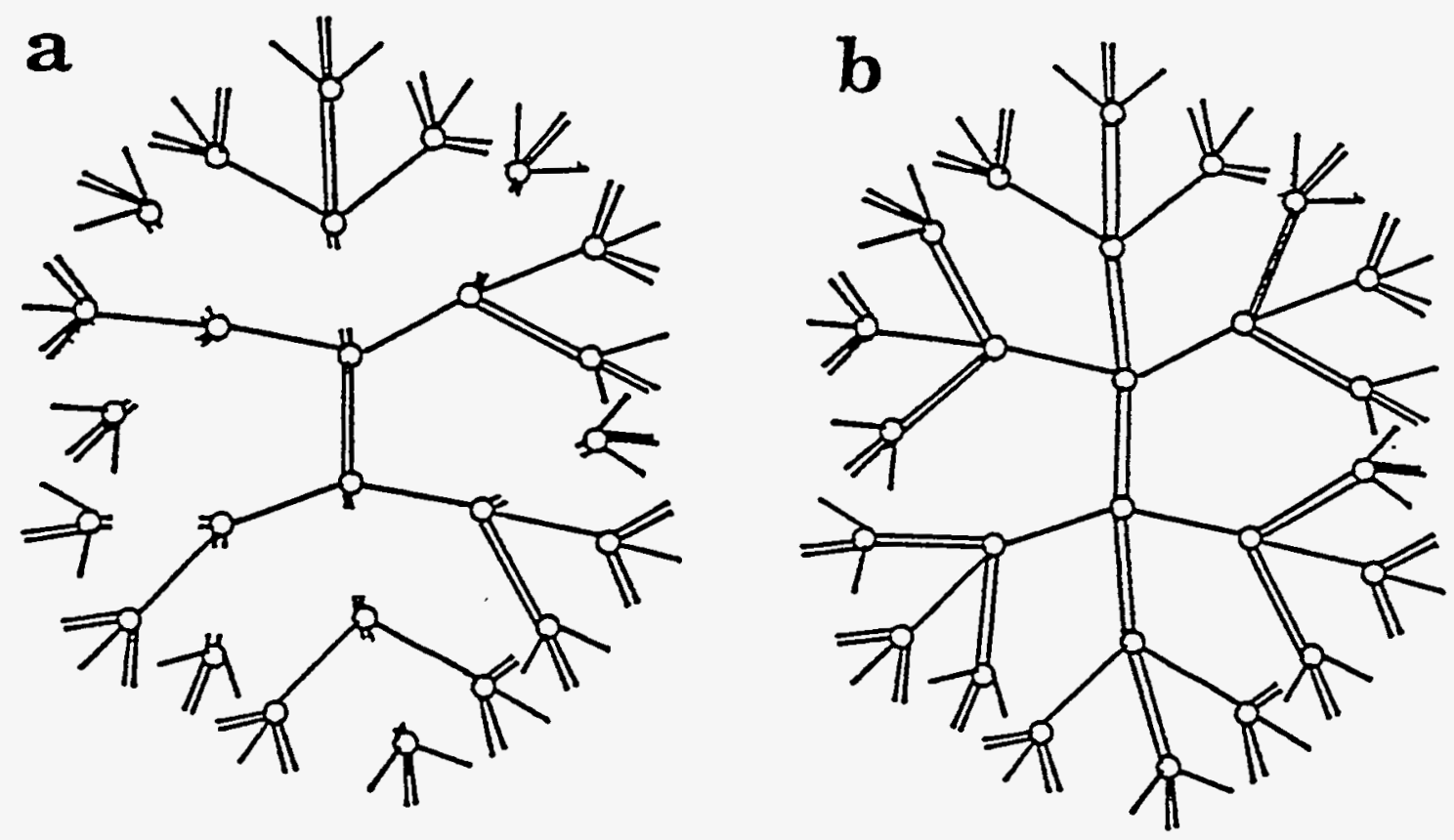

Figure 4.1 Bethe Lattice for two- $\sigma$ model with $\sigma_{1}=\sigma_{2}=1$ (shown as single bonds). a) With most double bonds representing the crosslinks not yet formed to represent the starting coal, the lattice is like one- $\sigma$ model with $\sigma=1$, linear chains. b) Fully linked case ( $p=q=1)$ is like one- $\sigma$ model with $\sigma=3$. 


\section{FG-DVC Solution Technique}

The "standard version" of the FG-DVC model implements the distributed rate kinetics by subdividing each functional group pool into 21 subpools, each with fixed rate parameters. This essentially decouples all the equations and allows us to integrate all $27 * 21$ equations analytically, thereby allowing us to have large time increments. For the fixed bed model presented in this manual, this approach cannot be used: we must use the actual differential equations, and we have therefore treated the distributed rate kinetics differently. We start out with a Gaussian distribution for each functional group, as indicated by Eq 4.8. In the "standard" FG-DVC, model this distribution evolves away from the Gaussian as the subpools with lower activation energies are consumed. In this version, we retain the Gaussian shape of the distribution for all time (or more accurately, we retain the error function shape), and allow only the lower energy limit on the error function to change with time. This implies one extra differential equation (for the lower energy limit) per functional group instead of 21 .

\section{FG Submodel}

As described in the previous Section, the model determines the weight loss of char to form tar and gas, where the initial char is a raw coal. The basic equations are Eqs. 4.7, 4.9, and 4.10. We start by defining a set of char fraction variables $f$ as follows:

$$
W_{i}(\text { char }, t)=W^{0}(\text { char }) \cdot f_{i}(t)
$$

where

$$
\begin{aligned}
W^{0}= & \text { the whole starting char (constant }=1.0) \\
f_{i}(t)= & \text { the mass fraction of the starting char (coal) which can form gas } \\
& \text { species } i \text { at time } t
\end{aligned}
$$

In order to implement the distributed activation energy reaction kinetics, each of these mass fraction functions, $f_{i}$, is described as a distribution function of activation energy, $E$ : 
$f_{i}(E, t) d E=$ the mass fraction of the starting char which, at time $t$, can evolve gas species $i$, with Arrhenius parameters given by frequency factor $A(i)\left[\mathrm{sec}^{-1}\right]$, and activation energy between $E$ and $E+d E$.

The integrals of these $f_{i}(E, t)$ 's over all $E$ are equal to the corresponding $f_{i}(t)$ 's in Equation 4.11. In the discussion which follows, we usually leave out the time variable, $t$ Note that $f_{i}(E)$ is zero for $E<E^{m i n}$.

To this definition, we add the following definitions:

$$
\begin{gathered}
W_{i}(\operatorname{char}, E)=W^{0} \cdot f_{i}(E) \\
W_{i}(\operatorname{char}, E)=W^{0} \cdot \int_{-\infty}^{\infty} f_{i}(E) d E
\end{gathered}
$$

and

$$
k_{i}(E)=A_{i} \exp \left(-\frac{E}{R T}\right)
$$

Eqs. 4.12 and 4.13 imply that

$$
W_{i}(\text { char }, E)=W^{0}(\text { char }) \cdot \frac{f_{i}(E)}{\int_{-\infty}^{\infty} f_{i}(E) d E}
$$

For cases where the tar also pyrolyzes to form gases, we assume the same form (since the tar is functionally similar to the parent char). 


$$
W_{i}(\operatorname{tar}, E)=W^{0}(\operatorname{tar}) \cdot \frac{f_{i}(E)}{\int_{-\infty}^{\infty} f_{i}(E) d E}
$$

With these definitions, Eq 4.7 becomes

$$
\frac{d W_{i}(\text { gas })}{d t}=\int_{-\infty}^{\infty} k_{i}(E) \cdot\left[W_{i}(\text { char }, E)+W_{i}(\operatorname{tar}, E)\left[\begin{array}{l}
0 \\
1
\end{array}\right]\right] d E
$$

Eqs. 4.9 and 4.10 are unchanged.

If we define average kinetic rate constants as follows,

$$
\left\langle k_{i}\right\rangle=\frac{\int_{-\infty}^{\infty} k_{i}(E) f_{i}(E) d E}{\int_{-\infty}^{\infty} f_{i}(E) d E}
$$

then Eq. 4.17 becomes

$$
\frac{d W_{i}(\text { gas })}{d t}=\left\langle k_{i}\right\rangle \cdot\left[W_{i}(\text { char })+W_{i}(\text { tar }) \cdot\left[\begin{array}{l}
0 \\
1
\end{array}\right]\right]
$$

Eqns. 4.9, 4.10, and 4.19, together with equations describing the time dependence of the distribution functions, are the basic differential equations needed for the FG submodel.

\section{Distributed Rate Submodel (the f's)}

As pointed out above, in the original versions of the FG and FG-DVC models, the $f(E) d E$ were approximated by initializing them to a Gaussian distribution and subdividing the range covering \pm 3 standard deviations into 21 equal energy increments. This approach gives 21 differential equations for each functional group and bridge (each subdivision decreases with the first order kinetics 
given by the rate constants determined by Equation 4.18. This means that the shape of the $f(E)$ changes with time. More recently, we have approximated this model by assuming that the shape remains fixed in time, but that the lower cutoff point changes in such a way as to give the same area under the $f(E) d E$ curve as before. This gives a single differential equation for each distribution instead of 21 . In this section, we derive this equation. For simplicity we assume that tar does not pyrolyze.

We start with the observation that

$$
\frac{d W_{i}(\text { gas })}{d t}=-\left(\frac{d W_{i}(\text { char })}{d t}\right)_{\text {tar, other held fxed }}
$$

and require that the distribution function is zero for the energies less than the minimum, and thus we differentiate Equation 4.13 with respect to time. Since only the lower bound of the integral, $E_{i}^{m}$, varies with time, the result is

$$
\frac{d W_{i}(g a s)}{d t}=W^{0} \cdot f_{i}\left(E_{i}^{m}(t)\right) \cdot \frac{d E_{i}^{m}(t)}{d t}
$$

Using Eqs. 4.15 and 4.19,

$$
\frac{d W_{i}(\text { gas })}{d t}=\left\langle k_{i}\right\rangle \cdot W_{i}(\text { char })=\frac{W_{i}(\text { char }) \cdot f_{i}\left(E_{i}^{m}(t)\right) \cdot \frac{d E_{i}^{m}(t)}{d t}}{\int_{E_{i}^{\prime \prime}(t)}^{\infty} f_{i}(E) d E}
$$

Solving for $d E / d t$, 


$$
\frac{d E_{i}^{m}(t)}{d t}=\left\langle k_{i}\right\rangle \cdot \frac{\int_{E_{i}^{m}(t)}^{\infty} f_{i}(E) d E}{f_{i}\left(E_{i}^{m}(t)\right)}
$$

For simplicity, we use a truncated Gaussian, so that

$$
\begin{gathered}
\int_{E_{i}^{m}(t)}^{\infty} f_{i}(E) d E=\operatorname{erfc}\left(z_{i}(t)\right) \\
z_{i}(t)=\frac{E_{i}^{m}(t)-E_{i}}{\sqrt{2 \sigma_{i}}}
\end{gathered}
$$

where $E_{i}$ and $\sigma_{i}$ are the parameters of the Gaussian distribution. We observe that the $f_{i}^{\prime}$ 's occur only in Eqns. 4.18 and 4.23, and only in normalized form, so the proportionality constant is not needed.

If there are source redistribution terms, then we must make suitable assumptions as to how these terms affect the distribution functions. For the cases considered to date, we have assumed that material removed from a given functional group, by mechanisms other than the bond breaking associated with freeing up the functional group, is removed from each activation energy subpool in proportion to its current quantity.

\section{DVC Submodel}

The DVC submodel is described fully in the General Model Paper (Solomon et al., 1988, see Appendix F). To implement this using the two coordination number percolation theory, as described in the Network Paper (Solomon et al., 1990, see Appendix E), we define two bond types (referred to as bridges and cross-links), each bond type having its own coordination number and probability of occupation. The bridges with probability of occupation, $p(t)$ are broken with a distributed kinetic rate (in much the same manner as the gas functional groups 
above). The cross-links (with probability of occupation, $q(t)$ ) are formed in conjunction with the evolution of $\mathrm{CO}_{2}$ and $\mathrm{CH}_{4}$. To cope with the problem of cross-linking tar to char, the cross-linking rate for $d q / d t$ is divided by $W_{i}$ (char), i.e., the percolation is done on the whole coal basis. The current version of the model assumes that bridges are only broken (never formed), and that crosslinks are only formed (never broken). To model the hydrogen abstraction, the population of bridges is subdivided into labile and nonlabile (unbreakable) bridges, so that each time a labile bridge is broken, an additional labile bridge is converted into a nonlabile bridge. Thus the differential equations needed for the percolation part of the model are

$$
\begin{gathered}
\frac{d p_{L}}{d t}=-2 \cdot\left\langle k_{B}\right\rangle \cdot p_{L} \\
\frac{d p}{d t}=-\left\langle k_{B}\right\rangle \cdot p_{L} \\
\frac{d p}{d t}=\sum_{C O_{2}, C H_{4}} \frac{d W_{i}(\text { gas })}{d t} \cdot \frac{X_{i}}{M_{i} \cdot \Sigma W_{i}(\text { char })}
\end{gathered}
$$

where

$$
\begin{aligned}
& p_{L}=\text { the number fraction of bridge sites containing labile bridges } \\
& p \quad=\quad \text { the number fraction of bridge sites containing either labile or } \\
& \text { nonlabile bridges } \\
& q=\text { the number fraction of occupied crosslink sites. } \\
& X_{i}=\text { the number of crosslinks formed for each molecule of gas } i \\
& \text { evolved [moles } \mathrm{x} \text {-link/mole gas } i) *(\mathrm{gm} \text { coal/crosslink site] } \\
& M_{i}=\text { the molecular weight of gas species } i[\mathrm{gm} / \mathrm{mole}]
\end{aligned}
$$


and

$$
\left\langle k_{B}\right\rangle=\frac{\int_{-\infty}^{\infty} k_{B}(E) f_{B}(E) d E}{\int_{-\infty}^{\infty} f_{B}(E) d E}
$$

with

$$
k_{B}(E)=A_{B} \cdot \exp \left(\frac{-E}{R T}\right)
$$

where

$$
\begin{aligned}
& f_{B}(E)=\quad \text { the fraction of the starting bridges which can break at time } t \text {, } \\
& \text { with Arrhenius parameters given by frequency factor } \\
& A(B)\left[\mathrm{sec}^{-1}\right] \text {, and activation energy between } E \text { and } E+d E \text {. }
\end{aligned}
$$

The $p(t)$ and $q(t)$ computed from these three differential equations are then used to compute the molecular weight distribution function, $F_{j}(p, q)$, from percolation theory. $F_{j}(p, q)$ is the weight fraction of $j$-mers in the char, and is a function only of $p, q$ and the two coordination numbers.

This $F_{j}(p, q)$ is converted to the mass distribution function needed for the tar submodel by using a molecular weight distribution of monomers.

$$
\begin{aligned}
& n_{i}(\text { char })=\sum_{j} \frac{G_{i j} \cdot F_{j}(p, q)}{M T_{i}} \\
& \chi_{i}=\frac{n_{i}(\text { char })-n_{i}(\text { tar })}{\sum_{i}\left[n_{i}(\text { char })-n_{i}(\text { tar })\right]}
\end{aligned}
$$

where

$$
F_{j}(p, q)=\text { mass fraction of coal in } j \text {-mers }[\mathrm{gm} \mathrm{j} \text {-mer/gm coal] }
$$




$$
\begin{aligned}
& n_{i} \text { (char) }=\text { fraction of coal in the } i \text {-th mass bin [mole } i / g m \text { coal] } \\
& n_{i} \text { (tar) }=\text { fraction of tar in the } i \text {-th mass bin [mole } i / g m \text { coal] } \\
& G_{i j} \quad=\quad \text { fraction of } j \text {-mers in the } i \text {-th mass bin [gm } i / g m j \text {-mer] } \\
& M T_{i} \quad=\quad \text { Effective Molecular weight of the } i \text {-th mass bin [gm } \\
& \text { i/mole i] } \\
& \chi_{i}=\text { the mole fraction of material in the } i \text {-th mass bin } \\
& \text { [mole } i / \text { mole coal] }
\end{aligned}
$$

To allow for the fact that some of the tar has already evolved from the char, we use for tar evolution only the difference $n_{i}$ (char)- $n_{i}(\operatorname{tar})$, (or zero if the difference is negative). Finally, we have

$$
\left(\frac{d W(\text { tar })}{d t}\right)_{D V C}=\sum_{j}\left(\frac{d n_{j}}{d t}\right) \cdot M_{j}
$$

Thus, from the differential equations for the $p$ 's and $q$, we compute (using Eqs. $4.13,4.17$ to 4.32 ) the quantities needed to evaluate the differential equation 4.6.

\section{Calculation of $F_{\mathbf{j}}(p, q)$ and $F(p, q)$}

The probability $F_{S, u}(p, q)$, that a site is a member of a cluster of $n$ sites with $s$ bridges and $u$ crosslinks, is given by

$$
F_{s, u}(p, q)=a_{s, u} p^{s}(1-p)^{\tau} q^{u}(1-q)^{v}
$$

where

$$
\begin{gathered}
n=u+s+1 \\
\tau=\left(\sigma_{i}+1\right) n-2 s \\
\nu=\left(\sigma_{2}+1\right) n-2 u
\end{gathered}
$$


and $\tau, v$ are the number of broken bridges and crosslinks, respectively, on the perimeter of the cluster, and $a_{s, u}$ is the number of different ways to form such a

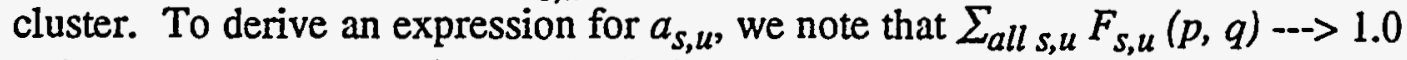
as both $p$ and $q$ go to 0 ; i.e., in the limit of no bonds, all sites are monomers with probability unity.

Second, define $Z$, $W$ such that

$$
Z=p(1-p)^{\left(\sigma_{1}-1\right)}(1-q)^{\left(\sigma_{2}+1\right)}
$$

and

$$
W=q(1-q)^{\left(\sigma_{2}-1\right)}(1-p)^{\left(\sigma_{1}+1\right)}
$$

then we have, after some rearrangement,

$$
\sum_{a, s} a_{s, u} Z^{s} W^{u}=\frac{1}{(1-p)^{\sigma_{1}+1} \cdot(1-q)^{\sigma_{2}+1}}
$$

Then, following Fisher and Essam (1961), this can be evaluated using a two variable version of Cauchy's theorem as

$$
a_{s, u}=\frac{1}{(2 \pi i)^{2}} \oint \frac{d Z}{Z^{s+1}} \cdot \oint \frac{d W}{W^{u+1}} \frac{1}{(1-p)^{\sigma_{1}+1} \cdot(1-q)^{\sigma_{2}+1}}
$$

to change variables in this double integral:

$$
d W / d Z=\frac{\partial(W, Z)}{\partial(p, q)} d p d q=\left[\frac{\partial W}{\partial p} \cdot \frac{\partial Z}{\partial q}-\frac{\partial W}{\partial q} \cdot \frac{\partial Z}{\partial p}\right] \cdot d p d q
$$

This can be evaluated from the definitions of $W, Z$; and we obtain the following integral for $a_{s, u}$ : 


$$
a_{s, u}=\frac{1}{(2 \pi i)^{2}} \oint d p \oint d q \frac{\left(1-\sigma_{1} p\right) \cdot\left(1-\sigma_{2} q\right)-p q\left(\sigma_{2}+1\right)}{p^{s+1} q^{u+1}(1-p)^{\tau+1}(1-q)^{\nu+1}}
$$

This is easily factored into integrals of the form given by Fisher and Essam (1961), leading to the following expression:

$$
a_{s, u}=\frac{\left(\sigma_{1}+1\right)\left(\sigma_{2}+1\right)}{(s+\tau)(u+v)}\left(\begin{array}{l}
s+\tau \\
s
\end{array}\right)\left(\begin{array}{l}
u+v \\
u
\end{array}\right)(u+s+1)
$$

Note that for $u=0$ (no cross-links), this reduces to the quantity $n b_{n}$ in Grant et al., (1989) and Fisher and Essam (1961). To determine the probability, $F_{n}(p, q)$, that a given site is a member of a cluster of $n$ sites, i.e., the fraction of $n$-mers, we must sum Eq. 4.34 over all possible values of $s$ and $u$ that give an $n$-site cluster:

$$
F_{n}(p, q)=\sum_{s=0}^{n-1} a_{s, u} p^{s}(1-p)^{\tau} q^{u}(1-q)^{\nu} ; \quad u=n-s-1
$$

The total fraction of sites, $F(p, q)$, in finite clusters is the sum over all $s$ and $u$

$$
F(p, q)=\sum_{s=0}^{\infty} \sum_{u=0}^{\infty} F_{s, u}(p, q)=\left(\frac{1-p}{1-p^{*}}\right)^{\sigma_{1}+1}\left(\frac{1-q}{1-q^{*}}\right)^{\sigma_{2}+1}
$$

where $p^{*}$ and $q^{*}$ are obtained by finding the smallest roots of

$$
\begin{aligned}
& p^{*} \cdot\left(1-p^{*}\right)^{\sigma_{1}-1} \cdot\left(1-q^{*}\right)^{\sigma_{2}+1}-p \cdot(1-p)^{\sigma_{1}-1} \cdot(1-q)^{\sigma_{2}+1}=0 \\
& q^{*} \cdot\left(1-q^{*}\right)^{\sigma_{2}-1} \cdot\left(1-p^{*}\right)^{\sigma_{1}+1}-q \cdot(1-q)^{\sigma_{2}-1} \cdot(1-p)^{\sigma_{1}+1}=0
\end{aligned}
$$

The quantity $F(p, q)$ is used as the fluid fraction in the full DVC model, which is not currently used in the fixed bed submodel. The critical point, where an infinite lattice begins to form (i.e., $F(p, q)$ begins to decrease), becomes a critical curve that divides the $p$ - $q$ plane into two regions. Note that for $q=0$, all the equations reduce to the single $\sigma$ case given in Grant et al., (1989) and Fisher and Essam (1961). 


\section{Summary of Active Differential Equations}

The Equation set that must be integrated for the FG-DVC submodel in the fixed bed code is thus

$$
\begin{aligned}
& \frac{d W_{i}(\text { tar })}{d t}=\left(\frac{d W(\text { tar })}{d t}\right)_{D V C} \cdot\left(\frac{W_{i}(\text { char })}{\sum_{i} W_{i}(\text { char })}\right)-k_{i} \cdot W_{i}(\text { tar }) \cdot\left[\begin{array}{l}
0 \\
1
\end{array}\right] \\
& \frac{d W_{i}(\text { char })}{d t}=\frac{d W_{i}(\text { gas })}{d t}-\frac{d W_{i}(\text { tar })}{d t}+\frac{d W_{i}(D V C)}{d t} \\
& \frac{d W_{i}(\text { gas })}{d t}=\left\langle k_{i}\right\rangle \cdot\left[W_{i}(\text { char })+W_{i}(\text { tar }) \cdot\left[\begin{array}{l}
0 \\
1
\end{array}\right]\right] \\
& \frac{d p_{L}}{d t}=-2 \cdot\left\langle k_{B}\right\rangle \cdot p_{L} \\
& \frac{d p}{d t}=-\left\langle k_{B}\right\rangle \cdot p_{L} \\
& \frac{d q}{d t}=\sum_{\mathrm{CO}_{2} \mathrm{CH}_{4}} \frac{d W_{i}(\text { gas })}{d t} \cdot \frac{X_{i}}{M_{i} \cdot \Sigma W_{i}(\text { char })} \\
& \frac{d n_{j}}{d t}=P_{j} \chi_{j}\left(\frac{d n_{i}}{d t}\right)_{g a s \text { only }}\left(\frac{1}{P_{0}+\Delta P-\sum_{\text {all tars }} P_{j} \chi_{j}}\right) \\
& \frac{d E_{i}^{m}(t)}{d t}=\left\langle k_{i}\right\rangle \cdot \frac{\int_{E_{i}^{m}(t)}^{\infty} f_{i}(E) d E}{f_{i}\left(E_{i}^{m}(t)\right)}
\end{aligned}
$$




\section{CHAPTER 5}

\section{SOLUTION METHOD}

The set of differential equations presented in Table 3.2, along with the auxiliary equations summarized in Tables 3.4 and 3.5, form the core of the FBED-1 model. These equations constitute a split boundary value problem, because the boundary conditions for all the dependent variables are not known at the same boundary. For the solid stream the boundary conditions are known at the top of the gasifier, whereas the boundary conditions for the gas stream are known at the bottom of the gasifier. Split boundary value problems can be solved by a shooting technique in which the unknown conditions at either boundary are guessed and then the system of equations is integrated to the other boundary. This requires iteration on the guessed conditions in order to satisfy the known boundary conditions.

In FBED-1, the two-zone, zero-dimensional submodel, FBED-0, as discussed in Chapter 2 is used to convert the split boundary value problem into an initial value problem by providing an initial estimate of the effluent gas composition and temperature. However, the gas exit temperature predicted by the two-zone submodel is always high due to the assumption that the devolatilization zone is at a single temperature. Likewise, the exit solid temperature may be high due to the uniform temperature and well-mixed assumption in the equilibrium zone. Thus after integrating from the top to the bottom of the reactor, the calculated temperature and compositions may be different from the input feed gas temperature and compositions. Therefore, a new guess for the product gas temperature and compositions must be obtained. This guess is obtained by integrating the gas phase quantities from the bottom to the top of the gasifier. This procedure can be repeated in an iterative manner until the calculated temperature and composition of the feed 
Solution Method

gas satisfy the input feed gas conditions within the specified tolerances. The details on this iterative solution method are discussed in the next section.

\section{Computational Algorithm}

The computational algorithm for the one-dimensional model, using the twozone submodel to obtain the initial guess for the gas phase quantities, is shown in Figures 5.1A to 5.1D. Subroutine names are shown in italics. The two-zone submodel solves overall energy and mass balances around the devolatilization and drying zone as well as the equilibrium (combustion and gasification) zone.

The initial conditions for the differential equations given in Table 3.2 are determined from the known values for the solid phase quantities and the values predicted by FBED-0 for the gas phase quantities. The integration of the system of equations is performed by LSODE (Hindmarsh, 1983). The derivatives of the dependent variables are evaluated by computing all the needed physical and thermodynamic quantities as given by the auxiliary equations listed in Table 3.7. The dependent variables are converted to the quantities of interest; e.g., total gas enthalpy is converted to gas temperature. The particle diameter and number density are calculated from Equations 3.22, and 3.24, respectively. Transport properties are calculated at the solid and gas temperatures and the corresponding particle diameter. Next, drying, devolatilization, oxidation and gasification rates are calculated.

The directions of the solid and gas flows are important. Flow in the positive axial direction, taken to be in the upward direction from the bottom to the top of the reactor, is considered positive, e. g., the gas flow rate. The flow rate of the solids is taken to be negative because the solid phase flows in the negative axial direction. 


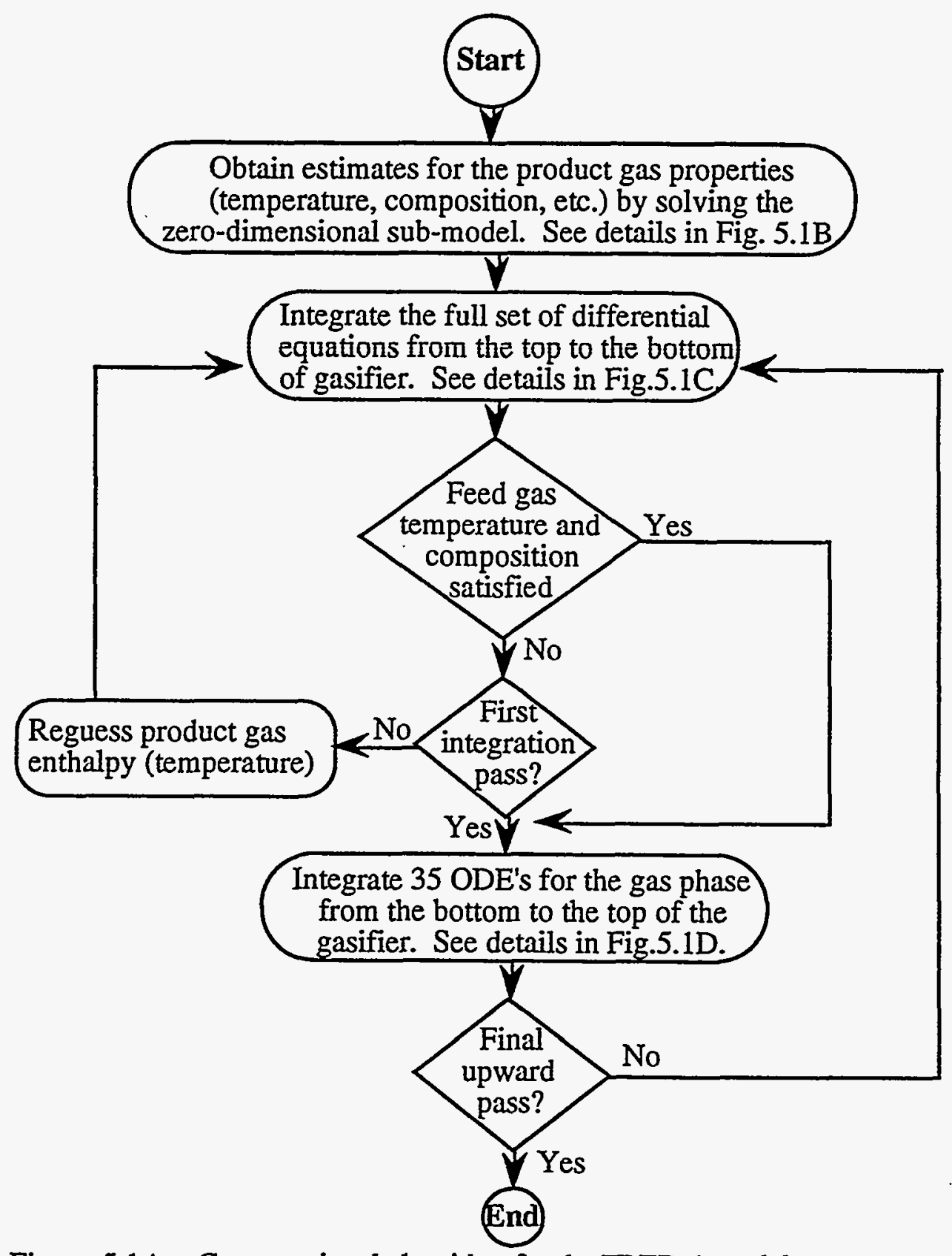

Figure 5.1A. Computational algorithm for the FBED-1 model. 


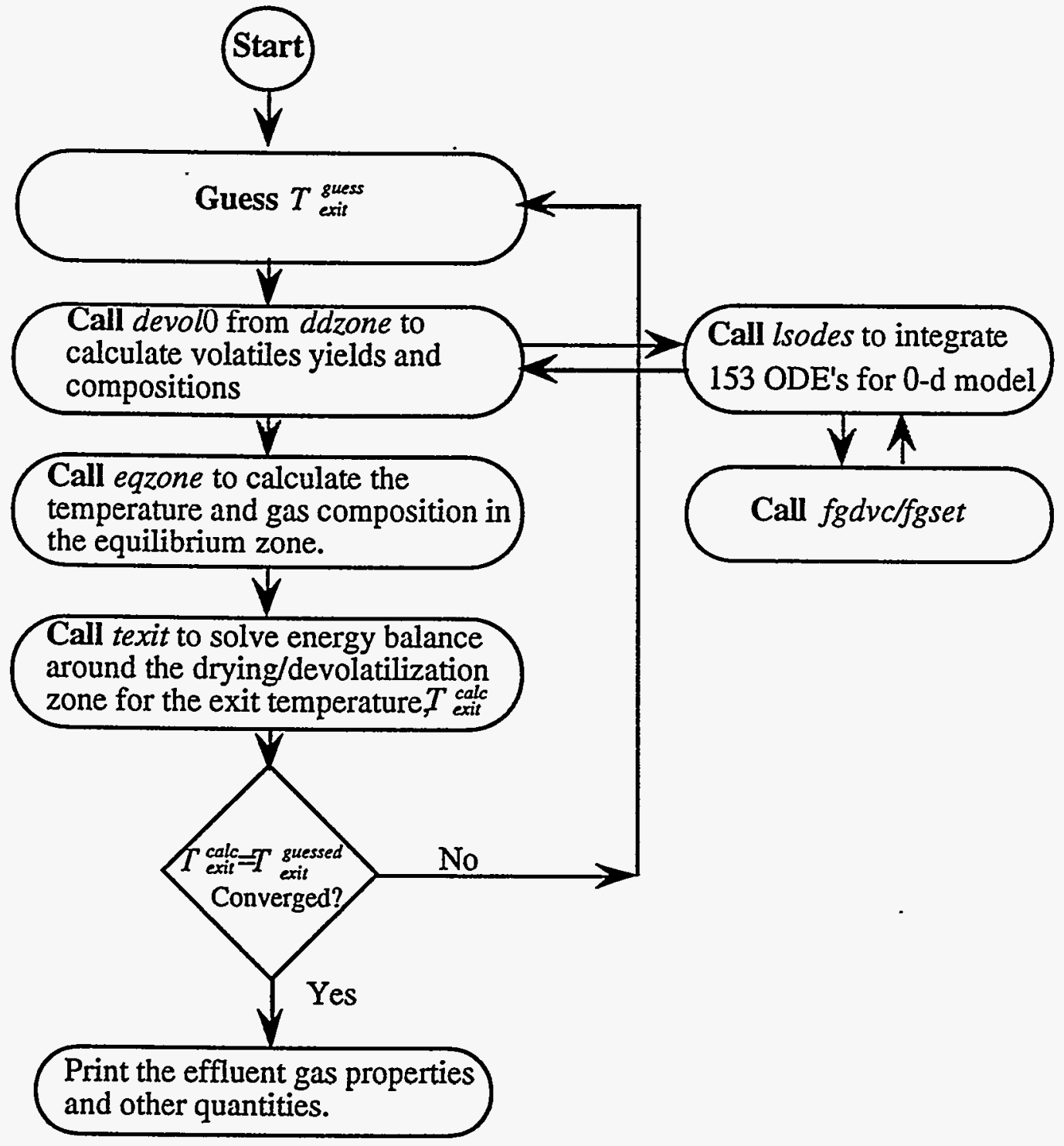

Figure 5.1B. Details of computational algorithm for the zero-dimensional submodel, FBED0D. 


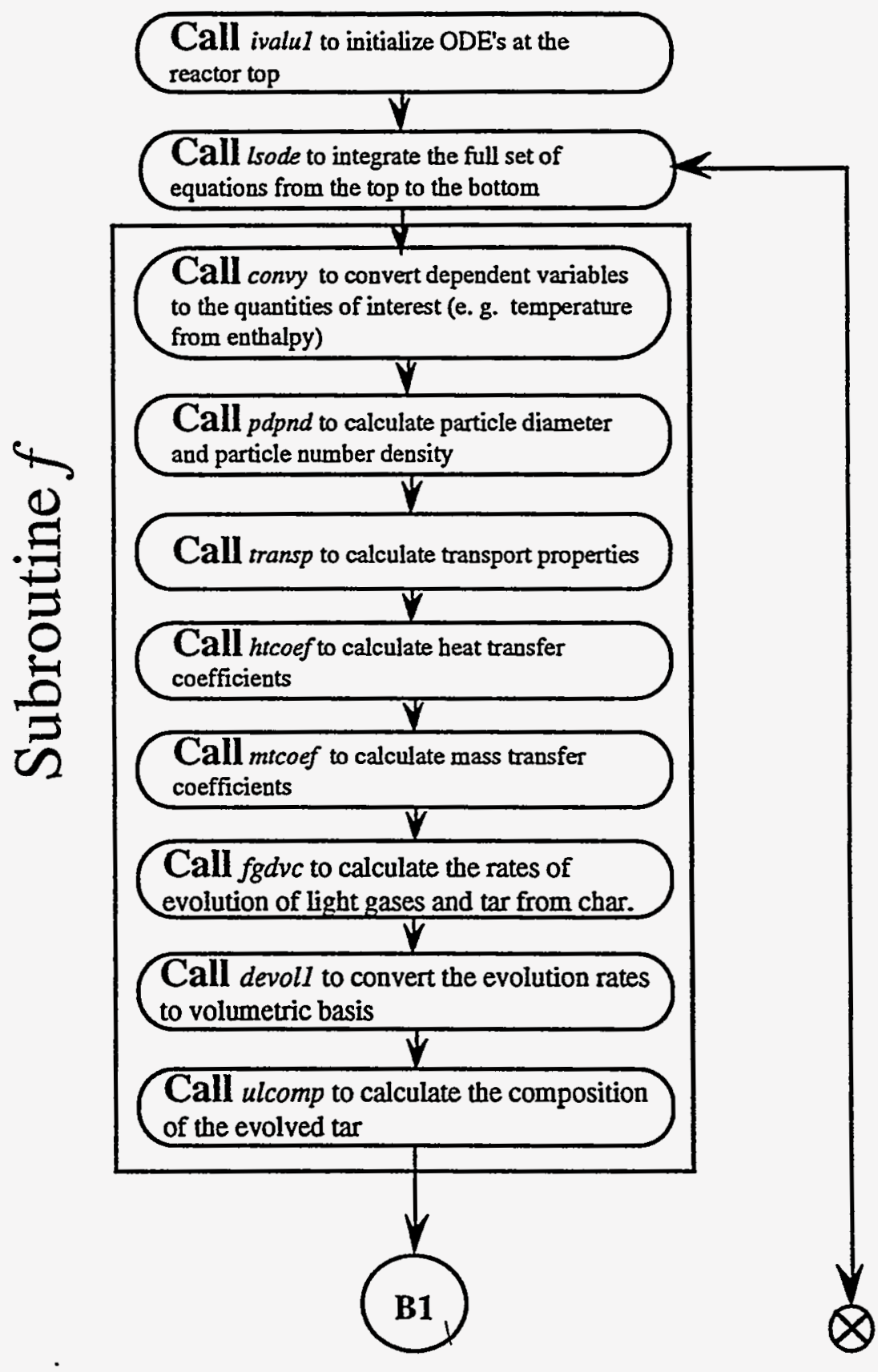




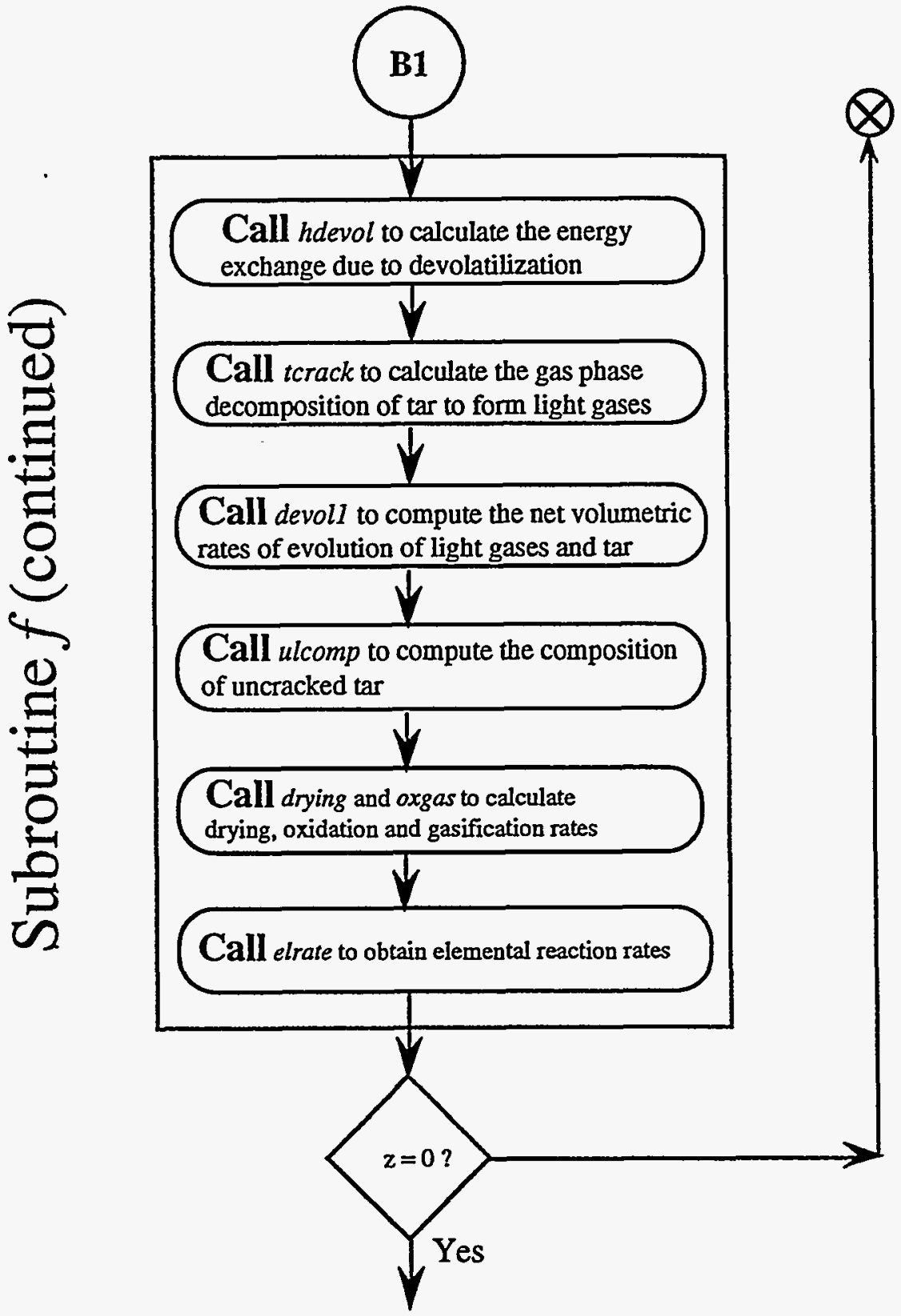

Figure 5.1C. Details of computational algorithm for the downward integration pass. 


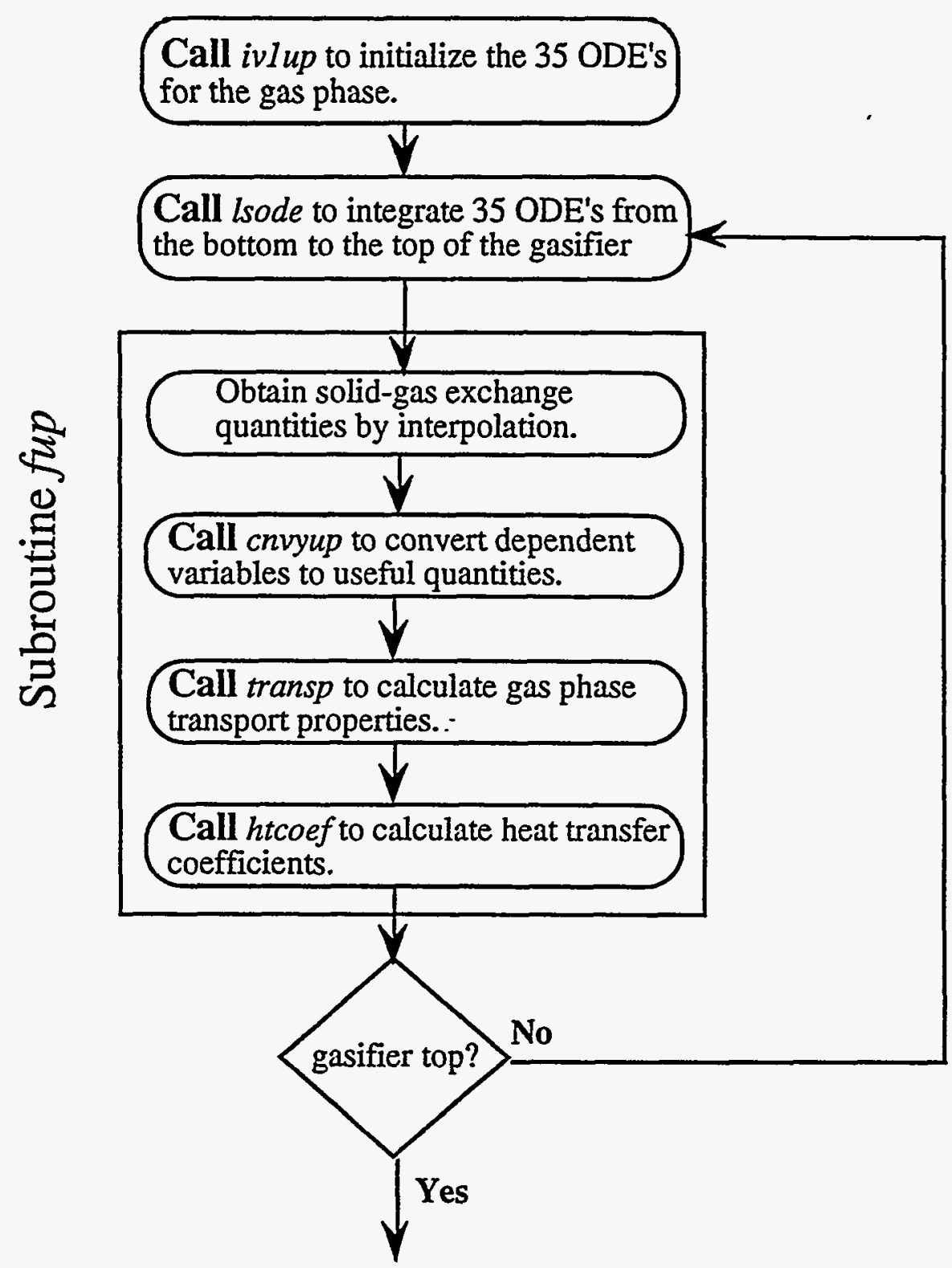

Figure 5.1D. Details of computational algorithm for the upward integration pass. 
After integrating from the top to the bottom of the reactor, the calculated feed gas temperature and composition are compared to the input feed gas temperature and composition. If the values do not agree within the specified tolerances, the temperature and composition of the product gases must be guessed again and the procedure repeated in an iterative manner. In order to obtain a new estimate for the product gas temperature and composition, 35 differential equations for the gas phase, which are a subset of the full system of governing equations, are integrated from the bottom to the top of the gasifier. In the upward integration pass, the solid phase variables are held constant, and the solid-gas exchange quantities are calculated from the values predicted during the downward integration pass. This yields a new guess for the gas phase quantities at the top of the gasifier; this guess is then used for the next downward integration pass. This procedure, called split back-and-forth integration, may be repeated untill all the boundary conditions have been satisfied. Simulations with consecutive back-and-forth integrations did not show much change in the composition of the product gas. The predicted feed gas composition was also found to be close to the input feed gas composition and did not show much change in subsequent iterations. However, this procedure did not yield a converged feed gas temperature. In order to satisfy the feed gas temperature, the split back-and-forth integration was coupled with the shooting method with the product gas enthalpy as the iteration variable. In this scheme, the product gas enthalpy is varied, while all other gas phase quantities are held constant, and the complete set of equations is integrated from the top to the bottom of the gasifier. Once the feed gas temperature is converged within the specified tolerance, an upward pass is taken to compute the final product gas composition and temperature.

In general, burnout should also be used as an iteration variable. The predicted burnout from the one-dimensional model should be used in the zerodimensioanl model to obtain new estimates for the product gas properties. However, burnout is typically high in fixed-bed gasifiers and is, therefore, assumed to be unity in our formulation. Iterations may be done both in an interactive mode and in an automatic mode. In the interactive mode, iterations are performed by adjusting the product gas temperature through the product gas enthalpy. A positive factor will increase the product gas temperature; a negative factor will lead to a lower product gas temperature. It is pointed out that a good set 
of input parameters is essential for this procedure to work properly. The code will successfully execute in a noninteractive mode, provided the predicted value of the feed gas temperature obtained in the first iterative pass is close to the input feed gas temperature. Convergence is typically obtained in 8-10 iterations; CPU time is typically around one hour on a CONVEX 220 computer, and around two hours on a SUN 4/310 computer. 


\section{PART TWO: CODE USER'S INSTRUCTIONS}

\section{CHAPTER 6}

\section{USER'S GUIDE}

FBED-1 was developed on a SUN 4/310 running under the UNIX operating system. The code is written in FORTRAN 77 and should be portable to machines with compatible compilers. Two plotting routines written for the DISSPLA graphics package are also distributed with the code and are discussed later. The source code occupies approximately 1.2 Megabytes of disk space. The executable and object code requires an additional 2 Megabytes of disk space. A total of 10 Megabytes may be needed for the additional graphics and output files.

The structure of the FBED-1 code and a description of the subroutines is presented in the next section. The functions performed by the subroutines used in the FBED-1 code are given in Appendix H. All input data to FBED-1 are in SI units. The input files are discussed later in this Chapter. All working variables within the program are either dimensionless or in SI units. Units associated with all variables are either given in the nomenclature or can be found in Appendix I.

CPU run time depends on the particular hardware on which the code has been implemented, as well as on the degree of compiler optimization. Typical CPU time on a CONVEX 220 is around one hour. The same simulation on a SUN 4/310 requires around two hours of $\mathrm{CPU}$ time. 
User's Guide

\section{Description of Subroutines}

The computational algorithm for FBED-1 was shown in Figure 5.1. A tree diagram of the structure of the program showing most of the actual subroutines is given in Figures 6.1 and 6.2. The differential equation solver LSODE or LSODES is represented by it's main routine, shown as a black box in Figures 6.1 and 6.2.

The main program is named FBEDID; its structure and the various routines called by it are shown in Figure 6.1. The routine CPUTIM is called to track the execution time. The first function performed by the main program FBEDID is the reading of the input data. This is accomplished through the subroutine READIN. The subroutine READIN reads the input data from the data file FB1IN. It also invokes the reading of the input data file FBITHM from the subroutine CREE0 and COAL0.ARGX from the subroutine COALIN. The X in the COAL0.ARGX corresponds to an appropriate value as input by the user. The subroutine COALIN also adjusts the input functional group composition, which is read from the data file COAL0.ARGX, to match the ultimate composition of the feed coal. This is achieved by a call to the subroutine ADJSTYO. In addition, subroutine READIN also computes the coefficients for the polynomial expression of ash heat capacity through a call to the module ASHCP and the input properties of the feed gas through a call to the module GASIN. The input files, which are read in the module READIN, are discussed further in the next section. Then the subroutine ECHOIN is called to echo the input data, as well as a number of computed quantities to the output file, FB1OUT, which is discussed in more detail in the next section. Then the main program FBED1D calls the routine FBEDOD, which provides the initial estimates of the effluent gas properties. The structure and the routines associated with subprogram FBED0D, shown in Figure 6.2, are discussed later in this section. To compute the CPU time for the zero-dimensional portion of the code, another call is made to the routine CPUTIM. Next, the dependent variables are initialized through a call to either IVALU1 or IV1UP, depending on the direction of integration. The routine IVALU1 also calls the routine IVALU0 to initialize the dependent variables that relate to the zero-dimensional portion of the code, i.e., for the devolatilization submodel. In order to initialize the derivatives of all the dependent variables at the start of the integration, a call is made to the subroutine $F$. 
Chapter 6

The subroutine $\mathrm{F}$ calls a number of other subroutines, which are discussed later. The integration of the system of differential equations is performed by the LSODE integration package. The main integration routine LSODE also calls the subroutine $\mathrm{F}$, as needed, to compute the derivatives. The control is transferred back to the main program FBED1D when the independent variable attains the final value tout, as specified in the main program. At this stage, another call is made, from the main program FBEDID, to either F or FUP, to compute the derivatives of the dependent variables at tout. The results are printed in the output files at each output location. A machine dependent routine FLUSH is used to write the buffer to the output files. This procedure is repeated iteratively following the logic discussed in the Chapter 5. Finally, after a converged solution is obtained, the mass and energy balance calculations are performed by calling the subroutine BALANC.

The subroutine $F$ is the most important of all because it computes the derivatives of the dependent variables, which is done through calls to a number of other routines. First, the routine CONVY is called, which converts the dependent variables to the quantities of interest such as temperature from enthalpy. This routine also calculates the equilibrium composition and temperature of the gas, and the temperature of the solid stream, by calling the equilibrium code CREE and the subroutine PTEMP. When the gas phase is kept out of chemical equilibrium, the gas temperature is determined by a call to the subroutine GTEMP. The routine PDPND is called to update the particle diameter as well as particle number density. Next, transport properties of the gas phase are computed by calling the routine TRANSP.

The next step is the calculations of the heat and mass transfer coefficients, accomplished through calls to the routines HTCOEF and MTCOEF respectively. Now the derivatives of the dependent variables related to the devolatilization model are computed by calling either the routine FGDVC or FGSET. Next, the volumetric rates of devolatilization are calculated by calling the routine DEVOL1. The ultimate composition of the evolved tar is computed by the routine ULCOMP. The heat of devolatilization is computed by the routine HDEVOL. The decomposition of tar to the gas is calculated by the subroutine TCRACK. The subroutines DEVOL1 and ULCOMP are called again to update the volumetric rates 


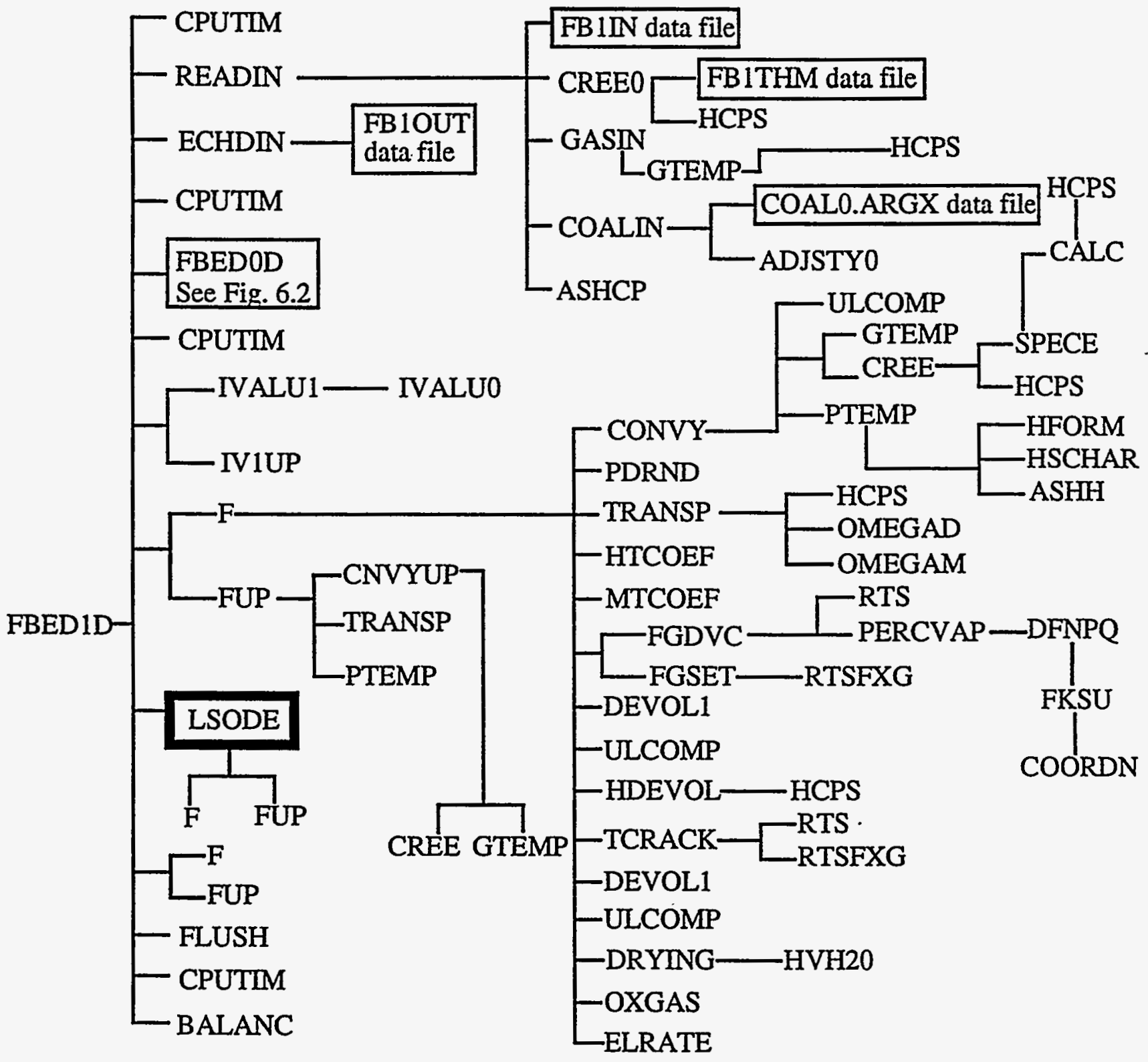

Figure 6.1. Structure of the main program FBED1D. 


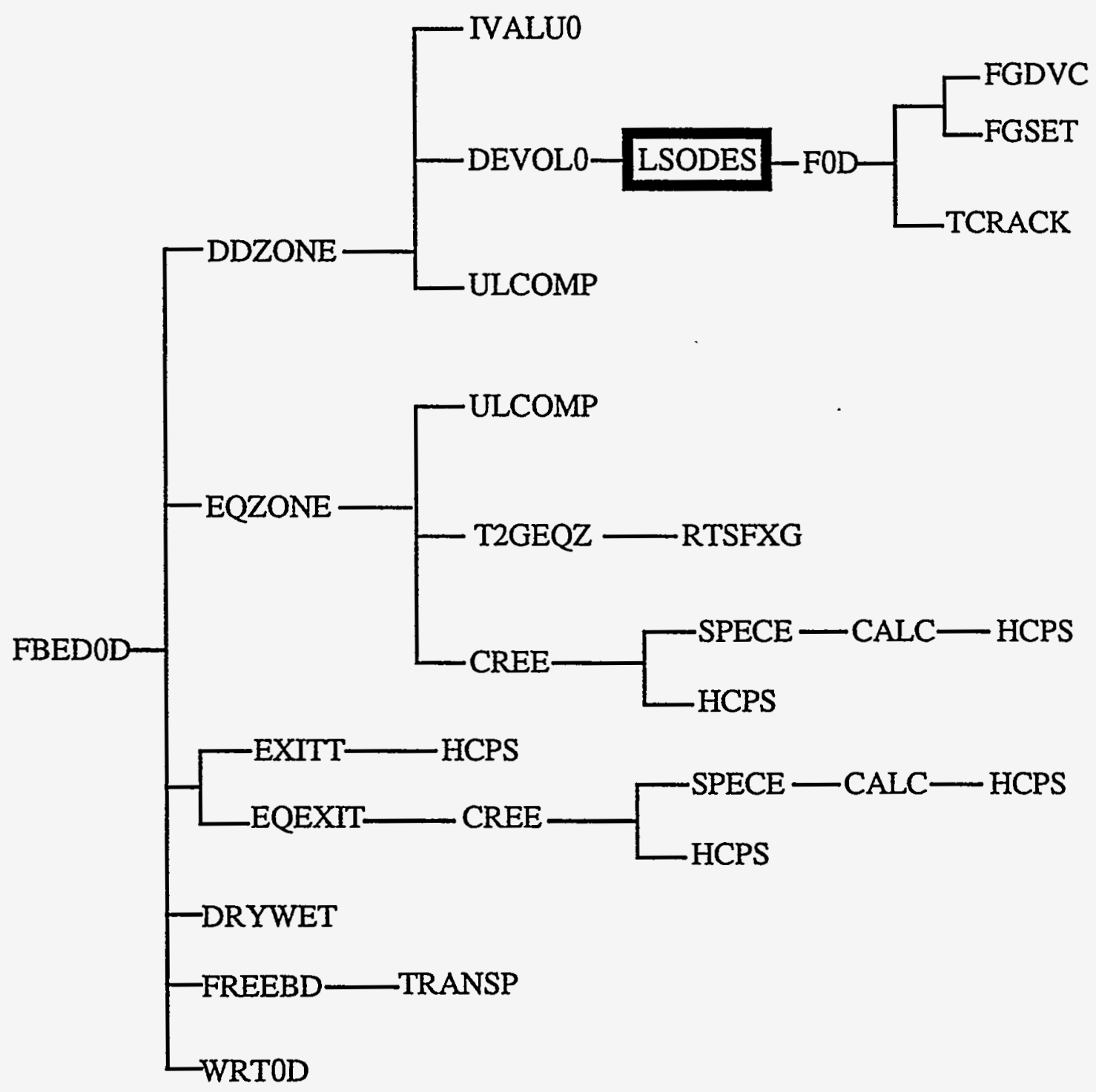

Figure 6.2. Structure of the subprogram FBED0D. 
User's Guide

of tar and gas evolution and the ultimate composition of the tar. Then volumetric rates of drying, oxidation and gasification are calculated by calling the routines DRYING and OXGAS. Finally, the subroutine ELRATE converts the volumetric rates to elemental basis. These computed quantities are then used to compute the derivatives of the quantities related to the one-dimensional model.

During the upward integration pass, a subset of the full differential equation set is integrated. The number of equations for the upward pass is 35; these are related to the gas and tar quantities. The corresponding routines for initialization, computations of the derivatives, and conversion of the dependent variables to the quantities of interest are IV1UP, FUP, and CNVYUP.

The subroutine FBEDOD calculates the effluent gas temperature and composition according to the two-zone submodel discussed in chapter 2 . The program structure for the FBEDOD is shown in Figure 6.2. First, it calls the subroutine DDZONE, which in turn calls the subroutines IVALUO, DEVOL0, and ULCOMP. The routine IVALUO initializes the variables for the zero-dimensional model. The subroutine DEVOLO calculates the ultimate volatiles yield and composition based on the selected devolatilization submodel. This is accomplished through the integration of the system of equations for the zero-dimensional submodel. The differential equations for this submodel are integrated using the sparse version of the integrator, i.e., LSODES, which is called from DEVOLO. The subroutine DDZONE also computes the ultimate composition of tar and char. Next, the subroutine EQZONE is called, which computes the composition and temperature of the gases leaving the equilibrium zone and entering the drying and devolatilization zone. Then the temperature of the gases leaving the drying and devolatilization zone is computed either by assuming all gases to be in thermal and chemical equilibrium, or by keeping the moisture and the gases released in the drying and devolatilization zone to be out of chemical equilibrium. This is accomplished by calls to the subroutines EQEXIT and EXITT respectively. This procedure is repeated iteratively following the logic explained in Figure 5.1B. Finally, the effluent gas composition is determined on both dry and wet basis, heat loss in the freeboard is calculated, and the results are written to the output file FB1OUT by calling the subroutines DRYWET, FREEBD, and WRTOD respectively. 
Chapter 6

\section{Program Input}

Three input data files are needed for the execution of FBED-1. These files are text files and can be generated or modified using any text editor. The first input data file, FBIIN, provides the essential input data, which include the operational parameters, e.g., gasifier dimensions, temperatures, flow rates and compositions of the gas and solid streams, flags for submodel selection, etc. In addition, values for parameters needed for integration routines, such as tolerances and operation mode (single-step/multi-step), are provided. All variables are fully explained in the input file. The second data file, FBITHM, provides the elemental and species data for thermodynamic calculations and parameters for the calculation of transport properties. The third data file provides the input data for the devolatilization submodel; data include the number of functional groups, the initial functional group composition, the kinetic parameters for rate coefficients based on the distributed activation energy model, and cross-linking efficiency for each functional group. In addition, input values for the DVC portion (percolation submodel) are provided. An optional input file EXPT.DAT is also needed when the experimental data for the effluent gas properties are available. A description of these data files is given in the next section.

\section{Main Input Data File, FB1IN}

A typical input data file (FB1IN) is shown in Table 6.1.

Table 6.1 Main data file, FB1IN.

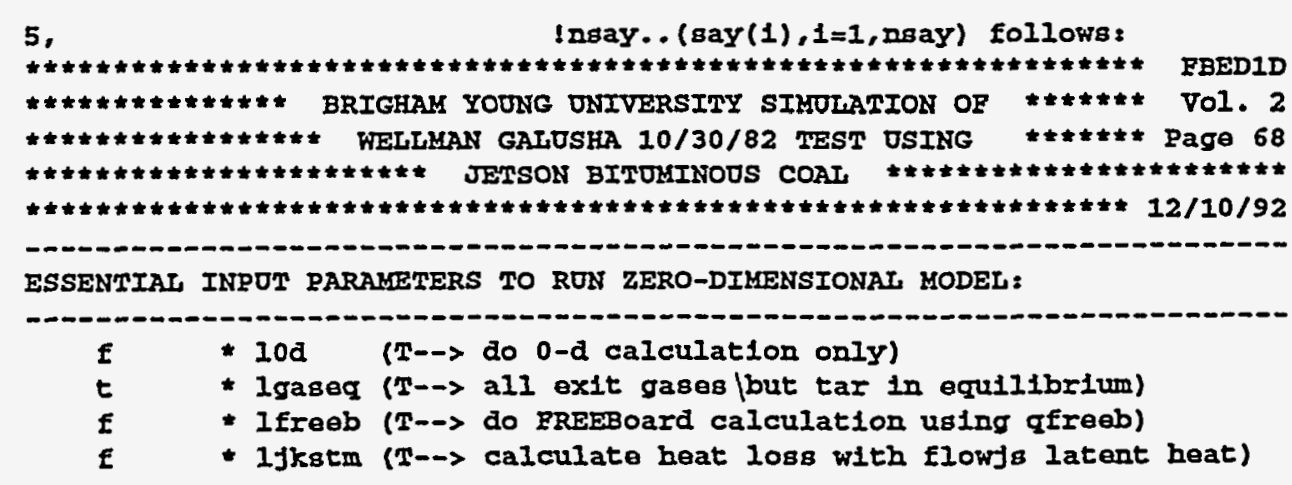


User's Guide

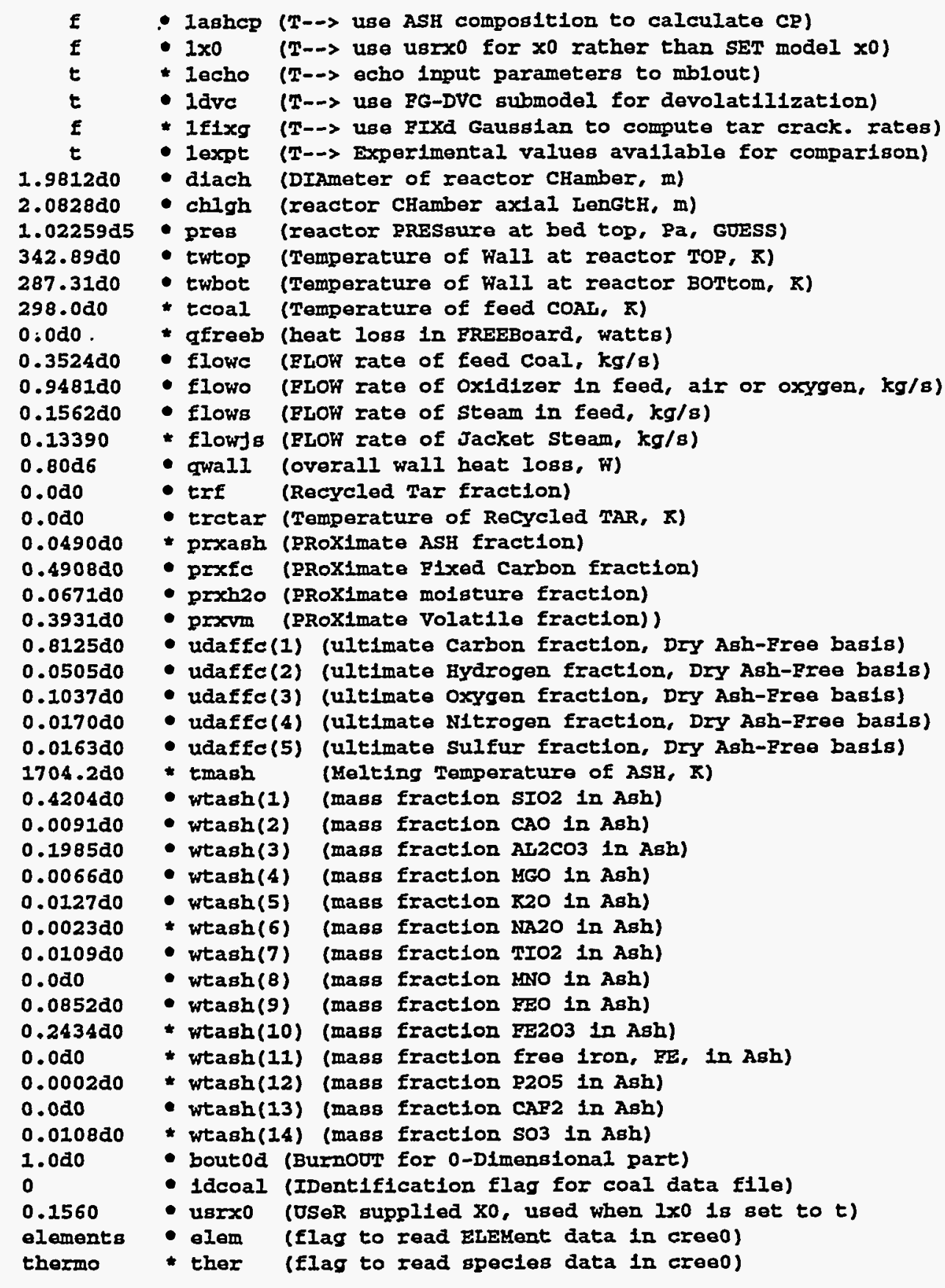


Chapter 6

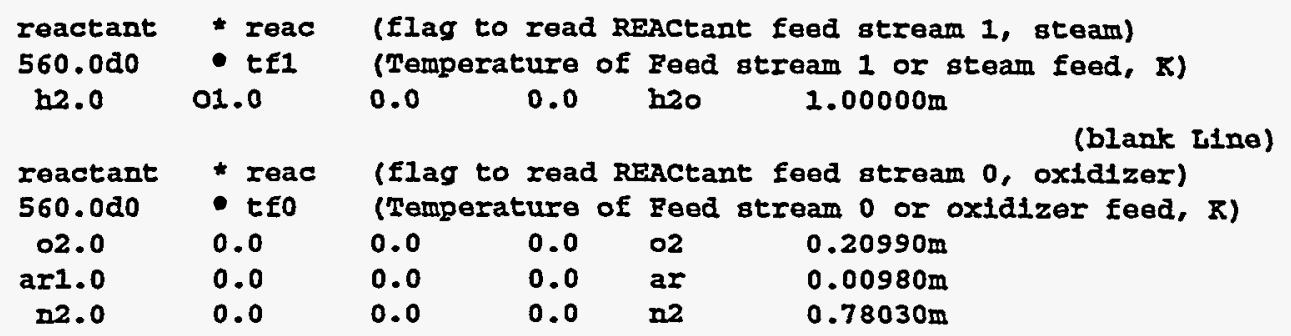

(blank Line)

ADDITIONAL INPOT PARAMETERS TO RON ONE-DIMENSIONAL MODEL:

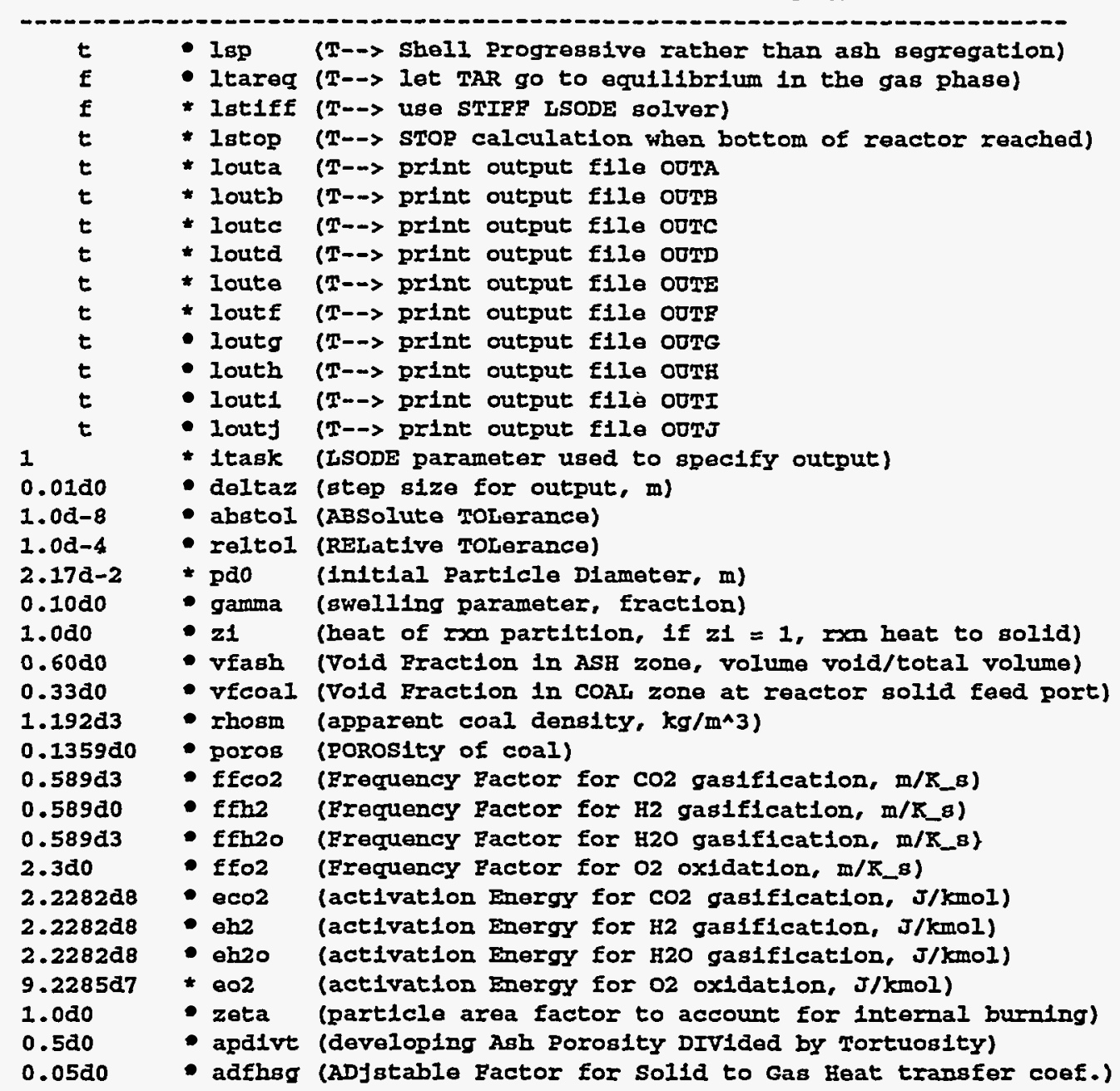




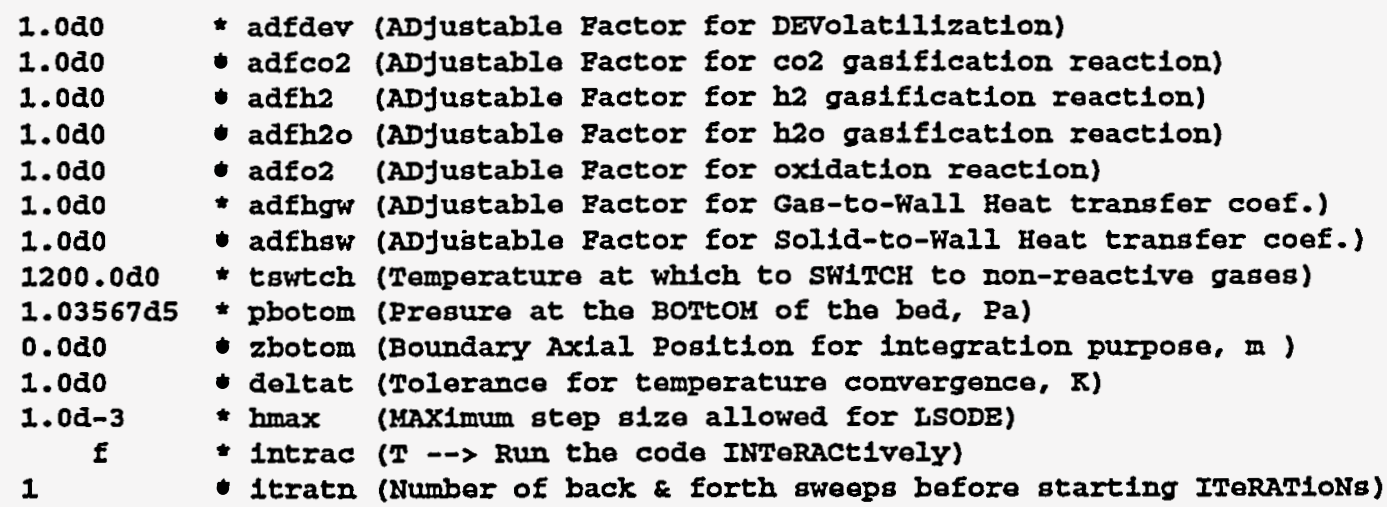

When setting up a new problem for simulation, it is easiest to start by editing the input files from a previous simulation, since much of the information remains unchanged. The main input file, FB1IN, is divided into two major sections. The first section contains the required data to run the two-zone, zerodimensional submodel; the second section contains the additional data required to run the one-dimensional model.

Most of the input data in FB1IN are single line, format free entries. After the data value, a comment statement is provided to describe the input variable. The variable is given in lower case, followed by its description and units, if required. Upper case is used in description to indicate the selection of the variable name (e.g., diach DIAmeter of reactor CHamber, $\mathrm{m}$ ).

The first entry in the file FB1IN is the integer nsay, which indicates the number of header statements that are printed at the top of the main output file FBIOUT. Any number of comment statements may be included in the header. In the sample file shown in Table 6.1, five comment lines are used to describe the simulation. Following the five comment lines, three more lines are used to differentiate the zero-dimensional input parameters from the one-dimensional input parameters. These three lines are required and must be included in the input file.

The next nine entries provide logical input parameters for the zerodimensional model in an L5 format: 10d, lgaseq, lfreeb, ljkstm, lashcp, 
Chapter 6

Ix0, lecho, Idvc, and Ifixg. These options are discussed in more detail later in this chapter. The next entry is the value of the logical variable lexpt in L5 format. If this variable is set to .true., then the optional input file EXPT.DAT must be provided. The next fourteen entries following the logical inputs are format free and describe the reactor geometry (diach and chlgh), reactor pressure at the bed top (pres), temperature of the wall at the top of the reactor (twtop), temperature of the wall at the bottom of the reactor (twbot), the feed coal temperature (tcoal), the freeboard heat loss (qfreeb), the flow rate of the feed coal (flowc), the flow rate of the oxidizer in the feed (flowo), the flow rate of the steam in the feed (flows), the flow rate of jacket steam (flowjs), the 0-D overall wall heat loss (qwall), the recycled tar fraction (trf), and the temperature of the tar recycle fraction (trctar). All of these fourteen parameters are not used in the zero-dimensional calculation. The recycled tar fraction and temperature are used only when the recycle stream is provided; otherwise these are set to zero. Similarly, the jacket steam flow rate is used to calculate the wall heat loss based on the heat of vaporization of steam, provided the logical variable ljkstm is set to .true.. When the wall heat loss is calculated based on the jacket steam flow rate, the estimated wall heat loss is set to zero.

The next nine entries provide the proximate and the ultimate analysis of feed coal. These are followed by the melting temperature and the composition of the mineral constituents of the ash. The next entry, bout0d, specifies the burnout of char for the zero-dimensional portion. The next two entries essential to run the zero-dimensional portion of the code are related to devolatilization. The integer variable, idcoal, governs the selection of input data file for the FG-DVC submodel; it is discussed later. The next variable, usr $\mathbf{0} 0$, is used to specify the potential tar-forming fraction when the flags Ix0 is set to .true. and the flag Idvc is set to .false..

The next two entries, elem and ther, invoke the reading of the data from the thermodynamic input data file FBITHM. The keyword reac invokes the reading of feed gas streams temperature and composition. In FBED-1 formulation, only two gaseous reactant streams are allowed; steam and oxidant. The oxidant stream may be either air or oxygen. A blank line signifies the end of data for a particular reactant stream and must be included. Following the 
keyword reac, the temperature of the stream is input in a format free manner. This is followed by the species atomic composition. A separate line is used for each species. The atomic composition of each species is provided by formatted input, $4(a 2, f 7.5)$. The character string, in a2 format, describes the element. For example, " $h$ " is used for hydrogen as shown in Table 5.1. Note the blank before the " $h$ " is required for the 22 format. The real value following the element character string, in f7.5 format, describes the number of atoms. For example, in Table 6.1, " h2.0 indicates two hydrogen atoms per water molecule. The four blanks following the 2.0 are consistent with the 77.5 format specification. Following the description of the atomic composition of the species, the species name is described in a $2 \mathrm{a} 4$ format. Finally, the composition of the species in the reactant stream is provided with a format specification of $(1 x, f 7.5,1 a)$. For example, pure steam is specified as " $1.00000 \mathrm{~m} "$ in Table 6.1 . A blank must precede the concentration fraction as required by the format specification. The character " $\mathrm{m}$ " following the concentration fraction specifies the fraction as a mole fraction. Weight fractions can be specified by using a "w" instead of an " $\mathrm{m}$ " after the species concentration fraction. The complete format specification for the species definition is $(4(\mathrm{a} 2, \mathrm{f} 7.5), 2 \mathrm{a} 4,1 \mathrm{x}, \mathrm{f} 7.5, \mathrm{a} 1)$.

The second half of the input file FBIIN is used to provide the additional input data that are necessary to run the one-dimensional fixed-bed model, as described in chapter 3. The additional one-dimensional parameters are separated from the essential zero-dimensional input parameters by the required three comment lines, which are followed by fifteen lines of logical input parameters: lsp, Itareq, Istiff, Istop, louta, loutb, loutc, loutd, loute, loutf, loutg, louth, louti, and loutj. Parameters essential to the differential equation solver then follow. The differential equation solver, LSODE, requires itask and deltaz to specify the mode of operation (single-step or multi-step) and output. Tolerances are specified with the variables abstol and reltol. For more information on the differential equation solver LSODE, the reader is referred to Hindmarsh (1983).

The diameter of the feed coal particles is then specified as pdo. In FBED-1, multiple particle sizes in the feed coal are not treated. Particle swelling is assumed to be proportional to the extent of devolatilization. The swelling parameter 
is input as gamma. The next entry, zi, specifies the heat of reaction partition, which assigns part of the heat of reaction to solid phase and remainder to the gas phase. The void fractions at the top and bottom of the bed are represented by vfcoal and vfash, respectively. Next, the format free apparent coal density and coal porosity are represented by rhosm and poros respectively. Likewise, the gasification and oxidation kinetics are also provided in format free manner. The frequency factors are provided first, followed by the activation energies: ffco2, ffh2, ffh 2o, ffo2, eco2, eh2, eh2o, and eo2. The remaining parameters required to run FBED-1 are used to study the sensitivities of various parameters. Some of these values represent the observed physical phenomena. The remaining format free parameters are the particle area factor, used to account for internal particle burning (zeta), the developing ash porosity divided by tortuosity squared (apdivt), the adjustable factor for the solid-to-gas heat transfer (adfhsg), the adjustable factor for devolatilization mass transfer (adfdev), the adjustable factor for $\mathrm{CO}_{2}$ gasification kinetics (adfco2), the adjustable factor for $\mathrm{H}_{2}$ gasification kinetics (adfh2), the adjustable factor for $\mathrm{H}_{2} \mathrm{O}$ gasification kinetics (adfh20), the adjustable factor for oxidation kinetics (adfo2), the adjustable factor for gas-towall heat transfer (adfhgw), and the adjustable factor for solid-to-wall heat transfer (adfhsw). The next entries are tswtch, the temperature below which the gases are assumed to be nonreactive; pbotom, the pressure at the bottom of the gasifier bed; zbotom, which specifies the axial position at the bottom of the gasifier; deltat, the tolerance for the feed gas temperature; hmax, which specifies the maximum step size allowed in integration routines; and intrac, a logical variable. When the flag intrac is set to .true., the code can be executed in an interactive mode. The final entry in this file is itratn, which species the number of back and forth integration sweeps before the iteration on the feed gas temperature is started.

\section{Thermodynamic Data File, FB1THM}

The second input data file, FB1THM, provides the data needed to calculate the thermodynamic and the transport properties of the gaseous species. A typical data file is shown in Table 6.2. In FBED-1, a maximum of twenty five gaseous species may be treated. This number, however, may be increased by changing the value of parameter maxsp in all the subroutines where it is used. In our 
User's Guide

formulation, twenty two species are actually treated, as shown in Table 6.2. This file does not change unless a new species or element is needed. The thermodynamic properties data file is read from the subroutine CREE0. The keywords elem and ther in the main data file FB1IN are used to invoke the reading of data from the thermodynamic input data file FB1THM.

The input file FB ITHM is divided into three major sections: element data, species thermodynamic data, and species transport data. The elemental data are read in by specifying the species name, molecular weight, and valence. The elemental data use the following format specification: a2,8x,2f10.6. The order in which the elements are listed must be $\mathrm{C}, \mathrm{H}, \mathrm{O}, \mathrm{N}, \mathrm{S}$, and Ar. A blank line is required between the elemental data and the species thermodynamic data, as shown in Table 6.2.

The thermodynamic data for each species are entered on three separate lines. The first line is used to specify the species name and the elemental composition. The second and third lines are used to specify the polynomial coefficients for the calculation of the thermodynamic properties (heat capacity, enthalpy and entropy) of the species. The format for specifying the species name and elemental composition is $3 a 4,12 x, 4(a 2, f 3.0)$. Following the species name, the source and date of the data are also provided, as shown in Table 5.2 (e. g., $\mathrm{j}$ 9/65) The " $\mathrm{j}$ " indicates the source of the data as being the JANAF thermochemical tables (Stull and Prophet, 1971). Also, the entry "g $300.0005000 .000 "$ indicates that the species is a gas over the temperature range 300 to $5000 \mathrm{~K}$. FBED-1 does not use the literature source and date, species physical state, and valid temperature range. This information, however, is available in Table 6.2 for reference.

A blank line is required between the species thermodynamic data and the species transport data. The format free input for the species transport data is read in by the subroutine READIN. The species transport data include the Stockmeyer collision diameter (s), Lennard-Jones temperature parameter (ek), and the nonpolar correction factor for the Lennard-Jones parameter (delta). 
Chapter 6

Table 6.2 Thermodynamic Input Data File, FB1THM.

$\begin{array}{llr}\text { c } & 12.01115 & 4.0 \\ \text { h } & 1.00797 & 1.0 \\ \text { o } & 15.9994 & -2.0 \\ \text { D } & 14.0067 & 0.0 \\ \text { B } & 32.06 & 4.0 \\ \text { ar } & 39.9480 & 0.0\end{array}$


User's Guide

nh3 I 9/65n $1 . \mathrm{h} \quad 3.00 \quad 0.00$ 0.g $300.000 \quad 5000.000$

$0.24165173 d+01 \quad 0.61871186 d-02-0.21785136 d-05 \quad 0.37599057 d-09-0.24448854 d-13$ $\begin{array}{lllll}-0.64747109 d+04 & 0.77043467 d+01 & 0.35912762 d+01 & 0.49388665 d-03 & 0.83449304 d-05\end{array}$ $-0.83833385 d-08 \quad 0.27299092 d-11-0.66717070 d+04 \quad 0.22520962 d+01$ J $6 / 63 \mathrm{n} \quad 1.0 \quad 1.00 \quad 0.00 \quad 0 . g \quad 300.000 \quad 5000.000$

$0.31889992 d+010.13382279 d-02-0.52899316 d-06 \quad 0.95919314 d-10-0.64847928 d-14$

$0.98283242 d+04 \quad 0.67458115 d+01 \quad 0.40459509 d+01-0.34181783 d-02 \quad 0.79819174 d-05$ $\begin{array}{llll}-0.61139254 d-08 & 0.15919072 d-11 & 0.97453867 d+04 & 0.29974976 d+01\end{array}$ 02 \begin{tabular}{llllllll}
\hline & $9 / 650$ & 2.0 & 0.0 & 0.0 & 0.9 & 300.000 & 5000.000
\end{tabular}

$0.36219521 d+01 \quad 0.73618256 d-03-0.19652219 d-06 \quad 0.36201556 d-10-0.28945623 d-14$ $-0.12019822 d+04 \quad 0.36150942 d+01 \quad 0.36255980 d+01-0.18782183 d-02 \quad 0.70554543 d-05$ $\begin{array}{lll}-0.67635071 d-08 & 0.21555977 d-11-0.10475225 d+04 & 0.43052769 d+01\end{array}$ oh $\quad J 3 / 660$ 1.h $1.00 \quad 0.00 \quad 0 . g \quad 300.000 \quad 5000.000$ $0.29106417 d+01 \quad 0.95931627 d-03-0.19441700 d-06 \quad 0.13756646 d-10 \quad 0.14224542 d-15$ $0.39353811 d+04 \quad 0.54423428 d+01 \quad 0.38375931 d+01-0.10778855 d-02 \quad 0.96830354 d-06$ $0.18713971 d-09-0.22571089 d-12 \quad 0.36412820 d+04 \quad 0.49370009 d+00$ $802 \quad$ I $6 / 61 \mathrm{~B} \quad 1.0 \quad 2.00 \quad 0.00 \quad 0.9 \quad 300.000 \quad 5000.000$

$0.52451363 d+01 \quad 0.19704204 d-02-0.80375759 d-06 \quad 0.15149969 d-09-0.10557998 d-13$

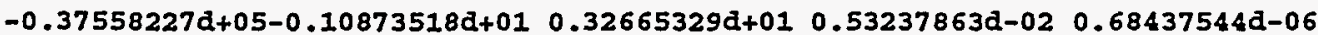
$\begin{array}{ll}-0.52809987 d-08 & 0.25590450 d-11-0.36908145 d+05 \quad 0.96513472 d+01\end{array}$ снзон I $4 / 80 \mathrm{C}$ 1.h $4.0 \quad 1.0$ 0.G $300.000 \quad 5000.000$

$0.40334730 D+01 \quad 0.93680508 D-02-0.30449373 D-05 \quad 0.43456994 D-09-0.22136329 D-13$ $\begin{array}{lllll}-0.26159859 D+05 & 0.23539820 D+01 & 0.26587849 D+01 & 0.73515214 D-02 & 0.71443337 D-05\end{array}$ $\begin{array}{lll}-0.87661114 D-08 & 0.23805116 D-11-0.25353684 D+05 & 0.11238121 D+02\end{array}$

$\begin{array}{llllllll}\text { C2H4 I } 4 / 80 \mathrm{C} & 2 . \mathrm{h} & 4.0 & 0 \mathrm{O} & \text { OG } & 300.000 & 5000.000\end{array}$

$0.43843679 D+010.96509494 D-02-0.31845530 D-05 \quad 0.46213655 D-09-0.24069342 D-13$

$0.41205039 D+04-0.23853559 D+01 \quad 0.11654673 D+01 \quad 0.13374992 D-01 \quad 0.25754771 D-05$ $\begin{array}{lllll}-0.10187399 D-07 & 0.43603341 D-11 & 0.53428867 D+04 & 0.15699718 D+02\end{array}$

C3H6 $120186 \mathrm{C} \quad 3 . \mathrm{h} \quad 6.0$ DO OG $300.000 \quad 5000.000$

$0.06732257 D+02 \quad 0.14908336 D-01-0.04949899 D-04 \quad 0.07212022 D-08-0.03766204 D-12$ $\begin{array}{llll}-0.09235703 D+04-0.13313348 D+02 & 0.14933071 D+01 & 0.02092517 D+00 & 0.04486794 D-04\end{array}$ $\begin{array}{lllll}-0.16689121 D-07 & 0.07158146 D-10 & 0.10748264 D+04 & 0.16145340 D+02\end{array}$ $\begin{array}{llllllll}\text { C4F8 } & 120186 \mathrm{C} & 4 . \mathrm{h} & 8.0 & 00 & \text { OG } & 300.000 & 5000.000\end{array}$

$0.02053584 D+02 \quad 0.03435050 D+00-0.15883196 D-04 \quad 0.03308966 D-07-0.02536104 D-11$ $\begin{array}{lllll}-0.02139723 D+05 & 0.15543201 D+02 & 0.11811380 D+01 & 0.03085338 D+00 & 0.05086524 D-04\end{array}$ $-0.02465488 D-06 \quad 0.11110192 D-10-0.01790400 D+05 \quad 0.02106247 D+03$

C3H8 $\quad$ I $4 / 80 \mathrm{C}$ 3.h 8.0 OO OG $300.000 \quad 5000.000$

$0.75252171 D+01 \quad 0.18890340 D-01-0.62839244 D-05 \quad 0.91793728 D-09-0.48124099 D-13$

$-0.16464547 D+05-0.17843903 D+02 \quad 0.89692080 D+00 \quad 0.26689861 D-01 \quad 0.54314251 D-05$ $-0.21260007 D-07 \quad 0.92433301 D-11-0.13954918 D+05 \quad 0.19355331 D+02$

I-C4H10 I-L $5 / 80 \mathrm{C}$ 4.h 10.00 00 OG 300.0005000 .000

$0.10845599 \mathrm{D}+02 \quad 0.23333851 \mathrm{D}-01-0.77793875 \mathrm{D}-05 \quad 0.11375818 \mathrm{D}-08-0.59640660 \mathrm{D}-13$ $\begin{array}{lllll}-0.21725719 D+05-0.35869400 D+02 & 0.50704670 D+00 & 0.38149782 D-01 & 0.46916175 D-05\end{array}$ $-0.29491598 D-07 \quad 0.13621288 D-10-0.18030996 D+05 \quad 0.21284882 D+02$

C2H2 J 3/61C $2 . \mathrm{h} \quad 2.0$ OO OG $300.000 \quad 5000.000$

$0.45751083 D+01 \quad 0.51238358 D-02-0.17452354 D-05 \quad 0.28673065 D-09-0.17951426 D-13$

$0.25607428 D+05-0.35737940 D+01 \quad 0.14102768 D+01 \quad 0.19057275 D-01-0.24501390 D-04$

$0.16390872 D-07-0.41345447 D-11 \quad 0.26188208 D+05 \quad 0.11393827 D+02$ 


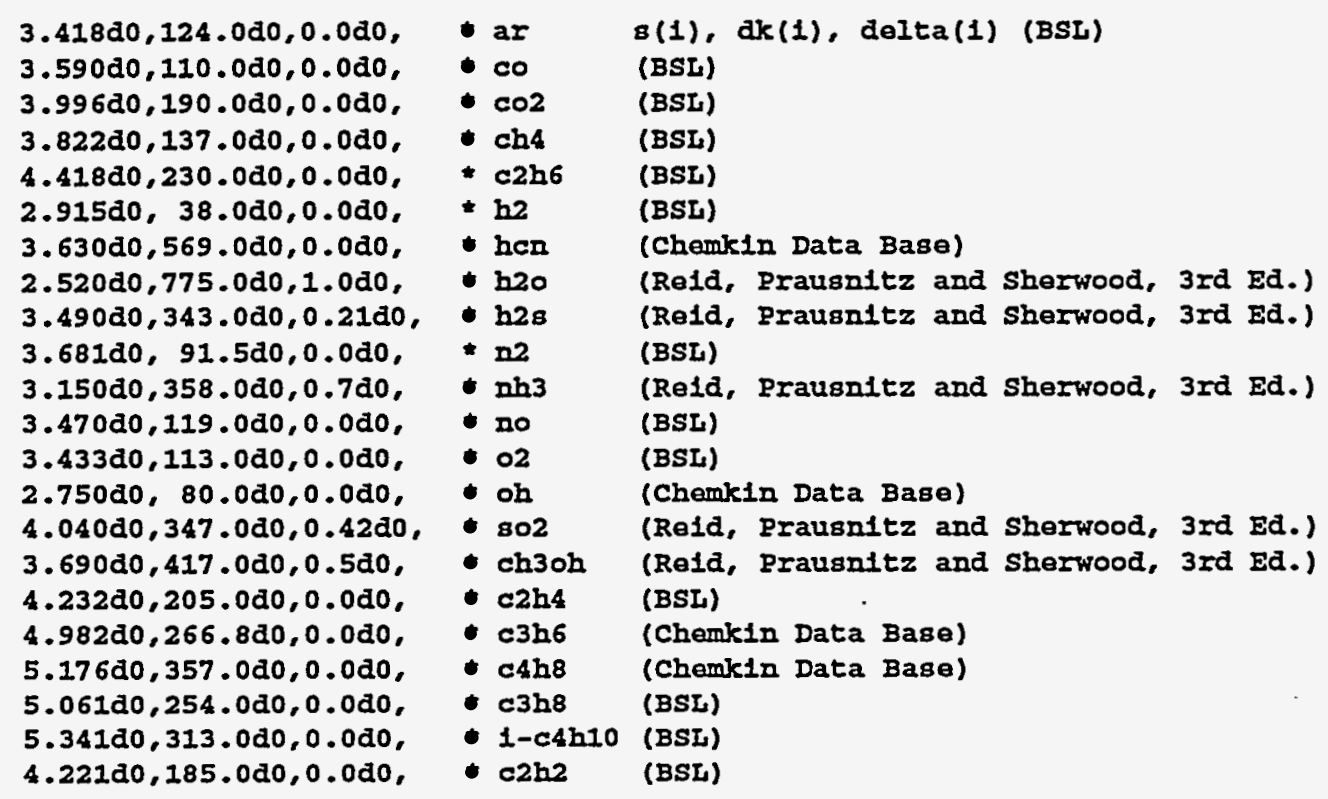

\section{FG-DVC Input Data File}

The input data for the FG-DVC submodel include the initial functional group composition of the coal, the rank dependent kinetic parameters, percolation submodel data for bond population, and probability distribution for the tar mass bins. Eight data files, COAL0.ARG1 to COAL0.ARG8, with the input values for the standard Argonne premium coals, are provided in Appendix A. Three options are provided in the code to select or provide an appropriate data file for the coal dependent parameters for the devolatilization submodel. These options are implemented through the integer variable idcoal, which is read from the main input data file FBIIN. The variable idcoal can take a value from 0-9. The first option is implemented when the value of the variable, idcoal, is 0 . This option allows the program to select the standard coal which is closest, on a oxygen/carbonhydrogen/carbon plot, to the feed coal. The second option allows the user to specify a standard coal based on specific considerations, e.g., the geographical origin of the feed coal. This option requires that the user specify the value of the 
User's Guide

variable idcoal from 1 to 8 . As an example, a value of 1 will invoke the reading of FG-DVC input data to be read form the file coal0.arg1 for the standard Argonne Premium coal 1. The third option allows the user to provide the data for the feed coal. The user must provide the data file as COALO.USER, and the value of the variable idcoal must be specified as 9. A typical input data file for the FG-DVC devolatilization submodel is shown in Table 6.3.

\section{Input Data File EXPT.DAT}

The final input data file for the FBED-1 code is an optional file EXPT.DAT This file must be provided when the logical variable lexpt, which is read from the input file FB1IN, is set to .true.. This file contains the experimental composition of $\mathrm{CO}, \mathrm{CO}_{2}, \mathrm{CH}_{4}, \mathrm{H}_{2}, \mathrm{H}_{2} \mathrm{O}$ and $\mathrm{N}_{2}$ in the product gas specified as percents. In addition, product gas temperature, product tar flow rate, experimental wall heat loss and burnout are specified. A sample data file is shown in Table 6.4.

Table 6.4 Sample Data file EXPT.DAT

$$
\begin{array}{r}
22.62 \mathrm{do} \\
5.19 \mathrm{do} \\
1.47 \mathrm{~d} 0 \\
15.75 \mathrm{~d} 0 \\
8.44 \mathrm{do} \\
45.43 \mathrm{do} \\
773.15 \mathrm{do} \\
0.0452 \mathrm{do} \\
0.4159 \mathrm{do} \\
0.9610 \mathrm{do}
\end{array}
$$

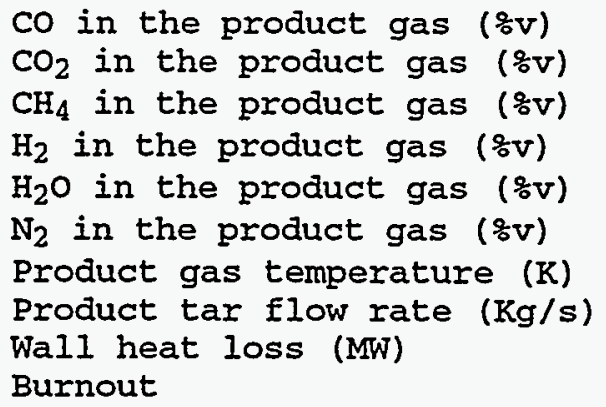

\section{Program Output}

The output of FBED-1 is available both in tabular and graphical form. The output data files provide the values for important variables, e.g., gas and solid 
27

$\begin{array}{llllll}0.10000 D-02 & 0.29240 D+02 & 0.20855 D+05 & 0.36000 D+04 & 0.204800+03\end{array}$

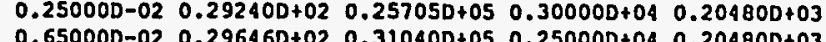

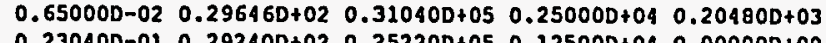
$\begin{array}{lllllllll}0.23040 \mathrm{D}-01 & 0.29240 \mathrm{D}+02 & 0.25220 \mathrm{D}+05 & 0.12500 \mathrm{D}+04 & 0.00000 \mathrm{D}+00\end{array}$

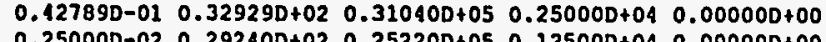
$\begin{array}{lllll}0.25000 D-02 & 0.29240 D+02 & 0.25220 D+05 & 0.12500 D+04 & 0.00000 D+00\end{array}$ $0.15231 D-01 \quad 0.29240 D+02 \quad 0.29828 D+05 \quad 0.19000 D+04 \quad 0.00000 D+00$ $0.390870-02 \quad 0.31369 D+02 \quad 0.291000+05 \quad 0.15000 D+04 \quad 0.00000 D+00$

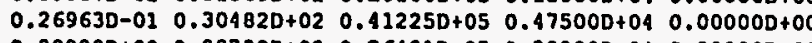
$\begin{array}{lllll}0.00000 D+00 & 0.28730 D+02 & 0.26481 D+05 & 0.30000 D+04 & 0.00000 D+00\end{array}$ $\begin{array}{llllll}0.15697 D+00 & 0.00000 D+00 & 0.00000 D+00 & 0.00000 D+00 & 0.00000 D+00\end{array}$ $0.00000 D+00 \quad 0.00000 D+00 \quad 0.00000 D+00 \quad 0.00000 D+00 \quad 0.00000 D+00$ $\begin{array}{llllll}0.18000 D-02 & 0.31032 D+02 & 0.27160 D+05 & 0.13000 D+04 & 0.102400+03\end{array}$

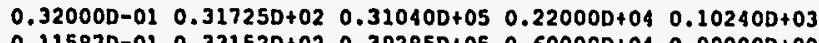

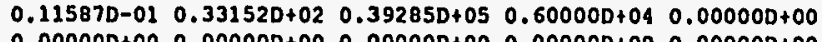
$0.00000 D+00 \quad 0.00000 D+00 \quad 0.00000 D+00 \quad 0.00000 D+00 \quad 0.00000 D+00$ $\begin{array}{lllll}0.23000 D-01 & 0.32929 D+02 & 0.38800 D+05 & 0.28000 D+04 & 0.00000 D+00\end{array}$ $0.562000+000.00000+000.00000+000.000000+000.00000 \mathrm{D}+00$

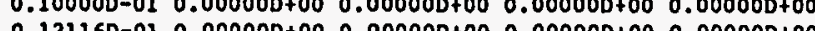
$0.121160-010.000000+000.00000+000.000000+000.000000+00$ $0.000000+000.292400+020.242500+050.150000+040.000000+00$ $0.000000010 .2924001020 .24250 D+050.150000+040.00000+00$

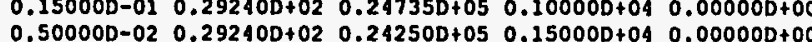
$0.00000 D+00 \quad 0.292400+020.24250 D+050.15000 D+040.0000000+00$ $0.250000-010.29240 D+020.24735 D+050.10000 D+04 \quad 0.00000 D+00$ $0.00000 \mathrm{D}+00 \quad 0.00000 \mathrm{D}+00 \quad 0.00000 \mathrm{D}+00 \quad 0.00000 \mathrm{D}+00 \quad 0.000000+00$ $\begin{array}{lll}0.00000 D+00 & 0.000000+00 & 0.00000 D+00 \\ 0.32236 D+02 & 0.266750+05 & 0.12500 D+04\end{array}$

$60.8202 D+00 \quad 0.8202 D+000.3600 D-010.1000 D+010.40000+01$

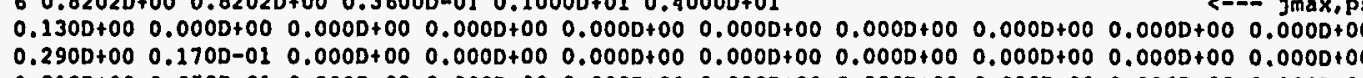
$0.310 \mathrm{D}+00 \quad 0.950 \mathrm{D}-01 \quad 0.2000-02 \quad 0.000 \mathrm{D}+00 \quad 0.000 \mathrm{D}+00 \quad 0.0000+00 \quad 0.0000+00 \quad 0.000 \mathrm{D}+00 \quad 0.0000+00 \quad 0.0000+00$

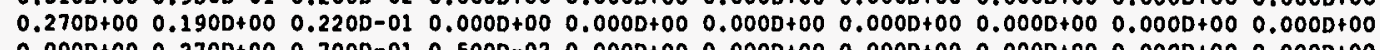

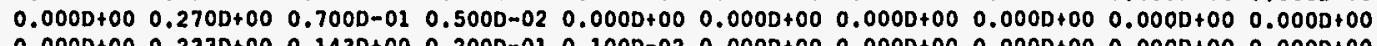
$\begin{array}{llllllllll}0.000 D+00 & 0.233 D+00 & 0.143 D+00 & 0.200 D-01 & 0.100 D-02 & 0.000 D+00 & 0.000 D+00 & 0.000 D+00 & 0.000 D+00 & 0.000 D+00 \\ 0.000 D+00 & 0.142 D+00 & 0.207 D+00 & 0.550 D-01 & 0.500 D-02 & 0.000 D+00 & 0.000 D+00 & 0.000 D+00 & 0.000 D+00 & 0.000 D+00\end{array}$

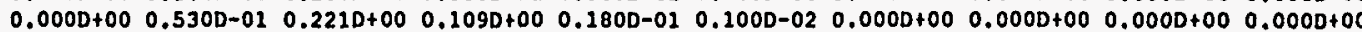

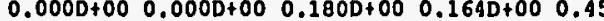
$\begin{array}{lllllllllll}0.000 D+00 & 0.000 D+00 & 0.104 D+00 & 0.195 D+00 & 0.860 D-01 & 0.150 D-01 & 0.000 D+00 & 0.000 D+00 & 0.000 D+00 & 0.000 D+00\end{array}$

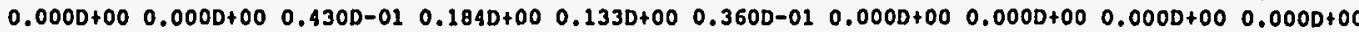

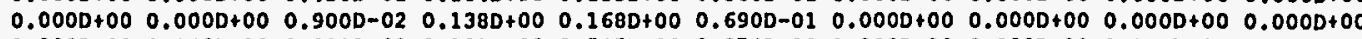

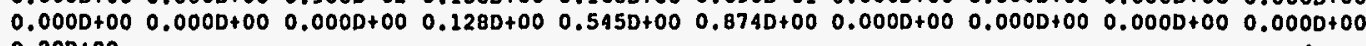
$0.200+00$

$0.83200+00 \quad 0.5300 D-01 \quad 0.8900 D-01 \quad 0.16000-01 \quad 0.10000-01$

File generated: Tue Apr 14 18:28:56 1992
- nfg (Number of Functional Groups)

- yo, a, b, slgmaa, and xeft for fg $1=$ = CO2XL - yo, a, b, slgmaa, and xeft for fg $4=$ = H2OL <-- yo, a, b, slgmaa, and xeff for fg $5=$ H2OT -- yo, a, b, slgmas, and xete for fg $7=\operatorname{cor}$ - yo, $a, b, s i g m a a$, and xeff for fg $8=$ = HCNL yo, $a, b$, sigmaa, and xeft for tg $9=$ HCNT , yo, $a, b$, sigmaa, and xeft for fg $10=$ NH3 - yo, a, b, slgmaa, and xeft for $f g$ 11 = CAL <o, $a, b$, slgmaa, and xeft for $29 / 14=$ CH4T c so, $a, b$, slgmaa, and xetf tor $\mathrm{fg} 16=\mathrm{CH}$ - 16 -.- yo, $a, b$, slgmad, and xeff for fg $17=$ coxT -- yo, $a, b$, slgmaa, and xete for $\mathrm{eg} / 18$ - CNV <-- yo, $a, b$, slgmaa, and xelt for $59,19=$ = SORG --- yo, $a, b$, slgmaa, and xoff for tg 20 - HAL -- yo, $a, b$, slgmaa, and xeff for fg $22=$ c3H6 -

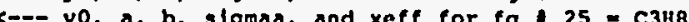
Jat $b$, and slgmas for bond klnetlcs t, ptotal, qtotal, slgmal, sigmaz (--- pdist $1,1: 10)$ -.- palst $(3,1: 10)$ (--- pdist $4,1: 10)$ <-- pdist $5,1: 10)$ <-- pd1st $6,1: 10)$ <-- pdist $7,1: 10)$ <--- pdist $0,1: 10)$ <-- pdist $(9,1: 10$ (-.- pd1st $(10,1: 10)$ <-- pdist $(11,1: 10)$ (--- pd1st(12,1:10) (--- pdist $(13,1: 10)$ <--- particle Internal pressure drop (--- ultimate composition (daf) coa10.arg 6 
User's Guide

temperature, pressure, gas phase composition, flow rates, char consumption rates, particle diameter and.burnout. Transport and thermodynamic properties are also available. The output files and their contents are discussed in the following sections.

\section{Main Output File, FB1OUT}

The main output file FB1OUT, given in Table 6.5, corresponds to the input data given in Tables 6.1 to 6.3. This file echos the input data, provides the values of the variables calculated from the input data, the effluent output data from the twozone, zero-dimensional submodel, and the axial output from the one-dimensional submodel. Overall heat loss and cpu time are also reported at the end of each integration pass. The axial output consists of the axial distance (m), the solid and gas temperatures $(\mathrm{K})$, and the mole percentage of $\mathrm{H}_{2}, \mathrm{CO}_{2}, \mathrm{CO}, \mathrm{CH}_{4}$, and $\mathrm{H}_{2} \mathrm{O}$. The results reported in Table 6.5 were obtained by executing the FBED-1 code on a SUN4/310 computer. The results may be slightly different on other computers, due to the differences in hardware and machine architecture.

Table 6.5 Main Output File, FB1OUT.

\footnotetext{
S1mulation started: Thu Jun 3 15:11:50 1993

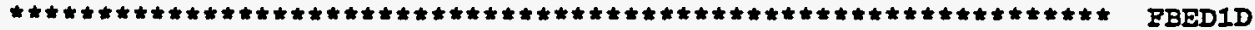

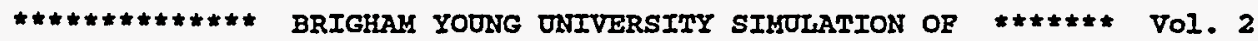

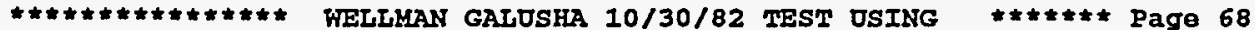

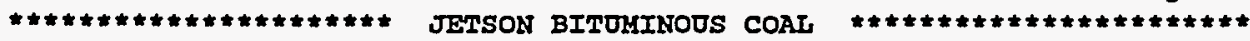

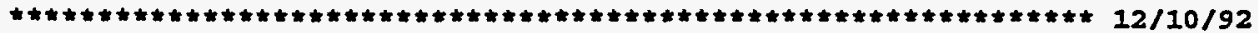

Zero-dimensional imput parameters:

- - - - - -

$P \quad T->$ do $0-d$ calculation only

T T-> all exit gases in equil. mainly for 0-d

F Ta do FREEBoard calculation using gfreeb

I T-> calculate heat loss with flowjs latent heat

I T-> UBe ASH composition to calculate CP

$P \quad T->$ use usrxo for $x 0$ rather than $S E T$ model $\times 0$

T $T \rightarrow$ use RG-DVC devolatilization submodel

F $T->$ compute tar cracking rates using FIXed Gaussian

T T-> acho 1nput parameters to fblout

$0.198 \mathrm{E}+01$ DIAmeter of reactor CHamber, m

$0.208 \mathrm{E}+01$ reactor Chamber axial Iength, m
} 


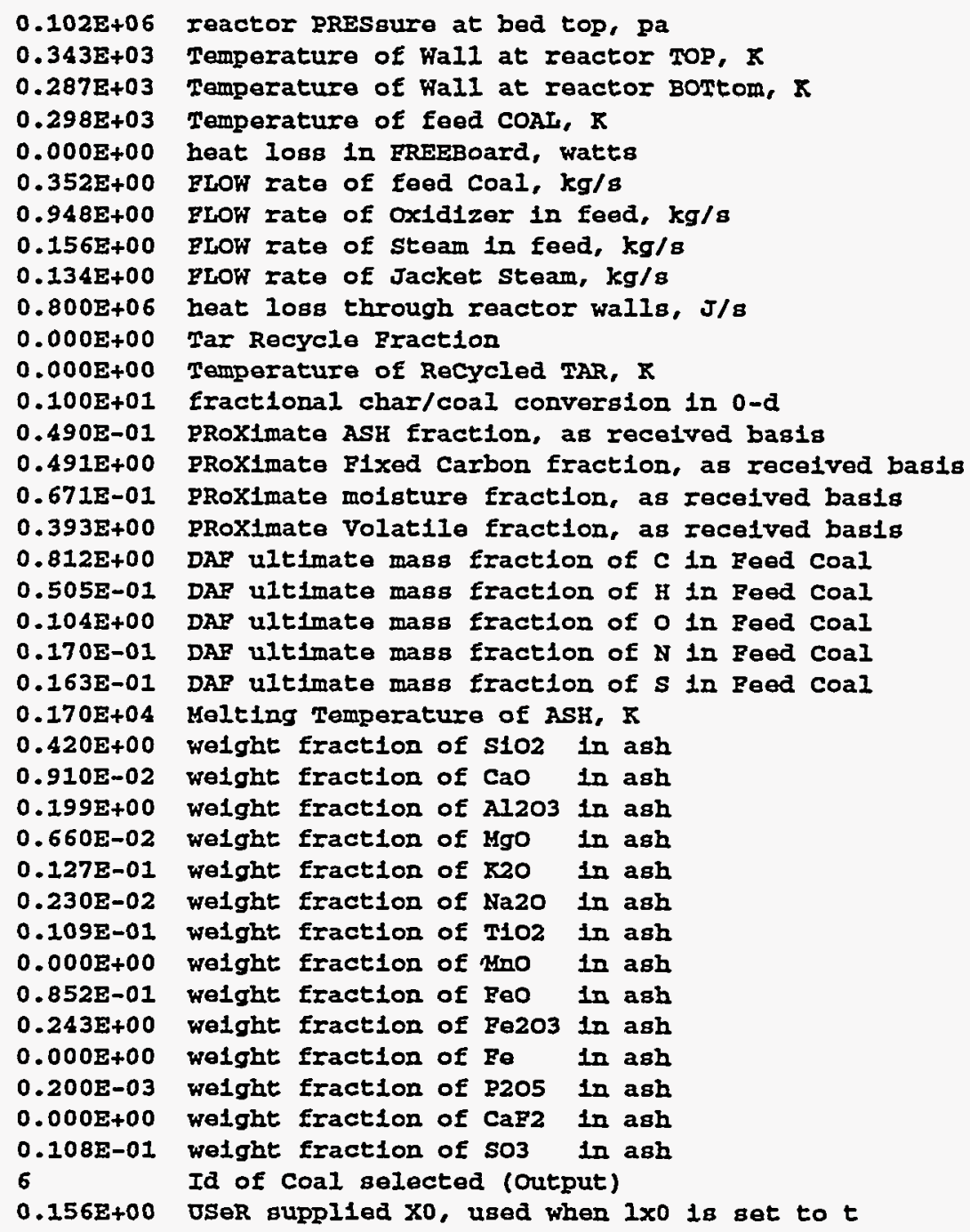

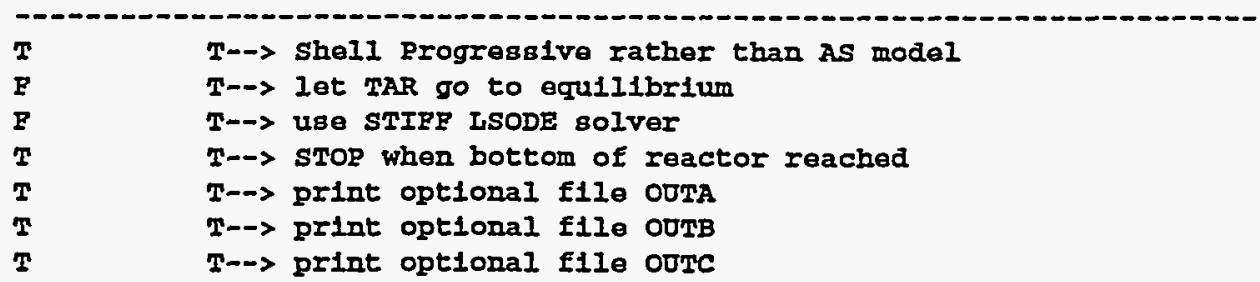


User's Guide

\begin{tabular}{|c|c|}
\hline $\mathbf{T}$ & r-> print optional f1le OJTD \\
\hline $\mathbf{T}$ & T--> print optional file OJTE \\
\hline $\mathbf{T}$ & T-> print optional file odTr \\
\hline$T$ & T-> print optional file odTe \\
\hline r & T- > print optional file oठTH \\
\hline T & Th print optional file oUTI \\
\hline $\mathbf{T}$ & T-> print optional file oUTJ \\
\hline 1 & uSODE parameter used to specify output \\
\hline $0.100 \mathrm{E}-01$ & step size for output, m \\
\hline $0.100 \mathrm{E}-02$ & maximum step size allowed in ISODE, $m$ \\
\hline $0.100 \mathrm{E}-07$ & ABSolute TOLerance used in ISODE \\
\hline $0.100 E-03$ & RELat ive TOLerance uBed in LSODE \\
\hline $0.217 \mathrm{E}-01$ & Inttial Particle Diameter, m \\
\hline $0.100 E+00$ & swelling parameter, fraction \\
\hline $0.100 E+01$ & heat of rxen partition \\
\hline $0.600 \mathrm{E}+00$ & Vold Eraction in AsH zone \\
\hline $0.330 \mathrm{E}+00$ & Vold Eraction in COAI zone \\
\hline $0.119 E+04$ & apparent coal density, $\mathrm{kg} / \mathrm{m}^{\wedge} 3$ \\
\hline $0.1362+00$ & POROSIty of coal \\
\hline $0.589 E+03$ & Frequency Pactor for $c 02$ gasification $\mathrm{rxm}, \mathrm{m} / \mathrm{s} \mathrm{K}$ \\
\hline $0.5895+00$ & Rrequency Factor for H2 gasification $\mathrm{rxm} . \mathrm{m} / \mathrm{s} \mathrm{K}$ \\
\hline $0.589 E+03$ & Frequency Factor for H2O gasification $\mathrm{rxn} ., \mathrm{m} / \mathrm{s} \mathrm{K}$ \\
\hline $0.230 E+01$ & Frequency Factor for 02 oxidation $\mathrm{rxh} ., \mathrm{m} / \mathrm{s} \mathrm{K}$ \\
\hline $0.223 E+09$ & Activation Energy for $\mathrm{CO} 2$ gasification rxn., J/kmol \\
\hline $0.223 E+09$ & Activation Energy for $\mathrm{H} 2$ gasification rxn., J/kmol \\
\hline $0.223 E+09$ & Activation Energy for H2O gasification $\mathrm{rxn} ., \mathrm{J} / \mathrm{kmol}$ \\
\hline $0.923 E+08$ & Activation Energy for 02 oxidation $2 \times 0 ., \mathrm{J} / \mathrm{kmol}$ \\
\hline $0.1005+01$ & particle area factor for internal burning \\
\hline $0.500 \mathrm{E}+00$ & developing Ash Porosity DIVIded by Tortuosity \\
\hline $0.500 \mathrm{E}-01$ & Adjustable Factor for Solld to Gas HT coef. \\
\hline $0.100 z+01$ & ADjustable Pactor for devolatillzation \\
\hline $0.100 \mathrm{E}+01$ & ADJustable Pactor $c 02$ gasification reaction \\
\hline $0.100 \mathrm{E}+01$ & ADJustable Factor H2 gasification reaction \\
\hline $0.100 \mathrm{E}+01$ & ADJustable Factor H2O gasification reaction \\
\hline $0.100 \mathrm{E}+01$ & ADJustable ractor 02 oxidation reaction \\
\hline $0.100 E+01$ & ADjustable Eactor for Gas-to-Wall kT coefficlent \\
\hline $0.100 \mathrm{E}+01$ & ADJustable ractor for solid-to-WaIl HT coefficlent \\
\hline $0.120 \mathrm{E}+04$ & Temperature to SWiTCH to/from equilibrium \\
\hline $0.104 \mathrm{E}+06$ & Pressure at the BOTtOH of the gasifler, Pa \\
\hline $0.000 \mathrm{E}+00$ & Axial position at the BOTtom of gasifler, a \\
\hline $\mathbf{F}$ & r--> Execute the code in INTeractive mode \\
\hline
\end{tabular}

Number of down \& upward integration passes before iterations

Calculated input parameters:

Calculated

$0.315 \mathrm{E}+03$ Temperature of Warl used, $\mathrm{K}$

$0.373 E+03$ sAturated water Temp. at reactor pressure, $R$

$0.244 E+07$ vaporization enthalpy of water at $T H, J / k g$

$0.800 \mathrm{E}+06$ heat $108 \mathrm{~s}$ through reactor walls, J/s 
Chapter 6

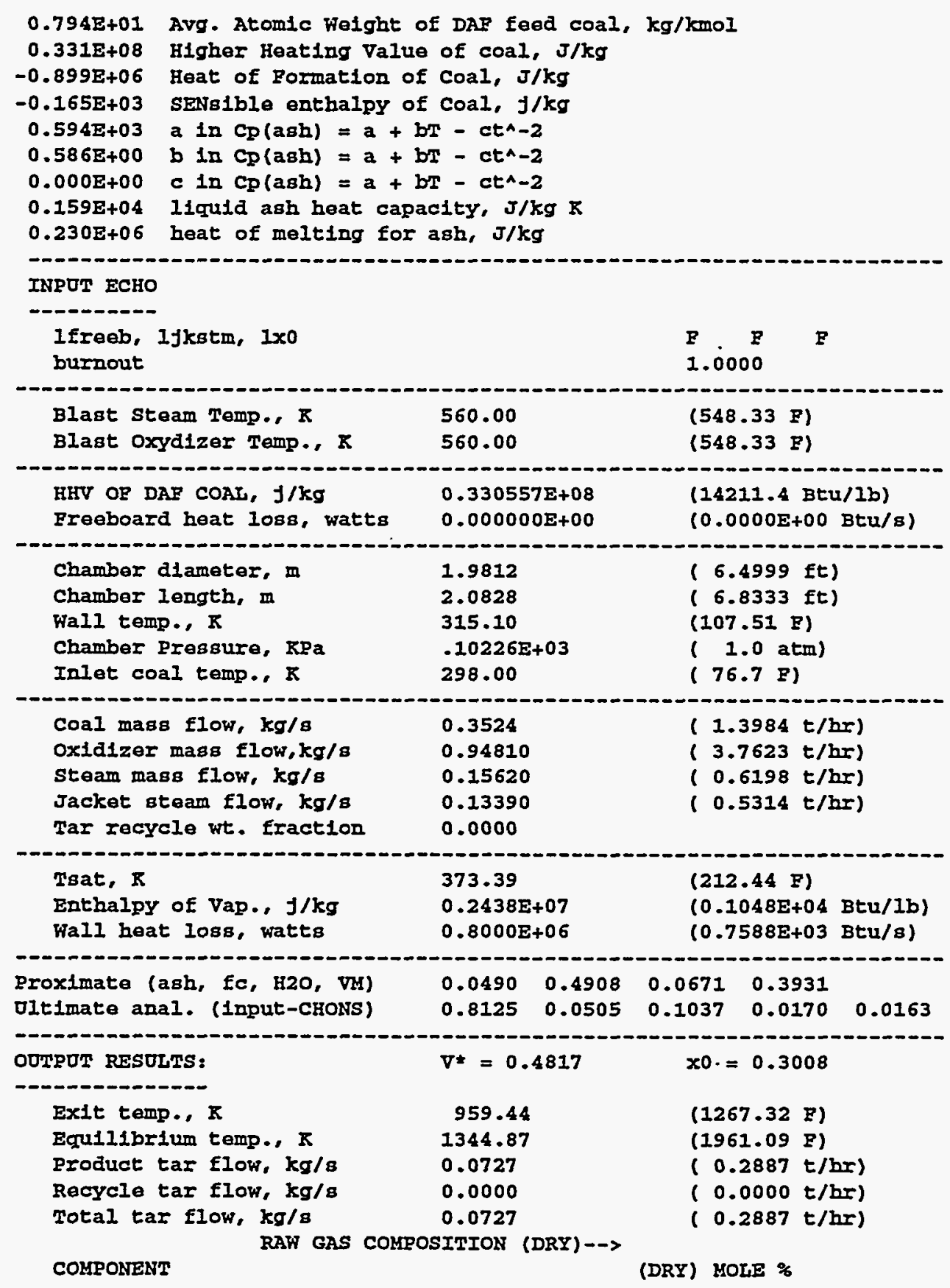


User's Guide

\begin{tabular}{|c|c|c|c|c|c|c|c|}
\hline \multicolumn{8}{|c|}{ 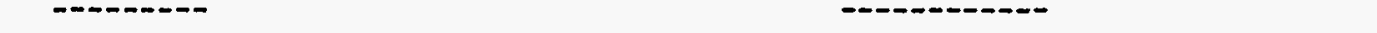 } \\
\hline \multicolumn{8}{|c|}{$\begin{array}{ll}\text { co } & 17.41\end{array}$} \\
\hline \multicolumn{8}{|c|}{11.08} \\
\hline \multicolumn{8}{|c|}{22.57} \\
\hline \multicolumn{8}{|c|}{0.26} \\
\hline \multicolumn{8}{|c|}{$\mathrm{C2H} 6$} \\
\hline \multicolumn{8}{|c|}{0.20} \\
\hline $\begin{array}{l}\text { INE } \\
\text { OTH }\end{array}$ & $\begin{array}{l}\text { TS } \\
\text { RS }\end{array}$ & $0.60 \% \& N$ & $N 2=47.87 \%$ & \%) & $\begin{array}{r}48.4 \\
0.0\end{array}$ & & \\
\hline \multicolumn{8}{|c|}{$\begin{array}{c}\text { RAW GAS COMPOSITION (WET) }--> \\
\text { RAW }\end{array}$} \\
\hline \multicolumn{8}{|c|}{ COMPONENT } \\
\hline \multicolumn{8}{|c|}{$---n-\infty-\infty$} \\
\hline \multicolumn{8}{|c|}{16.08} \\
\hline \multicolumn{8}{|c|}{10.23} \\
\hline \multicolumn{8}{|c|}{20.84} \\
\hline \multicolumn{8}{|c|}{0.24} \\
\hline \multicolumn{8}{|c|}{$\mathrm{C} 2 \mathrm{H} 6$} \\
\hline \multicolumn{8}{|c|}{7.68} \\
\hline \multicolumn{8}{|c|}{0.19} \\
\hline \multicolumn{4}{|c|}{$\begin{array}{l}\text { INERTS (AR }=0.55 \% \& N 2=44.19 \% \text { ) } \\
\text { OTHERS }\end{array}$} & \%) & $\begin{array}{r}44.7 \\
0.0\end{array}$ & & \\
\hline \multicolumn{8}{|c|}{ (CHX $=0.00 \%$, HCN $=0.00 \%$, NH3 $=0.00 \%$, etc. } \\
\hline \multicolumn{8}{|c|}{ S1mulation Results for dowrward Integration pass \# 1} \\
\hline Axial Pos. & ts $(\mathrm{x})$ & $\operatorname{tg}(\mathrm{R})$ & mol\% $\mathrm{F2}$ II & $\mathrm{mol} \% \mathrm{CO} 2$ & mol\% co & mol\% CH4 & mol\% H2O \\
\hline$--m-------$ & ----- & ------ & $-\infty-\infty-\infty$ & $-----\infty-$ & $---\infty--$ & $----\infty--$ & $---\cdots--$ \\
\hline $2.0828 \mathrm{E}+00$ & 298.0 & 959.4 & 20.84 & 10.23 & 16.08 & 0.24 & 7.68 \\
\hline $2.0800 \mathrm{E}+00$ & 321.9 & 966.0 & 20.86 & 10.24 & 16.09 & 0.24 & 7.59 \\
\hline $2.0700 E+00$ & 395.9 & 988.8 & 20.94 & 10.28 & 16.15 & 0.24 & 7.24 \\
\hline $2.0600 \mathrm{E}+00$ & 457.6 & 1010.1 & 21.02 & 10.32 & 16.22 & 0.24 & 6.88 \\
\hline $2.0500 \mathrm{E}+00$ & 510.0 & 1030.0 & 21.10 & 10.36 & 16.28 & 0.24 & 6.51 \\
\hline $2.0400 E+00$ & 558.7 & 1049.3 & 21.19 & 10.40 & 16.35 & 0.24 & 6.13 \\
\hline $2.0300 \mathrm{E}+00$ & 603.7 & 1067.9 & 21.27 & 10.45 & 16.41 & 0.24 & 5.75 \\
\hline $2.0200 \mathrm{E}+00$ & 644.9 & 1083.5 & 21.32 & 10.47 & 16.45 & 0.24 & 5.56 \\
\hline $2.0100 \mathrm{E}+00$ & 674.9 & 1094.1 & 21.32 & 10.47 & 16.45 & 0.24 & 5.56 \\
\hline $2.0000 \mathrm{E}+00$ & 690.8 & 1101.2 & 21.32 & 10.47 & 16.45 & 0.24 & 5.56 \\
\hline $1.9900 \mathrm{Z}+00$ & 702.0 & 1107.1 & 21.32 & 10.47 & 16.45 & 0.24 & 5.56 \\
\hline $1.9800 \mathrm{E}+00$ & 712.8 & 1112.8 & 21.32 & 10.47 & 16.45 & 0.24 & 5.56 \\
\hline $1.9700 \mathrm{E}+00$ & 724.6 & 1118.8 & 21.32 & 10.47 & 16.45 & 0.24 & 5.56 \\
\hline $1.9600 \mathrm{E}+00$ & 737.7 & 1125.4 & 21.32 & 10.47 & 16.45 & 0.24 & 5.56 \\
\hline $1.9500 \mathrm{E}+00$ & 751.0 & 1132.8 & 21.32 & 10.47 & 16.45 & 0.24 & 5.56 \\
\hline $1.9400 \mathrm{E}+00$ & 764.6 & 1141.0 & 21.32 & 10.47 & 16.45 & 0.24 & 5.56 \\
\hline $1.9300 \mathrm{E}+00$ & 778.0 & 1150.1 & 21.32 & 10.47 & 16.45 & 0.24 & 5.56 \\
\hline $1.9200 \mathrm{E}+00$ & 791.7 & 1159.9 & 21.32 & 10.47 & 16.45 & 0.24 & 5.56 \\
\hline
\end{tabular}




\begin{tabular}{|c|c|c|c|c|c|c|c|}
\hline $1.9100 \mathrm{E}+00$ & 805.3 & 1170.2 & 21.32 & 10.47 & 16.45 & 0.24 & 5.56 \\
\hline $1.9000 \mathrm{E}+00$ & 819.2 & 1181.1 & $21 \cdot 32$ & 10.47 & 16.45 & 0.24 & 5.56 \\
\hline $1.8900 \mathrm{E}+00$ & 833.1 & 1192.3 & 21.32 & 10.47 & 16.45 & 0.24 & 5.56 \\
\hline \multicolumn{8}{|l|}{ Switched to } \\
\hline $1.8800 \mathrm{E}+00$ & $848 \cdot 2$ & 1265.1 & 13.35 & 8.47 & 17.72 & 0.00 & $10 \cdot 28$ \\
\hline $1.8700 \mathrm{E}+00$ & 864.3 & 1279.4 & 13.09 & 8.44 & 17.71 & 0.00 & 10.40 \\
\hline $1.8600 \mathrm{E}+00$ & 881.1 & 1293.8 & 12.85 & 8.41 & 17.71 & 0.00 & 10.52 \\
\hline $1.8500 \mathrm{E}+00$ & 899.6 & 1308.2 & 12.61 & 8.37 & 17.71 & 0.00 & 10.62 \\
\hline \multicolumn{8}{|l|}{$\cdots$} \\
\hline \multicolumn{8}{|l|}{$\cdots$} \\
\hline $1.0000 \mathrm{E}-01$ & 387.8 & 388.0 & 0.00 & 0.00 & 0.00 & 0.00 & 23.15 \\
\hline $9.0000 \mathrm{E}-02$ & 387.8 & 388.0 & 0.00 & 0.00 & 0.00 & 0.00 & 23.15 \\
\hline $8.0000 \mathrm{E}-02$ & 387.9 & 388.1 & 0.00 & 0.00 & 0.00 & 0.00 & 23.15 \\
\hline $7.0000 \mathrm{E}-02$ & 388.0 & 388.2 & 0.00 & 0.00 & 0.00 & 0.00 & 23.15 \\
\hline $6.0000 \mathrm{E}-02$ & 388.0 & 388.2 & 0.00 & 0.00 & 0.00 & 0.00 & 23.15 \\
\hline 5.0000E-02 & $388 \cdot 1$ & 388.3 & 0.00 & 0.00 & 0.00 & 0.00 & 23.15 \\
\hline $4.0000 E-02$ & 388.2 & 388.4 & 0.00 & 0.00 & 0.00 & 0.00 & 23.15 \\
\hline $3.0000 E-02$ & 388.2 & 388.4 & 0.00 & 0.00 & 0.00 & 0.00 & 23.15 \\
\hline $2.0000 \mathrm{E}-02$ & 388.3 & 388.5 & 0.00 & 0.00 & 0.00 & 0.00 & 23.15 \\
\hline $1.0000 \mathrm{E}-02$ & 388.3 & 388.6 & 0.00 & 0.00 & 0.00 & 0.00 & 23.15 \\
\hline $0.0000 E+00$ & 388.4 & 388.6 & 0.00 & 0.00 & 0.00 & 0.00 & 23.15 \\
\hline
\end{tabular}

OveralI Heat Loss $=0.2776 \mathrm{E}+06 \mathrm{~J} / \mathrm{s}$

TOTAL CPU TIME: 0 hours, 30 minutes, 19.870 seconds!

Simulation Results for upward Integration pass \# 1

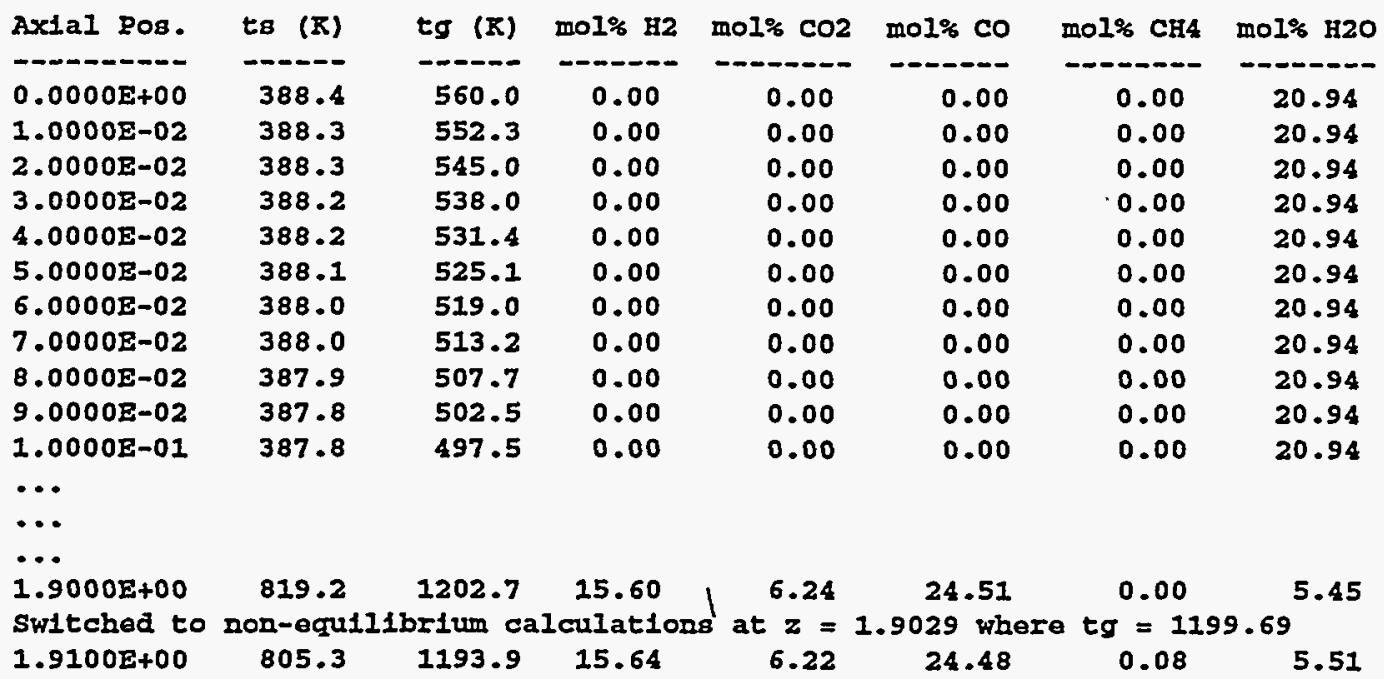


User's Guide

$\begin{array}{lrrrrrrr}1.9200 \mathrm{E}+00 & 791.7 & 1186.7 & 15.66 & 6.21 & 24.44 & 0.18 & 5.60 \\ 1.9300 \mathrm{E}+00 & 778.0 & 1180.3 & 15.66 & 6.19 & 24.39 & 0.29 & 5.70 \\ 1.9400 \mathrm{E}+00 & 764.6 & 1174.6 & 15.66 & 6.17 & 24.33 & 0.40 & 5.81 \\ 1.9500 \mathrm{E}+00 & 751.0 & 1169.6 & 15.66 & 6.15 & 24.27 & 0.51 & 5.93 \\ 1.9600 \mathrm{E}+00 & 737.7 & 1165.3 & 15.64 & 6.12 & 24.21 & 0.63 & 6.06 \\ 1.9700 \mathrm{E}+00 & 724.6 & 1161.3 & 15.62 & 6.10 & 24.15 & 0.74 & 6.19 \\ 1.9800 \mathrm{E}+00 & 712.8 & 1157.5 & 15.60 & 6.08 & 24.09 & 0.84 & 6.31 \\ 1.9900 \mathrm{E}+00 & 702.0 & 1153.3 & 15.57 & 6.06 & 24.03 & 0.94 & 6.43 \\ 2.0000 \mathrm{E}+00 & 690.8 & 1148.4 & 15.54 & 6.04 & 23.98 & 1.02 & 6.53 \\ 2.0100 \mathrm{E}+00 & 674.9 & 1141.1 & 15.52 & 6.03 & 23.93 & 1.08 & 6.61 \\ 2.0200 \mathrm{E}+00 & 644.9 & 1129.9 & 15.52 & 6.03 & 23.90 & 1.11 & 6.66 \\ 2.0300 \mathrm{E}+00 & 603.7 & 1113.3 & 15.48 & 6.01 & 23.84 & 1.11 & 6.87 \\ 2.0400 \mathrm{E}+00 & 558.7 & 1093.2 & 15.42 & 5.99 & 23.74 & 1.11 & 7.25 \\ 2.0500 \mathrm{E}+00 & 510.0 & 1072.6 & 15.36 & 5.97 & 23.65 & 1.11 & 7.63 \\ 2.0600 \mathrm{E}+00 & 457.6 & 1051.2 & 15.30 & 5.94 & 23.55 & 1.11 & 7.99 \\ 2.0700 \mathrm{E}+00 & 395.9 & 1028.7 & 15.24 & 5.92 & 23.46 & 1.10 & 8.35 \\ 2.0800 \mathrm{E}+00 & 321.9 & 1004.7 & 15.18 & 5.90 & 23.37 & 1.10 & 8.70 \\ 2.0828 \mathrm{E}+00 & 298.0 & 997.7 & 15.17 & 5.89 & 23.35 & 1.10 & 8.79\end{array}$

TOTAL CPO TIME: 0 hours, 38 minutes, 58.680 seconds!

Simulation Results for downward Integration pass \# 2

1teration $=1 \operatorname{yup}(2)=-2.77381 E+06$ factor $=0.000000 \quad Y(3)=-2.77381 E+06$ bbound $=\mathrm{F} \quad$ lbound $=\mathrm{F} \quad$ fuppas $=\mathrm{F}$

\begin{tabular}{|c|c|c|c|c|c|c|c|}
\hline AX1al РOB. & ts (K) & $\operatorname{tg}(\mathrm{K})$ & mol\% $\mathrm{g2}$ & mol\% CO2 & mol\% co & mo1\% CH4 & mo1\% H2O \\
\hline 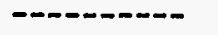 & 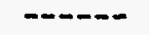 & ----- & ----n- & $---\infty---$ & - - - & - - & --- \\
\hline $2.0828 \mathrm{E}+00$ & 298.0 & 997.7 & 15.17 & 5.89 & 23.35 & 1.10 & 8.79 \\
\hline $2.0800 E+00$ & 322.1 & 1004.7 & 15.18 & 5.90 & 23.37 & 1.10 & 8.70 \\
\hline$\cdots$ & & & & & & - & \\
\hline$\cdots$ & & & & & & & \\
\hline & & & & & & & \\
\hline $1.9600 \mathrm{E}+00$ & 746.2 & 1183.8 & 15.52 & 6.03 & 23.89 & 1.12 & 6.69 \\
\hline $1.9500 \mathrm{E}+00$ & 760.8 & 1193.7 & 15.52 & 6.03 & 23.89 & 1.12 & 6.69 \\
\hline Sw1tched to & equilibr & a calcul & attons at & $z=1.942$ & 7 where $t$ & $=1200.41$ & \\
\hline $1.9400 E+00$ & 773.2 & 1147.5 & 16.58 & 6.36 & 24.44 & 0.00 & 5.06 \\
\hline$\cdots$ & & & & & & & \\
\hline & & & & & & & \\
\hline$\ddot{2.0000 E-02}$ & 552.3 & 552.9 & 0.00 & 0.00 & 0.00 & 0.00 & 20.80 \\
\hline $1.0000 \mathrm{E}-02$ & 552.5 & 553.1 & 0.00 & 0.00 & 0.00 & 0.00 & 20.80 \\
\hline $0.0000 E+00$ & 552.7 & 553.3 & 0.00 & 0.00 & 0.00 & 0.00 & 20.80 \\
\hline
\end{tabular}

Overall Heat IOBs $=0.3580 \mathrm{E}+06 \mathrm{~J} / \mathrm{s}$

TOTAL CPO TIME: 1 hours, 2 minutes, 20.480 seconds! 
Chapter 6

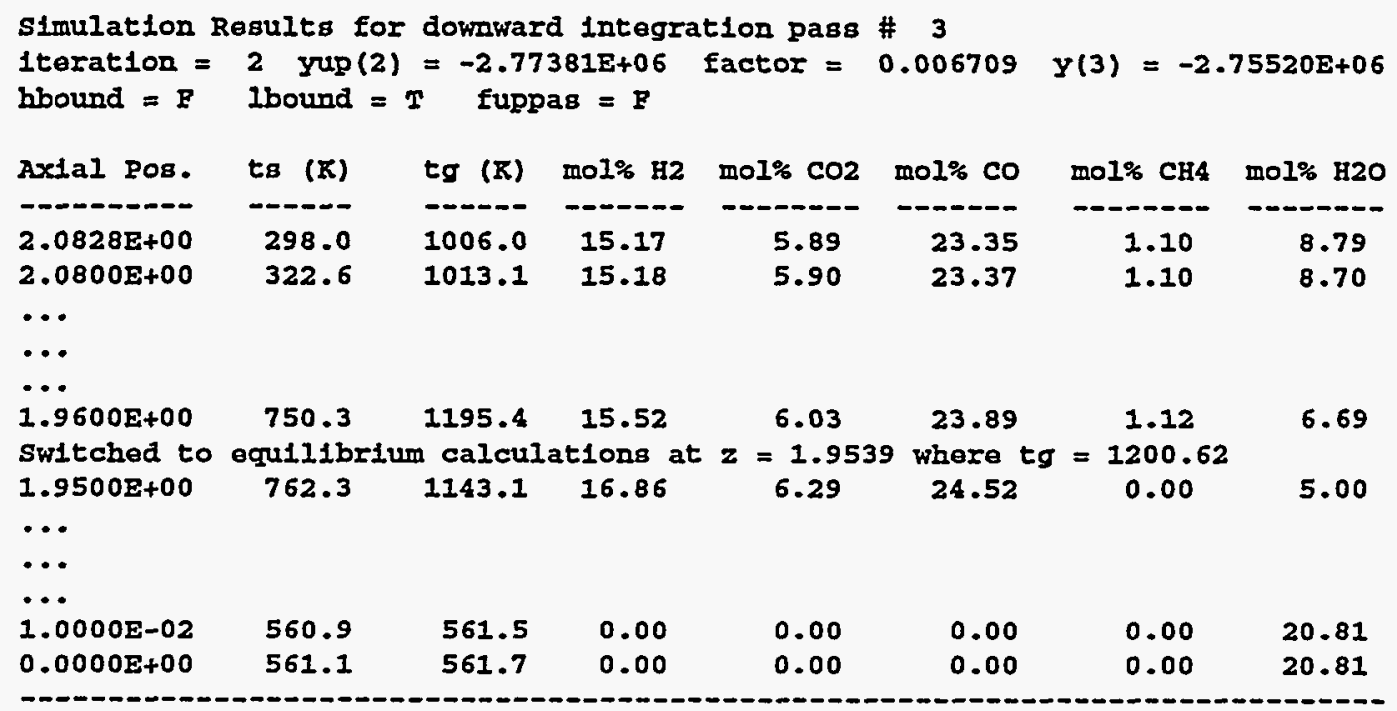

Overall Heat LO8s $=0.3483 \mathrm{E}+06 \mathrm{~J} / \mathrm{s}$

TOTAI CPU TIME: 1 hours, 25 minutes, 59.280 seconds!

Simulation Results for downward integration pass \# 4

1terat1on $=3 \operatorname{yup}(2)=-2.77381 E+06$ factor $=0.003354 \quad y(3)=-2.76451 E+06$ bbound $=T$ 1bound $=T$ fuppas $=F$

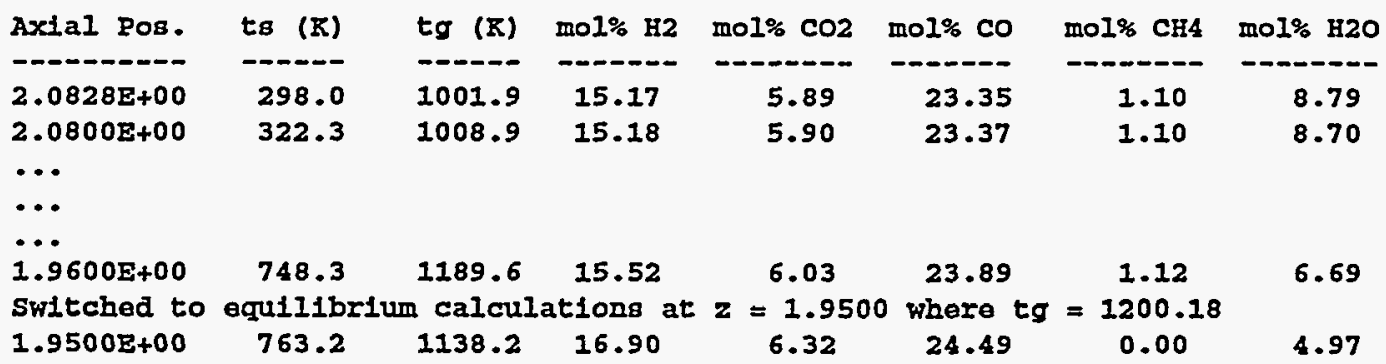

-..

-..

-...

$\begin{array}{llllllll}1.0000 \mathrm{E}-02 & 556.7 & 557.2 & 0.00 & 0.00 & 0.00 & 0.00 & 20.80 \\ 0.0000 \mathrm{E}+00 & 556.8 & 557.4 & 0.00 & 0.00 & 0.00 & 0.00 & 20.80\end{array}$

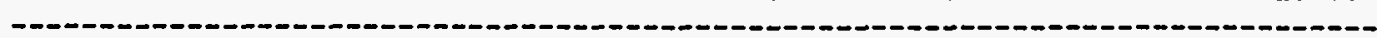

Overall Heat IOBS $=0.3530 \mathrm{E}+06 \mathrm{~J} / \mathrm{s}$ 
User's Guide

TOTAL CPO TIME: 1 hours, 50 minutes, 4.350 seconds!

Simulation Results for downward integration pass \# 5

1teration $=4$ yup $(2)=-2.77381 E+06$ factor $=0.005032$ y(3) $=-2.75985 E+06$ hbound $=T$ Ibound $=T$ fuppas $=F$

\begin{tabular}{|c|c|c|c|c|c|c|c|}
\hline Axlal Poв. & ts $(\mathrm{K})$ & tg $(\pi)$ & mo1\% $\mathrm{H2}$ & $\mathrm{mol} \% \mathrm{CO} 2$ & mol\% co & $\mathrm{mol} \% \mathrm{Cn} 4$ & mol\% 120 \\
\hline$-\infty-\infty-\infty-\infty$ & $---\infty--$ & $-\infty-\infty$ & $-\infty-\infty$ & 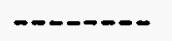 & ------ & $-\infty---\infty$ & $---\infty-\infty$ \\
\hline $2.0828 \mathrm{E}+00$ & 298.0 & 1003.9 & 15.17 & 5.89 & 23.35 & 1.10 & 8.79 \\
\hline $2.0800 \mathrm{E}+00$ & 322.4 & 1011.0 & 15.18 & 5.90 & 23.37 & 1.10 & 8.70 \\
\hline \multicolumn{8}{|l|}{$\cdots$} \\
\hline $1.9600 \mathrm{E}+00$ & 749.3 & 1192.5 & 15.52 & 6.03 & 23.89 & 1.12 & 6.69 \\
\hline Sw1tched to & equilibr & n calcul & attons at & $z=1.951$ & 2 where ts & $=1200.41$ & \\
\hline $1.9500 \mathrm{E}+00$ & 762.6 & 1140.8 & 16.88 & 6.31 & 24.51 & 0.00 & 4.98 \\
\hline \multicolumn{8}{|l|}{$\cdots$} \\
\hline$\ddot{2} .0000 \mathrm{E}-02$ & 558.7 & 559.2 & 0.00 & 0.00 & 0.00 & 0.00 & 20.81 \\
\hline $1.0000 \mathrm{E}-02$ & 558.9 & 559.4 & 0.00 & 0.00 & 0.00 & 0.00 & 20.81 \\
\hline $0.0000 \mathrm{E}+00$ & 559.0 & 559.6 & 0.00 & 0.00 & 0.00 & 0.00 & 20.81 \\
\hline
\end{tabular}

Overall Heat LOSB $=0.3506 \mathrm{E}+06 \mathrm{~J} / \mathrm{s}$

TOTAI CPO TIME: 2 hours, 13 minutes, 12.890 seconds!

Simulation Results for final upward integration pass

\begin{tabular}{|c|c|c|c|c|c|c|c|}
\hline Axtal Pos. & ts (K) & $\operatorname{tg}(\pi)$ & mo1\% Н2 & mol\% $\mathrm{CO} 2$ & mol\% $\mathrm{CO}$ & mo1\% CH4 & mol\% $\mathrm{E2O}$ \\
\hline $0.0000 \mathrm{E}+00$ & 559.0 & 560.0 & 0.00 & 0.00 & 0.00 & 0.00 & 20.94 \\
\hline $1.0000 \mathrm{E}-02$ & 558.9 & 559.8 & 0.00 & 0.00 & 0.00 & 0.00 & 20.94 \\
\hline $2.0000 E-02$ & 558.7 & 559.6 & 0.00 & 0.00 & 0.00 & 0.00 & 20.94 \\
\hline $3.0000 \mathrm{E}-02$ & 558.5 & 559.4 & 0.00 & 0.00 & 0.00 & 0.00 & 20.94 \\
\hline $4.0000 \mathrm{E}-02$ & 558.3 & 559.2 & 0.00 & 0.00 & 0.00 & 0.00 & 20.94 \\
\hline $5.0000 \mathrm{E}-02$ & 558.1 & 559.0 & 0.00 & 0.00 & 0.00 & 0.00 & 20.94 \\
\hline $6.0000 \mathrm{E}-02$ & 557.9 & 558.8 & 0.00 & 0.00 & 0.00 & 0.00 & 20.94 \\
\hline $7.0000 \mathrm{E}-02$ & 557.7 & 558.6 & 0.00 & 0.00 & 0.00 & 0.00 & 20.94 \\
\hline B. $0000 \mathrm{E}-02$ & 557.6 & 558.4 & 0.00 & 0.00 & 0.00 & 0.00 & 20.94 \\
\hline $9.0000 \mathrm{E}-02$ & 557.4 & 558.2 & 0.00 & 0.00 & 0.00 & 0.00 & 20.94 \\
\hline $1.0000 \mathrm{E}-01$ & 557.2 & 558.0 & 0.00 & 0.00 & 0.00 & 0.00 & 20.94 \\
\hline $1.1000 \mathrm{E}-01$ & 557.0 & 557.8 & 0.00 & 0.00 & 0.00 & 0.00 & 20.94 \\
\hline $1.2000 \mathrm{E}-01$ & 556.8 & 557.6 & 0.00 & 0.00 & 0.00 & 0.00 & 20.94 \\
\hline $1.3000 \mathrm{E}-01$ & 556.6 & 557.4 & 0.00 & 0.00 & 0.00 & 0.00 & 20.94 \\
\hline $1.4000 \mathrm{E}-01$ & 556.5 & 557.2 & 0.00 & 0.00 & 0.00 & 0.00 & 20.94 \\
\hline $1.5000 \mathrm{E}-01$ & 556.3 & 557.0 & 0.00 & 0.00 & 0.00 & 0.00 & 20.94 \\
\hline $1.6000 \mathrm{E}-01$ & 556.1 & 556.8 & 0.00 & 0.00 & 0.00 & 0.00 & 20.94 \\
\hline
\end{tabular}


Chapter 6

..

\begin{tabular}{|c|c|c|c|c|c|c|c|}
\hline $1.7000 \mathrm{E}-01$ & 555.9 & 556.7 & 0.00 & 0.00 & 0.00 & 0.00 & 20.94 \\
\hline $1.8000 \mathrm{E}-01$ & 555.7 & 556.5 & 0.00 & 0.00 & 0.00 & 0.00 & 20.94 \\
\hline $1.9000 \mathrm{E}-01$ & 555.5 & 556.3 & 0.00 & 0.00 & 0.00 & 0.00 & 20.94 \\
\hline $2.0000 \mathrm{E}-01$ & 555.4 & 556.1 & 0.00 & 0.00 & 0.00 & 0.00 & 20.94 \\
\hline $2.1000 \mathrm{E}-01$ & 555.2 & 555.9 & 0.00 & 0.00 & 0.00 & 0.00 & 20.94 \\
\hline $2.2000 \mathrm{E}-01$ & 555.0 & 555.7 & 0.00 & 0.00 & 0.00 & 0.00 & 20.94 \\
\hline 2.3000 E-0I & 554.8 & 555.5 & 0.00 & 0.00 & 0.00 & 0.00 & 20.94 \\
\hline $2.4000 E-01$ & 554.6 & 555.3 & 0.00 & 0.00 & 0.00 & 0.00 & 20.94 \\
\hline $2.5000 \mathrm{E}-01$ & 554.5 & 555.1 & 0.00 & 0.00 & 0.00 & 0.00 & 20.94 \\
\hline $2.6000 \mathrm{E}-01$ & 554.3 & 555.0 & 0.00 & 0.00 & 0.00 & 0.00 & 20.94 \\
\hline $2.7000 \mathrm{E}-01$ & 554.1 & 554.8 & 0.00 & 0.00 & 0.00 & 0.00 & 20.94 \\
\hline $2.8000 \mathrm{E}-01$ & 553.9 & 554.6 & 0.00 & 0.00 & 0.00 & 0.00 & 20.94 \\
\hline $2.9000 \mathrm{E}-01$ & 553.7 & 554.4 & 0.00 & 0.00 & 0.00 & 0.00 & 20.94 \\
\hline 3.0000 E-01 & 553.6 & 554.2 & 0.00 & 0.00 & 0.00 & 0.00 & 20.94 \\
\hline $3.1000 \mathrm{E}-01$ & 553.4 & 554.0 & 0.00 & 0.00 & 0.00 & 0.00 & 20.94 \\
\hline $3.2000 z-01$ & 553.2 & 553.9 & 0.00 & 0.00 & 0.00 & 0.00 & 20.94 \\
\hline 3.3000E-01 & 553.0 & 553.7 & 0.00 & 0.00 & 0.00 & 0.00 & 20.94 \\
\hline $3.4000 E-01$ & 552.9 & 553.5 & 0.00 & 0.00 & 0.00 & 0.00 & 20.94 \\
\hline $3.5000 E-01$ & 552.7 & 553.3 & 0.00 & 0.00 & 0.00 & 0.00 & 20.94 \\
\hline $3.6000 \mathrm{E}-01$ & 552.5 & 553.1 & 0.00 & 0.00 & 0.00 & 0.00 & 20.94 \\
\hline $3.7000 \mathrm{E}-01$ & 552.3 & $553.0^{\circ}$ & 0.00 & 0.00 & 0.00 & 0.00 & 20.94 \\
\hline $3.8000 \mathrm{E}-01$ & 552.2 & 552.8 & 0.00 & 0.00 & 0.00 & 0.00 & 20.94 \\
\hline $3.9000 \mathrm{E}-01$ & 552.0 & 552.6 & 0.00 & 0.00 & 0.00 & 0.00 & 20.94 \\
\hline $4.0000 \mathrm{E}-01$ & 551.8 & $55 \dot{2} .4$ & 0.00 & 0.00 & 0.00 & 0.00 & 20.94 \\
\hline $4.1000 \Sigma-01$ & 551.6 & 552.2 & 0.00 & 0.00 & 0.00 & 0.00 & 20.94 \\
\hline $4.2000 \mathrm{E}-01$ & 551.5 & 552.1 & 0.00 & 0.00 & 0.00 & 0.00 & 20.94 \\
\hline $4.3000 \mathrm{E}-01$ & 551.3 & 551.9 & 0.00 & 0.00 & 0.00 & 0.00 & 20.94 \\
\hline $4.4000 \mathrm{E}-01$ & 551.1 & 551.7 & 0.00 & 0.00 & 0.00 & 0.00 & 20.94 \\
\hline $4.5000 \mathrm{E}-01$ & 550.9 & 551.5 & 0.00 & 0.00 & 0.00 & 0.00 & 20.94 \\
\hline $4.6000 \mathrm{E}-01$ & 550.8 & 551.4 & 0.00 & 0.00 & 0.00 & 0.00 & 20.94 \\
\hline $4.7000 \mathrm{E}-01$ & 550.6 & 551.2 & 0.00 & 0.00 & 0.00 & 0.00 & 20.94 \\
\hline $4.8000 E-01$ & 550.4 & 551.0 & 0.00 & 0.00 & 0.00 & 0.00 & 20.94 \\
\hline $4.9000 \mathrm{E}-01$ & 550.3 & 550.8 & 0.00 & 0.00 & 0.00 & 0.00 & 20.94 \\
\hline $5.0000 \mathrm{E}-01$ & 550.1 & 550.7 & 0.00 & 0.00 & 0.00 & 0.00 & 20.94 \\
\hline $5.1000 \mathrm{E}-01$ & 549.9 & 550.5 & 0.00 & 0.00 & 0.00 & 0.00 & 20.94 \\
\hline $5.2000 \mathrm{E}-01$ & 549.7 & 550.3 & 0.00 & 0.00 & 0.00 & 0.00 & 20.94 \\
\hline $5.3000 \mathrm{E}-01$ & 549.6 & 550.1 & 0.00 & 0.00 & 0.00 & 0.00 & 20.94 \\
\hline $5.4000 \mathrm{E}-01$ & 549.4 & 550.0 & 0.00 & 0.00 & 0.00 & 0.00 & 20.94 \\
\hline $5.5000 \mathrm{E}-01$ & 549.2 & 549.8 & 0.00 & 0.00 & 0.00 & 0.00 & 20.94 \\
\hline $5.6000 \mathrm{E}-01$ & 549.1 & 549.6 & 0.00 & 0.00 & 0.00 & 0.00 & 20.94 \\
\hline $5.7000 \mathrm{E}-01$ & 548.9 & 549.5 & 0.00 & 0.00 & 0.00 & 0.00 & 20.94 \\
\hline $5.8000 \mathrm{E}-01$ & 548.8 & 549.3 & 0.00 & 0.00 & 0.00 & 0.00 & 20.94 \\
\hline $5.9000 \mathrm{E}-01$ & 555.3 & 549.3 & 0.00 & 0.00 & 0.00 & 0.00 & 20.94 \\
\hline $6.0000 \mathrm{E}-01$ & 839.5 & 556.7 & 0.00 & 0.00 & 0.00 & 0.00 & 20.94 \\
\hline $6.1000 \mathrm{E}-01$ & 1345.3 & 639.8 & 0.00 & 0.53 & 0.00 & 0.00 & 20.98 \\
\hline $6.2000 \mathrm{E}-01$ & 1390.4 & 785.0 & 0.00 & 1.59 & 0.00 & 0.00 & 21.06 \\
\hline $6.3000 E-01$ & 1428.2 & 922.5 & 0.00 & 2.63 & 0.00 & 0.00 & 21.14 \\
\hline $6.4000 \mathrm{E}-01$ & 1461.8 & 1053.8 & 0.00 & 3.67 & 0.00 & 0.00 & 21.21 \\
\hline $6.5000 \mathrm{E}-01$ & 1492.5 & 1180.1 & 0.00 & 4.70 & 0.00 & 0.00 & 21.29 \\
\hline
\end{tabular}




\begin{tabular}{|c|c|c|c|c|c|c|c|}
\hline $6.6000 \mathrm{E}-01$ & 1521.2 & 1302.2 & 0.00 & 5.73 & 0.00 & 0.00 & 21.37 \\
\hline $6.7000 \mathrm{E}-01$ & 1548.7 & 1420.9 & 0.00 & 6.76 & 0.00 & 0.00 & 21.44 \\
\hline $6.8000 \mathrm{E}-01$ & 1575.3 & 1536.4 & 0.00 & 7.80 & 0.00 & 0.00 & 21.51 \\
\hline $6.9000 \mathrm{E}-01$ & 1601.3 & 1648.9 & 0.00 & 8.84 & 0.00 & 0.00 & 21.58 \\
\hline 7.0000E-01 & 1626.7 & 1758.3 & 0.00 & 9.88 & 0.01 & 0.00 & 21.64 \\
\hline $7.1000 \mathrm{E}-01$ & 1651.3 & 1864.1 & 0.01 & 10.91 & 0.02 & 0.00 & 21.68 \\
\hline $7.2000 \mathrm{E}-01$ & 1674.7 & 1964.8 & 0.02 & 11.91 & 0.06 & 0.00 & 21.71 \\
\hline $7.3000 E-01$ & 1696.6 & 2058.0 & 0.06 & 12.84 & 0.16 & 0.00 & 21.69 \\
\hline 7.4000 E-01 & 1704.2 & 2137.8 & 0.12 & 13.61 & 0.39 & 0.00 & 21.64 \\
\hline $7.5000 \mathrm{E}-01$ & 1704.6 & 2197.3 & 0.24 & 14.12 & 0.81 & 0.00 & 21.52 \\
\hline $7.6000 \mathrm{E}-01$ & 1704.2 & 2232.6 & 0.42 & 14.31 & 1.49 & 0.00 & 21.32 \\
\hline 7.7000E-01 & 1703.0 & 2242.9 & 0.67 & 14.16 & 2.42 & 0.00 & 21.02 \\
\hline 7. $8000 \mathrm{E}-01$ & 1694.7 & 2233.9 & 0.99 & 13.79 & 3.52 & 0.00 & 20.65 \\
\hline $7.9000 \mathrm{E}-01$ & 1685.5 & 2214.8 & 1.34 & 13.32 & 4.63 & 0.00 & 20.22 \\
\hline $8.0000 \mathrm{E}-01$ & 1676.2 & 2191.9 & 1.69 & 12.86 & 5.70 & 0.00 & 19.79 \\
\hline $8.1000 \mathrm{E}-01$ & 1667.4 & 2167.9 & 2.05 & 12.41 & 6.69 & 0.00 & 19.35 \\
\hline 8.2000E-01 & 1659.0 & 2144.2 & 2.39 & 12.01 & 7.61 & 0.00 & 18.92 \\
\hline $8.3000 \mathrm{E}-01$ & 1651.1 & 2121.1 & 2.72 & 11.63 & 8.46 & 0.00 & 18.51 \\
\hline $8.4000 \mathrm{E}-01$ & 1643.8 & 2098.8 & 3.05 & 11.29 & 9.25 & 0.00 & 18.10 \\
\hline $8.5000 \mathrm{E}-01$ & 1636.9 & 2077.5 & 3.36 & 10.97 & 9.98 & 0.00 & 17.72 \\
\hline $8.6000 \mathrm{E}-01$ & 1630.4 & 2057.2 & 3.66 & 10.68 & 10.66 & 0.00 & 17.34 \\
\hline $8.7000 E-01$ & 1624.2 & 2037.8 & 3.95 & 10.42 & 11.29 & 0.00 & 16.98 \\
\hline $8.8000 \mathrm{E}-01$ & 1618.5 & 2019.2 & 4.23 & 10.18 & 11.89 & 0.00 & 16.64 \\
\hline $8.9000 \mathrm{E}-01$ & 1613.0 & 2001.6 & 4.50 & 9.95 & 12.44 & 0.00 & 16.31 \\
\hline $9.0000 \mathrm{E}-01$ & 1607.8 & 1984.7 & 4.76 & 9.75 & 12.96 & 0.00 & 15.99 \\
\hline $9.1000 \mathrm{E}-01$ & 1602.9 & 1968.6 & 5.00 & 9.56 & 13.44 & 0.00 & 15.68 \\
\hline $9.2000 \mathrm{E}-01$ & 1598.2 & 1953.2 & 5.24 & 9.38 & 13.90 & 0.00 & 15.39 \\
\hline $9.3000 \mathrm{E}-01$ & 1593.7 & 1938.5 & 5.46 & 9.22 & 14.33 & 0.00 & 15.11 \\
\hline $9.4000 \mathrm{E}-01$ & 1589.4 & 1924.4 & 5.68 & 9.07 & 14.74 & 0.00 & 14.84 \\
\hline $9.5000 \mathrm{E}-01$ & 1585.3 & 1910.9 & 5.88 & 8.93 & 15.13 & 0.00 & 14.58 \\
\hline $9.6000 \mathrm{E}-01$ & 1581.3 & 1898.0 & 6.08 & 8.80 & 15.49 & 0.00 & 14.33 \\
\hline 9.7000 E-01 & 1577.5 & 1885.6 & 6.27 & 8.67 & 15.84 & 0.00 & 14.09 \\
\hline $9.8000 \mathrm{E}-01$ & 1573.8 & 1873.7 & 6.45 & 8.56 & 16.17 & 0.00 & 13.86 \\
\hline $9.9000 \mathrm{E}-01$ & 1570.2 & 1862.2 & 6.62 & 8.45 & 16.49 & 0.00 & 13.64 \\
\hline $1.0000 \mathrm{E}+00$ & 1566.7 & 1851.1 & 6.79 & 8.35 & 16.79 & 0.00 & 13.43 \\
\hline $1.0100 \mathrm{E}+00$ & 1563.3 & 1840.4 & 6.95 & 8.26 & 17.08 & 0.00 & 13.22 \\
\hline $1.0200 \mathrm{E}+00$ & 1560.0 & 1830.0 & 7.11 & 8.16 & 17.35 & 0.00 & 13.02 \\
\hline $1.0300 \mathrm{E}+00$ & 1556.8 & 1819.9 & 7.26 & 8.08 & 17.62 & 0.00 & 12.83 \\
\hline $1.0400 E+00$ & 1553.6 & 1810.1 & 7.41 & 7.99 & 17.88 & 0.00 & 12.64 \\
\hline $1.0500 \mathrm{E}+00$ & 1550.5 & 1800.6 & 7.55 & 7.91 & 18.12 & 0.00 & 12.46 \\
\hline $1.0600 \mathrm{E}+00$ & 1547.5 & 1791.3 & 7.69 & 7.84 & 18.36 & 0.00 & 12.28 \\
\hline $1.0700 \mathrm{E}+00$ & 1544.5 & 1782.2 & 7.82 & 7.76 & 18.59 & 0.00 & 12.11 \\
\hline $1.0800 \mathrm{E}+00$ & 1541.6 & 1773.3 & 7.95 & 7.69 & 18.81 & 0.00 & 11.95 \\
\hline $1.0900 \mathrm{E}+00$ & 1538.7 & 1764.7 & 8.08 & 7.62 & 19.02 & 0.00 & 11.79 \\
\hline $1.1000 \mathrm{E}+00$ & 1535.9 & 1756.2 & 8.21 & 7.56 & 19.23 & 0.00 & 11.63 \\
\hline $1.1100 \mathrm{E}+00$ & 1533.1 & 1748.0 & 8.33 & 7.49 & 19.43 & 0.00 & 11.48 \\
\hline $1.1200 \mathrm{E}+00$ & 1530.3 & 1739.9 & 8.45 & 7.43 & 19.62 & 0.00 & 11.33 \\
\hline $1.1300 \mathrm{E}+00$ & 1527.6 & 1732.0 & 8.57 & 7.37 & 19.81 & 0.00 & 11.18 \\
\hline $1.1400 \mathrm{E}+00$ & 1524.9 & 1724.3 & 8.68 & 7.31 & 19.99 & 0.00 & 11.04 \\
\hline
\end{tabular}


Chapter 6

\begin{tabular}{|c|c|c|c|c|c|c|c|}
\hline $1.1500 \mathrm{E}+00$ & 1522.3 & 1716.8 & 8.79 & 7.25 & 20.16 & 0.00 & 10.91 \\
\hline $1.1600 E+00$ & 1519.7 & 1709.4 & 8.90 & 7.20 & 20.33 & 0.00 & 10.78 \\
\hline $1.1700 E+00$ & 1517.2 & 1702.2 & 9.00 & 7.15 & 20.49 & 0.00 & 10.65 \\
\hline $1.1800 \mathrm{E}+00$ & 1514.6 & 1695.2 & 9.11 & 7.09 & 20.65 & 0.00 & 10.52 \\
\hline $1.1900 \mathrm{E}+00$ & 1512.1 & 1688.3 & 9.21 & 7.05 & 20.80 & 0.00 & 10.40 \\
\hline $1.2000 E+00$ & 1509.7 & 1681.5 & 9.30 & 7.00 & 20.95 & 0.00 & 10.28 \\
\hline $1.2100 \mathrm{E}+00$ & 1507.2 & 1674.9 & 9.40 & 6.95 & 21.09 & 0.00 & 10.16 \\
\hline $1.2200 \mathrm{E}+00$ & 1504.8 & 1668.5 & 9.49 & 6.91 & 21.23 & 0.00 & 10.05 \\
\hline $1.2300 E+00$ & 1502.5 & 1662.2 & 9.58 & 6.86 & 21.37 & 0.00 & 9.94 \\
\hline $1.2400 \mathrm{E}+00$ & 1500.1 & 1656.0 & 9.67 & 6.82 & 21.50 & 0.00 & 9.83 \\
\hline $1.2500 \mathrm{E}+00$ & 1497.8 & 1649.9 & 9.76 & 6.78 & 21.63 & 0.00 & 9.73 \\
\hline $1.2600 \mathrm{E}+00$ & 1495.5 & 1644.0 & 9.84 & 6.74 & 21.75 & 0.00 & 9.63 \\
\hline $1.2700 \mathrm{E}+00$ & 1493.2 & 1638.2 & 9.93 & 6.70 & 21.87 & 0.00 & 9.53 \\
\hline $1.2800 \mathrm{E}+00$ & 1491.0 & 1632.5 & 10.01 & 6.67 & 21.98 & 0.00 & 9.43 \\
\hline $1.2900 E+00$ & 1488.8 & 1626.9 & 10.08 & 6.63 & 22.10 & 0.00 & 9.34 \\
\hline $1.3000 \mathrm{E}+00$ & 1486.6 & 1621.4 & 10.16 & 6.60 & 22.21 & 0.00 & 9.25 \\
\hline $1.3100 \mathrm{E}+00$ & 1484.4 & 1616.1 & 10.24 & 6.56 & 22.31 & 0.00 & 9.16 \\
\hline $1.3200 \mathrm{E}+00$ & 1482.2 & 1610.8 & 10.31 & 6.53 & 22.42 & 0.00 & 9.07 \\
\hline $1.3300 \mathrm{E}+00$ & 1480.1 & 1605.7 & 10.38 & 6.50 & 22.52 & 0.00 & 8.99 \\
\hline $1.3400 \mathrm{E}+00$ & 1477.9 & 1600.6 & 10.45 & 6.47 & 22.61 & 0.00 & 8.90 \\
\hline $1.3500 \mathrm{E}+00$ & 1475.8 & 1595.7 & 10.52 & 6.44 & 22.71 & 0.00 & 8.82 \\
\hline $1.3600 \mathrm{E}+00$ & 1473.8 & 1590.8 & 10.58 & 6.41 & 22.80 & 0.00 & 8.75 \\
\hline $1.3700 \mathrm{E}+00$ & 1471.7 & 1586.0 & 10.65 & 6.38 & 22.89 & 0.00 & 8.67 \\
\hline $1.3800 \mathrm{E}+00$ & 1469.6 & 1581.4 & 10.71 & 6.36 & 22.98 & 0.00 & 8.59 \\
\hline $1.3900 \mathrm{E}+00$ & 1467.6 & 1576.8 & 10.77 & 6.33 & 23.06 & 0.00 & 8.52 \\
\hline $1.4000 \mathrm{E}+00$ & 1465.6 & 1572.3 & 10.83 & 6.31 & 23.14 & 0.00 & 8.45 \\
\hline $1.4100 \mathrm{E}+00$ & 1463.6 & 1567.8 & 10.89 & 6.28 & 23.22 & 0.00 & 8.38 \\
\hline $1.4200 \mathrm{E}+00$ & 1461.6 & 1563.5 & 10.95 & 6.26 & 23.30 & 0.00 & 8.31 \\
\hline $1.4300 \mathrm{E}+00$ & 1459.6 & 1559.2 & 11.00 & 6.23 & 23.38 & 0.00 & 8.24 \\
\hline $1.4400 E+00$ & 1457.6 & 1555.0 & 11.06 & 6.21 & 23.45 & 0.00 & 8.18 \\
\hline $1.4500 E+00$ & 1455.7 & 1550.9 & 11.11 & 6.19 & 23.52 & 0.00 & 8.12 \\
\hline $1.4600 E+00$ & 1453.7 & 1546.8 & 11.16 & 6.17 & 23.59 & 0.00 & 8.05 \\
\hline $1.4700 \mathrm{E}+00$ & 1451.8 & 1542.8 & 11.22 & 6.15 & 23.66 & 0.00 & 7.99 \\
\hline $1.4800 \mathrm{E}+00$ & 1449.8 & 1538.9 & 11.27 & 6.13 & 23.72 & 0.00 & 7.93 \\
\hline $1.4900 \mathrm{E}+00$ & 1447.9 & 1535.0 & 11.32 & 6.11 & 23.79 & 0.00 & 7.88 \\
\hline $1.5000 \mathrm{E}+00$ & 1445.9 & 1531.2 & 11.36 & 6.09 & 23.85 & 0.00 & 7.82 \\
\hline $1.5100 \mathrm{E}+00$ & 1443.9 & 1527.5 & 11.41 & 6.07 & 23.91 & 0.00 & 7.76 \\
\hline $1.5200 \mathrm{E}+00$ & 1441.9 & 1523.8 & 11.46 & 6.06 & 23.97 & 0.00 & 7.71 \\
\hline $1.5300 \mathrm{E}+00$ & 1439.8 & 1520.1 & 11.50 & 6.04 & 24.03 & 0.00 & 7.66 \\
\hline $1.5400 \mathrm{E}+00$ & 1437.7 & 1516.5 & 11.55 & 6.03 & 24.08 & 0.00 & 7.60 \\
\hline $1.5500 \mathrm{E}+00$ & 1435.6 & 1513.0 & 11.59 & 6.01 & 24.14 & 0.00 & 7.55 \\
\hline $1.5600 \mathrm{E}+00$ & 1433.3 & 1509.5 & 11.63 & 6.00 & 24.19 & 0.00 & 7.50 \\
\hline $1.5700 \mathrm{E}+00$ & 1431.0 & 1506.0 & 11.67 & 5.98 & 24.24 & 0.00 & 7.46 \\
\hline $1.5800 \mathrm{E}+00$ & 1428.5 & 1502.6 & 11.71 & 5.97 & 24.29 & 0.00 & 7.41 \\
\hline $1.5900 \mathrm{E}+00$ & 1425.8 & 1499.1 & 11.75 & 5.95 & 24.34 & 0.00 & 7.36 \\
\hline $1.6000 \mathrm{E}+00$ & 1422.9 & 1495.7 & 11.79 & 5.94 & 24.38 & 0.00 & 7.32 \\
\hline $1.6100 \mathrm{E}+00$ & 1419.7 & 1492.3 & 11.83 & 5.93 & 24.42 & 0.00 & 7.28 \\
\hline $1.6200 \mathrm{E}+00$ & 1416.1 & 1488.9 & 11.86 & 5.92 & 24.46 & 0.00 & 7.23 \\
\hline $1.6300 \mathrm{E}+00$ & 1412.1 & 1485.5 & 11.90 & 5.91 & 24.50 & 0.00 & 7.19 \\
\hline
\end{tabular}


User's Guide

\begin{tabular}{|c|c|c|c|c|c|c|c|}
\hline $1.6400 \mathrm{E}+00$ & 1407.6 & 1482.0 & 11.94 & 5.90 & 24.54 & 0.00 & 7.15 \\
\hline $1.6500 \mathrm{E}+00$ & 1402.3 & 1478.4 & 11.97 & 5.90 & 24.57 & 0.00 & 7.11 \\
\hline $1.6600 \mathrm{E}+00$ & 1396.2 & 1474.7 & 12.01 & 5.89 & 24.60 & 0.00 & 7.08 \\
\hline $1.6700 \mathrm{E}+00$ & 1389.0 & 1470.9 & 12.04 & 5.89 & 24.62 & 0.00 & 7.04 \\
\hline $1.6800 \mathrm{E}+00$ & 1380.5 & 1466.9 & 12.08 & 5.89 & 24.64 & 0.00 & 7.01 \\
\hline $1.6900 \mathrm{E}+00$ & 1370.3 & 1462.6 & 12.11 & 5.89 & 24.66 & 0.00 & 6.97 \\
\hline $1.7000 \mathrm{E}+00$ & $1358: 1$ & 1458.0 & 12.15 & 5.89 & 24.67 & 0.00 & 6.94 \\
\hline $1.7100 \mathrm{E}+00$ & 1343.1 & 1452.8 & 12.19 & 5.90 & 24.68 & 0.00 & 6.90 \\
\hline $1.7200 \mathrm{E}+00$ & 1324.8 & 1446.9 & 12.24 & 5.90 & 24.68 & 0.00 & 6.87 \\
\hline 1.7300E+00 & 1302.1 & 1440.1 & 12.31 & 5.91 & 24.68 & 0.00 & 6.83 \\
\hline $1.7400 \mathrm{E}+00$ & 1273.8 & 1431.9 & 12.39 & 5.91 & 24.67 & 0.00 & 6.78 \\
\hline 1.7500E+00 & 1239.0 & 1422.1 & 12.50 & 5.92 & 24.66 & 0.00 & 6.73 \\
\hline $1.7600 \mathrm{E}+00$ & 1198.5 & 1410.5 & 12.65 & 5.92 & 24.65 & 0.00 & 6.67 \\
\hline $1.7700 E+00$ & 1155.5 & 1397.5 & 12.82 & 5.92 & 24.63 & 0.00 & 6.60 \\
\hline $1.7800 \mathrm{E}+00$ & 1113.7 & 1383.6 & 13.00 & 5.93 & 24.61 & 0.00 & 6.53 \\
\hline $1.7900 E+00$ & 1074.6 & 1369.1 & 13.19 & 5.94 & 24.58 & 0.00 & 6.46 \\
\hline $1.8000 \mathrm{E}+00$ & 1038.0 & 1354.4 & 13.38 & 5.97 & 24.55 & 0.00 & 6.39 \\
\hline 1. $8100 E+00$ & 1004.0 & 1339.7 & 13.56 & 5.99 & 24.52 & 0.00 & 6.32 \\
\hline $1.8200 \mathrm{E}+00$ & 973.3 & 1325.1 & 13.73 & 6.03 & 24.49 & 0.00 & 6.25 \\
\hline $1.8300 \mathrm{E}+00$ & 946.6 & 1310.6 & 13.92 & 6.06 & 24.46 & 0.00 & 6.18 \\
\hline $1.8400 E+00$ & 923.5 & 1296.2 & 14.10 & 6.10 & 24.44 & 0.00 & 6.10 \\
\hline $1.8500 \mathrm{E}+00$ & 903.5 & 1281.9 & 14.29 & 6.13 & 24.42 & 0.00 & 6.02 \\
\hline $1.8600 \mathrm{E}+00$ & 885.8 & 1267.6 & 14.49 & 6.17 & 24.40 & 0.00 & 5.94 \\
\hline $1.8700 \mathrm{E}+00$ & 869.7 & 1253.5 & 14.70 & 6.20 & 24.38 & 0.00 & 5.86 \\
\hline $1.8800 \mathrm{E}+00$ & 854.8 & 1239.4 & 14.92 & 6.24 & 24.37 & 0.00 & 5.77 \\
\hline $1.8900 \mathrm{E}+00$ & 840.6 & 1225.1 & 15.15 & 6.27 & 24.36 & 0.00 & 5.68 \\
\hline $1.9000 \mathrm{E}+00$ & 826.8 & 1210.4 & 15.40 & 6.29 & 24.37 & 0.00 & 5.57 \\
\hline \multicolumn{8}{|c|}{ Swltched to non-equilibrium calculations } \\
\hline $1.9100 \mathrm{E}+00$ & 813.3 & 1199.2 & 15.41 & 6.29 & 24.36 & 0.03 & 5.59 \\
\hline $1.9200 \mathrm{E}+00$ & 800.0 & 1191.7 & 15.41 & 6.27 & 24.33 & 0.12 & 5.68 \\
\hline $1.9300 \mathrm{E}+00$ & 786.8 & 1184.5 & 15.41 & 6.25 & 24.29 & 0.22 & 5.77 \\
\hline $1.9400 \mathrm{E}+00$ & 773.9 & 1177.5 & 15.40 & 6.24 & 24.24 & 0.32 & 5.86 \\
\hline $1.9500 \mathrm{E}+00$ & 762.6 & 1171.1 & 15.39 & 6.22 & 24.19 & 0.42 & 5.96 \\
\hline $1.9600 \mathrm{E}+00$ & 749.3 & 1166.5 & 15.37 & 6.20 & 24.12 & 0.53 & 6.08 \\
\hline $1.9700 z+00$ & 734.3 & 1163.5 & 15.37 & 6.17 & 24.06 & 0.66 & 6.22 \\
\hline $1.9800 \mathrm{E}+00$ & 720.5 & 1160.8 & 15.35 & 6.15 & 23.99 & 0.78 & 6.36 \\
\hline $1.9900 E+00$ & 708.2 & 1158.0 & 15.33 & 6.13 & 23.93 & 0.89 & 6.49 \\
\hline $2.0000 \mathrm{E}+00$ & 696.3 & 1154.5 & 15.30 & 6.10 & 23.87 & 0.99 & 6.62 \\
\hline $2.0100 \mathrm{E}+00$ & 681.6 & 1148.8 & 15.28 & 6.09 & 23.81 & 1.06 & 6.72 \\
\hline $2.0200 \mathrm{E}+00$ & 654.1 & 1138.8 & 15.27 & 6.08 & 23.78 & 1.10 & 6.78 \\
\hline $2.0300 \mathrm{E}+00$ & 612.9 & 1122.8 & 15.24 & 6.07 & 23.71 & 1.12 & 6.99 \\
\hline $2.0400 \mathrm{E}+00$ & 566.3 & 1102.8 & 15.18 & 6.04 & 23.61 & 1.12 & 7.37 \\
\hline $2.0500 \mathrm{E}+00$ & 515.8 & 1082.1 & 15.12 & 6.02 & 23.52 & 1.11 & 7.74 \\
\hline $2.0600 E+00$ & 461.6 & 1060.6 & 15.06 & 6.00 & 23.42 & 1.11 & 8.10 \\
\hline $2.0700 z+00$ & 398.3 & 1038.0 & 15.00 & 5.97 & 23.33 & 1.11 & 8.46 \\
\hline $2.0800 \mathrm{z}+00$ & 322.4 & 1013.7 & 14.95 & 5.95 & 23.25 & 1.10 & 8.80 \\
\hline $2.0828 E+00$ & 298.0 & 1006.7 & 14.93 & 5.95 & 23.22 & 1.10 & 8.89 \\
\hline
\end{tabular}

TOTAL CPO TIME: 2 hours, 21 minutes, 39.959 seconds! 
TOTAL CPO TIME: 2 hours, 21 mlnutes, 40.020 seconds!

Simulation Completed: Thu Jun 3 17:34:14 1993

\section{Output Files, OUTA through OUTJ}

FBED-1 output also includes ten optional output files OUTA through OUTJ. These files are created when the logical flags louta through loutj are set to "true". The contents of these optional output files are listed in Table 6.6.

Table 6.6 Contents of Output Files OUTA trough OUTJ.

\begin{tabular}{|c|c|c|c|c|c|c|c|c|c|}
\hline outa & outb & outc & outd & oute & outf & outg & outh & outi & outj \\
\hline$z$ & $\mathbf{z}$ & $z$ & $\mathbf{z}$ & $z$ & $\mathbf{z}$ & $z$ & $\mathbf{z}$ & $\mathbf{z}$ & $z$ \\
\hline$y(1)$ & $\operatorname{tg}$ & re & frco 2 & $\operatorname{trco} 2$ & pd & hrxal & hw & $x \operatorname{gas}(1)$ & ts \\
\hline$y(2)$ & pres & pr & frb2 & trb2 & pdu & hrxn2 & hgw & $\operatorname{xgas}(2)$ & pd \\
\hline$y(3)$ & $\operatorname{cpg}$ & $\operatorname{seco} 2$ & frb2o & $\operatorname{trh} 20$ & brnout & hrxn3 & hsw & $\mathrm{xgas}(3)$ & pnd \\
\hline$y(4)$ & gk & $\operatorname{sch} 2$ & fro2 & tro2 & $\mathrm{ICO} 2$ & hrxn4 & hsg & $\operatorname{xgas}(4)$ & areap \\
\hline$y \operatorname{dot}(1)$ & gmw & scb2o & $\operatorname{arco} 2$ & IW & $\operatorname{rh} 2$ & rlbl & hrs & $x \operatorname{gas}(5)$ & sk \\
\hline$y \operatorname{dot}(2)$ & gvisc & $s \operatorname{co} 2$ & arb2 & verate(1) & rh2o & $\mathrm{r} 2 \mathrm{~b} 2$ & hrv & $\operatorname{xgas}(6)$ & vsolid \\
\hline$y \operatorname{dot}(3)$ & vgas & difco2 & $\operatorname{arb} 20$ & verate(2) & ro2 & $\mathrm{r} 3 \mathrm{~h} 3$ & rskgk & $\operatorname{xgas}(7)$ & stime \\
\hline$y \operatorname{dot}(4)$ & gtime & difh2 & aro2 & verate(3) & risum & r $4 \mathrm{~h} 4$ & ergk & $\operatorname{xgas}(8)$ & solbr \\
\hline \multirow[t]{7}{*}{ fdafch } & gashr & $\operatorname{difh} 20$ & crco2 & verate(4) & & smribi & ersk & $\operatorname{xgas}(9)$ & \\
\hline & & difo2 & crh2 & verate(5) & & & qgw & $x \operatorname{gas}(10)$ & \\
\hline & & & $\operatorname{crb} 20$ & ziribi & & & qsw & $x \operatorname{gas}(11)$ & \\
\hline & & & cro2 & & & & qsg & xgas(12) & \\
\hline & & & & & & & & $\operatorname{xgas}(13)$ & \\
\hline & & & & & & & & $x \operatorname{xas}(14)$ & \\
\hline & & & & & & & & $x \operatorname{xas}(15)$ & \\
\hline
\end{tabular}

The output file OUTA contains the gas and solid mass flow rates $(y(1)$ and $y(2)$ ), gas and solid energy flow rates $(y(3)$ and $y(4)$ ), the derivatives of mass and energy flow rates for the gas and the solids (ydot(1) - ydot(4)), and the char mass flow rate on dry, ash-free basis (fdafch). 
The output file OUTB contains the transport properties and a few predicted quantities for the gas phase. These values are the gas temperature (tg), the gas phase pressure (pres), the gas heat capacity (cpg), the gas mixture conductivity (gk), the gas mixture molecular weight (gmw), the gas mixture viscosity (gvisc), the gas velocity (vgas), the gas residence time (gtime), and the gas heating rate (gashr).

The output file OUTC contains Reynolds and Prandtl numbers for the gas, the Schmidt number for the gasification and oxidation agents $\mathrm{CO}_{2}, \mathrm{H}_{2}, \mathrm{H}_{2} \mathrm{O}$, and $\mathrm{O}_{2}$ (scco2, sch2, sch2o, and sco2), and the diffusivities of the gasification and oxidation agents in the gas mixture (difco2, difh2, difh2o, and difo2).

The output file OUTD contains the film resistances for gasification and oxidation agents, (frco2, frh2, frh20, and fro2), the ash resistances for gasification and oxidation agents (arco2, $\operatorname{arh} 2, \operatorname{arh} 20$, and $\operatorname{aro2}$ ), and the chemical resistances to gasification and oxidation $(\operatorname{crco} 2, \operatorname{crh} 2, \operatorname{crh} 20$, and cro2).

The output file OUTE contains the total resistance to gasification and oxidation (trco2, trh2, trh20, and tro2), the volumetric drying rate (rw), the volumetric heterogeneous elemental reaction rates for carbon, hydrogen, oxygen, nitrogen, and sulfur (verate(1) - verate(5)), and the energy term associated with mass exchange between solid and gas phases (zirihi).

The output file OUTF contains the overall particle diameter (pd), the unreacted core diameter (pdu), the dry ash-free particle burnout (brnout), the volumetric char consumption rates by the $\mathrm{CO}_{2}, \mathrm{H}_{2}$ and $\mathrm{H}_{2} \mathrm{O}$ gasification reactions (rco2, rh2 and $\mathbf{r h 2 0}$ ), the volumetric char consumption rate by oxidation reaction (ro2), and the total volumetric char consumption rate by the gasification and oxidation reactions (risum).

The output file OUTG contains the net enthalpy production rate for the gasification and oxidation reactions (hrxn1 is for the $\mathrm{CO}_{2}$ gasification reaction, hrxn2 is for the $\mathrm{H}_{2}$ gasification reaction, hrxn3 is for the $\mathrm{H}_{2} \mathrm{O}$ gasification reaction, and hrxn4 is for the oxidation reaction), the products of reaction rates 
Chapter 6

and enthalpy production rate for these reactions ( $r 1 h 1, r 2 h 2, r 3 h 3$, and $r 4 h 4$ ), and the overall volumetric energy production from the gasification and oxidation reactions (smrihi).

The output file OUTH contains the bed-to-wall heat transfer coefficient (hw), the gas-to-wall heat transfer coefficient (hgw), the solid-to-wall heat transfer coefficient (hsw), the solid-to-gas heat transfer coefficient (hsg), the solid radiation coefficient (hrs), the void-to-void radiation coefficient (hrv), the ratio of the solid thermal conductivity to the gas mixture thermal conductivity (rskgk), the effective radial gas conductivity (ergk), the effective radial solid conductivity (ersk), the volumetric heat transfer from the gas to the wall (qgw), the volumetric heat transfer from the solid to the wall (qsw), and the volumetric heat transfer from the solid to the gas (qsg).

The output file OUTI gives the concentration in mole percent of the gas phase. The mole percentages for the first fifteen gas species in the FB1THM file are printed (xgas(1) - xgas(15)). The mole percentages listed in Table 6.6 are for AR, $\mathrm{CO}, \mathrm{CO}_{2}, \mathrm{CH}_{4}, \mathrm{C}_{2} \mathrm{H}_{6}, \mathrm{H}_{2}, \mathrm{HCN}, \mathrm{H}_{2} \mathrm{O}, \mathrm{H}_{2} \mathrm{~S}, \mathrm{~N}_{2}, \mathrm{NH}_{3}, \mathrm{NO}, \mathrm{O}_{2}, \mathrm{OH}$, and $\mathrm{SO}_{2}$ respectively.

The final output file OUTJ provides the properties and the quantities related to the solid phase. These values are temperature of the solid (ts), the overall particle diameter (pd), the unreacted core diameter ( $p d u)$, the external surface area of the particle (areap), the thermal conductivity of the solid (sk), the solid velocity (vsolid), the solid phase residence time (stime), and the solid heating rate (solhr).

\section{Other Output Files}

Additional output files generated by FBED-1 are: 1) BALANC.OUT, which presents the overall mass, energy and elemental balance for the gasifier, 2) PDIA.OUT, which is essentially the same as OUTF except the headings are not printed and only the results of the final downward pass are printed, 3) CCONV.OUT, which prints the drying, tar and total devolatilization rates, gasification and oxidation rates and finally the dry, ash-free char flow rate for the 
final downward pass, 4) COMPOS.OUT, which prints the composition of the first fifteen species for the final upward pass, and 5) TANDP.OUT, which prints the temperature and pressure profiles for the final upward integration pass. In addition, an optional file COMPAR.OUT is generated when the flag lexpt is .true.. These output files are then used to plot the comparison of the predicted and experimental results as well as the simulations results, as discussed in the next section.

\section{Graphics}

The output of the FBED-1 code is provided in a form suitable for use with popular spreadsheet packages. The user can use a spreadsheet package available at his/her site and generate the plots of the quantities of interest by importing the output files to the spreadsheet. In addition, two computer programs (PLOT1 and PLOT2), written in FORTRAN, are provided to be used with the DISSPLA graphics package. The first program generates the temperature, pressure, composition, char consumption and burnout profiles. The second program generates a plot that compares the available experimental data with the predictions. These comparisons include the feed gas properties (i.e., the temperature and the composition for the species of interest), the product gas properties, the temperature and pressure profiles, and some other quantities of interest, such as the burnout, the product tar flow rate and the wall heat loss. Figures 6.3 and 6.4 were generated using these two programs. The descriptions of the associated input files needed to run these plotting programs are provided in Appendix J. The procedure to compile and execute these programs is also explained in Appendix $\mathrm{J}$.

\section{Code Operation}

FBED-1 is a "user-specialist" code that requires familiarity with the theory and structure of the code in order to be used correctly and efficiently. This section provides the guidelines for the new users. A thorough understanding of the 
Chapter 6

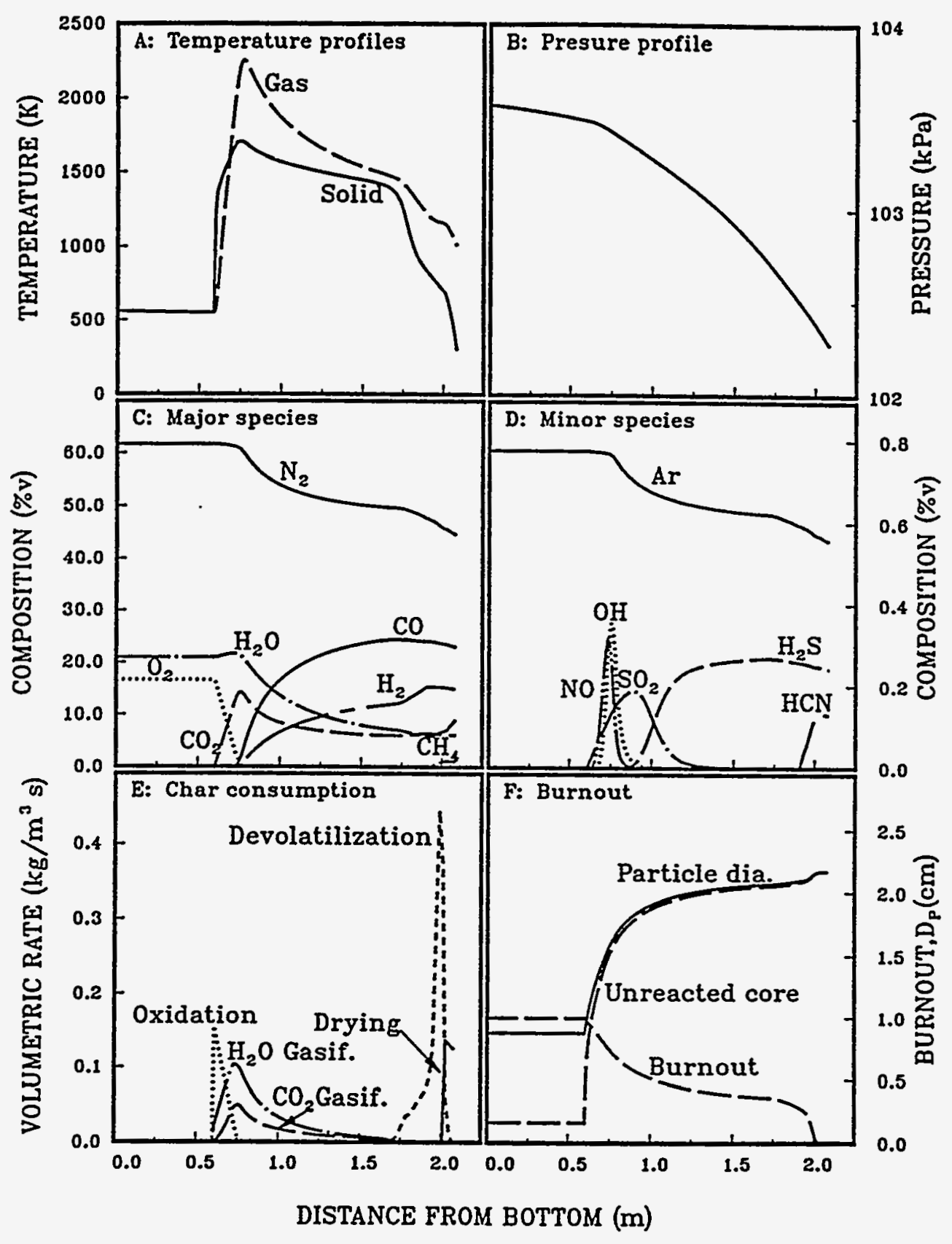

Figure 6.3. Plot generated by the program plotl showing the predicted axial profiles. 


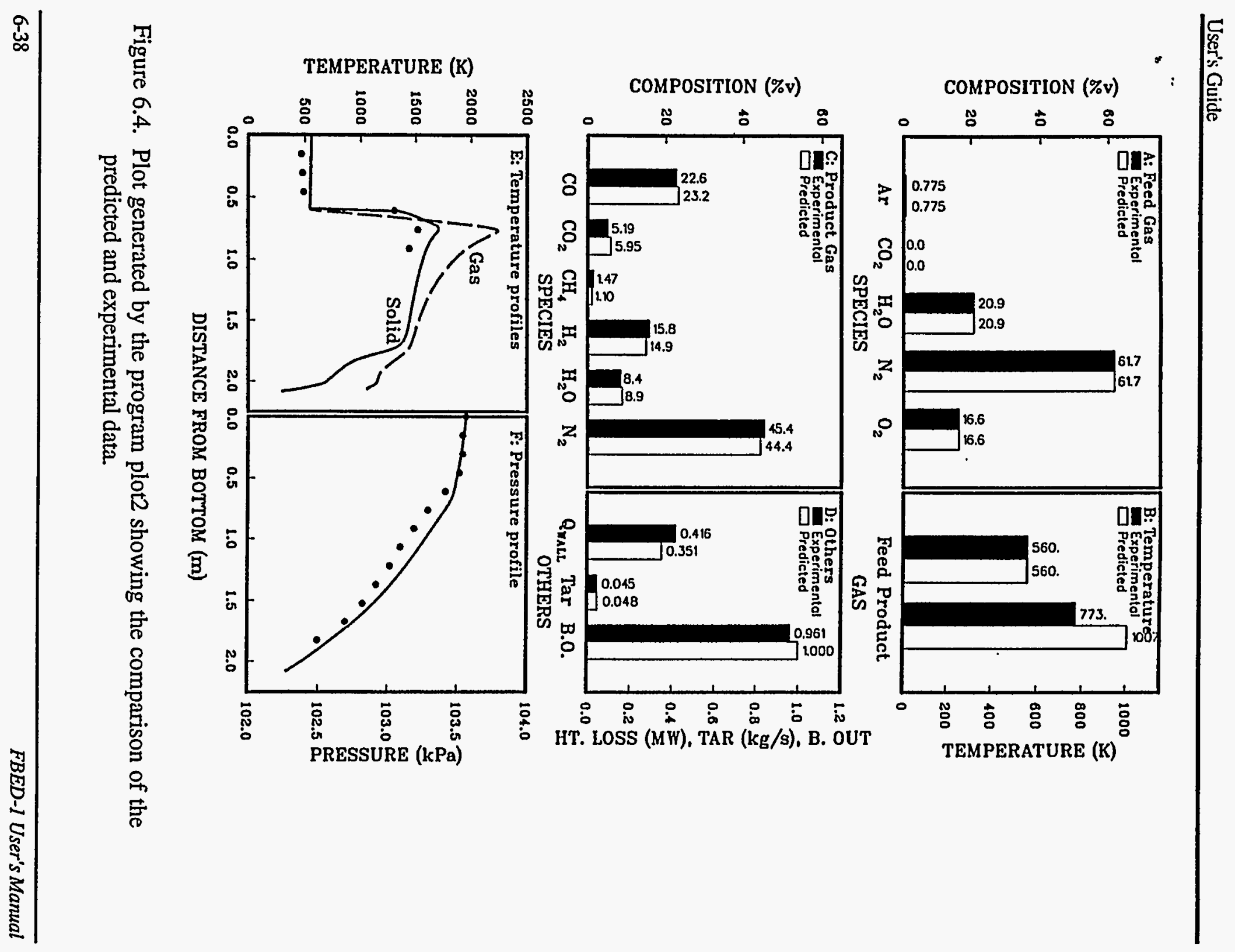


physics of the problem, assumptions, and numerical solution method is essential. Several iterations are required to obtain a converged solution. The iteration procedure is also discussed in this section.

\section{Setting the Logical Variables}

The various submodels and options provided in FBED-1 are exercised by the appropriate selection of the logical variables. Some of theses variables affect the operation of either zero-dimensional or one-dimensional portions of the model, whereas other variables affect the operation of both portions of the code. In addition to these logical variables, a few real variables are also needed for proper operation of the code. An example of such variables is tswtch, which governs the gas phase composition in the one-dimensional portion. A discussion of these variables is given below.

The flag $10 \mathrm{~d}$ is used to run either zero-dimensioanl or one-dimensional model. When 10d is set to .true., the zero-dimensioanl, two-zone submodel is executed as a stand-alone model. The composition, and temperature of the product gas are computed and reported, along with the flow rates of the product gas and the product tar. When this flag is set to false., these values serve as the initial guess for the one-dimensional model which is fully executed.

The flag Igaseq determines the gas phase chemistry submodel for the drying and devolatilization zone in the zero-dimensional portion. By setting this flag to .true., all the gases evolved in this zone are allowed to react to equilibrium with the gases from the equilibrium zone. When this flag is set to .false., all the gases but tar generated in the drying and devolatilization zone are considered to be non-reactive. Thus the product gas properties can be computed with either of these options, and their affect on the FBED-1 predictions can be studied.

The product gas temperature usually reported in the literature is at the gas off-take location and not at the top of the gasifier bed. In the freeboard, which is the space above the bed top, the gas stream losses some energy to the solid stream and the water jacket. If a reliable estimate of this energy loss is available, then the temperature drop in the freeboard zone for the gas stream can be computed. This 
option is then exercised by setting lfreeb to .true.. If this information is not available or cannot be estimated, then the temperature of the product gas is assumed to be same as at the bed top. This is achieved by either setting lfreeb to false. or assigning a value of zero to the variable qfreeb.

The logical flag ljkstm is used to estimate the wall heat loss, from the water jacket steam flow rate, for the zero-dimensional portion of the model. This option is exercised only if an estimate of the heat loss is not available. In case, neither an estimate of the wall heat loss nor jacket steam flow rate is available, the wall heat loss may also be computed from an overall heat transfer coefficient. This option is exercised by setting the flag ljkstm to .false., and assigning a value of zero to the variable qwall.

The flag lashcp provides the option to use either Kirov's correlation or Kopp-Neuman's formulation for the estimation of heat capacity of the ash.

The flag Idvc, perhaps the most important of all, selects the devolatilization submodel to be used in both the zero-dimensional and one-dimensional portions of the code. When this flag is set to .true., the FG-DVC submodel is used; otherwise the simpler FG-SET submodel is used.

The computation of the potential tar-forming fraction, when the FG-SET, submodel is used, is obtained either from the semiempirical correlation of Ko et al., (1988) or is specified by the user. If the flag $\mathbf{I x 0}$ is set to .true. then the user specified value usrx0 is used; otherwise Ko's correlation is used to determine the potential tar forming fraction.

The rate coefficients for the gas phase decomposition of tar may be determined by using a time dependent distribution for the activation energy. The time variation is approximated by keeping the activation energy function constant and changing the lower limit of the distribution function. This procedure is same as discussed for the FG-DVC submodel and the option can be exercised by setting the flag lfixg to .true.. When this flag is set to .false., the distribution function is kept constant throughout the decomposition process. 
The flag Isp is used to select between the shell progressive and the ash segregation submodel. When Isp is set to true., the oxidation and gasification of char is based on the shell progressive submodel, which takes into account the diffusion of the reactants and the products through the developing ash layer. Otherwise, the ash segregation submodel is used.

The variable Istiff, when set to .true., activates the stiff solver available in the LSODE package.

The gas phase chemistry in the one-dimensional portion of the code is governed by selecting an appropriate value of the variable tswtch. The gases are considered to be nonreactive when the gas temperature is below this value; otherwise full chemical equilibrium is assumed for the gas phase. The evolved tar in one-dimensional model can be either kept out of equilibrium, i.e., considered nonreactive, or allowed to react to complete equilibrium by setting the flag ltareq to .true..

The termination of the integration is exercised by assigning the axial position of the lower boundary to the variable zbotom and setting the flag Istop to .true.. Usually the variable zbotom is zero, but values less than zero may be specified to study the behavior of some coals that may need extended lengths of reaction zones for complete conversion. This option may be useful for design simulations when the gasifier's bed height is not known a priori. Required bed height may therefore be determined in an iterative manner.

Finally, the flags louta to loutj are used to invoke the printing of the optional output files.

\section{Iteration Procedure}

The nature of the governing equations for the FBED-1 requires an iterative solution. These equations are solved by a shooting method with split back and forth integration scheme, as discussed in chapter 5 . The code can be executed in both an interactive and a noninteractive mode. When executed in a noninteractive mode, the values of the input parameters must be such that they lead to a solution 
that is close to the final (converged) solution. The departure of the predicted feed gas temperature from the known boundary value is a measure of the closeness of the solution. Our simulation results suggest that the predicted feed gas temperature after the first iteration should not depart by more than $50 \mathrm{~K}$ from the input value. In case the difference is greater, a converged solution may be difficult to obtain.

The integration is started by obtaining an initial guess for the gas phase variables using the zero-dimensional submodel, FBED-0. Variables such as wall heat loss for the zero-dimensional portion (qwall), or the feed gas temperature may be changed to improve on the overall char conversion for the first downward integration pass. Once a reasonable burnout is obtained, the gas phase quantities are integrated from the bottom to the top of the reactor. This yields a new guess for the product gas properties. The complete set of differential equations is now integrated from the top to the bottom of the gasifier. This pass is treated as the first iteration pass, because the estimated product gas composition is more realistic. In the subsequent iterations, the product gas composition is held constant, but its enthalpy (and hence temperature) is varied until a converged solution is obtained. The enthalpy of the gas is lowered if the predicted temperature is higher than the input value and vice-versa. Once the solution is bracketed, a bisection techniques takes over to find the solution within the specified tolerance. It should be noted that the bracketing of the solution in a noninteractive mode may not always be possible, because high temperatures will be attained when the char conversion is not complete. This will mislead the iteration scheme, which will attempt to reduce the temperature by lowering the product gas enthalpy, resulting in even smaller char burnout. Thus the predicted feed gas temperature and the overall burnout must be closely monitored. If high feed gas temperature and less than adequate burnout are obtained, the user is urged to run the code in an interactive mode. 


\section{CHAPTER 7}

\section{IMPLEMENTATION GUIDE}

\section{Compiling, Linking, and Executing FBED-1 Code}

This chapter discusses the implementation of fbed using the UNIX operating system. By replacing the commands used for compilation, linkage and execution, the procedure discussed in this chapter is also applicable to other computer systems. The UNIX "make" command is used to compile and link the subroutines which comprise the FBED-1 code.

The make file, called MAKEFBED, which is used to compile and link the FBED-1 code, is shown in Table 7.1. This file is specific to the Sun Computers, which invoke the compilation by the command f77. On a CONVEX machine this should be replaced by $f c$. The compilation and linking of the code is accomplished by invoking the make command. A successful execution of the make command will produce the executable code specified as fbedl. The code can then be executed by typing "fbed1." Selected results are printed to the screen to monitor the progress of simulation. The steps required for this procedure are as shown below. "\%" represents the system's prompt; "al" is the name of our Sun computer.

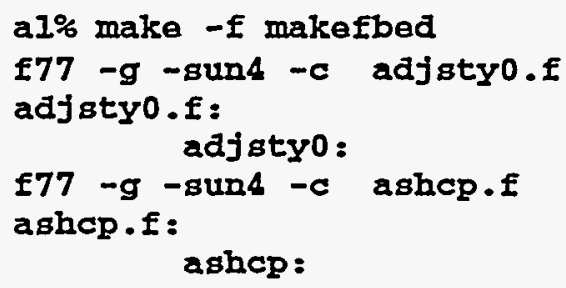

Generates the executable code fbed1. 
Implementation Guide

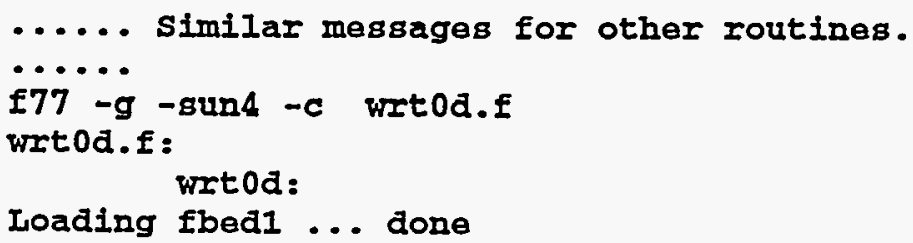

The code can now be executed by typing fbed1. Parts of the typical output obtained on the monitor during the execution of the code are shown below.

\section{al\% fbed1}

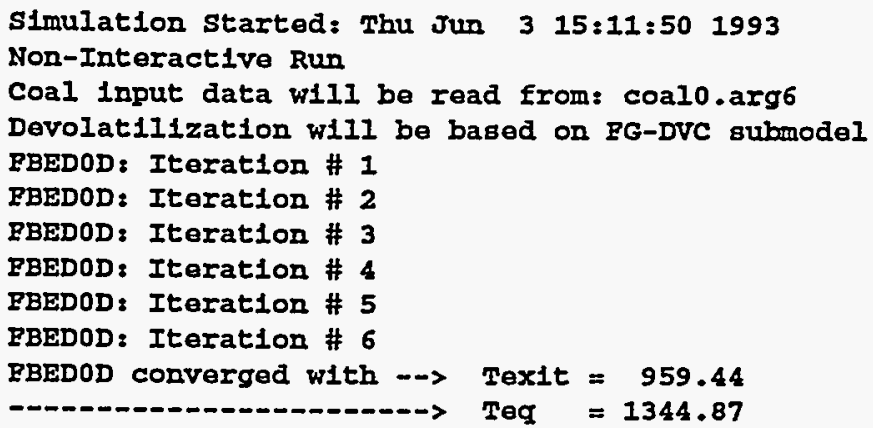




\begin{tabular}{|c|c|c|c|c|c|c|c|}
\hline $0.0000 \mathrm{E}+00$ & 388.4 & 388.6 & 0.00 & 0.00 & 0.00 & 0.00 & 23.15 \\
\hline Overall Heat & LOBB $=$ & $0.2776 E+$ & $06 \mathrm{~J} / \mathrm{s}$ & & & & \\
\hline \multicolumn{8}{|c|}{ ours, 30 minutes, 19.870 seconds! } \\
\hline \multicolumn{8}{|c|}{ Simulation Results for upward integration pass \# 1} \\
\hline Axtal 20s. & ts $(\mathrm{K})$ & $\operatorname{tg}(\mathrm{K})$ & mo1\% H2 & mol\% CO2 & mol\% Co & mo1\% CH4 & mo1\% H2O \\
\hline 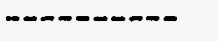 & $--\cdots$ & $-\cdots--$ & $--\infty--$ & ------ & ----- & ------ & ------ \\
\hline $0.0000 \mathrm{E}+00$ & $388 \cdot 4$ & 560.0 & 0.00 & 0.00 & 0.00 & 0.00 & 20.94 \\
\hline $1.0000 \mathrm{E}-02$ & 388.3 & 552.3 & 0.00 & 0.00 & 0.00 & 0.00 & 20.94 \\
\hline \multicolumn{8}{|l|}{... $\quad$ n } \\
\hline \multicolumn{8}{|l|}{$\cdots$} \\
\hline \multicolumn{8}{|l|}{-. } \\
\hline \multicolumn{8}{|c|}{ Swltched to non-equilibrium calculations at $z=1.9029$ where $\mathrm{tg}=1199.69$} \\
\hline $1.9100 \mathrm{E}+00$ & 805.3 & 1193.9 & 15.64 & 6.22 & 24.48 & 0.08 & 5.51 \\
\hline $1.9200 \mathrm{E}+00$ & 791.7 & 1186.7 & 15.66 & 6.21 & 24.44 & 0.18 & 5.60 \\
\hline \multicolumn{8}{|l|}{$\cdots$} \\
\hline \multicolumn{8}{|l|}{$\cdots$} \\
\hline \multicolumn{8}{|l|}{ … } \\
\hline $2.0828 \mathrm{E}+00$ & 298.0 & 997.7 & 15.17 & 5.89 & 23.35 & 1.10 & 8.79 \\
\hline \multicolumn{8}{|c|}{ TOTAL CPD TIME: 0 hours, 38 minutes, 58.680 seconds! } \\
\hline \multicolumn{8}{|c|}{ Simulation Results for downward 1ntegration pass \# 2} \\
\hline $\begin{array}{l}\text { 1terat1on = } \\
\text { hbound }=F\end{array}$ & $\begin{array}{l}1 \text { yupl } \\
\text { Ibound }\end{array}$ & $\begin{array}{r}=-2.77 \\
E \quad \text { fupp }\end{array}$ & $\begin{array}{l}381 E+06 \\
\text { pas }=E\end{array}$ & factor $=$ & 0.000000 & $y(3)=-2$ & 77381E+06 \\
\hline Axlal Pos. & $t_{B}(\pi)$ & $\operatorname{tg}(\mathrm{R})$ & mol\% $\mathrm{H2}$ & mol\% $\mathrm{CO} 2$ & mol\% co & mol\% CH4 & mol\% $\mathrm{H2O}$ \\
\hline$-\infty--\infty-\infty--$ & $-\infty----$ & $---\infty$ & $-\cdots-\infty$ & 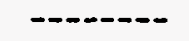 & $-\cdots--$ & $--\infty--\infty$ & ------ \\
\hline $2.0828 E+00$ & 298.0 & 997.7 & 15.17 & 5.89 & 23.35 & 1.10 & 8.79 \\
\hline $2.0800 \mathrm{E}+00$ & 322.1 & 1004.7 & 15.18 & 5.90 & 23.37 & 1.10 & 8.70 \\
\hline \multicolumn{8}{|l|}{$\cdots$} \\
\hline \multicolumn{8}{|l|}{$\cdots$} \\
\hline $1.9500 \mathrm{E}+00$ & 760.8 & 1193.7 & 15.52 & 6.03 & 23.89 & 1.12 & 6.69 \\
\hline Swltched to & equilibs & um calcul & Lations at & $t z=1.942$ & 27 where $t$ & $g=1200.41$ & \\
\hline $1.9400 E+00$ & 773.2 & 1147.5 & 16.58 & 6.36 & 24.44 & 0.00 & 5.06 \\
\hline \multicolumn{8}{|l|}{$\cdots$} \\
\hline \multicolumn{8}{|l|}{ - } \\
\hline$\ddot{1} \ddot{0} 0000 \mathrm{E}-02$ & 552.5 & 553.1 & 0.00 & 0.00 & 0.00 & 0.00 & 20.80 \\
\hline $0.0000 \mathrm{E}+00$ & 552.7 & 553.3 & 0.00 & 0.00 & 0.00 & 0.00 & 20.80 \\
\hline
\end{tabular}


Implementation Guide

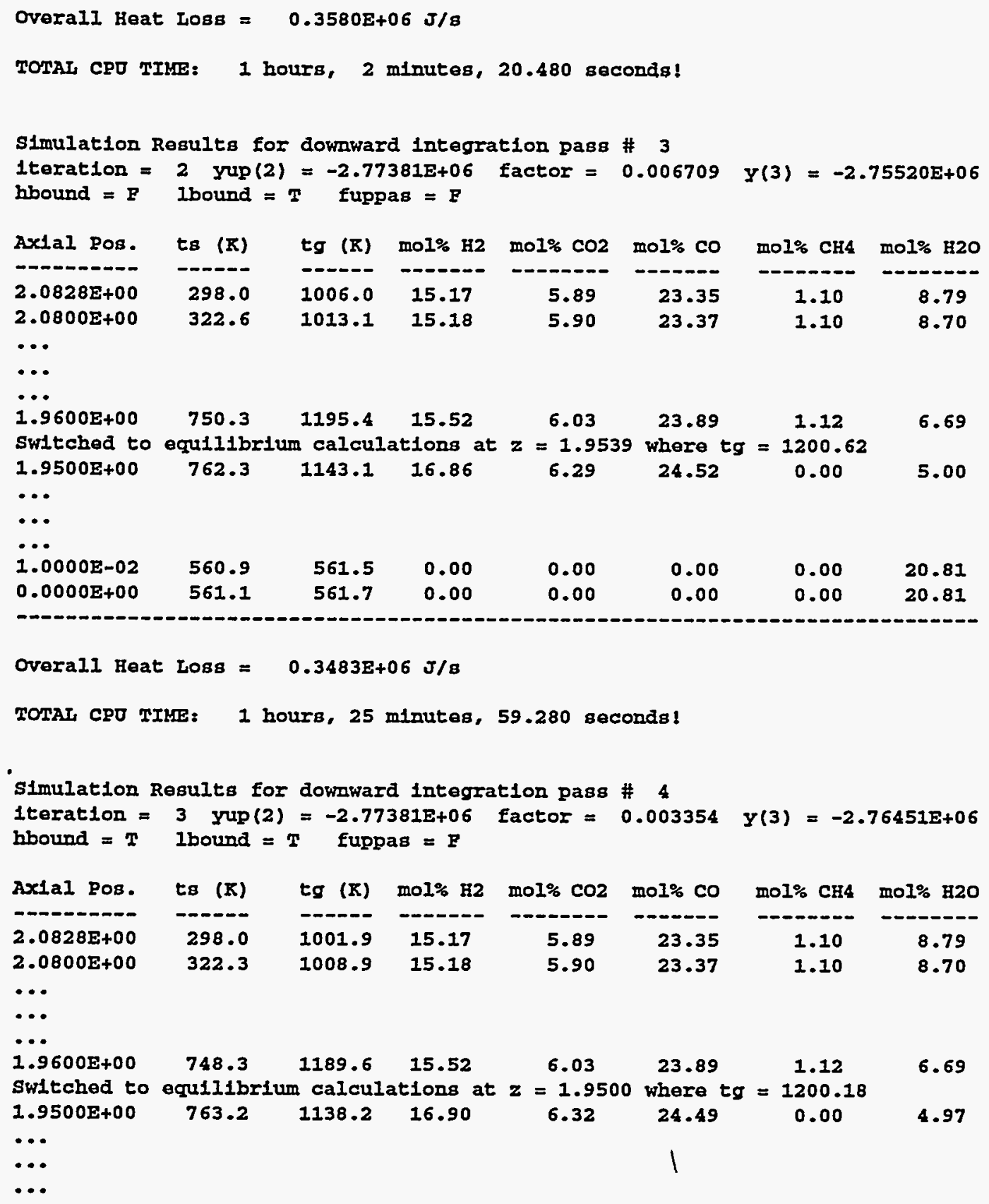


Chapter 7

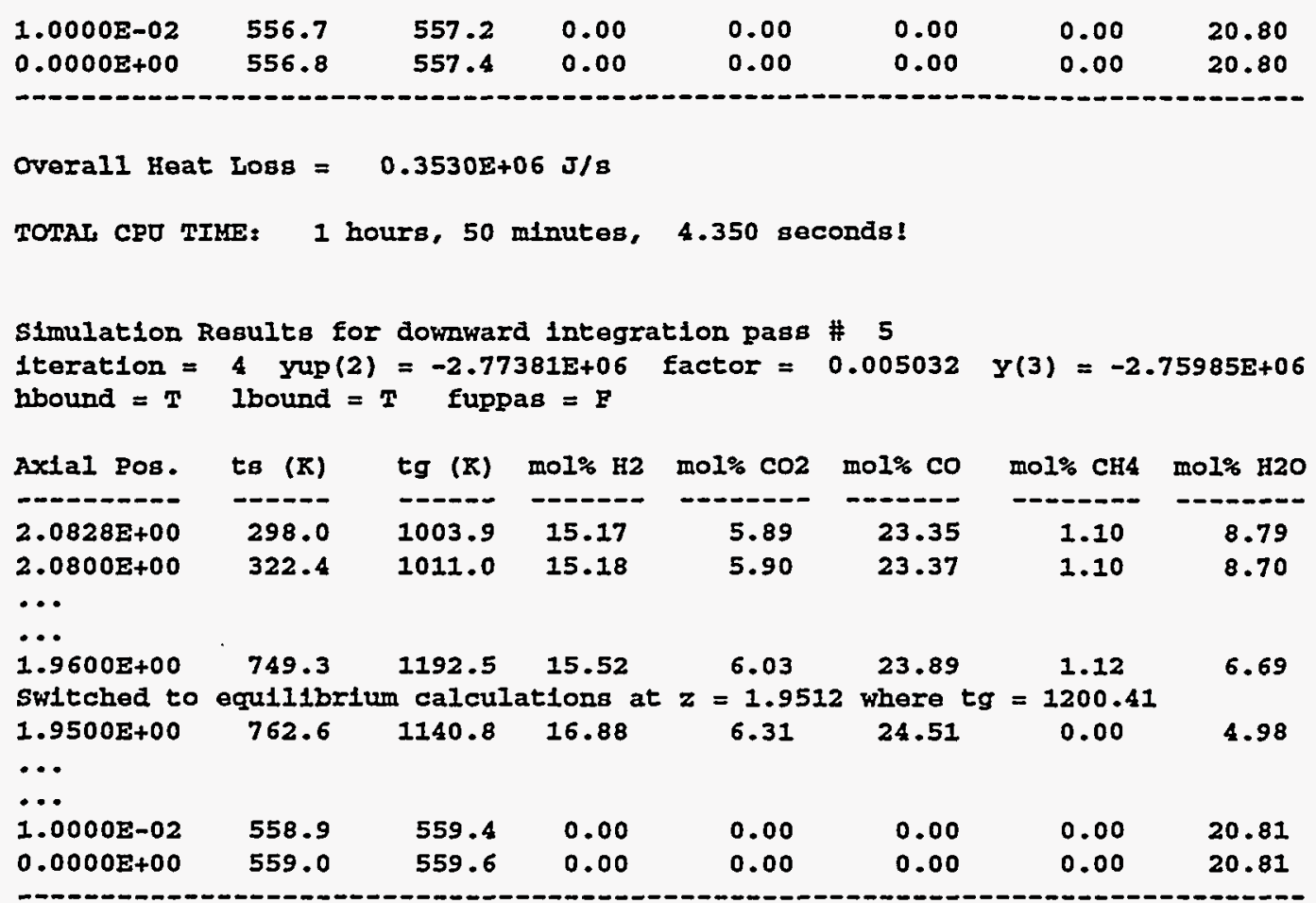

Overall Heat LOBS $=0.3506 \mathrm{E}+06 \mathrm{~J} / \mathrm{s}$

TOTAL CPD TIME: 2 hours, 13 minutes, 12.890 seconds!

simulation Results for final upward integration pass

\begin{tabular}{|c|c|c|c|c|c|c|c|}
\hline Axial Pos. & ts $(R)$ & $\operatorname{tg}(\mathrm{R})$ & mol\% $\mathrm{E2}$ & mol\% $\mathrm{CO} 2$ & mol\% co & mo1\% CH4 & mol\% $\mathrm{H} 2 \mathrm{O}$ \\
\hline - - & ----- & $---\infty$ & $----\infty$ & $--\infty-\infty$ & -----n- & $---n-n$ & - - \\
\hline $0.0000 \mathrm{E}+00$ & 559.0 & 560.0 & 0.00 & 0.00 & 0.00 & 0.00 & 20.94 \\
\hline $1.0000 \mathrm{E}-02$ & 558.9 & 559.8 & 0.00 & 0.00 & 0.00 & 0.00 & 20.94 \\
\hline \multicolumn{8}{|l|}{$\cdots$} \\
\hline \multicolumn{8}{|l|}{$\cdots$} \\
\hline \multicolumn{8}{|l|}{$\ddot{1} \ddot{9000 \mathrm{E}+00}$} \\
\hline switched to & non-equ1 & ortum ca & Lculatior & 8 at $z=$ & .9083 whe & $e t g=11$ & 9.71 \\
\hline $1.9100 \mathrm{E}+00$ & 813.3 & 1199.2 & 15.41 & 6.29 & 24.36 & 0.03 & 5.59 \\
\hline \multicolumn{8}{|l|}{$\cdots$} \\
\hline$\cdots$ & & & & & & & \\
\hline $2.0800 E+00$ & 322.4 & 1013.7 & 14.95 & 5.95 & 23.25 & 1.10 & 8.80 \\
\hline $.0828 \mathrm{E}+00$ & 298.0 & 1006.7 & 14.93 & 5.95 & 23.22 & 1.10 & 8.89 \\
\hline
\end{tabular}


Implementation Guide

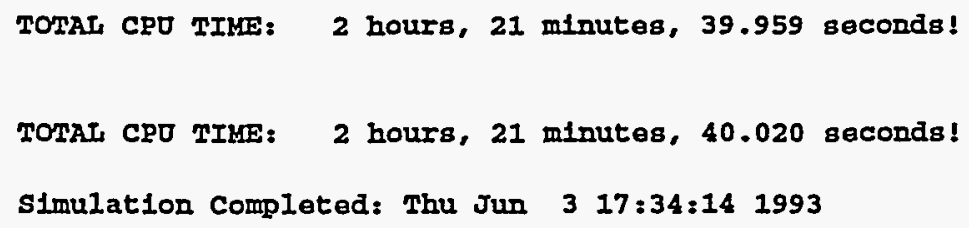

Table 7.1 Listing of the make file MAKEFBED

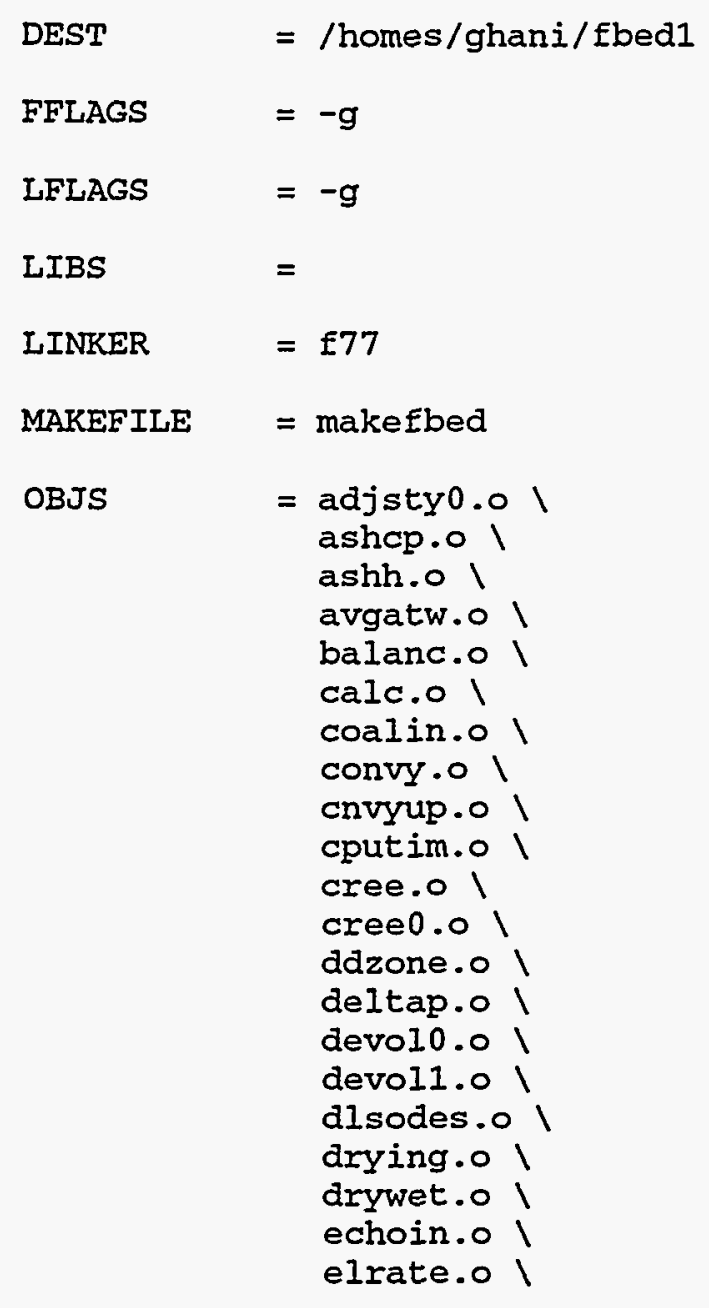




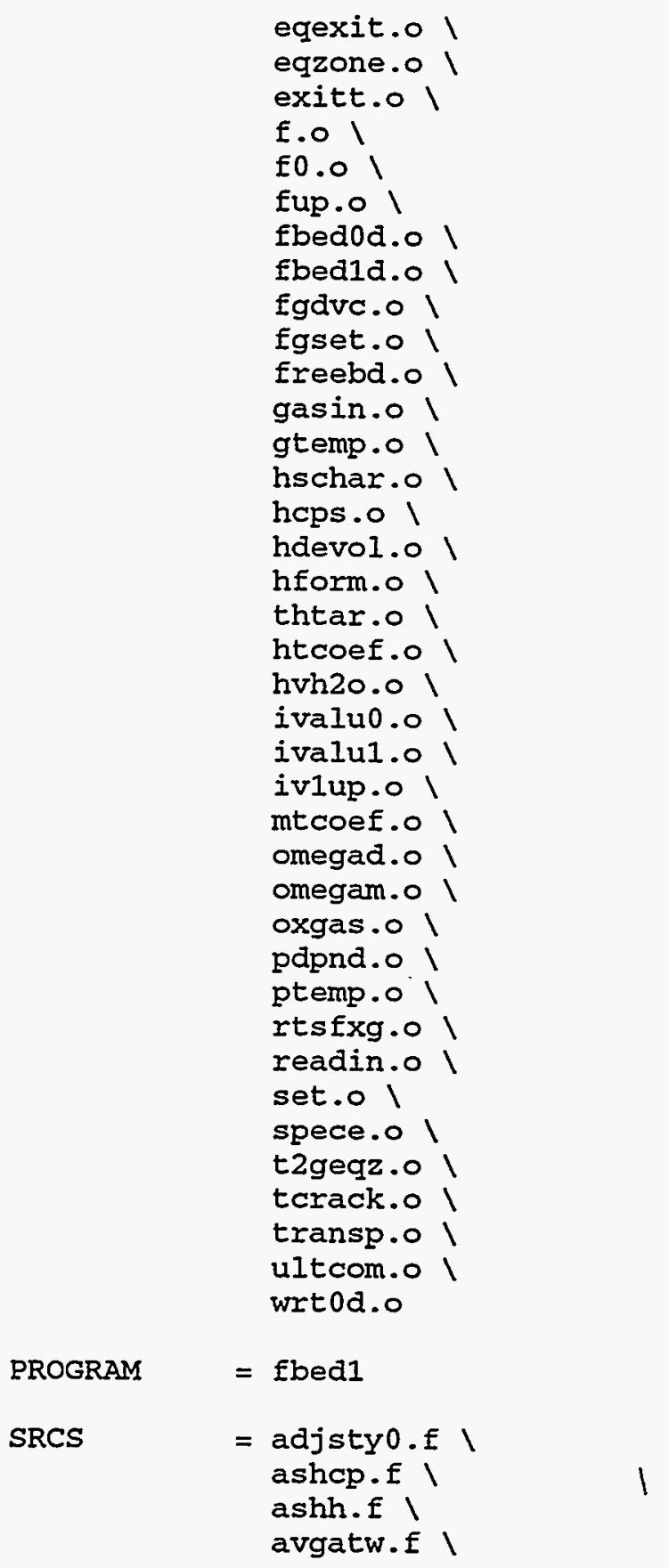


Implementation Guide

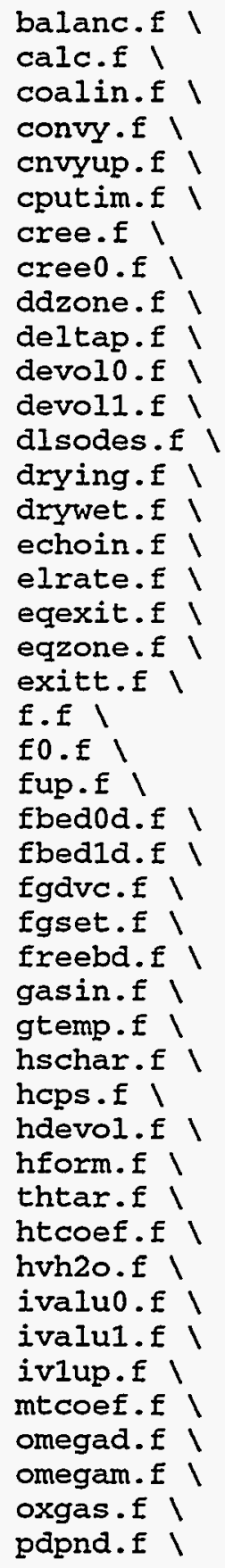




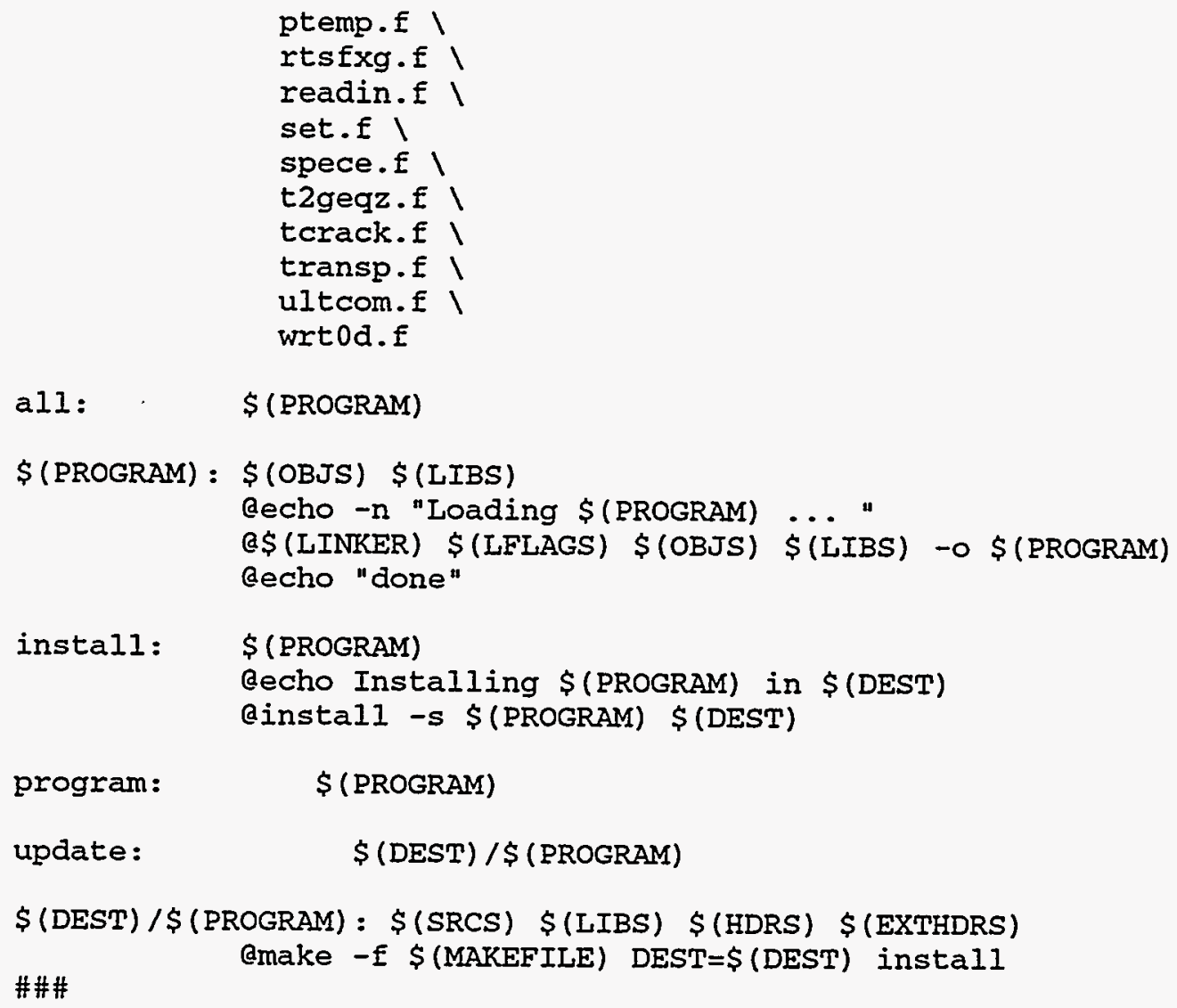

\section{FORTRAN and Machine Incompatibilities}

FBED-1 was developed on a SUN $4 / 310$ running under the UNIX operating system. Significant efforts have been expended to make the code compatible with FORTRAN-77 standards. However, a few statements may be specific to the SUN architecture. For example, FBED-1 frequently calls a subroutine called CPUTIM, which uses a system-dependent utility to monitor the cpu time used in various sections of the code. On the non-unix machines, the function CPUTIM may need to be replaced by the corresponding utility available on 
the host machine. Alternatively, the calls to the function CPUTIM may be commented out.

FBED-1 also uses calls to FLUSH, a UNIX utility, for flushing the contents of output buffers in a timely manner. These calls need to be replaced with calls to a similar utility available on the user's machine. Alternatively, they may be removed.

An ieee error trapping routine which is specific to the SUN system is also used in FBED-1. When compiled on a different system, statements relating to the SUN error trapping should be commented out. These statements are as follows:

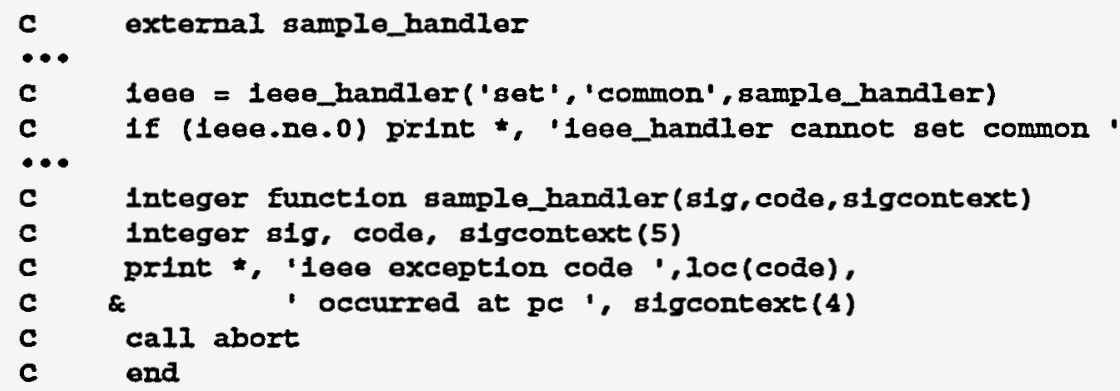

The error trapping routines can be activated when running in the debug mode on a SUN computer. To run in the debug mode, the UNIX "dbx" utility can be used. The error trapping is then activated by typing "catch FPE" at the $\mathrm{dbx}$ prompt and then executing the code. 


\section{CHAPTER 8}

\section{SAMPLE PROBLEM}

In this chapter, a sample simulation of the atmospheric, air-blown, dry-ash Wellman-Galusha gasifier fired with the Jetson bituminous coal is presented and discussed. The input data for this case were presented in Tables 6.1 to 6.4.

\section{Atmospheric Pressure, Air-Blown Gasification}

The FBED-1 predictions for the atmospheric, air-blown, dry-ash WellmanGalusha gasifier fired with the Jetson bituminous coal are shown in Figure 8.1. This case was selected because the experimental temperature profile show measurements in the ash zone. For other cases, either no or few data are available for the ash zone. The predicted profiles are shown for temperature, pressure, major and minor species composition, char consumption, and burnout. Both the solid and the gas temperatures show rapid change in the drying and devolatilization region. This is associated with the rapid addition of the devolatilized mass to the gas phase and the associated heat effects. In FBED-1, all coal conversion processes, namely drying, devolatilization, gasification and oxidation, are allowed to occur simultaneously. The results show that for atmospheric gasifiers, very little gasification occurs before devolatilization is complete, mainly due to the low solid temperatures. Oxidation and gasification reactions overlap in the region where oxygen is available. Due to high temperature in this zone, oxidation is dominant. The peak solid and gas temperatures occur when the oxygen becomes available to the char. The heat evolved due to the exothermic oxidation reaction causes the rapid heat-up of the gas stream. The temperatures drop sharply as soon as all char is 
Sample Problem

consumed and remain almost constant in the ash zone. $\mathrm{CO}$ and $\mathrm{H}_{2}$ are not observed in the oxidation zone, due to chemical equilibrium in the gas phase, which causes all carbon and hydrogen to form $\mathrm{CO}_{2}$ and $\mathrm{H}_{2} \mathrm{O}$. In the oxidation zone, $\mathrm{H}_{2} \mathrm{O}$ concentration also shows some increase due to the reaction of char oxygen and hydrogen, which is assumed to be proportional to the overall char consumption rate. Char consumption by hydrogen gasification reaction is insignificant in comparison with $\mathrm{CO}_{2}$ and $\mathrm{H}_{2} \mathrm{O}$ gasification reactions. Increase in $\mathrm{H}_{2} \mathrm{O}$ near the gasifier top is due to the release of moisture in the drying of feed coal. $\mathrm{CH}_{4}$ appears only in the low temperature devolatilization zone, where the gas phase is assumed to be out of chemical equilibrium. Char sulfur is reacted to form $\mathrm{H}_{2} \mathrm{~S}$ in the oxygen free zone and is converted to $\mathrm{SO}_{2}$ when the oxygen becomes available. $\mathrm{NO}$ and $\mathrm{OH}$ are observed only in the high temperature oxidation region. In addition, substantial amounts of $\mathrm{HCN}$ are observed in the product gas, with practically no $\mathrm{NH}_{3}$ and $\mathrm{NO}$. All of $\mathrm{HCN}$ is released in the devolatilization zone through HCN tight and extra-tight functional groups. The burnout profiles show rapid change in the devolatilization zone, followed by a gradual change in the gasification and oxidation zone. The particle size show a very rapid decrease in the oxidation zone, due to the high char consumption rates. Finally, the pressure profile shows a small drop in the ash zone, followed by a gradual change in the reactive zones of the gasifier. The small change in the ash zone is attributed to the higher bed void fraction offering little resistance to the gas flow.

Figure 8.2 presents the comparison of the FBED-1 predictions with the available data (Thimsen et al., 1984). The experimental data include the temperature and pressure profiles, and the product gas composition. Following Barriga and Essenhigh (1980), the experimental temperature profile is assumed to represent the solid temperature. The feed gas composition and temperatures are satisfied exactly. The product gas composition, the product tar flow rate, and the wall heat loss predicted by the model are in close agreement with the experimental data. The predicted pressure profile also shows good agreement with the experimental data. The predicted temperatures are slightly higher, but the trends in the devolatilization, gasification and oxidation zone are properly predicted. 
The overall mass and energy balances close within $1 \%$. The elemental mass balances close typically within 5\%. Overall, the comparison between the experimental and the predicted values is good. 


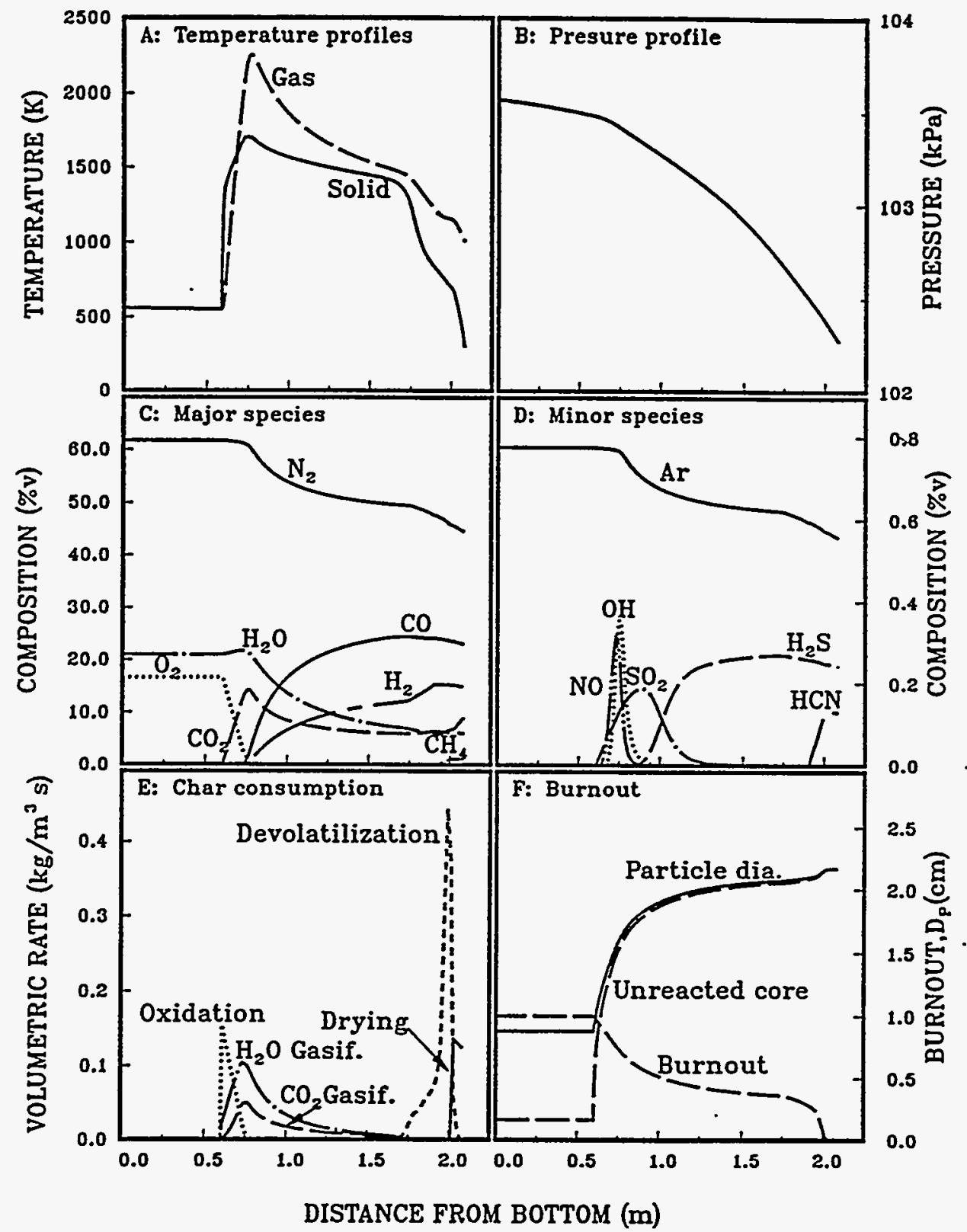

Figure 8.1. Predicted axial A) temperature, B) pressure, C) major species composition, D) minor species composition, E) volumetric char consumption rate, F) burnout and overall particle and unreacted core diameter in the atmospheric pressure, air-blown Wellman-Galusha gasifier fired with Jetson bituminous coal. 
Chapter 8
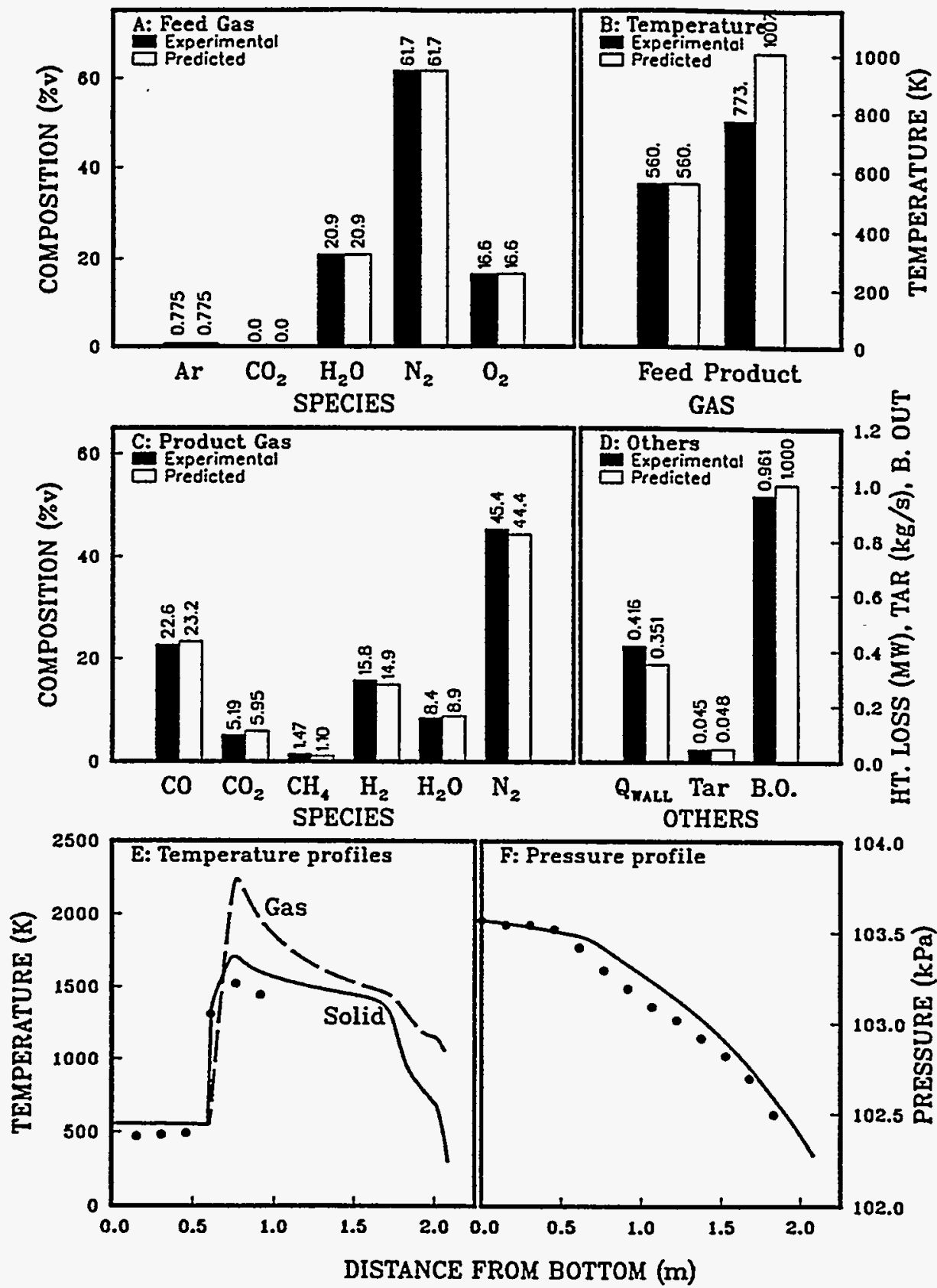

Figure 8.2. Comparison of FBED-1 predictions with experimental data for the gasification of the Jetson bituminuous coal in the atmospheric, airblown, dry-ash Wellman-Galusha gasifier. 


\section{NOMENCLATURE}

Symbol Definition and Units

$\begin{array}{ll}a & \text { Mean atomic weight, } \mathrm{kg} / \mathrm{kmol} \\ a_{s, u} & \text { Number of ways to form a cluster with } s \text { bridges and } u \text { crosslinks } \\ A & \text { Arrhenius Pre-exponential factor, } \mathrm{sec}^{-1} \\ A & \text { Cross sectional area of reactor, } \mathrm{m}^{2} \\ A & \text { Particle surface area, } \mathrm{m}^{2} \\ A & \text { Water wall surface area, } \mathrm{m}^{2} \\ C & \text { Integration constant } \\ C & \text { Molar concentration of oxidizer or gasification agent, } \mathrm{kmol} / \mathrm{m}^{3} \\ C_{p} & \text { Specific heat, } \mathrm{j} / \mathrm{kg} K \\ D & \text { Diffusivity, } \mathrm{m}^{2} / \mathrm{s} \\ D & \text { Reactor diameter, } m \\ d & \text { Particle diameter, } m \\ E & \text { Activation Energy, } \mathrm{j} / \mathrm{kmol} \\ F & \text { Fraction of original carbon } \\ F(p, q) & \text { Fraction of sites in finite clusters } \\ F_{n}(p, q) & \text { Probability that a site is member of cluster of } n \text { sites } \\ f & \text { Aromaticity of coal used in the tar correlation of Ko et al. (1988) } \\ f & \text { Distribution function } \\ f & \text { Friction factor }\end{array}$




$\begin{array}{ll}G & \text { Superficial gas mass flux, } \mathrm{kg} / \mathrm{m}^{2} \mathrm{~s} \\ G & \text { Fraction of j-mer in mass bins, } \mathrm{kg} / \mathrm{kg} \\ g & \text { Acceleration due to gravity, } 9.80665 \mathrm{~m} / \mathrm{s}^{2} \\ H & \text { Convective heat capacity flux, } \mathrm{j} / \mathrm{m}^{2} \mathrm{~s} \\ h & \text { Enthalpy, } j / \mathrm{kg} \\ h & \text { Heat transfer coefficient, watts } / \mathrm{m}^{2} \mathrm{~K} \\ \Delta H & \text { Reaction enthalpy, } \mathrm{j} / \mathrm{kg} \\ \Delta h & \text { Reaction enthalpy, } \mathrm{j} / \mathrm{kg} \\ H H V & \text { Higher heating value, } \mathrm{j} / \mathrm{kg} \\ k & \text { Arrhenius rate constant, } \mathrm{m} / \mathrm{s} \text { or } \mathrm{l} / \mathrm{s} \\ k & \text { Thermal conductivity, watts } / \mathrm{mK} \\ L & \text { Pressure parameter used in the tar correlation of Ko et al. (1988) } \\ L & \text { Reactor length, } m \\ \dot{m} & \text { Mass flow rate, } \mathrm{kg} / \mathrm{s} \\ M w & \text { Molecular weight, } \mathrm{kg} / \mathrm{s} \\ \bar{M} & \text { Weight average molecular weight of the ash or slag, or particle } \\ & \text { molecular weight, } \mathrm{kg} / \mathrm{kmol} \\ p & \text { Number fraction of bridges in coal } \\ P & \text { Pressure, } K P a, P a, \text { or } \mathrm{kg} / \mathrm{m}^{2} \\ P & \text { Equilibrium vapor pressure of tar, atm or } \mathrm{KPa}, \text { or } \mathrm{kg} / \mathrm{m}^{2} \\ P e & \text { Peclet number } \\ P r & \text { Prantl number } \\ q & \text { Heat release for daf coal, } \mathrm{J} / \mathrm{kg} \\ q & \text { Number fraction of cross-links in coal } \\ Q & \text { Heat loss, watts } \\ r & \text { Volumetric reaction rate, } \mathrm{kg} / \mathrm{m}^{3} s \\ R & \text { Universal gas constant, } j / \mathrm{kmolK} \\ R e & \text { Reynold's number } \\ s & \text { Number of bridges } \\ S c & \text { Schmidt number } \\ t & \text { Temperature, }{ }^{\circ} \mathrm{C} \\ t & \text { Time, } s \\ T & \text { Temperature, } K \\ u & \text { Gas velocity, } \mathrm{m} / \mathrm{s} \\ & \end{array}$




\begin{tabular}{|c|c|}
\hline$u$ & Number of crosslinks \\
\hline$U$ & Overall heat transfer coefficient, watts $/ \mathrm{m}^{2} K$ \\
\hline$V$ & Volatile matter, mass fraction \\
\hline$V$ & Volume, $m^{3}$ \\
\hline$V M$ & Volatile matter, mass fraction \\
\hline$w$ & Weight factor for Gausian quadrature \\
\hline$W$ & Mass flow rate, $\mathrm{kg} / \mathrm{s}$ \\
\hline$W$ & Functional group mass fraction, $\mathrm{kg} / \mathrm{kg}$ \\
\hline$x$ & Gas mole fraction \\
\hline$x$ & Mole fraction of ash constituents, mole fraction \\
\hline$x$ & Tar fraction, mass fraction \\
\hline$x$ & Tar parameter used in correlation of Ko et al. (1988) \\
\hline$x$ & Zero of Legendre polynomial \\
\hline$X$ & $\begin{array}{l}\text { Crosslink efficiency, (moles of crosslinks/mole of gas species } \\
\text { formed)*(gm coal/mole of crosslink sites). }\end{array}$ \\
\hline$y$ & functional group fraction, mass fraction \\
\hline$Y$ & Ultimate yield, mass fraction \\
\hline$z$ & Axial distance, $m$ \\
\hline$z$ & $\begin{array}{l}\text { Dummy variable representing the ratio of the Einstein temperature } \\
\text { and } T\end{array}$ \\
\hline$\alpha$ & Parameter used in tar correlation of Ko et al. (1988) \\
\hline$\beta$ & Parameter used in tar correlation of Ko et al. (1988) \\
\hline$\varepsilon$ & Bed void fraction, void volume/bed volume \\
\hline$\varepsilon$ & Emissivity \\
\hline$\varepsilon$ & Lennard-Jones parameter \\
\hline$\Phi$ & Heat of combustion of elements, $\mathrm{J} / \mathrm{kg}$ \\
\hline$\Phi$ & Interaction parameter \\
\hline$\phi$ & Packing parameter defined by Equation (10) in Table 3.5 \\
\hline$\phi$ & Porosity, volume/volume \\
\hline$\eta$ & Particle number density, $1 / m^{3}$ \\
\hline$\kappa$ & Ratio of solid conductivity and gas conductivity \\
\hline$\lambda$ & Stoichiometric coefficient for oxidation reaction, mole/mole \\
\hline
\end{tabular}




\begin{tabular}{ll}
$\mu$ & Atomic weight, $\mathrm{kg} / \mathrm{kmol}$ \\
$\mu$ & Viscosity, $\mathrm{kg} / \mathrm{ms}$ \\
$v$ & Number of broken crosslinks \\
$\nu$ & Stoichiometric coefficient \\
$\nu$ & Stoichiometric coefficient to identify the number of moles of product \\
& gas per mole of oxidant, mole/mole \\
$\rho$ & Density, $\mathrm{kg} / \mathrm{m}^{3}$ \\
$\sigma$ & Standard deviation of activation energy, $\mathrm{j} / \mathrm{kmol}$ \\
$\sigma$ & Stockmayer collision diameter, Angstrom \\
$\sigma$ & Network coordination number \\
$\tau$ & Number of broken bridges \\
$\tau$ & Residence time of coal in the gasifier, $s$ \\
$\Omega$ & Collision integral \\
$\Omega$ & Dry, ash-free mass fraction \\
$\omega$ & Functional group fraction or element fraction, mass fraction \\
$\zeta$ & Bed-to-wall heat transfer adjustable factor \\
$\zeta$ & Particle area factor to account for internal surface burning \\
$\zeta$ & Solid-to-gas heat transfer adjustable factor \\
$\ell$ & Boundary layer thickness, $m$ \\
$\chi$ & Mole fraction tar in mass bin \\
Subscripts & Definition \\
$a$ & Aromatic \\
$a$ & Ash \\
$a s h$ & Ash \\
$B$ & Labile bridge \\
$B G$ & Blast gas \\
$C$ & Carbon \\
$C$ & Char \\
$c$ & Coal \\
\hline
\end{tabular}




\begin{tabular}{|c|c|}
\hline char & Char \\
\hline $\mathrm{CO}_{2}$ & $\mathrm{CO}_{2}$ gasification \\
\hline devol & Devolatilization \\
\hline$e$ & Effective \\
\hline$e$ & Equilibrium \\
\hline eff & Effective \\
\hline exit & Exit \\
\hline$f$ & Formation \\
\hline$f b$ & Freeboard \\
\hline$F G$ & Functional group \\
\hline$g$ & Gas \\
\hline$g w$ & Gas-to-wall \\
\hline$H$ & Hydrogen \\
\hline$h_{2}$ & $\mathrm{H}_{2}$ gasification \\
\hline$h_{2} O$ & $\mathrm{H}_{2} \mathrm{O}$ gasification \\
\hline$i$ & Integer counter used to keep track of elements, species, or reactions \\
\hline$i$ & $\begin{array}{l}\text { index for drying, devolatilization, gasification and oxidation } \\
\text { reactions }\end{array}$ \\
\hline$i$ & index for tar monomer \\
\hline$i, k$ & index for functional groups \\
\hline in & Entering control volume \\
\hline$j$ & index for elements $\mathrm{C}, \mathrm{H}, \mathrm{O}, \mathrm{N}$, and $\mathrm{S}$ \\
\hline$j$ & index for tar fraction \\
\hline$j$ & index for tar mass bin \\
\hline$L$ & Labile bridge \\
\hline$m$ & mass transport \\
\hline moisture & Moisture \\
\hline$N$ & Nitrogen \\
\hline$n, m, h, j, k$ & Atomic stoichiometric coefficients \\
\hline nonvolatile $c$ & Carbon that is non-volatile \\
\hline nonvolatile s & Sulfur that is non-volatile \\
\hline 0 & Oxygen \\
\hline$o_{2}$ & Oxidation \\
\hline$O H$ & Hydroxyl \\
\hline out & Exiting control volume \\
\hline
\end{tabular}




$\begin{array}{ll}p & \text { Particle } \\ S & \text { Sulfur } \\ s & \text { Solid } \\ s g & \text { Solid-to-gas } \\ s m & \text { Solid measured (refers to the apparent density) } \\ s w & \text { Solid-to-wall } \\ t & \text { Tar } \\ t & \text { Total } \\ t a r & \text { Tar } \\ v & \text { Volatiles } \\ w & \text { Wall } \\ w & \text { Water } \\ x & \text { Tar } \\ z & \text { axial } \\ v & \text { Volatiles }\end{array}$

\section{Superscripts Definition}

$\begin{array}{ll}a & \text { Axial } \\ h & \text { Thermal } \\ m & \text { Mass } \\ o & \text { Initial } \\ 0 & \text { Reference temperature } \\ s & \text { Sensible enthalpy } \\ \text { ultimate } & \text { Yield at large times } \\ \text { vap } & \text { Vaporization } \\ \infty & \text { Infinity or ultimate }\end{array}$

Operators Definition
$\Delta$
Difference 


\section{REFERENCES}

Abramowitz, M. and Stegun, I. A., eds., Handbook of Mathematical Functions With Formulas, Graphs, and Mathematical Tables, $10^{\text {th }}$ printing, National Bureau of Standards, Applied Mathematics Series, 55, U. S. Government Printing Office, Washington, D. C. (1972).

Anthony, D. B., Howard, J. B., Hottel, H. C. and Meissner, M. P., "Rapid Devolatilization of Pulverized Coal," 15 th Symposium (International) on Combustion, The Combustion Institute, Pittsburgh, Pennsylvania, 1303 (1974).

Baxter, L. L., Condensed Phase Behavior in Combustion Environments, PhD Dissertation, Brigham Young University, Provo, Utah, 1987, see also Hedman, P. O., Smoot, L. D., Smith, P. J. and Blackham, A. U., "Entrainedflow Gasification at Elevated Pressure," Final Report, DOE/MC/22059-2570Vol.1, DE88001070, Brigham Young University, Provo, Utah (1987).

Bird, R. B., Stewart, W. E. and Lightfoot, E. N., Transport Phenomena, John Wiley \& Sons, New York (1960).

Bischoff, K. B., "A Note on Gas Dispersion in Packed Beds," Chem. Eng. Sci., 24, 607 (1962).

Brokaw, R. S., Ind. Eng. Chem. Process Design Develop., 8, 240, (1969).

Carslaw, H. S. and Jaegar, J. C., Conduction of Heat in Solids, Oxford Press, London, England, 61 (1959).

Cho, Y. S., Modeling and Simulation of Lurgi-type Gasifiers, M. S. Thesis, Washington University, Saint Louis, Missouri (1980). 
Computer Associates, CA-DISSPLA User's Manual, Release 11.0, Volume 1, Computer Associates International, Inc. Garden City, NY, (1989).

Crowe, C. T. and Smoot, L. D., "Multicomponent Conservation Equations," in Pulverized-Coal Combustion and Gasification, Smoot, L. D. and Pratt, D. T., editors, Plenum Press, New York (1979).

Denn, M., Wei, J., Yu, W., Cwiklinski, R., "Detailed Simulation of a MovingBed Gasifier, "Final Report, AP-2576, Research Project 1268-1, Electric Power Research Institute, Palo Alto, California (1982).

DeWasch, A. P. and Froment, G. F., "A Two-Dimensional Heterogeneous Model for Fixed Bed Catalytic Reactors," Chemical Engineering Science, 26, 629 (1971).

Dzhapbyev, K., Miropol'skii, A. L., and Mal'Kovskii, V. J., "Investigation of Unsteady Heat Transfer in a Packed Bed of Speheres Swept by Gas," Thermal Engineering, 33 (3), 159 (1986), translated from Teploenergetika, 33 (3), 70 (1986).

Elgin, D. C. and Perks, H. R., "Results of Trials of American Coals in Lurgi Pressure-Gasification Plant at Westfield, Scotland," Sixth Synthetic Pipeline Gas Symposium, Chicago, Illinois, October 18-20 (1974).

Ergun, S., "Fluid Flow Through Packed Columns," Chem. Eng. Prog. 48, 89 (1952).

Essenhigh, R. H., "Fundamentals of Coal Combustion," in Chemistry of Coal Utilization, Second Supplementary Volume, Elliott, M. A., editor, John Wiley \& Sons, New York, 1153 (1981).

Field, M. A., "Rate of Combustion of Size-Graded Fractions of Char from a LowRank Coal Between $1200 \mathrm{~K}$ and $2000 \mathrm{~K}$," Combustion and Flame, 13, 237 (1967).

Fisher, M.E. and Essam, J.W., J. Math. Phys., 1961, 2, 609.

Froberg, R. W., "The Carbon-Oxygen Reaction: An Experimental Study of the Oxidation of Suspended Carbon Spheres," PhD Dissertation, The Pennsylvania State University, University Park, Pennsylvania (1967). 
References

Froment, G. F. and Bischoff, K. B., Chemical Reactor Analysis and Design, John Wiley \& Sons, New York (1979).

Goetz, G. J., Nsakala, N. Y., Patel, R. L. and LaO, T. C., "Combustion and Gasification Characteristics of Chars from Four Commercially Significant Coals of Different Rank," Final Report, Project 1654-6, Electric Power Research Institute, Palo Alto, California (1982).

Grant, D.M., Pugmire, R.J., Fletcher, T.H. and Kerstein, A.R., " $C$ hemical Model of Coal Devolatilization using Percolation Lattice Statistics," Energy \& Fuels, 3 , 175 (1989).

Gray, D. D. and Stiles, J. M., "On the Consititutive Relation for Frictional Flow of Granular Materials," Topical Report DOE/MC/21353-2584, U. S. Department of Energy, Morgantown, West Virginia (1988).

Gupta, A. S. and Thodos, G., "Direct Analogy Between Mass and Heat Transfer to Beds of Spheres," AIChE J., 2, 751 (1963).

Hedman, P. O., Smoot, L. D., Smith, P. J. and Blackham, A. U. "Entrained-Flow Gasification at Elevated Pressure," DOE/MC/22059-2570, Combustion Laboratory, Chemical Engineering Department, Brigham Young University, Provo, Utah (1987).

Hindmarsh, A. C.: "ODEPACK, A Systematized Collection of ODE Solvers," in Scientific Computing (R. S. Stepleman, editor), Vol. 1. p. 55, IMACS Transactions on Scientific Computation, North-Holland, Amsterdam (1983).

Hobbs, M. L., Modeling Countercurrent Fixed-Bed Coal Gasification, PhD Dissertation, Brigham Young University, Provo, Utah (1990).

Hobbs, M. L., Radulovic, P. T., and Smoot, L. D., "Modeling Fixed-Bed Coal Gasifiers," AIChE J. 38, 681 (1992a).

Hobbs, M. L., Radulovic, P. T., and Smoot, L. D., "Predictions of effluent compositions for fixed-bed coal gasifiers," Fuel, 71, 1177 (1992b).

Johnson, J. L., "Fundamentals of Coal Gasification," in Chemistry of Coal Utilization, Second Supplementary Volume, M. A. Elliott, ed., John Wiley, New York (1981). 
Kee, R. J., Rupley, F. M., and Miller, J. A., The Chemkin Thermodynamic Data Base, Report No. SAND87-8215B, Sandia National Laboratories, Livermore, CA, (1987).

Khan, M. R., "A Literature Survey and an Experimental Study of Coal Devolatilization at Mild and Severe Conditions: Influences of Heating Rate, Temperature, and Reactor Type on Products Yield and Composition," Fuel, 68, 1522 (1989).

Khanna, R. and Seinfeld, J. H., "Mathematical Modeling of Packed Bed Reactors: Numerical Solutions and Control Model Development," in Advances in Chemical Engineering edited by Wei, J., Anderson, J. L., Bischoff, K. B., Denn, M. M. and Seinfeld, J. H., 13, Academic Press, Inc., 113 (1987).

Kobayashi, H., Howard, J. B. and Sarofim, A. F., "Coal Devolatilization at High Temperatures," 16th Symposium (International) on Combustion, The Combustion Institute, Pittsburgh, Pennsylvania, 411 (1977).

Ko, G. H., Sanchez, D. M., Peters, W. A. and Howard, J. B., "Correlations for Effects of Coal Type and Pressure on Tar Yields from Rapid Devolatilization," 22nd Symposium (International) on Combustion, The Combustion Institute, Pittsburgh, Pennsylvania, 115 (1988).

Kunii, D. and Smith, J.M., "Heat Transfer Characteristics of Porous Rocks," AIChE J., 6,71 (1960).

Kurylko, L., "The Unsteady and Steady Combustion of Carbon," PhD Dissertation, The Pennsylvania State University, University Park, Pennsylvania (1969).

Laidler, K. J. and Meiser, J. H., Physical Chemistry, The Benjamin/Cummings Publishing Compnay, Inc., Menlo Park, California (1982).

Laurendeau, N. M., "Heterogeneous Kinetics of Coal Char Gasification and Combustion," Prog. Energy Combust. Sci., 4, 221 (1978).

Lowry, H. H., Chemistry of Coal Utilization, Supplementary Volume, John Wiley \& Sons, Inc., New York (1963).

Mayers, A. M., "The Rate of Reduction of Carbon Dioxide by Graphite," J. Am. Chem. Soc., 56, 70 (1934). 
Merrick, D., "Mathematical Models of the Thermal Decomposition of Coal. 2. Specific Heats and Heats of Reaction," Fuel, 62, 540 (1983).

MeFayed, editor, Handbnok of Powder Science and Technology, Van Nostrand Reinhold Company, New York (1984).

Mills, K. C. and Rhine, J. M., "The Measurement and Estimation of the Physical Properties of Slags Formed During Coal Gasification: 2. Properties relevant to heat transfer," Fuel, 68, 201 (1989).

Nsakala, N., Patel, R. L., Lao, T. C., "Combustion Characteristics of Coals for Industrial Applications," Final Technical Report for U. S. Department of Energy, Pittsburgh, Combustion Engineering Inc., Kreisinger Development Laboratory, Windsor, CT, DOE/PC/402067-5 (DE85010673) (1985).

Nuttall, H. E., Stoddart, W. G. and Chen, W. J., "Pyrolysis of Subituminous New Mexico Coal," Journal of Petroleum Technology, 418 (1979).

Park, K. Y. and Edgar, T. F., "Modeling of Early Cavity Growth for Underground Coal Gasification," Ind. Eng. Chem. Res., 26, 237 (1987).

Perry, R. H. and Chilton, C. H., eds., Chemical Engineers' Handbook, Fifth Edition, McGraw-Hill Book Company, New York (1973).

Pratt, D. T., "Multicomponent Equilibrium," in Pulverized-Coal Combustion and Gasification, eds. Smoot, L. D. and Pratt, D. T., Plenum Press, New York (1979).

Reid, R. C. and Sherwood, T. K., The Properties of Gases and Liquids, McGrawHill Book Company, New York (1966).

Rohsenow, W. M., Hartnett, J. P. and Ganic, E. N., Handbook of Heat Transfer Applications, McGraw-Hill, New York (1985).

Saxena, S. C., "Devolatilization and Combustion Characteristics of Coal Particles," Prog. Energy Combust. Sci., 16, 55 (1990).

Serio, M. A., Advanced Fuel Research, Inc., New Haven, Connecticut, personal communication, telefax (1989). 
Serio, M. A., Hamblen, D. G., Markham, J. R. and Solomon, P. R., "Kinetics of Volatile Product Evolution in Coal Pyrolysis: Experiment and Theory," Energy \& Fuels, 1,138 (1987).

Smoot, L. D. and Smith, P. J., Pulverized-coal Combustion and Gasification (L. D. Smoot and D. T. Pratt, editors), Plenum Press, New York, 224 (1979).

Solomon, P. R., and Colket, M. B., "Coal Devolatilization," 17th Symposium (International) on Combustion, The Combustion Institute, Pittsburgh, Pennsylvania, 131 (1979).

Solomon, P. R. and Hamblen, D. G., "Pyrolysis," in Chemistry of Coal Conversion, ed. Schlosberg, R. H., Plenum Press, New York (1985).

Solomon, P. R., Hamblen, D. G., Carangelo, R. M., Serio, M. A. and Deshpande, G. Y., "General Model of Coal Devolatilization," Energy and Fuels, $\underline{2}, 405$ (1988).

Solomon, P. R., Hamblen, D. G., Zhen-Zhong Yu, and Serio, M. A., "Network models of coal thermal decomposition," Fuel, 69, 754 (1990).

Stull, D. R. and Prophet, H., JANAF Thermochemical Tables, Second Edition, National Bureau of Standards (1971).

Suuberg, E. M., Peters, W. A. and Howard, J. B., "Product Compositions and Formation Kinetics in Rapid Pyrolysis of Pulverized Coal--Implications for Combustion," 17th Symposium (International) on Combustion, The Combustion Institute, Pittsburgh, Pennsylvania, 177 (1979).

Thimsen, D., Maurer, R. E., Pooler, A. R., Pui, D. Y. H., Liu, B. Y. H. and Kittelson, D. B., "Fixed-Bed Gasification Research using U.S. Coals," 1-19, U.S. Bureau of Mines Contract H0222001 Final Report, (1984-1985).

Thorsness, C. B. and Kang, S. W., "Further Development of a General-Purpose, Packed-Bed Model for Analysis of Underground Coal Gasification Processes," Eleventh Annual Underground Coal Gasification Symposium, Denver, Colorado (1985).

Thorsness, C. B. and Kang, S. W., "A Method-of-line Approach to Solution of Packed-bed Flow Problems Related to Undergfound Coal Gasification 
Processes," Tenth Annual Underground Coal Gasification Symposium, Williamsburg, Virginia (1984).

Walker, P. L., Rusinko, F., and Austin, L. G., Advances in Catalysis (D. D. Eley, P. W. Selwood and P. B. Weisz, Editors), XI, Academic Press Inc., New York, 134 (1959).

Wang, S. C. and Wen, C. Y., "Experimental Evaluation of Nonisothermal SolidGas Reaction Model," AIChEJ., 18, 1231 (1972)

Yagi, S. and Kunii, D., "Studies on Heat Transfer Near Wall Surface in Packed Beds," AIChE J., 6, 97 (1960).

Yagi, S., Kunii, D. and Wakao, N., "Studies on Axial Effective Thermal Conductivities in Packed Beds," AIChE J., 6, 543 (1960).

Yagi, S. and Wakao, N., "Heat and Mass Transfer from Wall to Fluid in Packed Beds," AIChE J., 5, 79 (1959).

Yoon, H., Modeling and Analysis of Moving Bed Coal Gasifiers, PhD Dissertation, University of Delaware, Newark, Delaware (1978).

Yoon, H., Wei, J. and Denn, M. M., "A Model for Moving-bed Coal Gasification Reactors," AIChE J. 24, 885 (1978).

Young, L. L. and Finlayson, B. A., "Mathematical Methods of Monolith Catalytic Converter, Part I. Development of Model and Application of Orthogonal Collocation," AIChE J., 22, 331 (1976).

Yu, W., Dynamic Simulation of Moving Bed Coal Gasifiers, PhD Dissertation, University of Delaware, Newark, Delaware (1981). 


\section{APPENDIX A}

\section{Adjustment of Functional Group Composition}

When the functional group composition data are not available for a given coal, its functional group composition must be estimated from the available data for the standard coals. The ultimate composition of the given coal and the standard coal may be close but not identical. In order to close the elemental mass balance, the initial functional group composition and the ultimate composition of a coal must match. Serio (1989; See Hobbs, 1990) recommended the following procedure to adjust the functional group composition of a standard coal to match the ultimate analysis of a given coal.

1. The elemental analysis and the functional group pools cannot be changed independently.

2. The compositions of aliphatic carbon and hydrogen are calculated by the amount of leftover carbon and hydrogen respectively.

3. The total amount of $\mathrm{CH}_{4}$ and the fractions of $\mathrm{CH}_{4}-\mathrm{t}$ and $\mathrm{CH}_{4}$-xl are directly specified. The fraction of $\mathrm{CH}_{4}-\mathrm{l}$ is determined by difference.

4. The total amount of $\mathrm{CO}_{2}$ and the fractions of $\mathrm{CO}_{2}-\mathrm{t}$ and $\mathrm{CO}_{2}$-xl are directly specified. The fraction of $\mathrm{CO}_{2}-1$ is determined by difference.

5. The total amount of $\mathrm{H}_{2} \mathrm{O}$ and the fraction of $\mathrm{H}_{2} \mathrm{O}-\mathrm{t}$ are directly specified. The fraction of $\mathrm{H}_{2} \mathrm{O}-1$ is determined by difference. 
6. The amounts of CO-l and CO-xt are specified. The amount of CO-t is determined from the amount of unspecified leftover oxygen. This implies that a higher oxygen content coal will have a higher amount of CO-t functional group.

7. The amounts of $\mathrm{C}_{2} \mathrm{H}_{4}, \mathrm{C}_{2} \mathrm{H}_{6}, \mathrm{C}_{3} \mathrm{H}_{6}, \mathrm{C}_{3} \mathrm{H}_{8}$, paraffins $\left(\mathrm{C}_{4} \mathrm{H}_{10}\right)$ and olefins $\left(\mathrm{C}_{4} \mathrm{H}_{8}\right)$ are directly specified.

8. The amounts of aromatic hydrogen and aromatic carbon are also directly specified.

9. The evolution of nitrogen occurs through $\mathrm{NH}_{3}$ and $\mathrm{HCN}$. The amounts of $\mathrm{NH}_{3}$ and $\mathrm{HCN}-\mathrm{t}$ are directly specified. The amount of $\mathrm{HCN}-1$ is determined by the difference.

10. The amount of organic sulfur functional group must be identical to the ultimate sulfur (dry, ash-free basis), since only one sulfur containing functional group is considered in the current formulation.

11. The order for the adjustment of nitrogen containing functional groups is $\mathrm{HCN}-\mathrm{l}, \mathrm{HCN}-\mathrm{t}$ and finally $\mathrm{NH}_{3}$.

12. The adjustment of oxygen containing functional groups is accomplished as follows. The CO-t functional group is adjusted initially, since this group is used for specifying leftover oxygen. If this group is not sufficient to adjust the oxygen content, the CO-t group will be set to zero, and other oxygen containing functional groups must be adjusted to match the reported ultimate oxygen content. The $\mathrm{H}_{2} \mathrm{O}-\mathrm{l}$ and $\mathrm{H}_{2} \mathrm{O}-\mathrm{t}$ functional groups are adjusted if the $\mathrm{CO}$ groups are insufficient to match the ultimate analysis. The water functional groups are chosen for adjustment rather than $\mathrm{CO}_{2}$ groups, because $\mathrm{CO}_{2}$ may be important in the crosslinking submodel. If further adjustment is required, $\mathrm{CO}-\mathrm{t}, \mathrm{CO}_{2}-\mathrm{l}, \mathrm{CO}_{2}$-xl and $\mathrm{CO}_{2}$-t functional groups are adjusted sequentially.

Following the above guidelines, the functional group composition for any given coal can be determined from the available data for the standard coals. These 
data for eight standard coals are also included in this Appendix. This procedure is implemented through the subroutine ADJSTYO in the fixed-bed code. 
27

$\begin{array}{lll}0.74400 D-02 & 0.29240 D+02 \quad 0.17460 D+05 \quad 0.15000 D+04 & 0.20480 D+03\end{array}$ $0.33480 D-01 \quad 0.292400+02 \quad 0.227950+05 \quad 0.20000 D+040.20480 D+03$ $0.210800-010.296460+02 \quad 0.300700+050.300000+040.204800+03$ $0.18000 D-010.29240 D+02 \quad 0.23765 D+05 \quad 0.30000 D+04 \quad 0.00000 D+00$ $0.38767 D-02 \quad 0.31369 D+02 \quad 0.29100 D+05 \quad 0.15000 D+04 \quad 0.00000 D+00$ $0.96296 \mathrm{D}-02 \quad 0.304820+02 \quad 0.412250+05 \quad 0.47500 D+04 \quad 0.000000+00$ $0.17439 D+00 \quad 0.00000 D+00 \quad 0.00000 D+00 \quad 0.00000 D+00 \quad 0.000000+00$ $0.00000 D+00 \quad 0.00000 D+00 \quad 0.00000 D+00 \quad 0.00000 D+00 \quad 0.00000 D+00$

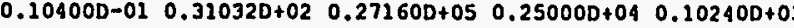

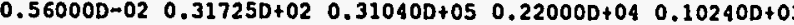

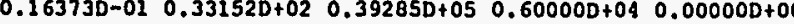
$0.12769 D-010.29240 D+02 \quad 0.16005 D+050.15000 D+040.00000 D+00$ $0.52500 D-010.329290+020.378300+050.25000 D+040.00000 D+00$ $0.44000 D+000.00000 D+000.000000+000.00000 D+000.00000 D+00$ $0.000000+000.00000 D+000.00000 D+000.00000 D+000.00000 D+00$ $0.63950020 .000000+000.000000+000.000000+000.00000 D+00$ $0.30000 D+020.292400+020.24250 D+050.15000 D+040.00000 D+00$ $0.00000+000.29240 D+020.24250 D+050.15000 D+040.00000 D+00$

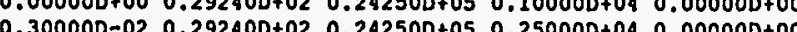

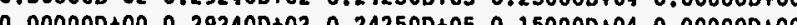
10000 0.252400102 0.24250D+05 $0.150000+040.000000+00$ $0.000000+000.000000+000.000000+000.000000+000.000000+00$ $0.322360+02 \quad 0.252200+05 \quad 0.10000 D+04.000000+00$ $0.44691 D-010.292400+02 \quad 0.223100+05 \quad 0.27000 D+040.00000 D+00$ $0.70229 \mathrm{D}-010.32929 \mathrm{D}+02 \quad 0.30070 \mathrm{D}+05 \quad 0.35000 \mathrm{D}+040.00000 \mathrm{D}+00$ $0.37139 D-02 \quad 0.29240 D+02 \quad 0.29100 D+050.30000 D+040.00000 D+00$ $0.00000 D+00 \quad 0.28730 D+02 \quad 0.26481 D+05 \quad 0.30000 D+04 \quad 0.00000 D+00$

(--- nfg (Number of Functional Groups)

yo, a, b, sigmaa, and xetf for fg 1 - co2xh C-C

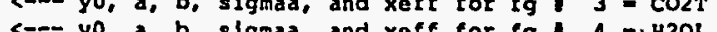
<-- yo, $a, b$, slgma, and xelf for gg 4 an 11202

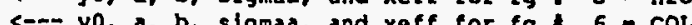
<-.- yo, $a, b$, stgaa, and xote for tg ? $<--y_{0} a b$, slgnaa, and xofe for fg -- yo, $a, b$, slgmaa, and xefe for fg <--- yo, $a b b$ signa, and xoff for fg $10=$ = <.-. yo, $a, b$, slgmaa, and xeft tor tg 11 = $c b_{1}$ (--) yo, $a, b$, slgmaa, and xeft for $f g, 12=$ CAL <-- yo, $a, b$, slgmaa, and xeff for fg 13 - CH4L <-m- yo, $a, b$, slgmaa, and xeff for fg 14 - CH4T <-- yo, $a, b, s$ lgmas, and xeff for tg 15 . HAROM s-.- yo, a, b, slgmaa, and xeff for fg $16=\mathrm{CH} 3 \mathrm{OH}$ <-- yo, a, b, slgmaa, and xeff for fg $17=$ COXT <-- yo, a, b, sigmaa, and xeff for fg 18 - CNV -- yo, a, b, slgmaa, and xeff for fg 19 = SORG <- yo, $a, b$, slgmaa, and xeff for tg 20 - HAL <- yo, a, b, slgmaa, and xetf for fg $21=\mathrm{C2H}$ <- yo, $a, b$, slgmaa, and xetf for fg $22=\mathrm{C} 3 \mathrm{H} 6$

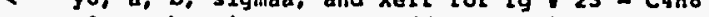
$<--y, a, b$, slgmaa, and xeft for tg $24=c 2 H 6$ <-- yo, a, b, sigmaa, and xeff for $\mathrm{fg} 25=\mathrm{c} 3 \mathrm{HB}$ <-- yo, $a, b$, sigmaa, and xoff for $\mathrm{fg} 26=\mathrm{C} 4 \mathrm{H} 10$ $7=\mathrm{C2H2}$

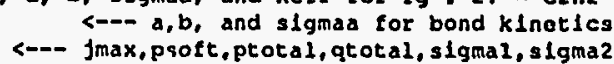

$0.230 D+00 \quad 0.000 D+00 \quad 0.000 D+00 \quad 0.000 D+00 \quad 0.000 D+00 \quad 0.000 D+00 \quad 0.000 D+00 \quad 0.000 D+00 \quad 0.000 D+00 \quad 0.000 D+00$

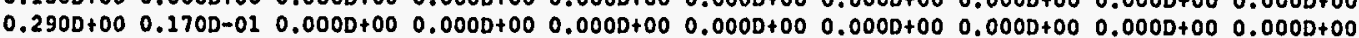

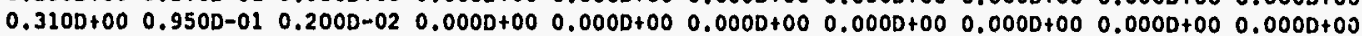

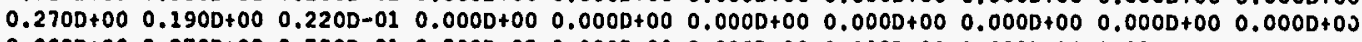
$0.000 D+00 \quad 0.270 D+00 \quad 0.700 D-01 \quad 0.500 D-02 \quad 0.000 D+00 \quad 0.000 D+00 \quad 0.0000+00 \quad 0.0000+00 \quad 0.0000+00 \quad 0.000 D+00$

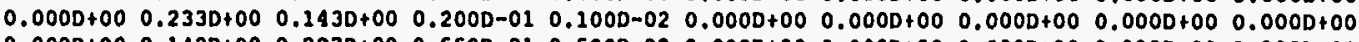
$\begin{array}{llllllllll}0.000 D+00 & 0.142 D+00 & 0.207 D+00 & 0.550 D-01 & 0.500 D-02 & 0.000 D+00 & 0.000 D+00 & 0.000 D+00 & 0.000 D+00 & 0.000 D+00\end{array}$

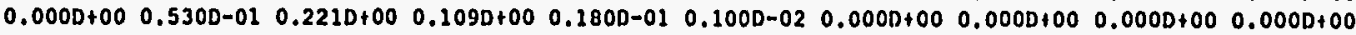
$\begin{array}{llllllllll}0.000 D+00 & 0.000 D+00 & 0.180 D+00 & 0.164 D+00 & 0.450 D-01 & 0.500 D-02 & 0.000 D+00 & 0.000 D+00 & 0.000 D+00 & 0.000 D+00\end{array}$ $0.000 D+00 \quad 0.000 D+00 \quad 0.104 D+00 \quad 0.195 D+00 \quad 0.860 D-01 \quad 0.150 D-01 \quad 0.000 D+00 \quad 0.000 D+00 \quad 0.000 D+00 \quad 0.000 D+00$ $\begin{array}{lllllllllll}0.000 D+00 & 0.000 D+00 & 0.130 D-01 & 0.184 D+00 & 0.1330+00 & 0.360 D-01 & 0.000 D+00 & 0.0000+00 & 0.000 D+00 & 0.000 D+00\end{array}$ $\begin{array}{llllllllll}0.000 D+00 & 0.0000+00 & 0.9000-02 & 0.138 D+00 & 0.1680+00 & 0.690 D-01 & 0.000 D+00 & 0.000 D+00 & 0.000 D+00 & 0.0000+00\end{array}$

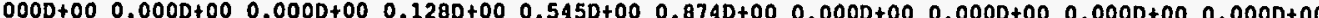
$0.100+02$

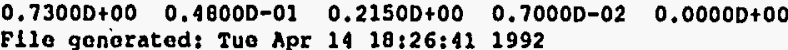
--- pdist $(12,1: 10)$ <-..- pdist $(1,1: 10)$ <-- pdist $(2,1: 10\}$ - pdlst $3,1: 10)$ c- palser 4, $4: 10)$ - palser $5,1: 10)$ - palat $7,1: 10)$ pdise 9 . (- palation --- particlo Internal pressure drop coa10.arg1 
Table A.2: FG-DVC input data for Argonne Premium coal \#2 (Wyodak-Anderson, Wyoming)

27 $0.32940 D-010.29240 D+02 \quad 0.23280 D+05 \quad 0.250000+040.204800+03$ $\begin{array}{lllll}0.15660 D-01 & 0.29646 D+02 & 0.31040 D+05 & 0.28000 D+04 & 0.20480 D+03\end{array}$ $\begin{array}{llllll}0.40248 D-01 & 0.29240 D+02 & 0.23522 D+05 & 0.25000 D+04 & 0.00000 D+00\end{array}$ $\begin{array}{lllll}0.43992 D-01 & 0.32929 D+02 & 0.30070 D+05 & 0.35000 D+04 & 0.00000 D+00\end{array}$ $\begin{array}{lllll}0.17000 D-01 & 0.29240 D+02 & 0.24008 D+05 & 0.25000 D+04 & 0.000000+00\end{array}$ $0.182960-010.30482 D+02 \quad 0.41225 D+05 \quad 0.47500 D+04 \quad 0.00000 D+00$

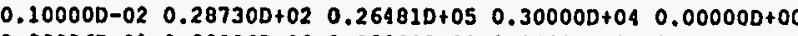
$0.00000 D+00 \quad 0.00000 D+00 \quad 0.000000+00 \quad 0.000000+00 \quad 0.00000 D+00$ $\begin{array}{lllll}0.126000-01 & 0.31032 D+02 & 0.271600+05 & 0.225000+04 & 0.10240 D+03\end{array}$ $\begin{array}{llllll}0.84000 D-02 & 0.31725 D+02 & 0.31040 D+05 & 0.20000 D+04 & 0.10240 D+03\end{array}$ $0.936000-020.3525+02 \quad 0.392850+050.60000 D+040.00000 D+00$ $0.500000-010.3290200+020.165500+050.15000 D+040.00000 D+00$ $0.50000-010.32929 D+02$ 0.385580+05 $0.250000+040.00000 D+00$ $0.15502 \mathrm{D}-010.000000+000.000000+000.000000+000.000000+00$ $\begin{array}{lllllll}0.00000 D+00 & 0.292400+02 & 0.24250 D+05 & 0.15000 D+04 & 0.000000+00\end{array}$ $\begin{array}{lllll}0.200000-02 & 0.292400+02 & 0.242500+05 & 0.150000+04 & 0.000000+00\end{array}$ $0.32236 \mathrm{D}+02 \quad 0.25220 \mathrm{D}+05 \quad 0.10000 \mathrm{D}+04$ (1)

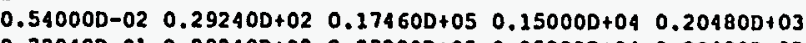
$0.41982 D-01 \quad 0.29240 D+02 \quad 0.29342 D+05 \quad 0.30000 D+04 \quad 0.00000 D+00$

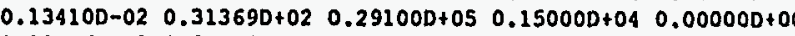

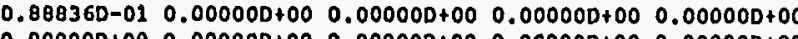
$0.400000-020.000000+000.000000+000.000000+000.000000+00$ $\begin{array}{lllllll}0.45000 D-02 & 0.29240 D+02 & 0.242500+05 & 0.15000 D+04 & 0.00000 D+00\end{array}$ $0.277000-010.292400+02 \quad 0.242500+05 \quad 0.75000 D+030.000000+00$ $\begin{array}{lllllll}0.000000+00 & 0.29240 D+02 & 0.24250 D+05 & 0.25000 D+04 & 0.00000 D+00\end{array}$ $\begin{array}{llllll}0.27300 D-01 & 0.29240 D+02 & 0.24250 D+05 & 0.75000 D+03 & 0.00000 D+00\end{array}$ $0.000000+00 \quad 0.000000+00 \quad 0.00000 D+00 \quad 0.00000 D+00 \quad 0.00000 D+00$

--- nig (Number of Eunct lonal Groups) --- yo, $a, b$, sigmaa, and xeff for $t g, 1=\cos \times L$ c-- yo, a, b, slgmaa, and xeff for tg, $2=\cos L$ -..- yo, a, b, slgmaa, and xetf for fg: $3=\operatorname{cost}$ yo, $a, b$, slgmad, and xeff for fg $4=\mathrm{H} 2 \mathrm{OL}$ - yo, $a, b$, slgmaa, and xeff for fg $5=$ H2OT - yo, $a, b$, slgmaa, and xeft for $f g / 6=\mathrm{COL}$ -.. -- yo, a, b, slgmaa, and xeft for to <-- yo, $a, b$, slgmaa, and xeft for $f g / 10=\mathrm{NH3}^{2}$ -- yo, $a, b$, sigmaa, and xeff for fg 11 = CAL <-- yo, a, b, slgmad, and xeff for $\mathrm{tg} / 12=\mathrm{CH} 4 \mathrm{XL}$ - yo, $a, b$, sigmad, and xeft tor fg $13=$ CH4L <-- yo, $a, b$, slgmad, and xeff for 59 14 = CH4T <-- yo, $a, b$, sigmad, and xeft for $f g$ 15 = HAROM <-- yo, a, b, slgmaa, and xeff for fg 16 - CH3OH <-- yo, a, b, slgmaa, and xeff for $\mathrm{fg} 17$ - COXI yo, $a, b$, lgmaa, and xeff for $1918=\mathrm{CNV}$

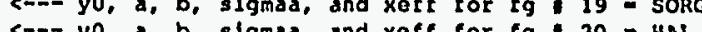
<-- yo, $a$, algmaa, and xols yor tg 20 = HAL

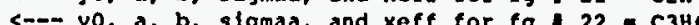

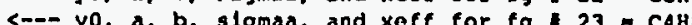
$<---$ yo, $a, b$, lgma, and xoff tor

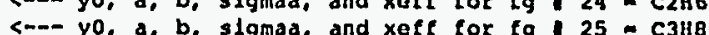
$<--$ yo, $a, b, g$ lgmas, and roff tor $g$ <-- yo, $a, b$, sigmaa, and xeft tor tg $27=c 2112$ - $a, b$, and slgmaa for bond kl natlcs <-..- Jmax, psoft, protal, qtotal, slgmal glgmas

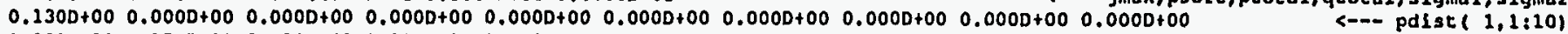

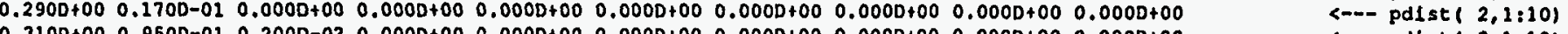

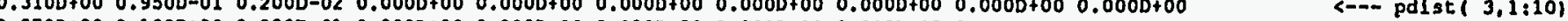

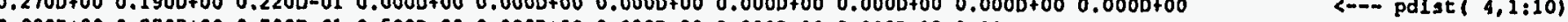

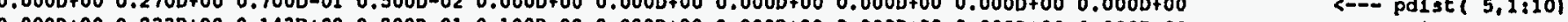

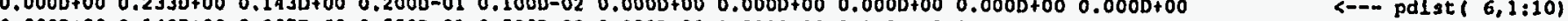

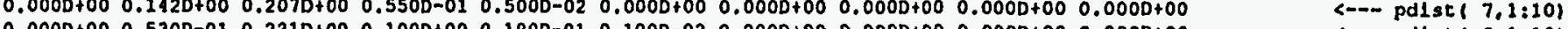

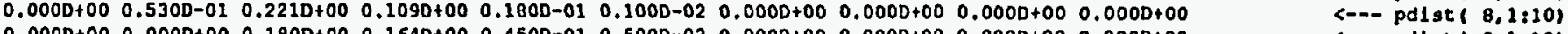

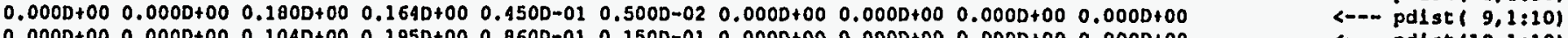

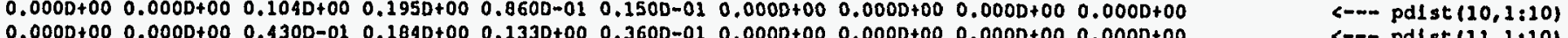
$0.0000+000.0000+000.4000-020.1380+000.1680+000.3000-010.0000+000.000 D+000.0000+000.0000+00 \quad<--$ pdist(11,1:10)

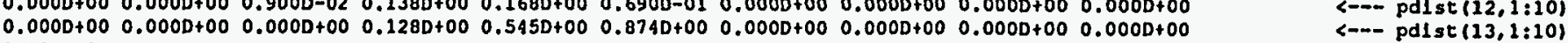
$0.100+02$
$0.1000+00$ $0.75000+00 \quad 0.54000-01 \quad 0.18100+00 \quad 0.11000-01 \quad 0.40000-02$ --- particle Internal pressure drop File generated: Tue Apr $14 \quad 18: 27: 48 \quad 1992$ 


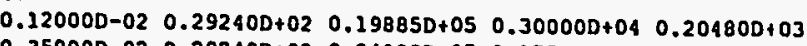
$\begin{array}{lllll}0.35000 D-02 & 0.29240 D+02 & 0.2400 B D+05 & 0.17500 D+04 & 0.20480 D+03\end{array}$ $0.360250-02 \quad 0.29646 \mathrm{D}+02 \quad 0.31040 \mathrm{D}+05 \quad 0.27500 \mathrm{D}+04 \quad 0.20480 \mathrm{D}+03$ $0.675470-010.329290+020.310400+050.2000004040 .000000+00$

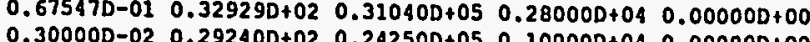
$0.30000 \mathrm{D}-020.29240 \mathrm{D}+020.24250 \mathrm{D}+050.10000 \mathrm{D}+040.00000 \mathrm{D}+00$ $0.232090-010.29240 D+020.291000+050.200000040 .00000 D+00$ $0.240740-010.304920+020.41225 D+050.475001040 .000000+00$ $0.000000+00 \quad 0.28730 \mathrm{D}+020.264010+050.30000 \mathrm{D}+040.00000 \mathrm{D}+00$ $0.105070+00 \quad 0.000000+000.00000+000.000000+000.000000+00$ $0.00000 \mathrm{D}+00 \quad 0.00000 \mathrm{D}+000.000000+000.00000+040.0000 \mathrm{D}+00$ $0.16340 \mathrm{D}-01 \quad 0.31032 \mathrm{D}+02 \quad 0.27160 \mathrm{D}+05 \quad 0.180000+040.102400+03$ $0.21660 D-010.31725 D+02 \quad 0.31040 D+05 \quad 0.22000 D+040.10240 D+03$ $0.15433 D-01 \quad 0.33152 D+02 \quad 0.39285 D+05 \quad 0.60000 D+04 \quad 0.00000 D+00$ $\begin{array}{lllll}0.32000 D-01 & 0.32929 D+02 & 0.38800 D+05 & 0.30000 D+04 & 0.00000 D+00\end{array}$ $\begin{array}{lllll}0.55000 \mathrm{D}+00 & 0.00000 \mathrm{D}+00 & 0.00000 \mathrm{D}+00 & 0.00000 \mathrm{D}+00 & 0.00000 \mathrm{D}+00\end{array}$

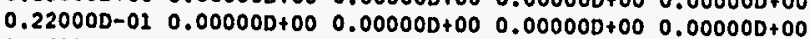

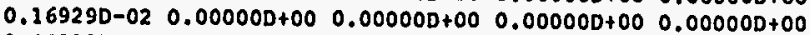
$\begin{array}{llllll}0.40000 D-02 & 0.29240 D+02 & 0.24250 D+05 & 0.15000 D+04 & 0.00000 D+00\end{array}$ $0.00000 D+00 \quad 0.29240 D+02 \quad 0.24250 D+05 \quad 0.15000 D+04 \quad 0.00000 D+00$

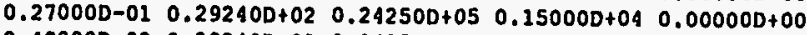
$0.400000-02 \quad 0.29240 D+02 \quad 0.24250 D+05 \quad 0.15000 D+04 \quad 0.00000 D+00$
$0.00000 D+00$ $0.00000 D+000.292400+02 \quad 0.24250 D+050.15000 D+040.00000 D+00$ $0.250000-010.29240 \mathrm{D}+020.24250 \mathrm{D}+050.15000 \mathrm{D}+04 \quad 0.00000 \mathrm{D}+00$ $0.00000 \mathrm{D}+00 \quad 0.00000 \mathrm{D}+000.00000 \mathrm{D}+00 \quad 0.00000 \mathrm{D}+00 \quad 0.00000 \mathrm{D}+00$ $0.32236 \mathrm{D}+02 \quad 0.25220 \mathrm{D}+05 \quad 0.75000 \mathrm{D}+03$

$60.5968 D+00 \quad 0.6560 D+00 \quad 0.1000 D+00 \quad 0.1000 D+010.4000 D+01$ $0.90063 \mathrm{D}-02 \quad 0.29240 \mathrm{D}+02 \quad 0.17460 \mathrm{D}+05 \quad 0.18000 \mathrm{D}+04 \quad 0.000000+00$

(--- nfg (Number of Funct lonal Groups)

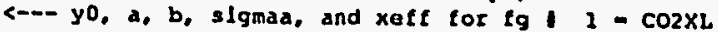

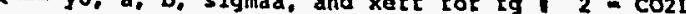

a, slgmaa, and xeff tor Ig $3=\mathrm{CO}$ T

, $a, b$, slgmaa, and xeff for tg a

yo, $a, b$, slgmaa, and xeff for $\mathrm{lg} / 5=\mathrm{H}=\mathrm{H}$

col

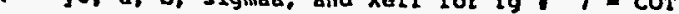

作

yo, a, b, slgmaa, and xetr lor tg

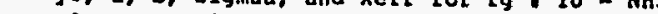
- yo, $a, b$, slgmaa, and xell ror fo 11 g $=$ CAL C < ( (a)

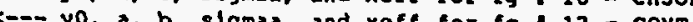
--- yo, $a, b$, slgmaa, and xose for tg $19=$ = sopo yo, $a, b$, slgmaa, and xofe tor fo 20 a c-- yo, $a, b$, sigmaa, and xoef for fg $21=$ cal

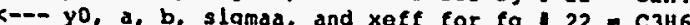
c-- yo, $a, b$, slgmaa, and xeff for fg $23=c 418$ c--. yo, $a, b$, slgmaa, and xeff for fg 24 - c246 <-- yo, $a, b$, slgmaa, and xoff for tg $25=$ - c3HB <-- yo, $a, b$, slgmaa, and xoff for $9 g, 26$ - c4H10 -- yo, a, b, slgmaa, and xeff for fg $27=$ c4H10 - $a, b$, and slgmaa for bond kinetics (---

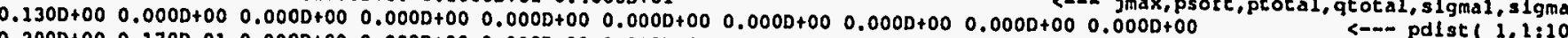

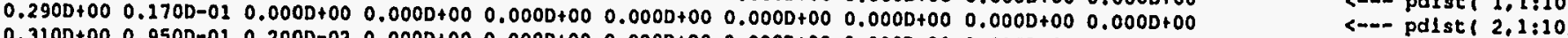

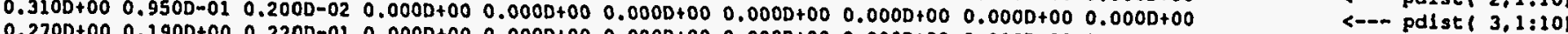

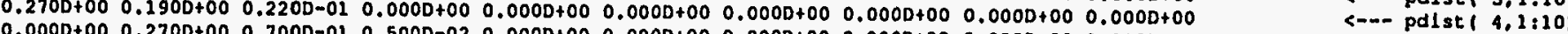
$\begin{array}{llll}0.0000 & 0.0000000\end{array}$

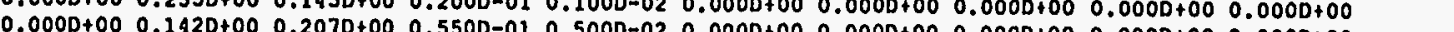

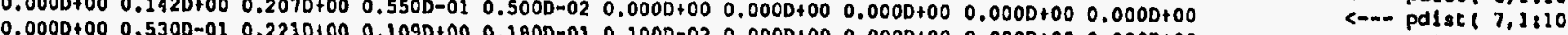

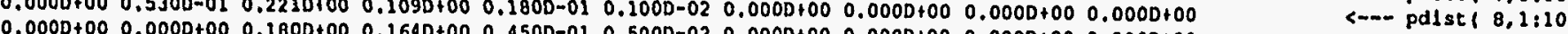

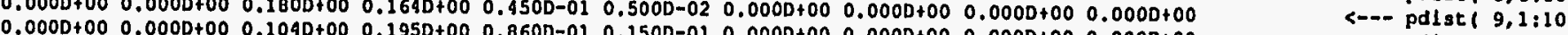

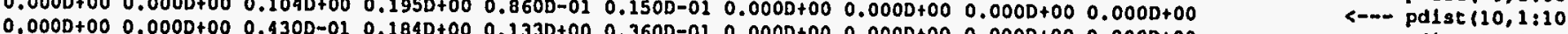

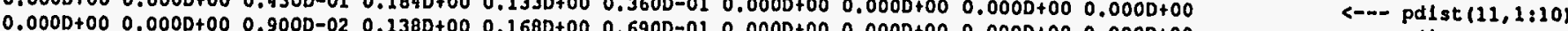
$0.0000+00 \quad 0.000 D+00 \quad 0.000 D+00 \quad 0.128 D+00 \quad 0.545 D+00 \quad 0.8740+00 \quad 0.0000+00 \quad 0.0000+00 \quad 0.0000+00 \quad 0.0000+00 \quad<-$ pdlst(12,1:10) $0.200+00$

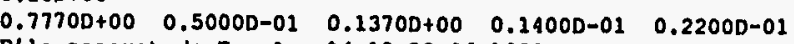

$<--$ particle Internal pressure drop composition (daf) 
Table A.4: FG-DVC input data for Argonne Premium coal \#4 (Utah Blind Canyon)

27

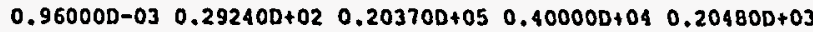

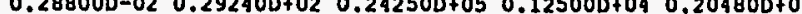

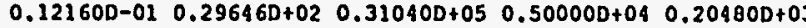
$\begin{array}{llllll}0.31122 D-01 & 0.29240 D+02 & 0.24250 D+05 & 0.12500 D+04 & 0.00000 D+00\end{array}$

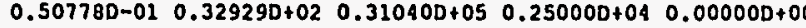

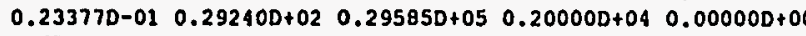

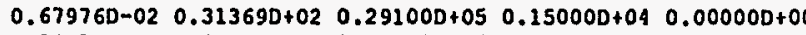
$0.00000 D+00 \quad 0.28730 D+02 \quad 0.26481 D+05 \quad 0.30000 D+04 \quad 0.00000 D+00$ $0.13001 D+00 \quad 0.00000 D+00 \quad 0.00000 D+00 \quad 0.00000 D+00 \quad 0.00000 D+00$

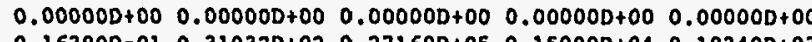

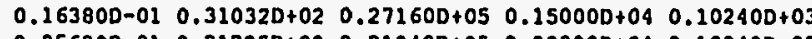

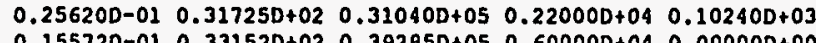
$0.000000+000.000000+000.000000+000.00000 D+000.00000 D+00$ $0.280000-010.329290+020.38000+050.250003+040.000000+00$ $0.300000-020.000000+000.000000+000.000000+000.000000+00$ $0.112730 .0 .000000+00.000000+00.000000+000.000000+00$ $0.000000+00 \quad 0.292400+02 \quad 0.24250 D+050.150000+040.000000+00$ $0.270000-010.292400+02 \quad 0.244920+050.120000+040.000000+00$

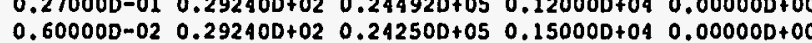
$0.25000 \mathrm{D}-010.29240 \mathrm{D}+02 \quad 0.24492 \mathrm{D}+05 \quad 0.12000 \mathrm{D}+040.00000 \mathrm{D}+00$ $0.322360+02 \quad 0.261900+050.125000+04$

$60.5835 D+00 \quad 0.77350+000.6000 D-010.10000+010.40000+01$ $0.40000 D-02 \quad 0.29240 D+02 \quad 0.24250 D+05 \quad 0.12500 D+04 \quad 0.00000 D+00$

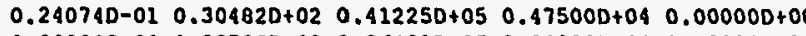
$0.155720-010.351520+020.392850+050.60000 D+040.000000+00$ $0.600000-02 \quad 0.292400+020.24250 D+050.15000 D+040.000000+00$ $0.00000 D+00 \quad 0.292400+020.242500+050.15000 D+040.000000+00$ $0.00000 D+00 \quad 0.00000 D+00 \quad 0.00000 D+00 \quad 0.00000 D+00 \quad 0.00000 D+00$

(-- nfg (Number of Functional Groups)

--. yo, a, b, slgmaa, and xeff for tg a $1=\cos x \mathrm{~L}$ <-.. yo, a, b, slgmaa, and xoft for tg a = co2t c-- yo, a, b, slgmaa, and xeff for $f g$, $3=\cos T$ c-- yo, a, b, slgmad, and xeff for tg | $=$-H2OL c- yo, a, b, slgmaa, and xeff for fg 5 - H2OT <- yo, $a, b$, sigmaa, and xeft for $f g \mid 6=\mathrm{cOl}$ <-- yo, a, b, slgmaa, and xeff for fg $7=$ = COT <-- yo, a, b, slgmad, and xeff for tg $8=$ HCNL < yo, $a, b$, sigmaa, and xeff for tg $10=$ NHT C c- yo, $a, b$, slgmaa, and xoff for tg $12=\mathrm{CH} \times \mathrm{KL}$ <-- yo, $a, b$, slgmaa, and xeff for tg $13=\mathrm{CH} L \mathrm{~L}$ <-- yo, $a, b$, slgmaa, and xeff for tg $14=$ CHAT -- Yo, $a, b$, sIgmaa, and xeff for $f g / 15=$ HAROM <-- yo, $a, b$, slgmaa, and xoff for $\mathrm{tg} 16$ - $\mathrm{CH} 3 \mathrm{OH}$ <-- yo, a, b, slgmaa, and xeff for tg $17=$ coxr $<-a, a, b l g m a a$, and xelf lor tg $18=C N V$ <- yo, $a, b$, slgmaa, and xocf for 99 I9 = SORG <-- yo, $a, b$, slgmaa, and xelt tor $c 9$, 20 - HAL $<---$ yo $a, b$, slgmaa, and xote for

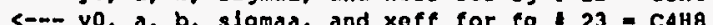
<--- yo, $a, b$, slgmea, and xete for tg 24 = call6 <-- yo, $a, b$, slgmaa, and xete for tg 25 - c3118 C--. yo, $a, b$, slgmaa, and xeff for tg $26=$ c c4llio s-- yo, $a, b$, slgmaa, and xeft for tg $27=\mathrm{C} 2 \mathrm{~Hz}$ - $a, b$, and slgmaa for bond $k l$ inerlcs (--- Imax, psoft, ptot al, qtot a

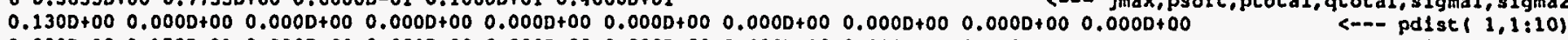

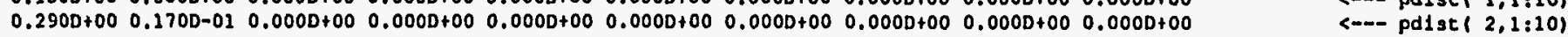

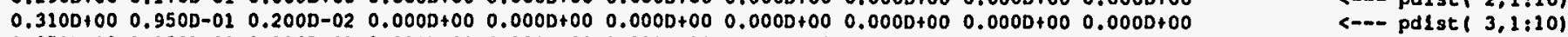

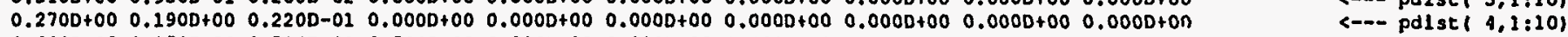

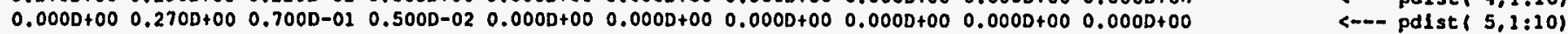

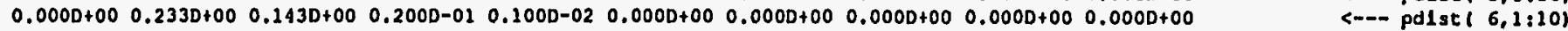

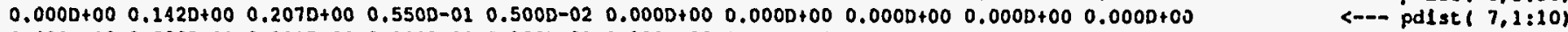

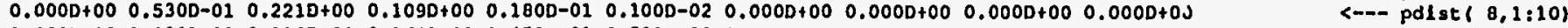

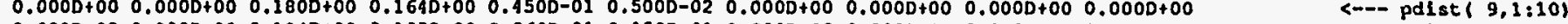

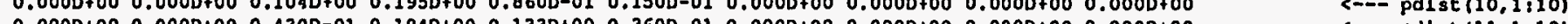

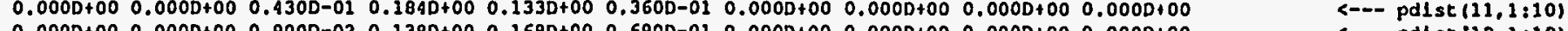

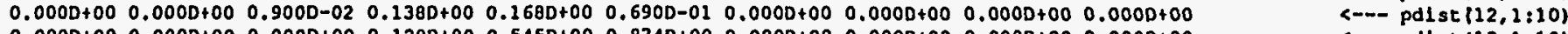
$0.200+00$

$0.59000-01 \quad 0.11600+00 \quad 0.36000-01 \quad 0.30000-02$ (--- ultimate composition (daf) coalo.arg4 
Table A.5: FG-DVC input data for Argonne Premium coal \#5 (Upkana-Stokton, WV)

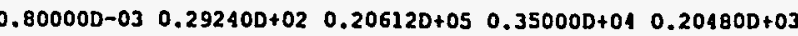

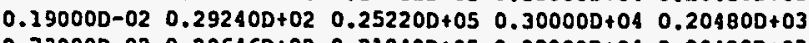
$0.730000-02 \quad 0.296460+02 \quad 0.31040 D+05 \quad 0.320000+04 \quad 0.204800+03$ $0.26264 D-01 \quad 0.29240 D+02 \quad 0.24735 D+05 \quad 0.12500 D+040.00000 D+00$

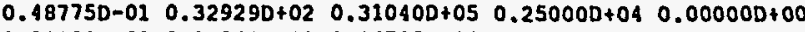
$0.20000 D-02 \quad 0.29240 D+02 \quad 0.24735 D+05 \quad 0.11000 D+04 \quad 0.00000 D+00$ $0.13671 D-010.29240 D+02 \quad 0.29585 D+05 \quad 0.360000+04 \quad 0.000000+00$ $0.19827 D-02 \quad 0.31369 D+02 \quad 0.29100 D+05 \quad 0.15000 D+040.00000 D+00$ $0.288890-010.30482 D+02 \quad 0.41225 D+05 \quad 0.47500 D+04 \quad 0.00000 D+00$ $0.00000 D+00 \quad 0.27813 D+02 \quad 0.26481 D+05 \quad 0.30000 D+04 \quad 0.00000 D+00$ $0.177010+000.000000+00 \quad 0.000000+00 \quad 0.00000 D+00 \quad 0.000000+00$ $0.00000 D+00 \quad 0.00000 D+00 \quad 0.00000 D+00 \quad 0.00000 D+00 \quad 0.00000 D+00$

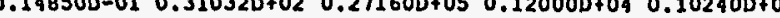
$0.30150 D-010.31725 D+02 \quad 0.310400+050.220000+040.10240 D+03$ $0.15640 D-010.33152 D+020.39285 D+050.60000 D+040.000000+00$ $0.000000+000.00000 D+00 \quad 0.000000+000.00000 D+000.000000+00$

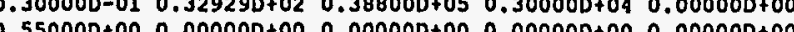

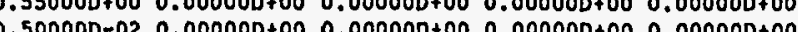

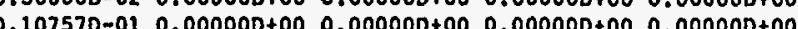
(1) $0.000000+000.292400+020.212500+050.150000+040.000000+00$ $0.100000-010.292400+020.245900+050.100000+040.00000+00$ $0.200000-020.292400+020.242500+050.150000+040.000000+00$ $0.000000+000.292400+020.242500+050.150000+040.000000+00$ $0.200000-020.292400+020.245900+050.100000+040.000000+00$ $0.00000 D+00 \quad 0.000000+00 \quad 0.000000+000.000000+00 \quad 0.000000+00$ $0.322360+020.26432 D+05 \quad 0.10000 D+04$

$6 \quad 0.83570+00 \quad 0.8357 D+00 \quad 0.6000 D-01 \quad 0.10000+01 \quad 0.40000+01$
$0.1300+00 \quad 0.000 D+00 \quad 0.0000+00 \quad 0.000 D+00 \quad 0.000 D+00 \quad 0.000 D$

$\begin{array}{lllllllllllll}0.2900+00 & 0.170 D-01 & 0.000 D+00 & 0.000 D+00 & 0.000 D+00 & 0.000 D+00 & 0.000 D+000 & 0.000 D+00 & 0.000 D+00 & 0.000 D+00 & 0.000 D+00 & 0.000 D+00 & 0.000 D+00\end{array}$

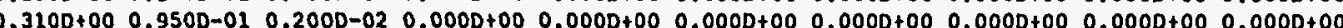

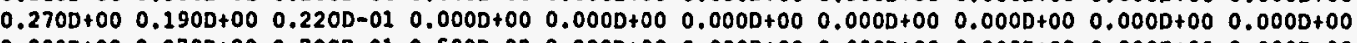
$\begin{array}{llllllllllll}0.000 D+00 & 0.270 D+00 & 0.700 D-01 & 0.500 D-02 & 0.000 D+00 & 0.000 D+00 & 0.000 D+00 & 0.000 D+00 & 0.000 D+00 & 0.000 D+00\end{array}$ $0.0000+000.233 D+000.1430+000.2000-010.1000-020.0000+000.0000+000.0000+000.0000+000.0000+00$ $0.0000+000.5300-010.2210+000.1090+00 \quad 0.1800-010.0000-020.0000+000.0000$ 0.0000+00 $0.000 D+000.1000+000.3640+000.4500-010.500 D-020.000 D+000.000$ (1) $0.00000+00 \quad 0.128 D+00 \quad 0.545 D+00 \quad 0.8740+00 \quad 0.000 D+00.000 D+000.000 D+000.0000+00$ $0.200+00$

1le generated: Tue Apr 14 16:28:40 1992

(-)- nfg (Number of Eunctional Groups

xolf lor fg : 1 - cosx <-- yo, a, b, sigmaa, and xeff for fg 1- H2OL C-- yo, a, b, sigmaa, and xeff for fg: 5 - H2OT <- yo, $a, b$, slgmaa, and xoff for $\mathrm{tg}, 7$ - coT $<--y_{0} a, b, s$ lgmaa, and xeff for fg $8=\mathrm{HCNL}$ - yo, $a, b$, sigmaa, and xeff for fg 9 - HCNT - yo, $a, b$, sigmaa, and xeft for tg $10=\mathrm{NH3}$ <-- yo, a, b, slgmaa, and xeff for fg 12 = CH4XL <- yo, a, b, slgmaa, and xeff for fg 13 - CH4L -- yo, $a, b$, sigma, and xeff for fg $14=$ - CHAT so, $a, b$, sigmad, and xetf for tg $15=$ HAROM c-.- yoo, a, b, slgmaa, and xetf for fg: $16=$ CH3OH cor $a, b$, slgmaa, and xeff for $f(18=C N V$

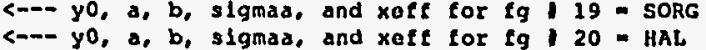
<-- yo, a bl slgmad, and xeff for tg 20 = HAL c-m- yo, a, b, slgmad, and xeff for fg $22=$ с --

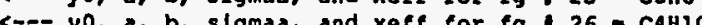
c-- yo $a$ bo slgmad and xese tor 99 slgna, and xore tor tg 127 a 242 $a, b$, and slgmaa for bond kinetics <-- pdist $1,1: 10)$ <-- pdist $(2,1: 10)$ <-- pdist ( 3,1:10) <-- pdist $8,1: 10)$ <-- pdist $(5,1: 10)$ <--- pdlst $6,1: 10)$ <-- pdlst $(8,1: 20)$ <-- pdlst $(9,1: 10)$ <-- pdlst $(10,1: 10)$ (-)- pdlst $(11,1: 10)$ <-- pdist $(12,1: 10)$ <-- pdist $(13,1: 10)$ - particle lnternal pressure drop -.- ultimate composition (dat) coalo.args 
Table A.6: FG-DVC input data for Argonne Premium coal \#6 (Pittsburgh \#8)

27

$\begin{array}{lllll}0.10000 D-02 & 0.29240 D+02 & 0.20855 D+05 & 0.36000 D+04 & 0.20480 D+03\end{array}$ $\begin{array}{llllll}0.250000-02 & 0.29240 D+02 & 0.25705 D+05 & 0.30000 D+04 & 0.20480 D+03\end{array}$

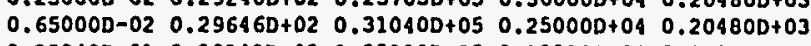
$\begin{array}{lllll}0.23040 D-01 & 0.292400+02 & 0.252200+05 & 0.12500 D+04 & 0.00000 D+00\end{array}$

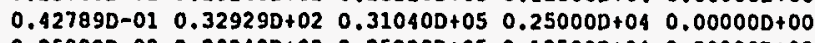

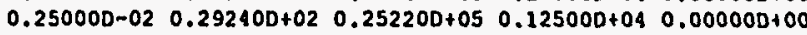
$0.15231 D-01 \quad 0.29240 D+02 \quad 0.29828 D+05 \quad 0.19000 D+04 \quad 0.00000 D+00$ $\begin{array}{llllll}0.26963 D-02 & 0.30482 D+02 & 0.41225 D+05 & 0.47500 D+04 & 0.00000 D+00\end{array}$ $\begin{array}{lllllll}0.00000 D+00 & 0.28730 D+02 & 0.264810+05 & 0.30000 D+04 & 0.00000 D+00\end{array}$

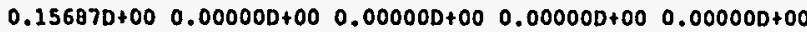
$0.00000 D+00 \quad 0.00000 D+00 \quad 0.00000 D+00 \quad 0.00000 D+00 \quad 0.00000 D+00$

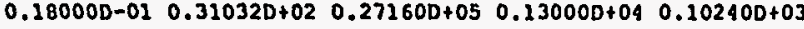
$\begin{array}{lllll}0.320000-01 & 0.31725 D+02 & 0.31040 D+05 & 0.22000 D+04 & 0.10240 D+03\end{array}$

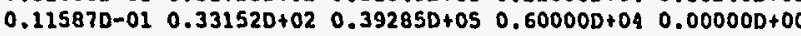
$0.000000+00 \quad 0.000000+00 \quad 0.00000 D+00 \quad 0.00000 D+00 \quad 0.00000 D+00$ $0.230000-010.329290+02 \quad 0.363000+050.280000+040.00000 D+00$ $\begin{array}{llllll}0.10000 \mathrm{D}-01 & 0.00000 \mathrm{D}+00 & 0.00000 \mathrm{D}+00 & 0.00000 \mathrm{D}+00 & 0.00000 \mathrm{D}+00\end{array}$ $0.12116 \mathrm{D}-01 \quad 0.00000 \mathrm{D}+00 \quad 0.00000 \mathrm{D}+00 \quad 0.00000 \mathrm{D}+00 \quad 0.00000 \mathrm{D}+00$

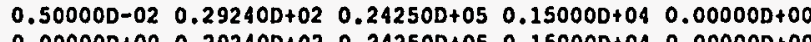
$0.000000+000.292400+02 \quad 0.242500+0550.15000 D+040.00000 D+00$ $0.5000 D-020.292400+02$ 0.24 $0.000000+00$ 0.292400+02 $0.242500+050.150000+040.000000+00$ $0.000000+000.000000+000.000000+000.000000+000.000000+00$

$$
0.322360+02 \quad 0.26675 D+050.125000+04
$$

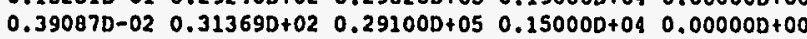

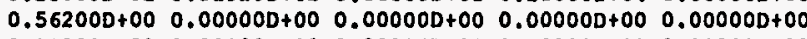

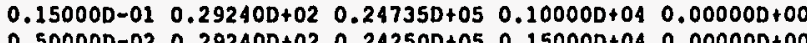

(-) nfg (Number of Functlonal Groups)

<-- yo, $a, b$, slgmaa, and xeff for fg $1=\cos \times L$ , $a, b$, slgmad, and xeff for fg: 2 - CO2L

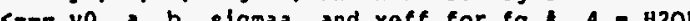
<

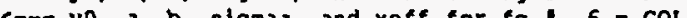
c-- yo, a, $b$, slama, and xeff for tg $7=\operatorname{cor}$ $<--\infty$ yo, $a, b$, stgmad, and xeft tor tg $B=$ - HCN $<-\ldots$ yo, $a, b$, sigmaa, and xeff for fg: $9=$ HCNT $<--$ yo, $a, b$, stgmas, and xoff for to: $10=$ NH3 <-- yo, a, b, stgmaa, and xetf for tg 11 = CAL <-- yo, $a, b$, sigmaa, and xeff tor $f g: 12$ = CH4XL <-- yo, $a, b$, slgmaa, and xetf for tg 13 = CH4L <-- yo, $a, b$, slgmaa, and xaff tor tg 14 - CH4T <-- yo, a, b, slgmaa, and xeff for fg 15 - HAROM <-- yo, $a, b$, sigmaa, and xeff for $\mathrm{tg} 16=\mathrm{CH} 3 \mathrm{OH}$ <n-- yo, $a, b$, slgmaa, and xete for tg $17=$ COXI

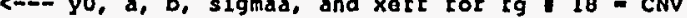
- yo, a, b, slgmad, and xeff for $\mathrm{tg} 19$ = SORG <-- yo, a, b, slgmaa, and xefe tor tg 20 = HAL -- yo, $a, b$, slgmaa, and xeff lor $\mathrm{fg}$ 21 = C2HA --- yo, $a, b, s i g m a s$, and xoff tor $\mathrm{gg} 22$ - c3H6 <-- yo, a, b, sigmaa, and xoff for $\mathfrak{g g} 23$ - catte yo, $a, b$, slgmas, and xeft lor fg 24 - C2HG < <- yo, $a, b$, slgmas, and xorr cor lg 26 - callio c-- $a, b$, and sigmaa for bond $x$ inotics $<-0$ Jmax a bort ptotal qtotal algnal algna

$60.8202 D+00 \quad 0.8202 D+00 \quad 0.36000-010.10000+010.40000+01$

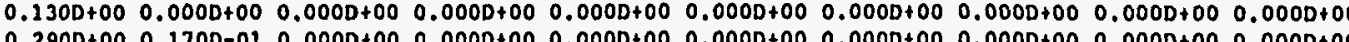
$0.3100+000.950 D-010.000 D-02 \quad 0.0000+000.0000+00 \quad 0.000 D+00 \quad 0.0000+00 \quad 0.000 D+00 \quad 0.0000+00 \quad 0.000 D+00$

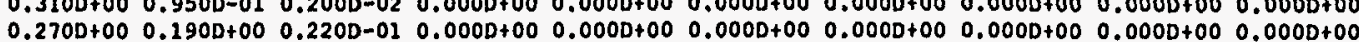
$0.000 D+00 \quad 0.270 D+00 \quad 0.700 D-01 \quad 0.500 D-02 \quad 0.000 D+00 \quad 0.000 D+00 \quad 0.000 D+00 \quad 0.000 D+00 \quad 0.0000+000.000 D+00$ $0.000 D+00 \quad 0.233 D+00 \quad 0.143 D+00 \quad 0.200 D-01 \quad 0.100 D-02 \quad 0.000 D+00 \quad 0.000 D+00 \quad 0.000 D+00 \quad 0.000 D+00 \quad 0.000 D+00$

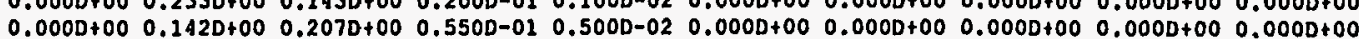
$\begin{array}{lllllllllll}0.000 D+00 & 0.530 D-01 & 0.221 D+00 & 0.109 D+00 & 0.180 D-01 & 0.100 D-02 & 0.000 D+00 & 0.000 D+00 & 0.000 D+00 & 0.000 D+00\end{array}$

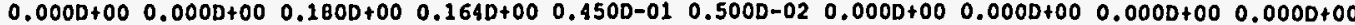
$\begin{array}{llllllllllll}0.000 D+00 & 0.000 D+00 & 0.104 D+00 & 0.195 D+00 & 0.860 D-02 & 0.150 D-02 & 0.000 D+00 & 0.000 D+00 & 0.000 D+00 & 0.000 D+00\end{array}$ $0.000 D+000.000 D+000.4300-010.1840+000.1330+000.360 D-010.0000+000.000 D+000.0000+000.000 D+00$ $0.000 D+00 \quad 0.000 D+00 \quad 0.900 D+02 \quad 0.138 D+00 \quad 0.152 D+00 \quad 0.690 D-01 \quad 0.000 D+00 \quad 0.000 D+00 \quad 0.000 D+00 \quad 0.000 D+00$

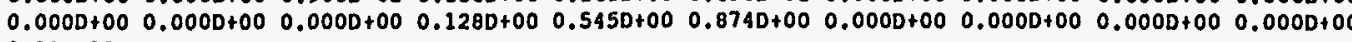
$0.200+00$

$0.83200+00 \quad 0.53000-01 \quad 0.89000-01 \quad 0.16000-01 \quad 0.10000-01$ File generated: Tue Apr 14 18:28:56 1992 c-a pdist $1,1,1: 10)$ -.-- pdist $(2,1: 10)$ --- pdist 4 ( $1: 10)$ C-.- pdist $4,1: 10)$ - polst ( $5,1: 10)$ --- pdist $7,1: 10)$ --- palset $8,1: 10)$ $<-\infty$ pdlst $9,1: 10$ <-- pdlst $(10,1: 10)$ <-- pdist $(11,1: 10)$ pdist $(12,1: 10)$ coalo.arg6 - particle internal pressure drop 


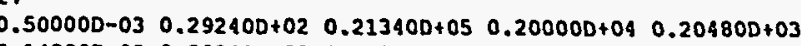
$\begin{array}{lllll}0.14000 D-02 & 0.29240 D+02 & 0.26190 D+05 & 0.30000 D+04 & 0.20480 D+03\end{array}$

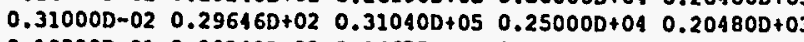
$\begin{array}{llllll}0.19300 D-01 & 0.29240 D+02 & 0.266750+05 & 0.12500 D+04 & 0.00000 D+00\end{array}$

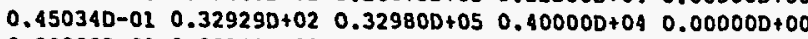
$\begin{array}{lllll}0.20000 D-02 & 0.29240 D+02 & 0.27160 D+05 & 0.75000 D+03 & 0.00000 D+00\end{array}$ $0.74604 \mathrm{D}-02 \quad 0.292400+02 \quad 0.30555 \mathrm{D}+05 \quad 0.140000+040.00000 \mathrm{D}+00$ $0.48681 D-02 \quad 0.313690+02 \quad 0.291000+05 \quad 0.150000+040.000000+00$ $0.24074 D-010.30482 D+020.43225 D+050.47500 D+040.00000 D+00$ $0.00000 D+00 \quad 0.28730 D+02 \quad 0.264810+05 \quad 0.300000+040.00000 D+00$ $0.19154 \mathrm{D}+00 \quad 0.000000+00 \quad 0.000000+00 \quad 0.00000 D+000.000000 D+00$ $\begin{array}{lllll}0.00000 D+00 & 0.00000 D+00 & 0.00000 D+00 & 0.00000 D+00 & 0.0000000+00\end{array}$

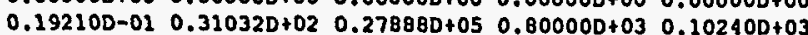
$\begin{array}{lllll}0.37290 D-01 & 0.31725 D+02 & 0.31040 D+05 & 0.20000 D+04 & 0.10240 D+03\end{array}$ $\begin{array}{llllll}0.11635 D-01 & 0.33152 D+02 & 0.39285 D+05 & 0.60000 D+04 & 0.00000 D+00\end{array}$ $0.00000 D+00 \quad 0.00000 D+00 \quad 0.00000 D+00 \quad 0.00000 D+00 \quad 0.00000 D+00$ $\begin{array}{lllll}0.13700 D-01 & 0.32929 D+02 & 0.38800 D+05 & 0.22500 D+04 & 0.00000 D+00\end{array}$

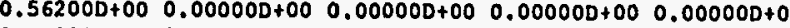

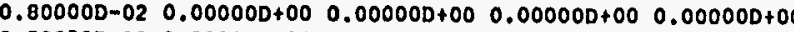
$\begin{array}{lllll}0.58852 D-02 & 0.00000 D+00 & 0.00000 D+00 & 0.00000 D+00 & 0.00000 D+00\end{array}$ $0.40000 \mathrm{D}-02 \quad 0.29240 \mathrm{D}+02 \quad 0.242500+05 \quad 0.15000 \mathrm{D}+04 \quad 0.00000 \mathrm{D}+00$

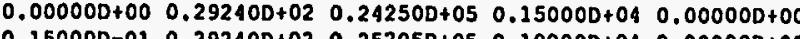
$0.15000 D-010.29240 D+020.25705 D+050.10000 D+040.00000 D+00$ $0.40000-020.292400+02 \quad 0.242500+050.150000+040.00000 D+00$ $0.00000+0.252400+020.242500+050.150000+040.00000 D+00$ $0.000000+000.000000+000.000000+000.000000+000.00000+00$ $0.322360+02 \quad 0.27402 D+05 \quad 0.12500 D+04$

$60.7683 D+00 \quad 0.8683 D+00.3000 D-010.10000+010.40000+01$

--- nfg (Number of Funcelonal Groups)

c-- yo, a, b, slgmaa, and xeff for fg 1 - co2x - yo, $a, b$, slgmad, and xelf tor tg 2 - Co2L

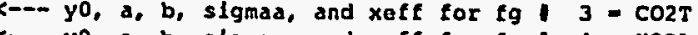
c- yo, $a, b$, slgmaa, and xetf for tg 4 -H2OL c - yo, $a, b$, slgmaa, and xete for tg 6 - CoL -- yo, $a, b$, slgmaa, and xerf for fg $7=$ cor --- yo, $a, b$, slgmaa, and xeff for tg a = HCNL --. yo, $a, b$, slgnaa, and xeft for fg a 9 - HCNT <-- $0, a, b$, slgna, and xelf for $f g$, $10=$ NH3

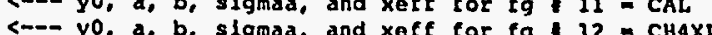
<-c- yo, $a, b$, signaa, and xofe for fg 13 = CHALL <-- yo, $a, b$, s lgmaa, and xoff tor tg 14 - CH4T $<---$ yo, $a, b$, slgmaa, and xeff tor $t g, 15=$ HAROM <-- yo, $a, b$, slgmaa, and xeff cor eg 16 = CH3OH $<---y 0, a, b$, ligma, and xofe for $g$ $<--$ yo, $a, b$, slgmaa, and xoff for $t g$ : 18 - CNV <-- yo, a, b, slgmaa, and xets for tg 19 = SORG <-- yo, a, b, slgmaa, and xoff for fg 20 . HAL <-- yo, $a, b$, slgmaa, and xeft for fg $21=$ = C2H4 c-- yo, a, b, slgmaa, and xotf for tg 22 = c3H6 <-- yo, a, b, slgmaa, and xotf for $\mathrm{fg} 23$ - $\mathrm{C4HB}$ <-- yo, a, b, slgmaa, and xetf for tg 24 - C2H6 <-- yo, $a, b$, sigmad, and xeff for $\mathrm{fg} 25=$ = 23 HB <-- yo, a, b, slgmaa, and xeff for $\mathrm{tg} 26=\mathrm{CAH10}$ and xalf tor 27 - $\mathrm{C2H}_{2}$ (-slgmaa, and xeff tor fo bond $x$ lnotics --- a,b, and sigmaa tor bond xinotics

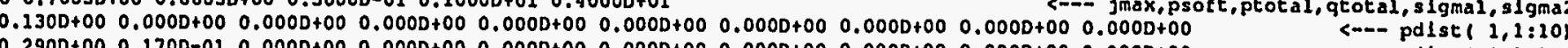

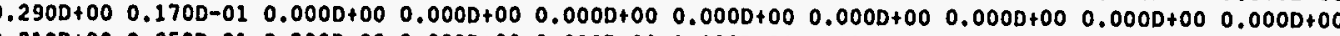

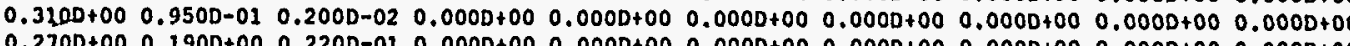

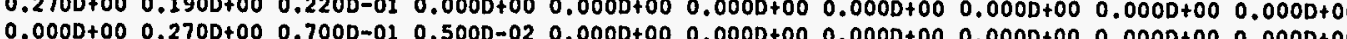

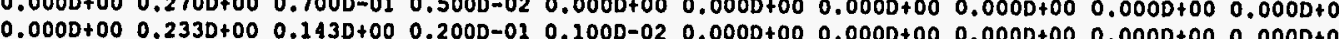
$0.0000+00.1420+00 \quad 0.2070+00 \quad 0.5500-010.5000-02 \quad 0.0000+00 \quad 0.000+000.0000+00 \quad 0.0000+000.000 D+00$ $0.000 D+00 \quad 0.530 D-01 \quad 0.2210+00 \quad 0.1090+00 \quad 0.180 D-010.1000-02 \quad 0.0000+00 \quad 0.000 D+00 \quad 0.000 D+000.0000+00$ $0.000 D+00 \quad 0.000 D+00 \quad 0.180 D+00 \quad 0.1640+00 \quad 0.450 D-010.500 D-02 \quad 0.0000+000.000 D+00 \quad 0.000 D+000.0000+00$ $0.000 D+00 \quad 0.000 D+00 \quad 0.4300-01 \quad 0.184 D+00 \quad 0.133 D+00 \quad 0.360 D-01 \quad 0.000 D+00 \quad 0.000 D+00 \quad 0.000 D+00 \quad 0.00000+00$

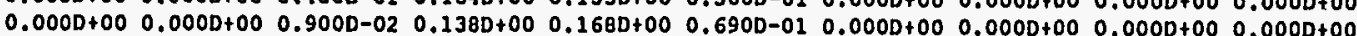

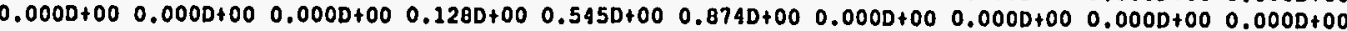
$0.200+00$

$0.85600+00 \quad 0.47000-01 \quad 0.74000-01 \quad 0.15000-01 \quad 0.80000-02$ Flle generated: Tue Apr 14 10:29:12 1992

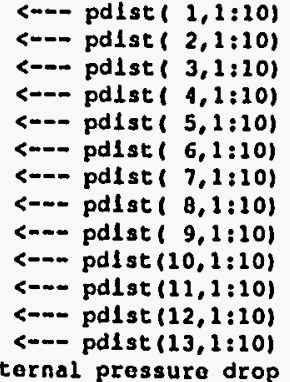

particle Internal pressure drop coa10.arg? 
Table A.8: FG-DVC input data for Argonne Premium coal \#8 (Pochahontes \#3, VA)

27

$\begin{array}{lllll}0.60000 D-03 & 0.29240 D+02 & 0.22310 D+05 & 0.25000 D+04 & 0.40960 D+03\end{array}$

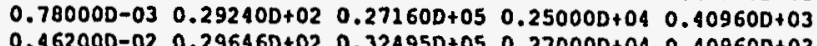
$\begin{array}{llllll}0.462000-02 & 0.296460+02 & 0.32495 \mathrm{D}+05 & 0.27000 \mathrm{D}+04 & 0.40960 \mathrm{D}+03\end{array}$

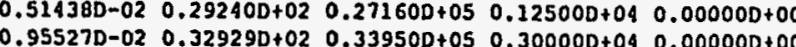

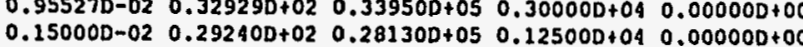

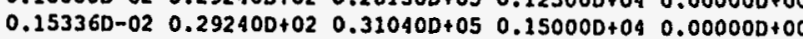
$0.19721 D-02 \quad 0.31369 D+02 \quad 0.29100 D+05 \quad 0.15000 D+04 \quad 0.00000 D+00$

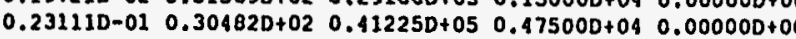
$0.000000+00 \quad 0.287300+02 \quad 0.26481 D+05 \quad 0.30000 D+040.00000 D+00$ $0.27615 \mathrm{D}+00 \quad 0.00000 \mathrm{D}+00 \quad 0.00000 \mathrm{D}+00 \quad 0.00000 \mathrm{D}+00 \quad 0.00000 \mathrm{D}+00$ $0.00000 D+00 \quad 0.00000 D+00 \quad 0.00000 D+00 \quad 0.00000 D+00 \quad 0.00000 D+00$

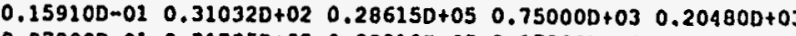
$\begin{array}{lllllll}0.27090 D-01 & 0.31725 D+02 & 0.320100+05 & 0.17000 D+04 & 0.204800+03\end{array}$ $0.118090-01 \quad 0.331520+02 \quad 0.392850+05 \quad 0.600000+04 \quad 0.000000+00$ . $0.190000-010.329290+02 \quad 0.388000+05 \quad 0.32000 D+040.00000 D+00$ $0.20000-020.000000+000.000000+000.000000+000.000000+00$ $0.152320-010.000000+000.000000+000.000000+000.00000000$ $0.200000-02 \quad 0.292400+02 \quad 0.242500+050.150000+040.000000+00$ $0.000000+00 \quad 0.292400+02 \quad 0.242500+050.150000+040.000000+00$ $0.800000-02 \quad 0.292400+02 \quad 0.266750+050.250000+030.000000+00$ $0.20000 D-02 \quad 0.29240 D+02 \quad 0.242500+05 \quad 0.150000+04 \quad 0.000000+00$ $0.000000+00 \quad 0.292400+02 \quad 0.242500+05 \quad 0.250000+04 \quad 0.000000+00$ $0.100000-01 \quad 0.292400+02 \quad 0.266750+05 \quad 0.750000+03 \quad 0.000000+00$ $0.000000+00 \quad 0.000000+00 \quad 0.00000 D+00 \quad 0.00000 D+00 \quad 0.000000+00$ $0.32236 \mathrm{D}+02 \quad 0.281300+05 \quad 0.750000+03$

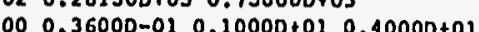
$\begin{array}{llllllllllllll}0.700 D-01 & 0.100 D-02 & 0.000 D+00 & 0.000 D+00 & 0.000 D+00 & 0.000 D+00 & 0.000 D+00 & 0.000 D+00 & 0.000 D+00 & 0.000 D+00 & 0.000 D+00 & 0.000 D+00 & 0.000 D+00 & 0.000 D+00\end{array}$

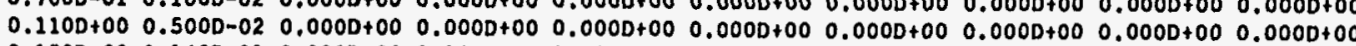

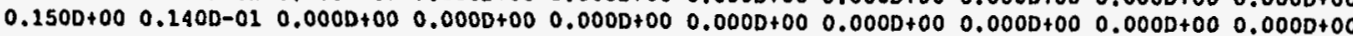
$\begin{array}{llllllllll}0.190 D+00 & 0.280 D-01 & 0.100 D-02 & 0.000 D+00 & 0.000 D+00 & 0.000 D+00 & 0.000 D+00 & 0.000 D+00 & 0.000 D+00 & 0.000 D+00\end{array}$ $\begin{array}{llllllllll}0.170 D+00 & 0.500 D-01 & 0.300 D-02 & 0.000 D+00 & 0.000 D+00 & 0.000 D+00 & 0.000 D+00 & 0.000 D+00 & 0.000 D+00 & 0.000 D+00\end{array}$

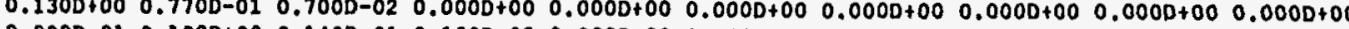

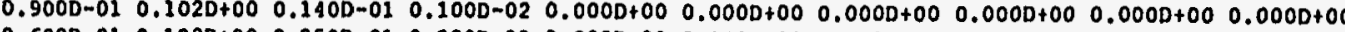

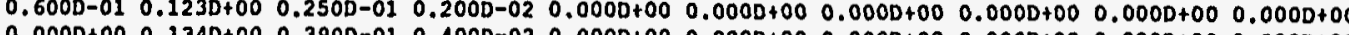

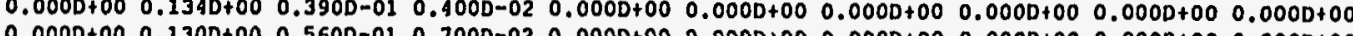

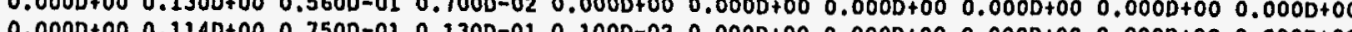

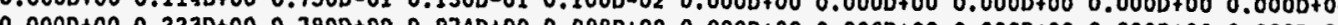
$0.91200+00$

$\begin{array}{lllll}0.30000-01 & 0.13000-01 \quad 0.20000-02\end{array}$

(-.- nfg (Number of Functional Groups! (1) 1 = CO2XL < -- \o, a, b, slgmaa, and xeft for tg a = H2OL - yo, $a, b$, sigmaa, and xeff for fg $5=1120$ yo, $a, b$, slgmaa, and xett for tg: $6=$ COL $<--y 0, a, b$, slomaa, and xeff tor tg $B$ - HCNE -- yo, $a, b, s l g m a a$, and xeff for fo $9=\mathrm{HCNT}$ - yo, a, b, slgmaa, and xeff for fg:10-NH3 --- yo, a, b, sigmaa, and xeff for tg $12=\mathrm{CH} \times \mathrm{CL}$ s-yo, $a, b$, slgmad, and xeff for eg $13=\mathrm{CHAL}$ <- yo, a, b, slgma, and xeft for tg $14=$ CH4T -- yo, a, b, slgmaa, and xeff for $t g$ I5 = HAROM yo, $a, b$, slgmaa, and xeft for fg: 17 - Coxt yo. a, b. slgmaa, and xeft for $2 \mathrm{~g} 18=\mathrm{CNV}$ - $a, b$, slgmaa, and xelf for fg 19 - SORG -- yo, a, b, slgmaa, and xeff for fg $21=$ C2H4 (a) 22 = c3lic (a)

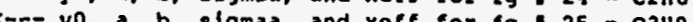

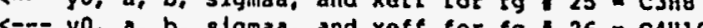
and and b, and slgma tor bond klnotlcs oft,ptotal, qtotal, slgmal, 31 gma2 <-- pdist $1,1: 101$ <-- pdist $3,1: 10)$ <-- pdist $4,1: 10)$ <-- pdstst $5,1: 10)$ (-)- pdist $6,1: 10)$ <-- pdist $7,1: 10)$ <-- pdist $B, 1: 10)$ <-.. pdiste $9,1: 101$ <-- pdist $(10,1: 10)$ <-- pdist $(11,1: 10)$ $<--$ pdist $(22,1: 10)$ <--- pdist $(13,1: 10)$ Flle generated: Tue Apr 14 18:29:33 1992 (--- particle internal pressure drop coalo.arg 8
con thaf 


\section{APPENDIX B}

\section{FG-SET DEVOLATILIZATION SUBMODEL}

\section{FG-SET Submodel Basis}

The FG-SET devolatilization submodel is similar to FG-DVC submodel, which was presented in Chapter 4. In FG-SET, the evolution of light gases is based also on the functional groups. The major difference is in the computation of tar evolution, which is based on a quantity called potential tar-forming fraction, denoted by $x^{0}$; it represents the maximum possible tar yield. In the FG-SET formulation, the potential tar-forming fraction is calculated from the empirical correlation proposed by Ko et al., (1980). A provision is also provided for the user to specify the value of $\mathrm{x}^{0}$ as input. Another difference between these two models is in the evaluation of rate coefficients. The FG-SET submodel also uses a distributed activation energy model for the calculation of rate coefficients but keeps the limits of normal distribution constant throughout the devolatilization process.

Figure B.1 shows a two-dimensional representation of this submodel. The dry, ash-free coal can be divided into various chemical functional groups, as shown in Figure B.1A. The $\mathrm{X}$ and $\mathrm{Y}$ dimensions represent the chemical functional group components (Solomon and Hamblen, 1985). The $Y$ dimension is divided into 
FG-SET Devolatilization Submodel

A) Functional Group Composition of Coal

B) Initial Stage of Decomposition

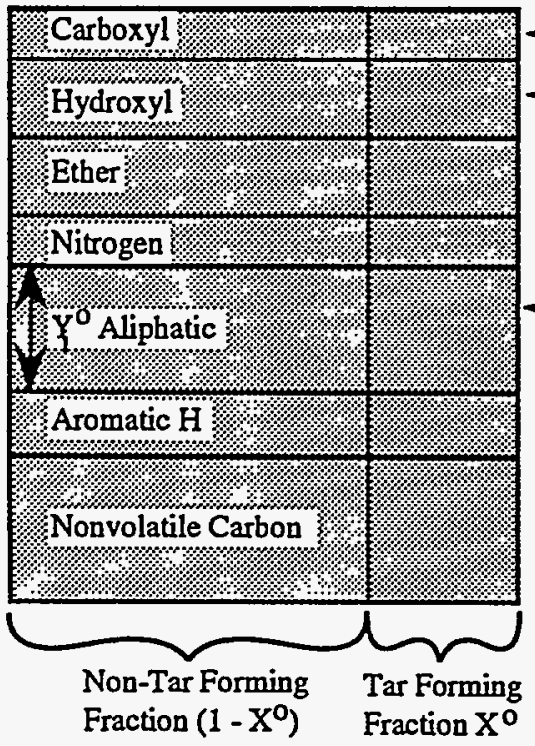

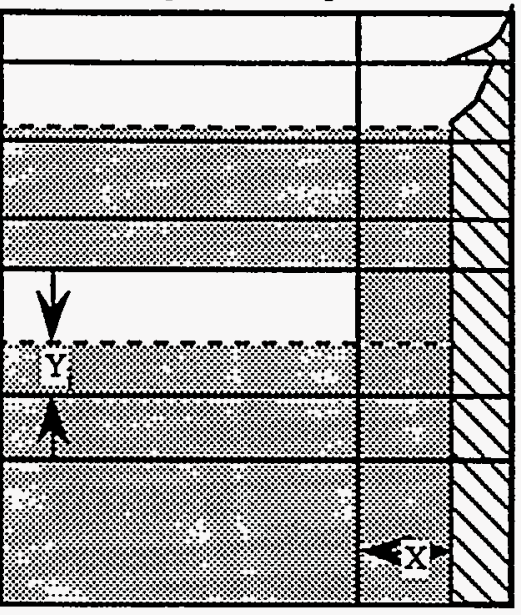

C) Later Stage of Decomposition

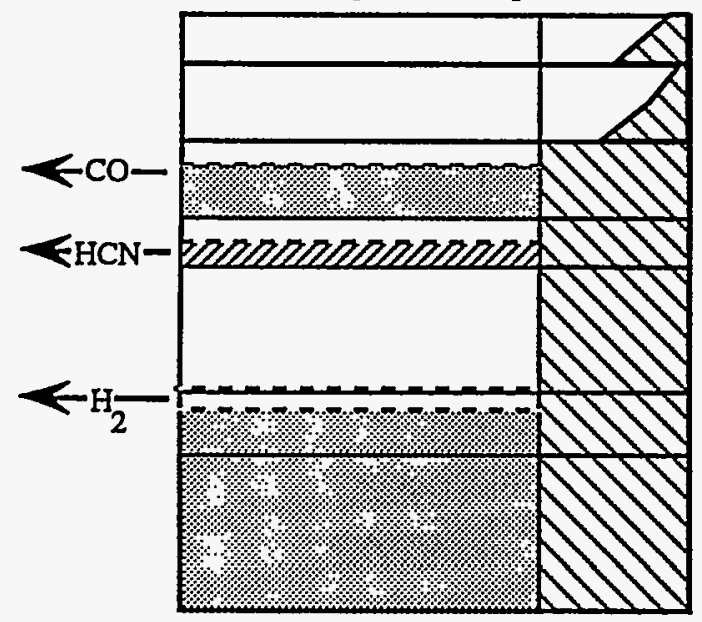

D) Completion of Decomposition

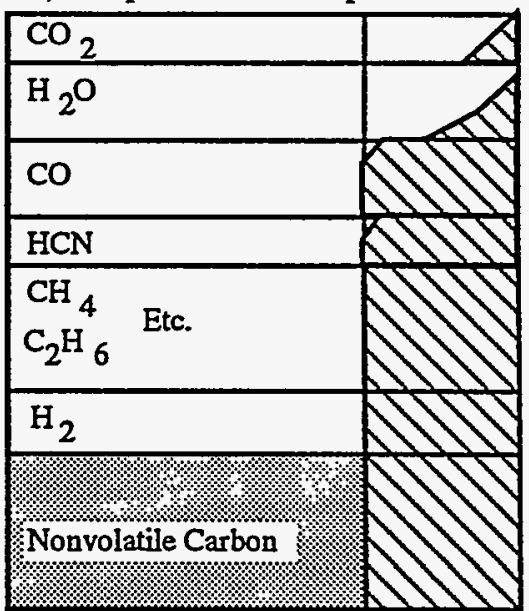

Figure B.1. Progress of thermal decomposition according to the functional group model: A) functional group composition of coal, B) initial state of decomposition, C) later stage of decomposition, D) completion of decomposition (after Solomon and Hamblen, 1985). 
fractions according to the chemical composition of the coal. The $X$ dimension represents non-tar-forming char, tar-forming char, i.e., instantaneous tar forming fraction $x$, and tar. The initial fraction of a particular functional group is represented by $y_{i}^{o}$, and the sum of $y_{i}^{o}$ s equals 1 . The initial functional group composition, $y_{i}^{o}$, for the formulation using nineteen functional groups, and the potential tar-forming fraction, $x^{o}$, for eight coals are given in Table B.1. The kinetic rate parameters can be either coal dependent or coal independent. The coal independent Arrhenius rate parameters for each functional group are also given in Table B.1 (from Solomon et al., 1988).

The evolution of each functional group into gas is represented by first order decay. The evolution of tar is also described by the first order decay of the dimension $\mathrm{X}$, i.e., the tar forming fraction. It is assumed that light gases do not evolve from the tar functional groups at the solid temperature, i.e., within the solid (Solomon and Hamblen, 1985), but decompose to form light gases in the gas phase at gas temperature. The Arrhenius rate coefficients are determined using a distributed activation energy model. The differential equations for the FG-SET devolatilization submodel are, therefore, summarized as follows:

$$
\begin{gathered}
\frac{d W_{g a s, i}}{d t}=k_{i}\left(T_{s}\right)\left(1-x^{0}+x\right) y_{i}+k_{i}\left(T_{g}\right) W_{\text {Tar, } i} \\
\frac{d W_{\text {Tar, },}}{d t}=k_{x}\left(T_{s}\right) W_{\text {Tar, }, i}-k_{i}\left(T_{g}\right) W_{\text {Tar, },} \\
\frac{d W_{\text {char }, i}}{d t}=-k_{i}\left(T_{s}\right)\left(1-x^{0}+x\right) y_{i}-k_{x}\left(T_{s}\right) W_{\text {Tar, } i} \\
\frac{d x}{d t}=-k_{x}\left(T_{s}\right) x
\end{gathered}
$$


Appendix B

\section{Arrhenius Rate Constants}

The rate constants for the evolution of char functional groups and tarforming fraction are determined by using a distributed activation energy model. According to this model, coal is viewed as a composite material consisting of a large number of components which decompose at different rates. Following Anthony et al., (1974), a multiple reactions model for each functional group is used to determine the Arrhenius rate constants. Here it is assumed that the total volatile mass production from the $i$-th functional group is normally distributed with respect to activation energy in the same manner as the total potential volatile mass production at large times. The resulting equation is

$$
k_{i}=\int_{-\infty}^{+\infty} k_{i}^{o} \exp \left(\frac{-E}{R T}\right) \frac{\exp \left[-\frac{1}{2}\left(\frac{E-\bar{E}_{i}}{\sigma_{i}}\right)\right]}{\sigma_{i} \sqrt{2 \pi}} d E_{i}
$$

Equation B.5 is solved using a seven-point Gaussian-Legendre quadrature (Abramowitz and Stegun, 1972). The derivation of the distributed Arrhenius rate expression is discussed by Hobbs (1990). In the FG-SET submodel, options are provided to use either rank dependent or rank independent kinetic data. For the rank independent option, the frequency factors, $k_{i}^{o}$, mean activation energies, $\bar{E}_{i}$, and standard deviations of activation energies, $\sigma_{i}$, are given in Table B.1; they were obtained from Solomon et al., (1988).

\section{Potential Tar-Forming Fraction}

The potential tar-forming fraction, $x^{0}$, represents the maximum possible tar yield. However, competition from light gas evolution prevents maximum tar yield from being realized, as shown in Eqns. B.1 and B.2. The potential tar-forming 
FG-SET Devolatilization Submodel

fraction can be adjusted to match experimentally determined tar yields, estimated from correlated experimental data (Khan, 1989, Ko et al., 1988 and Serio et al., 1987), predicted from simple ultimate yield models (Kobayashi et al., 1977), or predicted from coal-structure-dependent models (Solomon et al., 1988). The values for the potential tar-forming fraction can be chosen to match experimental tar yield data. The tar recirculated back to the oxidation and gasification zone can also be specified to match the reported tar recirculation rates. If tar yield data are not available, then the correlation of Ko et al., (1988) is recommended.

For comparison, the potential tar-forming fractions used in pulverized coal applications (Serio et al., 1987), experimental values obtained from TGA analysis, and calculated values at 1 and 25 atmospheres pressure from the semiempirical correlation of Ko et al., (1988) are given in Table B.1. The potential tar-forming fraction was calculated from the following equations (Ko et al., 1988):

$$
\begin{gathered}
x^{0}=0.01\left[\alpha+\beta x_{\text {tar }}\right] \\
x_{\text {tar }}=\frac{(\text { number of labile bridges })(\text { abstractable hydrogen })}{\text { (number of crosslinked bridges) }} \\
\text { number of labile bridges }=\left[\frac{\left(1-f_{a}\right)\left(100 \Omega_{c}\right)}{12}\right]^{1.8} \\
\text { abstractable hydrogen }=100\left[\left(\frac{\Omega_{H}}{1}\right)-\left(\frac{\Omega_{O H}}{17}\right)\right] \\
\text { number of crosslinked bridges }=\left\{\begin{array}{l}
100\left(\frac{\Omega_{O}}{16}+\frac{\Omega_{S}}{32}\right) \Omega_{O}>0.035 \\
100\left(\frac{0.035}{16}+\frac{\Omega_{S}}{32}\right) \Omega_{O}>0.035
\end{array}\right. \\
f_{a}=0.830526-2.008147 \Omega_{c}+2.241218 \Omega_{c}^{2}
\end{gathered}
$$




$$
\begin{gathered}
\Omega_{O H}=0.332-0.35 \Omega_{c} \\
\alpha= \begin{cases}2 & x_{\text {tar }}<15 \\
\frac{1}{\left(0.021533+0.028651 L_{p}\right)}-36 & 15 \leq x_{\text {tar }} \leq \\
11.24071+9.743707 L_{p}-0.91326 L_{p}^{2} & x_{\text {tar }}>31\end{cases} \\
\beta= \begin{cases}0 & x_{\text {tar }}<15 \\
0.0508030+0.696487 L_{p}-0.06959 L_{p}^{2} & 15 \leq x_{\text {tar }} \leq 31 \\
0 & x_{\text {tar }}>31\end{cases} \\
L_{p}= \begin{cases}-\log _{10} P+1 & P \leq 2.5 \mathrm{MPa} \\
2.5 & P>2.5 \mathrm{MPa} a\end{cases}
\end{gathered}
$$

where $\Omega_{i}$ represents the dry, mineral-matter-free weight fraction of the $i$-th component determined from the ultimate analysis, $f_{a}$ represents the aromaticity of the coal, and $\Omega_{O H}$ represents the dry, mineral-matter-free hydroxyl group fraction. This semiempirical correlation has been used to correlate tar yields over pressures ranging from vacuum to $9 \mathrm{Mpa}$ (about 90 atmospheres) and 37 coals ranging from lignites to anthracites, (Ko et al., 1988).

The potential tar-forming fractions determined for atmospheric, pulverized coal applications, TGA applications, and estimations based on the semiempirical tar model (SET model) of Ko et al. (1988) at 1 and 25 atmospheres are shown in Table B.1. The potential tar-forming fraction is smaller at high pressure due to mass transport effects, which are modeled empirically with the SET model. Also, the potential tar-forming fractions obtained from TGA experiments are smaller than the values using the SET model at atmospheric pressure. Mass transport through the coal bed, as well as secondary reactions with the hot char, may explain the 
FG-SET Devolatilization Submodel

difference. The potential tar-forming fractions determined for pulverized coal applications are closer to the SET results at atmospheric pressure than to the SET results at high pressure or to the TGA results. Yet the values determined for pulverized coal applications are still higher than the atmospheric SET results. The largest difference between the potential tar-forming fraction determined for pulverized coal applications and the atmospheric SET predictions was for the N. D. Zap lignite, which differs by a factor of 2.7. The Wyodak subbituminous case was in good agreement. However, all values were considered inadequate for predicting tar yield for fixed-bed gasifiers. Quantitative comparisons are discussed further by Hobbs (1990).

The overall rates of evolution of light gases and tar from coal can then be determined by

$$
r_{i}= \pm \rho_{s m}^{o}\left(1-\varepsilon^{o}\right)\left(1-\Omega_{\text {ash }}^{o}-\Omega_{\text {moisture }}^{o}\right) \frac{d \omega_{i,(\text { char, tar, or gas })}}{d t}
$$

where $\rho_{s m}^{o}$ is measured apparent density of the feed coal, $\varepsilon^{o}$ is the bed void fraction of the gasifier top, $\Omega_{a s h}^{o}$ and $\Omega_{\text {moisture }}^{o}$ are the proximate ash and moisture fractions of the feed coal, and $\omega_{i \text {, (char, tar, or gas) }}$ is the weight fraction of the $i$-th functional group in the char, tar, or gas. The time derivatives in Equation (B.16) are calculated by assuming that light volatile gases do not evolve from the gaseous tar:

$$
\begin{gathered}
\frac{d \omega_{i, g a s}}{d t}=\left(1-x^{0}+x\right) k_{i} y_{i} \text { and } \frac{d \omega_{i, \text { tar }}}{d t}=k_{x} x y_{i} \\
\frac{d \omega_{i, \text { char }}}{d t}=-\frac{d \omega_{i, \text { gas }}}{d t}-\frac{d \omega_{i, \text { tar }}}{d t}
\end{gathered}
$$

$\omega_{i, g a s}, \omega_{i, t a r}$, and $\omega_{i, \text { char }}$ represent the fractional amounts of a particular functional group that have evolved as light gas, tar or is remaining in the solid. 
Appendix B

The volumetric devolatilization rate expressions $r_{i}^{d}\left(\mathrm{~kg}_{i} / \mathrm{m}^{3} \mathrm{~s}\right)$ can be obtained by combining Equation B.17 and B.18 and by the use of the chain rule

$$
\begin{gathered}
r_{i}^{d}=\rho_{s m}^{o}\left(1-\varepsilon^{o}\right)\left(1-\Omega_{a s h}^{o}-\Omega_{\text {moisture }}^{o} \frac{d \omega_{i, c h a r}}{d t}\right. \\
\frac{d \omega_{i, c h a r}}{d t}=\frac{d\left(1-x^{o}+x\right) y_{i}}{d t}=\left(1-x^{o}+x\right) \frac{d y_{i}}{d t}+y_{i} \frac{d x}{d t} \\
\frac{d \omega_{i, c h a r}}{d t}=\left(1-x^{o}+x\right)\left(-k_{i} y_{i}\right)+y_{i}\left(-k_{x} x\right) \\
r_{i}^{d}=\rho_{s m}^{o}\left(1-\varepsilon^{o}\right)\left(1-\Omega_{a s h}^{o}-\Omega_{\text {moisture }}^{o}\right)\left[\left(1-x^{o}+x\right)\left(k_{i} y_{i}\right)+y_{i}\left(k_{x} x\right)\right]
\end{gathered}
$$

The volumetric devolatilization rate expressions $r_{i}^{d}\left(\mathrm{~kg}_{i} / \mathrm{m}^{3} \mathrm{~s}\right)$ include mass loss due to both light gas evolution and tar evolution which is required by the continuity equations listed in Table 3.2. However, the tar continuity equations require the tar volumetric rate equations $r_{i, t a r}^{d}\left(k g_{i} / m^{3} s\right)$, which are obtained similarly:

$$
r_{i, t a r}^{d}=\rho_{s m}^{o}\left(1-\varepsilon^{o}\right)\left(1-\Omega_{a s h}^{o}-\Omega_{m o i s t u r e}^{o}\right) k_{x} x y_{i}
$$

Coupling between mass transport of oxidation and gasification products and devolatilization complicates the mathematical description of both devolatilization and char oxidation. The devolatilization rate expressions given by the above equations do not include resistance due to mass transport. Saxena (1990) concluded that heat and mass transport limitations in large coal particles are significant. In addition, simulations with purely kinetic devolatilization equations as formulated above result in unrealistically fast product evolution and extremely steep temperature gradient, indicating the importance of the transport effects in large coal particles. To account for the mass transport effects, resistances through the film and particle are added to 
the Arrhenius rate expression as diffusional resistances, as shown by the following expression:

$$
k_{i, x}^{\text {effective }}=\left(\frac{1}{k_{i, x}}+\frac{1}{k_{m}}+\frac{1}{k_{\text {eff }}}\right)^{-1}
$$

where $k_{i, x}^{\text {effective }}, k_{i, x}, k_{m}$ and $k_{\text {eff }}$ represent the effective devolatilization rate constant, the distributed Arrhenius rate constant for devolatilization, the film mass transport coefficient, and the effective mass transport coefficient for the particle. The resistance through the particle is a function of the particle burnout and is negligible at the beginning of devolatilization. The mass transfer resistances used herein for devolatilization are identical to the mass transfer resistances used in the oxidation and gasification submodel, as discussed in chapter 3.

Diffusional mass transport may not be an appropriate mode of transport for devolatilization. Jets of volatile gases suggest that convection may dominate mass transport for small particle devolatilization. However, diffusion may be important for devolatilization of large particles at typical heating rates found in fixed-bed reactors. Further research is needed to address mass transport during large particle devolatilization. Internal particle temperature gradients may be significant during devolatilization; but experimental evidence using thermocouples embedded into large coal particles during devolatilization indicates that thermal equilibrium is reached rapidly (Nuttall, 1979). 


\section{APPENDIX C}

\section{PREDICTION OF EFFLUENT \\ COMPOSITIONS FOR FIXED-BED \\ COAL GASIFIERS}




\title{
Prediction of effluent compositions for fixed-bed coal gasifiers
}

\author{
Michael L. Hobbs*, Predrag T. Radulovic and L. Douglas Smoot \\ Advanced Combustion Engineering Research Center, Brigham Young University, Provo, \\ UT 84602, USA
}

(Received 12 July 1991; revised 24 January 1992)

\begin{abstract}
A two-zone, well-mixed, partial equilibrium model that provides efficient estimates of fixed bed coal gasifier effluent temperature, tar production and gas composition has been developed and evaluated. The model includes detailed treatment of devolatilization, partial equilibrium of volatile gases, treatment of a large number of gas-phase species, and estimation of tar production with potential for recirculation of effluent products. Predictions have been compared to measured effluent compositions from two large-scale fixed-bed reactors over a wide range of coal types and operating conditions. Model evaluation also includes determination of sensitivity of effiuent temperature and composition to model options, model parameters and operational parameters. The model can be used separately or as part of a large process simulator since execution times are on the order of seconds using an engineering workstation. Furthermore, the predicted effluent compositions can provide useful initial estimates for one- or two-dimensional fixed-bed models.
\end{abstract}

(Keywords: coal; effiluent; gasification)

A schematic diagram of a large-scale, fixed-bed gasifier is shown in Figure 1. Coal is fed to the top of the reactor and moves downward under gravity, countercurrent to the rising gas stream. The dry ash is removed at the bottom of the reactor. The feed gas is cumposed of air or oxygen and steam. Excess steam is supplied to the gasifier to control the ash temperature. Figure 1 also shows the reactor divided into four zones: (1) drying; (2) devolatilization; (3) gasification; and (4) combustion. As the coal slowly descends, the hot gases produced in the gasification and combustion zones exchange energy with the cooler solid fuel. Water and volatile matter are released when the solid reaches a sufficiently high temperature. After drying and devolatilization, the char enters the gasification zone where carbon reacts with steam, carbon dioxide and hydrogen. Endothermic reactions in this zone produce carbon monoxide and hydrogen. The slightly exothermic reaction of hydrogen with carbon produces methane. Differentiation between the gasification zone and the combustion zone is based on the presence or absence of free oxygen. Heterogeneous combustion and gasification reactions can occur simultaneously in the combustion zone. Combustible gases such as carbon monoxide or hydrogen react with gaseous oxygen. Solid residence time in the drying, gasification and oxidation zones may be on the order of several hours. Residence time in the ash layer may be even longer depending on the thickness of this zone. Gas residence times are on the order of seconds.

Gumz $z^{1}$ developed an equilibrium model for fixed-bed reactors. The effects of devolatilization, drying and sensible heat losses were not included in his model. Woodmansee ${ }^{2}$ extended Gumz's equilibrium model to

- Current address: Sandia National Laboratories, PO Box 5800. Division 1512, Albuquerque, NM 87185, USA include sensible heat losses. Kosky and Floess ${ }^{3}$ added devolatilization to the equilibrium model by assuming instantaneous devolatilization with yield based on proximate analysis and volatile composition based on the gas analysis of Loison and Chauvin ${ }^{4}$. Effluent temperature was calculated using overall energy balances. Compositions were calculated assuming water-gas shift equilibrium. Smoot and $\mathrm{Smith}^{\mathbf{5}}$ and more recently, Radulovic et al. ${ }^{6}$ have reviewed fixed-bed models.

No major advancement has been made in predicting effluent temperatures and compositions in fixed-bed gasification in recent years. Even one- and twodimensional, fixed-bed coal gasification models have failed to accurately predict effluent propertics. An assessment of the fixed-bed models of Amundson and Arri $^{7}$. Yoon et al. ${ }^{8}$, Desai and Wen ${ }^{9}$, Stillman ${ }^{10}$, Cho and Joseph ${ }^{11}, \mathrm{Yu}$ and Denn ${ }^{12}$, Earl and Islam ${ }^{13}$, Thorsness and Kang ${ }^{14}$, and Bhattacharya et al. ${ }^{15}$ indicates common assumptions such as axially uniform gas/solid phase plug flow, uniform porosity, instantaneous devolatilization (with volatile yield from proximate analysis and compositions assumed to be constant), char oxidation parameters from small particle data, and little or no gas-phase chemistry. Recently, Hobbs ${ }^{16}$ has relaxed most of these assumptions in a steady, one-dimensional model, while emphasizing the importance of devolatilization and gas-phase chemistry in the fixed-bed gasification processes.

An efficient two-zone, partial equilibrium model has been developed and is presented in this paper. The two-zone, fixed-bed model uses a coal-independent devolatilization submodel and a partial equilibrium gas-phase submodel to predict effluent temperatures and compositions. Effluent compositions can be determined for any number of species. Tar production can also be 
Prediction of effluent compositions: M. L. Hobbs et al.

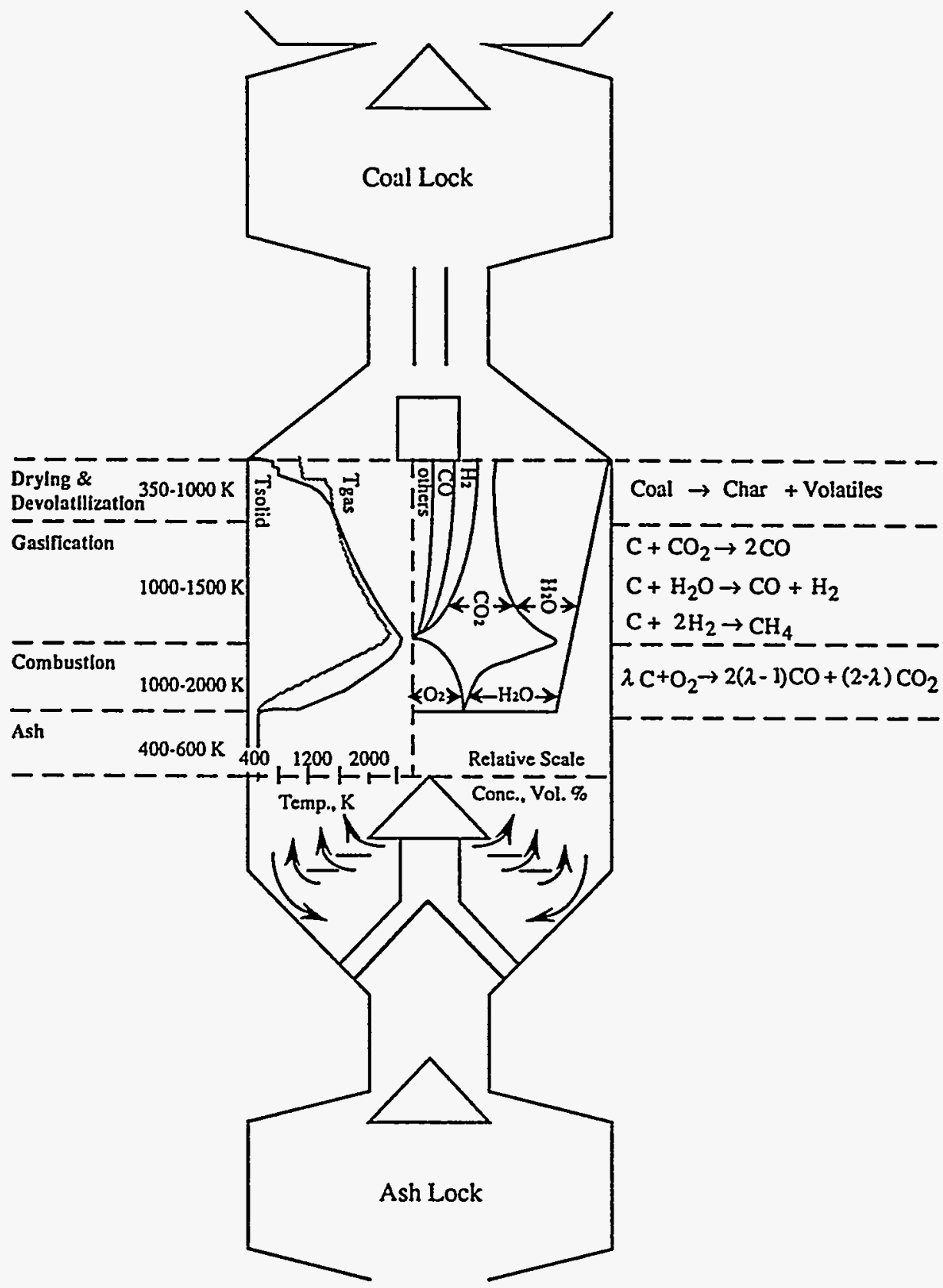

Figure 1 Schematic of large-scale, high pressure countercurrent fixed-bed gasifier. Temperature and concentration profiles are for illustration only

estimated and tar recycle can be taken into account. In many engineering applications, only effluent temperatures and compositions are of interest. For example, only effluent information is required from the gasifier in a comprehensive process simulation used for optimization of the overall coal conversion process. Also, since simulation of the gasifier is often only a small part of complete process calculations, computational time must be kept to a minimum. In such cases, the two-zone, partial equilibrium model is preferred to more complex and computationally demanding one- or two-dimensional models. Furthermore, this two-zone, partial equilibrium model can provide initial estimates for one- or two-dimensional models. Reliable initial estimates of fixed-bed reactor effluent temperatures and compositions have been found essential for solving the highly non-linear, fixed-bed equation set ${ }^{16}$.

TWO-ZONE PARTIAL EQUILIBRIUM MODEL

\section{Foundations and assumptions}

Typical temperature and concentration profiles in a large-scale, fixed-bed gasifier are illustrated in Figure 1 . Oxidation and gasification occur at relatively high temperatures compared to the cooler devolatilization zone. The temperature difference provides a natural division of the process into two zones. Higher temperatures in the oxidation/gasification section favour total equilibrium in the gas phase. Lower temperature 
and short gas residence time in the devolatilization zone favour partial equilibrium.

The primary assumption for the two-zone, partial equilibrium model is that the oxidation/gasification zone and drying/devolatilization zone can be assigned different uniform temperatures, $T_{\text {equilibrium }}$ and $T_{\text {exit, }}$ respectively. All gases are assumed to be in thermal equilibrium. Furthermore. the gases produced in the higher temperature oxidation/gasification zone are assumed to be in chemical equilibrium in this zone, but non-reactive in the lower temperature devolatilization/ drying zone. The coal volatiles and water vapour produced in the lower temperature devolatilization zone are also assumed to be non-reactive in this zone. Coals are assumed to be composed of various percentages of chemical functional groups ${ }^{17}$. Rcuctor gcometry, operational parameters, heat loss or overall heat transfer coefficient, coal properties, coal burnout and recycle tar fraction must be specified.

\section{Energy balance}

Control volumes for three equilibrium model options are shown in Figure 2. The one-zone models shown in Figures $2 a$ and $2 b$ are included for comparison and show the evolution of the two-zone model. The energy balance around any control volume shown in Figure 2 is:

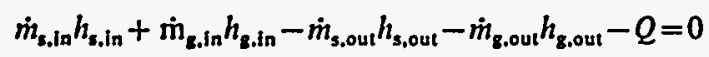

where $\dot{m}$ is mass flow rate $\left(\mathrm{kg} \mathrm{s}^{-1}\right), h$ is total enthalpy $\left(\mathrm{J} \mathrm{kg}^{-1}\right)$ and $Q$ is heat loss $\left(\mathrm{J} \mathrm{s}^{-1}\right)$; the subscripts $\mathrm{s}, \mathrm{g}$, in and out refer to solid, gas, entering control volume and exiting control volume, respectively. The total equilibrium model shown in Figure $2 a$ assumes complete reaction of the dry ash free coal by either devolatilization, gasification or oxidation. The total equilibrium model does not differentiate among mechanisms for these chemical processes as do the one- and two-zone partial equilibrium models. The only difference between the one- and two-zone partial equilibrium models relates to devolatilization and drying. The one-zone model assumes that drying and devolatilization occur in the same zone as oxidation and gasification. This assumption is relaxed in the two-zone partial equilibrium model of Figure $2 c$. Equation (1) can be solved by iteration as discussed in detail by $\mathrm{Hobbs}^{16}$. Computation time for the two-zone partial equilibrium model is less than $1 \mathrm{~s}$ on an engineering workstation.

The total enthalpy is composed of the enthalpy of formation and the sensible enthalpy:

$$
h=h_{i}^{\circ}+h^{\mathbf{s}}
$$

where the superscript o refers to the reference temperature $(298.15 \mathrm{~K})$, and superscript $\mathrm{s}$ to sensible enthalpy from the reference temperature to the stream temperature. The heat of formation of coal, $h_{f, c}^{\circ}$, is based on the following coal reaction:

$$
\begin{aligned}
\sim_{n} \mathrm{H}_{m} \mathrm{O}_{h} \mathrm{~S}_{j} \mathrm{~N}_{k}+ & \left(n+\frac{m}{4}-\frac{h}{2}+j\right) \mathrm{O}_{2} \rightarrow \\
& n \mathrm{CO}_{2}+\frac{m}{2} \mathrm{H}_{2} \mathrm{O}+\frac{k}{2} \mathrm{~N}_{2}+j \mathrm{SO}_{2}
\end{aligned}
$$

The heat of formation of the coal can be obtained from the higher heating value of the coal $\left(\mathrm{HHV}, \mathrm{J} \mathrm{kg}^{-1}\right)$, which can be measured or estimated from the ultimate analysis with Dulong's formula ${ }^{18}$. The higher heating value is the negative of the heat of reaction (i.e. $H H V=-\Delta h_{\text {teaction }}$ ). Ash effects on the coal heat of formation are included via the experimentally determined $H H V$. The heat of formation of the coal is determined as the sum of the heats of formation of the products and the higher heating value of the coal:

$$
h_{\mathrm{f}, \mathrm{c}}^{0}=\sum_{i=1}^{4} v_{i} h_{\mathrm{f}, \mathrm{i}}^{0}+H H V
$$
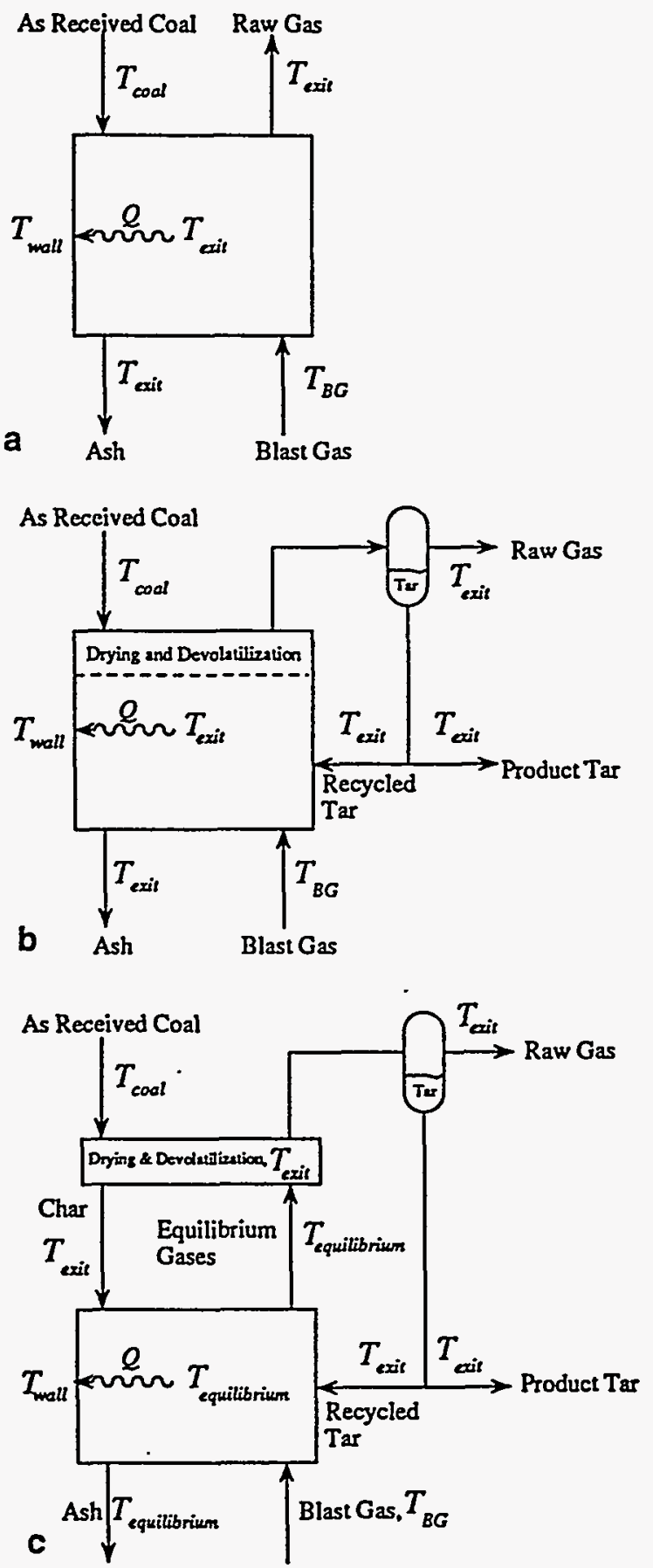

Figure 2 Control volume for (a) one-zone total equilibrium model; (b) one-zone partial equilibrium model; (c) two-zone partial equilibrium mode! 
wherc $v_{\text {i }}$ represents the stoichiometric coefficient for the $i$ th pr.)duct given in Equation (3) and $i$ represents the formation of $\mathrm{CO}_{2}, \mathrm{H}_{2} \mathrm{O}, \mathrm{N}_{2}$ and $\mathrm{SO}_{2}$. Error results in calculiting the heat of formation of coal, which is a relatiscly small number, as the difference of two much larger numbers of limited accuracy ${ }^{19}$. Fortunately, the enthalpy of the feed coal is small in comparison to the feed gis enthalpy for dry-ash, fixed-bed gasifiers and has little influence on the equilibrium calculations.

The sensible enthalpy for the feed coal, $h_{\mathrm{c}}^{\mathrm{s}}\left(\mathrm{J} \mathrm{g}^{-1}\right)$, can be determined using Merrick's enthalpy correlation evaluated at $298.15 \mathrm{~K}$ and the coal feed temperature ${ }^{20}$.

$$
\begin{aligned}
& h_{\mathrm{c}}^{s}=\frac{R}{a}\left[380 g_{0}\left(\frac{380}{T}\right)+3600 g_{0}\left(\frac{1800}{T}\right)\right] \\
& g_{0}(z)=\frac{1}{\exp (z)-1}
\end{aligned}
$$

where $R$ is the gas constant ( $8314.4 \mathrm{~J} \mathrm{kmol}^{-1} \mathrm{~K}^{-1}$ ), $T$ is solid temperature (K), 380 and 1800 are characteristic Einstein temperatures and $z$ is a dummy variable representing the ratio of the Einstein temperature and $T$. The mean atomic weight, $a$, may be defined as:

$$
\frac{1}{a}=\sum_{i=1}^{5} \frac{\Omega_{i}}{\mu_{i}}
$$

where $\mu_{l}$ and $\Omega_{l}$ represent the atomic weight and the dry ash free mass fraction, respectively, of carbon, hydrogen, oxygen, nitrogen and sulphur.

The specific heat of the ash, $C p_{\mathrm{a}}\left(\mathrm{J} \mathrm{kg}^{-1} \mathrm{~K}^{-1}\right)$, can be determined from ${ }^{20}$ :

$$
C p_{\mathrm{a}}=754+0.586 t
$$

where $t$ is the ash temperature $\left({ }^{\circ} \mathrm{C}\right)$. The KoppNeumann rule also gives reliable estimates of the ash heat capacity if the various constituents of the ash are known ${ }^{21}$. The heats of formation and sensible enthalpies for the feed gases are found with a generalized chemical equilibrium $\operatorname{code}^{22}$ which uses polynomial fits of data in the JANAF thermochemical tables ${ }^{23}$.

The final term to define in Equation (1) is the heat loss through the reactor wall, $Q(\mathrm{~W})$. This quantity can be calculated if the jacket steam and cooling water flow rates and temperatures are known, or by the following equation if the overall heat transfer coefficient, $U$ (W $\mathrm{m}^{-2} \mathrm{~K}^{-1}$ ), is known:

$$
Q=U A\left(T_{e}-T_{w}\right)
$$

where $A$ is the water wall surface area and $T_{c}$ and $T_{w}$ represent the well mixed exit temperature and the wall temperature, respectively. Values for the overall heat transfer coefficient, $U$, range from 50 to $200 \mathrm{~W} \mathrm{~m}^{-2} \mathrm{~K}^{-1}$ which is in the forced convection regime. Calculations herein use measured heat loss based on jacket steam and cooling water flow rates.

\section{Gas-phase chemistry}

Partial equilibrium exists when certain reactions or species are in chemical equilibrium while other species concentrations are determined by other means such as no reaction, sometimes termed 'frozen'. In other words, partial equilibrium for the mixture is assumed if any species is considered to be out of equilibrium. The species concentration may be found by assuming full equilibrium, by using heterogeneous kinetics (devolatilization, gasification or oxidation), by using homogeneous gas-phase kinetics, or by 'freezing' the species to disallow further reaction. Most fixed-bed models in the literature have assumed the major gaseous species $\left(\mathrm{CO}, \mathrm{CO}_{2}, \mathrm{H}_{2}\right.$ and $\mathrm{H}_{2} \mathrm{O}$ ) to be in equilibrium, by the water-gas shift reaction, while neglecting all minor species.

The approach taken herein is to allow major species to be in partial equilibrium or full equilibrium depending on the zone. Minor species are not neglected, but taken to be in equilibrium. Gas temperature is determined by assuming all gas species to be in thermal equilibrium even though chemical equilibrium may not exist. The equilibrium composition and temperature is determined by Gibbs's free energy minimization ${ }^{22}$, given total gas-phase static enthalpy, pressure and atomic composition. No account is made for secondary homogeneous chemistry, since little is yet known on this subject.

The gases produced in the hot oxidation/gasification zone are assumed to be in complete equilibrium at the oxidation/gasification zone temperature. However, when these gases are cooled to the temperature of the drying/devolatilization zone, the oxidation/gasification zone gases are not allowed to react further. Also, the coal volatile gases and coal moisture produced in the cooler devolatilization/drying zone can be assumed to be non-reactive in the gas phase. In countercurrent, fixed-bed gasifiers, the assumptions regarding nonreacting species may be justified by the low temperature of the drying/devolatilization zone which prevents further reactions of the volatile gases and coal moisture with the gases produced in the oxidation/gasification zone. However, the two-zone model also has the option to allow all gases in the drying/devolatilization zone to react to equilibrium, or to allow all gases but tar to react to equilibrium.

\section{Devolatilization}

Basis. Obtaining accurate coal volatiles yield and composition is important since as much as $40-60 \%$ of the dry ash free mass can be lost by devolatilization. Further, devolatilization products are carried off the top of the reactor by the countercurrent gas flow shortly after their release from the coal. Thus, specification of gas product composition depends upon the devolatilization model. The primary difference between the total equilibrium model and the partial equilibrium models is the addition of yield computations for coal volatile matter. Devolatilization is assumed to take place instantaneously with the yield and composition equal to the ultimate volatile yield and composition. Large solid particle residence time, compared to the time required for complete devolatilization, justifies this assumption when estimating fixed-bed effluent compositions.

The ultimate volatile composition, including coal gas, tar and chemically formed water yields, is predicted with a functional group (FG) model ${ }^{17}$ and a semi-empirical $\operatorname{tar}$ (SET) formation model ${ }^{24}$. The devolatilization model uses coal-general devolatilization parameters. Also, the devolatilization submodel was used in a recently developed one-dimensional model ${ }^{16}$.

The devolatilization submodel also predicts the amount and composition of the ultimate char fraction. Dulong's formula is used to calculate the heating value of the char wtich can be used to calculate the char heat of formation. Merrick's correlations ${ }^{20}$ are used to 
Prediction of effluent compositions: M. L. Hobbs et al.

Table 1 Devolatilization kinetic parameters, functional group compositions, potential tar-forming fractions and ultimate analysis*

\begin{tabular}{|c|c|c|c|c|c|c|c|c|c|c|c|c|}
\hline \multirow{2}{*}{\multicolumn{2}{|c|}{$\begin{array}{l}\text { Functional groups } \\
\text { (daf) }\end{array}$}} & \multicolumn{3}{|c|}{ Kinetic parameters } & \multirow{2}{*}{$\begin{array}{l}\text { Gillette } \\
y_{i}^{i}\end{array}$} & \multirow{2}{*}{$\begin{array}{l}\text { IL no. } 6 \\
y_{i}^{\circ}\end{array}$} & \multirow{2}{*}{$\begin{array}{l}\text { KY no. } 9 \\
j_{i}^{\circ}\end{array}$} & \multirow{2}{*}{$\begin{array}{l}\text { ND Zap } \\
y_{i}^{\circ}\end{array}$} & \multirow{2}{*}{$\begin{array}{l}\text { Pilt no. } 8 \\
\text { li }^{\circ}\end{array}$} & \multirow{2}{*}{$\begin{array}{l}\text { Rose } \\
3^{\circ}\end{array}$} & \multirow{2}{*}{$\begin{array}{l}\text { Utah } \\
\text { ji }\end{array}$} & \multirow{2}{*}{$\begin{array}{l}\text { Wyodak } \\
y_{i}^{\circ}\end{array}$} \\
\hline & & $k_{i}^{0}\left(s^{-1}\right)$ & $E_{l} / R(K)$ & $\overline{\sigma_{1} / R(\mathrm{~K})}$ & & & & & & & & \\
\hline $\mathrm{CO}_{2}$ extra loose & & $0.81 \times 10^{13}$ & 22500 & 1500 & 0.018 & 0.022 & 0.000 & 0.065 & 0.000 & 0.035 & 0.003 & 0.028 \\
\hline $\mathrm{CO}_{2}$ loose & \multirow{2}{*}{.. } & $0.65 \times 10^{17}$ & 33850 & 1500 & 0.053 & 0.022 & 0.006 & 0.300 & 0.007 & 0.035 & 0.007 & 0.020 \\
\hline $\mathrm{CO}_{2}$ tight & & $0.11 \times 10^{16}$ & 38315 & 2000 & 0.028 & 0.030 & 0.005 & 0.005 & 0.005 & 0.030 & 0.017 & 0.021 \\
\hline $\mathrm{H}_{2} \mathrm{O}$ loose & & $0.22 \times 10^{19}$ & 30000 & 1500 & 0.031 & 0.045 & 0.011 & 0.061 & 0.012 & 0.051 & 0.025 & 0.030 \\
\hline $\mathrm{H}_{2} \mathrm{O}$ light & & $0.17 \times 10^{14}$ & 32700 & 1500 & 0.031 & 0.000 & 0.011 & 0.033 & 0.012 & 0.051 & 0.020 & 0.022 \\
\hline CO ether loose & & $0.14 \times 10^{19}$ & 40000 & 6000 & 0.080 & 0.060 & 0.050 & 0.060 & 0.050 & 0.055 & 0.025 & 0.050 \\
\hline CO ether tight & & $0.15 \times 10^{16}$ & 40500 & 1500 & 0.043 & 0.063 & 0.026 & 0.044 & 0.021 & 0.013 & 0.034 & 0.055 \\
\hline HCN loose & & $0.17 \times 10^{14}$ & 30000 & 1500 & 0.007 & 0.100 & 0.026 & 0.006 & 0.009 & 0.005 & 0.006 & 0.001 \\
\hline HCN tight & & $0.69 \times 10^{13}$ & 42500 & 4750 & 0.015 & 0.016 & 0.009 & 0.012 & 0.023 & 0.015 & 0.024 & 0.018 \\
\hline $\mathrm{NH}_{3}$ & & $0.12 \times 10^{13}$ & 27300 & 3000 & 0.000 & 0.000 & 0.000 & 0.001 & 0.000 & 0.001 & 0.000 & 0.001 \\
\hline $\mathrm{CH}_{\mathbf{x}}$ aliphatic & & $0.84 \times 10^{25}$ & 30000 & 1500 & 0.158 & 0.081 & 0.183 & 0.095 & 0.207 & 0.127 & 0.188 & 0.152 \\
\hline $\mathrm{CH}_{4}$ extra loose & & $0.84 \times 10^{15}$ & 30000 & 1500 & 0.000 & 0.011 & 0.020 & 0.000 & 0.000 & 0.000 & 0.000 & 0.000 \\
\hline $\mathrm{CH}_{a}$ loose & & $0.75 \times 10^{14}$ & 30000 & 2000 & 0.026 & 0.011 & 0.015 & 0.016 & 0.020 & 0.022 & 0.022 & 0.017 \\
\hline $\mathrm{CH}_{4}$ tight & & $0.34 \times 10^{12}$ & 30000 & 2000 & 0.017 & 0.022 & 0.015 & 0.009 & 0.015 & 0.012 & 0.022 & 0.008 \\
\hline $\mathrm{H}$ aromatic & & $0.10 \times 10^{15}$ & 40500 & 6000 & 0.012 & 0.016 & 0.012 & 0.017 & 0.013 & 0.013 & 0.017 & 0.013 \\
\hline $\mathrm{CH}_{3} \mathrm{OH}$ & & 0.00 & NA & NA & 0.000 & 0.000 & 0.000 & 0.000 & 0.000 & 0.000 & 0.000 & 0.000 \\
\hline CO extra tight & & $0.20 \times 10^{14}$ & 45500 & 1500 & 0.031 & 0.000 & 0.020 & 0.090 & 0.020 & 0.000 & 0.040 & 0.043 \\
\hline$C_{\text {non-volatile }}$ & & 0.00 & NA & NA & 0.440 & 0.550 & 0.562 & 0.440 & 0.562 & 0.520 & 0.548 & 0.518 \\
\hline S organic & & 0.00 & NA & NA & 0.005 & 0.038 & 0.024 & 0.011 & 0.024 & 0.012 & 0.003 & 0.004 \\
\hline Total, $\Sigma y_{i}^{*}$ & & NA & NA & NA & 1.000 & 1.000 & 1.000 & 1.000 & 1.000 & 1.000 & 1.000 & 1.000 \\
\hline Tar, $x^{\circ}$, entrained & & $0.85 \times 10^{15 b}$ & $27700^{b}$ & $1500^{3}$ & 0.200 & 0.430 & 0.430 & 0.160 & 0.430 & 0.160 & 0.430 & 0.160 \\
\hline Tar, $x^{\circ}$, TGA & & NA & NA & NA & NA & $\mathrm{NA}$ & NA & 0.040 & 0.110 & 0.060 & NA & NA \\
\hline Tar, $x^{*}$, SET $1 \mathrm{~atm}^{\bullet}$ & & NA & NA & NA & 0.071 & 0.116 & 0.270 & 0.043 & 0.270 & 0.098 & 0.270 & 0.160 \\
\hline Tar, $x^{\circ}$, SET $25 \mathrm{~atm}^{\circ}$ & & NA & NA & NA & 0.067 & 0.092 & 0.168 & 0.031 & 0.168 & 0.082 & 0.168 & 0.117 \\
\hline Coal type & & & & & Subbit. & Bit. & Bit. & Lig. & Bit. & Subbit. & Bit. & Subbit. \\
\hline Ultimate analysis (wt & $\%$ daf) & & & & 0770 & 0736 & 0817 & 0665 & 087 & 0774 & 0887 & 0750 \\
\hline H & & & & & 0.047 & $\begin{array}{l}0.130 \\
0.047\end{array}$ & 0.056 & $\begin{array}{l}0.002 \\
0.048\end{array}$ & $\begin{array}{l}0.821 \\
0.056\end{array}$ & $\begin{array}{l}0.124 \\
0.049\end{array}$ & 0.058 & 0.054 \\
\hline $\mathbf{N}$ & & & & & 0.012 & 0.014 & 0.019 & 0.011 & 0.017 & 0.012 & 0.016 & 0.011 \\
\hline $\mathbf{0}$ & & & & & 0.216 & 0.165 & 0.084 & 0.265 & 0.082 & 0.203 & 0.116 & 0.181 \\
\hline $\mathbf{S}$ (organic) & & & & & 0.005 & 0.038 & 0.024 & 0.011 & 0.024 & 0.012 & 0.003 & 0.004 \\
\hline
\end{tabular}

'NA, data not applicable or not available. Functional group data are from experimental measurements of Solomon and co-workers ${ }^{17.27 .34}$. Potential tar-forming fraction from small particle entrained-jlow experiments and TGA experiments are from Serio et al. ${ }^{27}$. The semi-empirical tar model of Ko et al. ${ }^{24}$ was used to determine tar-forming fractions at 1 and 25 atm $(0.1$ and $2.5 \mathrm{MPa}$, respectively)

Kinetic parameters $(k i, E / R$ and $\sigma / R)$ are for tar formation

calculate the sensible enthalpy of the char. The tar fraction recirculated back to the oxidation/gasification zone is specified. Any recirculated tar is assumed to react to equilibrium with the other gases in this zone. The gases produced in the oxidation/gasification zone, and the coal volatiles and water vapour produced in the devolatilization/drying zone, can be assumed to be either non-reactive or reactive in the devolatilization/drying zone.

Ultimate composition. Dry ash free coal can be divided into various chemical functional groups as discussed by Solomon and Hamblen ${ }^{25}$ and includes such species as carboxyl, hydroxyl, ether, etc. (see Table 1). The initial fraction of a particular functional group component is represented by $y_{i}^{\circ}$ and the sum of the $y_{i}^{\circ}$ values equals 1 . The fraction of a functional group that can potentially form tar is represented by $x^{\circ}$. The initial functional group components, $y_{i}^{\circ}$, and the potential tar-forming fraction, $x^{\circ}$, for eight coals are given in Table 1 . The coal-independent Arrhenius rate parameters for each functional group are also given in Table 1 .
The evolution of each functional group into the gas is represented by first-order decay, $(\mathrm{d} / \mathrm{d} t) y_{i}=-k_{i} y_{i}$. The evolution of tar is also described by first-order decay, $(d / d t) x=-k_{x} x$. Since the Arrhenius rate coefficients, $k_{i}$ and $k_{x}$, are constant under isothermal conditions, $y_{t}$ and $x$ for volatile functional groups can be determined by integration:

$$
y_{t}=y_{i}^{0} \exp \left(-k_{i} t\right) \text { and } x=x^{0} \exp \left(-k_{x} t\right)
$$

Non-volatile functional group fractions are constant, $y_{i}=y_{i}^{\circ}$.

The fractional amounts of a particular functional group component that remain in the solid phase as char, or evolve as tar or gas, $\omega_{i, e}, \omega_{t, l}$ and $\omega_{i, z}$ can be determined by assuming that light gases do not evolve from the tar vapour ${ }^{16,25}$ :

$$
\begin{aligned}
& \omega_{i, \mathrm{c}}=\left(1-x^{0}+x\right) y_{i} \\
& \omega_{i, \mathrm{l}}=\left(x^{0} y_{i}^{0}-x y_{i}\right) \frac{k_{x}}{k_{i}+k_{x}}
\end{aligned}
$$




$$
\omega_{i, 8}=\left(1-x^{0}\right)\left(y_{i}^{0}-y_{i}\right)+\omega_{i, 8}\left(\frac{k_{i}}{k_{x}}\right)
$$

The ultimate volatile composition can then be determined by taking the limit of Equations (10) to (12) as time becomes large and by accounting for the Arrhenius rate constants of non-volatile functional groups being zero, to give:

$\omega_{i, \mathrm{c}}^{\infty}=\left\{\begin{array}{cl}0 & \text { for volatile functional groups } \\ \left(1-x^{\circ}\right) y_{i}^{\circ} & \text { for non-volatile functional groups }\end{array}\right.$

$\omega_{i, 1}^{\infty}=\left\{\begin{array}{cc}x^{0} y_{i}^{0}\left(\frac{k_{x}}{k_{i}+k_{x}}\right) & \text { for volatile functional groups } \\ x^{0} y_{i}^{0} & \text { for non-volatile functional groups }\end{array}\right.$

$\omega_{i, 8}^{\infty}=\left\{\begin{array}{c}\left(1-x^{0}\right) y_{i}^{0}+x^{0} y_{i}^{0}\left(\frac{k_{i}}{k_{i}+k_{x}}\right) \text { for volatile functional groups } \\ 0 \text { for non-volatile functional groups }\end{array}\right.$

The ultimate char composition, $\omega_{i, c}^{\infty}$, predicted by the functional group model, is not a function of temperature when the potential tar-forming fruction, $x^{\circ}$, is specified. However, the ultimate tar and gas compositions, $\omega_{i, k}^{\infty}$ and $\omega_{i, g}^{\infty}$ are functions of temperature even when $x^{\circ}$ is specified because of the temperature-dependent Arrhenius rate constants $k_{i}$ and $k_{x}$.

Ultimate yields. The ultimate char, tar and gas yields, $\omega_{\mathrm{c}}^{\infty}, \omega_{\mathrm{s}}^{\alpha}$, and $\omega_{\mathrm{s}}^{\alpha}$, are readily found from Equations (13) to (15) by summing over functional groups:

$$
\begin{aligned}
& \omega_{c}^{\infty}=\left(1-x^{\circ}\right) \sum_{i=\text { in }} y_{i}^{\circ} \\
& \omega_{i}^{\infty}=x^{\circ} \sum_{i=i v} \frac{y_{i}^{0} k_{x}}{k_{i}+k_{x}}+x^{\circ} \sum_{i=\text { in }} y_{i}^{0} \\
& \omega_{i}^{\infty}=\left(1-x^{\circ}\right) \sum_{i=\text { iv }} y_{i}^{\circ}+x^{\circ} \sum_{i=i v} \frac{y_{i}^{0} k_{i}}{k_{i}+k_{x}}
\end{aligned}
$$

where the subscripts iv and in refer to volatile and non-volatile functional groups, respectively. The ultimate volatiles yield, $\omega_{y}^{\infty}$, is by definition a sum of the tar and the gas ultimate yields, $\omega_{1}^{\infty}$ and $\omega_{8}^{\infty}$; thus, from Equations (17) and (18):

$$
\omega_{v}^{\infty}=x^{0}+\left(1-x^{\circ}\right) \sum_{i=\mathrm{iv}} y_{i}^{\circ}
$$

Potential tar-forming fraction. The potential tarforming fraction, $x^{0}$, represents the maximum possible tar yield. However, competition from light gas evolution prevents maximum tar yield from being realized, as shown in Equations (17) and (18). The potential tar-forming fraction can be: (1) determined from experimental tar yields; (2) estimated from correlated experimental data (e.g. Refs 24,26 , and 27 ); (3) predicted from simple ultimate yield models (e.g. Ref. 28); or (4) predicted from coal-structure-dependent models (e.g. Ref. 17). The potential tar-forming fraction from Ko et calculated from the following equations:

$$
x^{0}=0.01\left(x+\beta x_{\text {tar }}\right)
$$

$x_{t a r}=\frac{\text { (number of labile bridges) (abstractable hydrogen ) }}{\text { (number of cross-linked bridges) }}$

where $\alpha$ and $\beta$ are pressure-dependent correlations. The number of labile bridges is a function of the aromaticity of the coal; the abstractable hydrogen is determined from the difference in ultimate analysis hydrogen and the hydroxyl functional group content; and the number of cross-linked bridges is determined from the ultimate analysis oxygen and sulphur content. This semi-empirical correlation has been used to correlate tar yields over pressures ranging from vacuum to $9 \mathrm{MPa}$ ( $\sim 90 \mathrm{~atm}$ ) for 37 coals ranging from lignites to anthracites ${ }^{24}$.

The potential tar-forming fractions determined from entrained-flow experiments, TGA experiments, and estimations based on the semi-empirical tar model of Ko et al. ${ }^{24}$ at 1 and $25 \mathrm{~atm}(0.1$ and $2.5 \mathrm{MPa}$, respectively) are shown in Table 1. The potential tar-forming fraction is smaller at high pressure due to mass transport effects which are modelled empirically with the SET model. Also, the potential tar-forming fractions obtained from TGA experiments are smaller than the values using the SET model at atmospheric pressure. Mass transport through the coal bed as well as secondary reactions with the hot char may explain the difference. The potential tar-forming fractions determined from entrained-flow experiments are closer to the atmospheric SET results than to the high pressure SET results or to the TGA results. Yet, the measured entrained-flow values are still higher than the atmospheric SET results. The largest difference between the potential tar-forming fraction determined from entrained-flow experiments and the atmospheric SET predictions was for the N. D. Zap lignite, which differs by a factor of 3.7. Corresponding values for Wyodak subbituminous coal were in good agreement. However, all of these estimates were considered inadequate for predicting tar yield for fixed-bed gasifiers, when compared to measured values. For the simulations discussed in this paper, the potential tar-forming fraction was determined from the tar yields reported for commercial scale fixed-bed gasifers. The two-zone model discussed herein was used to determine the potential tar-forming fraction from experimental fixed-bed tar yields. The potential tar-forming fractions obtained from experimental fixed-bed data are closest to the values obtained with the semi-empirical correlation discussed above. However, the values from Ko et al..$^{24}$ are still higher, as discussed further.

Arrhenius rate constants. A multiple reactions model for each functional group (similar to that of Anthony et al. ${ }^{28}$ ) is used to distribute the Arrhenius rate constants. The total volatile mass production from the $i$ th functional group is assumed to be normally distributed to activation energy in the same manner as the total potential volatile mass production at large times. Seven point GaussianLegendre quadrature was used to integrate the distributed rate equation ${ }^{16,29}$. Frequency factors, $k_{i}^{\circ}$, mean activation energies, $\bar{E}_{i}$, and standard deviations of activation energies, $\sigma_{i}$, were obtained from Solomon et al. ${ }^{17}$ and are given in Table 1. The rationale for using the distributed rate 

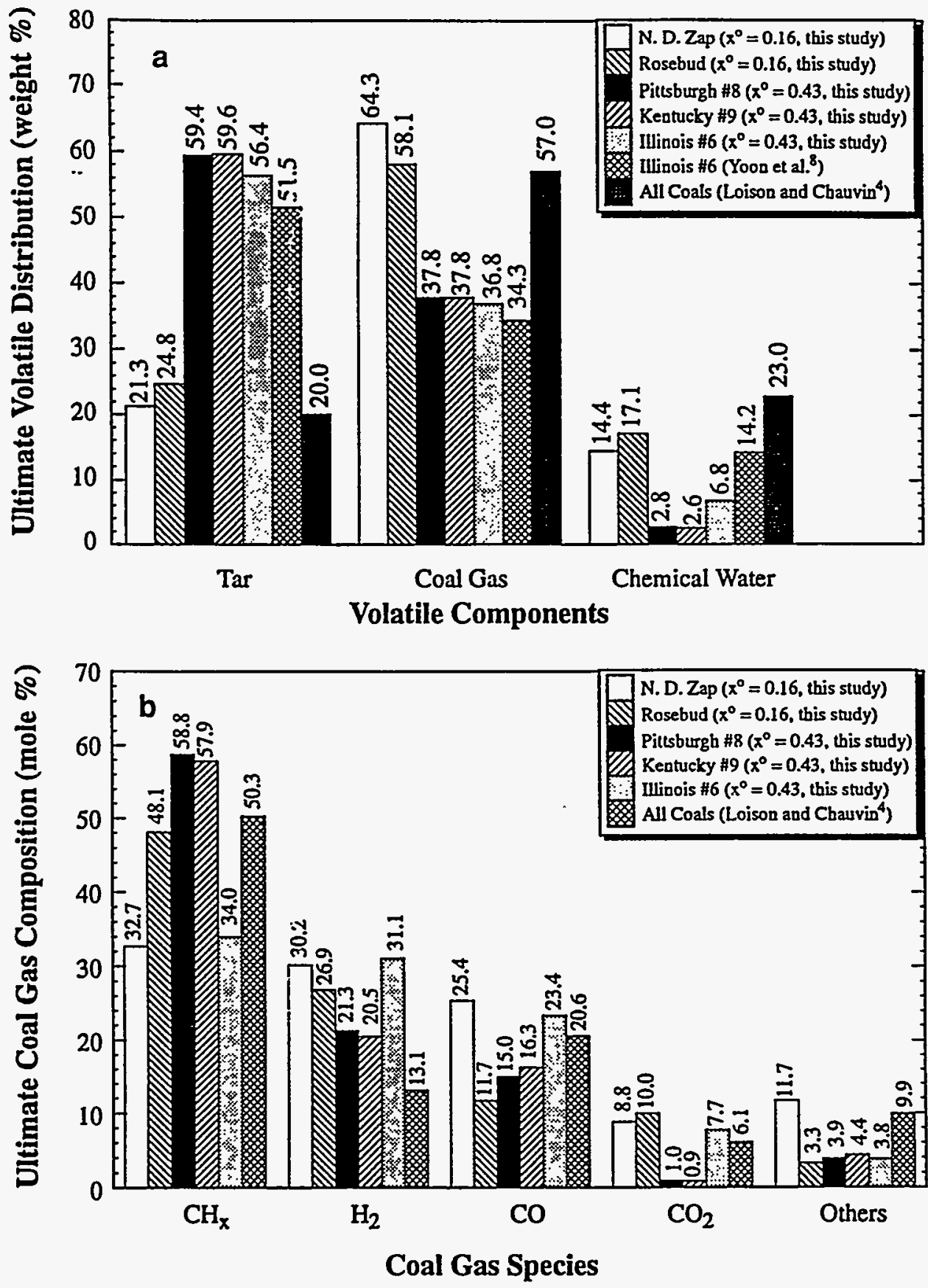

Figure 3 Comparison of predicted (a) ultimute coal volatiles distribution and (b) ultimate coul gas composition for various coals. Temperature is assumed to be $1000 \mathrm{~K}$. Distributions from Loison and Chauvint and Yoon et al..$^{8}$ at unspecified conditions are also shown

equation was to keep the functional group model consistent with the original development by Solomon et $a$ al. $^{17.25}$.

\section{Oxidation and gasification}

Oxidation and gasification are treated by specifying char bumout percentage following devolatilization. Temperature and composition sensitivity to specified burnout is discussed further in the sensitivity analysis. "omplete burnout was specified for the predictions herein. arge solid residence times and high oxygen concentrations and temperatures in the oxidation/gasification zone often justify this assumption for fixed beds. If more information is needed on carbon conversion, a one- or twodimensional model with kinetic rates for oxidation and gasification is required ${ }^{16}$.

\section{Freeboard}

The freeboard is the void space directly above the coal bed. The sampling point for the offtake gas may be significantly downstream from the top of the coal bed. The two-zone model predicts the temperature at the top of the coal bed which is greater than the temperature at the higher sampling point due to heat loss in the 

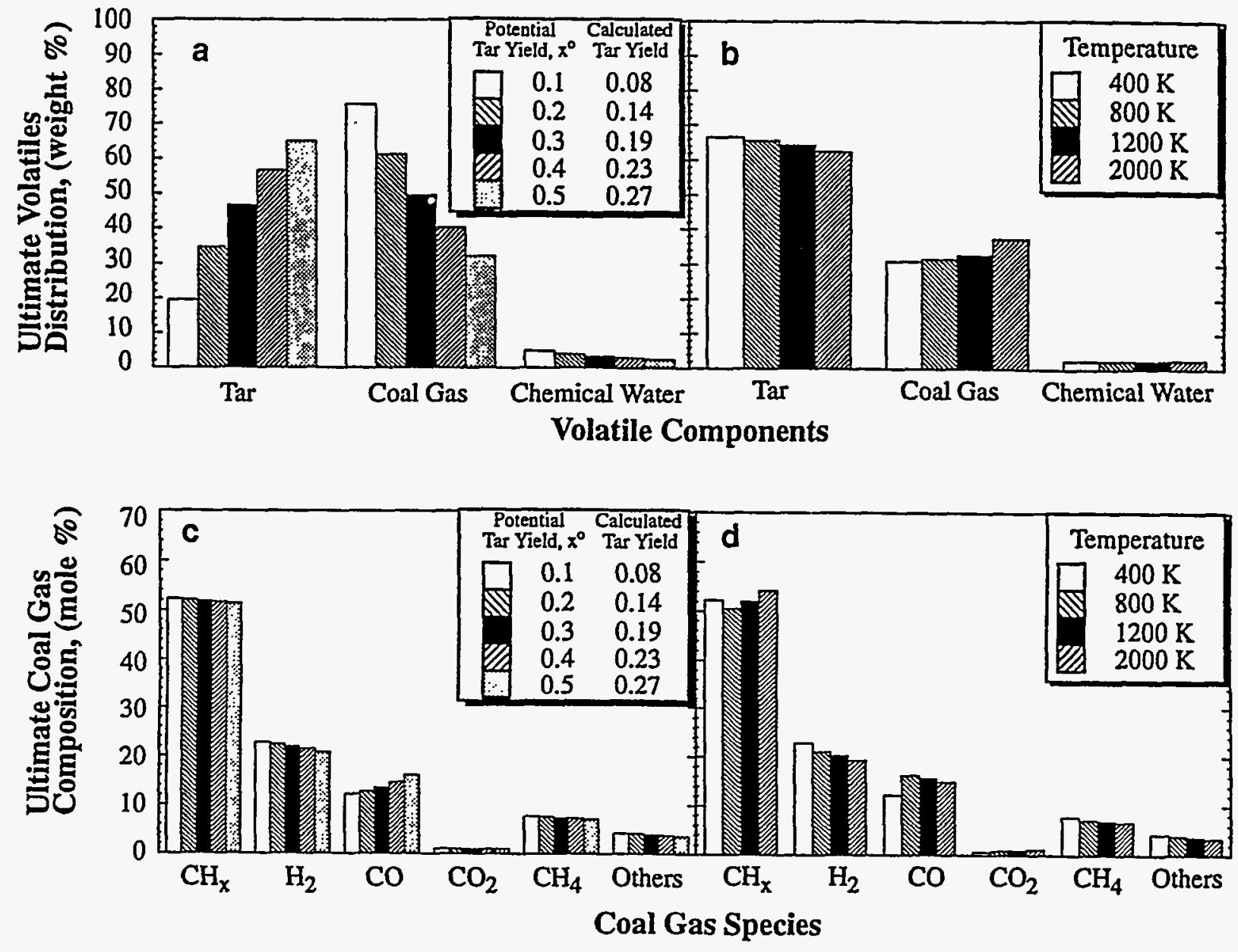

Figure 4 Predicted sensitivity of ultimate coal volatiles distribution to (a) potential tar-forming fraction and (b) temperature. Predicted sensitivity of ultimate coal gas composition to (c) potential tar-forming fraction and (d) temperature. Coal is assumed to be Pittsburgh no. 8. Temperature is assumed to be $1000 \mathrm{~K}$ for (a) and (c). Potential tar-forming fraction is asstmed to be 0.5 for (b) and (d)

water-cooled freeboard. The freeboard temperature, $T_{\mathrm{f}}$ (K), can be determined by doing a simple energy balance around the freeboard zone and accounting for heat loss in this section, $Q_{\mathrm{fb}}(\mathrm{W})$, as follows:

$$
T_{\mathrm{fb}}=T_{\text {exit }}+\frac{Q_{\mathrm{rb}}}{\dot{m}_{\mathrm{gas}} C p_{\mathrm{gas}}}
$$

where $T_{\text {exit }}$ is the exit temperature $(\mathrm{K}), \dot{m}_{\text {gas }}$ is the exit gas mass flow rate $\left(\mathrm{kg} \mathrm{s}^{-1}\right)$ and $C p_{\text {yas }}$ is the exit gas heat capacity $\left(\mathrm{J} \mathrm{kg}^{-1} \mathrm{~K}^{-1}\right)$. The frecboard tcmperature is the temperature usually reported in the literature. For the air-fired Wellman-Galusha gasifier, the estimated temperature drop from the bed top to the sampling point is on the order of $10-20 \mathrm{~K}$. Quantitative temperature comparisons were not attempted herein since: (1) the zero-dimensional assumption gives an average temperature in the drying/devolatilization zone which is significantly higher than the exit temperature; (2) there are no temperature measurements immediately above the bed and the freeboard heat loss was not predicted; and (3) measured temperature values are observed to scatter significantly and are lower than might be expected. However, qualitative comparisons are possible and are discussed.

\section{SENSITIVITY ANALYSIS}

Effuent product composition

Predicting accurate coal volatiles distribution and coal gas composition is important since as much as $40-60 \%$ of the dry ash free mass can be lost by devolatilization. The ultimate distribution of volatile matter into tar, water and coal gas is the final distribution, as time becomes large. The predicted ultimate coal volatile distribution and composition are primarily dependent on coal rank, temperature and potential tar-forming fraction.

Effects of coal runk. Comparisons of predicted ultimate volatile products distribution and ultimate coal gas composition for five of the coals listed in Table 1 are shown in Figure 3. The predictions are from Equations (17), (18) and (19) at $1000 \mathrm{~K}$. The temperature was chosen to be representative of the average temperature in the devolatilization zone. In addition, distributions often reported by other investigators are presented for comparison (e.g. the data of Loison and Chauvin ${ }^{4}$, compiled from a wide range of coal types and assumed valid for all coals, as used by Yoon et al. ${ }^{8}$, Kosky and Floess $^{3}$, Cho and Joseph ${ }^{11}, Y u$ and Denn ${ }^{12}$ ). The 
Prediction of effluent compositions: M. L. Hobbs et al.
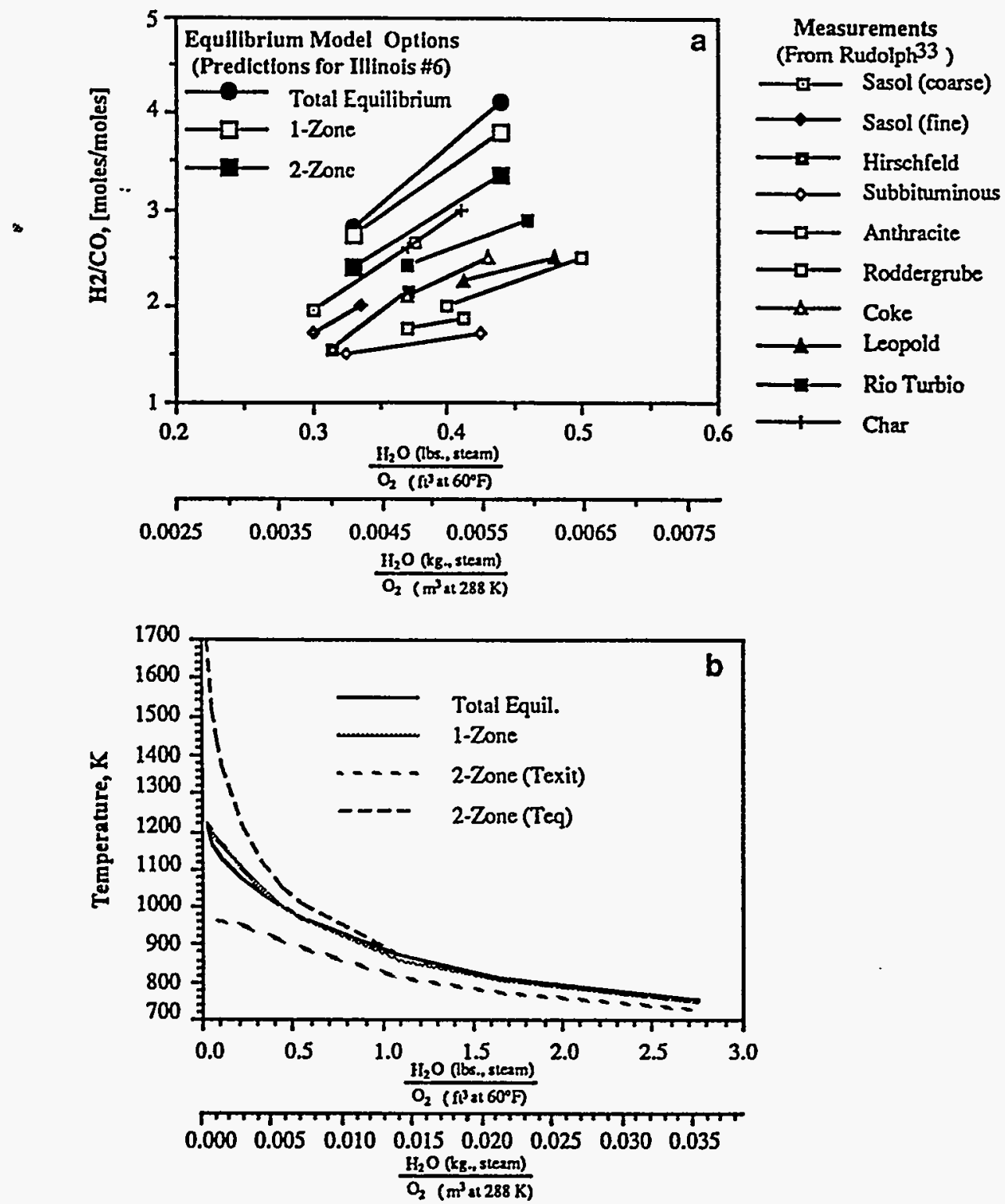

Figure 5 Temperature and composition sensitivity to steam-to-oxygen ratio. (a) Comparison of the effect of steam-to-oxygen feed ratio on hydrogen-to-carbon monoxide ratio for several fixed-bed test results and various equilibrium model options. Comparison is with Rudolph ${ }^{33}$. (b) Predicted temperature sensitivity to steam-to-oxygen ratio for various equilibrium model options using Illinois no. 6 in Westfield gasifier

ultimate volatiles distribution and ultimate coal gas composition strongly depend on coal rank. The lower ultimate tar yields for lignites and subbituminous coals are consistent with expected values. The lower tar yields may be caused by crosslinking associated with $\mathrm{CH}_{4}$ and $\mathrm{CO}_{2}$ evolution ${ }^{17}$. The distribution of Loison and Chauvin ${ }^{4}$ should not be used for all coal types as is commonly assumed, but the ultimate coal volatiles distribution should be calculated for each coal rank. In fact, Yoon et al. ${ }^{8}$ assumed a different coal volatiles distribution from the Loison and Chauvin distribution for the Illinois no. 6 case to get reasonable predictions. Yoon's volatiles distribution used for Mllinois no. 6 is also given in Figure $3 a$.

Effects of temperature and tar frattion. Predicted sensitivities of ultimate coal volatile products distribution and ultimate coal gas composition for Pittsburgh no. 8 to temperature and potential tar-forming fraction are shown in Figure 4. The calculated tar yield is also shown. As much as $27 \%$ of the dry ash free mass of the coal may evolve as tar. In a fixed bed, the tar may also crack to form smaller light gas species or repolymerize to form char. The ultimate coal volatiles distribution is more sensitive to the potential tar-forming fraction than to the temperature. Furthermore, the ultimate coal gas composition is not very sensitive to either, which emphasizes the importance of predicting accurate volatile distributions into tar, coal gas and chemical water as opposed to the coal gas composition. The lack of sensitivity of the ultimate coal gas composition to the potential tar-forming fraction and temperature comes from competition between tar evolution and light gas evolution. When the tar rate constant, $k_{x}$, is significantly 


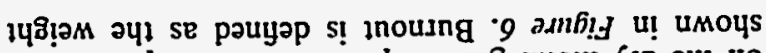

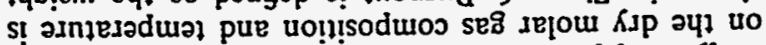

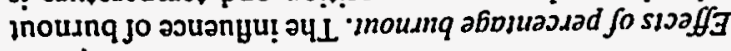

-aseajoap suo!̣do

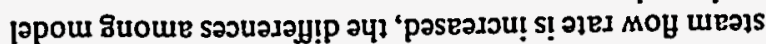

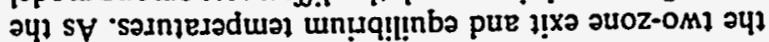

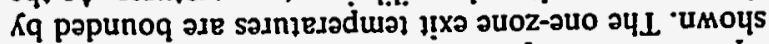

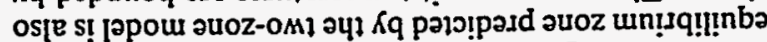

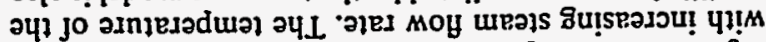

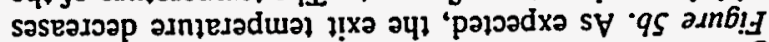

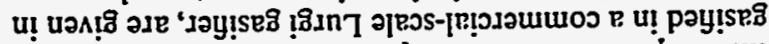

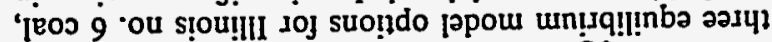

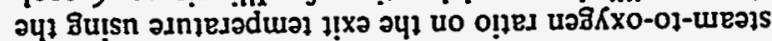

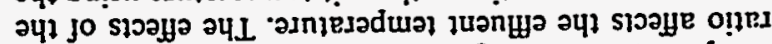

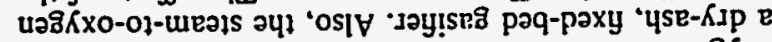

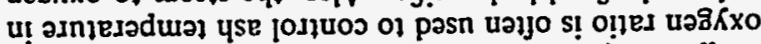

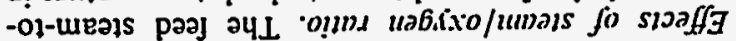

aมmusadual נuanßg

-uo!̣t!soduros

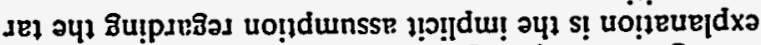

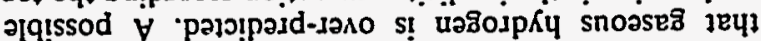

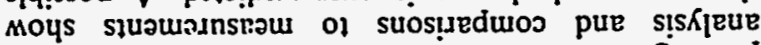
К\}! !!! !

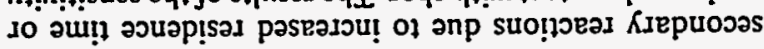

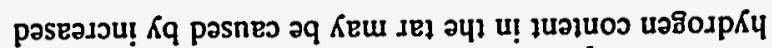

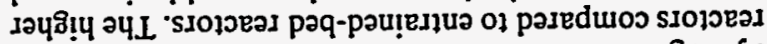

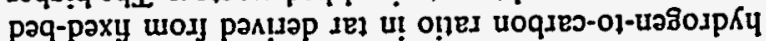

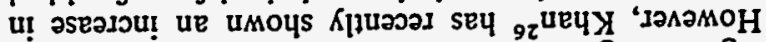

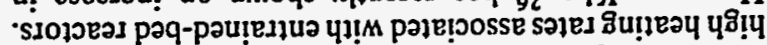

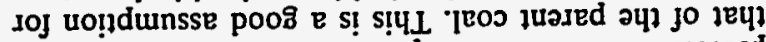

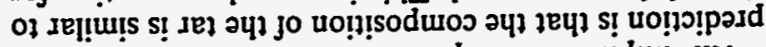
sə|lle

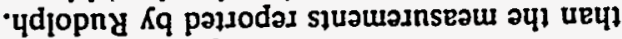

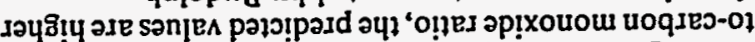

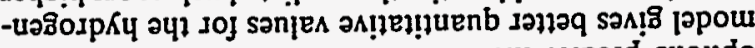

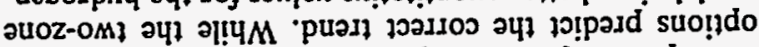

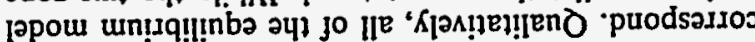

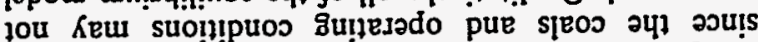

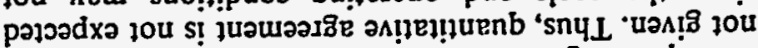

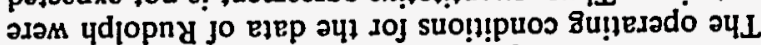

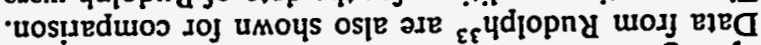

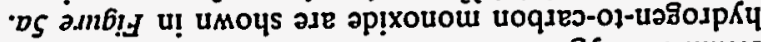

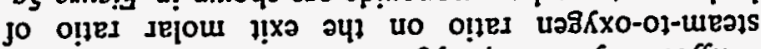

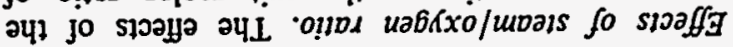

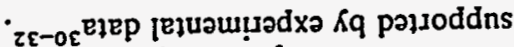

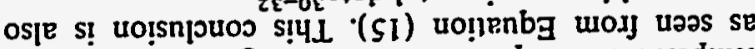

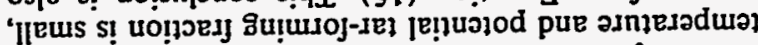

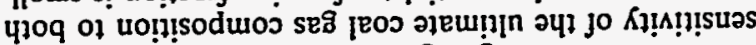

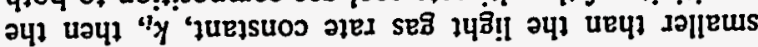

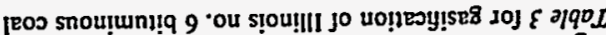

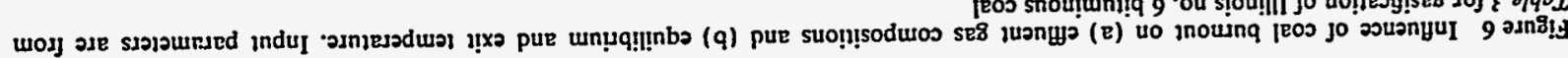

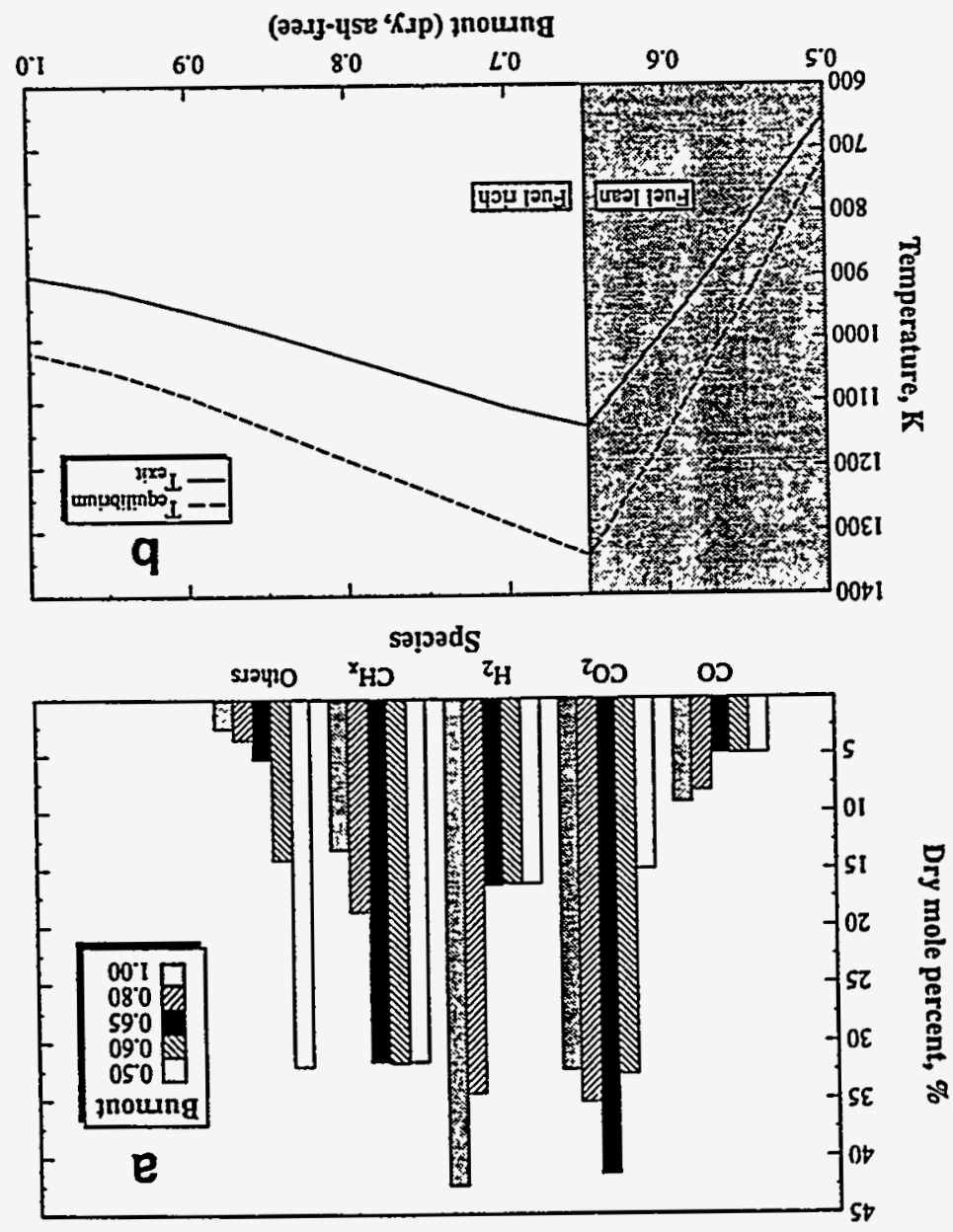

je ta sqgoh $7 \mathrm{~W}$ :suo!!!sodwos juan!fja to uo!lo!padd 
fraction (daf) of the organic matter in the raw coal that has reacted. The input conditions for Figure 6 are for Illinois no. 6 gasified at Westfield, UK. As burnout declines, $\mathrm{CO}$ and $\mathrm{H}_{2}$ steadily decreuse and hydrocarbons increase while $\mathrm{CO}_{2}$ reaches a maximum at $65 \%$ burnout. Temperature also reaches a maximum at this burnout level, where the-gas-phase concentration is nearly stoichiometric. For fuel-rich operation, increasing burnout in the gasification/oxidation zone causes temperature to decrease. However, if burnout is small, the gas-phase becomes oxygen rich and increasing burnout causes the temperature of the oxidation zone 10 increase.

\section{EXPERIMENTAL}

Thirteen cases have been simulated by the two-zone model. The ultimate analyses of coals for the test cases were performed several years ago and do not correspond exactly to the ultimate analysis of the eight coals of similar rank with measured functional groups as listed in Table 1. The functional group composition must be consistent with the ultimate analysis to maintain elemental continuity. Both the measured ultimate analysis for the test coals (Table 2) and measured functional group compositions from similar coals (Table J) were used to estimate the functional group composition of the test coals based on the method of Serio ${ }^{34}$. Functional groups with the highest experimental uncertainty were used to balance functional group compositions with the ultimate analysis. For example, the amount of $\mathrm{CO}$-tight is based on the amount of leftover unspecified oxygen. Increasing the oxygen content of the coal will increase the amount of $\mathrm{CO}$ in this pool. The method of detailed chemical characterization of the coals is given by $\mathrm{Hobbs}^{16}$. Table 2 lists the recommended values of the functional group composition and corresponding ultimate analysis for each of the 13 coals.

\section{Lurgi data}

Detailed experimental data on three high volatile bituminous coals and one subbituminous coal gasified in a high pressure, dry-ash, fixed-bed Lurgi gasifier in Westfield, UK, include flare-gas analysis and gas offtake temperature $\mathrm{s}^{35.36}$. The four coals are Illinois no. 6 (greatest reserve for Eastern caking coals), Illinois no. 5 (equivalent to Kentucky no. 9), Pittsburgh no. 8 (major Eastern coal with a high swelling index), and Rosebud subbituminous (from Southern Montana). The properties of gasified Illinois no. 5 coal are taken to be those of

Table 2 Recommended functional group compositions, calculated potential tar-forming fractions, and ultimate analysisa

\begin{tabular}{|c|c|c|c|c|c|c|c|c|c|c|c|c|c|}
\hline \multirow[b]{2}{*}{$\begin{array}{l}\text { Functional groups } \\
\text { (daf) }\end{array}$} & \multicolumn{4}{|c|}{ Lurgi cases } & \multicolumn{9}{|c|}{ Wellman-Galusha cases } \\
\hline & $\begin{array}{l}\text { Il no. } 6 \\
y_{i}^{\circ}\end{array}$ & $\begin{array}{l}\text { IL no. } 5 \\
y_{i}^{\circ}\end{array}$ & $\begin{array}{l}\text { Pitt no. } 8 . \\
y_{i}^{0}\end{array}$ & $\begin{array}{l}\text { Rose } \\
y_{i}^{\circ}\end{array}$ & $\begin{array}{l}\text { Absal } \\
y_{i}^{i}\end{array}$ & $\begin{array}{l}\text { Benton } \\
y_{i}^{\circ}\end{array}$ & $\begin{array}{l}\text { Elk } \\
y_{i}^{0}\end{array}$ & $\begin{array}{l}\text { IL no. } 6 \\
y_{i}\end{array}$ & $\begin{array}{l}\text { Jetson } \\
y_{i}^{\circ}\end{array}$ & $\begin{array}{l}\text { Kemer } \\
y_{i}\end{array}$ & $\begin{array}{l}\text { Leucite } \\
y_{i}\end{array}$ & $\begin{array}{l}\text { Rose } \\
y_{i}^{\circ}\end{array}$ & $\begin{array}{l}\text { Utah } \\
y_{i}^{\circ}\end{array}$ \\
\hline $\mathrm{CO}_{2}$ extra loose & 0.022 & 0.000 & 0.000 & 0.035 & 0.028 & 0.065 & 0.000 & 0.022 & 0.000 & 0.028 & 0.028 & 0.035 & 0.003 \\
\hline $\mathrm{CO}_{2}$ loose & 0.022 & 0.006 & 0.007 & 0.035 & 0.020 & 0.030 & 0.006 & 0.022 & 0.006 & 0.020 & 0.020 & 0.035 & 0.007 \\
\hline $\mathrm{CO}_{2}$ tight & 0.030 & 0.005 & 0.005 & 0.030 & 0.021 & 0.005 & 0.005 & 0.030 & 0.005 & 0.021 & 0.021 & 0.030 & 0.017 \\
\hline $\mathrm{H}_{2} \mathrm{O}$ loose & 0.015 & 0.011 & 0.000 & 0.000 & 0.030 & 0.000 & 0.011 & 0.016 & 0.011 & 0.027 & 0.024 & 0.000 & 0.025 \\
\hline $\mathrm{H}_{2} \mathrm{O}$ tight & 0.000 & 0.011 & 0.004 & 0.051 & 0.022 & 0.027 & 0.011 & 0.000 & 0.011 & 0.022 & 0.022 & 0.040 & 0.020 \\
\hline CO ether loose & 0.060 & 0.050 & 0.050 & 0.055 & 0.050 & 0.060 & 0.050 & 0.060 & 0.050 & 0.050 & 0.050 & 0.055 & 0.025 \\
\hline CO ether tight & 0.000 & 0.037 & 0.000 & 0.000 & 0.043 & 0.000 & 0.044 & 0.000 & 0.059 & 0.000 & 0.000 & 0.000 & 0.036 \\
\hline HCN loose & 0.013 & 0.021 & 0.007 & 0.010 & 0.000 & 0.005 & 0.022 & 0.011 & 0.025 & 0.003 & 0.016 & 0.005 & 0.000 \\
\hline HCN tight & 0.016 & 0.009 & 0.023 & 0.015 & 0.013 & 0.013 & 0.009 & 0.016 & 0.009 & 0.018 & 0.018 & 0.015 & 0.022 \\
\hline $\mathrm{NH}_{3}$ & 0.000 & 0,000 & 0.000 & 0.001 & 0.001 & 0.001 & 0.000 & 0.000 & 0.000 & 0.001 & 0.001 & 0.001 & 0.000 \\
\hline $\mathrm{CH}_{\mathrm{z}}$ aliphatic & 0.176 & 0.164 & 0.245 & 0.182 & 0.169 & 0.212 & 0.185 & 0.169 & 0.161 & 0.201 & 0.195 & 0.202 & 0.189 \\
\hline $\mathrm{CH}_{4}$ extra loose & 0.011 & 0.020 & 0.000 & 0.000 & 0.000 & 0.000 & 0.020 & 0.011 & 0.020 & 0.000 & 0.000 & 0.000 & 0.000 \\
\hline $\mathrm{CH}_{4}$ loose & 0.011 & 0.015 & 0.020 & 0.022 & 0.017 & 0.017 & 0.015 & 0.011 & 0.015 & 0.017 & 0.017 & 0.022 & 0.022 \\
\hline $\mathrm{CH}_{4}$ tight & 0.022 & 0.015 & 0.015 & 0.012 & 0.008 & 0.009 & 0.015 & 0.022 & 0.015 & 0.008 & 0.008 & 0.012 & 0.022 \\
\hline H aromatic & 0.016 & 0.012 & 0.013 & 0.013 & 0.013 & 0.017 & 0.012 & 0.016 & 0.012 & 0.013 & 0.013 & 0.013 & 0.017 \\
\hline $\mathrm{CH}_{3} \mathrm{OH}$ & 0.000 & 0.000 & 0.000 & 0.000 & 0.000 & 0.000 & 0.000 & 0.000 & 0.000 & 0.000 & 0.000 & 0.000 & 0.000 \\
\hline CO extra tight & 0.000 & 0.020 & 0.020 & 0.000 & 0.043 & 0.090 & 0.020 & 0.000 & 0.020 & 0.043 & 0.043 & 0.000 & 0.040 \\
\hline C non-volatile & 0.552 & 0.565 & 0.562 & 0.522 & 0.518 & 0.440 & 0.565 & 0.552 & 0.565 & 0.518 & 0.518 & 0.522 & 0.548 \\
\hline S organic & 0.035 & 0.039 & 0.029 & 0.017 & 0.004 & 0.009 & 0.009 & 0.042 & 0.016 & 0.010 & 0.006 & 0.013 & 0.006 \\
\hline Total & 1.001 & 1.000 & 1.000 & 1.000 & 1.000 & 1.000 & 0.999 & 1.000 & 1.000 & 1.000 & 1.000 & 1.000 & 0.999 \\
\hline Calculated $x^{0 e}$ & 0.177 & 0.168 & 0.168 & 0.110 & 0.137 & 0.227 & 0.270 & 0.270 & 0.222 & 0.219 & 0.167 & 0.150 & 0.270 \\
\hline Source coal for yi & IL no. 6 & KYno.9 & Pitt no. 8 & Rose & Wyo & Zap & KY no. 9 & IL no. 6 & KYno. 9 & wyo & Wyo & Rose & Utah \\
\hline Coal type & Bit. & Bit. & Bit. & Subbit. & Subbit. & Lig. & Bit. & Bit. & Bit. & Subbit. & Subbit. & Subbit. & Bit. \\
\hline $\begin{array}{l}\text { Ultimate analysis ( } \\
\text { C }\end{array}$ & $\begin{array}{l}\text { (wt } \% \text { daf) } \\
0.795\end{array}$ & 0.802 & 0.846 & 0.771 & 0.763 & 0.737 & 0.825 & 0.785 & 0.814 & 0.775 & 0.781 & 0.787 & 0.803 \\
\hline $\mathbf{H}$ & 0.054 & 0.054 & 0.058 & 0.049 & 0.051 & 0.062 & 0.057 & 0.057 & 0.051 & 0.056 & 0.050 & 0.049 & 0.062 \\
\hline $\mathbf{N}$ & 0.015 & 0.015 & 0.016 & 0.014 & 0.008 & 0.010 & 0.016 & 0.014 & 0.018 & 0.012 & 0.019 & 0.011 & 0.012 \\
\hline $\mathbf{0}$ & 0.101 & 0.089 & 0.053 & 0.150 & 0.174 & 0.182 & 0.093 & 0.102 & 0.102 & 0.147 & 0.144 & 0.140 & 0.117 \\
\hline S (organic) & 0.035 & 0.039 & 0.029 & 0.017 & 0.004 & 0.009 & 0.009 & 0.042 & 0.016 & 0.010 & 0.006 & 0.013 & 0.006 \\
\hline
\end{tabular}

-Functional group compositions from Table I were adjusted to match reported ultimate analysis using the criterion discussed by Hobbs ${ }^{16}$. The semi-empirical tar correlation of Ko et al. ${ }^{24}$ was used to calculate $x^{\circ}$ at pressures listed in Tubles 3 and 4 
Prediction of effluent compositions: M. L. Hobbs et al.

Table 3 Operating parameters and yield data for four coals in Lurgi gasifier simulations

\begin{tabular}{|c|c|c|c|c|}
\hline & $\begin{array}{l}\text { Illinois no. } 6 \\
\text { (Bituminous) }\end{array}$ & $\begin{array}{l}\text { Illinois no. } 5^{e} \\
\text { (Bituminous) }\end{array}$ & $\begin{array}{l}\text { Pittsburgh no. } 8 \\
\text { (Bituminous) }\end{array}$ & $\begin{array}{l}\text { Rosebud } \\
\text { (Subbituminous) }\end{array}$ \\
\hline \multicolumn{5}{|l|}{ Proximate analysis ( $w t \%$ ) } \\
\hline Ash & 9.1 & 8.1 & 7.7 & 9.7 \\
\hline Fixed carbon & 46.0 & 44.7 & 50.3 & 36.4 \\
\hline Moisture & 10.2 & 11.9 & 4.6 & 24.7 \\
\hline Volatile & 34.7 & 35.2 & 37.4 & 29.2 \\
\hline \multicolumn{5}{|l|}{ Ultimate analysis (wt\%) } \\
\hline Carbon & 79.5 & 80.2 & 84.6 & 77.1 \\
\hline Hydrogen & 5.4 & 5.4 & 5.8 & 4.9 \\
\hline Nitrogen & 1.5 & 1.5 & 1.6 & 1.4 \\
\hline Sulphur & 3.5 & 3.9 & 2.9 & 1.7 \\
\hline Oxygen & 10.1 & 8.9 & 5.3 & 15.0 \\
\hline \multicolumn{5}{|l|}{ Operating parameters } \\
\hline Chamber inside diameter $(\mathrm{m})$ & 2.74 & 2.74 & 2.74 & 2.74 \\
\hline Chamber length $(m)$ & 3.05 & 3.05 & 3.05 & 3.05 \\
\hline Chamber pressure (kPa) & 2410 & 2450 & 2510 & 2560 \\
\hline Inlet coal temperature $(\mathrm{K})$ & 298 & 298 & 298 & 298 \\
\hline Feed gas temperature (K) & 644 & 644 & 644 & 644 \\
\hline Wall temperature $(\mathrm{K})$ & 438 & 438 & 496 & 498 \\
\hline Coal mass flow $\left(\mathrm{kg} \mathrm{s}^{-1}\right)$ & 1.67 & 1.80 & 1.02 & 2.23 \\
\hline Oxygen mass flow $\left(\mathrm{kg} \mathrm{s}^{-1}\right)$ & 0.81 & 0.88 & 0.63 & 0.58 \\
\hline Steam mass flow $\left(\mathrm{kg} \mathrm{s}^{-1}\right)$ & 4.20 & 4.04 & 3.29 & 2.80 \\
\hline Jacket steam mass flow $\left(\mathrm{kg} \mathrm{s}^{-1}\right)$ & 0.89 & 0.73 & 0.66 & 0.31 \\
\hline Wall heat loss $(\mathrm{MW})^{b}$ & 2.40 & 1.97 & 1.78 & 0.836 \\
\hline Predicted $T_{\text {etil }}, T_{\text {equil }}(\mathrm{K})$ & 898,1020 & 941,1100 & 1040,1110 & 728,1020 \\
\hline Measured $d T_{\mathrm{fb}}(\mathrm{K})$ & $863-895$ & $879-908$ & $911-928$ & $635-664$ \\
\hline$x^{\circ}$ obtained from measured tar & 0.106 & 0.132 & 0.065 & 0.128 \\
\hline $\begin{array}{l}\text { Measured tar data' } \\
\text { Product tar }\left(\mathrm{kg} \mathrm{s}^{-1}\right)\end{array}$ & 0.070 & 0.107 & 0.054 & 0.123 \\
\hline Recycle tar $\left(\mathrm{kg} \mathrm{s}^{-1}\right)$ & 0.058 & 0.068 & 0.000 & 0.046 \\
\hline Total tar $\left(\mathrm{kg} \mathrm{s}^{-1}\right)$ & 0.128 & 0.175 & 0.054 & 0.169 \\
\hline Predicted dry gas yield $\left(\mathrm{kg} \mathrm{s}^{-1}\right)$ & 3.13 & 3.26 & 2.07 & 2.92 \\
\hline Measured dry gas yield $\left(\mathrm{kg} \mathrm{s}^{-1}\right)$ & 2.99 & 3.26 & 2.13 & 2.91 \\
\hline Predicted water yield $\left(\mathrm{kg} \mathrm{s}^{-1}\right)$ & 3.39 & 3.28 & 2.74 & 2.39 \\
\hline Measured water yield $\left(\mathrm{kg} \mathrm{s}^{-1}\right)$ & 3.53 & 3.18 & 2.64 & 2.18 \\
\hline Predicted $\mathrm{CO}$ yield $\left(\mathrm{kg} \mathrm{s}^{-1}\right)$ & 0.392 & 0.605 & 0.262 & 0.487 \\
\hline Measured $\mathrm{CO}$ yield $\left(\mathrm{kg} \mathrm{s}^{-1}\right)$ & 0.694 & 0.772 & 0.484 & 0.608 \\
\hline Predicted $\mathrm{H}_{2}$ yield $\left(\mathrm{kg} \mathrm{s}^{-1}\right)$ & 0.131 & 0.132 & 0.077 & 0.114 \\
\hline Measured $\mathrm{H}_{2}$ yield $\left(\mathrm{kg} \mathrm{s}^{-1}\right)$ & 0.113 & 0.122 & 0.081 & 0.119 \\
\hline
\end{tabular}

Illinois no. 5 is reported to be from the same basin as Kentucky no. 9, and they are taken to be equivalent coals

-Calculated from jacket steam flow rate (cooling water losses not reported)

ePredictions are from the two-zone model with light gases held out of equilibrium in the drying and devolatilization zone

Measurements are from ref. 35

-Values obtained using the two-zone model to match measured tar data

'Values obtained with the two-zone model using the $x^{\circ}$ value above

Kentucky no. 9 coal in Table 1 since the two coals are reportedly from the same basin. The Illinois no. 6 coal has been used for comparison with predictions from several fixed-bed models (e.g. Refs 3, 8, 11, 12 and 37). Operational data for all four Lurgi cases are given in Table 3.

\section{Wellman-Galusha data}

The most extensive set of fixed-bed data was reported by Thimsen et al. ${ }^{38}$. These data have been compiled recently by Thimsen and Maurer ${ }^{39}$. Data for 17 coals (bituminous, subbituminous and lignite) peat and coke were reported. The data include gasifier operating conditions, coal properties, tar and water yield, ash and dust data, and gas composition. Some profile data for temperature and pressure were also reported as discussed further by Hobbs ${ }^{16}$. Of the 17 gasified coals, nine coals were chosen for simulation based on how well the coal gasified during the experimental test. The simulated coals are Absaloka/Robinson subbituminous $C$ (from Powder River Basin, Montana), Benton lignite (from Arkansas), Elkhorn high volatile A bituminous (from Eastern Kentucky), Illinois no. 6 high volatile $C$ bituminous (from the Illinois Basin), Jetson high volatile B bituminous (mined in Kentucky's Illinois Basin), Kemmerer subbituminous B (from Southwest Wyoming), Leucite Hills subbituminous A (from Sweetwater County, Wyoming), Rosebud subbituminous B (from the 
Prediction of effluent compositions: M. L. Hobbs et al.

Table 4 Operating parameters and yield data for nine coals in Wellman-Galusha gasifier simulations

\begin{tabular}{|c|c|c|c|c|c|c|c|c|c|}
\hline & $\begin{array}{l}\text { Absaloka } \\
\text { (Subbitum.) }\end{array}$ & $\begin{array}{l}\text { Benton } \\
\text { (Lignite) }\end{array}$ & $\begin{array}{l}\text { Elkhom } \\
\text { (Bituminous) }\end{array}$ & $\begin{array}{l}\text { Illinois no. } 6 \\
\text { (Bituminous) }\end{array}$ & $\begin{array}{l}\text { Jetson } \\
\text { (Bituminous) }\end{array}$ & $\begin{array}{l}\text { Kemmerer } \\
\text { (Subbitum.) }\end{array}$ & $\begin{array}{l}\text { Leucite } \\
\text { (Subbitum.) }\end{array}$ & $\begin{array}{l}\text { Rosebud } \\
\text { (Subbitum.) }\end{array}$ & $\begin{array}{l}\text { Utah B.C. } \\
\text { (Bituminous) }\end{array}$ \\
\hline \multicolumn{10}{|l|}{ Proximate analysis (wt\%) } \\
\hline Ash & 6.3 & 6.4 & 4.7 & 9.3 & 4.3 & 5.7 & 9.0 & 11.8 & 11.1 \\
\hline Fixed carbon & 40.7 & 25.9 & 53.7 & 45.1 & 49.5 & 42.4 & 44.9 & 40.1 & 43.9 \\
\hline Moisture & 23.5 & 32.8 & 4.6 & 10.3 & 6.3 & 16.8 & 17.4 & 21.3 & 6.1 \\
\hline Volatile & 29.6 & 34.9 & 36.9 & 35.3 & 39.9 & 35.1 & 28.8 & 26.8 & 38.9 \\
\hline \multicolumn{10}{|l|}{ Ulimate analysis (wt\%) } \\
\hline Carbon & 76.3 & 73.7 & 82.5 & 78.5 & 81.4 & 77.5 & 78.1 & 78.7 & 80.3 \\
\hline Hydrogen & 5.1 & 6.2 & 5.7 & 5.7 & 5.1 & 5.6 & 5.0 & 4.9 & 6.2 \\
\hline Nitrogen & 0.8 & 1.0 & 1.6 & 1.4 & 1.8 & 1.2 & 1.9 & 1.1 & 1.2 \\
\hline Sulphur & 0.4 & 0.9 & 0.9 & 4.2 & 1.6 & 1.0 & 0.6 & 1.3 & 0.6 \\
\hline Oxygen & 17.4 & 18.2 & 9.3 & 10.2 & 10.2 & 14.7 & 14.4 & 14.0 & 11.7 \\
\hline \multicolumn{10}{|l|}{ Operating parameters } \\
\hline Chamber diameter (m) & 1.98 & 1.98 & 1.98 & 1.98 & 1.98 & 1.98 & 1.98 & 1.98 & 1.98 \\
\hline Chamber length (m) & 1.90 & 1.90 & 1.90 & 1.90 & 1.90 & 1.90 & 1.90 & 1.90 & 1.90 \\
\hline Chamber pressure (kPa) & 101.3 & 101.3 & 101.3 & 101.3 & 101.3 & 101.3 & 101.3 & 101.3 & 101.3 \\
\hline Inlet coal temperature $(K)$ & 298 & 298 & 298 & 298 & 298 & 298 & 298 & 298 & 298 \\
\hline Feed gas temperature (K) & 332 & 340 & 331 & 335 & 300 & 332 & 334 & 336 & 335 \\
\hline Wall temperature (K) & 310 & 310 & 310 & 310 & 310 & 310 & 310 & 310 & 310 \\
\hline Coal mass flow $\left(\mathrm{kg} \mathrm{s}^{-1}\right)$ & 0.4201 & 0.7491 & 0.3243 & 0.3178 & 0.3524 & 0.2844 & 0.2928 & 0.1790 & 0.3373 \\
\hline Air mass flow $\left(\mathrm{kg} \mathrm{s}^{-1}\right)$ & 0.7637 & 0.9310 & 0.7889 & 0.7451 & 0.9481 & 0.5734 & 0.5323 & 0.3350 & 0.6730 \\
\hline Steam mass flow $\left(\mathrm{kg} \mathrm{s}^{-1}\right)$ & 0.1143 & 0.2312 & 0.1128 & 0.1347 & 0.1562 & 0.0881 & 0.0883 & 0.0500 & 0.1187 \\
\hline $\begin{array}{l}\text { Jacket steam mass flow } \\
(\mathrm{kg}=-1)\end{array}$ & 0.0034 & 0.0510 & 0.0082 & 0.1257 & 0.1339 & 0.0030 & 0.0620 & 0.0174 & 0.0261 \\
\hline $\begin{array}{l}\text { Wall heat loss (jacket steam) } \\
\text { (MW) }\end{array}$ & 0.1554 & 0.2258 & 0.2602 & 0.6049 & 0.4159 & 0.1672 & 0.2907 & 0.1694 & 0.2947 \\
\hline Predicted $T_{\text {rine }} T_{\text {equll }}(K)$ & 872,1360 & 723. 1340 & 1140,1420 & 1000.1260 & 1150,1440 & 1010,1400 & 801.1120 & 929.1310 & 980.1230 \\
\hline Measured $T_{r b}(\mathrm{~K})$ & 579 & 432 & 711 & 729 & 699 & 646 & 544 & sol & 699 \\
\hline$x^{\circ}$ obtained messured tar & 0.0690 & 0.2093 & 0.1726 & 0.1910 & 0.1560 & 0.1175 & 0.0775 & 0.0730 & 0.1670 \\
\hline Measured tar yield $\left(\mathrm{kg} \mathrm{s}^{-1}\right)^{*}$ & 0.0181 & 0.0832 & 0.0464 & 0.0435 & 0.0451 & 0.0230 & 0.0149 & 0.0079 & 0.0435 \\
\hline Predicted dry gas yield $\left(k_{B} s^{-1}\right)$ & 1.09 & 1.39 & 1.09 & 1.01 & 1.28 & 0.807 & 0.784 & 0.470 & 0.970 \\
\hline Measured dry gas yield $\left(\mathrm{kg} \mathrm{s}^{-1}\right)$ & 1.09 & 1.39 & 1.10 & 1.00 & 1.25 & 0.813 & 0.780 & 0.469 & 0.962 \\
\hline Prediced water yield $\left(\mathrm{kg} \mathrm{s}^{-1}\right)$ & 0.159 & 0.390 & 0.074 & 0.114 & 0.120 & 0.0995 & 0.0876 & 0.0652 & 0.0779 \\
\hline Measured water yield $\left(\mathrm{kg} \mathrm{s}^{-1}\right)$ & 0.147 & 0.441 & 0.065 & 0.107 & 0.094 & 0.103 & 0.090 & 0.0607 & 0.0863 \\
\hline Predicted CO yield (kB s-1) & 0.286 & 0.271 & 0.258 & 0.155 & 0.253 & 0.185 & 0.189 & 0.0982 & 0.233 \\
\hline Measured CO yield $\left(\mathrm{kg} \mathrm{s}^{-\mathrm{t}}\right)$ & 0.398 & 0.373 & 0.358 & 0.264 & 0.360 & 0.278 & 0.280 & 0.164 & 0.305 \\
\hline Predicted $\mathrm{H}_{2}$ yield $\left(\mathrm{kg} \mathrm{s}^{-1}\right)$ & 0.0112 & 0.0167 & 0.0095 & 0.0092 & 0.0102 & 0.0076 & 0.0093 & 0.0044 & 0.0120 \\
\hline Measured $\mathrm{H}_{2}$ yield $\left(\mathrm{kB} \mathrm{s}^{-1}\right)$ & 0.0166 & 0.0236 & 0.0167 & 0.0127 & 0.0181 & 0.0111 & 0.121 & 0.0064 & 0.0149 \\
\hline Reference $38^{f}$ & 12,53 & 10.55 & 9,71 & 8.68 & 2,68 & 14.49 & 4,59 & 15,50 & 13.57 \\
\hline
\end{tabular}

- Calculated from jacket steam and cooling water heat loss

"Predictions ane from the two-zone model with light gases held out of equilibrium in the drying and devolatilization zone

'Measurements are from rel. 38

4 Values obtained using the two-zone model to match measured tar data

-Values obtained with the two-zone model using the $x^{\bullet}$ value above

solume (bold) and page number

Powder River Basin, Montana), and Utah Blind Canyon high volatile B bituminous (from Central Utah). The recommended functional group compositions and corresponding ultimate analysis for each of these nine coals are listed in Table 2. Operational jata for all nine Wellman-Galusha cases are given in Table 4.

\section{MODEL COMPARISONS WITH DATA}

\section{Lurgi effluent predictions}

Measured and predicted effluent compositions are shown in Figures 7 and 8 for Illinois no. 6, Pittsburgh no. 8, Illinois no. 5, and Montana Rosebud coals. Measured and predicted tar yields and temperatures are given in Table 3. Complete burnout was assumed for all of the predictions in Figures 7 and 8 . Heat loss was calculated based on the jacket steam flow rate; the potential tar-forming fraction, $x^{\circ}$, was determined from experimental tar yields. For the Lurgi cases, a portion of the tar was recycled back to the reactor and injected into the top of the reactor. For the simulations, the recycled tar fraction was chosen to match the measured tar recycle rate ${ }^{35}$.

Tar. The predicted tar-forming fractions using the correlation of Ko et al. ${ }^{24}$ are high for the three bituminous coals and slightly low for the subbituminous coal when compared to those obtained from experimental 
Prediction of effluent compositions: M. L. Hobbs et al.

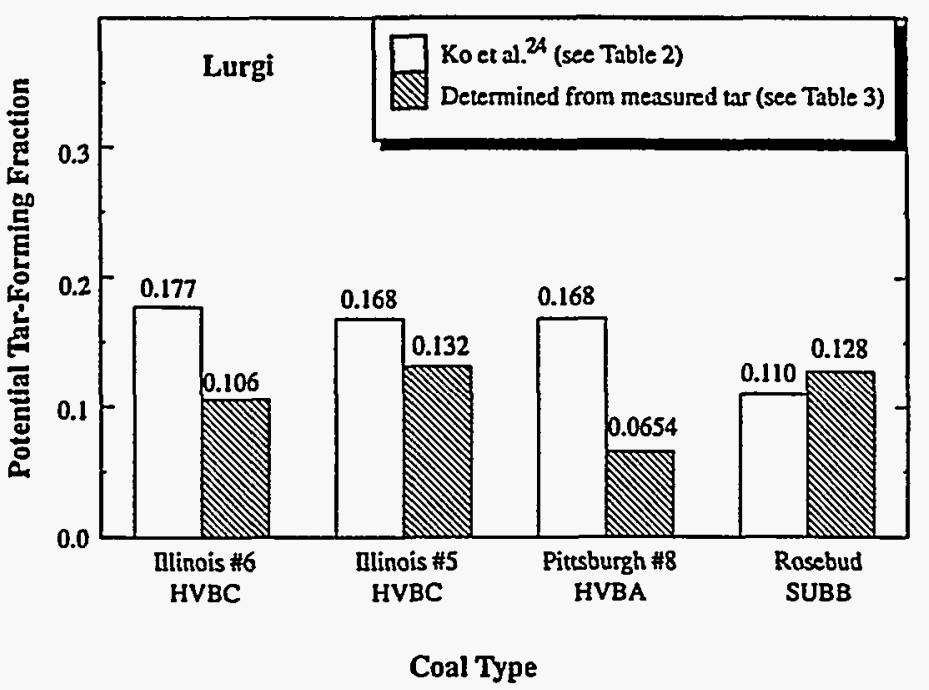

Figure 7 Potential tar-forming fractions calculated from the semi-empirical tar model of Ko et al. ${ }^{24}$ and determined from experimental tar data ${ }^{3 s}$

data (see Figure 7). The correlation of Ko et al. ${ }^{24}$ is reportedly good for coal particles smaller than $500 \mu \mathrm{m}$. Particle size effects may account for the observed discrepancy. More rigorous methods for tar prediction that depend on coal structure are being developed by Solomon et al. ${ }^{17.40}$, Grant et al. ${ }^{41}$ and Niksa ${ }^{42}$.

Gas compositions. The measured effluent compositions and those predicted from the total equilibrium, one-zone partial equilibrium, and two-zone partial equilibrium models are shown in Figure 8. Both partial equilibrium models assume that the gases produced during drying and devolatilization are non-reactive. The Illinois no. 6 case also shows predictions from Yoon et al.' $\mathrm{s}^{8}$ one-dimensional model, which are probably the best predictions of effluent composition in the literature. The two-zone model predictions are as good as these predictions and better than the one-zone model predictions. The predicted $\mathrm{CH}_{x}$ concentrations are high compared to measured values. The measured $\mathrm{CH}_{x}$ group was reported as $\mathrm{CH}_{4}$. The predicted $\mathrm{CH}_{x}$ group consists of both $\mathrm{CH}_{4}$ and other $\mathrm{CH}_{x}$ species which account for leftover aliphatic carbon and hydrogen in the functional group compositions. Predicted carbon monoxide concentrations for both one-zone models are low, although the partial equilibrium model predictions are closer to measured values. Carbon monoxide predictions from the two-zone model are closer to measured values. Other species are also predicled more closely to measured values with the two-zone partial equilibrium model.

Temperatures. Predicted exit temperatures using the 1 partial equilibrium model, given in Table 3, were higher than the total equilibrium predictions for the bituminous coals. Since the gas phase exiting the reactor is fuel rich, separating tar out from the light gas species causes the gas mixture to become more fuel lean, thereby causing the exit temperature to increase slightly. This effect was more pronounced when comparing the total equilibrium model results to the two-zone partial equilibrium model.

The most interesting results are the Rosebud predictions. The one-zone models did not give reasonable exit temperature predictions or exit composition predictions for the Rosebud subbituminous coal case (see Table 3 and Figure 8). The exit temperatures for the Rosebud one-zone equilibrium predictions were about $100 \mathrm{~K}$ higher than the two-zone model predictions. The energy required to dry the coal and heat up the feed coal causes the temperature in the drying/devolatilization zone to be lower. The predicted compositions for the Rosebud case with the two-zone partial equilibrium model are in close agreement with measured compositions. The improved agreement obtained by using the two-zone partial equilibrium model is attributed partially to dividing the process into two zones and also to using partial equilibrium in the gas phase rather than allowing the species to react to complete equilibrium.

\section{Wellman-Galusha effuent predictions}

Measured and predicted tar fractions and effluent compositions are shown in Figures 9-11 for the nine coals. Predicted exit temperature and predicted equilibrium temperatures are given in Table 4. Complete burnout was assumed for all of the predictions in Figures 9-11. The heat loss was calculated from the jacket steam and cooling water flow rate; the potential tar-forming fraction, $x^{\circ}$, was determined from experimental tar yields. For the Wellman-Galusha cases, tar was not recycled back to the reactor ${ }^{38}$.

Tar. The potential tar-forming fractions for the Wellman-Galusha cases calculated with the correlation of Ko et al. ${ }^{24}$ are compared with values determined from measurements in Figure 9. This correlation gives consistently high tar values for the Wellman-Galusha cases, although the trends seem to be predicted correctly. Quantifying tar yields from fixed-bed gasifier product gas streams is difficult. Liquid loadings are often underreported due to difficulties in collecting liquid droplets smaller than $1 \mu \mathrm{m}$ in diameter ${ }^{39}$.

Gas compositions. Measured effluent gas compositions from the atmospheric, air-blown Wellman-Galusha gasifier are shown in Figures 10 and 11 for the nine coals with results from two sets of model predictions: (1) all gases in the drying and devolatilization zone are 
Prediction of effluent compositions: M. L. Hobbs et al.

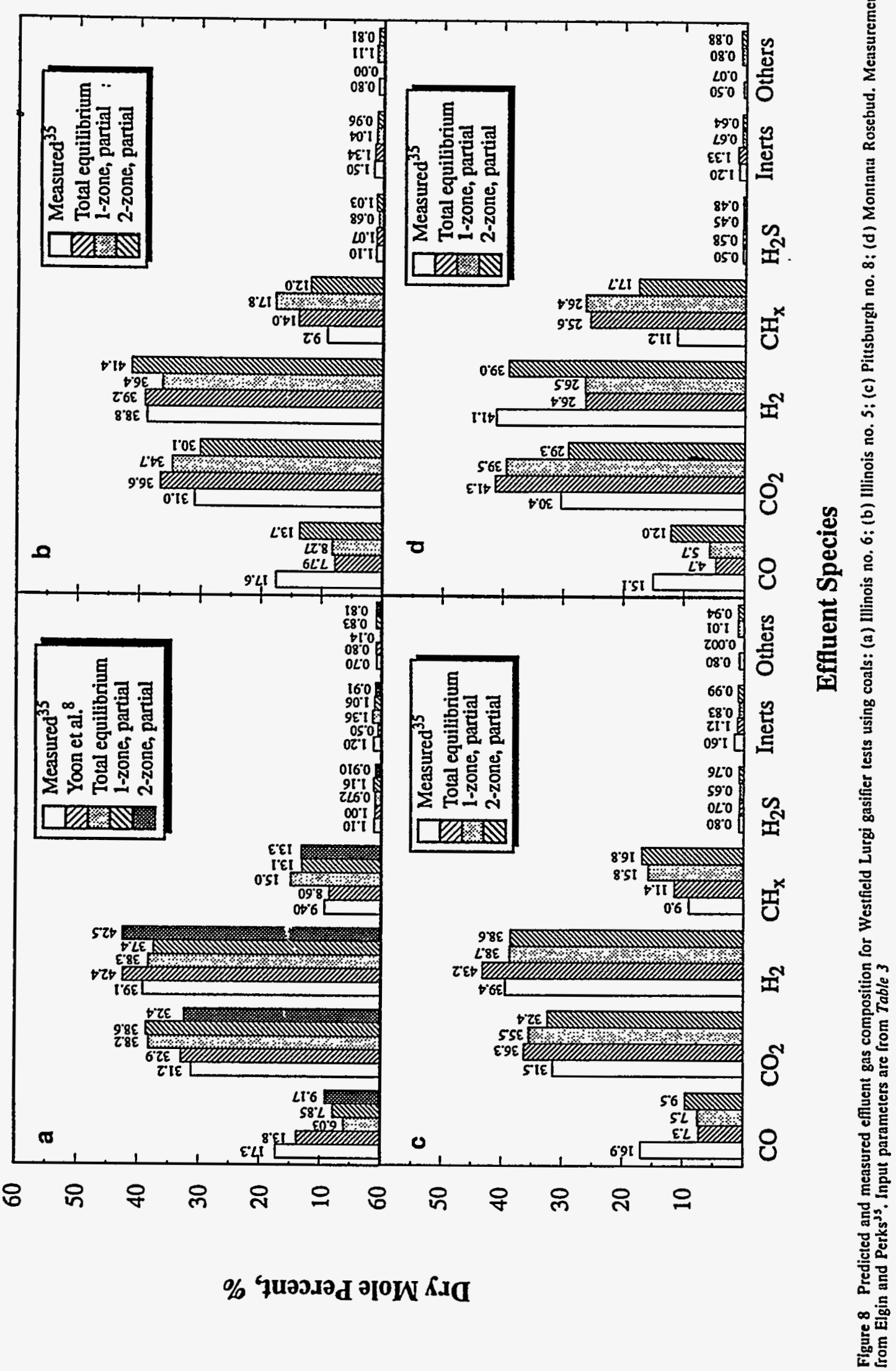




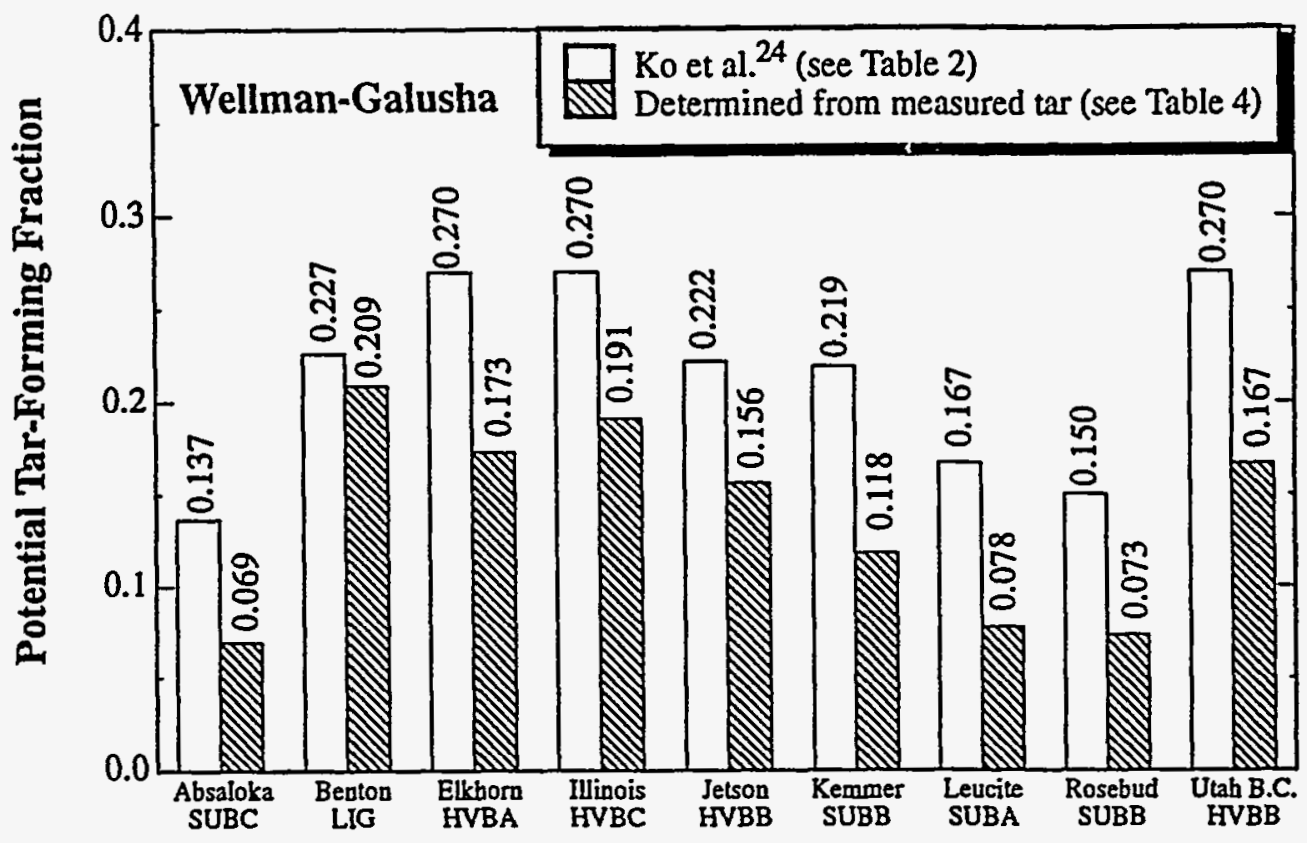

\section{Coal Type}

Figure 9 Potential tar-forming fractions calculated from the semi-empirical tar model of Ko et al. ${ }^{24}$ and determined from experimental tar data ${ }^{38}$

non-reactive; and (2) all gases in the drying and devolatilization zone, except tar, are in chemical equilibrium. In general, predictions using either gasphase assumption are acceptable; however, the assumption of non-reactive gases may give better results for low rank coals. For example, all of the subbituminous and lignite cases which assume complete equilibrium, predict high $\mathrm{CO}_{2}$, low $\mathrm{CO}$, and high $\mathrm{H}_{2}$ effluent concentrations in comparison to the data. These concentration differences may be attributed to higher oxygen content in the lower rank coals. Also, the lower rank coals contain more moisture which results in lower exit temperatures favouring partial equilibrium.

\section{Yields}

Comparisons of predicted and measured dry gas yields, water yields, $\mathrm{CO}$ yields and $\mathrm{H}_{2}$ yields are shown in Tables 3 and 4. In general, predictions of dry gas and water yields are acceptable. However, predicted $\mathrm{CO}$ yields are lower than measured values. Predicted $\mathrm{CO}$ values about 0.9 times measured values were also reported by Watkinson et al. ${ }^{43}$. Two factors are thought to relate to the observations that measured $\mathrm{CO}$ concentrations are consistently higher than predicted values, by about 3-10\%. First, experimentally measured tar values were used in predictions. Yet, it has been noted that these tar values are thought to be low because of the difficulty in tar collection ${ }^{39}$. Carryover of coal fines could also add to the underestimate of the carbonaceous product. Thus actual tar values may have been somewhat higher, possibly closer to the predictions of Ko et al. ${ }^{24}$. As shown in Figures $4 a$ and $4 c$, higher values of tar yield lead to higher values of $\mathrm{CO}$ in the equilibrium mixture. Secondly, the equilibrium temperature, in zone $2, T_{\text {exit, }}$ is only a global temperature and is higher than the local exit temperature. The $\mathrm{CO} / \mathrm{CO}_{2}$ ratio is dependent on this calculated temperature. As $T_{\text {exit }}$ declines, $\mathrm{CO}$ increases. This is shown in Figure 8 by comparing one-zone and two-zone partial equilibrium values. For two-zone calculations, the equilibrium temperature is lower and the predicted $\mathrm{CO}$ is higher while the $\mathrm{CO}_{2}$ is lower. Thus, higher tar yields and lower exit temperatures may explain the differences tetween observed $\mathrm{CO}$ and predicted $\mathrm{CO}$ percentages.

\section{SUMMARY AND CONCLUSIONS}

The two-zone partial equilibrium model uses a coal-general devolatilization submodel and a partial equilibrium gas-phase submodel to provide estimates of effluent temperature and composition for fixed-bed gasification and combustion. Effluent composition is determined for all the major species and a number of minor species. Tar production can be estimated, with some uncertainty, using a semi-empirical tar model and tar recycle is taken into account.

The key assumption for the two-zone model is that oxidation and gasification occur at relatively high temperatures compared to the cooler devolatilization and drying zones. The temperature difference provides a natural division of the process into two zones. High temperatures in the oxidation/gasification section favour total equilibrium in the gas phase. Lower temperature and small gas residence time in the devolatilization zone favour partial equilibrium. Limitations to the two-zone model are the need to specify burnout and uncertainty in wall heat loss.

The two-zone, partial equilibrium predictions show that the ultimate volatiles distribution and coal gas composition depend strongly on coal rank. The ultimate volatiles distribution is more sensitive to the potential 


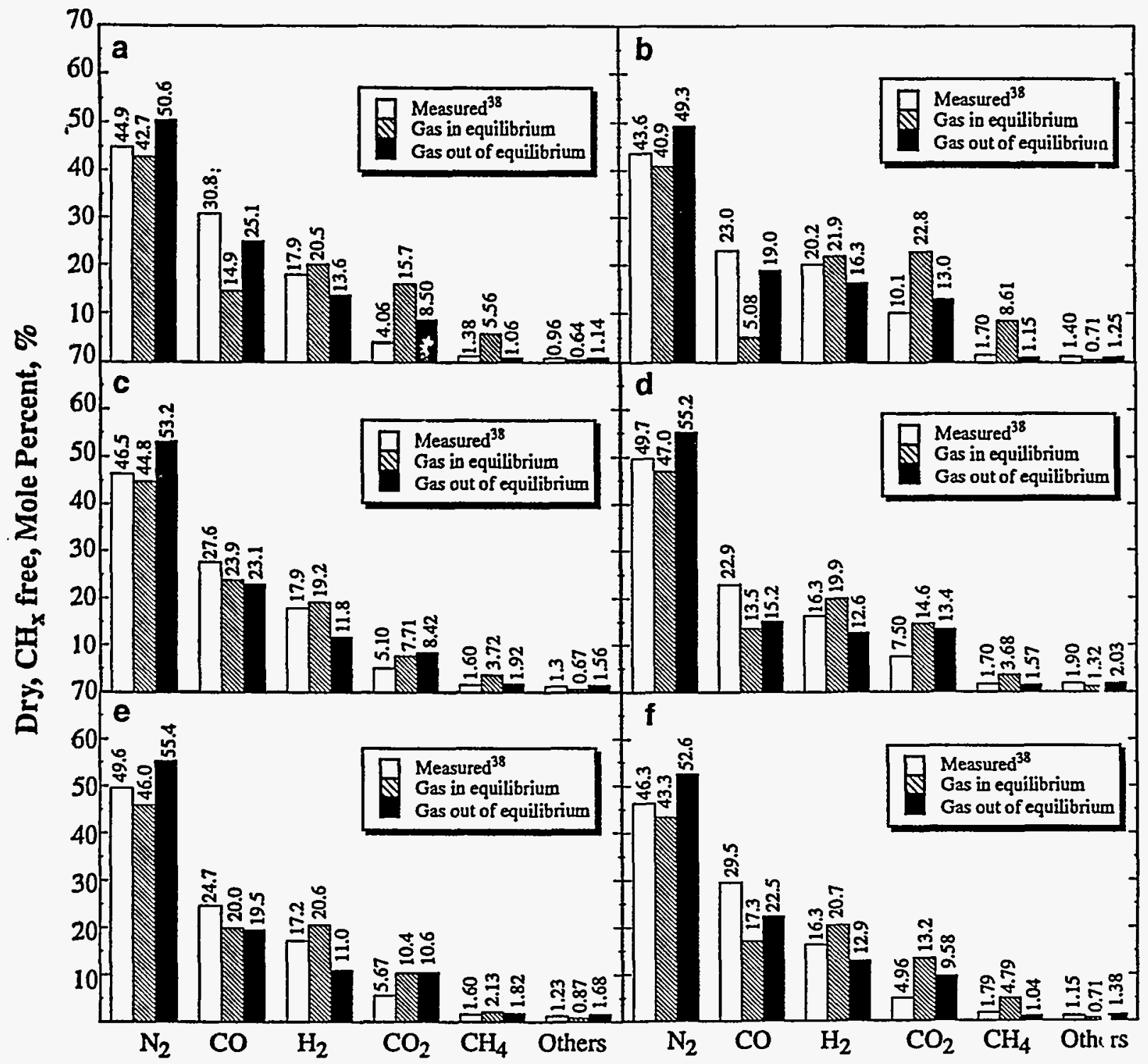

\section{Effluent Species}

Figure 10 Predicted and measured effiuent gas composition for Wellman-Galusha gasifier tests: (a) Absaloka/Robinson subbituminous: (b) Benton lignite; (c) Elkhorn bituminous; (d) Illinois no. 6 bituminous: (e) Jetson bituminous: (f) Kemmerer subbituminous. Input data are from Tables 2 and 4

tar-forming fraction than to the temperature. The coal gas composition is not very sensitive to either. This emphasizes the importance of predicting accurate volatiles distribution into tar, coal gas and chemical water.

Predicted fixed-bed effluent gas compositions compare reasonably well with experimental data for gasification of 13 coal types in two commercial-scale, fixed-bed gasifiers. However, the predicted tar yields from the correlation of Ko et al..$^{24}$ are high in comparison to the values measured in two commercial-scale, fixed-bed gasifiers. Part of the differences may be due to uncertainties in measurement of tar. More rigorous methods for predicting tar that depend on coal structure may improve these estimates.
The two-zone, partial equilibrium model may be preferred to more complex and computationally demanding one- or two-dimensional models when only effluent temperatures and composition are required and computational times must be minimized as in large process optimizations.

\section{ACKNOWLEDGEMENTS}

This work was sponsored principally by the US Department of Energy, Morgantown Energy Technol igy Center (Contract No. DE-AC21-86MC23075, Dr Richard Johnson, project officer) under subcont act from Advanced Fuel Research, Inc., East Hartford, Connecticut, and also in part by the Advanced 


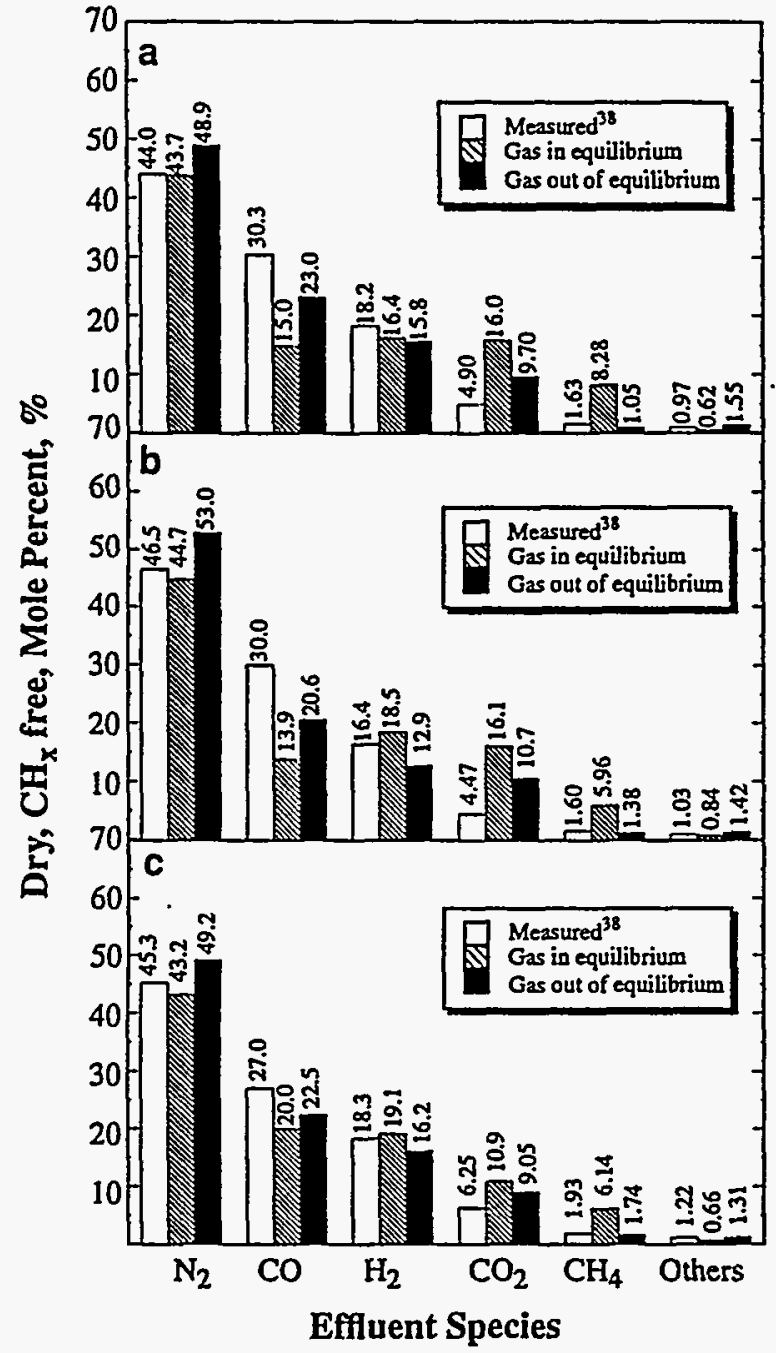

Figure 11 Predicted and measured effluent gas composition for Wellman-Galusha gasifier tests: (a) Leucite bituminous: (b) Rosebud subbituminous; (c) Utah Blind Canyon bituminous. Input data are from Tables 2 and 4

Combustion Engineering Research Center at Brigham Young University. Funds for this Center are received from the National Science Foundation's Engineering Centers Division (Dr Tapan Mukherjee, project officer), the State of Utah, and 25 industrial participants. Help from M. Usman Ghani is also acknowledged.

\section{REFERENCES}

1 Gumz, W. 'Gas Producers and Blast Furnaces', Wiley, New York, 1950

2 Woodmansee, E. E. Energy Commun. 1976, 2, 13

3 Kosky, P. G. and Floess, J. K. Ind. Eng. Chem. Process Des. Dev. 1980, 19, 586

4 Loison, R. and Chauvin, F. Chem. Ind. (Paris) 1964, 91, 269

5 Smott, L. D. and Smith, P. J. 'Coal Combustion and Gasification : Plenum Press, New York, 1985

6 Radulovic, P. T., Hobbs, M. L. and Smoot, L. D. Prog. Energy Combust. Sci. submitted

7 Amundson, N. R. and Arri, L. E. AlChE J. 1978, 24, 87

8 Yoon, H., Wei, J. and Denn, M. M. AICHE J. 1978, 24, 885

9 Desai, P.R. and Wen, C. Y. "Computer Modeling of the MERC Fixed Bed Gasifier', MERC/CF-78/3, US Department of
Energy, Murgantown. 1978

10 Stillman. R. IBM J. Res. Dev. 1979, 23. 240

11 Cho, Y. S. and Joseph. B. Ind. Eng. Cliem. Process Des. Der. $1981,20,314$

12 Yu, W. and Denn, M. M. Chem. Eng. Sci. 1983, 38, 1467

13 Eari. W. B. and Islım. K. A. 'CHEMCA 85', Thirteenth Australian Chemical Engineering Conference. Institute of Engineers. Barton. Atstralia, 1985, p. 289

14 Thorsness, C. B. and Kang. S. W. 'A General-Purpose Packed-Bed Model for Analysis of Underground Coal Gasifica'ion Processes', UCID-20731, Lawrence Livermort National Laboratory. University of California, Livermore, 1986

15 Bhattacharya, A., Salam, L.. Dudukovic, M. P. and Joseph, B. Ind. Ene. Chem. Process Des. Dev. 1986, 25, 988

16 Hobbs, M. L. PhD Dissertation Brigham Young University. Provo, :990 (see also Hobbs, M. L., Radulovic, P. T. and Smoot. L. D. AIChE J. in press)

17 Solomon, P. R., Hamblen, D. G., Carangelo, R. M. Serio, M. A. and Deshpande. G. Y. Energy Fuels 1988, 2, 405

18 Perry, R. H. and Chilton. C. H. (Eds), 'Chemical Engineering Handbook', 5th Edn, McGraw-Hill, New York, 1973

19 Johnson, J. L. in 'Chemistry of Coal Utilization' (Ed. M. A. Elliott ) 2nd suppl. vol., John Wiley, New York, 1981 Merrick, D. Fuel $1983,62,540$

21 Mills, K. C. and Rhine. J. M. Fuel 1989, 68, 20

22 Pratt, D. T. in 'Pulverized-Coal Combustion and Gasification (Eds L. D. Smoot and D. T. Pratt), Plenum Press, New York, 1979

23 Stull, D. R. and Prophet, H. 'JANAF Thermochemical Tables' 2nd Edn, National Bureau of Standards, Washington, DC, 1971

24 Ko, G. H., Sanchez, D. M., Peters, W. A. and Howard, J. B. 'Twenty-Second Symposium (International) on Combustion" The Combustion Institute, Pittsburgh, 1988, p. 115

25 Solomon, P. R. and Hamblen, D. G. in "Chemistry of Coal Conversion' (Ed. R. H. Schlosberg), Plenum Press, New York, 1985

26 Khan, M. R. Fuel 1989, 68, 1522

27 Serio, M. A., Hamblen, D. G., Markham, J. R. and Solomon P. R. Energy Fuels 1987, 1, 138

28 Anthony, D. B., Howard, J. B. Hottel, H. C. and Meissner. M. P. 'Fifteenth Symposium (International) on Combustion", The Combustion Institute, Pitssburgh, 1974, p. 1303

29 Abramowitz, M. and Stegun, 1. A. (Eds), 'Handbook of Mathematical Functions with Formulas Graphs, and Mathematical Tables', Applied Mathematics Series no. 55, National Bureau of Standards, US Government Printing Office. Washington, DC, 1972

30 Blair, D. W., Wendt, J. O. L. and Bartok, W. 'Sixteenth Symposium (International) on Combustion', The Combustion Institu:c, Pittsburgh, 1977. p. 475

31 Solomon, P. R, and Colket, M. B. 'Seventeenth Symposium (International) on Combustion'. The Combustion Institute. Piltsburgh, 1979, p. 131

32 Suuberg, E. M., Peters, W. A. and Howard. J. B. 'Seventeenth Symposium (International) on Combustion'. The Combustion Institute, Pittsburgh. 1979, p. 177

33 Rudolph, P. 'Fourth Synthetic Pipeline Gas Symposium" American Gus Institute, Chicago, 1972, p. 175

34 Serio, M. A. personal communication, Advanced Fuel Research, Inc.. East Hartford, 1989

35 Elgin, D. C. and Perks, H. R. 'Sixth Synthetic Pipeline Gas Symposium', American Gas Institute, Chicago, 1974, p. 247

36 Elgin, D. C. and Perks, H. R. 'Fifth Synthetic Pipeline Gas Symposium', American Gas Institute, Chicago, 1973, p. 145

$37 \mathrm{Kim}, \mathrm{M}$. and Joseph, B. Ind. Eng. Chem. Process Des. Dev. 1983, 22,212

38 Thimsen, D., Maurer, R. E., Pooler, A. R., Pui, D. Y. H., Liu, B. Y. H. and Kittelson. D. B. 'Fixed-Bed Gasification Research Using U.S. Coals', Vols 1-19, US Bureau of Mines Contract H0222001 Final Report, Houston, 1984-1985

39 Thimsen, D. P. and Maurer, R. E. Coal Devolatilization in a Moving-Bed Gasifier', GS-6797, Research Project 2524-14 Final Report, Electric Power Research Institute, Palo Alto, 1990

40 Solomon, P. R., Hamblen, D. G., Carangelo, R. M., Setio, M. A. and Deshpande, G. Y. Fuel 1990, 69, 754

41 Grant, D. M., Pugmire, R. J., Fletcher, T. H. and Kerstein, A. R. Energy Fuels 1989, 3, 175

42 Niksa, S. AIChE J. 1988, 34, 790

43 Watkinsun, A.P., Lucas, J. P. and Lim, C.J. Fuel 1991, 70, 519 


\section{APPENDIX D}

\section{MODELING FIXED-BED COAL GASIFIERS}




\title{
Modeling Fixed-Bed Coal Gasifiers
}

\author{
Michael L. Hobbs, Predrag T. Radulovic, and L. Douglas Smoot \\ Advanced Combustion Engineering Research Center, Brigham Young University, Provo, UT 84602
}

\begin{abstract}
A one-dimensional model of countercurrent fixed-bed coal gasification has been developed, and results have been compared to experimental data from commercialscale gasifiers. The steady-state model considers separate gas and solid temperatures, axially variable solid and gas flow rates, variable bed void fraction, coal drying, devolatilization based on chemical functional group composition, oxidation and gasification of char, and partial equilibrium in the gas phase. Generalized treatment of gas-phase chemistry and accounting for variable bed void fraction were necessary to predict realistic axial temperature and pressure profiles in an atmospheric fixedbed gasifier. Model evaluation includes sensitivity of axial temperature profiles to model options, model parameters and operational parameters. Model predictions agree reasonably well with experimental temperature and pressure profile data for gasification of eight coal types ranging from lignite to bituminous. The relative importance of char oxidation resistances to bulk film diffusion, ash diffusion, and chemical reaction is identified.
\end{abstract}

\section{Introduction}

Fixed-bed systems are simple and reliable, have high thermal efficiency, and require minimal pretreatment of feed coal. Combustion and gasification of coal in fixed or slowly moving beds of packed coal particles are of substantial commercial interest. Such beds can be operated at high pressure, providing opportunity for increased power generation efficiency through combined cycle processes. Fixed beds are also a popular choice for mild gasification since, by their countercurrent nature, the liquids can be quickly removed before being altered by substantial reaction. The U.S. Clean Coal Technology demonstration program includes two fixed-bed gasifier processes undergoing commercial demonstration.

In a large-scale, fixed-bed gasifier, Figure 1, coal is fed to the top of the reactor and moves downward under gravity, countercurrent to the rising gas stream. The dry or slagging ash is removed at the bottom of the reactor. The feed gas is commonly composed of air or oxygen and steam. Excess steam is supplied to the gasifier to control the ash temperature. Figure 1 also shows the reactor divided into four overlapping zones: drying, devolatilization, gasification, and combustion. As the coal slowly descends, the hot gases produced in the gasification and combustion zones exchange energy with the colder solid. Water and subsequently volatile matter are released when the

Correspondence concerning this article should be addressed $h$, L. D. Smoot.

M. L. Hobbs is presently at the Sendia National Laboratories, P. O. Box 5800, Div. 1512. tibuquerque, NM 87185.

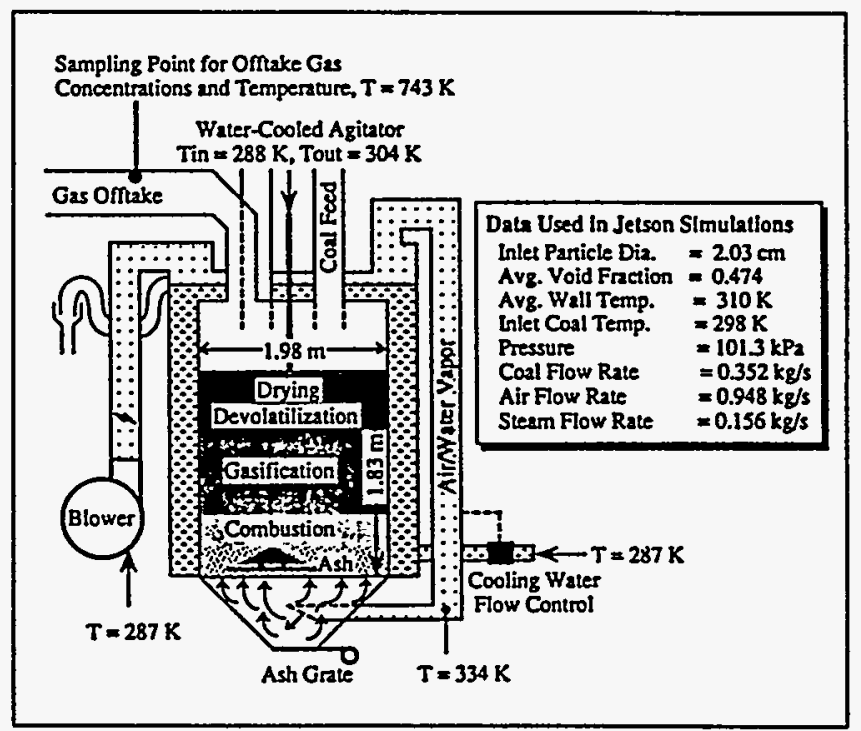

Figure 1. Typical atmospherlc fixed-bed gasifier (Wellman-Galusha).

Temperatures are for gasification of Jetson coal blown with air. Configuration and data taken from Thimsen et al. (1984).

solid reaches sufficiently high temperatures. After drying and devolatilization, the char enters the gasification zone where carbon reacts with steam, carbon dioxide, and hydrogen. Endothermic reactions in this section produce carbon monoxide 
and hydrogen. The slightly exothermic reaction of hydrogen with carbon produces methane. Differentiation between the "gasification zone" and "combustion zone" is based on the presence or absence of free oxygen. Combustion and gasification reactions occur simultaneously in the "combustion zone." Combustible gases such as carbon monoxide or hydrogen may react with oxygen. The exothermic combustion reactions provide the necessary energy for the endothermic gasification reactions and drying. Blast gas, which is composed of steam and oxidant (air or oxygen), is preheated by the hot ash. Part of the process steam is produced by a water jacket surrounding the gasification chamber.

Solid residence times in the drying, gasification and oxidation zones may be on the order of several hours. Residence time in the ash layer may be even higher depending on the thickness of this zone. Large solid residence times indicate significant settling resulting in variable axial velocities. Gas residence times are on the order of seconds. Both solid and gas heating rates are most dramatic in the devolatilization and the oxidation zones. Typical solid heating rates are smaller than $10 \mathrm{~K} / \mathrm{s}$, while gas heating rates are up to $10,000 \mathrm{~K} / \mathrm{s}$.

Existing fixed-bed models in the open literature show that all fixed-bed models were qualitative until the late 1970s. At that time, Winslow (1976) presented a detailed model for underground coal gasification. Soon afterward, Amundson and Arri (1978), Yoon et al. (1978), Desai and Wen (1978), and Stillman (1979) presented detailed models of a fixed-bed gasifier. Cho and Joseph (1981) extended Yoon's model to include unequal gas-solid temperatures. Yoon's model was further extended by Kim and Joseph (1983) to account for transient effects. Yu et al. (1983) extended Yoon's model to two space dimensions. More recent models include the one-dimensional, steady-state model of Earl and Islam (1985) and the two-dimensional, transient models of Thorsness and Kang (1986) and Bhattacharya et al. (1986). Khanna and Seinfeld (1987) discussed recent advances in catalytic fixed-bed reactor models which have many of the features of coal gasification/combustion fixed-bed models discussed earlier. Smoot and Smith (1985), Hobbs (1990), and Radulovic et al. (1992) have reviewed fixed-bed modeling.

Previous fixed-bed models share common assumptions such as equal gas/solid temperatures, axially uniform gas/solidphase plug flow, uniform bed porosity, instantaneous devolatilization (with percent volatiles from proximate analysis), combustion/gasification by shell progressive or ash segregation submodels, kinetic parameters from small particle data, and little or no gas-phase chemistry. The fixed-bed model discussed here eliminates most of these assumptions. The primary contribution of this article is the development and evaluation of an improved, comprehensive fixed-bed model that utilizes an advanced, coal-general devolatilization submodel.

\section{One-Dimensional Fixed-Bed Model Conservation equations}

The conservation equations for mass and energy form the foundation of the model. The derivation of the two-phase conservation equations can be found in Crowe and Smoot (1979). The source terms in the continuity and energy equations are described by physical and chemical submodels. Input parameters are reactor dimensions, operating conditions, inlet solid and gas temperatures, pressure, concentrations, flow rates, and wall temperature. Calculated quantities include axial variation in gas temperature, solids temperature, pressure, species concentration, gas-flow rate, solid flow rate, and wall heat loss. Plug flow is assumed in both the solid and gas phases with variable axial velocities. Gas-phase pressure drop is calculated with the Ergun equation for packed beds (Ergun, 1952). An effective heat-transfer coefficient is used for heat loss to the wall, including both stagnant and dynamic contributions for convective and radiative transfer. Large coal particle devolatilization is allowed to occur simultaneously with char oxidation and gasification. Shell progressive or ash segregation, shrinking core char submodels describe oxidation and gasification. Chemical equilibrium is used to calculate gas concentrations and temperatures. Turbulence is not treated formally in the slowly moving bed with low gas velocities, but is included implicitly through model correlations such as the effective heat-transfer coefficient. Primary assumptions for the one-dimensional fixed-bed model include negligible aerodynamic drag, ideal gases, and particles locally isothermal throughout; one particle size and type exist in the feed coal. This model will be referred to as MBED-1D (moving-bed, onedimensional) here. The reactor, however, is frequently referred to as a fixed-bed reactor because of the large difference in gasand solid-phase residence times. Assumptions are discussed in detail by Hobbs (1990).

The conservation equations and boundary conditions for the one-dimensional, fixed-bed model are given in Table 1. These can be classified as gas and solid overall continuity, gas and solid energy equations, and gas and solid species or elemental continuity equations. The constitutive relations for solid flow have been proposed only recently, and no solution for these equations has been attempted (Gray and Stiles, 1988). Thus, only differential equations for continuity and energy are treated in the model described here.

The overall gas and solid species continuity equations are given in Eqs. 1 and 2 in Table 1 . The gas and solid flow rates are represented by $W_{\varepsilon}(\mathrm{kg} / \mathrm{s})$ and $W_{s}(\mathrm{~kg} / \mathrm{s})$, respectively. The axial distance and cross-sectional areas are represented by $z$ (m) and $A\left(\mathrm{~m}^{2}\right)$, respectively. The volumetric reaction rate is represented by $r_{i}\left(\mathrm{~kg} / \mathrm{m}^{3} \cdot \mathrm{s}\right)$ where $i$ depicts the different heterogeneous reactions such as drying, char oxidation, char gasification, and devolatilization. Reaction rates are written as the rate of mass addition to the gas phase per unit volume per unit time; thus, a positive rate indicates a source of mass for the gas phase and a sink for the particle phase.

The gas and solid energy equations are given by Eqs. 3 and 4 in Table 1. The total gas and solid enthalpies are represented by $h_{g}(\mathrm{~J} / \mathrm{kg})$ and $h_{s}(\mathrm{~J} / \mathrm{kg})$. The energy exchange between solid and gas is represented by $Q_{s z}\left(\mathrm{~W} / \mathrm{m}^{3}\right)$. Heat losses to the walls from the gas and solid phases are represented by $Q_{z w}\left(\mathrm{~W} / \mathrm{m}^{3}\right)$ and $Q_{s w}\left(\mathrm{~W} / \mathrm{m}^{3}\right)$, respectively. Calculation of $Q_{s z}, Q_{z w}$, and $Q_{s w}$ will be discussed subsequently. The last term in the energy equation represents the energy exchange due to chemical reaction and accounts for mass loss from the particle phase and mass addition to the gas phase. This reaction term can be calculated by performing an energy balance around the particle/gas interface as discussed in detail by Hobbs (1990). The gaseous reactants are assumed to be at the gas temperature, and the reaction is assumed to take place at the solid temperature. A sample calculation associated with the term $r_{i} h_{i z}$ for the oxidation reaction is given by Hobbs (1990). 
Table 1. Differential Equation Set and Boundary Conditions for MBED-1D

\begin{tabular}{|c|c|c|}
\hline Overall Gas Species Continuity & $\frac{d W_{g}}{d z}=\mathrm{A} \sum_{n_{1}}^{n} r_{1}$ & (1) \\
\hline $\begin{array}{l}\text { Overall Solid Species Continuity } \\
\text {.. }\end{array}$ & $\frac{d W_{s}}{d z}=-A \sum_{i=1}^{n} r_{i}$ & (2) \\
\hline Gas-Phase Energy & $\frac{d W_{z} h_{g}}{d z}=A\left(Q_{s z}-Q_{z w}+\sum_{i=1}^{n} r h_{i 2}\right)$ & (3) \\
\hline Solid-Phase Energy & $\frac{d W_{s} h_{s}}{d z}=A\left(-Q_{s z}-Q_{s w}+\sum_{i=1}^{n} r_{s} h_{i z}\right)$ & (4) \\
\hline $\begin{array}{l}\text { Solid Species Continuity } \\
\text { Moisture }\end{array}$ & $\frac{d W_{\text {moixture }}}{d z}=-A r_{\text {moisture }}$ & (5) \\
\hline Nonvolatile carbon & $\frac{d W_{\text {Nonvolutik }} \mathrm{c}}{d z}=-A r_{\text {Noaroltetik } \mathrm{c}}$ & (6) \\
\hline Nonvolatile sulfur & $\frac{d W_{\text {Nonvolutile }} \mathrm{S}}{d z}=-A r_{\text {Nonvolutile } \mathrm{S}}$ & (7) \\
\hline Organic functional groups & $d y_{1} / d z=-\left(1 / u_{s}\right) k_{2} y_{1}$ & $(8-26)$ \\
\hline $\begin{array}{l}\text { Tar fraction } \\
\text { Gas-Phase Elemental Continuity* }\end{array}$ & $\begin{array}{l}d x / d z=-\left(1 / u_{s}\right) k_{x} x \\
\frac{d W_{g} \omega_{j}}{d z}=A \sum_{i=1}^{n} r_{i J}\end{array}$ & $\begin{array}{r}(27) \\
(28-32)\end{array}$ \\
\hline Gas-Phase Tar Species Continuity & $\frac{d W_{\text {tar }}}{d z}=A \sum_{i=1}^{n} r_{\text {lus }}$ & (33) \\
\hline Gas-Phase Tar Elemental Continuity* & $\frac{d W_{\mathrm{tar}} \omega_{\mathrm{ur} J j}}{d z}=A \sum_{j=1}^{n} r_{i j}^{\mathrm{tur}}$ & $(34-38)$ \\
\hline $\begin{array}{l}\text { Boundary Conditions } \\
\text { Overall solid/gas species continuity } \\
\text { Solid/gas-phase energy } \\
\text { Solid species continuity } \\
\text { Gas-phase elemental and tar continuity }\end{array}$ & $\begin{array}{l}\text { Feed coal/gas weight flow rate } \\
\text { Feed coal/gas enthalpy or temperature } \\
\text { Proximate and functional group analysis } \\
\text { Feed gas and tar composition }\end{array}$ & \\
\hline
\end{tabular}

- $j$ represents carbon, hydrogen, nitrogen, oxygen, and sulfur.

\section{Auxiliary equations}

Pressure Drop. The pressure drop in the reactor is calculated from Ergun's equation (Ergun, 1952) since Reynolds numbers are typically less than 500 in fixed-bed coal gasifiers. The small pressure drop in the reactor is sensitive to the bed void fraction, $\epsilon$. Void fractions of the feed coal at the bed top and the product ash at the bed bottom were estimated based on coal and ash bulk and apparent density measurements. The void fraction was assumed to vary linearly from the top to the bottom of the bed. In two sets of independent measurements, Krishnudu et al. (1989) stopped coal bed reaction and measured bed void fraction along the bed length, which was shown to vary linearly from the top to the bottom of the bed. Subsequent comparisons with pressure drop measurements provide support for this approach.

Gas-Phase Chemistry. Gas temperature is determined by assuming all gas species to be in thermal equilibrium and partial or total chemical equilibrium. Gas-phase composition is determined by Gibbs free energy minimization. Solid temperature is determined from the solid enthalpy and the elemental composition of the coal. All gas-phase transport properties (such as conductivity, viscosity, and diffusivity) are considered to be functions of both temperature and composition. Partial equilibrium refers to a gaseous mixture where at least one species is held out of chemical equilibrium. Hobbs et al. (1992) investigated three options regarding equilibrium: total equilibrium, partial equilibrium where tar was held out of chemical equilibrium, and partial equilibrium where all gases in the drying and devolatilization zone were assumed to be nonreactive. Since gas-phase kinetic models for coal systems are com- plex, the one-dimensional fixed-bed model assumes partial equilibrium by holding tar out of chemical equilibrium.

Mass and Heat Transfer. Mass- and heat-transfer processes in fixed-bed gasifiers are affected by complex solids flow and chemical reactions. Coarsely crushed coal settles while undergoing heating, drying, devolatilization, gasification, and combustion. Coal particles change in diameter, shape, and porosity. Nonideal behavior may result from coal bridges, gas bubbles, and channels. Variable bed void fraction may also change heat- and mass-transport properties. Correlations for solid-to-gas heat-transfer coefficients are questionable, since they are typically obtained under ideal conditions. Mass transfer occurs by diffusion and convection. Heat transfer is by conduction, convection, and radiation in the gas and solid phases.

Several physical properties of the gas and particle phases are required to obtain mass- and heat-transfer coefficients needed to solve the differential equation set in Table 1. Chapman-Enskog theory has been used to calculate multicomponent gas mixture viscosity and diffusivity (Bird et al., 1960). Conventional gas species and mixture conductivity, viscosity, and diffusivity methods used here are documented by Hobbs (1990). The particle is assumed to swell linearly with the extent of devolatilization. Euken's formula is used to calculate the conductivity of individual gaseous species (Bird et al., 1960). Furthermore, the gaseous tar diffusivity is assumed to be 0.1 $\mathrm{cm}^{2} / \mathrm{s}$ at standard temperature and pressure (Suuberg et al., 1979). The pressure and temperature dependences for the tar diffusivity are assumed to follow Chapman-Enskog theory. The JANAF tables were used to provide values for calculating gas-phase enthalpy, entropy and heat capacity (Stull and 
Prophet, 1971). Dulong's formula (Perry and Chilton, 1973) was used for heating value of the char, Merrick's correlations (1983) were used for char enthalpy and heat capacity, and the Kopp-Neumann rule was used for ash heat capacity (Mills and Rhine, 1989).

- The heat- and mass-transfer correlations used in the onedimensional model are summarized in Table 2. Effective axial and radial conductivities are correlated by Yagi et al. (1960), Bischoff (1962), and Froment and Bischoff (1979), respectively. Both the axial and radial effective conductivities take into account molecular as well as turbulent contributions. The effective radial conductivities of the gas and solid phases, which account for radiation, are correlated by DeWasch and Froment (1971). The mass-transfer coefficient in Table 2 is used to calculate the film resistance and the effective particle resistance to mass transport.

The effective bed-to-wall heat-transfer coefficient as well as the gas- and solid-phase contributions are determined by the correlations suggested by DeWasch and Froment (1971). The heat transfer to the wall is treated by Yagi and Wakao (1959), Yagi and Kunii (1960), and Rohsenow et al. (1985). There are no direct experimental data available on the gas- and solidphase contributions to the bed-to-wall heat transfer. The volumetric heat-transfer rate from solid to gas, $Q_{s z}\left(\mathrm{~J} / \mathrm{m}^{3} \cdot \mathrm{s}\right)$, can be written as:

$$
Q_{s z}=\zeta h_{s z} \pi d_{p}^{2} \eta_{p}\left(T_{s}-T_{s}\right)
$$

where $\zeta, h_{s z}\left(\mathrm{~J} / \mathrm{s} \cdot \mathrm{m}^{2} \cdot \mathrm{K}\right)$, and $\eta_{p}\left(1 / \mathrm{m}^{3}\right)$ represent deviations from nonreactive solid-to-gas heat transfer (ranges from 0.02 to 1.0), the nonreactive solid-to-gas heat-transfer coefficient (in Table 2), and the particle number density, respectively. The volumetric wall heat losses from the solid and gas phase, $Q_{z w}$ and $Q_{s w}\left(\mathrm{~J} / \mathrm{m}^{3} \cdot \mathrm{s}\right)$, can be calculated as follows:

$$
Q_{z w}=\frac{4 h_{w}^{g}}{D}\left(T_{z}-T_{\text {wall }}\right)
$$

Table 2. Heat- and Mass-Transport Correlations Used in MBED-1D

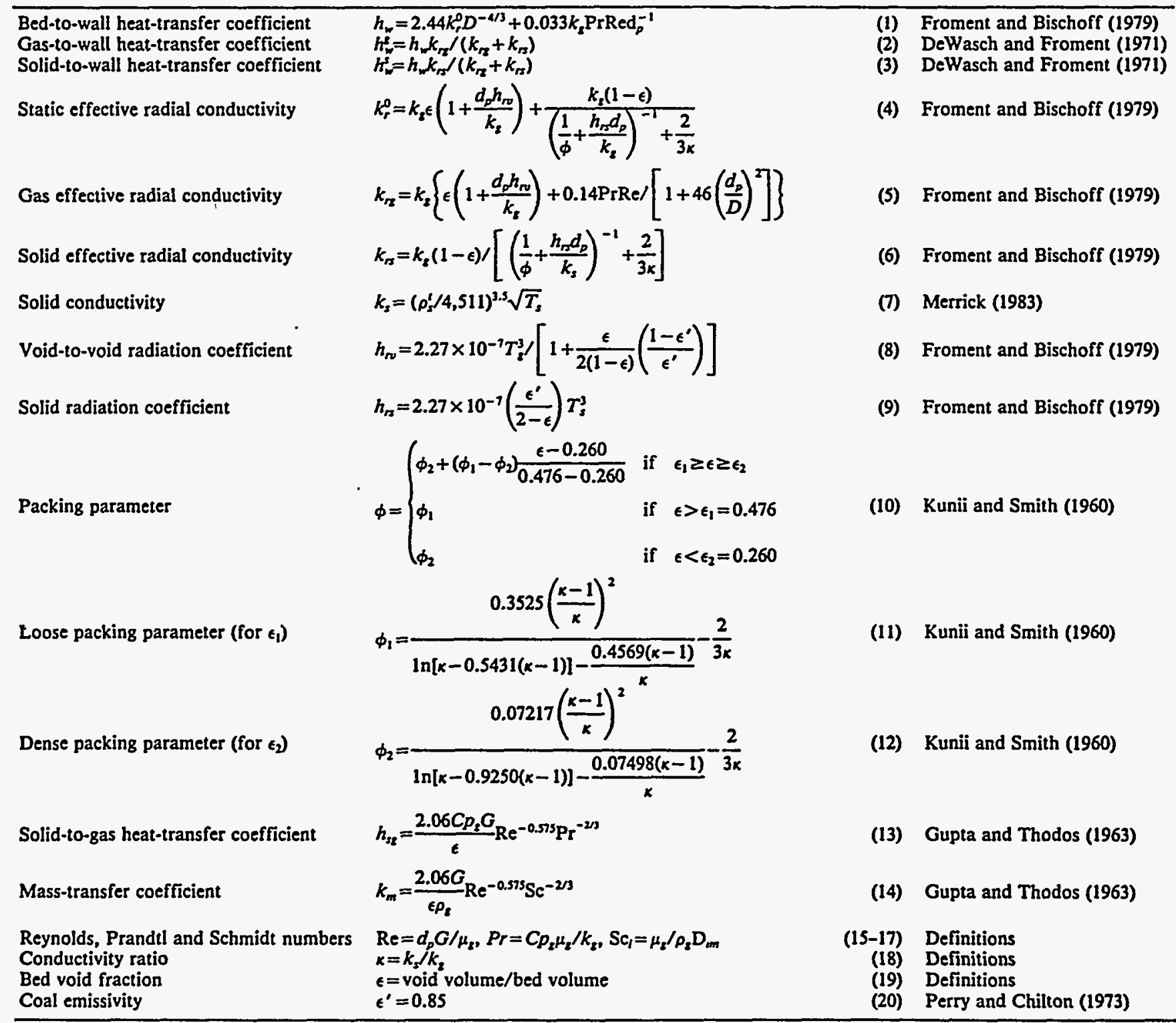




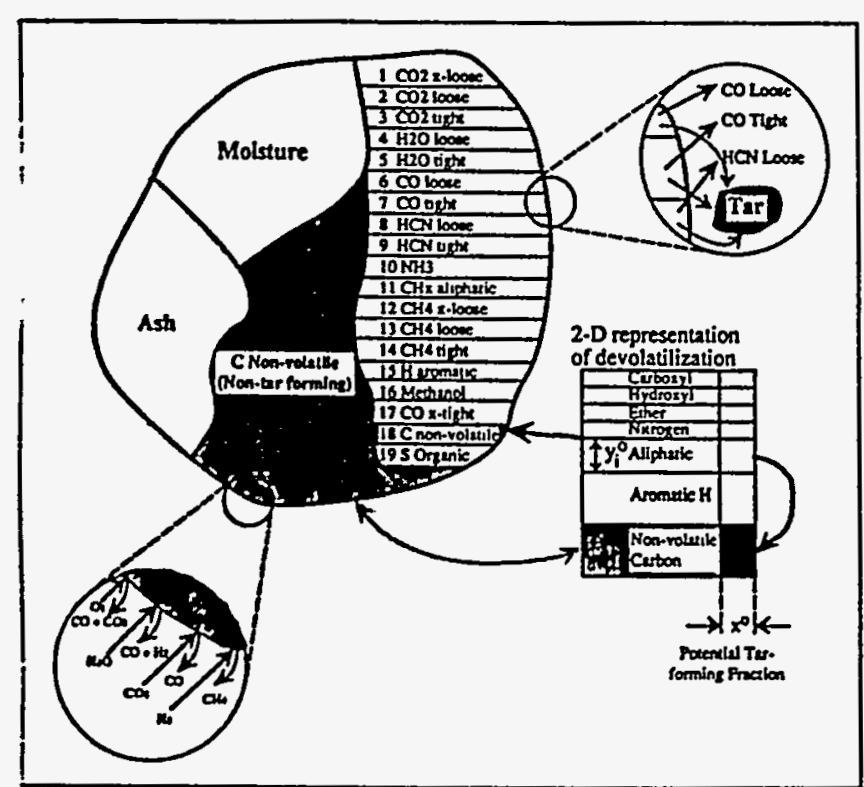

Figure 2. Coal particle with devolatilization model based on chemical functional groups (Solomon et al., 1988).

The potential tar-forming fraction of the nonvolatile carbon functional group evolves as tar. The nontar-forming $C$ and $S$ organic groups evolve via heterogeneous char oxidation or gasification.

$$
Q_{s w}=\frac{4 h_{w}^{s}}{D}\left(T_{s}-T_{w 211}\right)
$$

ae wall temperature can be varied from the bottom of the reactor to the top of the reactor.

Coal Drying and Devolatilization. Coal reaction source terms represent drying, devolatilization, char oxidation, and gasification. These chemical and physical processes in Figure 2 show a conceptual coal particle divided into various functional groups, including moisture and ash, which is taken to ie inert. Drying is assumed to be diffusion-limited (Smoot and imith, 1979):

$$
r_{n}=k_{w m}\left(\rho_{w p}-\rho_{w z}\right)
$$

Blowing effects have been neglected.

As shown in Figure 2, devolatilization is described by assuming that the organic portion of the coal particle is composed of various functional groups, such as carboxyl, hydroxyl, ether, and nitrogen. A functional group model (FG model) has been used to describe the devolatilization process (Serio et al., 1987; Solomon et al., 1988). The kinetics for functional group evolution are considered to be independent of the type of coal used.

Application of the functional group devolatilization model of Solomon et al. (1988), used here, is discussed in detail by Hobbs (1990). This section describes the devolatilization rate equations which appear as source terms in the continuity equations in Table 1. The change in functional group composition and tar fraction is obtained by Eqs. 8 through 27 in Table 1. The evolution of each functional group into the gas phase is represented by first-order decay. The equations representing this first-order decay are not truly solid species conservation equations; however, Eqs. 8 through 27 are used to keep track of the functional groups in the solid phase throughout the reactor. The values for Arrhenius rate coefficients, $k_{i}$ and $k_{x}$, and the initial functional group compositions, $y_{i}^{\circ}$, can be found in Hobbs (1990) or Hobbs et al. (1992).

The mass rate of change of each reactive element in the gas phase is depicted by Eqs. 28 through 32 in Table 1. Gases such as argon are inert to reaction, and a differential equation is not required to track such gases. The sources for gas-phase elements are the heterogeneous oxidation, gasification and devolatilization reactions. The only gas-phase species that is tracked throughout the reactor is tar. Tar was either assumed to be nonreactive or to react to equilibrium in the gas phase.

Predicting accurate coal volatiles yield and composition is important since as much as $40-60 \%$ of the dry, ash-free mass can be lost by devolatilization. The coal particle in Figure 2 is divided into various chemical functional groups. The $X$ and $Y$ values represent the two-dimensional description of coal. The $Y$ dimension is divided into fractions according to the chemical composition of the coal. The initial fraction of a particular functional group component is represented by $y_{i}^{\circ}$ and the sum of $y_{i}^{\text {is }}$ equals 1 . The evolution of each functional group into the gas is represented by the first-order decay of the $Y$ dimension, $d y_{i} / d t=-k_{i} y_{i}$. The $X$ dimension represents nontar-forming char, tar-forming char, and tar. The evolution of tar is represented by the first-order decay of the $X$ dimension, $d x / d t=-k_{x} y_{1}$. The potential tar-forming fraction, $x^{\circ}$, was calculated with the semi-empirical correlation of Ko et al. (1988) as discussed by Hobbs et al. (1992). Values for $y_{i}$ can be found subsequently in Table 3. Normally distributed Arrhenius rate coefficients for 19 functional groups, $k_{i}$, and tar, $k_{x}$, were obtained from Solomon et al. (1988) for the organic functional groups in Figure 2.

The volumetric devolatilization rate expressions, $r_{i}^{4}\left(\mathrm{~kg} / \mathrm{m}^{3} \cdot \mathrm{s}\right)$ are derived by Hobbs (1990):

$$
\begin{aligned}
& r_{i}^{d}= \\
& \quad \rho_{s m}^{o}\left(1-\epsilon^{0}\right)\left(1-\Omega_{\text {sch }}^{o}-\Omega_{\text {molsure }}^{o}\right)\left[\left(1-x^{\circ}+x\right)\left(k_{i} y_{i}\right)+y_{l}\left(k_{x} x\right)\right]
\end{aligned}
$$

$r_{i}^{d}$ includes mass losses due to both light gas evolution and tar evolution, which are required by the continuity equations in Table 1. However, the tar continuity equations require the tar volumetric rate equations, $r_{l, u r}^{d}\left(\mathrm{~kg} / \mathrm{m}^{3} \cdot \mathrm{s}\right)$, which are also found in Hobbs (1990):

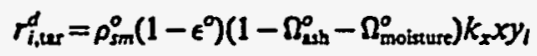

Equations 5 and 6 do not explicitly include resistance due to mass transport. Saxena (1990) concludes that heat- and mass-transport limitations in large coal particles are significant. Coupling between mass transport of oxidation and gasification products and devolatilization complicates the mathematical description of both devolatilization and char oxidation. Simulations with the purely kinetic devolatilization equations as formulated above can result in unrealistically fast product evolution for large coal particles. To introduce mass transport effects, transport resistance through the film and particle is added to the Arrhenius rate expression as diffusional resistances: 
Table 3. Functional Group Compositions, Calculated Potential Tar-Forming Fractions, and Ultimate Analysis*

\begin{tabular}{|c|c|c|c|c|c|c|c|c|c|c|c|}
\hline & $\begin{array}{l}\text { Reactor"- } \\
\text { Functional Groups } \\
\text { (Dry, Ash-Free) }\end{array}$ & $\begin{array}{c}\text { Lurgi } \\
\text { Rose } \\
y_{i}^{0}\end{array}$ & $\begin{array}{c}\text { WG } \\
\text { Absal } \\
y_{i}^{0}\end{array}$ & $\begin{array}{c}\text { WG } \\
\text { Benton } \\
y_{i}^{0}\end{array}$ & $\begin{array}{l}\text { WG } \\
\text { Elk } \\
y_{i}^{0}\end{array}$ & $\begin{array}{c}\text { WG } \\
\text { Jetson } \\
y_{i}^{0}\end{array}$ & $\begin{array}{c}\text { WG } \\
\text { Kemer } \\
y_{i}^{0}\end{array}$ & $\begin{array}{c}\text { WG } \\
\text { Leucite } \\
y_{i}^{0}\end{array}$ & $\begin{array}{l}\text { WG } \\
\text { Rose } \\
y_{i}^{0}\end{array}$ & $\begin{array}{l}\text { WG } \\
\text { Utah } \\
y_{i}^{0}\end{array}$ & \\
\hline$\cdots$ & $\begin{array}{l}\mathrm{CO}_{2} \text { extra loose } \\
\mathrm{CO}_{2} \text { loose } \\
\mathrm{CO}_{2} \text { tight } \\
\mathrm{H}_{2} \mathrm{O} \text { loose } \\
\mathrm{H}_{2} \mathrm{O} \text { tight } \\
\mathrm{CO} \text { ether loose } \\
\mathrm{CO} \text { ether tight } \\
\mathrm{HCN} \text { loose } \\
\mathrm{HCN} \text { tight } \\
\mathrm{NH}_{3} \\
\mathrm{CH}_{\mathrm{x}} \text { aliphatic } \\
\mathrm{CH}_{4} \text { extra loose } \\
\mathrm{CH}_{4} \text { loose } \\
\mathrm{CH}_{4} \text { tight } \\
\mathrm{H}_{\text {aromatic }} \\
\mathrm{CH}_{3} \mathrm{OH} \\
\mathrm{CO} \text { extra tight } \\
\mathrm{C} \text { nonvolatile } \\
\mathrm{S} \text { organic } \\
\text { Total } \\
\text { calculated } x^{\circ *} \\
\mathrm{Coal} \text { type } \\
\text { DAF ultimate } \\
\mathrm{C} \\
\mathrm{H} \\
\mathrm{N} \\
\mathrm{O} \\
\mathrm{S} \text { (organic) }\end{array}$ & $\begin{array}{l}0.035 \\
0.035 \\
0.030 \\
0.000 \\
0.051 \\
0.055 \\
0.000 \\
0.010 \\
0.015 \\
0.001 \\
0.182 \\
0.000 \\
0.022 \\
0.012 \\
0.013 \\
0.000 \\
0.000 \\
0.522 \\
0.017 \\
1.000 \\
0.110 \\
\text { Subbit } \\
\\
0.771 \\
0.049 \\
0.014 \\
0.150 \\
0.017\end{array}$ & $\begin{array}{l}0.028 \\
0.020 \\
0.021 \\
0.030 \\
0.022 \\
0.050 \\
0.043 \\
0.000 \\
0.013 \\
0.001 \\
0.169 \\
0.000 \\
0.017 \\
0.008 \\
0.013 \\
0.000 \\
0.043 \\
0.518 \\
0.004 \\
1.000 \\
0.137 \\
\text { Subbit } \\
\\
0.763 \\
0.051 \\
0.008 \\
0.174 \\
0.004\end{array}$ & $\begin{array}{l}0.065 \\
0.030 \\
0.005 \\
0.000 \\
0.027 \\
0.060 \\
0.000 \\
0.005 \\
0.013 \\
0.001 \\
0.212 \\
0.000 \\
0.017 \\
0.009 \\
0.017 \\
0.000 \\
0.090 \\
0.440 \\
0.009 \\
1.000 \\
0.227 \\
\text { Lig } \\
0.737 \\
0.062 \\
0.010 \\
0.182 \\
0.009\end{array}$ & $\begin{array}{l}0.000 \\
0.006 \\
0.005 \\
0.011 \\
0.011 \\
0.050 \\
0.044 \\
0.022 \\
0.009 \\
0.000 \\
0.185 \\
0.020 \\
0.015 \\
0.015 \\
0.012 \\
0.000 \\
0.020 \\
0.565 \\
0.009 \\
0.999 \\
0.270 \\
B i t \\
0.825 \\
0.057 \\
0.016 \\
0.093 \\
0.009\end{array}$ & $\begin{array}{l}0.000 \\
0.006 \\
0.005 \\
0.011 \\
0.011 \\
0.050 \\
0.059 \\
0.025 \\
0.009 \\
0.000 \\
0.161 \\
0.020 \\
0.015 \\
0.015 \\
0.012 \\
0.000 \\
0.020 \\
0.565 \\
0.016 \\
1.000 \\
0.222 \\
\text { Bit }\end{array}$ & $\begin{array}{c}0.028 \\
0.020 \\
0.021 \\
0.027 \\
0.022 \\
0.050 \\
0.000 \\
0.003 \\
0.018 \\
0.001 \\
0.201 \\
0.000 \\
0.017 \\
0.008 \\
0.013 \\
0.000 \\
0.043 \\
0.518 \\
0.010 \\
1.000 \\
0.219 \\
\text { Subbit }\end{array}$ & $\begin{array}{l}0.028 \\
0.020 \\
0.021 \\
0.024 \\
0.022 \\
0.050 \\
0.000 \\
0.016 \\
0.018 \\
0.001 \\
0.195 \\
0.000 \\
0.017 \\
0.008 \\
0.013 \\
0.000 \\
0.043 \\
0.518 \\
0.006 \\
1.000 \\
0.167 \\
\text { Subbit } \\
\\
0.781 \\
0.050 \\
0.019 \\
0.144 \\
0.006\end{array}$ & $\begin{array}{c}0.035 \\
0.035 \\
0.030 \\
0.000 \\
0.040 \\
0.055 \\
0.000 \\
0.005 \\
0.015 \\
0.001 \\
0.202 \\
0.000 \\
0.022 \\
0.012 \\
0.013 \\
0.000 \\
0.000 \\
0.522 \\
0.013 \\
1.000 \\
0.150 \\
\text { Subbit }\end{array}$ & $\begin{array}{l}0.003 \\
0.007 \\
0.017 \\
0.025 \\
0.020 \\
0.025 \\
0.036 \\
0.000 \\
0.022 \\
0.000 \\
0.189 \\
0.000 \\
0.022 \\
0.022 \\
0.017 \\
0.000 \\
0.040 \\
0.548 \\
0.006 \\
0.999 \\
0.270 \\
\text { Bit } \\
\\
0.803 \\
0.062 \\
0.012 \\
0.117 \\
0.006\end{array}$ & \\
\hline
\end{tabular}

"Functional group compositions were determined to match reported ultimate analysis using the criterion presented in Hobbs (1990). The semi-empirical correlation of Ko et al. (1988) was used to calculate $x^{2}$ at pressures in Table 7.

-WG represents the atmospheric Wellman-Galusha Gasifier.

$$
k_{i, x}^{\text {effective }}=\left(\frac{1}{k_{i, x}}+\frac{1}{k_{m}}+\frac{1}{k_{\text {efr }}}\right)^{-1}
$$

where $k_{i x}^{\text {effective, }} k_{i, x}, k_{m}$, and $k_{\text {efr }}$ represent the effective devolatilization rate constant, the distributed Arrhenius devolatilization rate constant, the film mass-transport coefficient, and the effective internal mass-transport coefficient for the particle. The resistance through the particle is a function of the particle burnout and is negligible at the beginning of devolatilization. The mass-transfer resistances used here for devolatilization are identical to those used in the oxidation and gasification submodel and will be discussed in more detail in the following section.

Diffusional mass transport may not be an appropriate mode of transport for devolatilization. Jets of volatile gases suggest that convection may dominate mass transport for small-particle devolatilization. However, diffusion may be important for devolatilization of large particles at typical heating rates found in fixed-bed reactors. Internal particle temperature gradients may be significant during devolatilization (Phuoc and Mathur, 1991), but experimental evidence using thermocouples embedded into large coal particles during devolatilization indicates that thermal equilibrium is reached rapidly (for example, Nuttall et al., 1979).

Tar. Estimates of the tar production rate from a fixed-bed gasifier using the functional group devolatilization model have not been reliable (Hobbs, 1990). More recently, Solomon et al. (1988) and Fletcher et al. (1992) have developed network models of organic coal structure that lead to promising methods for estimating tar production rates. This new work, however, had not advanced to the point where it could be considered here. Ko et al. (1988) have published a correlation of a large quantity of tar production data, mostly on small coal particles. In our fixed-bed model, tar is either estimated from Ko et al. or provided from experimental data. In a separate study, we (Hobbs, et al., 1992) show that tar production estimated from Ko et al. was consistently higher than measured data for several coals in the Wellman-Galusha gasifier.

Oxidation and Gasification. Oxidation and gasification reactions consume the char that is assumed to be composed of nonvolatile functional groups. As shown in Figure 2, three gasification agents are considered: steam, carbon dioxide, and hydrogen. Light gases and tar competitively evolve, resulting in char. Both tar and char are treated as single species that have variable compositions depending on the location in the reactor.

The two most common char oxidation submodels used in fixed-bed coal gasification modeling are the Shell Progressive model (SP model) and the Ash Segregation model (AS model). The differences between the two models are in the description of the ash. The ash in the SP model remains intact. The oxidant is required to diffuse through the gas film boundary layer and the ash layer. The ash in the AS model is assumed to crumble and fall away from the char particle with the oxidant required to diffuse only through the gas film boundary layer. An ash layer may collect around the particle during oxidation as observed during the burning of large coal slabs (Park and Edgar, 1987). 
The char oxidation/gasification submociels assume global reactions and depend on the external particle surface area. The SP or AS submodels are used to obtain the rates of char oxidation and gasification. Derivation of the rate equation for a single particle is given by Hobbs (1990):

$$
r_{i}^{0 s}=\frac{A_{p} \nu_{s} M w_{p} C_{i g}}{\frac{1}{k_{r} \zeta}+\frac{1}{k_{m}}+\frac{1}{k_{\mathrm{efr}}}}
$$

The resistances in the denominator represent surface reaction, molecular diffusion through the gaseous film and diffusion through the ash layer. Equation 8 neglects the effects of diffusion-induced convective transport and assumes that the reactions are first order in oxidizer concentration. The validity of Eq. $B$ has not been demonstrated for groups or packed beds of oxidizing particles. Values obtained for reaction order among various investigators vary between 0 and 1 , with most investigators correlating their data on the basis of first order. Gray et al. (1974) explain the variation in terms of the controlling chemical reaction step: if adsorption is controlling, $n=1$, whereas if desorption controls, $n=0$, where $n$ is the order of the reaction.

Equation 8 also explicitly neglects pore diffusion, in which case these effects are lumped into $k_{\text {efr. }}$. The diffusional resistance through the ash layer is set to zero if the AS model is used. Oxidation and gasification kinetic rate constants are given in Table 4. Given the lack of more reliable steam-char reaction rate data, it has been assumed, based on the data of Walker (1959), Yoon (1978), Wen et al. (1982), Wen and Chaung (1979), and Blackwood (1959), that the steam gasification rate is the same as the carbon dioxide gasification rate: $A_{\mathrm{H}_{2} \mathrm{O}}=A_{\mathrm{CO}_{2}}$, and $E_{\mathrm{H}_{2} \mathrm{O}}=E_{\mathrm{CO}_{2}}$. Based on the same references, the hydrogen gasification rate is taken to be three orders of magnitude smaller than the carbon dioxide rate: $A_{\mathrm{H}_{2}}=10^{-3} A_{\mathrm{CO}_{2}}$ and $E_{\mathrm{H}_{2}}=E_{\mathrm{CO}_{2}}$. Although the parameters in Table 4 were derived from smallparticle experimentation, the kinetic rate constants are assumed to be applicable to large-particle oxidation and gasification. Large-particle oxidation and gasification data are scarce. The model uses measured char oxidation rate data for various coals correlated to first-order kinetics, Table 4. Acceptable predictions can be obtained with these correlations without a precise knowledge of the mechanism or the intrinsic reaction order.
This is particularly effective when correlated rates are for the same or similar coal of the appropriate size covering the appropriate temperature range. Use of this simpler expression makes the addition of important diffusional effects more straightforward.

Several studies have been done on large spherical carbon particles (for example, Froberg, 1967; Kurylko, 1969) and may not be applicable to coal. Mass transport, however, may dominate the oxidation and gasification processes. For example, diffusion through an ash layer was shown to be significant when predicting burning rates for large coal slabs (see Hobbs, 1990). For the slab, the film resistance was 10 orders of magnitude higher than the chemical resistance for oxidation using the parameters in Table 4. Similar results were obtained from the large particle rates of Froberg (1967) and Kurylko (1969). Although chemical rates for small particles may differ from large particle rates, film diffusion and internal diffusion dominate, at least for oxidation. Additional experimental data are needed to make this same conclusion for the gasification reactions.

The last resistance in the denominator of Eq. 8 can be determined using an effective mass-transfer coefficient (Thorsness and Kang, 1984):

$$
\frac{1}{k_{\mathrm{eft}}}=\frac{(1-F) d_{p}}{2 D_{\mathrm{eft}}}
$$

Walker et al. (1959) and Laurendeau (1978) discuss methods for calculating effective diffusivities. Park and Edgar (1987) show the effect of a developing ash layer on the burning rate of a core sample of coal. The core burning rate can be predicted by using an effective diffusivity based on the molecular diffusivity multiplied by a constant $\left(D_{\mathrm{efr}}=\phi D_{l m}\right)$. The molecular diffusivity, $D_{i m}$, was calculated from Chapman-Enskog theory for gas mixtures (Bird et al., 1960). The constant, $\phi$, is based on the porosity of the developing ash layer. Thorsness and Kang (1985) have used 0.35 for $\phi$. Wang and Wen (1972) have measured porosity of a fire clay ash which varied from 0.4 to 0.8 . Laurendeau (1978) shows that $\phi$ can be estimated by the ash porosity divided by the tortuosity, which was taken to be 2. Using Wang and Wen's values for the ash porosity $(0.4$ to $0.8), \phi$ should range between 0.2 and 0.4 . However, lower values of ash porosity were determined for ash originating

Table 4. Oxidation and Gasification Kinetic Rate Constants (after Hedman et al., 1987)

\begin{tabular}{clcccc}
\hline & \multicolumn{5}{c}{$k_{r}=A_{r} T \exp \left(-E_{r} / R T\right), \mathrm{m} / \mathrm{s}$} \\
\hline Reaction & Rank & $A_{r, \mathrm{~m} / \mathrm{s} \cdot \mathrm{K}}$ & $E_{r} / R_{r} \mathrm{~K}$ & Source of Data & Source of Correlation \\
\hline $\mathrm{C}+0.5 \mathrm{O}_{2}-\mathrm{CO}$ & All ranks & 2.30 & 11,100 & Field et al. (1967) & Baxter (1987) \\
& HVBA & 1.03 & 9,010 & Goetz et al. (1982) & Baxter (1987) \\
& HVBC & 0.50 & 6,310 & Goetz et al. (1982) & Baxter (1987). \\
& SUBC & 10.4 & 11,200 & Goetz et al. (1982) & Baxter (1987) \\
& Lignite A & 1.22 & 10,300 & Nsakala et al. (1985) & Nsakala et al. (1985) \\
\hline $\mathrm{C}+\mathrm{CO}_{2}-2 \mathrm{CO}$ & All ranks & 589.0 & 26,800 & Goetz et al. (1982) & This study \\
& HVBA & $1,160.0$ & 31,200 & Goetz et al. (1982) & Baxter (1987) \\
& HVBC & $4,890.0$ & 31.300 & Goetz et al. (1982) & Baxter (1987) \\
& SUBC & $6,190.0$ & 28,900 & Goetz et al. (1982) & Baxter (1987) \\
& Lignite A & 3.42 & 15,600 & Goetz et al. (1982) & Baxter (1987) \\
\hline
\end{tabular}

- Base case parameters used in sensitivity analysis.

- Baxter's (1987) rate constants were obtained by nonlinear analysis of Field et al. (1967) and Goetz (1982) data.

tThe $\mathrm{CO}_{2}$ rate constants for all ranks were obtained in this study by averaging Baxter's (1987) rate constants for four coal ranks. 
from the Lurgi and Wellman-Galusha gasifiers (Hobbs, 1990). Porosities for these ashes ranged from 0.06 to 0.60 , indicating a lower range for $\phi$ of 0.03 to 0.3 for fixed-bed gasifiers.

The single-particle model can be related to the bed by using particle number density and the unreacted core particle sur face area. The particle diameter, unreacted core diameter, and number density for the SP model were obtained by mass balance, assuming spherical particles and constant solids density:

$$
\begin{gathered}
d_{p}=\left[\left(1-\Omega_{c h}^{\circ}\right) d_{u}^{3}+\Omega_{c L h}^{\circ} d_{o}^{3}\right]^{1 / 3} \\
d_{u}=F^{1 / 3} d_{o} \\
\eta_{p}=\frac{6(1-\epsilon)}{\pi d_{p}^{3}}
\end{gathered}
$$

The heterogeneous oxidation of carbon produces both $\mathrm{CO}$ and $\mathrm{CO}_{2}$ as primary products as shown in the following reaction:

$$
\lambda \mathrm{C}+\mathrm{O}_{2} \rightarrow 2(\lambda-1) \mathrm{CO}+(2-\lambda) \mathrm{CO}_{2}, \quad 1 \leq \lambda \leq 2
$$

Carbon monoxide may be favored at higher temperatures, if $\mathrm{CO}$ is formed at carbon edges and $\mathrm{CO}_{2}$ is formed at inorganic sites. Lower temperatures may favor $\mathrm{CO}_{2}$ due to catalytic activity. The $\mathrm{CO} / \mathrm{CO}_{2}$ ratio has been correlated by Laurendeau (1978):

$$
\frac{\mathrm{CO}}{\mathrm{CO}_{2}}=A \exp \left(-\frac{E}{R T}\right)=\frac{2(\lambda-1)}{(2-\lambda)}
$$

where $A \approx 10^{2.5}$ and $E \approx 25-38 \mathrm{~kJ} / \mathrm{mol}$ for low pressures, and $A \approx 10^{3.5}$ and $E \approx 50-80 \mathrm{~kJ} / \mathrm{mol}$ at high pressures. The stoichiometric coefficient for Eq. 13 can be determined as a function of temperature from Eq. 14:

$$
\lambda=\frac{2\left[A \exp \left(-\frac{E}{R T}\right)+1\right]}{A \exp \left(-\frac{E}{R T}\right)+2}
$$

For oxidation, $\lambda$ is equal to $\nu_{s}$ in Eq. 8. Values for $\lambda$ at 500 , $1,000,1,500$ and $2,000 \mathrm{~K}$ are $1.08,1.78,1.93$, and 1.96 , respectively, using Eq. 15 with the low-pressure parameters; 1.00 , $1.06,1.47$, and 1.76, respectively, using the high-pressure parameters. Thus, $\mathrm{CO}$ is favored at higher temperatures.

\section{Model equations, options and parameters}

The one-dimensional fixed-bed model equations are summarized in Table 5 . The 38 ordinary differential equations in Table 1 were solved simultaneously from the top to the bottom of the reactor with LSODE (Livermore Solver for Ordinary Differential Equations, Hindmarsh, 1983). Drying, devolatilization, combustion, and gasification are described by Eqs. 4, 5 and 8 . The volumetric heat-transfer rates from solid to gas, solid to wall, and gas to wall are described by Eqs. 1, 2 and 3. The heat- and mass-transfer correlations are in Table 2.
Table 5. Summary of the One-Dimensional Fixed-Bed Model Equations

\begin{tabular}{ll}
\hline Basic Equations and Boundary Conditions & \multicolumn{1}{c}{ Reference } \\
\hline Overall gas species continuity & Table 1 \\
Overall solid species continuity & Table 1 \\
Gas-phase energy & Table 1 \\
Solid-phase energy & Table 1 \\
Solid species continuity & Table 1 \\
Gas-phase elemental continuity & Table 1 \\
Gas-phase tar species continuity & Table 1 \\
Gas-phase tar elemental continuity & Table 1 \\
\hline Auxiliary Equations & \multicolumn{1}{c}{ Reference } \\
\hline Ash heat capacity & Mills and Rhine (1989) \\
Devolatilization rates & Eqs. 5 and 6 \\
Diffusive radiation coefficients & Table 2 \\
Drying rate & Eq. 4 \\
Effective gas and solid conductivities & Table 2 \\
Enthalpy exchange due to reaction & Hobbs (1990) \\
Formation enthalpies & Hobbs et al. (1992) \\
Gas conductivity, viscosity, and diffusivity & Bird, et al. (1960) \\
Gas-to-wall heat-transfer coefficient & Table 2 \\
Mass-transfer coefficient & Table 2 \\
Oxidation and gasification rates & Eq. 8 \\
Particle diameter and surface area & Hobbs (1990) \\
Particle number density & Eq. 12 \\
Pressure drop & Ergun (1952) \\
Reynolds, Prandl and Schmidt numbers & Table 2 \\
Sensible enthalpies & Hobbs et al. (1992) \\
Solid conductivity & Table 2 \\
Solid-to-gas heat-transfer coefficient & Table 2 \\
Solid-to-wall heat-transfer coefficient & Table 2 \\
Total enthalpies & Hobb et al. (1992) \\
Volumetric solid-to-gas heat-transfer rate & Eq. 1 \\
Volumetric wall heat loss from gas & Eq. 2 \\
Volumetric wall heat loss from solid & Eq. 3 \\
\hline &
\end{tabular}

Elemental reaction rates can be determined from reaction stoichiometry. The tar reaction rates can be determined from Eq. 6.

The one-dimensional fixed-bed model parameters and options are summarized in Table 6. Parameters that are difficult to obtain and subject to the most uncertainty include the solidto-gas heat-transfer coefficient, effective diffusivity, and bed void fraction. It has been reported (Lowry, 1963) that the solidto-gas heat-transfer coefficient typically ranges between $\mathbf{0 . 0 2}$ and 0.1 times that for a nonreacting fixed-bed system; this is discussed in detail in the sensitivity analysis. The effective diffusivity can be estimated from measured ash porosity. Bed void fraction for the feed coal can also be determined experimentally. However, the bed void fraction is not constant throughout the reactor. Values at the top and bottom of the reactor are required in the one-dimensional model.

\section{Solution technique}

The set of equaiions in Table 5 with boundary conditions describes a split boundary value problem. The term "split boundary" has been used to describe the partially known and unknown boundary conditions at both the top and the bottom of the moving-bed reactor. Although split boundary value problems have been solved satisfactorily for homogeneous models (equal solid and gas temperatures), heterogeneous models are more difficult to solve. For example, converged results from Cho's heterogeneous model (1980) indicate that the solid temperature is close to the gas temperature at the top 
Table 6. Summary of One-Dimensional Fixed-Bed Model Parameters and Options

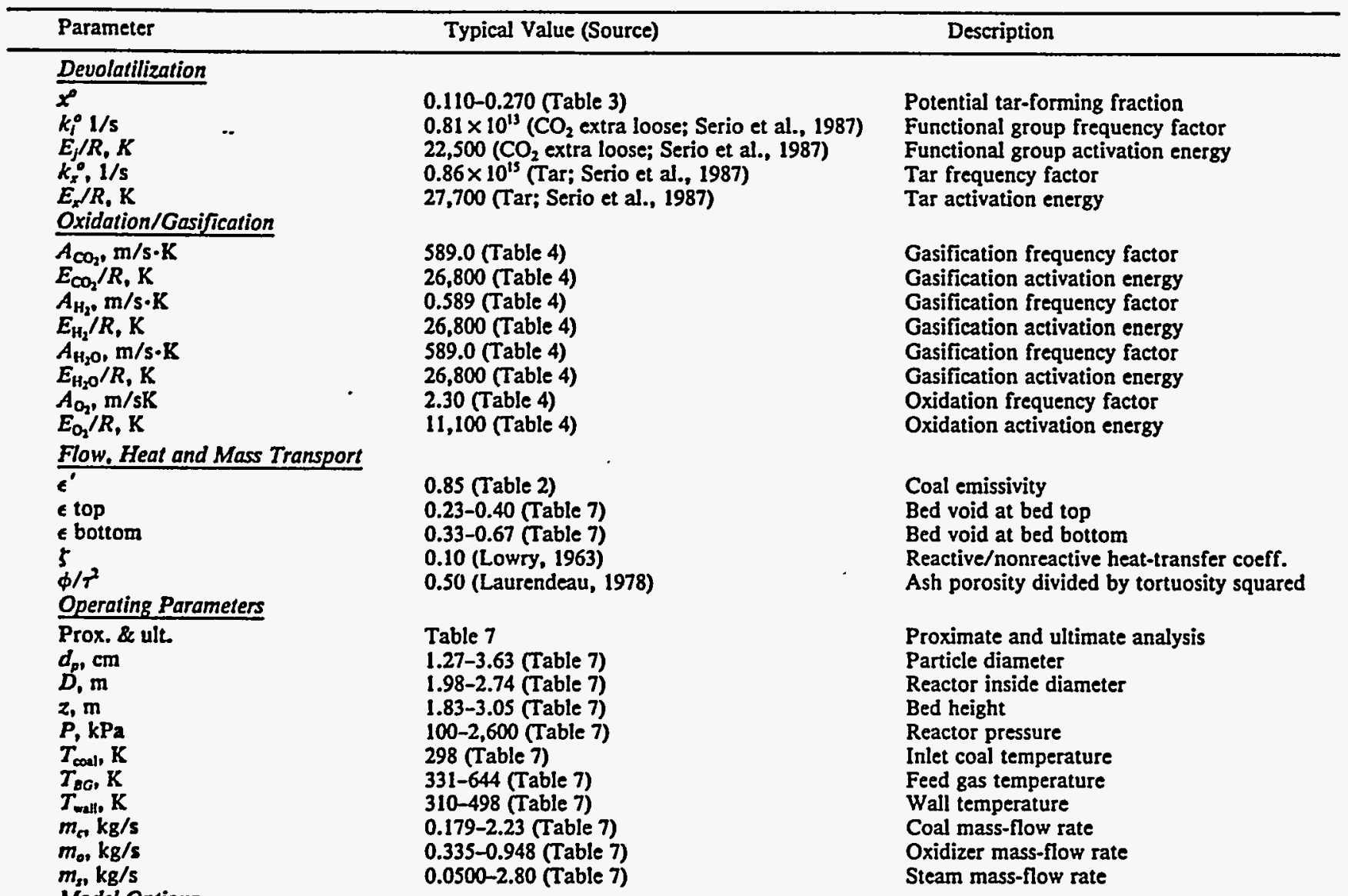

Model Options

(1) AS or SP char submodel; (2) gas-phase tar reaction equilibrium option; (3) volatiles mass-transport option; (4) combustion product distribution.

of the reactor, which was $1,050 \mathrm{~K}$. However, the initial temperature of the solid at the top of the reactor was specified to be $370 \mathrm{~K}$. In this study, iterative methods were used to satisfy temperature boundary conditions.

A two-zone, well-mixed, equilibrium-based, fixed-bed model (Hobbs et al., 1992) has been used here to provide an initial estimate of the effluent gas composition and temperature. However, the gas exit temperature predicted by the two-zone model is generally high due to the assumption that the devolatilization zone is at a single temperature. Likewise, the exit solid temperature is high due to the well-mixed assumption. Thus, after integrating from the top to the bottom of the reactor, the calculated feed gas temperature will be higher than the input feed gas temperature. Therefore, a new exit gas temperature must be estimated which is smaller than the temperature predicted by the two-zone model. This procedure can be repeated in an iterative manner until the calculated feed gas temperature is equal to the input feed gas temperature. In general, burnout should also be used as an iteration variable. However, burnout is typically high in fixed-bed gasifiers, and thus it was assumed to be unity for all calculations reported here and was not used as an iteration variable. Iterations using temperature converged to a burnout equal to unity. Typically, two to four iterations through the gasifier are required for convergence. Hobbs (1990) provides details on the computational algorithm. Calculation times for one sweep through the reactor using an engineering workstation for the two-zone model and one-dimensional model are on the order of seconds and minutes, respectively.

\section{Parametric Analysis}

The parametric sensitivity analysis was divided into three major categories: model options, model parameters, and operational parameters. Four model options were investigated: tar vapor reaction equilibrium, volatiles mass transport, char ash layer formation and combustion product distribution. Seven model parameters were also examined: solid-to-gas heattransfer coefficient, effective diffusivity, bed-to-wall heattransfer coefficient, potential tar-forming fraction, functional group composition, coal density, and oxidation and gasification kinetic parameters. Furthermore, eight operational parameters were examined: feed gas temperature, reactor pressure, coal mass flow rate, steam mass flow rate, air mass flow rate, proximate ash content of the feed coal, coal particle diameter, and bed void fraction.

The response to parametric changes in input parameters can be observed in various output parameters such as solid temperature, gas temperature, gas concentrations, and pressure drop. Solid temperature was chosen as the primary response variable for all the parametric simulations, since it is indicative of the extent of heterogeneous reaction. The base case for the parametric sensitivity simulations was gasification of Jetson bituminous coal in an air-blown Wellman-Galusha gasifier 
Table 7. Operating Parameters for Lurgi and Wellman-Galusha Gasifiers

\begin{tabular}{|c|c|c|c|c|c|c|c|c|c|}
\hline $\begin{array}{l}\text { Gasifier } \\
\text { Coal } \\
\text { Type }\end{array}$ & $\begin{array}{c}\text { Lurgi } \\
\text { Rosebud } \\
\text { Subbitum. }\end{array}$ & $\begin{array}{c}\text { WG } \\
\text { Absaloka } \\
\text { Subbitum. }\end{array}$ & $\begin{array}{c}\text { WG } \\
\text { Benton } \\
\text { Lignite }\end{array}$ & $\begin{array}{c}\text { WG } \\
\text { Elkhorn } \\
\text { Bituminous }\end{array}$ & $\begin{array}{c}\text { WG } \\
\text { Jetson } \\
\text { Bituminous }\end{array}$ & $\begin{array}{l}\text { WG } \\
\text { Kemmerer } \\
\text { Subbitum. }\end{array}$ & $\begin{array}{c}\text { WG } \\
\text { Leucite } \\
\text { Subbitum. }\end{array}$ & $\begin{array}{c}\text { WG } \\
\text { Rosebud } \\
\text { Subbitum. }\end{array}$ & $\begin{array}{c}\text { WG } \\
\text { Utah B.C. } \\
\text { Bituminous }\end{array}$ \\
\hline $\begin{array}{l}\text { Proximate, wt. \% } \\
\text { Ash } \\
\text { Fixed carbon } \\
\text { Moisture } \\
\text { Volatile }\end{array}$ & $\begin{array}{r}9.7 \\
36.4 \\
24.7 \\
9.2\end{array}$ & $\begin{array}{r}6.3 \\
40.7 \\
23.5 \\
29.6\end{array}$ & $\begin{array}{r}6.4 \\
25.9 \\
32.8 \\
34.9\end{array}$ & $\begin{array}{r}4.7 \\
53.7 \\
4.6 \\
36.9\end{array}$ & $\begin{array}{r}4.3 \\
49.5 \\
6.3 \\
39.9\end{array}$ & $\begin{array}{r}5.7 \\
42.4 \\
16.8 \\
35.1\end{array}$ & $\begin{array}{r}9.0 \\
44.9 \\
17.4 \\
28.8\end{array}$ & $\begin{array}{l}11.8 \\
40.1 \\
21.3 \\
26.8\end{array}$ & $\begin{array}{r}11.1 \\
43.9 \\
6.1 \\
38.9\end{array}$ \\
\hline $\begin{array}{l}\text { Ultimate, wt. } \% \\
\text { Carbon } \\
\text { Hydrogen } \\
\text { Nitrogen } \\
\text { Sulfur } \\
\text { Oxygen } \\
\text { Operating Parameters }\end{array}$ & $\begin{array}{r}77.1 \\
4.9 \\
1.4 \\
1.7 \\
15.0\end{array}$ & $\begin{array}{r}76.3 \\
5.1 \\
0.8 \\
0.4 \\
17.4\end{array}$ & $\begin{array}{r}73.7 \\
6.2 \\
1.0 \\
0.9 \\
18.2\end{array}$ & $\begin{array}{r}82.5 \\
5.7 \\
1.6 \\
0.9 \\
9.3\end{array}$ & $\begin{array}{r}81.4 \\
5.1 \\
1.8 \\
1.6 \\
10.2\end{array}$ & $\begin{array}{r}77.5 \\
5.6 \\
1.2 \\
1.0 \\
14.7\end{array}$ & $\begin{array}{r}78.1 \\
5.0 \\
1.9 \\
0.6 \\
14.4\end{array}$ & $\begin{array}{r}78.7 \\
4.9 \\
1.1 \\
1.3 \\
14.0\end{array}$ & $\begin{array}{r}80.3 \\
6.2 \\
1.2 \\
0.6 \\
11.7\end{array}$ \\
\hline $\begin{array}{l}\text { Chamber inside dia., m } \\
\text { Bed Height, } \mathrm{m} \\
\text { Chamber pres., } \mathrm{kPa} \\
\text { Apparent density, } \mathrm{kg} / \mathrm{m}^{3} \\
\text { Particle dia., } \mathrm{cm} \\
\text { Void fraction at bed top.. } \\
\text { Void fraction at bed bottom } \\
\text { Inlet coal temp., } \mathrm{K} \\
\text { Feed gas temp., } \mathrm{K} \\
\text { Wall temp., } \mathrm{K} \\
\text { Coal mass flow, } \mathrm{kg} / \mathrm{s} \\
\text { Air-mass flow, } \mathrm{kg} / \mathrm{s}^{\dagger} \\
\text { Steam mass flow, } \mathrm{kg} / \mathrm{s} \\
\text { Jacket steam mass flow, } \mathrm{kg} / \mathrm{s} \\
\text { Wall heat loss, } \mathrm{MW} W^{\ddagger} \\
\text { Wall heat loss, } \mathrm{MW}^{\mathrm{s}} \\
\text { Reference }\end{array}$ & $\begin{array}{l}2.74 \\
3.05 \\
2,560 \\
1,270 \\
1.61 \\
0.40 \\
0.50 \\
298 \\
644 \\
498 \\
2.23 \\
0.58 \\
2.80 \\
0.31 \\
0.836 \\
0.888\end{array}$ & $\begin{array}{c}1.98 \\
2.11 \\
101.3 \\
1,260 \\
2.29 \\
0.36 \\
0.50 \\
298 \\
332 \\
310 \\
0.420 \\
0.764 \\
0.114 \\
0.0034 \\
0.155 \\
0.343 \\
12(53)\end{array}$ & $\begin{array}{c}1.98 \\
2.13 \\
101.3 \\
1,200 \\
3.05 \\
0.25 \\
0.45 \\
298 \\
340 \\
310 \\
0.749 \\
0.931 \\
0.231 \\
0.051 \\
0.226 \\
0.481 \\
10(55)\end{array}$ & $\begin{array}{c}1.98 \\
2.13 \\
101.3 \\
1,300 \\
3.63 \\
0.35 \\
0.46 \\
298 \\
331 \\
310 \\
0.324 \\
0.789 \\
0.113 \\
0.0082 \\
0.260 \\
0.518 \\
9(71)\end{array}$ & $\begin{array}{c}1.98 \\
1.83 \\
101.3 \\
1,190 \\
2.03 \\
0.33 \\
0.60 \\
298 \\
334 \\
310 \\
0.352 \\
0.948 \\
0.156 \\
0.134 \\
0.416 \\
0.257 \\
2(68)\end{array}$ & $\begin{array}{c}1.98 \\
2.13 \\
101.3 \\
1,260 \\
1.27 \\
0.30 \\
0.52 \\
298 \\
332 \\
310 \\
0.284 \\
0.573 \\
0.0881 \\
0.0030 \\
0.167 \\
0.197 \\
14(49)\end{array}$ & $\begin{array}{c}1.98 \\
2.00 \\
101.3 \\
1,310 \\
2.54 \\
0.32 \\
0.46 \\
298 \\
334 \\
310 \\
0.293 \\
0.532 \\
0.0883 \\
0.0620 \\
0.291 \\
0.255 \\
4(59)\end{array}$ & $\begin{array}{c}1.98 \\
2.00 \\
101.3 \\
1,310 \\
2.29 \\
0.23 \\
0.33 \\
298 \\
336 \\
310 \\
0.179 \\
0.335 \\
0.050 \\
0.0174 \\
0.169 \\
0.126 \\
15(50)\end{array}$ & $\begin{array}{c}1.98 \\
2.13 \\
101.3 \\
1,220 \\
2.16 \\
0.33 \\
0.67 \\
298 \\
335 \\
310 \\
0.337 \\
0.673 \\
0.119 \\
0.0261 \\
0.295 \\
0.341 \\
13(57)\end{array}$ \\
\hline
\end{tabular}

-WG represents the atmospheric Wellman-Galusha Gasifier.

- Estimated from measured bulk and apparent densities.

'Oxygen mass-flow rate for the Lurgi case, $\mathrm{kg} / \mathrm{s}$.

'Heat loss calculated from reported jacket steam and cooling water heat loss.

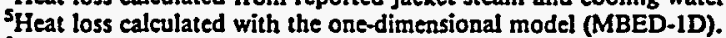

'Volume (underlined) and page number (in parenthesis) of Thimsen et al. (1984).

(Thimsen et al., 1984). Operational data for the Jetson case are given in Table 7. Additional parameters required by the one-dimensional model for the base case (Jetson) are given in Table 3. The sensitivity of temperature to the solid-to-gas heattransfer coefficient was completed for eight different cases, rather than just the Jetson case. Input parameters for these eight cases are also reported in Table 7.

\section{Model options}

Tar Vapor Chemistry. The one-dimensional, fixed-bed model has two options for treating tar vapor chemistry: 1 . the tar is allowed to react in the gas phase to completion (chemical equilibrium assumption); or 2. the tar vapor is nonreactive (in thermal equilibrium, but "frozen" chemically). Condensedphase tar remaining in the solid is considered as part of the char. If option 1 is chosen, all gases including tar are assumed to be in chemical equilibrium. If option 2 is chosen, all gases except for tar are assumed to be in chemical equilibrium.

The predicted sensitivity of the axial solid temperature profile to the tar gas-phase equilibrium assumption for gasification of Jetson bituminous coal in an atmospheric, air-blown, Wellman-Galusha gasifier is shown in Figure 3A. Use of the equilibrium assumption caused a small shift in the temperature peak toward the bottom of the reactor. This small effect can be explained by noting that gasifiers operate fuel-rich near the top of the gasifier. When the tar reacted to equilibrium in the drying and devolatilization zones, the gas phase became more fuel-rich, which caused the temperature to decrease slightly. The decrease in temperature in the devolatilization zone caused the devolatilization zone length to increase, which consequently caused the entire temperature profile to shift toward the reactor bottom. The transition between the devolatilization zone and the gasification zone was more gradual when the tar was allowed to react to equilibrium. Again, the gradual transition was caused by lower temperatures.

Volatiles Mass Transport. The predicted sensitivity of axial solid temperatures to the devolatilization mass transport (Eq. 7) for gasification of Jetson bituminous coal in an atmospheric, air-blown Wellman-Galusha gasifier is shown in Figure 3B. Inclusion of mass-transport resistance caused only a small effect on the solid temperature profile. The more rapid release of volatile matter near the reactor top caused the gas phase to become fuel-richer, which consequently caused the temperature of the devolatilization zone to decrease. The decreased temperature in the devolatilization zone caused the devolatilization zone length to increase, which caused the location of the maximum temperature to move toward the bottom of the reactor.

Char Ash Layer. The predicted sensitivity of axial solid temperature to the SP and AS ash assumptions for gasification 


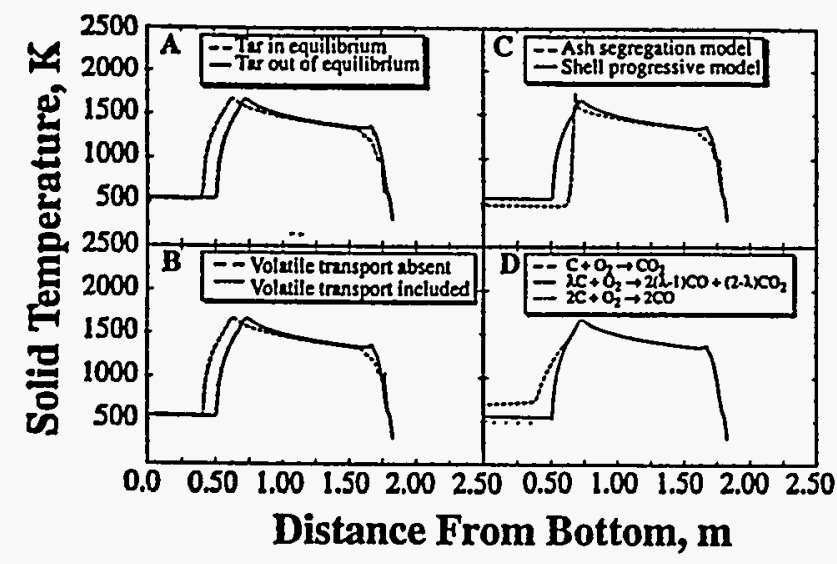

Figure 3. Predicted sensitivity of axial solid temperature to model optlons: A) tar gas.phase equilibrium assumplion; B) volatlle mass-transport as. sumption; C) char model ash assumption; and D) distribution of $\mathrm{CO}$ and $\mathrm{CO}_{2}$ for the char oxIdation reaction.

Simulation is for gasification of Jetson bituminous coal in an airblown Wellman-Galusha gasifier. Input parameters are in Tables $3,4,7$ and 8 .

of Jetson bituminous coal in an atmospheric air-blown Wellman-Galusha gasifier is shown in Figure $3 C$. Once again, relatively small differences are observed between the two options. The shape of the solid temperature profile using the AS model (no ash layer) was sharper than the broad peak predicted by the SP model. Also, the maximum temperature was higher when the AS model option was chosen. The two char models represent extremes in ash behavior, although the SP model may be closer to actual ash behavior. This observation is based on 1) comparing predicted temperature profiles to measured temperature profiles and 2) experimental observations of largeparticle oxidation which show ash-layer accumulation (Park 3nd Edgar, 1987). However, more specific data on oxidation II large particles in packed beds is required to confirm this suggestion.

Use of the AS submodel option resulted in an increased rate of carbon being added to the gas phase, which caused the gas phase to become fuel-richer. In the drying, devolatilization and gasification zones, a decrease in temperature was calculated. The combined effects of a shorter oxidation zone due to more rapid reaction and a lower devolatilization zone temperature caused the location of the maximum temperature to remain nearly unchanged.

Oxidation Product Distribution. Predicted sensitivity of axial solid temperature and gas concentration to the distribution of combustion products $\mathrm{CO}$ and $\mathrm{CO}_{2}$ during gasification of Jetson bituminous coal in an atmospheric, air-blown Wellman-Galusha gasifier is shown in Figure 3D. Three assumptions are shown: 1) $\mathrm{CO}_{2}$ as the sole primary combustion product; 2) $\mathrm{CO}$ as the sole primary combustion product; and 3) a distribution of $\mathrm{CO}_{2}$ and $\mathrm{CO}$ combustion products that depends on solid temperature (Eq. 14). As expected, only the oxidation zone was affected by the combustion product distribution assumption. Different temperatures resulted from the energy exchange at the particle-to-gas interface due to reaction.

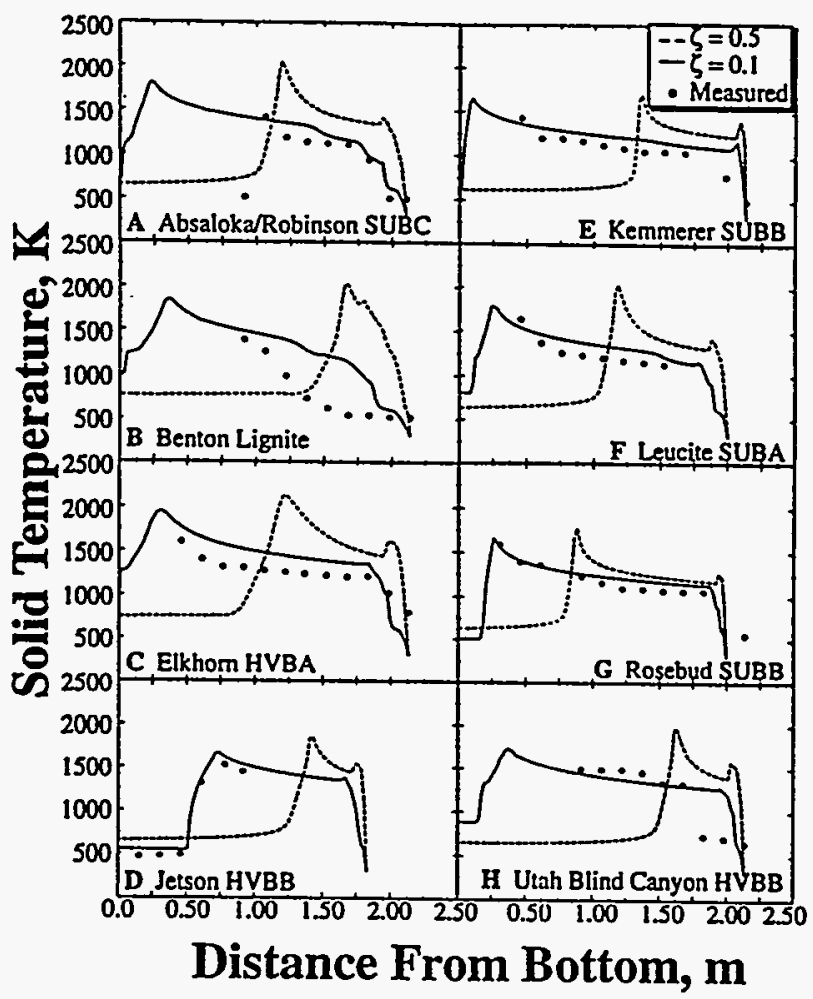

Figure 4. Predicted sensitlitity of axial solid temperature to solid-to-gas heat-transfer correction factor, $\zeta$, in an atmospheric pressure Wellman-Galu. sha gasifier fired with: A) Absaloka/Robinson subbituminous; B) Benton lignite; C) Elkhorn bituminous; D) Jetson bituminous; E) Kem. merer subbltuminous, F) Leucite subbitumlnous; G) Rosebud subbituminous; and $H$ ) Utah Blind Canyon bituminous coals.

Measurements from Thimson et al. (1984). Input conditions are given in Tables 3, 4, 7 and 8 .

Close agreement between the sole $\mathrm{CO}$ distribution and the combined $\mathrm{CO} / \mathrm{CO}_{2}$ distribution suggests that carbon monoxide may be assumed to be the primary product at typical combustion temperatures, which complements the findings of Laurendeau (1978).

\section{Model parameters}

Solid-to-Gas Heat-Transfer Coefficient. Effects of key model parameters that have not been reported experimentally and have a significant impact on the solid temperature profile were investigated. The solid-to-gas heat-transfer coefficient for a nonreacting system may be 10 to 50 times higher $(\zeta=0.02-$ $0.1)$ than that for a reacting system (Lowry, 1963). Dzhaphyev et al. (1986) attribute the observed difference to unsteady heat transfer. Vigorous reactions as well as nonsphericity and transpiration cooling may also contribute to this difference. The solid temperature is sensitive to this ratio, $\zeta$. Predicted sensitivity of axial solid temperature to $\zeta$ for the Wellman-Galusha cases is shown in Figure 4, where measured temperatures are also shown. None of the measured and predicted profiles correspond when $\zeta$ is greater than 0.1. All profiles in Figure 4 


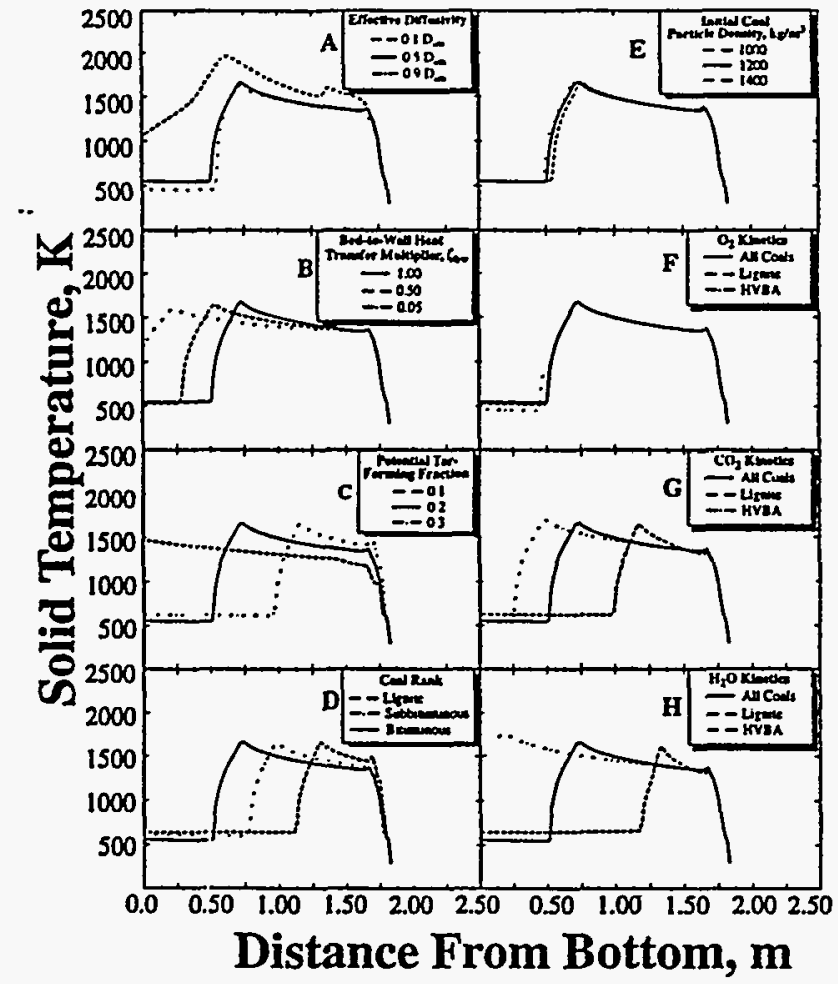

Figure 5. Predicted sensitivity of axial solid temperature to model parameters; A) effective diffusivity; B) bed-to-wall heat transfer, C) potential tar. forming fraction; D) coal rank; E) initial coal particle density; F) $\mathrm{O}_{2}$-char oxidation kinetic parameters; G) $\mathrm{CO}_{2}$-char gasification kinetic parameters; and $\mathrm{H}) \mathrm{H}_{2} \mathrm{O}$-char gasification kinetics.

Input conditions are for atmospheric gasification of Jetson bituminous coal in an air-blown Wellman-Galusha gasifier. Input conditions are in Tables $3,4,7$ and 8 .

also show the importance of treating devolatilization as a kinetic process. The Benton lignite case with $33 \%$ moisture shows that the drying zone was about $0.5 \mathrm{~m}$, thus discounting the common assumption of instantaneous drying.

Effective Diffusivity. The predicted sensitivity of the solid temperature to the effective diffusivity (Eq. 9) is shown in Figure 5A. The effective diffusivity affects the location of the maximum solid temperature, the magnitude of the maximum solid temperature, and the shape of the solid temperature profile. Lower values of $\phi\left(D_{\mathrm{efl}} / D_{l m}\right)$ cause the location of the maximum solid temperature to shift toward the bottom of the reactor and the size of the oxidation zone to increase, because the char oxidizes more slowly.

Bed-to-Wall Heat Transfer. The sensitivity of solid temperature to the bed-to-wall heat-transfer coefficient is shown in Figure 5B. For the calculations, $h_{w}$ from Eq. 1 in Table 2 was multiplied by a constant, $\zeta_{\text {hw }}$, to see the effect of lowering bed-to-wall heat transfer. Lower values of $\zeta_{h m}$ caused the solid temperature to decrease in the gasification and oxidation regions of the gasifier. The shift in location of the maximum solid temperature and the change in the shape of the curve were attributed to a redistribution of solid enthalpy.

Tar Fraction. Predicted sensitivity of solid temperature to the potential tar-forming fraction, $x^{\circ}$ (maximum tar yield), for the base case is shown in Figure 5C. To provide a consistent method of calculating tar, a semi-empirical model of Ko et al. (1988) was used to predict tar yield. As $x^{\circ}$ was increased, the ultimate volatile yield also increased and the corresponding ultimate char yield decreased, which resulted in a smaller gasification/oxidation zone. The shift in the location of the maximum solid temperature was attributed to the smaller oxidation and gasification zones.

Coal Rank. The predicted sensitivity of solid temperature to coal rank is shown in Figure 5D. Three different coal types were investigated: lignite, subbituminous, and bituminous coals. The solid temperature sensivity to rank may be attributed to the rank dependence of volatile matter (that is, lower-rank coals contain more volatile matter than higher rank coals). The increase in ultimate volatile yield and corresponding decrease in ultimate char yield resulted in a smaller gasification/ oxidation zone.

Coal Density. During devolatilization, coal particles evolve tars and gases, and the density can decline. The particles can also soften and swell depending on coal rank. Thus, it is expected that solids density will decline during devolatilization. The rate equations for devolatilization (Eqs. 5 and 6 ) depend on initial coal density which is specified. However, rates of char reaction (with $\mathrm{O}_{2}, \mathrm{CO}_{2}, \mathrm{H}_{2} \mathrm{O}$, and $\mathrm{H}_{2}$ ) depend on the char density, not on the original coal density. Further, the char density can change during oxidation (Smith, 1983). In this study, the solid density was taken to be constant throughout the bed at the initial coal density value. Sensitivity of solids density was tested by comparing temperature and pressure with different initial solids density values of $1,000-1,400 \mathrm{~kg} / \mathrm{m}^{3}$. Effects of solid density are shown in Figure SE to be modest.

Oxidation and Gasification Kinetics. Sensitivity of solid temperature to oxidation and gasification parameters (Table 4 ) is shown in Figures $5 F$ through $5 \mathrm{H}$. The solid temperature was not sensitive to the $\mathrm{H}_{2}$ gasification kinetics because of the slow reaction rate and is therefore not shown. The solid temperature, however, was sensitive to the $\mathrm{O}_{2}, \mathrm{CO}_{2}$ and $\mathrm{H}_{2} \mathrm{O}$ gasification kinetic parameters as shown in Figures $5 \mathrm{~F}$ and $5 \mathrm{H}$. Differences among coals were small for oxygen rates, but large for $\mathrm{CO}_{2}$ and $\mathrm{H}_{2} \mathrm{O}$ rates, with faster rates shortening the gasification zone. This suggests that reliable gasification rates for specific coals are required to make reliable predictions.

\section{Operational parameters}

Sensitivity of solid temperature to operational parameters is shown in Figure 6. Operational parameters that can be changed readily are the feed gas temperature, the reactor pressure, the coal mass-flow rate, the steam mass-flow rate, and the air-mass-flow rate. Other operational parameters are not as easily modified during daily operation. Such parameters include the proximate ash content, coal particle diameter, and the bed void fraction. Coal selection or pretreatment such as washing or crushing may be necessary to change these parameters.

Feed Gas Temperature. The location and magnitude of the maximum solid temperature were substantially affected by feed gas temperature (Figure 6A). As expected, the maximum temperature decreased when feed gas temperature was lowered, while the location of the maximum temperature at steady operating conditions shifted toward the bottom of the reactor. 


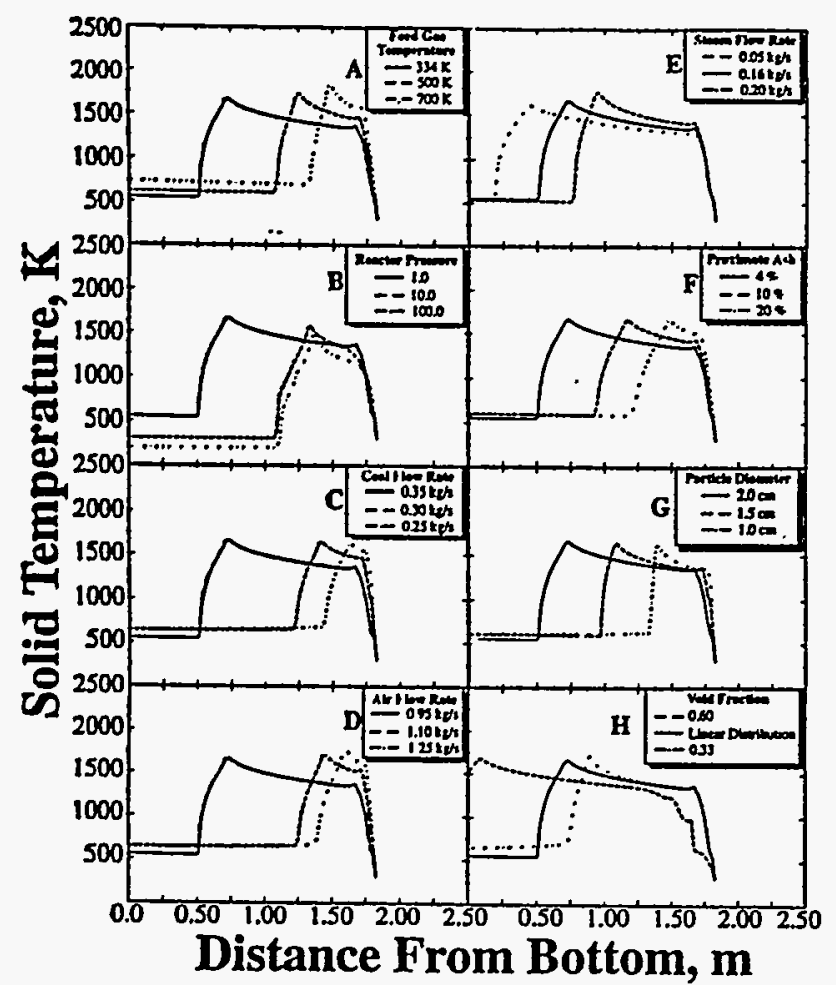

Figure 6. Predicted sensitivity of axial solid temperature to operational variables: $A$ ) feed gas temperature; B) reactor pressure; C) feed coal mass. flow rate; D) feed air-mass-flow rate; E) feed steam mass-flow rate; $F$ ) feed coal proximate ash content; G) feed coal mean particle di. ameter; and $H$ ) bed vold fraction.

Input conditions are for atmospheric gasification of Jetson bituminous coal in an air-blown Wellman-Galusha gasifier. Input conditions are in Tables 3, 4, 7 and 8.

The lower feed gas temperature caused the effluent gas temperature to decrease. The lengths of the drying, the devolatilization, the gasification, and the oxidation zones were all increased, which caused the location of the maximum temperature to move toward the bottom of the reactor.

Reactor Pressure. The predicted sensitivity of solid temperature to reactor pressure is shown in Figure 6B. Increasing pressure at lower pressure levels caused the location of the maximum temperature to shift toward the top of the reactor. The change in temperature gradient in the oxidation zone of the high-pressure simulation was due to competition between the highly exothermic oxidation reaction and the endothermic steam gasification reaction. This effect was substantial in the high-pressure Lurgi case discussed subsequently. Once the solid temperature is sufficiently high for the oxidation reaction to begin, the rapid oxidation of carbon causes the solid temperature to increase dramatically. Once the temperature reaches about $1,000 \mathrm{~K}$, the steam reaction begins. Although the steam reaction is not as fast as the oxidation reaction, the concentration of steam is significantly higher than the oxygen concentration. Thus, carbon consumption associated with the steam reaction approaches the carbon consumption associated with the oxidation reaction. The endothermic steam gasifisation reaction quenches the rapid increase in solid temperature and causes a sudden change in solid temperature, which was observed in the high-pressure case in Figure 6B.

When pressure is increased, the partial pressure of the water increases. Although the partial pressure of oxygen is also increased, the greater abundance of steam causes a substantial increase in the steam gasification reaction. The effect is to magnify the competition between the endothermic and exothermic reactions, producing a small peak shown subsequently in Figure 7A. Also, the diffusivity of water is greater than that of oxygen. Therefore, the steam gasification reaction is amplified at higher pressures.

Coal Flow Rate. The predicted sensitivity of solid temperature to coal mass-flow rate is shown in Figure 6C. All parameters in these simulations were held constant except for the coal mass-flow rate. The substantial shift in the location of the maximum temperature profile was attributed to a decrease in time required to consume all of the organic matter in the coal. The low coal mass-flow rates correspond to combustion occurring at the top of the gasifier. Thus, both combustion and gasification runs are depicted in Figure 6C.

Air Flow Rate. The predicted sensitivity of solid temperature to air-mass-flow rate is shown in Figure 6D. The influence of air-mass-flow rate was similar to that of coal mass flow rate. Again, the higher air-mass-flow rate corresponded to a combustion case. The shift in location of the maximum temperature was attributed to increased oxidation rates.

Steam Flow Rate. The predicted sensitivity of the solid temperature to the steam mass-flow rate is shown in Figure 6E. As the steam mass-flow rate was increased, both the lo-

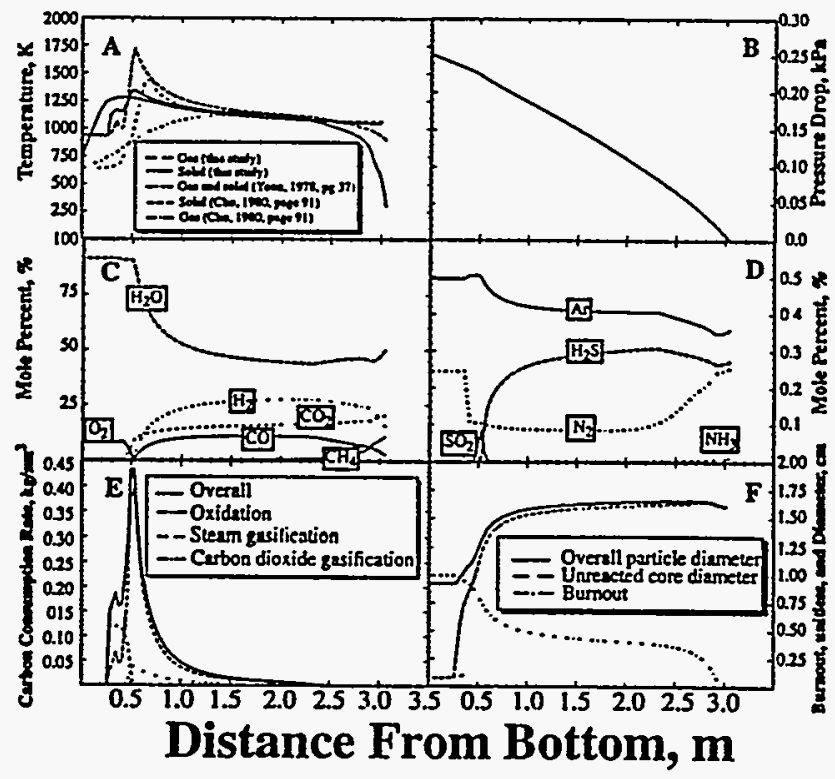

Figure 7. Predicted axial: A) temperature; B) pressure drop; C) major species concentration; D) $\mathrm{mi}$. nor species concentration; E) oxidation/gas. Ification carbon consumption rate; F) burnout, overall and unreacied particle diameter in a high-pressure, oxygen-blown Lurgi gasifler fired with Rosebud subbituminous coal.

Input parameters are in Tables 3, 4, 7 and 8. Figure $A$ shows results from the homogeneous model of Yoon (1979) and the heterogeneous model of Cho (1980). 
cation and the magnitude of the maximum temperature were affected. Increasing feed steam caused the maximum temperature to decrease. The shift in the location of the maximum temperature is caused by lower exit temperatures associated with higher steam mass-flow rates. This operational parameter is used frequently to control the temperature to avoid significant ash melting.

Ash Content. The predicted sensitivity of solid temperature to proximate ash content of the feed coal is shown in Figure 6F. The shift in the location of the maximum solid temperature is analogous to the temperature shift caused by the coal mass-flow rate. Lower proximate ash content is equivalent to an increase in the coal mass-flow rate, which results in a larger gasification zone. Similar results were obtained when the proximate moisture content was changed.

Particle Diameter. The predicted sensitivity of solid temperature to feed coal particle diameter is shown in Figure 6G. Small particles heat up faster and react sooner than large particles. The increased solid temperature promotes all reactions, which are completed much earlier for the smaller particles than for the larger particles. Also, mass transport resistances are less for small particles than for large particles. Particle-size effects are approximately linear as shown in Figure 6G. The influence of a distribution of particle sizes is more difficult to determine. Heat- and mass-transport rates are affected significantly, and the bed void fraction may also change dramatically, as discussed in the following section. Wide particle- size distributions tend to decrease the bed void fraction (Fayed and Otten, 1984).

Void Fraction. The predicted sensitivity of solid temperature to bed void fraction is shown in Figure $6 \mathrm{H}$. The void fraction significantly affects the particle number density, which in turn affects the overall bed consumption rate. Increasing void fraction causes $a$ decrease in the overall bed reaction rate. Drying, devolatilization, gasification and oxidation rates are all decreased significantly when the void fraction is increased. Thus, all prominent reaction zones increase with increasing bed void fraction, and the location of the maximum temperature moves toward the bottom of the reactor.

Rapid increase in the solid temperature at the reactor top is due, for the most part, to the rising hot gases from the oxidation zone heating the descending coal particles. The solid heat capacity is reduced significantly by the release of the volatiles in the devolatilization zone making the solid temperature increase more rapidly. The gas temperature, at the same time, is reduced sharply due to the mixing with colder volatiles released from the solids.

Model parameters with the most uncertainty are the solidto-gas heat-transfer coefficient and the effective diffusivity coefficient. Methods used for estimates of these parameters are summarized in Table 8. If experimental values are unavailable, the approach of Table 8 can be used with caution to estimate bed void fraction, $\epsilon$, solid-to-gas heat-transfer ratio, $\zeta$, and the ash porosity, $\phi$, and consequently, the effective diffusivity.

Table 8. Procedures Used to Estimate Key Model Parameters

\begin{tabular}{|c|c|c|}
\hline Parameter & $\begin{array}{l}\text { Independent } \\
\text { Calculation } \\
\end{array}$ & $\begin{array}{l}\text { Selection } \\
\text { Procedure } \\
\end{array}$ \\
\hline $\begin{array}{l}\text { Bed void fraction at coal inlet, } \epsilon_{c o} \\
\text { and ash outlet, } \epsilon_{a}\end{array}$ & $\begin{array}{l}\text { Void fractions can be measured or } \\
\text { estimated from coal and ash proper- } \\
\text { ties. Typical values may range be- } \\
\text { tween } 0.2 \text { and } 0.8 \text { depending on } \\
\text { particle distribution, sphericity and } \\
\text { particle roughness. }\end{array}$ & $\begin{array}{l}\text { Bed void fraction is selected to } \\
\text { match measured pressure profile. Ini- } \\
\text { tially, } \epsilon_{c} \text { and } \epsilon_{e} \text { are adjusted in equal } \\
\text { increments; however, top or bottom } \\
\text { void fractions are adjusted independ- } \\
\text { ently if the measured profile indi- } \\
\text { cates a larger or smaller void } \\
\text { fraction. }\end{array}$ \\
\hline $\begin{array}{l}\text { Solid-to-gas heat-transfer correction } \\
\text { factor, } \zeta\end{array}$ & $\begin{array}{l}\text { Selection of } \zeta \text { is based on experience } \\
\text { with the sensitivity analysis shown in } \\
\text { Figures } 4 \text { and } 5 \text {. A value of } 0.1 \text { is } \\
\text { recommended if no other data are } \\
\text { available. }\end{array}$ & $\begin{array}{l}\zeta \text { is selected to match the location of } \\
\text { the maximum measured temperature. }\end{array}$ \\
\hline $\begin{array}{l}\text { Effective diffusivity parameter, } \\
\phi=\phi_{N} / \tau^{2} \approx \phi_{N} / 2\end{array}$ & $\begin{array}{l}\phi \text { can be measured or estimated from } \\
\text { the ash porosity, } \phi_{e} \text {. Ash porosities } \\
\text { range from } 0.06 \text { to } 0.60 \text { for ash ob- } \\
\text { tained from fixed-bed gasifiers (see } \\
\text { Hobbs, } 1990 \text { ). An acceptable range } \\
\text { for } \phi \text { might be } 0.02 \text { to } 0.5 \text {. A value } \\
\text { of } 0.5 \text { is recommended if no other } \\
\text { data are available. }\end{array}$ & $\begin{array}{l}\phi \text { can be selected to match the loca- } \\
\text { tion of the maximum measured tem- } \\
\text { perature. } \phi \text { is usually adjusted after } \zeta \\
\text { is chosen. If adjusting } \zeta \text { is insuffi- } \\
\text { cient to adjust the location of the } \\
\text { maximum temperature, adjust } \phi .\end{array}$ \\
\hline SP or AS model option & $\begin{array}{l}\text { Selection of the char model is based } \\
\text { on experience with the sensitivity } \\
\text { analysis as shown in Figure } 3 C \text {. The } \\
\text { SP model option is recommended if } \\
\text { no other data are available. }\end{array}$ & $\begin{array}{l}\text { The AS model can be chosen, if a } \\
\text { discontinuity is observed in the meas- } \\
\text { ured pressure profile, if the oxidation } \\
\text { zone is very small with a correspond- } \\
\text { ing steep temperature gradient, or if } \\
\text { burnout is high simultaneously with } \\
\text { a large drying zone as is common for } \\
\text { lignites with high moisture contents. }\end{array}$ \\
\hline
\end{tabular}

\footnotetext{
-Fayed and Otten (1984) discuss bed void fraction. For spherical particles, bed void fraction for close randon packing ranges from 0.359 to 0.375 ; for hexagonal close packing, bed void fraction is 0.26 . The effect of nonsphericity is to inerease bed void fraction. For example, for a sphere with a sphericity of 1.0 , it is 0.4 ; for a cube with sphericity 0.8 , it is 0.5 ; and for a particle with sphericity of 0.2 it is 0.85 .
} 


\section{Comparisons with Fixed-Bed Data Selection of model variables}

While effluent gas composition is predicted by MBED-1D, comparison of these predicted and measured values does not provide a strong evaluation of a generalized fixed-bed model. Once coal burnout is established, which is often near unity, and tar production is estimated (for example, Ko et al., 1988), effluent gas composition can be readily estimated without recourse to a generalized, fixed-bed model (Hobbs et al., 1992). Thus, evaluation of MBED-1 was performed by comparison with measured axial profiles of temperature and pressure in fixed beds. No data for composition profiles were located for this purpose, and experimental temperature and pressure profiles within laboratory or commercial-scale fixed-bed gasifiers are limited. Eight low-pressure, air-blown Wellman-Galusha cases (Thimsen et al., 1984) were simulated together with one high-pressure, oxygen-blown Lurgi gasifier case with Rosebud subbituminous coal. No measured profiles were available for the dry-ash Lurgi gasifier.

These simulations are also compared to predictions from other one-dimensional models. Measured and predicted solid temperatures for several operating conditions are also compared for some of the Wellman-Galusha tests. Comparisons of predicted and experimental temperature profiles have helped to determine recommended values of model parameters. Caution must be exercised, however, when making conclusions based on profiles from experimental fixed-bed data. Thimsen (1990) warns that temperature profiles for the Wellman-Galusha cases be used for qualitative comparisons only, since the temperature probe [1/2-in. (13-mm) schedule 40304 SS pipe with six sheathed type-K thermocouples placed 6 in. (152 $\mathrm{mm}$ ) apart] was retracted from the gasifier when any junction approached 1,600 K. Typically, the temperature probe was allowed 10 minutes to reach steady state (Thimsen et al., 1984). Furthermore, the temperature profiles may represent transient conditions if the probes were retracted early. The usual disadvantages related to intrusive probes may also cause problems in fixed-bed temperature measurements: physical processes may be altered by flow disturbances; catalytic perturbations may be caused by the probe; and radiation and/or conduction losses may be significant. The measured temperature profiles were assumed to be closer to the solid temperature following Barriga and Essenhigh (1980). Additional simulations predicted concentration profiles of major and minor species, carbon consumption rates due to oxidation and gasification, burnout, and particle diameter.

A solid-to-gas heat-transfer correction factor of 0.1 was used for all simulations. The effective diffusivity was assumed to equal 0.5 times the molecular diffusivity. The bed void distribution was assumed to vary linearly between the top and bottom of the bed, as supported by the data from Krishnudu et al. (1989). Bed void fractions were not measured directly for any of the cases. Void fractions of the feed coal at the bed top and the product ash at the bed bottom were estimated based on coal and ash bulk and apparent density measurements.

\section{Wellman-Galusha dry-ash gasifier}

Predicted axial variations in temperature and pressure pro-

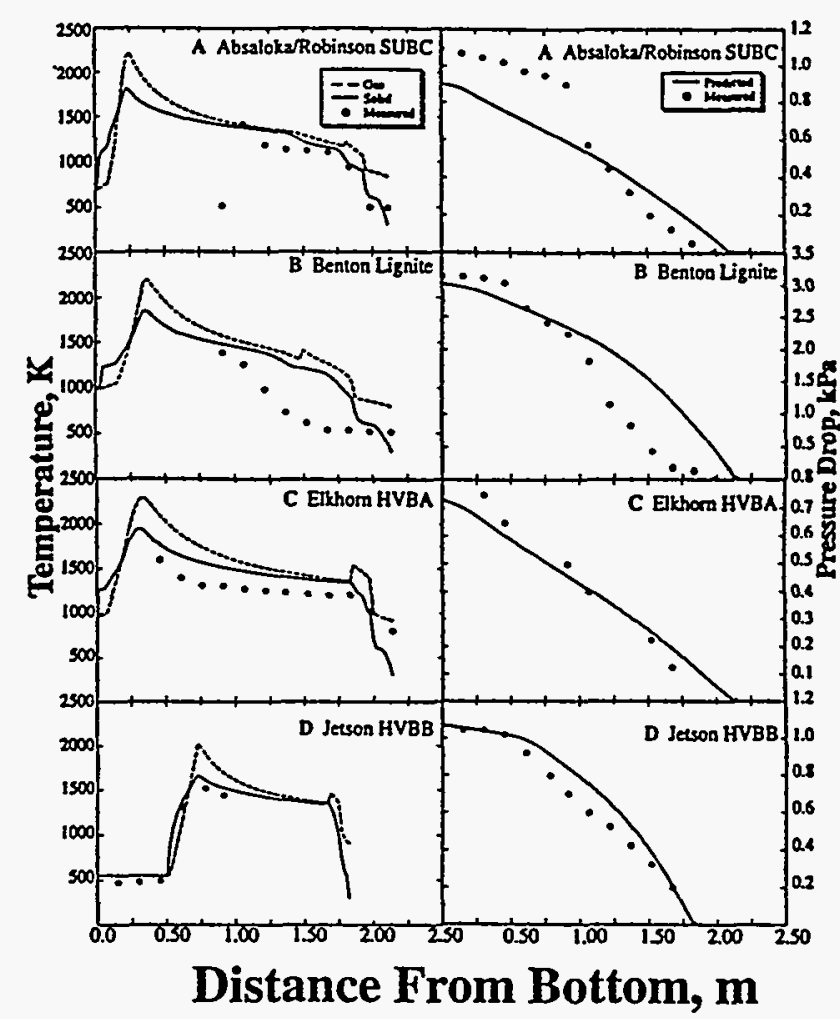

Figure 8. Predicted and measured axial variations in temperature and pressure drop during gasi. fication of: A) Absaloka/Robinson subbituminous; B) Benton lignite; C) Elkhorn bituminous; and D) Jetson bituminous coals in an air-blown, atmospheric Weliman-Galusha gasifier.

Measurements are from Thimson et al. (1984). Input parameters are in Tables 3, 4, 7 and 8.

files are compared to measurements for gasification of the eight coals in the Wellman-Galusha gasifier in Figures 8 and 9. Profile comparisons for both temperature and pressure are considered to be quite good for all, but the lignite case. Measurements for the Jetson case indicate the location of the maximum temperature. Predicted axial variations in temperature, pressure drop, gas concentration, oxidation/gasification carbon consumption rate, burnout, and particle diameter for atmospheric gasification of the Jetson bituminous coal are shown in Figure 10. This case is presented in detail since it was used as the base case in the sensitivity analysis. In the Jetson case, burnout (daf) was predicted to be unity as shown in Figure 10F. Thus, carbon is not available for oxidation in the ash zone. The ash zone temperature remains approximately $\mathbf{5 5 0}$ $\mathrm{K}$. Carbon becomes available for oxidation approximately 0.5 $\mathrm{m}$ from the bottom of the reactor where oxidation begins. The highly exothermic oxidation reaction increases the solid temperature dramatically. The oxidation reaction occurs before the steam gasification reaction, as shown by the carbon consumption rates in Figure 10E. Once initiated, the endothermic steam gasification reaction causes the positive solid temperature gradient to decrease. This competition between exothermic and endothermic reactions describes the initial step in the temperature profile going from the ash zone to the oxidation zone. 


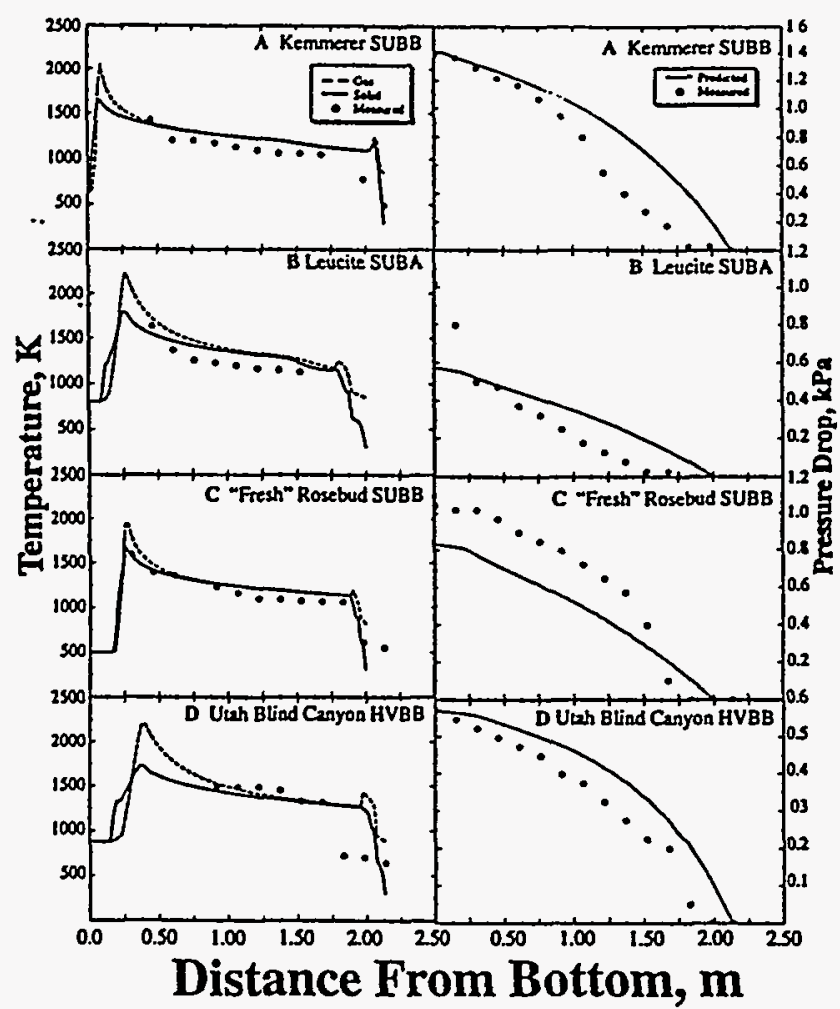

Figure 9. Predicted and measured axial variations in temperature and pressure drop during gasification of: A) Kemmerer subbltuminous; B) Leuclte subbituminous; C) "fresh" Rosebud subbituminous; and D) Utah Blind Canyon bi. tuminous coals in an air-blown, atmospheric Wellman.Galusha gasifier.

Measurements are from Thimsen et al. (1984). Input parameters are in Tables 3, 4, 7 and 8.

The temperature is highest at the end of the oxidation zone and starts to decrease due to predominantly endothermic gasification reactions of carbon with steam and carbon dioxide in the gasification zone.

Steam gasification overlaps oxidation as shown in Figure 10E. For example, at $0.65 \mathrm{~m}$ from the reactor bottom, the carbon consumption rate due to steam gasification is approximately $0.05 \mathrm{~kg} / \mathrm{s} \cdot \mathrm{m}^{3}$. At this location in the reactor, steam is reacting with carbon to produce hydrogen and carbon monoxide. However, no depletion of steam is observed in the concentration profile at this reactor location as shown in Figure $10 \mathrm{C}$. Steam is being replenished by the homogeneous oxidation of hydrogen. Also, carbon monoxide produced from both oxidation and steam gasification is being oxidized in the presence of oxygen to produce carbon dioxide. Carbon dioxide reaches a maximum at the end of the oxidation zone and decreases in the gasification zone. In the gasification zone, carbon dioxide reacts heterogeneously with carbon to produce carbon monoxide. Carbon monoxide is not present in the gas phase until oxygen is depleted. As with carbon monoxide, hydrogen does not appear in the gas phase until oxygen is depleted. Although agreement between the predicted and measured pressure profiles was acceptable, the void distribution may not be linear throughout the reactor. For example, the

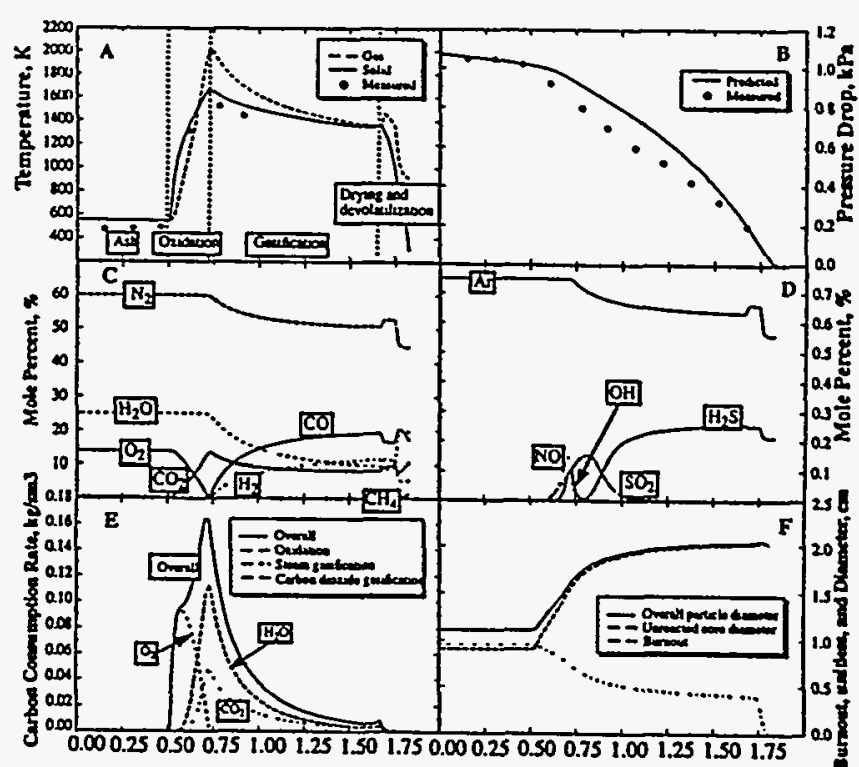

\section{Distance From Bottom, $m$}

Figure 10. Predicted axial: A) temperature; B) pressure drop; C) major species concentration; D) $\mathrm{ml}$ nor species concentration; 日 oxidation/gasIfication carbon consumption rate; and F) burnout, overall and unreacted particle di. ameter in an atmospheric alr.blown Wellman. Galusha gasifier with Jetson bituminous coal.

Input parameters are in Tables 3, 4,7 and 8. Measurements are from Thimsen et al. (1984, Vol. 2, p. KS).

measured pressure profile in Figure 10B indicated that the void fraction changed markedly near the ash zone.

The predicted increase in the particle diameter at the top of the reactor was due to coal particle swelling. Swelling was assumed to be proportional to the extent of devolatilization. For the Jetson case, the predicted particle diameter of the ash was about $1.1 \mathrm{~cm}$. The measured geometric mean diameter of the ash was $1.02 \mathrm{~cm}$ as reported by Thimsen et al. (1984). The excess nitrogen and high temperatures caused the equilibrium quantities of $\mathrm{NO}$ and $\mathrm{OH}$ to form in the oxygen-rich, hightemperature zone of the reactor. These quantities decayed to zero as temperature decreased. Sulfur dioxide also formed in the presence of oxygen and was converted to $\mathrm{H}_{2} \mathrm{~S}$ in the colder, fuel-rich regions of the gasifier.

Several of the Wellman-Galusha experimental test cases included temperature profiles at different operating conditions. Predicted temperature profiles were compared to measurements for the Elkhorn, the Jetson, the Leucite Hills and the Utah Blind Canyon coals in Figure 11. A shift in the measured temperature profile due to changing reactant feed rates during gasification of Elkhorn bituminous coal is shown in Figure 11A. The predicted trends agreed with the direction of the measured temperature shifts in each case. From the sensitivity analysis, an increase in coal flow rate caused the location of the maximum temperature to move closer to the bottom of the reactor. In general, an increase in either the steam flow rate or air flow rate caused the location of the maximum temperature to move closer to the top of the reactor. In this 


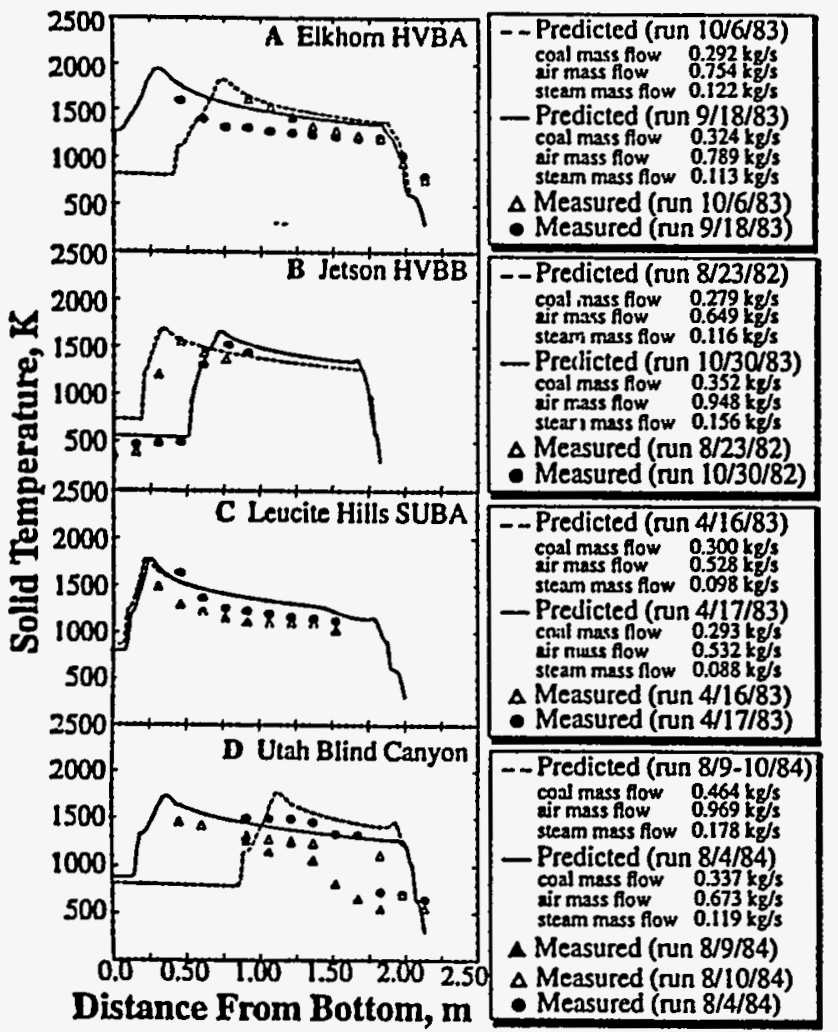

Flgure 11. Measured temperature vs. predicted solld temperature for several operating conditlons for gasification of: A) Elkhorn bituminous; B) Jetson bituminous; C) Leucite Hills subbltuminous; and D) Utah Blind Canyon bituminous coals in an air.blown, low.pres. sure Wellman-Galusha gasifler.

Experimental data are in Thimsen et al. (1984). Input parameters are listed in the legend and in Tables 3, 4, 7 and 8.

case, the coal and the air flow rates were both increased, the steam flow rate was decreased, and the location of the maximum temperature moved toward the reactor bottom. Although the increased air flow rate should have caused the location of the maximum temperature to move toward the reactor top, changes in coal and steam flow rates were more significant for the Elkhorn case.

The effect of varying feed rates on the location of the maximum temperature is shown in Figure 11B for gasification of Jetson bituminous coal. The direction of the temperature shift was predicted adequately by the one-dimensional model. An increase in the coal, air and steam mass-flow rates caused the location of the maximum temperature to move toward the top of the reactor. For the Jetson case, the increase in steam and air mass-flow rates was more significant than the increase in the coal mass flow rate. Although the low-rank coals were more difficult to simulate, predictions from the one-dimensional model agreed with the experimental data for the Leucite Hills subbituminous coal as shown in Figure 11C. The increase in coal flow rate and decrease in steam flow rate caused the location of the maximum temperature to shift toward the bottom of the reactor for the Leucite Hills case. The Utah Blind Canyon case in Figure 11D also showed the effect of increased coal and gas throughputs. Trends in measured and predicted profiles do not agree for this case. However, the measurements were repeated on two separate days, but only one set of operational data was reported for this time period (Thimsen et al., 1984), possibly explaining variability in the data.

\section{Lurgi dry-ash gasifier}

Predicted axial temperature, pressure drop, gas concentration, carbon consumption rates due to oxidation and gasification, burnout, and particle diameter are shown in Figure 7 for the Lurgi gasifier fired with Rosebud subbituminous coal. The most obvious difference compared to results from the atmospheric air-blown gasifier was the absence of a carbon dioxide peak. The shape of the carbon dioxide profile was due to the low temperature of the solid in the gasification section, resulting from large quantities of steam in the feed gas stream. The temperature was low enough that the only significant heterogeneous reaction in this section of the gasifier was the steam gasification reaction. With only hydrogen and carbon monoxide being produced in the gasification section, the hydrogen and carbon monoxide profiles should have been similar. However, gas-phase reactions such as the water-gas-shift reaction produced a slight increase in carbon dioxide concentration.

Temperature predictions from two other one-dimensional models are also shown in Figure 7A. These cases were reported to be the Illinois \#6 Westfield case (Yoon, 1978; Cho, 1980). However, the input conditions differed from those reported by Elgin and Perks (1974) and seemed to be closer to the Rosebud case. The source of Yoon's input data is uncertain. Also, Cho (1980) used the values provided by Yoon (1978). Yoon assumed equal solid and gas temperature, instantaneous devolatilization with a fixed composition, and gas-phase chemistry dominated by the water-gas-shift reaction. Cho essentially extended the model of Yoon to include separate solid and gas temperatures. The Yoon prediction was similar to the prediction made in our work. The shape of the solid temperature profile predicted by Cho is similar to the solid profile predicted here. Cho's gas temperature profile, however, does not correspond to the predictions by Yoon or this study. Cho's gas temperatures were less than the solid temperature in the oxidation zone. The gas temperature will be less when important gas-phase reactions are neglected. For example, steam gasification will produce $\mathrm{H}_{2}$ and $\mathrm{CO}$ in the oxidation zone, and $\mathrm{CO}$ will be produced from oxidation. In the presence of oxygen, the homogeneous reactions of $\mathrm{H}_{2}$ and $\mathrm{CO}$ with $\mathrm{O}_{2}$ will react to produce $\mathrm{H}_{2} \mathrm{O}, \mathrm{CO}_{2}$, and heat. These exothermic gas-phase reactions will cause a dramatic increase in gas temperature as shown in the predictions of this study.

\section{Insights Into Fixed-Bed Gasification \\ Resistances to char oxidation and gasification}

Chemical reaction resistance $\left.\left(\propto 1 / k_{r}\right\}\right)$, ash diffusion resistance $\left(\propto 1 / k_{\mathrm{eff}}\right)$, and film diffusion resistance $\left(\propto 1 / k_{m}\right)$ illustrate the dominant chemical and physical processes occurring in fixed-bed reactors. Resistances for char oxidation and gasification of the base case are shown in Figure 12. Chemical resistances dominate at the top and bottom of the reactor for the oxidation and gasification reactions. The ash resistance was highest at the reactor bottom. At the top of the reactor, 


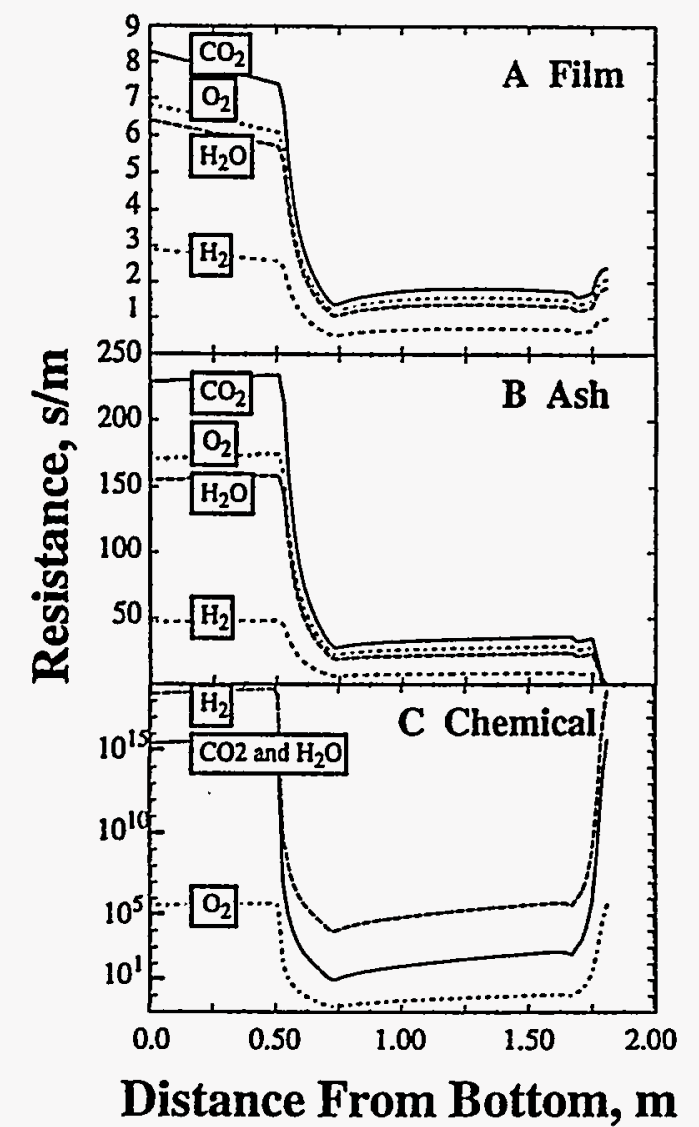

Figure 12. Resistances for char oxidation and gasiffcation of Jetson coal in an atmospheric fixed. bed gasifier: A) film resistance; B) ash re. sistance; and C) chemical resistance to char oxidation and gasification reactions.

These plots correspond to the Jetson case in Figure 10.

no ash layer was present. Once the ash layer was sufficiently thick, ash diffusion competed with the chemical reaction resistance in the gasification section of the gasifier as shown in Figures 12B and 12C. Although confidence in calculated masstransfer coefficients was generally greater than confidence in chemical reaction coefficients, ash porosity was not measured throughout the reactor, and the effective ash diffusivity was difficult to predict.

\section{Transport properties}

Predicted heat-transfer and -transport coefficients for the base case are shown in Figure 13. Typical values for the overall bed-to-wall heat-transfer coefficient range from 15-35 $\mathrm{W} / \mathrm{m}^{2} \cdot \mathrm{K}$. The bed-to-wall heat-transfer coefficient can be divided into contributions from the gas-to-wall coefficient and solid-to-wall coefficient, as shown in Figure 13A. Over 95\% of the heat loss to the wall for this case was due to the gas phase. The large heat transfer from the gas phase was caused by a high effective radial gas conductivity. The gas conductivity was dominated by a large dynamic contribution due to the high gas velocities. Gas Reynolds numbers throughout the reactor are shown in Figure 13F. The solid and gas conductivities are shown in Figure 13C. The solid conductivity was

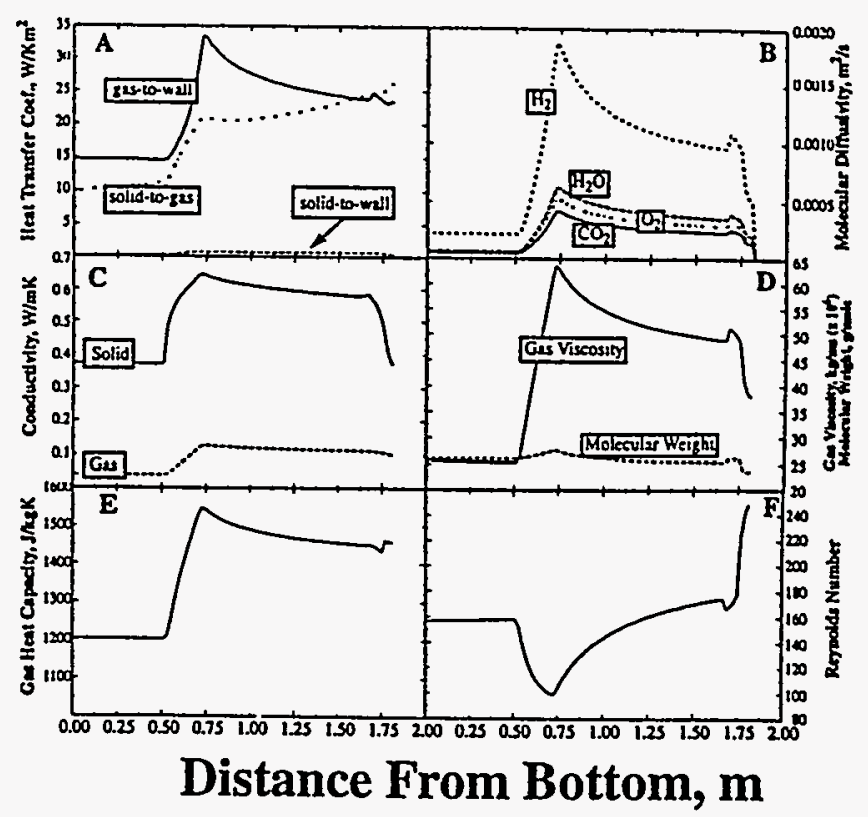

Figure 13. Predicted heat-transfer coefficients and transport properties for gasification of Jetson coal in an atmospheric Wellman-Galu. sha gasifier with air: A) heat-transfor coefficients; B) molecular diffusivities; C) solid and gas conductivitles; D) gas viscos. ity and molecular weight; E) gas heat ca. pacity; and F) Reynolds numbers.

These plots correspond to the Jetson case in Figure 10.

assumed to be proportional to the square root of solid temperature (Table 2, Eq. 7). The gas mixture conductivity was calculated from classical methods. Changes in gas conductivity, gas heat capacity, and gas viscosity were somewhat similar through the bed, all rising sharply with rapid increase in gas temperature, as shown in Figures 13C-13E.

\section{Solid and gas residence time, velocity and heating rate}

Solid residence time for the base case was on the order of hours as shown in Figure 14A. The corresponding gas residence time was on the order of seconds. The solid residence time increases in the ash zone due to significant settling resulting in variable axial velocity. Axial solid and gas velocities were shown in Figure 14B. Gas velocities were less than $3 \mathrm{~m} / \mathrm{s}$. Solid velocities were less than $0.1 \mathrm{~mm} / \mathrm{s}$. Solid velocity was affected by mass loss due to drying and devolatilization. The solid velocity decreases as heterogeneous oxidation and gasification reactions consumed the solid mass. With mass loss, the particle diameter decreased and the corresponding particle number density increased. Once all reactions were quenched, the solid velocity remained constant. Although mass addition to the gas phase contributed to increased gas velocity, temperature effects probably were more significant.

Gas and solid heating rates depend on the axial temperature profile and solid and gas residence times. Gas heating rates are approximately 4 orders of magnitude higher than the solid heating rate due to different residence times. Significant changes in heating rate occur at the oxidation temperature spike and 


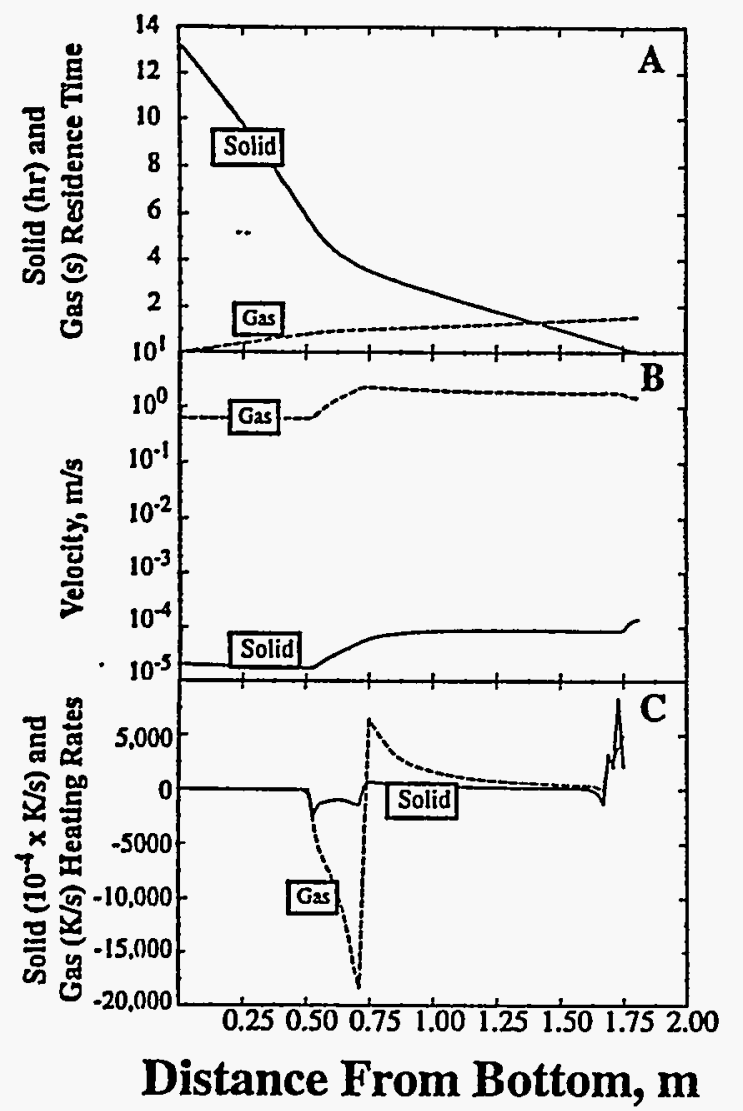

Figure 14. Predicted solid and gas: A) residence times; $B$ ) velocities; and $C$ ) heating rates during gas. Ification of Jetson bituminous coal in an alr. blown, atmospheric Wellman.Galusha gasi. fler.

These plots correspond to the Jetson case in Figure 10.

in the devolatilization zone where rapid heterogeneous reactions occur.

\section{Summary and Conclusions}

A generalized, one-dimensional fixed-bed gasification/combustion model (MBED-1D) has been formulated, solved numerically, evaluated through parametric sensitivity analysis, and compared with measured fixed-bed axial temperature and pressure profiles. Model advances and/or characteristics include separate gas and particle temperatures, simultaneous drying, coal devolatilization, char oxidation and gasification zones, generalized gas-phase chemistry, axially variable bed void fraction and gas/solid flow rates, and generalized, coalindependent rate-controlled devolatilization. Solution times on a midlevel computer workstation are only a few minutes.

Predictions from the MBED-1D are compared to temperature and pressure measurements from a commercial-scale Wellman-Galusha dry-ash gasifier. Predicted axial temperature and pressure profiles, using a common set of model parameters, compared well with the measured values for many coals. A suitable estimate of bed void distritution was shown to be necessary to predict the pressure drop throughout the ieactor. A linear increase in bed void fraction from the top to the bottom of the reactor was found to be sufficient for most calculations. The void distribution dramatically influences volumetric reaction rates through the particle number density.

From temperature profile comparisons, higher coal feed rates or processes, which cause the gas phase to become fuel-richer, also cause the location of the maximum temperature to move toward the bottom of the reactor. Such processes include: cracking of tar in the gasification and devolatilization zones; increasing devolatilization rate by neglecting volatiles transport; and increasing the volatile content through changes in coal type, moisture content, or ash content. Devolatilization is not instantaneous, and volatile yield significantly affects the temperature profile and the location of the maximum temperature. Various dips and peaks in the axial temperature profile correspond to different functional groups that evolve at different temperatures. Furthermore, a dual temperature peak may result during high-pressure gasification as a result of competing endothermic and exothermic reactions. Oxidation and gasification do not occur in separate zones, but simultaneously in the reactor bed. Furthermore, results suggest that solid-togas heat transfer for reacting fixed beds is significantly smaller than for nonreacting fixed beds. Diffusional resistance of oxidizer through the ash layer during oxidation and gasification was predicted to be significant.

Important model parameters whose values are not well established were identified. These include the solid-to-gas heattransfer coefficient, ash layer thickness and oxidizer diffusivity through the char-ash layer, and the variable bed void fraction. The effective diffusivity of oxidizer through the ash layer is a complex function of the developing ash porosity and tortuosity which is not likely to be constant throughout the reactor. Future research should include the effect of coal structure, mineral matter, devolatilization and bed burden on the effective diffusivity.

\section{Acknowledgment}

This work was sponsored principally by the U. S. Department of Energy, Morgantown Energy Technology Center (Contract No. DEAC21-86MC23075) under subcontract from Advanced Fuel Research, Inc., East Hartford, and in part by the Advanced Combustion Engineering Research Center at Brigham Young University. Funds for this center are received from the National Science Foundation's Engineering Centers, the State of Utah, 25 industrial participants, and the U.S. Department of Energy. Help from Dr. M. Usman Ghani is also acknowledged. Helpful suggestions and comments by the reviewers are also acknowledged.

$$
\begin{aligned}
& \text { Notation } \\
& \qquad \begin{aligned}
A & =\text { cross-sectional area of reactor, } \mathrm{m}^{2} \\
A & =\text { particle surface area, } \mathrm{m}^{2} \\
A & =\text { preexponential factor, } \mathrm{m} / \mathrm{s} \cdot \mathrm{K} \\
A & =\text { water wall surface area, } \mathrm{m}^{2} \\
C & =\text { carbon } \\
C & =\text { molar concentration of oxidizer or gasification agent, } \\
& =\mathrm{kmol} / \mathrm{m}^{3} \\
C_{p} & =\text { specific heat, } \mathrm{J} / \mathrm{kg} \cdot \mathrm{K} \\
d & =\text { particle diameter, } \mathrm{m} \\
D & =\text { diffusivity, } \mathrm{m}^{2} / \mathrm{s} \\
D & =\text { reactor diameter, } \mathrm{m} \\
E & =\text { activation energy, J/kmol } \\
F & =\text { fraction of original carbon }(\mathrm{Eq} .9), \text { mass fraction } \\
g & =\text { acceleration of gravity, } 9.80665 \mathrm{~m} / \mathrm{s}^{2} \\
G & =\text { superficial gas mass flux }, \mathrm{kg} / \mathrm{m}^{2} \cdot \mathrm{s} \\
h & =\text { enthalpy, J/kg } \\
h & =\text { heat-transfer coefficient, } \mathrm{W} / \mathrm{m}^{2} \cdot \mathrm{K}
\end{aligned}
\end{aligned}
$$


$H=$ convective heat capacity flux, $\mathrm{J} / \mathrm{m}^{2} \cdot \mathrm{s}$

$H=$ hydrogen

$k=$ rate and Arrhenius rate constant, $\mathrm{m} / \mathrm{s}$ or $1 / \mathrm{s}$

$k=$ thermal conductivity, $\mathrm{W} / \mathrm{m} \cdot \mathrm{K}$

$k=$ mass transport coefficient, $\mathrm{m} / \mathrm{s}$

$L=$ reactor length, $\mathrm{m}$

$\dot{m}=$ mass flow rate, $\mathrm{kg} / \mathrm{s}$

$M w=$ molecular weight, $\mathrm{kg} / \mathrm{kmol}$

$N=$ nitrogen

$O=$ oxygen

$P=$ pressure, $\mathrm{kPa}, \mathrm{Pa}$

$P e=$ Peclet number (Table 2)

$\operatorname{Pr}=$ Prandtl number (Table 2)

$Q=$ heat loss, $\mathrm{W} / \mathrm{m}^{3}$

$r=$ volumetric reaction rate, $\mathrm{kg} / \mathrm{m}^{3} \cdot \mathrm{s}$

$\boldsymbol{R}=$ ideal gas constant, $\mathrm{J} / \mathrm{kmol} \cdot \mathrm{K}$

$R e=$ Reynold's number (Table 2)

$S=$ sulfur

Sc $=$ Schmidt number (Table 2)

$t=$ time, $s$

$T=$ temperature, $\mathrm{K}$

$u=$ velocity, $\mathrm{m} / \mathrm{s}$

$V=$ volatile matter, mass fraction

$V=$ volume, $\mathrm{m}^{3}$

$V M=$ volatile matter, mass fraction

$W=$ mass-flow rate, $\mathrm{kg} / \mathrm{s}$

$x=$ gas mole fraction

$x=$ tar fraction, mass fraction

$x=$ tar parameter used in correlation of Ko et al. (1988)

$y=$ functional group fraction, mass fraction

$z=$ axial distance, $m$

\section{Greek letters}

$\epsilon=$ bed void fraction, void vol./bed vol.

$\epsilon^{\prime}=$ emissivity

$\phi=$ packing parameter defined by Eq. 10 in Table 2

$\phi=$ porosity, vol./vol.

$\eta=$ particle number density, $1 / \mathrm{m}^{3}$

$x=$ ratio of solid conductivity and gas conductivity

$\lambda=$ stoichiometric coefficient for oxidation reaction, mol/ mol

$\mu=$ viscosity, $\mathrm{kg} / \mathrm{m} \cdot \mathrm{s}$

$y=$ stoichiometric coefficient, $\mathrm{mol}$ carbon/mol oxidant

$\rho=$ density, $\mathrm{kg} / \mathrm{m}^{3}$

$\mathbf{a}=\mathbf{d r y}$, ash-free mass fraction

$\omega=$ functional group fraction or element fraction, mass fraction

$\zeta=$ bed-to-wall heat-transfer multiplier (for sensitivity analysis)

$\zeta=$ particle area factor to account for internal surface burning

$\zeta=$ reacting to nonreacting solid-to-gas heat-transfer ratio

$\tau=$ tortuosity

\section{Subscripts}

$a=$ ash

$B G=$ blast gas

$c=$ char

$c=$ coal

$\mathrm{CO}_{2}=\mathrm{CO}_{2}$ gasification

$e=$ effective

$e=$ equilibrium

eff = effective

$g=g a s$

$\mathbf{g w}=$ gas-to-wall

$\mathrm{H}_{2}=\mathrm{H}_{2}$ gasification

$\mathrm{H}_{2} \mathrm{O}=\mathrm{H}_{2} \mathrm{O}$ gasification

$h w=$ heat transfer to the wall

$i=$ index for elements, species, or reactions

$j=$ index for solid species

$\boldsymbol{m}=$ mass transport

$\boldsymbol{m}=$ mixture

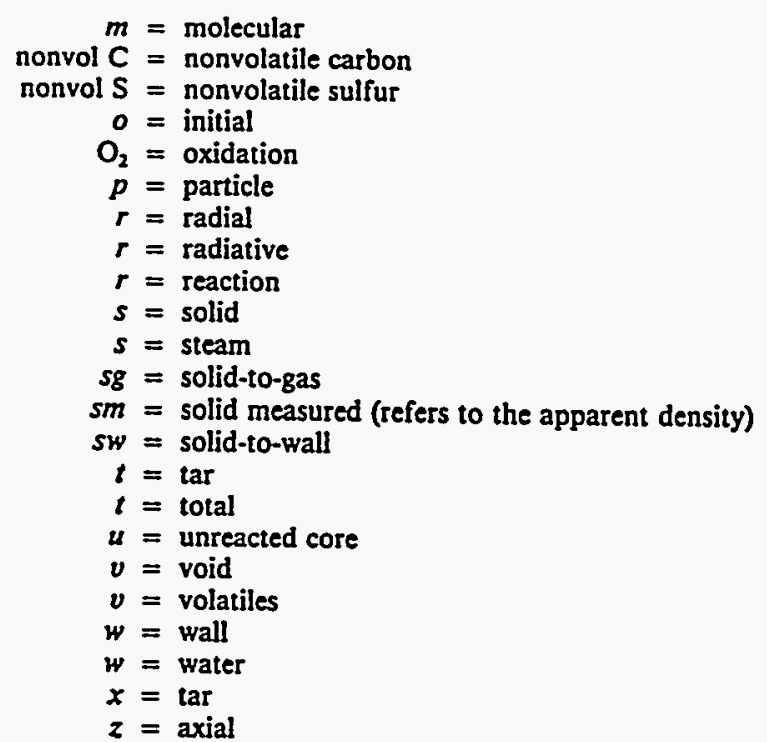

Superscripts

$a=$ axial thermal

$d=$ devolatilization

$\boldsymbol{g}=$ gas

$h=$ thermal

$m=$ axial mass

$0=$ initial

$0=$ reference temperature

0 = static

$z=$ sensible enthalpy

$s=$ solid

$t=$ true

ultimate $=$ yield at large times

$\infty=$ infinity or ultimate

\section{Literature Cited}

Amundson, N. R., and L. E. Arri, "Char Gasification in a Countercurrent Reactor," AIChE J., 24, 87 (1978).

Barriga, A., and R. H. Essenhigh, "A Mathematical Model of a Combustion Pot: Comparison of Theory and Experiment," Paper 80-WA/HT-32, ASME Meeting, Chicago (1980).

Baxter, L. L., personal communication, Brigham Young Univ., Provo (1987).

Bhattacharya, A., L. Salam, M. P. Dudukovic, and B. Joseph, "Experimental and Modeling Studies in Fixed-Bed Char Gasification," Ind. Eng. Chem. Process Des. Dev., 25, 988 (1986).

Bird, R. B., W. E. Stewart, and E. N. Lightfoot, Transport Phenomena, Wiley, New York (1960).

Bischoff, K. B., "A Note on Gas Dispersion in Packed Beds," Chem. Eng. Sci., 24, 607 (1962).

Blackwood, J. D., "The Reaction of Carbon with Hydrogen at High Pressure," Aust. J. Chem., 12, 14 (1959).

Cho, Y. S., Modeling and Simulation of Lurgi-type Gasifiers, MS Thesis, Washington Univ., St. Louis, MO (1980).

Cho, Y. S., and B. Joseph, "Heterogeneous Model for Moving-Bed Coal Gasification Reactors," Ind. Eng. Chem. Process Des. Dev, 20, 314 (1981).

Crowe, C. T., and L. D. Smoot, "Multicomponents Conservation Equations," Pulverized-Coal Combustion and Gasification, L. D. Smoot and D. T. Pratt, eds., Plenum Press, New York (1979).

Desai, P. R., and C. Y. Wen, "Computer Modeling of the MERC Fixed Bed Gasifier," MERC/CR-78/3, U. S. Dept. of Energy, Morgantown, WV (1978).

DeWasch, A. P., and G. F. Froment, "A Two-Dimensional Heterogeneous Model for Fixed Bed Catalytic Reactors," Chem. Eng. Sci., 26, 629 (1971). 
Dzhapbyev, K., A. L. Miropol'skii, and V. J. Mal'kovskii, "Investigation of Unsteady Heat Transfer in a Packed Bed of Spheres Swept by Gas," Thermal Eng., 33(3), 159 (1986); translated from Teploenergetika, 33(3), 70 (1986).

Earl, W. B., and K. A. Islam, "Steady-state Model of a Lurgi-type Coal Gasifier," Innovation in the Process and Resource Industry, CHEMCA, Proc. Australian Chem. Eng. Conf., Perth, Australia, Paper c2b, 289 (1985).

Elgin, D. C., and H. R. Perks, "Results of Trials of American Coals in Lurgi Pressure-Gasification Plant at Westfield, Scotland," Synthetic Pipeline Gas Symp., Chicago (Oct. 18-20, 1974).

Ergun, S., "Fluid Flow Through Packed Columns," Chem. Eng. Prog., 48, 89 (1952).

Fayed, M. E., and L. Otten, eds., Handbook of Powder Science and Technology, Van Nostrand Reinhold, New York (1984).

Field, M. A., D. W. Gill, B. B. Morgan, and P. G. W. Hawksley, Combustion of Pulverized Coal, The British Coal Utilization Res. Assoc., Leatherhead, England (1967).

Fletcher, T. F., A. R. Kerstein, R. J. Pugmire, M. S. Solem, and D. M. Grant, "A Chemical Percolation Model for Devolatilization: 3. Chemical Structure as a Function of Coal Type," Energy and Fuels, submitted (1992).

Froberg, R. W.. "The Carbon-Oxygen Reaction: an Experimental Study of the Oxidation of Suspended Carbon Spheres," PhD Diss., Pennsylvania State Univ., University Park (1967).

Froment, G. F., and K. B. Bischoff, Chemical Reactor Analysis and Design, Wiley, New York (1979).

Goetz, G. J., N. Y. Nsakala, R. L. Patel, and T. C. Lao, "Combustion and Gasification Characteristics of Chars from Four Commercially Significant Coals of Different Rank," final report 165-6, EPRI, Palo Alto, CA (1982).

Gray, D. D., and J. M. Stiles, "On the Constitutive Relation for Frictional Flow of Granular Materials," topical report DOE/MC/ 21353-2584, U. S. Dept. of Energy, Morgantown, WV (1988).

Gray, D., J. G. Cogoli, and R. H. Essenhigh, "Problems in Pulverized Coal and Char Combustion," Adv. Chem. Ser., 131, 72, (1974).

Gupta, A. S., and G. Thodos, "Direct Analogy Between Mass and Heat Transfer to Beds of Spheres," AIChE J., 9(6), 751 (1963).

Hedman, P. O., L. D. Smoot, P. J. Smith, and A. U. Blackham, "Entrained-Flow Gasification at Elevated Pressure," DOE/MC/ 22059-2570, Combustion Laboratory, Chemical Engineering Dept., Brigham Young Univ., Provo (1987).

Hindmarsh, A. C., "ODEPACK, A Systematized Collection of ODE Solvers," Scientific Computing, R. S. Stepleman, ed., Vol. 1, p. 55, IMACS Trans. on Scientific Computation, North-Holland, Amsterdam (1983).

Hobbs, M. L.. "Modeling Countercurrent Fixed-Bed Coal Gasification," PhD Diss., Brigham Young Univ., Provo (1990).

Hobbs, M. L., P. T. Radulovic, and L. D. Smoot, "Prediction of Effluent Temperatures and Compositions for Fixed-Bed Coal Gasifiers," Fuel, accepted (1992).

Khanna, R., and J. H. Seinfeld, "Mathematical Modeling of Packed Bed Reactors: Numerical Solutions and Control Model Development," Advances in Chemical Engineering, J. Wei, J. L. Anderson, K. B. Bischoff, M. M. Denn, and J. H. Seinfeld, eds., Vol. 13, p. 113. Academic Press (1987).

Kim, M., and B. Joseph, "Dynamic Behavior of Moving-Bed Coal Gasifiers," Ind. Eng. Chem. Process Des. Dev., 22, 212 (1983).

Ko, G. H., D. M. Sanchez, W. A. Peters, and J. B. Howard, "Correlations for Effects of Coal Type and Pressure on Tar Yields from Rapid Devolatilization," Symp. Int. on Combustion, p. 115, The Combustion Institute, Pittsburgh (1988).

Krishnudu, T., B. Madhusudhan, S. N. Reddy, V. S. R. Sastry, K.S. Rao, and R. Vaidyeswaren, "Studies in a Moving Bed Pressure Gasifier: Prediction of Reaction Zones and Temperature Profile," Ind. Eng. Chem. Res., 28, 438 (1989).

Kunii, D., and J. M. Smith, "Heat Transfer Characteristic of Porous Rocks," AIChE J., 6(1), 71 (1960).

Kurylko, L.." "The Unsteady and Steady Combustion of Carbon," PhD Diss., The Pennsylvania State Univ., University Park (1969). Laurendeau, N. M., "Heterogeneous Kinetics of Coal Char Gasification and Combustion," Prog. Energy Combust. Sci., 4, 221 (1978). Lowry, H. H., Chemistry of Coal Utilization, Supple. Vol. Wiley, New York (1963).

Merrick. D., "Mathematical Models of the Thermal Decomposition of Coal: 2. Specific Heats and Heats of Reaction," Fuel, 62, 540 (1983).

Mills, K. C., and J. M. Rhine, "The Measurement and Estimation of the Physical Properties of Slags Formed During Coal Gasification: 2. Properties Relevant to Heat Transfer," Fuel, 68, 201 (1989).

Nsakala, N., R. L. Patel, and T. C. Lao, "Combustion Characterization of Coals for Industrial Applications," Final Technical Report for U. S. Dept. of Energy, Pittsburgh, Combustion Engineering Inc., Kreisinger Development Laboratory, Winsor, CT, DOE/PC/ 402067-5 (DE85010673) (Mar. 1985).

Nuttall, H. E., W. G. Stoddart, and W. J. Chen, "Pyrolysis of Subbituminous New Mexico Coal," J. of Petr. Tech., 31, 418 (1979).

Park, K. Y., and T. F. Edgar, "Modeling of Early Cavity Growth for Underground Coal Gasification," Ind. Eng. Chem. Res., 26, 237 (1987).

Perry, R. H., and C. H. Chilton, eds., Chemical Engineers' Handbook, Sth ed., McGraw-Hill, New York (1973).

Phuoc, T. X., and M. P. Mathur, "Transient Heating of Coal Particles Undergoing Pyrolysis," Combust. and Flame, 85, 380 (1991).

Radulovic, P. T., M. L. Hobbs, and L. D. Smoot, "Fixed-Bed Coal Combustion and Gasification Review," Prog. Energy Combust. Sci., submitted (1992).

Rohsenow, W. M., J. P. Hartnett, and E. N. Ganic, Handbook of Heat Transfer Applications, McGraw-Hill, New York (1985).

Saxena, S. C., "Devolatilization and Combustion Characteristics of Coal Particles," Prog. Energy Combust. Sci., 16, 55 (1990).

Serio, M. A., D. G. Hamblen, J. R. Markham, and P. R. Solomon, "Kinetics of Volatile Product Evolution in Coal Pyrolysis: Experiment and Theory," Energy \& Fuels, 1, 138 (1987).

Smith, I. W., "The Combustion Rates of Coal Chars: a Review," Int. Symp. on Combustion, p. 1045. The Combustion Institute, Pittsburgh (1983).

Smoot, L. D., and P. J. Smith, Coal Combustion and Gasification, Plenum Press, New York (1985).

Smoot, L. D., and P. J. Smith, Pulverized-Coal Combustion and Gasification, p. 224, L. D. Smoot and D. T. Pratt, eds., Plenum, Press, New York (1979).

Solomon, P. R., D. G. Hamblen, R. M. Carangelo, M. A. Serio, and G. Y. Deshpande, "General Model of Coal Devolatilization," Energy and Fuels, 2, 405 (1988).

Stillman, R., "Simulation of a Moving Bed Gasifier for a Western Coal," IBM J. Res. Dev., 23, 240 (1979).

Stull, D. R., and H. Prophet, JANAF Thermochemical Tables, 2nd ed., National Bureau of Standards (1971).

Suuberg, E. M., W. A. Peters, and J. B. Howard, "Product Compositions and Formation Kinetics in Rapid Pyrolysis of Pulverized Coal-Implications for Combustion," Int. Symp. on Combustion, p. 177, The Combustion Institute, Pittsburgh (1979).

Thimsen, D., personal communications (1990).

Thimsen, D., R. E. Maurer, A. R. Pooler, D. Y. H. Pui, B. Y. H. Liu, and D. B. Kittelson, "Fixed-Bed Gasification Research using U. S. Coals," 1-19, U. S. Bureau of Mines Contract H0222001, final report (1984).

Thorsness, C. B., and S. W. Kang, "Further Development of a General-Purpose, Packed-Bed Model for Analysis of Underground Coal Gasification Processes," Underground Coal Gasification Symp., Denver (1985).

Thorsness, C. B., and S. W. Kang, "A General-Purpose, Packed-Bed Model for Analysis of Underground Coal Gasification Processes," UCID-20731, Lawrence Livermore National Laboratory, Univ. of California, Berkeley (1986).

Thorsness, C. B., S. W. Kang, "A Method-of-line Approach to Solution of Packed-bed Flow Problems Related to Underground Coal Gasification Prokesses," Underground Coal Gasification Symp., Williamsburg, VA (1984).

Walker, P. L., F. Rusinko, and L. G. Austin, Advances in Catalysis, p. 134, D. D. Eley, P. W. Selwood, and P. B. Weisz, eds., XI, Academic Press, New York (1959).

Wang, S. C., and C. Y. Wen, "Experimental Evaluation of Nonisothermal Solid-Gas Reaction Model," AIChE J., 18(6), 1231 (1972).

Wen, C. Y., and T. Z. Chaung, "Entrainment Coal Gasification Modeling," Ind. Eng. Chem. Process Des. Dev., 18(4), 684 (1979).

Wen, C. Y., H. Chen, and M. Onozaki, User's Manual for Computer Simulation and Design of the Moving Bed Coal Gasifier, final report 
prepared for Morgantown Energy Technology Center, U. S. Dept. of Energy, DOE/MC/16474-1390, Morgantown, WV (1982).

Winslow, A. M., "Numerical Model of Coal Gasification in a Packed Bed," Int. Symp. on Combustion, p. 503, The Combustion Institute, Pittsburgh, (1976).

Yagi, S., and D. Kunii, "Studies on Heat Transfer Near Wall Surface in Packed Beds," AIChE J., 6(1), 97 (1960).

Yagi, S., D. Kunii, and N. Wakao, "Studies on Axial Effective Thermal Conductivities in Packed Beds, AIChE J., 6(4), 543 (1960).

Yagi, S., and N. Wakao, "Heat and Mass Transfer from Wall to
Fluid in Packed Beds," AIChE J., 5(1), 79 (1959).

Yoon, H., "Modeling and Analysis of Moving Bed Coal Gasifiers," PhD Diss., Univ. of Delaware, Newauk (1978).

Yoon, H., J. Wei, and M. M. Denn, "A Model for Moving-bed Coal Gasification Reactors," AIChE, J., 24(5), 885 (1978).

Yu, W., M. M. Denn, and J. Wei, "Radial Effects in Moving Bed Coal Gasifiers," Chem. Eng. Sci., 38, 1467 (1983).

Manuscript received Aug. 9, 1991, and revision received Mor. S, 1992. 


\title{
APPENDIX E
}

\author{
NETWORK MODELS OF COAL \\ THERMAL DECOMPOSITION
}




\title{
Network models of coal thermal decomposition
}

\author{
Peter R. Solomon, David G. Hamblen, Zhen-Zhong Yu and \\ Michael A. Serio \\ Advanced Fuel Research, Inc., 87 Church Street, East Hartford, CT 06108, USA \\ (Received 24 July 1989; revised 2 February 1990)
}

\begin{abstract}
Several groups have considered statistical network fragmentation models to describe coal thermal decomposition. In these models, the coal macromolecule is viewed as a collection of fused aromatic rings (monomers) linked by bridges. During thermal decomposition, existing bridges break and new bridges are formed. The parameters of the models are the geometry of the network, which is expressed as the number of attachments per monomer (the coordination number, $\sigma+1$ ), and the chemistry of bridge breaking and formation. Given $\sigma+1$ and the instantaneous number of unbroken and formed bridges. the molecular weight distribution can be predicted. The different groups have employed both Monte Carlo methods and percolation theory to describe the network statistics. The former approach has advantages in terms of describing both the depolymerization and crosslinking processes in coal decomposition, since it does not require a constant coordination number. The latter method provides closed form solutions and is computationally less demanding. The models differ in the geometry of the network, the chemistry of bridge breaking and bridge formation (crosslinking) and the mass transport assumptions. This paper considers for three such models: the mathematical schemes; the assumed network geometries; the assumed bond breaking and bond formation chemistries; and the mass transport assumptions. The predictions of three models were compared by comparing the oligomer populations as a function of the number of unbroken bridges per ring cluster. This paper also presents results from a new model which combines the geometry, chemistry and mass transport assumptions of the FG-DVC model with the mathematics of a modified percolation theory.
\end{abstract}

(Keywords: thermal decomposition; coal; modelling)

Many recent studies have proposed that coal can be thought of as having a macromolecular network structure to which concepts of crosslinked polymers can be applied $^{1-10}$. These concepts have been employed to understand and model such properties of coal as: the insolubility; the equilibrium swelling and penetration of solvents; the viscoelastic properties; similarities between the parent coal and products of hydrogenolysis, or mild oxidation; crosslinking during char formation ${ }^{11.12}$; and the formation of coal tar in pyrolysis ${ }^{13-1:}$. With the success of these concepts in describing coal properties, it appears logical to extend macromolecular network concepts to completely describe the thermal decomposition behaviour of coal.

A number of investigators have used statistical methods to predict how the network behaves when subjected to thermally induced bridge breaking, crosslinking, and mass transport processes ${ }^{17-30}$. Gavalas et al. ${ }^{20}$ employed statistical methods to predict the release of monomers from a randomly connected network. The model of Niksa and Kerstein employed percolation theory in a model called DISARAY ${ }^{28}$, which extended their previous model built on chain statistics ${ }^{24.25}$. Grant et al. $^{30}$ employed percolation theory in a model called chemical percolation devolatilization (CPD). Solomon et al. ${ }^{21-23,27}$ used Monte Carlo methods in a network model called the depolymerization, vaporization, and crosslinking (DVC) model. This was an extension of their previous model for linear polymers ${ }^{17.20}$. The DVC model was recently combined with their functional group (FG) model $^{27.29}$ to produce the general FG-DVC pyrolysis model. This model is currently being applied to model the devolatilization behaviour of Argonne premium coals $^{31}$ and to predict the fluidity of coals $s^{32}$. Other statistical methods for $n$ 'twork behaviour have been employed in the polymer literature ${ }^{33-38}$.

In applying network models to coal thermal decomposition, one considers the coal to consist of aromatic ring clusters linked together by bridges in some geometry. The geometry is described by the degree of branching in the network. An unbranched linear network will have one bridge per ring cluster attaching it to the next cluster. Thus each cluster has two attachments and is said to have a coordination number $(\sigma+1)$ of two. A highly branched 'fish net' would have two bridges per cluster, attaching it to the neighbouring four clusters and thus a coordination number of four. A branch point is considered to occur at any cluster connected to more than two neighbours (i.e. having more than two attachments).

When the coal is heated, the bridges can break and new bridges can form. Various statistical methods can be employed to predict the concentration of individual aromatic ring clusters (monomers) and linked clusters (oligomers of $n$ clusters, ' $n$-mers') up to a totally linked network. By assigning an average or distribution of molecular weights to the monomers, the amounts of tar, extractables, liquids or char can then be defined from the distribution of oligomer sizes. The models vary in the assumed chemistry of bridge breaking and crosslinking, 
in the definition of tar, extracts. liquids, and char and in the statistical methods used.

In view of the importance of macromolecular network models to the accurate predictions of coal processing behaviour, this paper assesses the assumptions and limitations of the proposed models. It appears that the way one performs the statistics (Monte Carlo, percolation theory, or other statistical methods) makes little difference. For example, percolation theory methods were substituted for Monte Carlo calculations in the FG-DVC model, and comparable predictions were obtained for appropriately restricted cases. The important differences among models are in the assumptions for: the network geometry (i.e. the degree of branching or coordination number); the chemistry of bridge breaking; the chemistry of crosslink formation; hydrogen utilization; and mass transport. This paper compares the three most recent models (DISARAY, CPD, and FG-DVC) and considers how the assumed network properties relate to behaviours observed for coal.

\section{MACROMOLECULAR NETWORKS}

\section{General properties of networks}

Figures 1 and 2 present the networks employed in the FG-DVC Monte Carlo calculations and percolation theory, respectively. For the FG-DVC Monte Carlo calculation, linear oligomers of $l$ clusters (shown as the horizontal chains of clusters) of a molecular weight distribution defined by $M_{\text {avg }}$ and deviation. $\Delta M$, are linked by $m_{0}$ 'crosslinks' per monomer 26.29 . These 'crosslinks' are indicated by the vertical double lines as shown in Figure 1. The branch points in the network are defined to occur at those clusters where more than two attachments connect a cluster to another cluster. Unless the crosslink occurs at the end of the oligomer, it forms at least one branch point. The term 'crosslink' has previously been used to mean the extra bridges that can form a branch point, and this is the definition employed here ${ }^{27.29}$. During thermal decomposition, bridges break, new crosslinks are added and the molecular weight of the oligomers is calculated by randomly distributing these changes.

For the percolation theory, a Bethe lattice is employed ${ }^{28,30,39}$. Lattices are characterized by the coordination number, $(\sigma+1)$, which is the number of attachment sites for bridges per cluster and the probability, $p$, that an unbroken bridge occupies the site. Figure 2 shows lattices at $p=1$ (i.e. all possible bridges

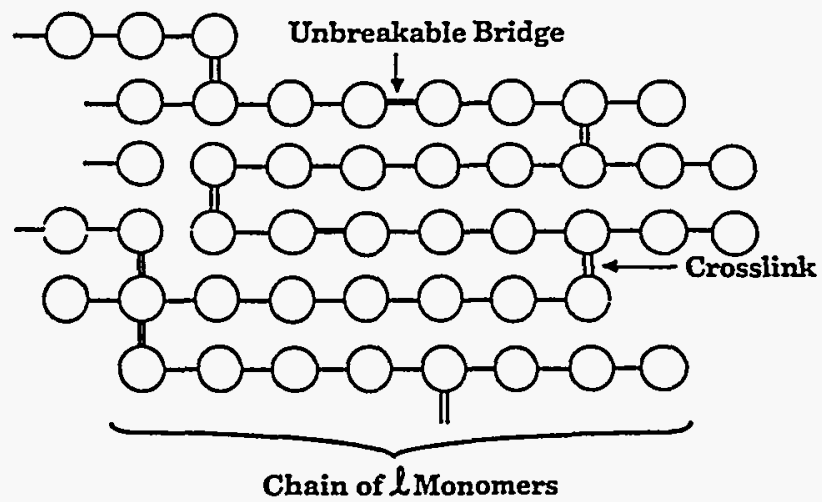

Figure 1 Macromolecular network used in Monte Carlo simulation a

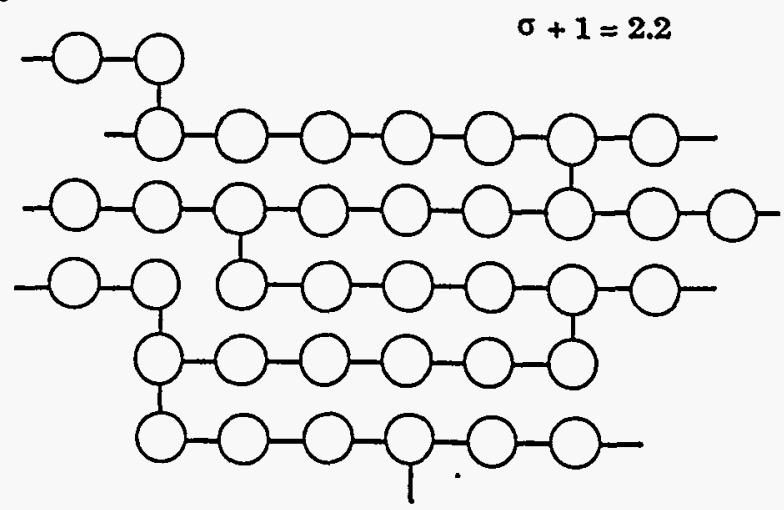

b

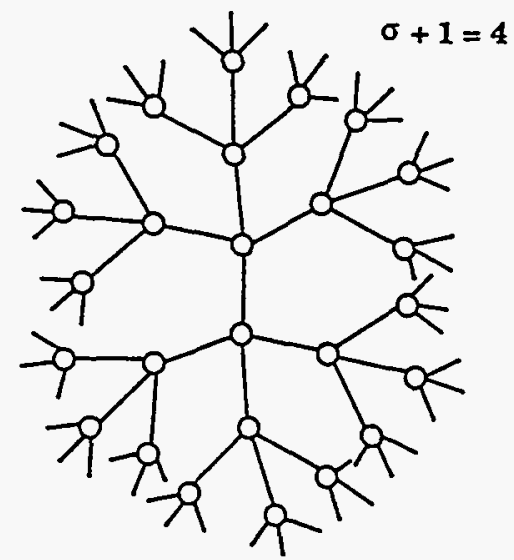

Figure 2 Bethe lattice for a, coordination number 2.2, $p=l$ : $b$. coordination number $4, p=1$

occupied) for $\sigma+1=2.2$ and $\sigma+1=4$. The Bethe lattice has no loops, but it has been demonstrated that this lattice is a good approximation to a lattice of equivalent coordination number containing loops ${ }^{39}$. As shown in Figure 2, the $\sigma+1=2.2$ lattice has branch points every 5 th cluster. This network is close to a linear polymer $\sigma+1=2$ which has no branch points. The $\sigma+1=4$ lattice is much more highly branched, having double branch points on each cluster (this would be the Bethe lattice analogue of the 'fish net'). For values of $p$ less than 1 , the bridges are randomly removed from the network. It is important to realize that the statistical distribution of unbroken bridges means that even when the average number of unbranched bridges per cluster is identical for the two networks in Figure 2, the $\sigma+1=4$ network can never look like the $\sigma+1=2.2$ network (except for the case $p=0$ ).

The loop free geometry of the Bethe lattice allows for the number of free oligomers to be analytically expressed as a function of $\sigma+1$ and the probability $p$ of bonds being unbroken. This is the feature which makes the percolation theory so attractive from the standpoint of computer efficiency and for understanding the behaviour of networks under conditions of varying bridge populations. Figure 3 shows calculations using percolation theory (for three values of $\sigma+1$ ) for the monomer, the sum of oligomers up to 3 , up to 10 , and the sum of all free oligomers as a function of the number of unbroken bridges per ring cluster $\alpha$, where $\alpha=1 / 2 p(\sigma+1)$. The $1 / 2$ enters because $\sigma+1$ is the number of attachments per cluster, which is twice the number of bridges (i.e. each 

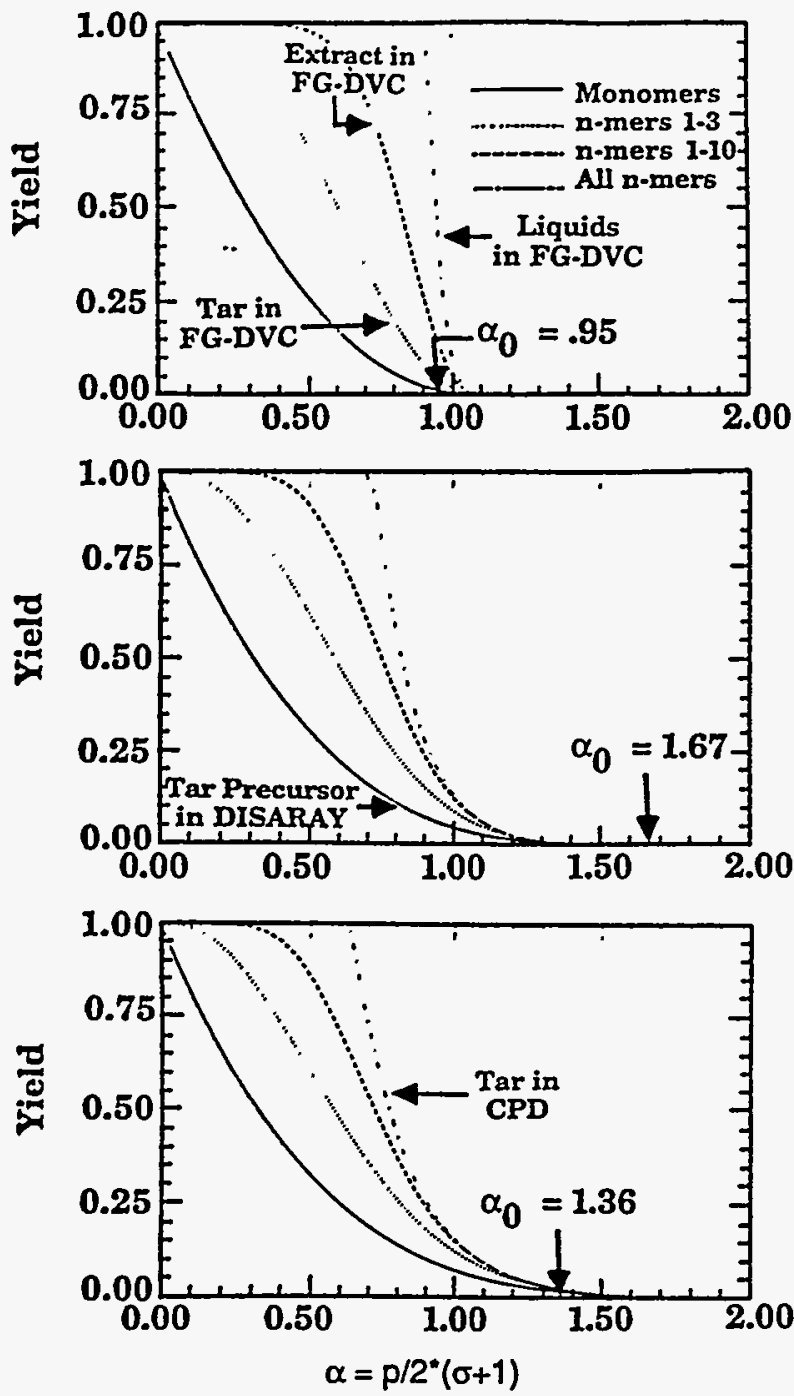

Figure 3 Percolation theory predictions for pyrolysis products (monomers. tar, extracts and total liquids) for three values of the coordination number $(\sigma+1): a, \sigma+1=2.2 ; b, \sigma+1=3.25 ; c, \sigma+1=4.6$

bridge forms two attachments). If $\sigma+1$ remains constant during pyrolysis, the molecular weight distribution is a single valued function of $\alpha$. For ring clusters of molecular weight $300 \mathrm{amu}$, the sum of 1-3 $n$-mers corresponds roughly to the potential tar fraction (up to $900 \mathrm{amu}$ ), the sum of $1-10 n$-mers corresponds to the extractable fraction (up to $3000 \mathrm{amu}$ ), and the sum of all oligomers corresponds to the liquids fraction (all free oligomers). It can be seen that, with increasing $\sigma+1$, more broken bridges are required to achieve equivalent fractions of free oligomers. Also, at a fixed value of $\alpha$, the relative amounts of ta, extracts, and liquids vary with $\sigma+1$.

\section{Network geometries representative of coal}

The three important parameters of the network are the average ring cluster size $M_{\text {avg }}$, the coordination number $(\sigma+1)$, and the starting probability of bridges being unbroken, $p_{0}$. To compare networks of different coordination numbers, it is convenient to use $\alpha$ and $\alpha_{0}$ rather than $p$ and $p_{0}$. The assumptions of DISARAY, CPD, and FG-DVC are considered below.

Ring cluster size. Ring cluster sizes have been estimated from n.m.r. alone ${ }^{40}$. n.m.r. and FT-i.r. ${ }^{41}$, mild degradation ${ }^{42}$. and the molecular weight distribution of $\operatorname{tar}^{15.16 .29}$. Based on these results. the average ring cluster size for coals with less than $90 \%$ carbon is expected to be between 2 and 3 aromatic rings or a total molecular weight per cluster, including peripheral groups, of $200-400 \mathrm{amu}$. DISARAY assumes a value of $1400 \mathrm{amu}$ for the monomer which can split into two $700 \mathrm{amu}$ tar fragments. CPD does not specify the monomer molecular weight. For coals with less than $90 \%$ carbon. FG-DVC employs a distribution of monomers with an $M_{\text {avg }}$ of 256 amu.

Coordination number. Information on the coordination number comes from solvent swelling measurements and recent estimates made using n.m.r. of the number of non-peripheral group attachments to the cluster ${ }^{40}$. There is some controversy about the meaning of the measurements of the solvent swelling ratio. Network theories have, however, been applied ${ }^{2-9}$ to relate the solvent swelling ratio, $Q$, and the average molecular weight of a cluster, $M_{\text {2vg }}$, to the average molecular weight between branch points, $M_{\mathrm{c}}$. The $M_{\mathrm{c}}$ determinations ${ }^{2-9}$ suggest that there are between 4 and 8 repeating units (ring clusters) between branch points for coals with less than $90 \%$ carbon. (In this work the term crosslink has been used to indicate the bridge which makes a ring cluster into a branch point). This indicates a value for $\sigma+1$ between 2.13 and 2.25 . since $\sigma+1$ is the average number of attachments per cluster ( 1 bridge makes two attachments). The n.m.r. data suggest that there are between 2 and 3 bridge or loop attachments per cluster (see Figure 8 of Ref. 40). This suggests $\sigma+1$ is between 2 and 3 . Based on these two measurements, the coordination number for the starting coal for describing the break up of the network by bridge cleavage should be less than 3, and probably between 2.2 and 2.5. A different value of $\sigma+1$ might be appropriate for describing crosslinking (branch point formation at higher temperature). To model a high volatile bituminous coal, the different models used networks with $(\sigma+1)=3.25$ (DISARAY), 4.6 (CPD), and $\approx 2.1$ (FG-DVC).

Initial bridge population, $\mathrm{p}_{0}$. The starting macromolecular network for FG-DVC is chosen to match the measured extract yield and molecular weight between crosslinks by picking two parameters: the length of the linear oligomer chain, $l$; and the number of initial crosslinks (branch points) per monomer, $m_{0}$. First $m_{0}$ is picked such that $m_{0}=M_{\mathrm{avg}} / M_{\mathrm{c}}$, where $M_{\mathrm{avg}}$ is the average ring cluster (monomer) molecular weight and $M_{\mathrm{c}}$ is the molecular weight between crosslinks determined from solvent swelling ${ }^{2-9}$. Then $l$ is chosen so that when the molecule is randomly constructed, the weight per cent of oligomers less than $3000 \mathrm{amu}$ matches the measured extract yield. There is the implicit assumption that the extract yield is due to the unpolymerized fraction of a homogeneous network. Polymethylenes or highly fluid macerals (e.g. exinites), which can be a significant portion of the extracts in coal, should really be treated as separate components but were not in the first version of the FG-DVC model. The initial value of $\alpha$ is approximately $\left((l-1) / l+m_{0}\right)$. which for the Pittsburgh seam coal modelled in Ref. 29 is $\alpha_{0} \approx 0.95$. This initial value is indicated in Figure $3 a$. In DISARAY, $p_{0}$ is set equal to $1\left(\alpha_{0}=1.63\right)$. This is illustrated in Figure $3 b$. 
The starting macromolecular network in the CPD model is chosen by picking two parameters: the coordination number $\sigma+1$, picked to match the average number of all connections (including peripheral groups in addition to bridges and loop attachments) per ring determined by n.m.r. ${ }^{30.40}$; and $p_{0}$. the starting probability of unbroken bonds. For the high volatile bituminous coal simulated in Ref. 30. $z_{0}=1 / 2 p_{0}$ $(\sigma+1)=1.36$. This initial value is indicated in Figure 3 .

\section{PROCESSES CONTROLLING THE NETWORK DECOMPOSITION}

This section considers the important processes in pyrolysis: bridge breaking, and hydrogen utilization: crosslinking (branch point formation): and the mass transport processes which control the distribution of oligomers into tar, extracts, liquids. and solids. The processes are summarized in Figure 4.

\section{Bridge breaking and hydrogen utilization}

Figure $4 a$ summarizes the bridge breaking assumptions of the three models. Both the FG-DVC and CPD models assume similar (within a factor of 3) bridge breaking rates, $0.86 \times 10^{15} \exp -(55400 / R T) \mathrm{s}^{-1}$ for FG-DVC* and $2.6 \times 10^{15} \exp -(55400 / R T) \mathrm{s}^{-1}$ for CPD. Both models employ rank independent kinetics. The FG-DVC model rate was determined in experiments in which particle temperatures were directly measured ${ }^{43}$. The rate was recently confirmed within a factor of 2 by Fletcher et al. ${ }^{44}$ in a second experiment to directly measure particle temperatures. The DISARAY model* assumes a bridge dissociation rate which can produce monomers of $6 \times 10^{8} \exp -(30000 / \mathrm{RT}) \mathrm{s}^{-1}$. The monomers subsequently decompose at $1.4 \times 10^{7} \exp -(31000 / \mathrm{RT}) \mathrm{s}^{-1}$ to form tar.

In DISARAY, all the initial bridges can break. In FG-DVC and CPD, there is a process for creating unbreakable bridges associated with the bridge breaking process. The FG-DVC model includes three kinds of bonds: labile bridges, 'unbreakable bridges' (which do not form branch points), and crosslinks (which do form branch points). The unbreakable bridges are represented by the heavy horizontal lines in Figure 1. For each broken labile bridge, FG-DVC requires that hydrogen be available to stabilize the free radicals. It is assumed that all the donatable hydrogen (aliphatic plus hydroaromatic) is located in the labile bridges, so that only half the labile bridges can break with the other half becoming unbreakable with the donation of their hydrogen (i.e. there is a 1:1 ratio between the occurrence of bridge breaking and the formation of additional 'unbreakable bridges'). In the FG-DVC model. crosslinks are also considered to be bridges which cannot be broken. but are not in the 'unbreakable bridges' pool. The weight fraction of the initial bridges in the chain of length $l$ which are labile is given by the parameter $W_{B}$; the rest are assumed to be unbreakable bridges. $W_{B}$ is a fitting parameter chosen to make the model fit the pyrolysis data.

In a similar manner, in CPD there are both unbreakable bridges with probability $c_{0}$ and labile bridges with probability $L_{0}\left(L_{0}+c_{0}=p_{0}\right)$. As pyrolysis

* Both FG-DVC and DISARAY employ distributed activation energy expressions. The rates quoted above are for the centre of the distribution proceeds, the labile bridges can break and react by two possible routes to form unbreakable 'char' bridges or broken bridges. CPD assumes a 0.9:1.0 ratio of broken bridge to char bridge formation. That assumption is almost identical to the $1: 1$ ratio used in FG-DVC. In

\section{a Bridge Breaking}

DISARAY
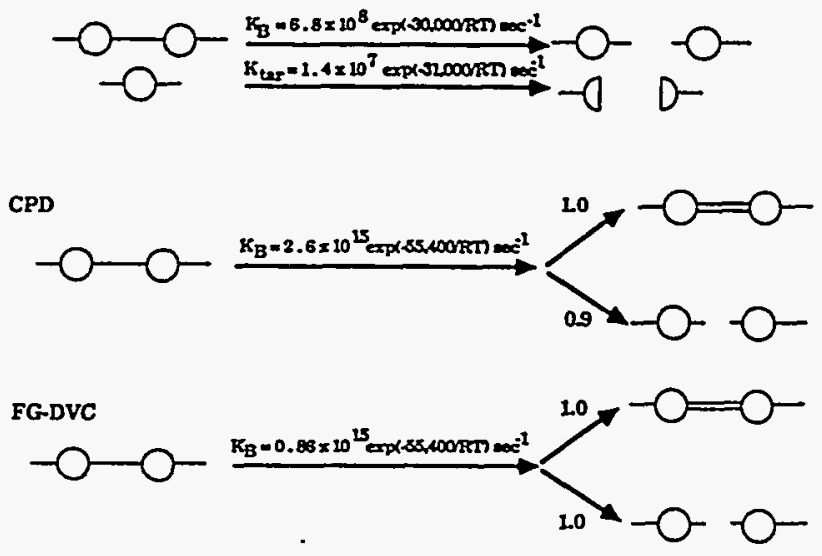

b Crosslinking

DISARAY

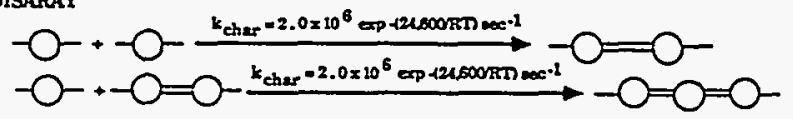

CPD

No Independent Crosedinling Proceses

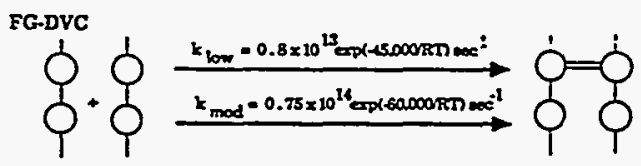

c ProductDistribution

DISARAY

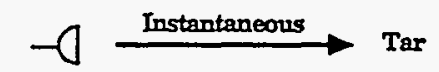

CPD
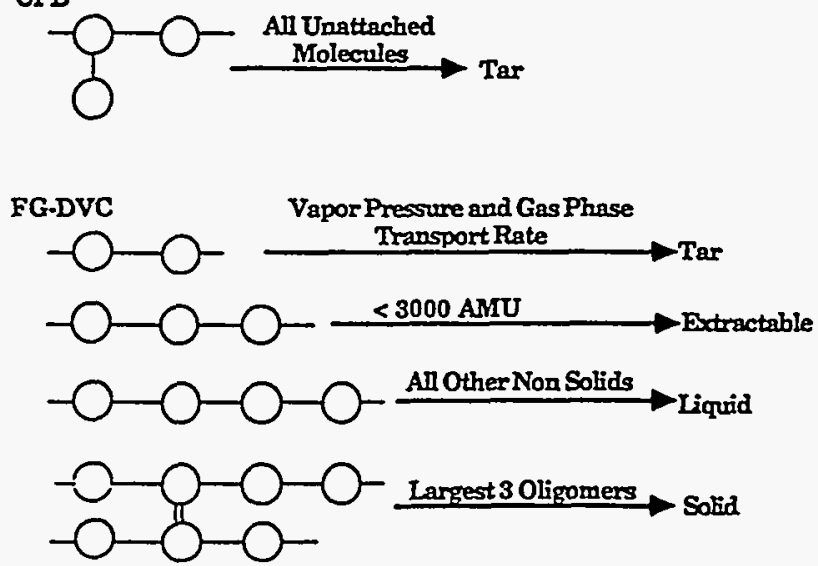

Figure 4 Summary of model assumptions for a, bridge breaking; $b$, crosslinking; c, product distribution 
CPD there is no distinction between unbreakable bridges and crosslinks. A branch point occurs any place there are three or more attachments per cluster.

\section{Crosslinking}

The crosslinking (branch point forming) reactions are summarized in -Figure $4 b$. CPD does not define any distinct crosslinking processes. Char forming reactions produce unbreakable bridges as a consequence of bridge breaking. These reactions form branch points (three or more attachments per cluster) randomly depending on the coordination number, $\sigma+1$ and probability, $p$.

DISARAY assumes char formation occurs at a rate $2 \times 10^{6} \exp -(24600 / \mathrm{R} T) \mathrm{s}^{-1}$. Char formation is assumed to occur by monomers attaching to the original lattice or to each other.

FG-DVC assumes two independent crosslinking reactions. These are the only reactions which can form branch points in the Monte Carlo formulation. One process occurs at low temperature (below that for bridge breaking) for low rank coals and is associated with $\mathrm{CO}_{2}$ evolution ${ }^{11.12,45}$. Crosslinking also occurs at moderate temperatures, slightly higher than bridge breaking, and is associated with the evolution of $\mathrm{CH}_{4}$. The model assumes one crosslink is formed for each $\mathrm{CH}_{4}$ or $\mathrm{CO}_{2}$ evolved ${ }^{29}$. The mechanistic basis for these assumptions has been discussed elsewhere ${ }^{45}$.

\section{Product distribution}

The product distribution assumptions are summarized in Figure 4c. The identification of different size oligomers with tar, extracts, liquids, and solids is related to their molecular weight. The oligomers which can form tar are the lightest fraction. Tar formation is controlled in part by the vapour pressure of the components. This idea is supported by the observation that tar yields are strongly influenced by external pressure 29.46 .47 .

Only oligomers with molecular weights less than 1000 amu have sufficient vapour pressure to become gas at typical pyrolysis tempratures. so tar is roughly limited to $<1000 \mathrm{amu}^{47-51}$. The extract yield is controlled by the solubility of the oligomers. For coal fragments in pyridine this limit is roughly $3000 \mathrm{amu}^{47-49.52}$. Larger fragments appear to be important to the fluid properties of $\mathrm{Coal}^{32}$.

In FG-DVC, the Monte Carlo calculation is employed to determine the molecular weight distribution in the decomposing char. Then, a mass transport equation is applied to determine the probability of the light $n$-mers evolving as tar. The transport equation assumes that a molecular weight dependent vapour pressure controls the appearance of these molecules in the gas phase and that they escape the coal particles by convective transport of the $\operatorname{gas}^{29}$. Tar is thus the light end of the molecular weight spectrum, i.e. those with sufficiently high vapour pressures. This produces tar with number average molecular weights of $300-400 \mathrm{amu}$ and maximum weights of $800-1000 \mathrm{amu}$. Thus, in FG-DVC, tar is approximately the sum of 1-3 n-mers in Figure $3 a$. Extractable material is defined as all molecules up to $3000 \mathrm{amu}$ (sum of 1-10 $n$-mers) and liquids are defined as all molecules not attached to the starting network. An alternative definition, which has been employed here, is to assume the largest three molecules constitute the solid, and all the rest are liquid.

In DISARAY, tar is defined as half the monomer. and the monomer is taken as $1400 \mathrm{amu}$. Consequently, the tar would be defined as some fraction of the monomer curve in Figure $3 b$. No transport equations are employed in CPD. Tar is defined as all molecules not attached to the infinite lattice. Thus tar is represented by the highest line in Figure 3c.

One advantage of the Monte Carlo method over the percolation theory is that, when tar is produced, molecules can be removed from the network. In percolation theory, there is no consistent way to remove molecules from the network and to allow the formation of new bridges such as the crosslinking events in FG-DVC. CPD avoids this problem by excluding any independent crosslinking which would reconnect oligomers. This presents the limitation that independent crosslinking and mass transport cannot be treated with the exact percolation theory expressions.

\section{EXAMPLES OF MODEL CALCULATIONS}

\section{Formation of p!rolysis products}

The evolution of the macromolecular network in the CPD model is illustraied in Figure 5. Figure $5 a$ shows the percolation theory predictions for the total of
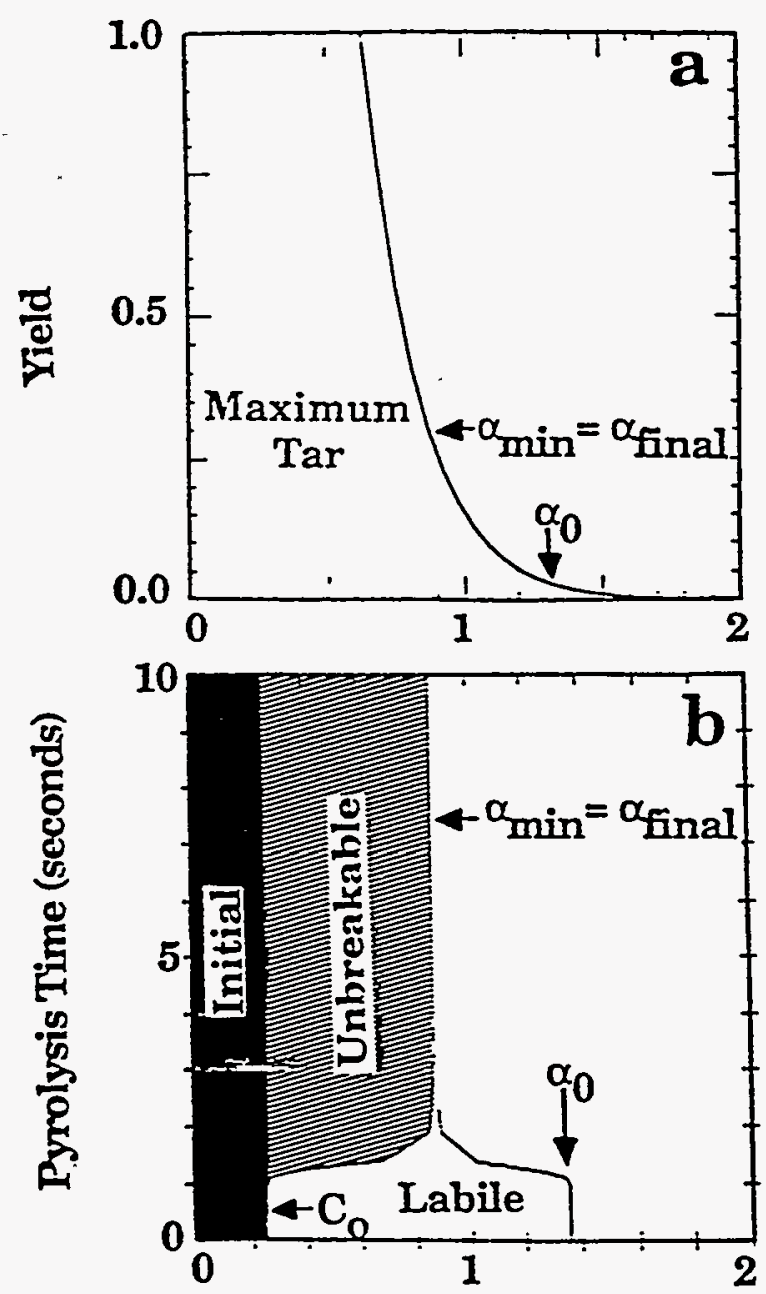

No. of UnbrokenBridges per Bead, $\alpha$

Figure 5 Tar yield for a bituminous coal predicted by the CPD model: a. tar yield versus $x$ : b. variation in $\alpha$ with time. heating at $450^{\circ} \mathrm{C} \mathrm{s}^{-1}$ to $936 \mathrm{~K}$. The shaded areas show the relative amounts of the two types of crosslinks (initial: unbreakable bridge formation) 

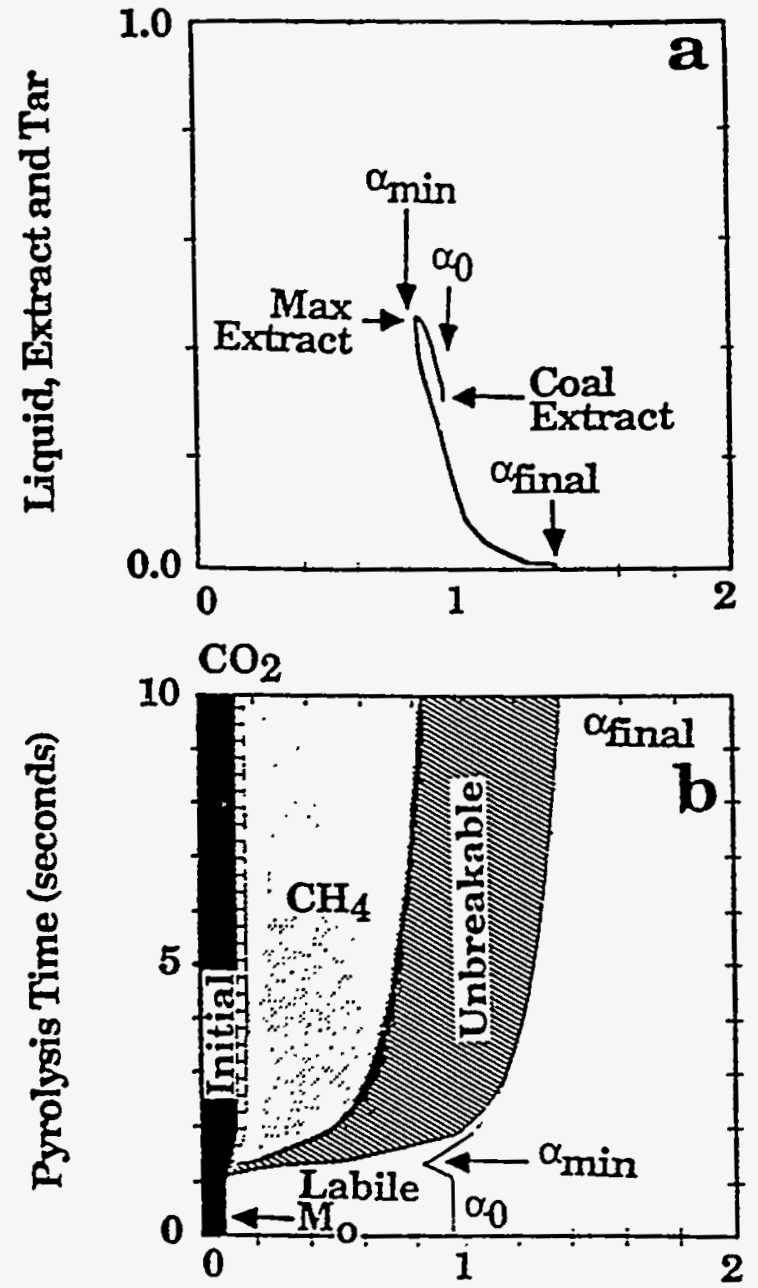

No. of UnbrokenBridges per Bead, $\alpha$

Figure 6 Extract yield for a bituminous coal predicted by the FG-DVC model: a, extract yield versus $\alpha: b$, variation in $z$ with time. heating at $450^{\circ} \mathrm{C} \mathrm{s}^{-1}$ to $936 \mathrm{~K}$. The shaded areas show the relative amounts of the three types of crosslinks (initial: crosslinks related to gas evolution; unbreakable bridge formation)

unattached oligomers (defined to be the tar) as a function of $\alpha$. The coal is represented at $p_{0}=0.59$ or $\alpha_{0}=1 / 2 p_{0}$ $(\sigma+1)=1.36$. During pyrolysis, the labile bridges form either broken bridges or unbreakable char bridges in the ratio 0.9 to 1.0. Figure $5 b$ shows how $\alpha$ changes during pyrolysis. Pyrolysis proceeds until $\alpha_{\min }$ is reached, and all the labile bridges are either broken or have formed unbreakable bridges. Thus

$$
x_{\min }=1 / 2(\sigma+1)\left(c_{0}+(1.0 / 1.9) L_{0}\right)=0.83
$$

and the change in $\alpha$ during pyrolysis was 0.53 . Note that $\alpha$ can only decrease in the CPD model.

The evolution of the macromolecular network for FG-DVC computed using the Monte Carlo method for a bituminous coal is illustrated in Figure 6. Figure $6 a$ shows the calculated extract yield as a function of $\alpha$. The initial probability of unbroken bridges, $\alpha_{0}$, starts out at close to 1.0 to produce the measured extract yield $(30 \%)$. Figure $6 b$ shows the computed value of $\alpha$ with its contributions from the initial crosslinks $m_{0}$, the conversion of labile bridges to broken bridges and unbreakable bridges and the added crosslinks related to gas evolution. For the bituminous coal, the added crosslinks are almost all due to $\mathrm{CH}_{4}$ related processes. $x$ goes back up in the FG-DVC model to resolidify the lattice. This is necessary to model fluidity effects ${ }^{32}$.

Results of the FG-DVC model applied to a lignite are presented in Figure 7. For the lignite, the formation of low temperature crosslinks from $\mathrm{CO}_{2}$ evolution prevents $x$ from being reduced due to bridge breaking. Thus pyrolysis produces no additional extract yield. The thermosetting behaviour of the low rank coal, and the release of little tar or extracts is related to this low temperature crosslinking process.

\section{Utilization of donatable hydrogen}

As discussed abo:e, $W_{B}$, the initial fraction of labile bridges, is a parameter of the FG-DVC model. This parameter is related to the fraction of donatable hydrogen by $H(d)=2: 28 W_{\mathrm{B}}$, i.e. there are two donatable hydrogens per labile bridge. This parameter has a strong effect on $x_{\min }$ and hence the yield of tar, extracts, and liquids.

There are two ways to estimate the amount of hydrogen donated. During pyrolysis the donation of hydrogen converts two aliphatic or hydroaromatic
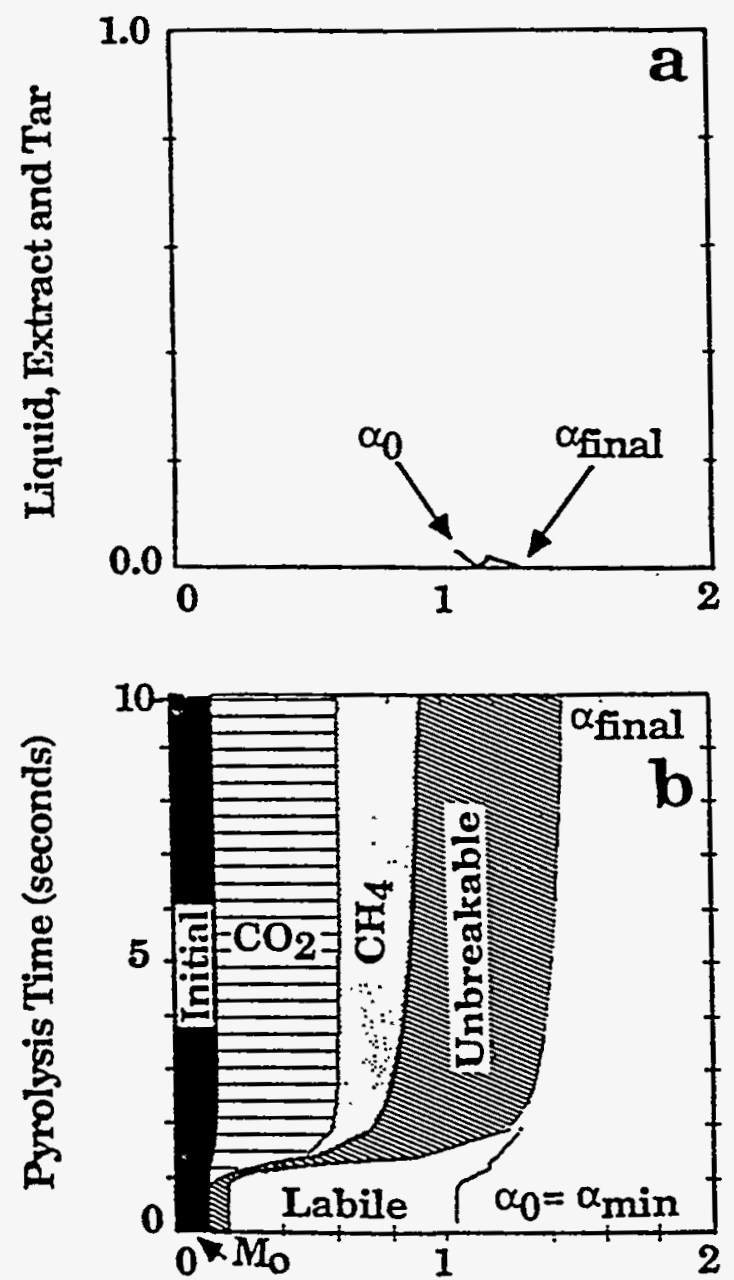

No. of UnbrokenBridges per Bead, $\alpha$

Figure 7 Extract yield for a lignite predicted by the FG-DVC model: a. extract vield versus $z: b$, variation in $\alpha$ with time, heaing at $450^{\circ} \mathrm{C} \mathrm{s}^{-1}$ to $9: 5: \therefore$. The shaded $2: 20 s$ show the relative amounts of the three types of crosslinks (initial: crosslinks related to gas evolution: unbreakable bridge formation) 


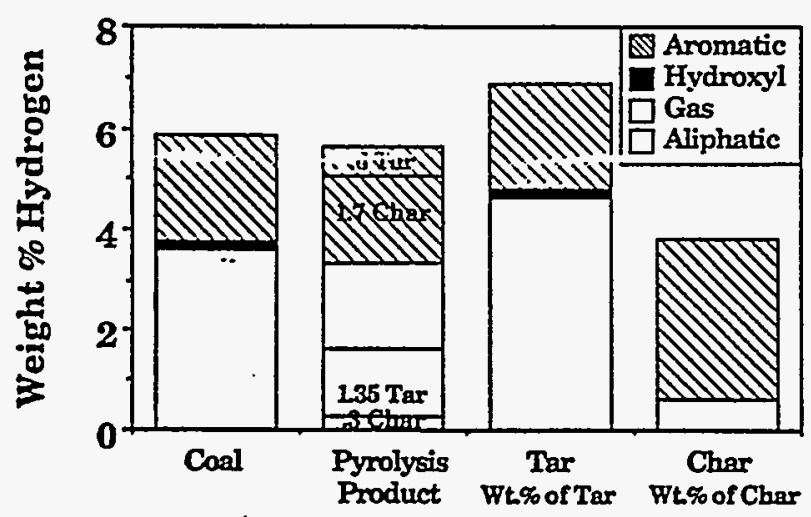

Figure 8 Distribution of hydrogen in coal and pyrolysis products. Pyrolysis produced approximately $53 \%$ char, $30 \%$ tar and $21 \%$ gas

hydrogens into a donated aliphatic hydrogen plus a newly formed aromatic hydrogen. The increase in aromatic hydrogen in the pyrolysis products. and the increase in aliphatic hydrogen in the tar. can both be measured using quantitative $\mathrm{FT}-\mathrm{i} . \mathrm{r}$. analysis ${ }^{53.5:}$. The results for a Pittsburgh seam coal are summarized in Figure 8 . They show that the aromatic hydrogen in the total pyrolysis products increased from 2.1 to $2.4 \%$, or an increase of $0.3 \%$ on a starting coal basis. This increased aromatic content is all in the char. The aromatic content in the tar remains about the same. The tar, which is approximately $30 \%$ of the starting coal, increases its aliphatic hydrogen content by about $1 \%$ or $0.3 \%$ on the starting coal basis. The two numbers are thus consistent; $0.6 \%$ aliphatic or hydroaromatic hydrogens in the coal are converted to $0.3 \%$ new aromatics plus $0.3 \%$ donated aliphatics. If it is assumed that a monomer has a molecular weight of $300 \mathrm{amu}$, then one breakable bridge per monomer with four aliphatic hydrogens is $1.33 \%$. Half the bridges can break $(0.67 \%)$ and the other half $(0.67 \%)$ can donate half its hydrogen $(0.34 \%)$. in reasonable agreement with the experimentally estimated value of $0.3 \%$ hydrogens actually donated. The vaiue assumed ${ }^{29}$ in FG-DVC for $H(d)$ for the Pittsburgh seam coal is $0.67 \%$.

The value of $H(d)$ has implications for the CPD model. If there is only one labile bridge per monomer. then

$$
\begin{aligned}
x_{\min } & =1 / 2(\sigma+1)\left(c_{0}+(1.0 / 1.9) L_{0}\right) \\
& =1 / 2(4.6)(0.37+(1.0 / 1.9) 0.22)=1.11
\end{aligned}
$$

rather than 0.83 . In this case, the value of $\sigma+1$ would have to be reduced to match the data. Also. the average molecular weight for the unattached molecules is too high to be identified as tar. If a more reasonable definition of tar is used (e.g. the sum of oligomers up to 3), then $\sigma+1$ has to be reduced still further.

\section{Comparison of Monte Carlo calculation with percolation theory}

To further illustrate some of the differences between the FG-DVC Monte Carlo model and percolation theory calculations, the extract yield calculated for a case similar to that in Figure 6a, but with tar evolution not permitted. is plotted in Figure 9 along with the predictions of percolation theory for several values of $\sigma+1$. The FG-DVC Monte Carlo prediction is not a single valued function of $\alpha$. As pyrolysis proceeds, the increase in extract yield follows $\sigma+1 \approx 2.2$ while the decrease in extract yield follows $\sigma+1 \approx 4$.

It is important to know whether this result is an artefact of the Monte Carlo calculation or a real feature of pyrolysis. Based on what is known to happen in pyrolysis, the result does make sense. For a bituminous coal, the initial process occurring in pyrolysis is bridge breaking. This occurs by breaking bridges in the network described by $\sigma+1$ between 2.1 and 2.5. No crosslinking occurs initially as the solvent swelling ratio is observed to increase during this period ${ }^{45}$. Thus. the coordination number used in CPD. which includes all connections to the ring cluster (bridges, loops, and peripheral groups) ${ }^{30.40}$. may not be appropriate to this phase of pyrolysis. Eventually crosslinks start forming, resulting in an increase in the coordination number and in $\alpha$. There is evidence that crosslinks form at peripheral group sites so that the coordination number used in CPD (the sum of branches and peripheral groups) is appropriate for this phase of the process. Consequently, the network cannot adequately be described by one type of bridge site with a single coordination number. There are bridge sites for labile bridges and for crosslinks, each with their own coordination number. This observation motivated the development of a more general percolation theory model.

\section{LATTICE MODEL WITH TWO BRIDGE BOND TYPES}

\section{Two- $\sigma$ model}

To deal with a structure with a time dependent coordination number, a Bethe lattice with two types of bridging bonds is considered. with coordination numbers and probabilities of occupation given by $\sigma_{1}+1, p$, and $\sigma_{2}+1 . q$, for the two types, respectively. Such a lattice for $\sigma_{1}+1=\sigma_{2}+1=2$ is illustrated in Figure 10. The analysis can be carried through using the procedures described previously ${ }^{30.39}$, but with extensions to deal with the extra variables. The equations are presented in Appendix A.

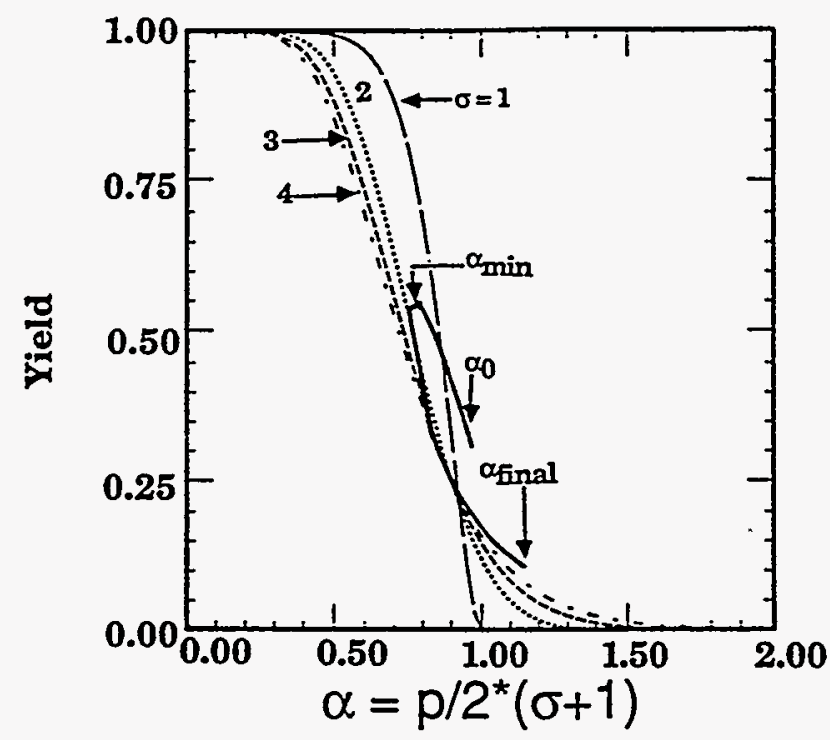

Figure 9 Comparison of extract yield predictions from FG-DVC model with percolation theory for $\sigma=1,2,3$ and 4. FG-DVC predictions are for Pittsburgh seam coal heated at $450^{\circ} \mathrm{C} \mathrm{s}^{-1}$ to $936 \mathrm{~K}$ with no tar evolved 


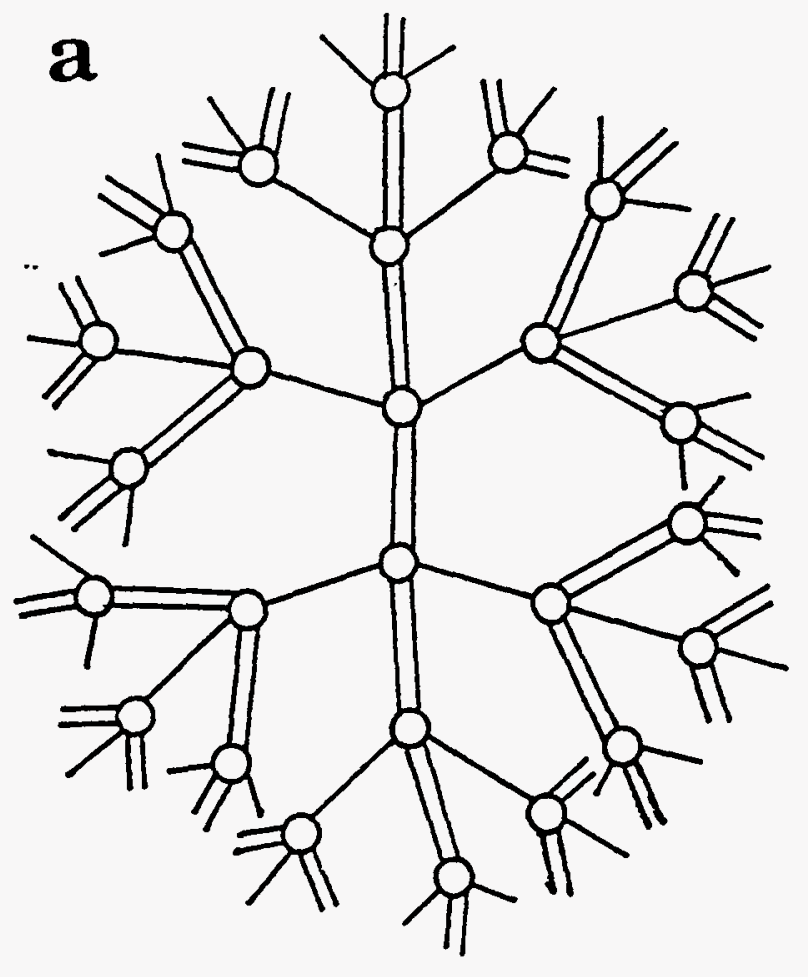

b

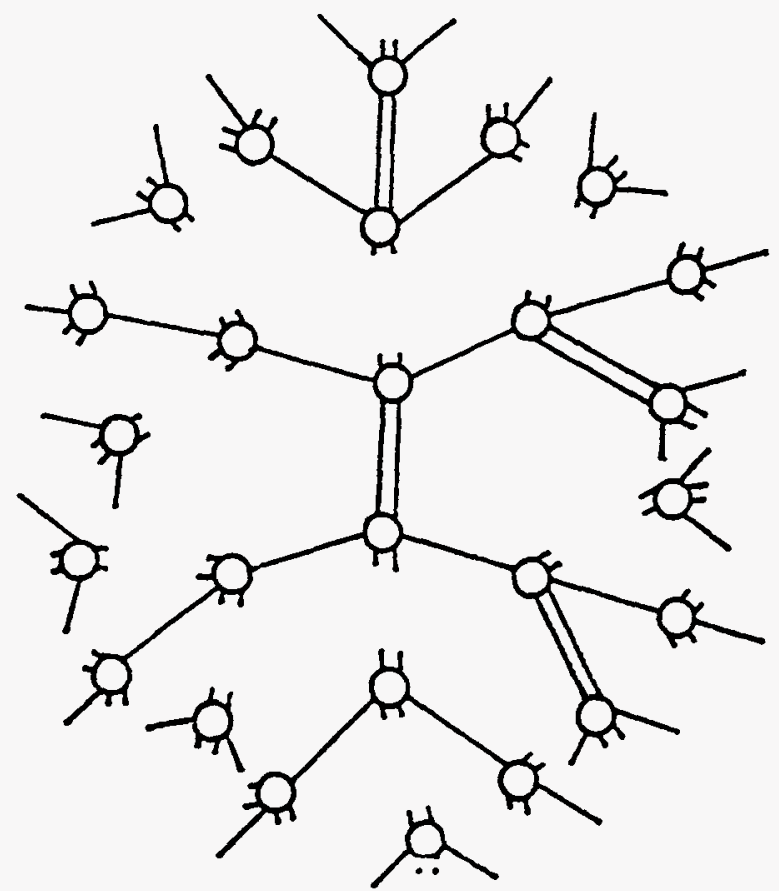

Figure 10 Bethe lattice for two- $\sigma$ model with $\sigma_{1}=1$ (shown as single bonds) and $\sigma_{2}=1$ (shown as double bonds): a. fully linked case $(p=q=1)$ is like one $\sigma$ model with $\sigma=3 ; b$, shown with most double bonds (representing the crosslinks) not yet formed to represent the starting coal. This lattice is like a one- $\sigma$ model with $\sigma=1$, linear chains

\section{Application of two- $\sigma$ model}

Figure 11 presents a comparison of the predictions for pyrolysis assuming the FG-DVC chemistry, using: the Monte Carlo calculation; the two- $\sigma$ percolation calculations $\left(\sigma_{1}+1=2, \sigma_{2}+1=2\right)$; and two cases of the one- $\sigma$ percolation calculation $(\sigma+1=2.2$ and $\sigma+1=3.2)$. The calculations are made under the assumption that no tar is evolved. The tar values in Figure 11 are the sum of 1-3n-mers remaining in the char. The Monte Carlo calculation in Figure $11 a$ is matched best by the two- $\sigma$ model if liquids are assumed to be the sum of the first 100 n-mers (i.e. up to $300000 \mathrm{amu}$ ). The two- $\sigma$ model has a reasonable value for the initial extract yield but predicts slightly more initial tar. Neither of the one- $\sigma$ cases is a good match. Use of $\sigma+1=2.2$ is good at low temperature. but overpredicts the maximum values of extracts and liquids and resolidifies the network very abruptly at too low a temperature. Use of $\sigma+1=3.2$ does a much better job at predicting the maximum values of tar, extract and liquids. and resolidifies the network more slowly at a higher temperature, but the initial ratio of tar to extract is not consistent with that observed for coal and the rate of increase of $n$-mers is too slow. It thus appears that the two- $\sigma$ model can be used instead of the Monte Carlo calculations when no tar is evolved, while one- $\sigma$ calculations are less accurate.

The real test, however, is how well the models fit the data for coal. A comparison of tar yield is not a sufficient test since $\alpha_{0}$ and $\Delta x$ can always be selected in conjunction with the network geometry to fit the data. A critical test requires a careful comparison of how $\alpha_{0}$ and $\alpha(t)$ match with measurement of functional group changes in the char (e.g. the transformation of hydrogen functional groups and bridges), solvent swelling behaviour (i.e. crosslink density), and the complete molecular weight distribution as reflected in the amounts of tar, extracts, and fluidity.

\section{COMPARISON OF NETWORK MODELS}

A summary of the processes predicted by the three recent network models, CPD, DISARAY and FG-DVC is presented in Table 1. All the models predict their primary objective, i.e. the variations in tar and gas yield with time and temperature. All three are capable of predicting
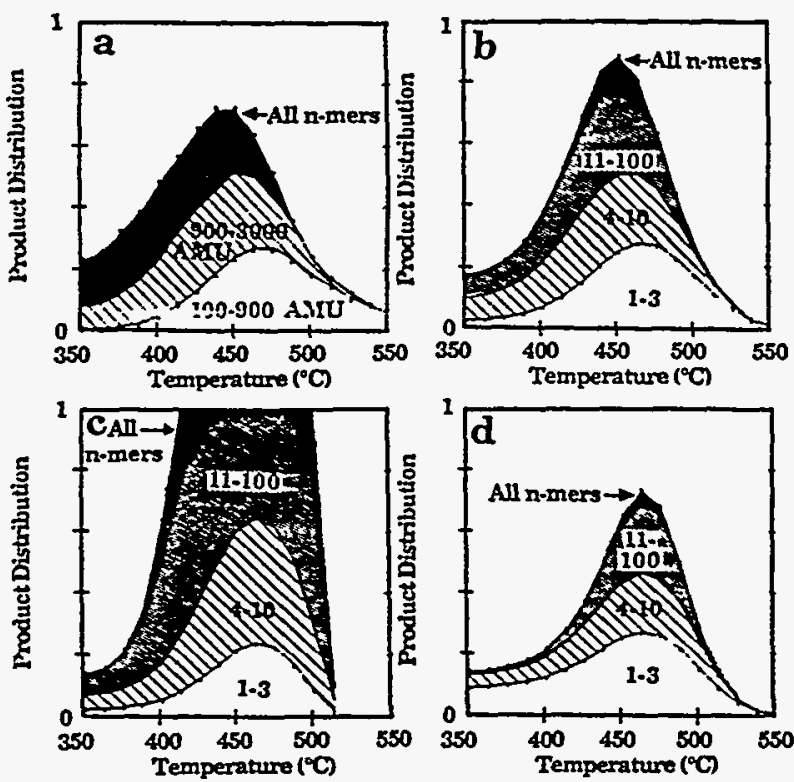

Figure 11 Comparison of distribution of $n$-mers for pyrolysis of upper Freeport coal at $3^{\circ} \mathrm{C} \mathrm{min}^{-1}:$ a. Monte Carlo calculation; $b$, two- $\sigma$ model $\left(\sigma_{1}=1, \sigma_{2}=1\right) ; c$, one- $\sigma$ model $(\sigma=1.2): \mathrm{d}$, one- $\sigma$ model $(\sigma=2.2)$ 
Network models of coal thermal decomposition: P. R. Solomon et al.

Table 1 Comparison of network models

\begin{tabular}{|c|c|c|c|c|}
\hline & CPD & DISARAY & $\begin{array}{l}\text { FG-DVC } \\
\text { Monte-Carlo } \\
\text { or } 2 \sigma\end{array}$ & Relevant model process \\
\hline Tar yield versus time & Yes & Yes & Yes & Bond breaking \\
\hline Extract yield versus. time & No & No & Yes & Bond breaking \\
\hline Gas yield versus time & Yes & Yes & Yes & From peripheral groups \\
\hline Tar yield versus heating rate & Not yet & Yes & Yes & Relative rates of bond breaking and crosslinking \\
\hline Variation of tar molecular weight with heating rate & No & No & Yes & Relative rates of bond breaking and crosslinking \\
\hline Molecular weight of tar & No & No & Yes & Mass transport limitation \\
\hline Tar yields versus pressure & No & No & Yes & Mass transport limitation \\
\hline Molecular weight versus pressure & No & No & Yes & Mass transport limitation \\
\hline Solvent swelling of char & No & No & Yes & Crosslinking \\
\hline
\end{tabular}

- All oligomers are defined as tar

variations of tar yield with heating rate, but $C P D$ has not yet done this. All three models are capable of predicting the complete molecular weight distributions of fragments, but only FG-DVC uses this information to predict the extract yield, the tar yield and the tar molecular weight distribution. DISARAY uses only the prediction for monomers (defined as tar precursor) and CPD uses only the prediction for all oligomers (defined as tar). In a recent paper ${ }^{32}$, the total oligomer population computed by the FG-DVC model was used to predict coal fluidity behaviour. Only FG-DVC employs a mass transport equation which is necessary to predict tar molecular weights and the variations of yield and molecular weights with pressure. Only FG-DVC predicts the solvent swelling ratio, which is determined by the crosslink density in the char.

\section{CONCLUSIONS}

This paper examines the extension of macromolecular network concepts to describe coal thermal decomposition. The statistical methods (Monte Carlo calculations and percolation theories) and the classes of chemical reactions (labile bridge breaking, hydrogen utilization, crosslinking) and mass transport (vaporization and convection) employed by different researchers have been compared. The conclusion are as follows:

1. The application of macromolecular network concepts appears to be a very promising and versatile approach.

2. Monte Carlo methods for computing the network statistics are the most versatile, but are computationally demanding.

3. The use of percolation theory is computationally efficient and helps provide insight into network behaviour, but the use of a fixed coordination number may be inadequate to accurately describe coal thermal decomposition. The network appears to require a coordination number between 2.2 and 2.5 during labile bridge breaking and greater than 3 during crosslinking.

4. Alternatively, a more general percolation theory model for a network with two types of bridging bonds was developed, each with their own $\sigma+1$.

5. When the two- $\sigma$ percolation model is applied using the FG-DVC chemistry to cases in which tar is not removed, it is much more flexible in matching the Monte Carlo calculations. The one- $\sigma$ models either decompose at too low a temperature for large values of $\sigma+1$, or decompose too much and resolidify at too low a temperature for small values of $\sigma+1$. Applying percolation theory to cases where tar is removed requires additional approximations.

6. Of the three models compared (CPD, DISARAY, and FG-DVC), FG-DVC is the most complete in treating the molecular weight distribution of network fragments and the processes of vaporization and mass transport to define tar, the tar molecular weight distribution and the extract yield.

7. Of the three models, FG-DVC is the most closely related with the previous concepts of coal as a macromolecular network by requiring that the model predict the coal solvent swelling ratios and measured extract yields. The assumptions which define the parameters of the starting network are open to question and must be explored.

8. Future efforts should focus on identifying the chemistry for the processes of bridge breaking, low temperature crosslinking, moderate temperature crosslinking, and hydrogen utilization.

\section{ACKNOWLEDGEMENTS}

The authors acknowledge support for the work provided by the Morgantown Energy Technology Center of the Department of Energy under Contract No. DE-AC2186MC23075.

\section{REFERENCES}

1 van Krevelen. D. W. in 'Coal', Elsevier, Amsterdam, The Netherlands. 1961

2 Green, T. K.. Kovac. J. and Larsen, J. W. Fuel 1984, 63, 935

3 Brenner. D. Fuel 1985. 64, 167

4 Lucht. L. M. and Peppas. N. A. Fuel 1987. 66. 803

5 Lucht. L. M., Larsen. J. M. and Peppas, N. A. Energy \& Fuels 1987, 1, 56

6 Larsen, J. W. Am. Chem. Soc. Div. Fuel Chem. Prepr. 1985, 30(4). 444

7 Green. T.. Kovac. J.. Brenner, D. and Larsen. J. in 'Coal Structure" (Ed. R. A. Meyers). Academic Press, NY. USA, 1982. p. 199

8 Hall. P. J.. Marsh. H. and Thomas. K. M. Fuel 1988. 67, 863

9 Sanada. Y. and Honda, H. Fuel 1966, 45. 295

10 Suuberg. E. M.. Yoshi. O. and Deevo. S. Am. Chent. Soc. Dir. Fuel Chem. Prepr. 1988, 33(1). 387

11 Suuberg. E. M.. Lee. D. and Larsen.J. W. Fuel 1985.64. 1668

12 Suuberg, E. M., Unger, P. E. and Larsen, J. W. Energy \& Fuels 1987, 1, 305

13 Brown, J. K.. Dryden, I. G. C., Dunevein, D. H. et al. J. Inst. Fuel 1958, 31, 259 
Orning, A. A. and Greifer, B. Fuel 1956, 35, 318

Solomon, P. R. and Hamblen, D. G. in 'Chemistry in Coal Conversion' (Ed. R. H. Schlosberg), Plenum Press, New York, USA, chapter 5, p. 121

Solomon, P. R. 'New Approaches in Coal Chemistry', ACS Symposium Series 169, ACS, Washington, DC, USA, 1981, p. 61 Solomon, P. R. and King, H. H. Fuel 1984, 63, 1302

Gavalas, G. R. in 'Coal Pyrolysis' Elsevier, NY, USA, 1982, p. 51

Gavalas, G. R. Cheong, P. H. and Jain, R. Ind Eng Chem. Fundam. 1981, 20, 122

Squire, K. R., Solomon, P. R., Carangelo, R. M. and DiTaranto, M. B. Fuel 1986, 65.833

Squire, K. R., Solomon P. R.. DiTaranto. M. B. and Carangelo, R. M. Am. Chem. Soc. Div. Fuel Chem. Prepr. 1985 , 30(1), 386

Solomon. P. R., Squire, K $\mathrm{S}$ and Carangelo, R. M. 'Proc. Int. Conf. on Coal Science:. Pergamon, Sydney, Australia, 1985 , p. 945

Solomon, P. R. and Squire, K. R. Am. Chem. Soc. Div. Fuel Chem. Prepr. 1985, 30(4), 347

Niksa. S. and Kerstein. A. R. Comb. and Flame 1986. 66.95

Niksa, S. Comb. and Flame 1986, 66, 111

Solomon, P. R., Hamblen, D. G., Deshpande, G. V. and Serio, M. A. 'Proc. Int. Coal Science Conference'. Elsevier. NY, USA, 1987, p. 601

Solomon, P. R., Hamblen, D. G., Carangelo, R. M. et al. Comb. and Flame 1988, 71, 137

Niksa, S. and Kerstein, A. R. Fuel 1987, 66, 1389

Solomon, P. R. Hamblen, D. G.. Carangelo. R. M. et al. Energy' \& Fuels 1988, 2, 405

Grant, D. M.. Pugmire. R. J.. Fletcher. T. H. and Kerstein, A. R. Energy \& Fuels 1989, 3, 175

Serio, M. A., Solomon, P. R., Yu, Z. Z. et al. Am. Chem. Soc. Div. Fuel Chem. Prepr. 1988, 33(3), 91

Solomon, P. R., Best, P. E., Yu, Z. Z. and Deshpande. G. V. Am. Chem. Soc. Div. Fuel Chem. Prepr. 1989, 34(3), 895

Nielsen, L. E. in Mechanical Properties of Polvmers and Composites", Volume 2, Marcel Dekker, Inc., NY. USA, 1974 Bartels, C. R., Crist, B., Felters, L. J. and Graessley, W. W. Macromolecules 1986, 19, 785

Nazem, F. F. Fuel 1980. 59, 851

Macosko, C. W. Brit. Polymer Journ. 1985, 17. 239

Flory, P. J. J. Am. Chem. Soc. 1941. 63. 3083, 3097; 'Principles of Polymer Chemistry', Cornell University Press, Ithaca. NY. USA, 1953, chapter

Stockmayer, W. H. J. Chem. Phys. 1943, 11, 45; 1944, 12, 125 Fisher, M. E. and Essam, J. W. J. Math. Phys. 1961, 2. 609

Solum. M., Pugmire, R. J. and Grant, D. M. Energy \& Fuels 1989. 3, 40

Gerstein, B. C., Murphy. D. P. and Ryan. L. M. in "Coal Structure' (Ed. R. A. Meyers), Academic Press, NY, USA, 1982. chapter 4, p. 87

Hooker, D. T. II, Lucht, L. M. and Peppas, N. A. Ind. Eng. Chem. Fundam. 1986. 25. 103

Solomon, P. R.. Serio, M. A., Carangelo, R. M. and Markham, J. R. Fuel 1986, 65, 182

Fletcher, T. H. Comb. Sci. and Tech. 1989, 63, 87

Solomon, P. R., Serio, M. A., Deshpande, G. Y. and Kroo, E. Energy \& Fuels submitted for publication; Deshpande, G. V., Solomon, P. R. and Serio, M. A. Am. Chem. Soc. Div. Fuel Chem. Prepr. 1988, 33(2), 310

Unger, P. E. and Suuberg, E. M. "18th Symposium (Int) on Combustion:, The Combustion Institute, Pittsburgh, PA, USA, 1981 , p. 1203

Suuberg, E. M., Unger, P.E. and Lilly, W.D. Fuel 1985, 64,956

Unger. P. E. and Suuberg. S. E. Fuel 1984. 63. 606

Oh, M. S., Peters, W. A. and Howard, J. B. AIChE J. 1989, 35(5), 775

Serio. M. A. Ph.D. Thesis Massachusetts Institute of Technology, Cambridge, MA, USA, 1984
51 Khan. M. R., Serio, M. A., Malhotra, R. and Solomon, P. R. Am. Chem. Soc. Div. Fuel Chem. Prepr. 1989, 34(4), 1054 Fong. W.S., Peters, W.A. and Howard, J. B. Fuel 1986, 65.251 Solomon. P. R. and Carangelo, R. M. Fuel 1982, 61. 663 Solomon. P. R. and Carangelo, R. M. Fuel 1988, 67, 949

\section{APPENDIX A}

The probability $F_{s, u}(p, q)$, that a site is a member of a cluster of $n$ sites with $s$ type 1 bridges and $u$ type 2 bridges is given by

$$
F_{s . u}(p, q)=a_{s . u} p^{s}(1-p)^{v} q^{u}(1-q)^{v}
$$

where

$$
\begin{aligned}
& n=u+s+1 \\
& \tau=\left(\sigma_{1}+1\right) n-2 s \\
& v=\left(\sigma_{2}+1\right) n-2 u
\end{aligned}
$$

$\tau$ and $v$ are the number of broken bridges of type 1 and 2 , respectively, on the perimeter of the cluster, and $a_{\text {s.u }}$ is the number of different ways to form such a cluster. Following the procedure used by Fisher and Essam, an expression for the configuration coefficient can be derived

$$
a_{s, u}=\frac{\left(\sigma_{1}+1\right)\left(\sigma_{2}+1\right)}{(s+\tau)(u+v)} \Gamma(s+\tau+1) \Gamma(u+v+1)(u+\bar{s}+1)
$$

where $\Gamma$ is a gamma function. Note that for $u=0$ (no type 2 bonds), this reduces to the quantity $n b_{n}$ in Ref. 30 . To determine the probability, $F_{n}(p, q)$ that a given site is a member of a cluster of $n$ sites, i.e. the fraction of $n$-mers, Equation (1) must be summed over all possible values of $s$ and $u$ that give an $n$-site cluster:

$F_{\mathrm{n}}(p, q)=\sum_{s=0}^{n-1} a_{\mathrm{s}, \mathrm{u}} p^{\mathrm{s}}(1-p)^{\mathrm{x}} q^{\mathrm{u}}(1-q)^{v} \quad u=n-s-1$

The total fraction of sites, $F(p, q)$ in finite clusters is the sum over all $s$ and $u$

$$
F(p, q)=\sum_{s=0}^{\infty} \sum_{u=0}^{\infty} F_{s, u}(p, q)=\left(\frac{1-p}{1-p^{*}}\right)^{\sigma_{1}+1}\left(\frac{1-q}{1-q^{*}}\right)^{\sigma_{2}+1}
$$

where $p^{*}$ and $q^{*}$ are obtained by finding the least roots of

$$
\begin{aligned}
& p^{*}\left(1-p^{*}\right)^{\sigma_{1}-1}\left(1-q^{*}\right)^{\sigma_{2}+1}-p(1-p)^{\sigma_{1}-1}(1-q)^{\sigma_{2}+1}=0 \\
& q^{*}\left(1-q^{*}\right)^{\sigma_{2}-1}\left(1-p^{*}\right)^{\sigma_{1}+1}-q(1-q)^{\sigma_{2}-1}(1-p)^{\sigma_{1}+1}=0
\end{aligned}
$$

The critical point at which an infinite lattice begins to form (i.e. $F(p, q)$ begins to decrease) becomes a critical curve which divides the $p-q$ plane into two regions. Note that for $q=0$, the equations all reduce to the single $\sigma$ case given in Ref. 30 . 


\section{APPENDIX F}

\section{GENERAL MODEL OF COAL DEVOLATILIZATION}


Reprinted from Journal of ENERGY \& FUELS, 1988, $2,405$.

Copyright (c) 1988 by the American Chemical Society and reprinted by permission of the copyright owner.

..

\title{
General Model of Coal Devolatilization ${ }^{\dagger}$
}

\author{
P. R. Solomon,* D. G. Hamblen, R. M. Carangelo, M. A. Serio, and \\ G. V. Deshpande \\ Advanced Fuel Research, Inc., 87 Church Street, East Hartford, Connecticut 06108 \\ Received December 21, 1987. Revised Manuscript Received April 21, 1988
}

A general model for coal devolatilization, which combines a functional group model for gas evolution and a statistical model for tar formation, has been presented. The tar formation model includes depolymerization, cross-linking, external transport, and internal transport. The cross-linking is related to the evolutions of $\mathrm{CO}_{2}$ and $\mathrm{CH}_{4}$, with one cross-link formed per molecule evolved. The model predictions compare favorably with a variety of data for the devolatilization of Pittsburgh Seam coal and North Dakota (Beulah) lignite, including volatile yields, extract yields, cross-link densities, and tar molecular weight distributions. The variations with pressure, devolatilization temperature, rank, and heating rate were accurately predicted. Comparison of the model with several sets of data employing alternative assumptions on transport suggests that assuming that the particle is well mixed (i.e. the surface concentration of tar molecules is the same as the bulk) overpredicts the transport rate. For $50-\mu \mathrm{m}$ particles, assuming that the internal-transport limitation dominates (i.e. neglecting the external transport) provides a good fit to the data. The rank dependence of tar formation, extract yields, cross-linking, and viscosity appears to be explained by the rank dependence of $\mathrm{CO}_{2}$ yields and its associated cross-linking. High $\mathrm{CO}_{2}$ yields in low-rank coals produce rapid cross-linking at low temperatures and hence thermosetting behavior, low tar yields, low extract yields, loss of solventswelling properties, and high viscosities. The relative importance of cross-linking compared to bond breaking is, however, sensitive to heating rate, and this effect is predicted by the model. Areas for improving the model include (1) refinement of the internal and external transport assumptions, (2) accounting for hydroaromatic structures and bridge structures besides ethylene, and (3) including polymethylene "guest" molecules.

\section{Introduction}

Coal devolatilization is a process in which coal is transformed at elevated temperatures to produce gases, tar, and char. (Tar is defined as the room-temperature condensibles formed during coal devolatilization.) The combined chemical and physical processes in devolatilization have been reviewed by a number of investigators..$^{1-6}$ Gas formation can often be related to the thermal decomposition of specific functional groups in the coal and can be predicted with reasonable accuracy by models employing first-order reactions with ultimate yields. ${ }^{.-15}$ On the other hand, tar and char formation are more complicated, and success in mechanistic modeling of tar formation has been more limited.

Predicting tar formation is, however, important for several reasons. Tar is a major volatile product (up to $40 \%$

\footnotetext{
- To whom correspondence is to be addressed

1 Presented at the Symposium on Coal Pyrolysis: Mechanisms and Modeling, 194th National Meeting of the American Chemical Society, New Orleans, LA, August 31-September 4, 1987.
}

of the DAF coal weight for some bituminous coals). Tar yields vary substantially depending on reactor conditions

(1) Howard, J. B.; Peters, W. A.; Serio, M. A. "Coal Devolatilization Information for Reactor Modeling"; Final Report on EPRI Project No. 986-5, 1981.

(2) Howard, J. B. In Chemistry of Coal Utilization; Elliott, M. A., Ed.; Wiley: New York, 1981; Chapter 12, p 665.

(3) Gavalas, G. R. Coal Pyrolysis; Elsevier: Amsterdam, The Nethexlands, 1981.

(4) Suuberg, E. M. In Chemistry of Coal Conversion; Schlosberg, $R$. H., Ed.; Plenum: New York, 1985; Chapter 4.

(5) Solomon, P. R.; Hamblen, D. G. In Chemistry of Coal Conversion; Schlosberg, R. H., Ed.; Plenum: New York, 1985; Chapter 5, p 121.

(6) Serio, M. A.; Hamblen, D. G.; Markham, J. R.; Solomon, P. R. Energy Fuels 1987, 1, 138.

(7) Suuberg, E. M.; Peters, W. A.; Howard, J. B. Seventeenth Symposium (International) on Combustion; The Combustion Institute: Pittsburgh, PA, 1979; p 117.

(8) Juntgen, H.; van Heek, K. H. Fuel Process. Technol. 1979, 2, 261.

(9) Weimer, R. F.: Ngan, D. Y. Prepr. Pap.-Am. Chem. Soc., Div. Fuel Chem. 1979, 24(3), 129.

(10) Campbell, J. H. Fuel 1978, 57, 217.

(11) Solomon, P. R.; Colket, M. B. Seventeenth Symposium (International) on Combustion; The Combustion Institute: Pittsburgh, PA, 1979; p 131 . 
(pressure, heating rate, final temperature, bed geometry, particle size, etc.). In combustion or gasification, tar is often the volatile product of highest initial yield and thus controls ignition and flame stability. It is a precursor to soot, which is important to radiative heat transfer. The process of tar formation is linked to the char viscosity ${ }^{16-19}$ and the subsequent physical and chemical structure of the char and so is important to char swelling and reactivity. Also, because tar molecules are sometimes minimally disturbed coal molecular fragments, primary tars provide important clues to the structure of the parent coal.5,6,20

It is generally agreed that the tar formation includes the following steps: (1) depolymerization by rupture of weaker bridges in the coal macromolecule to release smaller fragments that make up the "metaplast"; ;,6,7,16,21-33 (2) repolymerization (cross-linking) of metaplast molecules; $35,7,1621-33$ (3) transport of lighter molecules away from the surface of the coal particles by combined vaporization and gas phase diffusion; ${ }^{23,32}(4)$ internal transport of lighter molecules to the surface of the coal particles by convection and diffusion in the pores of nonsoftening coals $24,27,34,35$ and liquid-phase or bubble transport in softening coals. ${ }^{17.36-38}$ Char is formed from the unreleased or recondensed fragments. Varying amounts of loosely bound "guest" molecules, usually associated with the extractable material, are also released in devolatilization.

(12) Solomon, P. R.; Hamblen, D. G.; Carangelo, R. M.; Krause, J. L. Nineteenth Symposium (International) on Combustion; The Combustion Institute: Pittsburgh, PA, 1982; 1139.

(13) Solomon, P. R.; Serio, M. A.; Carangelo, R. M.; Markham, J. R. Fuel 1986, 65, 182

(14) Xu, W.C.; Tomita, A. Fuel 1987, 66, 627.

(15) Juntgen, H. Fuel 1984, 63, 731.

(16) Fong, W. S.; Peters, W. A.; Howard, J. B. Fuel 1986, 65, 251.

(17) Oh, M. S.; Peters, W. A.; Howard, J. B. Proceedings of the 1983 Intermational Conference on Coal Science; Center for Conference Management: Pittsburgh, PA, 1983; p 483.

(18) Fong, W. S.; Khalil, Y. F.; Peters, W. A; Howard, J. B. Fuel 1986, 65,195 .

(19) Van Krevelen, D. W. Properties of Polymers; Elsevier. Amsterdam, 1976.

(20) Solomon, P. R. New Approaches in Coal Chemistry; ACS Symposium Series 169; American Chemical Society: Washington, DC, 1981; pp 61-71.

(21) van Krevelen, D. W.; Schuyer, J. Coal Science; Elsevier: Amaterdam, 1957.

(22) Anthony, J). B.; Howard, J. B.; Hottel, H. C.; Meissner, H. P. Fifteenth Symposium (International) on Combustion; The Combustion Institute: Pittsburgh, PA, 1974; p 1303.

(23) Unger, P. E.; Suuberg, E. M. Eighteenth Symposium (International) on Combustion; The Combustion Institute: Pittsburgh, PA, 1981; p 1203.

(24) Russel, W. B.; Saville, D. A.; Greene, M. I. AIChE J. 1979, 25, 65.

(25) James, R. K.; Mills, A. F. Lett. Heat Mass Transfer 1976, 3, 1.

(26) Lewellen, P. C. S.M. Thesis, Department of Chemical Engineering, MIT, 1975.

(27) Chen, L. W; Wen, C. Y. Prepr. Pap.-Am. Chem. Soc., Div. Fuel Chem. 1979, 24(3), p 141 .

(28) Niksa, S.; Kerstein, A. R. Combust. Flame 1986, 66, 95

(29) Niksa, S. Combust. Flame 1986, 66, 111.

(30) Solomon, P. R.; Squire, K. R.; Carangelo, R. M. Proceedings of the International Conference on Coal Science; Pergamon: Sydney, Australia, 1985; p 945.

(31) Solomon, P. R.; Squire, K. R. Prepr. Pap.-Am. Chem. Soc., Div.

Fuel Chem. 1985, 30(4), 347.

(32) Suuberg, E. M.; Unger, P. E.; Lilly, W. D. Fuel 1985, 64, 956.

(33) Solomon, P. R.; Hamblen, D. G.; Carangelo, R. M.; Serio, M. A.; Deshpande, G. V. Prepr. Pap.-Am. Chem. Soc., Div. Fuel Chem. 1987, 32(3), 83 .

(34) Gavalas, G. R.; Wilks, K. A. AIChE J. 1980, 26, 201.

(35) Simons, G. A. Prog. Energy Combust. Sci. 1983, 9, 269.

(36) Suuberg E M. Sezen, Y. Proceedings of the International Conference on Coal Science; Pergamon: Sydney, Australiz, 1985; 913

(37) Melia, P. F.; Bowman, C. T. Combust. Sci. Technol. 1983, 31, 195; Paper presented at the Western States Section of the Combustion Intitute, Salt Lake City, UT, 1982

(38) Oh, M. S. Sc.D. Thesis, Department of Chemical Engineering, MIT, Cambridge, MA, 1985.
The complexity of proposed devolatilization models varies substantially. They can be divided into four categories. The simplest are the "weight loss models" employing a single rate, ${ }^{6,22,39-12}$ two rates, ${ }^{39.43}$ multiple parallel rates, or distributed rates. 9,22 These models do nut account for the variations in tar yield with reaction conditions, and a number of "tar formation models" incorporating retrogressive char-forming reactions and mass transport have been proposed that account for such variations. ${ }^{16,21-30,37,41-49}$ A recent innovation has been the description of the decomposition and repolymerization of the macromolecular network by using statistical methods. ${ }^{28,29,44-46,50,51}$

Most of the above models do not consider the evolution of gas species, which have been treated in a number of "species evolution/functional group models" as parallel first-order reactions. ${ }^{5-13}$ More complicated "comprehensive chemical models" also describe the composition of the char and tar. $3,5,6,11-13,33,48,49,51$

The level of detail required in a model depends on its application. In the modeling of combustion and gasification, the simple "weight loss models" have often been employed. However, to predict the variations in yield with reactor conditions, the more complicated "tar formation models" must be used. A case can also be made for employing "species evolution/functional group models" or "comprehensive chemical models". For example, in predicting the energy released from combustion of the volatiles, it is important to know that for low-rank coals a high percentage of the volatiles may be noncombustible $\mathrm{H}_{2} \mathrm{O}$ and $\mathrm{CO}_{2}$. For a North Dakota lignite, the total of these two components can be as high as $35 \%$ of the rapidly released volatiles which are important for ignition. ${ }^{6}$ In addition, the swelling, particle agglomeration properties, char reactivity, and char fragmentation are functions of the char composition. Soot formation (which can dominate radiative energy transport) is controlled by the tar amount.

In the modeling of liquefaction and mild gasification, -knowledge of the chemical makeup and molecular weight distribution of the soluble and volatile products is essential, requiring the more complete "comprehensive chemical models".

This paper presents a "comprehensive chemical model" for coal devolatilization that considers the evolution of gas, tar, char, and guest molecules. The model is general in its applicability to bituminous coals, subbituminous coals,

(39) Kobayashi, H.; Howard, J. B.; Sarofim, A. F. Sixteenth Symposium (International) on Combustion; The Combustion Institute: Pittsburgh, PA, 1977; p 411. Kobayashi, H. Ph.D. Thesis, Department of Mechanical Engineering, MIT, Cambridge, MA. 1976.

(40) Nikss, S.; Heyd, L. E.; Russel, W. B.; Saville, D. A. Twentieth Symposium (International) on Combustion; The Combustion Institute: Pittsburgh, PA, 1984; 1445.

(41) Badzioch, S.; Hawksley, P. G. W. Ind. Eng. Chem. Process Des. Dev. 1970, 9, 521 .

(42) Maloney, D. J.; Jenkins, R. G. Twentieth Symposium (International) on Combustion; The Combustion Institute: Pittsburgh, PA, 1984; p 1435.

(43) Witte, A. B.; Gat, N. Presented at the DOE Direct Utilization AR\&TD Contractor's Meeting, Pittsburgh, PA, 1983.

(44) Solomon, P. R. King H. H. Fuel 1984, 63, 1302.

(45) Solomon, P. R.; Squire, K. R.; Carangelo, R. M. Prepr. Pap.-Am. Chem. Soc., Div. Fuel Chem. 1984, 29(1), 10.

(46) Squire, K. R.; Solomon, P. R.; Carangelo, R. M.; DiTaranto, M.

B. Fuel 1986, 65, 833

(47) Squire, K. R.; Solomon, P. R.; DiTaranto, M. B.; Carangelo, R. M. Prepr. Pap. - Am. Chem. Soc., Div. Fuel Chem. 1985, 30(1), 386. (48) Gavalas, G. R.; Cheong, P. H.; Jain, R. Ind Eng. Chem. Fundam. $1981,20,113$.

(49) Gavalas, G. R.; Cheong, P. H.; Jain, R. Ind. Eng. Chem. Fundam. $1981,20,122$.

(50) Solomon, P. R.; Hamblen, D. G.; Carangelo, R. M.; Serio, M. A.; Deshpande, G. V. Combust. Flame 1988, 71, 137.

(5i) Solomon, P. R.; Hamblen, D. G.; Deshpande, G. V.; Serio, M. A. Coal Sci. Technol. 1987, 11,601. 
and lignites (employing rank-independent kinetic parameters), in its application to reactors of widely differing heating rates $\left(0.05\right.$ to $\left.20000^{\circ} \mathrm{C} / \mathrm{s}\right)$, and in its ability to predict the variations in tar yield with reactor conditions.

Two previously developed models, a functional group (FG) model $1^{5,6,11-13}$ (a "species evolution/functional group model ${ }^{\text {) }}$ and a depolymerization-vaporization-cross-linking (DVC) model ${ }^{30,31,44-77}$ ( $\mathrm{a}$ "tar formation model") have been combined as subroutines of what is now called the "FGDVC" model. $^{33,50,51}$ The DVC subroutine is employed to determine the yield of tar and the mole zular weight distribution of the tar and char. The FG subroutine is used to describe the gas evolution and the elemental and functional group compositions of the tar and char. Cross-linking in the DVC subroutine is computed by assuming that this event is correlated with $\mathrm{CO}_{2}$ and $\mathrm{CH}_{4}$ evolutions predicted in the FG subroutine. The dependence of the yield of rapidly released $\mathrm{CO}_{2}$ (which is related to coal rank or weathering) is the factor that controls the thermosetting or thermoplastic behavior of coals.

The combined FG-DVC model was described in two previous publications, ${ }^{50,51}$ and comparisons were made to a limited set of data. In this paper, a description of internal transport has been added to the model. The model equations are presented, and comparisons are made to a wider set of data. The paper also includes a discussion of the assumptions, approximations, and exceptions to the model and a sensitivity analysis for the parameters of the DVC subroutine. The model describes the processes of (1) depolymerization and hydrogen consumption, (2) cross-linking, (3) external transport, (4) internal transport, (5) gas formation for all principal species, (6) tar composition, and (7) char composition.

The work presented here is limited to dilute phase reactions of small coal particles where internal temperature gradients can be neglected. Secondary gas phase reactions have been discussed elsewhere, ${ }^{6}$ and reactions of pyrolysis products with a char bed and large particle effects have not yet been included. Only reactions involving $\mathrm{C}, \mathrm{H}$, and $O$ are discussed here.

A number of coal composition parameters and reactor parameters (pressure, particle time-temperature profile) gre required to predict the pyrolysis behavior. A sub:tantial reduction in the number of parameters that must te measured for each coal is made by the use of rank-insependent kinetic rates. These parameters have already been determined for a wide variety of coals and reactors. This simplification is a good first approximation to describe the kinetics of individual evolved species and the functional group decompositions. $5,6,49,52-55$ The properties predicted as a function of time, include the following: for tar, molecular weight distribution, elemental and functional group composition, and yield; for char, molecular weight distribution, elemental and functional group composition, yield, cross-link density, and extract yield; for gas, yields of individual light gas species. Results are presented for a Pittsburgh Seam bituminous coal and a North Dakota lignite.

\section{Experimental Section}

Coals Examined. The two coals described in this paper are a Pittsburgh Seam bituminous coal and a Nor'h Dakota (Beulah, Zap) lignite. Samples of the Pittsburgh Seam coal were obtained

(52) Solomon, P. R.; Hamblen, D. G. Prog. Energy Combust. Sci. 1983, 9.323.

(53) Xu, W. C.: Tomita, A. Fuel 1987, 66, 632.

(54) Agarwal, P. K. Fuel 1985, 64, 870

(55) Agarwal, P. K.; Agnew, J. B.; Ravindran, N.; Weimann, R. Fuel $1987,66,1097$. from the Pittsburgh Energy Technology Center and the Argonne National Laboratory premium coal sample collection. Samples of the North Dakota (Beulah, Zap) lignite were obtained from the University of North Dakota Energy Research Center and the Argonne National Laboratory premium coal sample collection. Data on the premium samples are presented in ref 56, and data on the other two samples, in ref. 6 . The FG-DVC model was also compared to data on Pittsburgh coal samples from ref 7,16 , and 22 , and characterizations of these samples are presented therein.

Coal Characterization. The cross-link density was estimated by using the volumetric swelling technique developed by Larsen and co-workers. ${ }^{67-59}$ Pyridine extract yields were obtained by using a Soxhlet apparatus. Molecular weight distributions of tars were obtained at SRI International on the field-ionization mass spectrometry (FIMS) apparatus described by St. John et al. ${ }^{00}$ Tar samples were collected from the pyrolysis apparatus and vaporized from a heated probe into the FIMS apparatus. In addition, coal samples were pyrolyzed directly in the FIMS apparatus.

Apparatus. Pyrolysis experiments were performed in several apparatuses that have been described previously including a heated-grid pyrolyzer, ${ }^{5,12}$ a heated-tube reactor, ${ }^{6,13}$ and a thermogravimetric analyzer with analysis of evolved products by Fourier transform infrared (FT-IR) spectroscopy (TG-FTIR). ${ }_{1,61}$

\section{General Model}

Any general model of a process as complicated as coal devolatilization must of course be a gross approximation. However, there are many general trends that have been observed in devolatilization. The trick in developing a model is to pick a set of first approximations that best match the majority of these trends. There will of course be exceptions to the trends. These exceptions can be treated as perturbations to the first-order approximation. Differences in models occur because of the subjective choice of what is a general trend and what is an exception. The following discussion presents the authors' view of the general trends and the exceptions.

General Trends in Devolatilization. The general model of coal pyrolysis is based on a number of observations that have been previously made concerning coal pyrolysis. These are as follows: (i) Pyrolysis species kinetics are insensitive to rank. $5,6,11-13,62-55$ (ii) Species amounts vary with coal rank and can be correlated with the coal's functional group compositions. $5.6,14,15,48,49,62$ The evolution of each species can be correlated with the change in the corresponding functional group composition in the char. $5,6,62$ (iii) The primary tar composition is similar (except for a higher concentration of methyl groups) to that of the parent coal for bituminous coals and rapidly heated low-rank coal. $0,20,45,62-64$ (iv) Tar yields are controlled by the amount of donatable hydrogen and how efficiently it is used. $5,6,20,46$ (v) Cross-linking correlates with $\mathrm{CO}_{2}$ and $\mathrm{CH}_{4}$ evolution. ${ }^{50,51}$

The general outline of devolatilization based on these observations was presented by Solomon and Hamblen ${ }^{5}$ and Serio et al. ${ }^{6}$ Figure 1 (adapted from ref 6) presents a hypothetical picture of the coal's or char's organic structure at successive stages of devolatilization. The figure represents (a) the raw coal, (b) the formation of tar and light

(56) Vorres, K. S. Prepr. Pap.-Am. Chem. Soc., Div. Fuel Chem. 1987, $32(4), 221$.

(57) Green, T. K.; Kovac, J.; Larsen, J. W. Fuel 1984, 63, 935.

(58) Green, T. K.; Kovac, J.; Larsen, J. W. In Cocl Structure; Meyers,

R. A., Ed.; Academic: New York, 1982

(59) Suuberg, E. M.; Lee, D.; Larsen, J. W. Fuel 1985, 64, 1668.

(60) St. John, G. A.; Butrill, S. E., Jr.; Anbar, M. ACS Symp. Ser. 1978. No. $71,223$.

(61) Carangelo, R. M.: Solomon, P. R.: Gerson, D. J. Fuel 1987, 66, 960

(62) Solomon, P. R.; Colket, M. B. Fuel 1978, 57, 748.

(63) Brown, J. K.; Dryden, I. G. C.; Dunevein, D. H.; Joy, W. K.;

Pankhurst, K. S. J. Inst. Fuel 1958, $31,259$.

(64) Orning, A. A.; Greifer, B. Fuel 1956, 35, 318. 

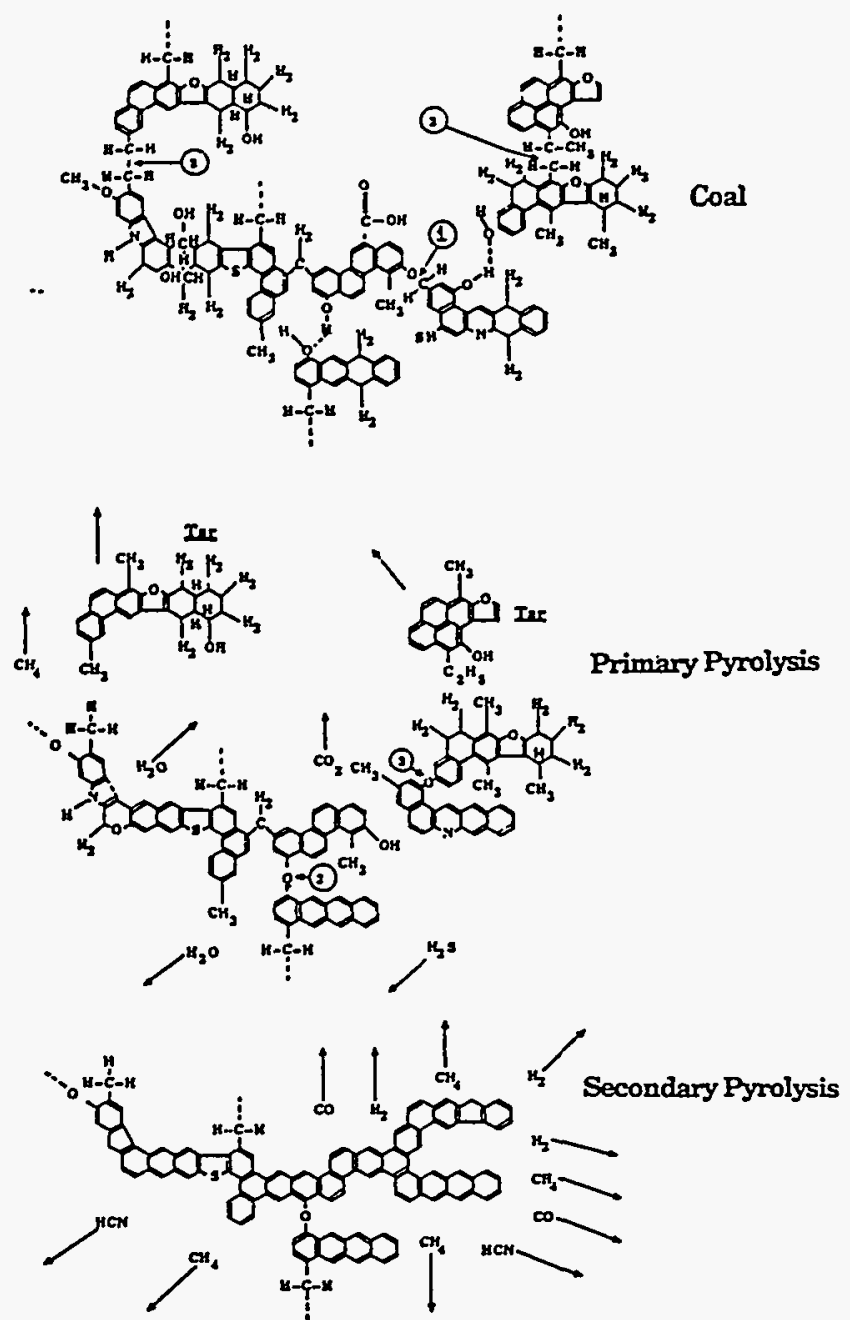

Figure 1. Hypothetical coal molecule during stages of pyrolysis. Adapted from ref 6.

hydrocarbons during primary pyrolysis, and (c) char condensation and cross-linking during secondary pyrolysis. The hypothetical structure in Figure 1a represents the chemical and functional group compositions for a Pittsburgh Seam bituminous coal as discussed by Solomon. ${ }^{20}$ It consists of aromatic and hydroaromatic clusters linked by aliphatic bridges. During pyrolysis, the weakest bridges, labeled 1 and 2 in Figure $1 a$, can break producing molecular fragments (depolymerization). The fragments abstract hydrogen from the hydroaromatics or aliphatics, thus increasing the aromatic hydrogen concentration. These fragments will be released as tar if they are small enough to vaporize under typical pyrolysis conditions and do not undergo retrograde reactions before escaping from the particle. The two lightest fragments are labeled tar. The other two fragments are shown to have repolymerized, producing a molecule that is too large to vaporize.

The other events during primary pyrolysis are the decomposition of functional groups to release $\mathrm{CO}_{2}$, light aliphatic gases, and some $\mathrm{CH}_{4}$ and $\mathrm{H}_{2} \mathrm{O}$. The release of $\mathrm{CH}_{4}, \mathrm{CO}_{2}$, and $\mathrm{H}_{2} \mathrm{O}$ may produce cross-linking, $\mathrm{CH}_{4}$ by a substitution reaction in which the attachment of a larger molecule releases the methyl group, $\mathrm{CO}_{2}$ by condensation after a radical is formed on the ring when a carboxyl is removed, and $\mathrm{H}_{2} \mathrm{O}$ by the condensation of two $\mathrm{OH}$ groups to produce an ether link (labeled 3 in Figure 1b). The cross-linking is important to determine the release of tar and the viscoelastic properties of the char.

The end of primary pyrolysis occurs when the donatable hydrogens from hydroaromatic or aliphatic portion of the coal are depleted. During secondary pyrolysis (Figure 1c) there is additional methane evolution (from methyl groups), $\mathrm{HCN}$ rrom ring nitrogen compounds, $\mathrm{CO}$ from ether links, and $\mathrm{H}_{2}$ from ring condensation. These general concepts are incorporated into the combined FG-DVC model.

Exceptions to the General Trends. a. Polymethylene. The major exception to the trends described above is the presence of varying amounts (typically $0-9 \%$, but in some cases as high as $18 \%$ ) of long-chain aliphatics (polymethylenes). These have recently been reported in pyrolysis products by Nelson ${ }^{65}$ and by Calkins and coworkers ${ }^{66-69}$ and references quoted therein. The chains appear alone and attached to aromatic nucleii. ${ }^{65}$ During devolatilization, the smaller molecules may be released without bond breaking and the heavier molecules with bond breaking to contribute to the tar. The presence of these polymethylenes makes the tar more aliphatic than the parent coal. Further cracking of this material under more severe devolatilization conditions produces ethylene, propylene, and butadiene from which the concentration of polymethylenes may be determined. ${ }^{68}$ Presently, the polymethylenes are included in the FG model as part of the aliphatic functional group pool, which is assumed to decompose to produce gas products, not tar. If the amount of heavy polymethylenes is determined, these can be computed as a separate functional group pool with an appropriate release rate and added to the tar. The modeling of polymethylene evolution will be the subject of a subsequent publication.

b. Tar/Coal Similarities. The general model assumed, as a first approximation, that tar is derived from material of the same average composition as that of the parent coal. The model predicts that the tar is richer than the parent coal in methyl groups (due to hydrogen stabilization) and poorer in the rapidly removed functional groups. Evidence for this assumption is the similaritits in elemental composition, infrared spectra and NMR spectra ${ }^{5,20,45,62-64}$ between the primary tar and parent coal observed for bituminous coals. It was, however, noted $d^{5,45,70}$ that the infrared spectrum for a lignite tar was significantly different from that of the parent coal. The tar is much richer in aliphatic groups and poorer in oxygen functional groups. Freihaut et al. have recently reported a systemaric increase in the tar hydrogen concentration with decreasing rank that suggests a similar trend. ${ }^{71}$

There are at least two reasons for this variation with rank. One reason is the influence of the polymethylene groups. As noted by Calkins, ${ }^{68}$ the concentration of polymethylenes increases with decreasing rank $(\sim 4 \%$ for high volatile bituminous coals compared to $\sim 8 \%$ for lignites). In addition, the tar yield decreases with decreasing rank ( $6 \%$ for the North Dakota lignite compared to $30 \%$ for the Pittsburgh Seam bituminous coal). The relative contribution of the polymethylenes to the tar is therefore increased with decreasing rank. This will lead to a higher aliphatic conter.t and lower oxygen content for the lowrank coal tar. This effect can be treated in the FG-DVC model by the addition of polymethylenes to the tar as discussed above.

(65) Nelson, P. F. Fuel 1987, 66, 1264

(66) Calkins, W. H.; Hagaman, E; Zeldes, H. Fuel 1984, 63, 1113.

(67) Calkins, W. H.; Tyler, R. J. Fuel 1984, 63, 1119.

(68) Calkins, W. H. Fuel 1985, 63, 1125.

(69) Calkins, W. H.; Hovsepian, B. K.; Drykacz, G. R.; Bloomquist, C. A. A.; Ruscic, L. Fuel 1984, 63, 1226.

(70) Solomon, P. R. Coal Structure; Advances in Chemistry Series 192; American Chemical Society: Washington, DC, 1981; p 7.

(71) Freihaut, J. D.; Proscia, W. M.; Seery, D. J. Presented at the 194th National Meeting of the American Chemical Suciety, New Orleans, LA, Aug 31-Sept 4, 1987. 
A second reason for differences in structure between the tar and parent coal is that the extensive cross-linking in low-rank coals is related to the carboxyl group concentration, which increases with decreasing rank. This cross-linking will thus selectively repolymerize the fragments rich in oxygen, while those poorer in oxygen are more likely to be released as tar. This effect has not as yet been included in the model.

It is interesting to note an exception to the above observations. At very high heating rates, the North Dakota (Beulah, Zap) lignite is observed to melt and swell and produce a higher yield of tar that resembles the parent coal. ${ }^{13,30,31}$ The high heating rate appears to reduce the effect of cross-linking, leading to higher oxygen concentrations in the tar and to increase yields. Both effects enhance the resemblance to the parent coal.

c. Variations of Kinetic Rates with Rank. While the model assumes rank-independent kinetic rates, there is a systematic variation of rate with rank. As reported by Solomon and Hamblen, ${ }^{52}$ the variation between a lignite and bituminous coal results in a $50-75^{\circ} \mathrm{C}$ difference in the peak evolution temperature for most species (at a heating rate of $30 \mathrm{~K} / \mathrm{min}$ ). Systematic rank variations in the rate constants can be added to the model if increased accuracy is desired.

d. Macerals. Individual macerals are not considered in this model. The influence of the maceral concentration is assumed to occur through its effect on the average elemental and functional group composition. If details on macerals are desired, then each maceral must be treated as a distinct molecular population with its own functional group composition and molecular weight distribution.

e. Physical Properties of Molecular Fragments. The general model has assumed that the vaporization and solubility of the molecular fragments are functions of molecular weight alone. Both properties are expected to depend on functional group composition. Such effects can be included as corrections to the vaporization law and solubility assumptions.

Depolymerization-Vaporization-Cross-Linking (DVC) Subroutine Formulation. The DVC model has been described in a number of publications. ${ }^{30,31,44-47,50,51}$ It predicts the tar yield, the tar molecular weight distribution, the char yield, the char molecular weight distribution, the extract yield, and the cross-link density. The model had its beginning in a study of polymers representative of structural features found in coal. ${ }^{44}$ The objective of that study was to develop an understanding of coal pyrolysis by studying a simpler, more easily interpretable system. The polymers were studied in a series of pyrolysis experiments in which tar amounts and molecular weights were measured. The theory which was developed describes the combined effects of (1) depolymerization and hydrogen consumption, (2) cross-linking, and (3) external transport. Recently, an expression to describe (4) internal transport has been added to the model. ${ }^{33}$ These processes, which are described below, are incorporated into a computer code that employs a Monte Carlo method for performing the statistical analysis.

Process 1. Depolymerization and Hydrogen Consumption. Bond cleavage in coal is likely to be very complicated, including homolytic cleavage, ipso substitution, ${ }^{16}$ and hydrogen-transfer-induced bond-scission reactions $^{72}$ for a variety of bond types. However, it has been observed that tar evolution is consistent with a narrow distribution of activation energies, ${ }^{5,6,12}$ which allows con-

(72) McMillen, D. F.; Malhotra, R.; Hum, G. P.; Chang, S. J. Energy Fuels 1987, 1, 193. sideration in the model of a single type of bridge (while acknowledging that other types may be present). Also, the rate for tar formation from coal, $k_{\text {tar }}{ }^{6,13}$ is in good agreement with the rate determined for the breaking of ethylene bridges between naphthalene rings, $k_{\mathrm{B}}$. This kinetic rate, $k_{\mathrm{B}}{ }^{46}$ employs an activation energy that is in agreement with resonance-stabilization calculations ${ }^{73,74}$ and an overall rate that agrees with previous measurements on model compounds. ${ }^{75}$ In view of these observations, a single type of bond (ethylene) undergoing homolytic cleavage is assumed for coal as a simple approximation of the complicated behavior.

Bond cleavage is accompanied by the consumption of donatable hydrogens, $H($ al), to cap free radicals, along with corresponding carbon-carbon double-bond formation at the donor site. In the polymers that were studied, the ethylene bridges were identified as a source of donatable hydrogen with the formation of a double bond between the bridge carbons. ${ }^{46,47}$ The double-bond formation was assumed to remove a breakable bond. It should be noted that hydroaromatic groups are also a source of donatable hydrogen with aromatization of the ring. However, for simplicity, the DVC model assumes all the coal's donatable hydrogens, whether in bridges or in hydroaromatic rings, are in bridges, i.e., $H(\mathrm{al})=(2 / 28) W_{\mathrm{B}}$. This approximation will produce some error in tar yield since a broken bond in a hydroaromatic ring will not be as effective as a broken bond in a bridge in fragmentating the coal. But this effect will be compensated for, since $H(a)$ is a parameter that is determined for each coal from a selected pyrolysis experiment. $H$ (al) could, in principle, be determined by FT-IR or NMR, but not with sufficient accuracy.

The equation describing the disappearance of labile bridges in the char, $W_{\mathrm{B}}$ (char), due to bond breaking and hydrogen donation is

$$
\mathrm{d} W_{\mathrm{B}} / \mathrm{d} t=-2 k_{\mathrm{B}} W_{\mathrm{B}}
$$

The value for $k_{\mathrm{B}}$ is taken as the previously determined $k_{\mathrm{tar}}{ }^{6}$ The rate of decrease of labile bridges is twice the rate of bond breaking since for each broken bond, an additional labile bridge is converted to a nonlabile bridge with the donation of hydrogen. By assuming that all the donatable hydrogens are in the labile bridges, the consumption of labile bridges and donatable hydrogens occur simultaneously. The redistribution of hydrogen creates source and loss terms, $\mathrm{d} W_{\mathrm{i}}(\mathrm{DVC}) / \mathrm{d} t$, in the equations for the char functional groups $W_{\mathrm{i}}$ (char), as will be discussed with the FG part of the model (see eq 7).

Equation 1 only describes the loss due to bond breaking and hydrogen donation. The loss of labile bridges due to evolution with the tar is computed in the Monte Carlo calculation by using the transport equations (eq 3 and 4) discussed below.

Process 2. Cross-Linking. Cross-linking reactions are important in describing the rank and heating rate dependence of the tar molecular weight distributions and yields. While cross-linking reactions were originally included in the DVC model, using adjustable parameters for the rate and amount, $30,31,46$ work has recently been performed to define the reactions that cause cross-linking. $33,50,51$ Under the assumption that the cross-linking reactions may also

(73) Stein, S. F. New Approaches in Coal Chemistry; Blaustein, B. D., Bockrath, B. C.. Friedman, S., Eds.; ACS Symposium Series 169; American Chemical Society: Washingtin, DC, 1981; p 208.

(74) Stein, S. E. “Multistep Bond Breaking and Making Processes of Relevance to Thermal Coal Chemistry"; Annual Report for GRI Contract No. 5081-261-0556; Accession No. GRI-81/0147, 1983.

(75) Stein, S. E.; Robauch, D. A.; Alfieri, A. D.; Miller, R. E. J. Am. Chem. Soc. 1982, 104, 6567. 

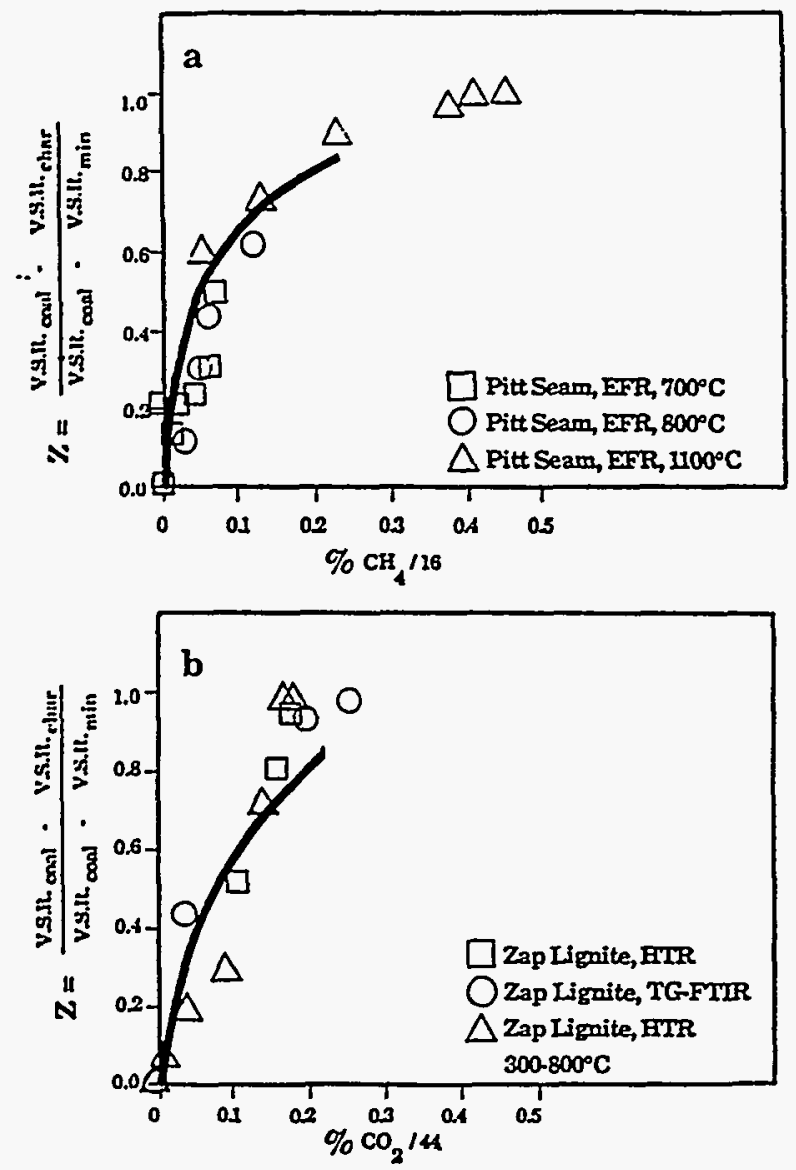

Figure 2. Measured and calculated normalized volumetric swelling ratio (VSR) for coal and chars: (a) Pittsburgh Seam bituminous coal plotted against the methane yield; (b) Zap North Dakota lignite plotted against the $\mathrm{CO}_{2}$ yield. VSR $\mathrm{VR}_{\min }$ is the value achieved when crosslinking is complete. The chars were prepared in an entrained-flow reactor (EFR), a heated-tube reactor (HTR), and $a$ thermogravimetric analyzer with evolved product analysis by FT-IR (TG-FTIR) as described in ref 61 .

release gas species, the molecular weight between crosslinks (or cross-link density) measured by solvent swelling was correlated with the observed evolution of all the major gas species during pyrolysis. Likely candidates were $\mathrm{CO}_{2}$ formation from carboxyl groups or methane formation from methyl groups. Suuberg et al..$^{59}$ also noted that cross-linking in low-rank coals is correlated with $\mathrm{CO}_{2}$ evolution. Both $\mathrm{CO}_{2}$ and $\mathrm{CH}_{4}$-forming reactions may leave behind free radicals that can be stabilized by cross-linking. Condensation of hydroxyl groups to form water and an ether link is also a possible reaction.

For a series of chars, the reduction in the volumetric swelling ratio in pyridine was compared with $\mathrm{CO}_{2}$ evolution for a North Dakota (Beulah) lignite and $\mathrm{CH}_{4}$ evolution for a Pittsburgh Seam bituminous coal. ${ }^{60}$ The results are presented in Figure 2. The abscissa (parameter $Z$ ), which is the change in the volumetric swelling ratio (VSR) between coal and char divided by the maximum change, is given by

$$
Z=\left(V_{S R} R_{\text {coll }}-V_{S R_{\text {char }}}\right) /\left(V_{S R_{\text {coll }}}-V_{\text {min }}\right)
$$

$\mathrm{Z}$ is $\mathbf{0}$ for coal and 1 for fully cross-linked char. Since the lignite reaches maximum cross-linking before the start of methane evolution and the Pittsburgh Seam bituminous coal evolves little $\mathrm{CO}_{2}$, correlations can be made separately between cross-linking and $\mathrm{CO}_{2}$ evolution in the lignite and cross-linking and $\mathrm{CH}_{4}$ evolution in the Pittsburgh seam bituminous coal. On a molar basis, the evolution of $\mathrm{CO}_{2}$ from the lignite and the evolution of $\mathrm{CH}_{4}$ from the bitu- minous coal appear to have similar effects on the volumetric swelling ratio. The results suggest that one cross-link is formed for each $\mathrm{CO}_{2}$ or $\mathrm{CH}_{4}$ molecule evolved. No correlation was observed between the volumetric. swelling ratio and tar yield for either coal. A correlation with water yield appears valid for the North Dakota (Beulah) lignite but not for the Pittsburgh Seam bituminous coal.

It therefore appears that a correlation exists between gas evolution and cross-linking which permits the rates for cross-linking and the number of cross-link sites to be related to rates and yields for gas evolution. The model assumes the following expression for the rate of increase of the number of cross-links per gram of coal, $m$

$$
\frac{\mathrm{d} m}{\mathrm{~d} t}=N_{0}\left[\frac{\mathrm{d} W_{\mathrm{Co}_{2}} \text { (gas) } / \mathrm{d} t}{44}+\frac{\mathrm{d} W_{\mathrm{CH}_{4}} \text { (gas) } / \mathrm{d} t}{16}\right]
$$

where the rates, $d W_{i} / d t$, of evolution per gram of coal of $\mathrm{CO}_{2}$ and $\mathrm{CH}_{4}$ are calculated in the $\mathrm{FG}$ subroutine. $N_{0}$ is Avogadro's number.

Again, a caution should be added that the reactions which have been assumed must be a gross simplification of a very complicated set of chemical reactions. This is especially true for the cross-links occurring during methane formation, during which time there is extensive bond breaking and cross-linking accompanying tar formation. The inaccuracy in the description of this higher temperature cross-linking event is one of the present weaknesses in the model.

Process 3. External Transport. The external transport of tars from the particle surface to the bulk gas by vaporization and diffusion through a gas boundary layer as in the original DVC model ${ }^{44-47,50,51}$ is described with the model of Unger and Suuberg. ${ }^{23}$ However, in the current paper, the modified expression for the vapor pressure law of Suuberg et al..$^{32}$ is now used to replace that in the model of Unger and Suuberg. The rate of evolution per gram of coal, $\left(\mathrm{d} n_{\mathrm{j}} / \mathrm{d} t\right)_{\mathrm{ET}}$, of oligomers of molecular weight $M_{\mathrm{j}}$ is given by

$$
\left(\mathrm{d} n_{\mathrm{j}} / \mathrm{d} t\right)_{\mathrm{ET}}=\left(3 / r_{0}^{3} \rho\right) r D_{\mathrm{j}} \chi_{\mathrm{j}}\left(P_{\mathrm{j}} / R T\right)
$$

where $r$ is the particle radius assumed to shrink with the cubic root of its mass $r_{0}$ is the initial particle radius, $\rho$ is the particle density, $\chi^{s}$ is the mole fraction of species of molecular weight $M_{\mathrm{j}}$ in the metaplast at the surface of the particle, $P_{j}$ is the vapor pressure for oligomers of molecular weight $M_{\mathrm{j}}$ (given by Suuberg et al. ${ }^{32}$ ), $D_{\mathrm{j}}$ is the gas-phase diffusivity of species of molecular weight $M_{\mathrm{j}}{ }^{38} R$ is the gas constant, and $T$ is the particle temperature.

In the previous work, it was assumed that the surface mole fraction, $\chi^{s}{ }_{j}$, was the same as that in the bulk, $\chi_{j}^{b}$. That is, mass transport to the surface was not a limiting factor.

Process 4. Internal Transport. When comparing the predictions of the model to available data assuming $X_{j}^{s}$ $=X^{b}{ }_{j}$ it was found that tar yields were overpredicted when devolatilization occurred at low temperatures. This was observed for either low-heating-rate experiments ${ }^{6}$ or experiments with rapid heating to relatively low temperatures. ${ }^{16}$ As discussed in the Results, it appears that the lower yields were the result of the additional transport limitations within the particle.

For softening coals, the internal transport mechanisms include (i) the transport of tar molecules through the liquid to the surface, (ii) the transit of bubbles containing tar from the interior of the particle to the surface, (iii) the transport of tars within the liquid to the bubbles, and (iv) 
the stirring action of the bubble evolution. For nonsoftening coals, transport occurs by (v) convection and diffusion within the pores.

Mechanism i was treated by Suuberg and Sezen. ${ }^{36}$ The unknown factor is the diffusion coefficient of the tar molecules in the liquid. The detailed mudeling of mechanisms ii and iii has been undertaken by several investigators. ${ }^{426,37,38}$ Calculations for mechanism $v$ have also been published. 242534,35 The models are complicated and require many assumptions. A common feature of mechanisms iii and $v$ is that tars are transported out of the particle with the light devolatilization products that exit the coal via bubbles or pores. In ref 33, the upper limit for this process was calculated. This limit, which occurs when the tars achieve their equilibrium vapor pressure in the evolving gases, can be computed with few assumptions. In this case, the rate of transport per gram of coal, $\left(\mathrm{d} n_{\mathrm{j}} / \mathrm{d} t\right)_{\mathrm{IT}}$, for tar component $j$ is proportional to the volume of gases evolved, $\mathrm{d} V / \mathrm{d} t$. That is

$$
\left(\mathrm{d} n_{\mathrm{j}} / \mathrm{d} t\right)=P_{\mathrm{j}} x_{\mathrm{j}}^{\mathrm{b}}(\mathrm{d} V / \mathrm{d} t)(1 / R T)
$$

The volume of gases is proportional to the number of gas molecules and the temperature. It is inversely proportional to the pressure within the particle, $P_{0}+\Delta P$ where $P_{0}$ is the ambient pressure and $\Delta P$ is the average pressure difference between the surface and the particle's interior. Then

$$
\mathrm{d} V / \mathrm{d} t=\sum_{i}\left(\mathrm{~d} n_{\mathrm{i}} / \mathrm{d} t\right)_{\mathrm{gas}}\left(\frac{R T}{P_{0}+\Delta P}\right)
$$

where $\sum_{\mathrm{i}}\left(\mathrm{d} n_{i} / \mathrm{d} t\right)_{\text {gas }}$ is the rate of production per gram of coal of gas components i summed over all gas and light tar species. For gas molecules, $\mathrm{d} n_{\mathrm{j}} / \mathrm{d} t$ is taken as the rate of production given by the FG model. For light tar molecules, $\mathrm{d} n_{\mathrm{j}} / \mathrm{d} t$ is taken as the total amount transported out of the particle as tar computed in the previous time step. For computational efficiency, the sum has been limited to molecular weights less than $300 \mathrm{amu}$, since this accounts for over $90 \%$ of the volume. Combining the two equations with this approximation gives

$$
\left(\mathrm{d} n_{\mathrm{j}} / \mathrm{d} t\right)_{\mathrm{IT}}=P_{\mathrm{j}} \chi^{\mathrm{b}}{ }_{\mathrm{i}} \sum_{\mathrm{i}<300}\left(\mathrm{~d} n_{\mathrm{i}} / \mathrm{d} t\right)_{\mathrm{gas}}\left[\frac{1}{P_{0}+\Delta P}\right]
$$

$\Delta P$ is used as an adjustable parameter that varies with the coal and experimental conditions. For the highly fluid Pittsburgh Seam bituminous coal, in cases where $P_{0}$ is 1 atm or greater, we have considered the upper limit to this rate where $P_{0} \gg \Delta P$. Then all the terms in eq 4 can be determined by the combined FG-DVC model. This limit coincides with assumptions recently used by Niksa in his FLASHKIN model for Pittsburgh Seam bituminous coal. ${ }^{76}$

While $\Delta P=0$ appears to be a good approximation for fluid coals at one atmosphere or more, $\Delta P>0$ is expected for some coals and situations. $\Delta P$ is proportional to the coal's viscosity and so will become important for less fluid coals. $\Delta P$ is also important when $P_{0}$ is small, when particles are large, and when the heating rates are very high.

Two possibilities have been considertd for combining the internal and external transportation. In an earlier publication, ${ }^{33}$ the internal-transport term and externaltransport term (with $X_{j}^{\prime}=X_{j}^{b}$ ) were assumed to be in series. Then the transport was controlled by the smaller term. The internal-transport term was the smaller for all

(76) Niksa, S. Presented at the Western States Section/The Combustion Institute, Spring Meeting, Salt Lake City, UT, March 1988; Paper BB-4. pyrolysis cases that were considered and so it dominated. In fact, calculations performed by neglecting the external transport limitation where almost identical with those made by assuming the two terms to be in series.

Alternatively, a case can be made that the total transport should be the sum of eq 3 and 4. The reasoning is that internal transport assumes the tars to be in equilibrium with the escaping light gases. It is more likely that this mechanism will transport the tars to the ambient gas than to the surface. In this case, the mechanism considered in eq 4 transports the tars away from the surface in parallel with the surface vaporization and gas diffusion considered in eq 3 .

If the two terms are taken in parallel, it is again obvious that $X_{j}^{s}=X_{j}^{b}$ is a bad assumption. Since we did not have a good method to determine $X^{s}$, calculations were made by assuming that the external-transport term can be neglected, i.e.

$$
\left(\mathrm{d} n_{\mathrm{i}} / \mathrm{d} t\right)_{\mathrm{tot}}=\left(\mathrm{d} n_{\mathrm{i}} / \mathrm{d} t\right)_{\mathrm{rT}}
$$

This provides an excellent fit to the data for $50-\mu \mathrm{m}$-diameter particles.

Therefore, for either parallel or series combinations of the transport terms, it appears best to neglect the external transport. It is likely that the external transport term will be increasingly important for smaller particles, but this will require better knowledge of the liquid-phase diffusion coefficient (mechanism i), and the stirring action of bubbles (mechanism iv). The relative importance of the various internal- and external-transport mechanisms is the subject of ongoing research.

Schematic Representation of DVC Model. In the current DVC model, the parent coal is represented as a two-dimensional network of monomers (condensed ring clusters) linked by strong and weak bridges as shown in Figure 3a. The monomers are linked to form unbranched oligomers of length " $l$ " by breakable and nonbreakable bridges (shown as horizontal single or double lines, respectively, in Figure 3a). The monomers are represented by circles with molecular weights shown in each circle. The molecular weight distribution of the monomers is assumed to be Gaussian and is described by two parameters, $M_{\text {av }}$ (mean) and $\sigma$ (standard deviation). The breakable bridges (assumed to be ethylene) are represented by single lines, the unbreakable bridges by double lines. " $m_{0}$ " cross-links per monomer are added (as vertical double lines in Figure $3 a)$ to connect the oligomers of length $l$ so that the molecular weight between cross-links, $M_{\mathrm{c}}$, corresponds to the value reported in the literature ${ }^{77}$ for coals of similar rank. The cross-links form the branch points in the macromolecule. Unconnected "guest" molecules (the extract yield) are obtained by choosing the value of $l$. A large value of $l$ will mean that a completely connected macromolecule will be formed when even a small number of cross-links are added, leaving no extractable material. For smaller values of $l$ some of the oligomers will be unattached after the cross-links are added, and these are the guest molecules. The number of ethylene bridges, $W_{B}$, (two donatable hydrogens per bridge) is chosen to obtain the appropriate value for total donatable hydrogen (i.e., to fit a selected laboratory pyrolysis experiment). The remainder are nonbreakable bridges whose carbons are counted with the aromatic carbons.

The parameters $M_{\mathrm{c}}, l, M_{\mathrm{av}}$, and $a$ determine the molecular weight distribution of oligomers in the starting coal molecule. A histogram showing the distribution created 
Table I. Coal Structure Parameters for DVC Subroutine

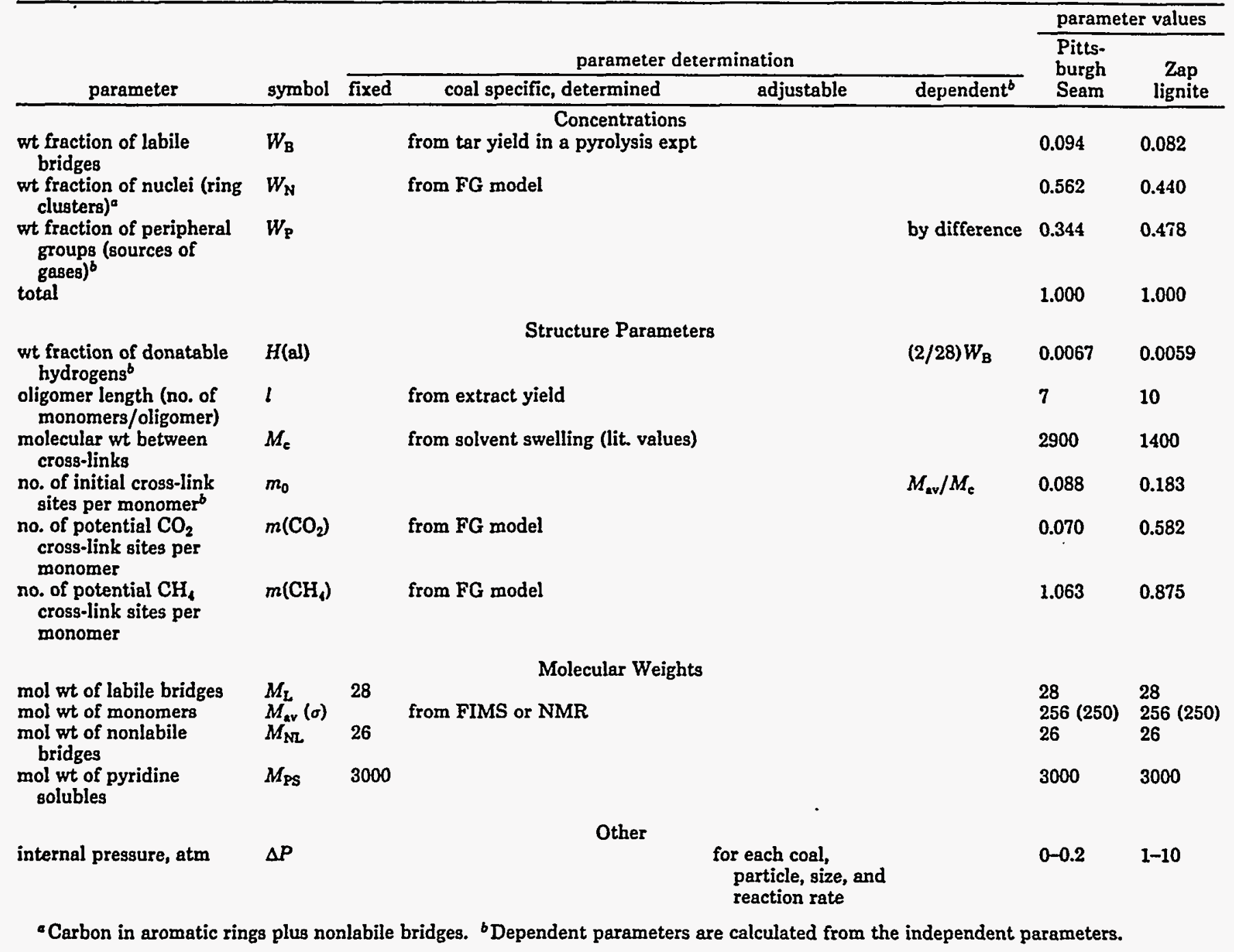

by randomly picking monomers to form oligomers of length $l$ and randomly cross-linking them to achieve an average molecular weight between cross-links, $M_{c}$, is presented at the right of Figure 3a. The distribution is divided into a pyridine-soluble portion below 3000 amu (light shading) and a pyridine-insoluble portion above 3000 amu (dark shading).

Figure $3 b$ shows the molecule during pyrolysis. The rates for bond breaking and cross-linking are from the FG model and are the same for all coals and all experiments. Some bonds have broken, other bonds have been converted to unbreakable bonds by the abstraction of hydrogen to stabilize the free radicals, and new cross-links have been formed. To determine the change of state of the computer molecules during a time step, the number of cross-links formed is determined by using the FG subroutine and passed to the DVC subroutine. These cross-links are distributed randomly throughout the char, assuming that the cross-linking probability is proportional to the molecular weight of the monomer. Then the DVC subroutine breaks the appropriate number of bridging bonds and calculates the quantity of tar evolved for this time step by using the internal and external transport equations. The result is the coal molecule representation and the molecular weight distributions shown in Figure $3 \mathrm{~b}$. The lighter "tar molecules", which leave the particle according to the transport equations, are shown as crosshatched. A fraction of the donatable hydrogen is used to stabilize the free radicals formed by bridge breaking, creating two new methyl groups per bridge and the same fraction of breakable bridges is converted into (unbreakable) double bonds.

Figure $3 c$ shows the final char, which is highly crosslinked with unbreakable bonds and has no remaining donatable hydrogen. The histogram now shows only tar and pyridine-insoluble fractions. The extractables have been eliminated by tar formation and cross-linking.

The output of the DVC subroutine is the molecular weight distribution in the coal, its time-dependent transformation during devolatilization, and the evolution of tar determined by the transport of the lighter components.

Selection of DVC Parameters. The DVC composition parameters employed for a Pittsburgh Seam coal and North Dakota lignite are summarized in Table I. The FG composition parameters and the kinetic parameters, which are fixed for all coals and experiments, are presented in Table II. In Table 1 , there are 11 independent composition parameters. Three parameters are fixed, the molecular weight of the labile bridges, $M_{\mathrm{L}}$, the nonlabile bridges, $M_{\mathrm{NL}}$, and the pyridine-extractable limit, $M_{\mathrm{PS}}$.

Eight parameters are coal specific (i.e., fixed for each coal, for all conditions) and must be determined by some measurement. $M_{c}$ and $l$ are determined experimentally for each coal by the measured molecular weight between cross-links and the pyridine extract yield, respectively. The weight fraction of carbon in nucleii and nonbreakable bridges, $W_{N}$, is obtained from the FG model and is equal to the amount of nonvolatile carbon. This value is, in 
a. Starting Molecule
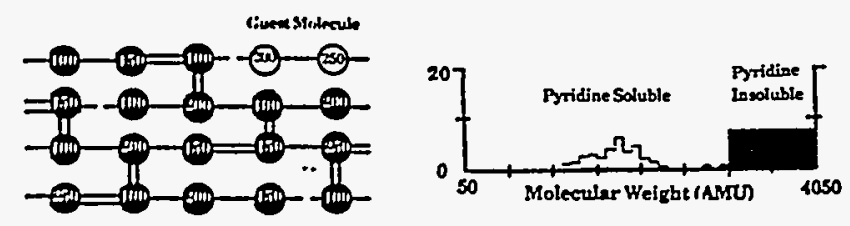

b. During Tar Formation

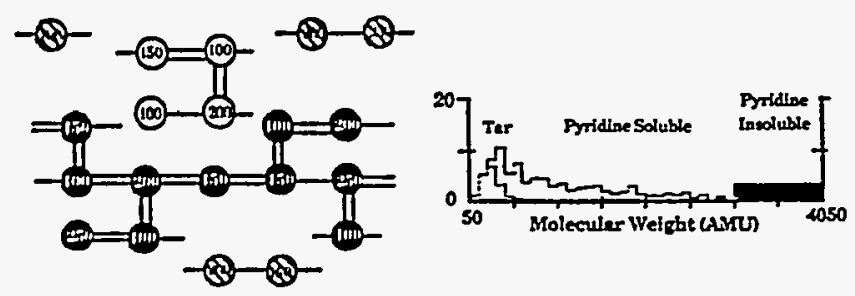

c. Char Formed

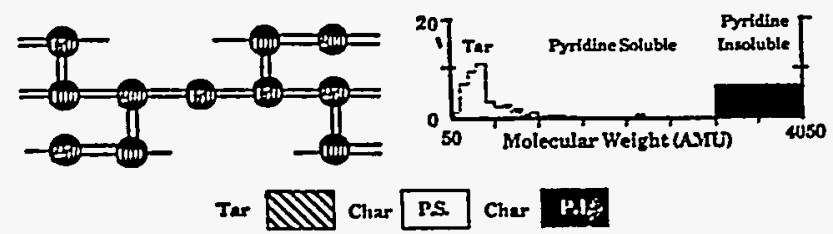

Figure 3. Representation of coal molecule in the DVC simulation and corresponding molecular weight distribution. In the molecule, the circles represent monomers (ring clusters and peripheral groups). The molecular weight shown by the numbers is the molecular weight of the monomer including the attached bridges. The single-line bridges are breakable and can donate hydrogen. The Double-line bridges are unbreakable and do not donate hydrogen. The molecular weight distribution of the coal, tar, and chars are shown as a histogram at the right. The histogram is divided into tar and char with pyridine-soluble and pyridine-insoluble fractions. The area under the histogram corresponds to the weight percent of the oligomers.

principle, determined for each coal for a single pyrolysis experiment. In practice, several experiments are performed. The number of potential cross-link sites, $m\left(\mathrm{CO}_{2}\right)$ and $m\left(\mathrm{CH}_{4}\right)$, are proportional to the total yield of $\mathrm{CO}_{2}$ and the total yield of $\mathrm{CH}_{4}$, respectively. $W_{\mathrm{B}}, M_{\mathrm{av}}$, and $\sigma$ are determined by using the model to fit selected pyrolysis experiments. The value of $W_{B}$ is adjustable to fit the tar yield or total volatile yield from one or two selected experiments. In principle, $W_{B}$ could be measured by FT-IR or NMR but not with sufficient accuracy for this highly sensitive parameter. The values of $M_{\mathrm{av}}$ and $\sigma$ are chosen based on FIMS analysis of the coal. $M_{\mathrm{av}}$ can be determined from the average cluster size determined by NMR. ${ }^{78,79}$ The value of 256 chosen for both the lignite and bituminous coal is in reasonable agreement with these reported by Solum, et al., ${ }^{79} 290$ for Zap and 300 for the Pittsburgh Seam coal.

One parameter, $\Delta P$, is adjustable and can vary with each type of experiment. For fluid coals at pressures above one atmosphere, $\Delta P \approx 0$. For low external pressures, less fluid coals, large particles, or high heating rates, $\Delta P>0$.

There are three dependent parameters that are computed from the other parameters: the weight fraction of peripheral groups, $W_{p}$; the donatable hydrogen, $H(a)$; and the number of initial cross-link sites per monomer, $\boldsymbol{m}_{0}$.

Functional Group (FG) Model Formulation. The Functional Group (FG) model has been described in a

(78) Gerstein, B. C.; Murphy, P. D.; Ryen, L. M. Coal Structure; Meyers, R. A., Ed.; Academic Press: New York, 1982; Chapter 4.

(79) Solum, M. A.; Pugmire, R. J.; Grant, D. M.; Wolfenden, W. R. submitted for publication in Energy Fuels. a
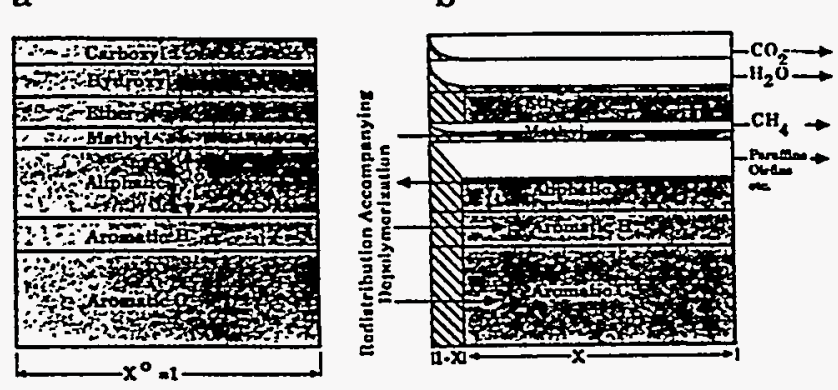

c

d

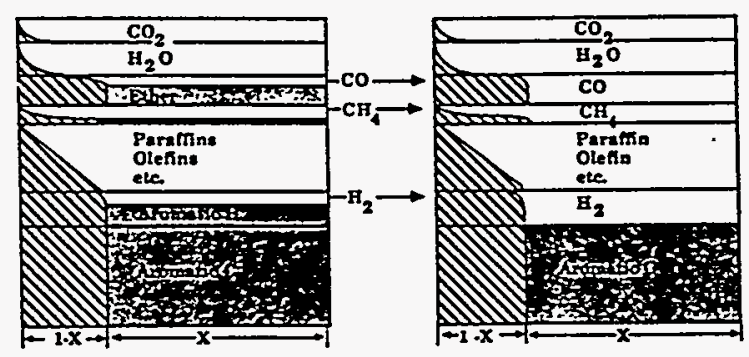

cubrea

Figure 4. Schematic representation of functional group ( $F G$ ) model: (a) Initial Coal Composition; (b) composition during tar formation; (c) composition after completion of tar formation; (d) composition after completion of devolatilization.

number of publications. ${ }^{5,6,11-13}$ It permits the detailed prediction of the composition of volatile species (gas yield, tar yield, and tar functional group and elemental composition) and of char (elemental and functional group composition). It employs coal-independent rates for the decomposition of individual assumed functional groups in the coal and char to produce gas species. The ultimate yields of each gas species are related to the coal's functional group composition. Tar evolution is a parallel process which competes for all the functional groups in the coal. In the original FG model, the potential tar forming fraction of the coal, $X^{0}$, was an input parameter that was adjusted for each coal and type of experiment. In the combined FG-DVG model, the DVC subroutine provides this parameter.

Schematic Representation of FG Model. The mathematical description of the functional group pyrolysis model has been presented previously. $6,6,11-13$ The evolution of tar and light-gas species provides two competing mechanisms for removal of a functional group from the coal: evolution as a part of a tar molecule and evolution as a distinct gas species. This process is shown schematically in Figure 4. To model these two paths, with one path yielding a product that is similar in composition to the parent coal, the coal is represented as a rectingulir area with $X$ and $Y$ dimensions. As shown in Figure 4a, the $Y$ dimension is divided into fractions according to the chemical composition of the coal. $Y_{i}^{0}$ represents the initial fraction of a particular component (carboxyl, aromatic hydrogen, etc.), and the sum of the $Y_{i}^{0}$ 's equal 1 . The evolution of each component into the gas (carboxyl into $\mathrm{CO}_{2}$, aromatic hydrogen into $\mathrm{H}_{2}$, etc.) is represented by the first-order diminishing of the $Y_{\mathrm{i}}$ dimension, $\mathrm{d} Y_{\mathrm{i}} / \mathrm{d} t=$ $-k_{\mathrm{i}} Y_{\mathrm{i}}$.

The $X$ dimension is divided into char, $X$, and tar, $(1-$ $X)$; initially $X=1$. The evolution of the tar is represented by the decreasing of the $X$ dimension, $\mathrm{d} X / \mathrm{d} t$, coinputed in the DVC subroutine as

$$
\mathrm{d} X / \mathrm{d} t=-\sum_{\mathrm{j}}\left(\mathrm{d} n_{\mathrm{j}} / \mathrm{d} t\right)_{\mathrm{tot}} M_{\mathrm{j}}
$$


Table II. Kinetic Rate Coefficients and Species Composition Parameters for FG Subroutine

\begin{tabular}{|c|c|c|c|c|c|}
\hline $\begin{array}{c}\text { compusition } \\
\text { params }\end{array}$ & gas & $\begin{array}{c}\text { primary } \\
\text { functional } \\
\text { group source }\end{array}$ & rate $\mathrm{eq}^{\alpha}$ & $\begin{array}{l}\text { Pittshurgh No. } \\
8 \text { bituminous } \\
\text { coal }\end{array}$ & $\begin{array}{l}\text { North } \\
\text { Dakota } \\
\text { Zap } \\
\text { lignite }\end{array}$ \\
\hline $\begin{array}{ll} & \mathrm{C} \\
\mathrm{H} \\
\mathrm{N} \\
\mathrm{S}(\text { org) } \\
\mathrm{O}\end{array}$ & & & & $\begin{array}{l}0.821 \\
0.056 \\
0.017 \\
0.024 \\
0.082\end{array}$ & $\begin{array}{l}0.665 \\
0.048 \\
0.011 \\
0.011 \\
0.265\end{array}$ \\
\hline total & & & & 1.000 & 1.000 \\
\hline 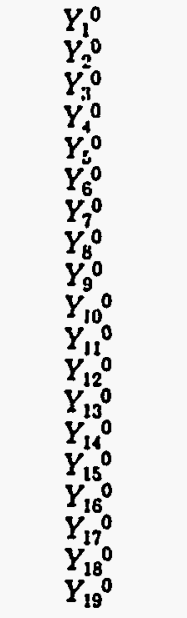 & $\begin{array}{l}\mathrm{CO}_{2} \text { extra loose } \\
\mathrm{CO}_{2} \text { loose } \\
\mathrm{CO}_{2} \text { tight } \\
\mathrm{H}_{2} \mathrm{O} \text { loose } \\
\mathrm{H}_{2} \mathrm{O} \text { tight } \\
\mathrm{CO} \text { ether loose } \\
\mathrm{CO} \text { ether tight } \\
\mathrm{HCN} \text { loose } \\
\mathrm{HCN} \text { tight } \\
\mathrm{NH}_{3} \\
\mathrm{CH}_{x} \text { aliphatic } \\
\text { methane extra loose } \\
\text { methane loose } \\
\text { methane tight } \\
\mathrm{H} \text { aromatic } \\
\text { methanol } \\
\text { CO extra tight } \\
\mathrm{C} \text { nonvolatile } \\
\mathrm{S} \text { organic }\end{array}$ & $\begin{array}{l}\text { carboxyl } \\
\text { carboxyl } \\
\text { hydroxyl } \\
\text { hydroxyl } \\
\text { ether } 0\end{array}$ & $\begin{array}{l}k_{1}=0.81 \mathrm{E}+13 \exp (-(22500 \pm 1500) / T) \\
k_{2}=0.65 \mathrm{E}+17 \exp (-(33850 \pm 1500) / T) \\
k_{3}=0.11 \mathrm{E}+16 \exp (-(38315 \pm 2000) / T) \\
k_{4}=0.22 \mathrm{E}+19 \exp (-(30000 \pm 1500) / T) \\
k_{5}=0.17 \mathrm{E}+14 \exp (-(32700 \pm 1500) / T) \\
k_{6}=0.14 \mathrm{E}+19 \exp (-(40000 \pm 6000) / T) \\
k_{7}=0.15 \mathrm{E}+16 \exp (-(40500 \pm 1500) / T) \\
k_{8}=0.17 \mathrm{E}+14 \exp (-(30000 \pm 1500) / T) \\
k_{9}=0.69 \mathrm{E}+13 \exp (-(42500 \pm 4750) / T) \\
k_{10}=0.12 \mathrm{E}+13 \exp (-(27300 \pm 3000) / T) \\
k_{11}=0.84 \mathrm{E}+15 \exp (-(30000 \pm 1500) / T) \\
k_{12}=0.84 \mathrm{E}+15 \exp (-(30000 \pm 1500) / T) \\
k_{13}=0.75 \mathrm{E}+14 \exp (-(30000 \pm 2000) / T) \\
k_{14}=0.34 \mathrm{E}+12 \exp (-(30000 \pm 2000) / T) \\
k_{16}=0.10 \mathrm{E}+15 \exp (-(40500 \pm 6000) / T) \\
k_{16}=0 \\
k_{17}=0.20 \mathrm{E}+14 \exp (-(45500 \pm 1500) / T) \\
k_{18}=0\end{array}$ & $\begin{array}{l}0.000 \\
0.007 \\
0.005 \\
0.012 \\
0.012 \\
0.050 \\
0.021 \\
0.009 \\
0.023 \\
0.000 \\
0.207 \\
0.000 \\
0.020 \\
0.015 \\
0.013 \\
0.000 \\
0.020 \\
0.562 \\
0.024\end{array}$ & $\begin{array}{l}0.065 \\
0.030 \\
0.005 \\
0.062 \\
0.033 \\
0.060 \\
0.038 \\
0.007 \\
0.013 \\
0.001 \\
0.102 \\
0.000 \\
0.017 \\
0.009 \\
0.017 \\
0.000 \\
0.090 \\
0.440 \\
0.011\end{array}$ \\
\hline total & & & & 1.000 & 1.000 \\
\hline$X^{0}$ & tar & & $k_{\mathrm{B}}=k_{\mathrm{T}}=0.86 \mathrm{E}+15 \exp (-(2770 \mathrm{~N} \pm 1500) / T)$ & & \\
\hline
\end{tabular}

- The rate equation is of the form $k_{n}=k_{0} \exp (-(E / R \pm \sigma / R) / T)$, with $k_{0}$ in $\mathrm{s}^{-1}, E / R$ in $\mathrm{K}$, and $\sigma / R$ in $\mathrm{K}$. $\sigma$ designates the spread in activation energies in a Gaussian distribution. The notation for $k_{0}$ is defined as follows: $0.81 E+13$ is equivalent to $0.81 \times 10^{13}$ etc.

The fractional amount of a particular functional group component in the char is

$$
W_{\mathrm{i}}(\mathrm{char})=X Y_{\mathrm{i}}
$$

and the amounts in the gas and tar may be obtained by integration with respect to time starting from $t=0$.

Secondary reactions such as further decomposition of aliphatic species to form olefins, acetylene, and soot modify the basic equations. Some of these have been described elsewhere. ${ }^{6}$ These types of secondary reactions are not considered in the current paper.

Figure $4 a$ shows the initial state of the coal. Values for $Y_{i}^{0}$ are obtained from elemental analysis and FT-IR analysis of the raw coal or from analysis of the products of one or two selected pyrolysis experiments. Figure $4 \mathrm{~b}$ shows the initial stage of devolatilization, during which the most volatile components, $\mathrm{H}_{2} \mathrm{O}, \mathrm{CO}$ (loose), and $\mathrm{CO}_{2}$ evolve from the hydroxyl, ether-loose, and carboxyl groups, respectively, along with aliphatics and tar. At a later stage (Figure 4c) $\mathrm{CO}$ (tight), $\mathrm{HCN}$ and $\mathrm{H}_{2}$ are evolved from the ether-tight, ring nitrogen, and aromatic hydrogen groups. Figure $4 \mathrm{~d}$ shows the final state of the char, tar, and gas.

The evolution of gas and the composition of the char and tar are then described mathematically as follows.

Process 5. Gas Formation. The evolution of each gas species is assumed to be a first-order reaction

$$
\mathrm{d} W_{\mathrm{i}} \text { (gas) } / \mathrm{d} t=k_{\mathrm{i}} W_{\mathrm{i}} \text { (char) }=k_{\mathrm{i}} X Y_{\mathrm{i}}
$$

where, $\mathrm{d} W_{\mathrm{i}}$ (gas)/ $\mathrm{d} t$ is the rate of evolution of speices $\mathrm{i}$ into the gas phase, $k_{\mathrm{i}}$ is a distributed rate for species $i$ and $W_{i}$ (char) is the functional group source remaining in the char. The concept of the distributed rate was introduced by Pitt $^{80}$ and subsequently employed by Rennhack ${ }^{81}$ and
Anthony et al. ${ }^{22}$ to describe weight loss. Hanbaba et al., ${ }^{82}$ van Heek et al., ${ }^{83}$ Weimer and Ngan, ${ }^{9}$ and Solomon et al. ${ }^{12}$ employed distributed rates for individual species. In the FG subroutine, $k_{\mathrm{i}}$ is given by an Arrhenius expression $k_{\mathrm{i}}$ $=k_{\mathrm{i}}{ }^{0} \exp \left(-\left(E_{\mathrm{i}} \pm \sigma_{\mathrm{i}}\right) / R T\right)$, where $\pm \sigma_{\mathrm{i}}$ indicates that a Gaussian distribution is employed to describe the product sources, $W_{\mathrm{i}}\left(E_{\mathrm{i}}\right)$, as a function of the activation energies $E_{\mathrm{i}}{ }^{5,9,12,22} W_{\mathrm{i}}\left(E_{\mathrm{i}}\right)=\left(W_{\mathrm{i}}^{0} / \sigma_{\mathrm{i}}(2 \pi)^{1 / 2}\right) \exp \left(-\left(E_{\mathrm{i}}-E_{\mathrm{i}}^{0}\right)^{2} / 2 \sigma_{\mathrm{i}}^{2}\right)$. $E_{i}^{0}$ is the average activation energy, and $\sigma_{i}$ is the width of the Gaussian distribution.

Note that $W_{\mathrm{i}}$ (char) also is decreased by its evolution with the tar.

Process 6. Tar Formation. The tar composition is tracked by summing the functional group contributions evolved with the tar. The rate of evolution of each contribution is

$$
\mathrm{d} W_{\mathrm{i}}(\operatorname{tar}) / \mathrm{d} t=-(\mathrm{d} X / \mathrm{d} t) Y_{\mathfrak{i}}
$$

where $\mathrm{d} W_{\mathrm{i}}(\operatorname{tar}) / \mathrm{d} t$ is the rate of evolution of each functional group component with the tar.

Process 7. Char Formation. The change in the ith char pool, $W_{i}$ (char), is computed by summing the losses to the gas and tar and the redistributions determined in the DVC subroutine

$$
\begin{aligned}
& \mathrm{d} W_{\mathrm{i}}(\text { char }) / \mathrm{d} t= \\
& \quad-\mathrm{d} W_{\mathrm{i}}(\mathrm{gas}) / \mathrm{d} t-\mathrm{d} W_{\mathrm{i}}(\text { tar }) / \mathrm{d} t+\mathrm{d} W_{\mathrm{i}}(\mathrm{DVC}) / \mathrm{d} t
\end{aligned}
$$

where $d W_{i}(D V C) / d t$ includes the source and loss terms from the DVC model, given by $(30 / 28) k_{\mathrm{B}} W_{\mathrm{B}},(2 / 28) k_{\mathrm{B}} W_{\mathrm{B}}$

(81) Rennhack, R. Brennst.-Chem. 1964, 45, 300 368.

(82) Hanbaba, P.; Juntgen, H.; Peters, W. Brennst.-Chem. 1968, 49

(83) Ven Heek, K. H.; Juntgen, H.; Peters, W. Ber. Bunsen-Ges. Phys Chem. 1967, 71, 113. 
Ditul COAL COALOSITIO:

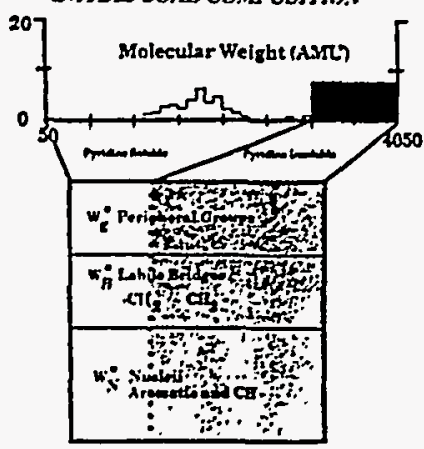

PRINLARY DEVOLATILIZATION
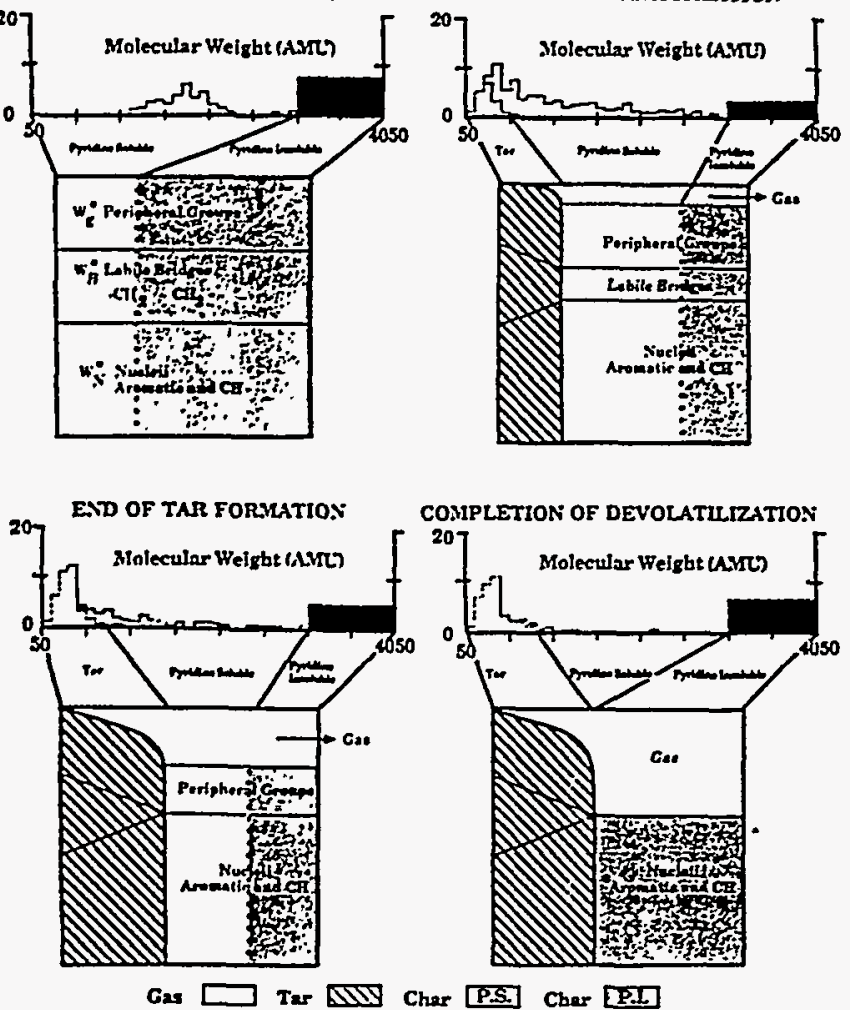

Figure 5. Schematic representation of the FG-DVC model combining the DVC and FG subroutines. The FG subroutine is illustrated for a single gas species only. The area under the histogram corresponds to the weight percent of the oligomers.

$(24 / 28) k_{\mathrm{B}} W_{\mathrm{B}}$, and $-2 k_{\mathrm{B}} W_{\mathrm{B}}$ for methyl, aromatic $\mathrm{H}$, aromatic $C$, and labile bridge functional groups, respectively.

The general rates and specific composition parameters for Pittsburgh Seam coal and North Dakota lignite are presented in Table II.

Schematic and Execution of FG-DVC Model. Figure 5 presents a schematic of the linked model for a simple case of only one gas species. The combined model connects the upper (DVC portion) and lower (FG portion) parts of Figure $5 \mathrm{a}-\mathrm{d}$. The model is initiated by specifying the functional group composition parameters $\left(W_{B}, W_{N}\right.$, and, in this case, only one gas species parameter, $W_{p}$ ) and the coal structure parameters (starting oligomer length, $l$, number of added cross-links per monomer, $m_{0}$, and the monomer molecular weight distribution parameters, $M_{\mathrm{av}}$ and $\sigma$ ). The starting molecular weight distribution of oligomers is presented at the top of Figure $5 \mathrm{a}$. The monomers are assumed to have the average elemental and functional group composition given by the FG parameters. The functional groups are divided into pyridine-soluble and pyridine-insoluble parts. Each computer simulation considers coal to consist of a network made from 2100-2400 monomers.

Once the starting distribution of oligomers in the coal is established, it is then subjected to a time-temperature profile made up of a series of isothermal time steps. Each time step is chosen so the temperature rise in each step does not exceed a fixed maximum. During each step, the gas yields, elemental composition, and functional group composition are computed by using the FG subroutine. The $\mathrm{CO}_{2}$ and $\mathrm{CH}_{4}$ yields are used to determine the number of new cross-links to be randomly added to the molecule. The molecular weight distribution, the escape of tar molecules, and the redistribution of hydrogens and carbons from the labile groups is computed with the DVC subroutine. Figure 5 b illustrates tar formation simultaneous with gas formation. The labile bridges are either evolved with the tar, converted to methyl groups (and thus added to the peripheral groups), or converted to unbreakable bridges (and thus added to aromatic $\mathrm{C}$ and $\mathrm{H}$ groups). Tar formation is complete (Figure 5c) when all the labile bridges are consumed. Devolatilization is completed (Figure 5d) when all volatile functional groups (in this case the single gas species represented as peripheral groups) are removed from the char.

The model has been programmed in Fortran 77 and runs on the Sun Microsystems $3 / 260$ and $3 / 50$ computers. Run times on a Sun $3 / 260$ are between 83 and 550 s/simulation for 2100-2400 monomers. A streamlined version of the code designed to run as a subroutine in a comprehensive combustion or gasification reactor simulation employs from 400 to 800 monomers and requires approximately $10 \mathrm{~s} /$ simulation for the pyrolysis of a single particle.

Summary of FG Subroutine Assumptions. (a) Light-gas species are formed from the decomposition of specific functional groups with rate coefficients that depend on the functional group but are independent of coal rank. The evolution rate is first order in the remaining functional group concentration in the char. The rates follow an Arrhenius expression with a Gaussian distribution of activation energies. $5,12,22$

(b) Simultaneous with the production of light-gas species is the thermal cleavage of bridge structures in the coal to release molecular fragments of the coal, which consist of a representative sampling of the functional group ensemble. These fragments may be transported out of the coal particle to form tar. The instantaneous tar yield is given by the DVC subroutine.

(c) Under conditions where pyrolysis products remain hot (such as an entrained-flow reactor), pyrolysis of the functional groups in the tar continues at the same rates used for functional groups in the char (e.g., the rate for methane formation from methyl groups in tar is the same as from methyl groups in the char).

Summary of DVC Subroutine Assumptions. (d) The oligomer length, $l$, the number of cross-links per monomer, $m_{0}$, and the fraction of labile bridges, $W_{\mathrm{B}}$, are parameters of the model, chosen to be consistent with the coal's measured extract yield, cross-link density, and volatile yield in selected calibration experiments.

(e) The molecular weight distribution is adjusted so that the model predictions fit the observed molecular weight distribution for that coal, measured by pyrolysis of the coal (in vacuum at $3^{\circ} \mathrm{C} / \mathrm{min}$ to $500^{\circ} \mathrm{C}$ ) in a FIMS apparatus. ${ }^{60}$ Molecular weights 106, 156, 206, 256, 306, 356, and 406 (which are aromatic one-ring through seven-ring compounds with two methyl substituents) are considered as representative of typical monomer molecular weights.

(f) During pyrolysis, the breakable bonds are assumed to rupture randomly at a rate $k_{\mathrm{B}}=k_{\mathrm{tar}}$, described by an Arrhenius expression with a Gaussian distribution of sources as a function of activation energies. Each rupture creates two free radicals that consume two donatable hydrogens to form two new methyl groups and convert two more donatable hydrogens to two aromatic $\mathrm{CH}$ groups. Oxymethylene bridges, which may be important for lowrank coals, have not been modeled although a second class of labile bridges could easily be added.

(g) All the donatable hydrogens are assumed to be located in the labile bridges. Two donatable hydrogens are available at each bridge. The consumption of the donatable hydrogen converts the bridge into an unbreakable bridge by the formation of a double bond. The unbruakable bridges are included in the aromatic hydrogen and 
aromatic carbon functional groups.

(h) Tar formation continues until all the donatable hydrogens are consumed.

(i) During pyrolysis, additional unbreakable cross-links ar $e$ added at a rate determined by the evolution of $\mathrm{CH}_{4}$ and $\mathrm{CO}_{2}$. One cross-link is created for each evolved molecule. The rates of $\mathrm{CH}_{4}$ and $\mathrm{CO}_{2}$ evolution are given by: the FG subroutine.

(j) The cross-links are distributed randomly, with the probability of attachment on any one monomer being proportional to the molecular weight of the monomer.

(k) Tar molecules are assumed to vaporize from the surface of the coal particle (or into bubbles) with a molet:ular weight and temperature dependence based on the vapor pressure correlation of Suuberg et al. ${ }^{32}$ The external-transport model is based on the surface-evaporation model of Unger and Suuberg. ${ }^{23}$

(I) To describe internal transport, a simple empirical expression (eq 4) is used to describe both bubble transport in softening coals and convective transport through pores in nonsoftening coals. The tar is assumed to be transported at its equilibrium vapor pressure in the light-gas species. The pressure increase that drives the transport within the particle, $\Delta P$, is between 0 and 0.2 atm for the bit uminous coal and between 0 and 10 atm for the lignite, depending on the experimental conditions.

im) Extractable material (in boiling pyridine) in the char is issumed to consist of all molecules less than $3000 \mathrm{amu}$. This limit can be adjusted depending on the solvent and exiraction conditions.

(n) The molecular weight between cross-links, $M_{c}$, is computed to be the total molecular weight of the computer molecule divided by the total number of cross-links. This assumption will underestimate $M_{c}$ since broken bridges are not considered.

\section{Results}

The model predictions have been compared to the results obtained from a number of experiments on the pyrolysis of a Pittsburgh Seam coal $10,16,22$ and a North Dakota (Beulah, Zap) lignite. ${ }^{6,51}$ The coal composition and kinetic parameters are presented in Tables I and II. It should be noted that different samples of Pittsburgh seam coal from different sources were employed. While the elemental compositions were similar, extract yields varied depending on the sample source. The oligomer length in Table I was chosen to fit an extract yield of $30 \%$ for the Pittsburgh Seam coal and $1 \%$ for the lignite. Comparisons are considered for gas yields, tar yields, tar molecular weight distributions, extract yields, and volumetric swelling ratios.

Volatile Yields. Extensive comparisons of the FG model with gas yields have been presented previously for high- and low-heating-rate devolatilization experiments. ${ }^{5,6,11-13}$ The evolution of gases for the combined model is similar to results of the FG model and will not be repeated here. There is good agreement between the measured and predicted results. The functional group parameters and the kinetic rates used for this work for the Pittsburgh Seam coal and North Dakota (Zap) lignite are principally those determined previously and published in ref 6 . The methane parameters for the Pittsburgh Seam coal were, however, adjusted (methane $\mathrm{X}-\mathrm{L}=0.0$, methane- $\mathrm{L}=0.02$, methane- $\mathrm{T}=0.015$, unchanged) to better match yields of refs. 5-7 (see Figure 20c in ref 6). Also note that the $\mathrm{CH}_{x}$ - aliphatic rate in ref 6 applies to the observed gas species (paraffins, olefins, $\mathrm{C}_{2} \mathrm{H}_{6}, \mathrm{C}_{2} \mathrm{H}_{4}$ ) only. The aliphatic material in the labile bridge part of the aliphatic groups is assumed to be made up of bridges that volatilize only when attached to a tar molecule (i.e., $k_{i}=0$ ). Also,

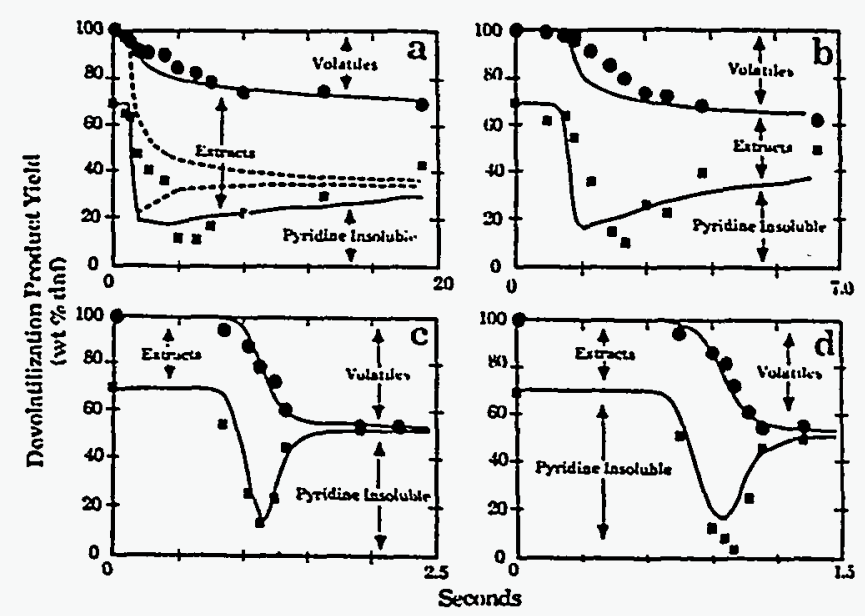

Figure 6. Comparison of FG-DVC model predictions with the data of Fong et al. ${ }^{16}$ (symbols) for Pittsburgh Seam coal: (a) 813 $\mathrm{K}$ at $470 \mathrm{~K} / \mathrm{s}$; (b) $858 \mathrm{~K}$ at $446 \mathrm{~K} / \mathrm{s}$; (c) $992 \mathrm{~K}$ at $514 \mathrm{~K} / \mathrm{s}$; (d) $1018 \mathrm{~K}$ at $640 \mathrm{~K} / \mathrm{s}$. $P=0.85 \mathrm{~atm}$. The solid line assumes transport by eq $4(\Lambda P=0 \mathrm{~atm})$ and no external transport. The dashed line in part a shows the predicted yield assuming $X_{j}^{2}=$ $x_{j}{ }_{j}$ in eq 3 and no internal-transport limitations.

the rate for $\mathrm{CO}_{2}$ (loose) has been adjusted to improve the predictions of the change in tar molecular weight distributions and yield with heating rate. The predictions of gas yield due to this change have not been changed noticeably. The predicted values of $X^{0}$ from the DVC subroutine vary with heating rate and final temperature and are in good agreement with the values of $X^{0}$ used in the original FG model.

Extract Yields. Figure 6 compares the FG-DVC predictions to the data of Fong et al..$^{16}$ on total volatile yield and extract yield as a function of temperature in pyrolysis at $0.85 \mathrm{~atm}$. The experiments were performed in a heated-grid apparatus at heating rates of approximately 500 ${ }^{\circ} \mathrm{C} / \mathrm{s}$, with variable holding times and rapid cooldown. The predictions at the two higher temperatures (Figures $6 c, d$ ) are in excellent agreement with the data.

The initial predictions for the two lower temperature cases, which neglected interal-transport limitations, were not good. The dashed line in Figure 6a shows the predicted yield in the absence of internal transport limitations (i.e., $\left(\mathrm{d} n_{j} / \mathrm{d} t\right)_{\mathrm{IT}}=0$ and with $X^{{ }_{j}}=X^{\mathrm{b}}{ }_{\mathrm{j}}$ in eq 3$)$. The predicted ultimate yield is clearly too high. The data suggest that the low yields are not a result of unbroken bonds (which would result from a lower bond breaking rate, $k_{\mathrm{B}}$ ), since the extract yields at low temperatures are equivalent to those at the higher temperatures. The coal molecule thus appears to be well decomposed, the low yields resulting from poor transport out of the coal. This suggested an additional transport limitation in getting molecules to the surface, so $X_{j}^{*}=X_{j}^{b}$ appears to be a bad assumption.

Equation 4 was employed for the internal-transport rate, and surface evaporation by eq 3 was assumed to be unimportant $\left(\chi_{\mathrm{j}}^{s_{\mathrm{j}}}=0\right)$.' Then, $W_{\mathrm{B}}$ had to be slightly readjusted from 0.096 in ref 50 to 0.094 to match the $1018 \mathrm{~K}$ case. This new value of $W_{B}$ was used for subsequent cases. The predictions with this assumption are the solid lines in Figure 6. The internal-transport limitation is most important when pyrolysis occurs at low temperatures and $\sum_{\text {light }} \mathrm{d} n_{\mathrm{i}} / \mathrm{d} t$ in eq 4 is small.

There still is a discrepancy between the prediction and the data at early times for the two lower temperature cases (Figure 6a,b). While it is possible that the rate $k_{\mathrm{b}}$ for bond breaking is too high, adjustment of this rate alone would significantly lower the extractable yield, since the lower depolymerization rate is closer to the methane cross-linking 

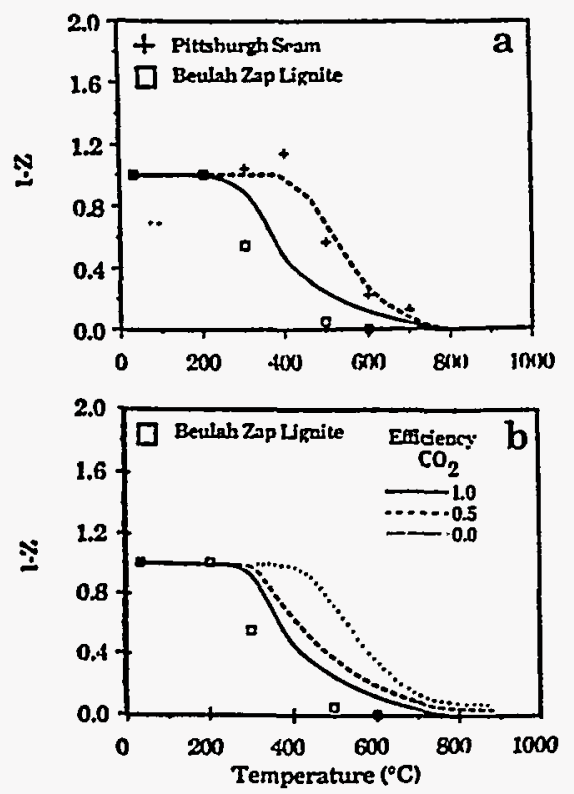

Figure 7. (a) Comparison of measured and predicted normalized volumetric swelling ratio as a function of temperature. The solid line is the prediction of Beulah lignite; the dashed line is for Pittsburgh Seam coal. The cross-link efficiency of $\mathrm{CO}_{2}$ is 1.0. (b) Effect of cross-link efficiency of $\mathrm{CO}_{2}$ on the normalized volumetric swelling ratio profile with temperature. For parts $a$ and $b$ the heating rate is $0.5^{\circ} \mathrm{C} / \mathrm{s} . \Delta P=0$ atm.

rate. In addition, both the methane and depolymerization rates appear to be in good agreement with the data at even lower temperatures. ${ }^{6}$ Another possibility is that the coal particles heat more slowly than the nominal temperatures given by Fong et al. ${ }^{16}$ Such an effect could be caused by having some clumps of particle that would heat more slowly than isolated particles, by reduction in the convective heat transfer due to the volatile evolution (blowing effect) or by endothermic tar forming reactions. A firm conclusion as to the source of this remaining discrepancy cannot be drawn without further investigation.

It is also seen in Figure $6 \mathrm{a}, \mathrm{b}$ that the cross-linking rate is higher than predicted. This can be due to other crosslinking events not considered. These possibilities are currently under investigation.

Cross-Link Density. To examine the effect of coal rank on cross-linking, the volumetric swelling ratios (VSR) for North Dakota (Beulah, Zap) lignite and Pittsburgh Seam bituminous coal were measured as a function of temperature at $0.5^{\circ} \mathrm{C} / \mathrm{s}$. The VSR can be related to the cross-link density. ${ }^{77}$ The swelling data are plotted in Figure $7 a$ as $1-Z$, where $Z$ is the change in VSR between coal and char normalized by the maximum change. For coal, $Z$ is 0 and for completely cross-linked char, $Z$ is 1 . While the weight loss profiles of the two samples look similar at $0.5^{\circ} \mathrm{C} / \mathrm{s}$, the swelling behaviors in Figure $7 \mathrm{a}$ are quite different. The Pittsburgh Seam coal starts to cross-link during tar evolution, and the Beulah lignite cross-links well before tar evolution. Similar results have been reported by Suuberg et al., ${ }^{59}$ who also suggested a correlation between cross-linking in lignites and $\mathrm{CO}_{2}$ evolution. The coals that undergo early cruss-linking are less fluid, produce less tar, and produce lower molecular weight tar compared with coals that do not experience early cross-linking. ${ }^{30,31,41}$

As discussed previously, under the assumption that the cross-linking reactions may also release gas species, the VSR was correlated with the observed evolution of gas species during pyrolysis. Correlations presented in Figure 2 show that on a molar basis, the evolution of $\mathrm{CO}_{2}$ from the lignite and $\mathrm{CH}_{4}$ from the bituminous coal appear to have similar effects on the VSR. Reactions that form these gases, leave behind free radicals that can be stabilized by cross-linking.

Assuming that one cross-link is formed for each $\mathrm{CO}_{2}$ or $\mathrm{CH}_{4}$ evolved from the char, the FG-DVC model predictions are presented as the lines in Figures 2 and 7a. The agreement between theory and experiment is good except that the increase in $1-Z$ for the Pittsburgh Seam coal in Figure $7 \mathrm{a}$ is not predicted. This may be related to the restrictions of assumption $n$ (see summary of DVC subroutine assumptions). The predictions in Figure $2 \mathrm{a}$ are different from those originally presented in ref 50 . In ref 50 , the value used for $\mathrm{VSR}_{\min }$ was not appropriate for the fully cross-linked molecule. This error has now been corrected.

In Figure $7 \mathrm{~b}$, the effect of varying the $\mathrm{CO}_{2}$ cross-linking efficiency is considered. The figure shows cases calculated for the lignite assuming $0,0.5$, and 1.0 cross-links are formed per $\mathrm{CO}_{2}$ evolved. Varying this assumption has a major effect on the early cross-linking of the lignite. Assuming that the cross-linking efficiency per $\mathrm{CO}_{2}$ is 1.0 gives the best agreement with the data.

The difference in crosslinking behavior between the two coals is manifested in several areas. At low heating rates, the Pittsburgh Seam chars soften; the Beulah, Zap chars do not. This is in agreement with the high predicted maximum extract yields in the Pittsburgh char (70\%) compared to the low extract yields in the Beulah, Zap lignite $(7 \%)$. The measured values are $71 \%^{16}$ and $\sim 6 \%$, respectively. The predicted yield of tar plus aliphatic gases at 1 atm, $0.5^{\circ} \mathrm{C} / \mathrm{s}$ to $900^{\circ} \mathrm{C}$, of $26 \%$ is in good agreement with the measured value of $28 \%$ for the Pittsburgh Seam coal. The predicted value of $11 \%$ (for $\Delta P=10 \mathrm{~atm}$ ) is in good agreement with the measured value of $10 \%$ for the Beulah, Zap lignite.

Molecular Weight Distribution. A sensitive test of the general model is the ability to predict the tar molecular weight distribution and its variations with rank, pressure, and heating rate. The input to the model is the distribution of monomer molecular weights. The tar, which consists of oligomers, has a different distribution from the monomer distribution and is controlled by the relative effects of bond breaking, cross-linking, and transport. The tar molecular weight distribution is not highly sensitive to the choice of $M_{\mathrm{av}}$ and $\sigma$. For Pittsburgh Seam coal, the average monomer was assumed to be a three-ring compound $\left(M_{\mathrm{av}}=256\right)$ and a fairly broad distribution $(\sigma=250)$ was chosen. The same values appeared to work for the lignite. These are in reasonable agreement with the measured values of $\sim 300$ reported by Solum et al. ${ }^{79}$ for both coals.

Figure 8c,d show results for the Pittsburgh Seam bituminous coal and the Beulah, Zap lignite pyrolyzed in the FIMS apparatus. The data have been summed over 50 amu intervals. While the Pittsburgh bituminous coal shows a peak intensity at about $400 \mathrm{amu}$, the lignite peak is at 100 amu. The predicted average tar molecular weight distributions are in good agreement with FIMS data as shown in Figure 8a,b. Since both tar distributions are froin the same monomer distribution, the enhanced drop off in amplitude with increased molecular weight for the lignile compared to the bituminous coal must be due to early cross-linking and transport effects in the lignite.

Pressure Effects. The predicted effect of pressure on the tar molecular weight distribution is illustrated in Figure 9a,b. Pressure enters the model through the transport eq 3 and 4 . The internal-transport rate (eq 4), which is assumed to dominate, is inversely proportional to the am- 
Table III. Comparison of Measured and Predicted Yields for Pittsburgh Seam Bituminous Coal ( $\perp P=0$ atm)

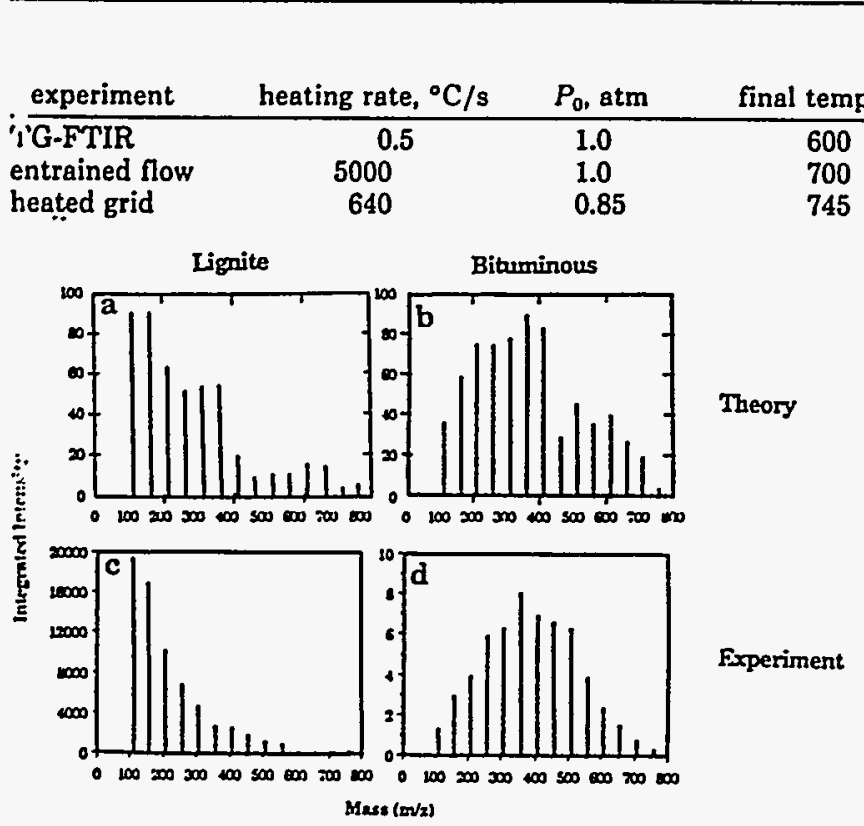

Figure 8. Comparison of measured and predicted tar molecular weight distributions for lignite and bituminous coals. The experiments are performed by pyrolysis of coal samples in a FIMS apparatus. Intensities have been summed over 50 amu intervals. For part a $\Delta P=10 \mathrm{~atm}$, and for part b $\Delta P=0.0 \mathrm{~atm}$.

bient pressure $P_{0}$. The reduced transport rate reduces the evolution rate of the heavier molecules. Therefore, the average molecular weight and vaporization "cutoff" decrease with increasing pressure. The trends are in agreement with observed tar molecular weight distributions shown in Figure 9c,d. The spectra are for previously formed tar that has been collected and analyzed in a FIMS apparatus. ${ }^{60}$ The low values of intensity between 100 and 200 mass units are believed to be due to loss of these components due to their higher volatility.

Pressure effects on yields have also been examined. Figure 10 compares the predicted and measured pressure dependence on yield for a Pittsburgh Seam coal. Figure 10 a compares the prediction to the total volatile yield data of Anthony et al. ${ }^{22}$ while Figure $10 \mathrm{~b}$ compares the prediction to the tar plus liquids data of Suuberg et al. ${ }^{7}$ The agreement between theory and experiment is good at 1 atm and above, but the theory with $\Delta P=0$ (solid line) overpredicts the yields at low pressure. Below $1 \mathrm{~atm}$, it is expected that $\Delta P$ within the particle will become important compared to the ambient pressure, $P_{0}$. The dashed lines, which agree with the data, were obtained by assuming $\Delta P$ $=0.2 \mathrm{~atm}$, which is physically reasonable.

Heating-Rate Effects. It is well-known that the heating rate can affect the amount of volatiles produced. ${ }^{29,76,84-86}$ Heating rate can also affect the melting and swelling behavior of low-rank coals. ${ }^{13}$ Considering the mechanisms proposed for pyrolysis (including those in this paper), it is the relative rates of competing processes for tar formation (e.g., bond breaking, cross-linking, and mass transport) that provide the heating-rate effects. The relative rates of these processes change with temperature, and it is the heating rate that determines the temperature at which the controlling reactions occur. So it is really the

(84) Gibbins-Matham, J.; Kandiyoti, R. Prepr. Pap.-Am. Chem. Soc., Div. Fuel Chem. 1987, 32(4), 318.

(85) Freihaut, J. D.; Seery, D. J. Prepr. Pap.-Am. Chem. Soc., Div. Fuel Chem. 1983, 28(4), 265.

(86) Serio, M. A.; Peters, W. A.; Sawada, K.; Howard, J. B. Prepr.

Pop.-Am. Chem. Soc., Div. Fuel Chem. 1984, $29(2), 65$.

\section{max yield of tar +}

aliphatic gases, \%

\begin{tabular}{cc}
\hline measd & predicted \\
\hline 25 & 29 \\
36 & 37 \\
& 40
\end{tabular}

total max volatiles, wt \%

\begin{tabular}{cc}
\hline measd & predicted \\
\hline 35 & 37 \\
43 & 43 \\
47 & 47
\end{tabular}
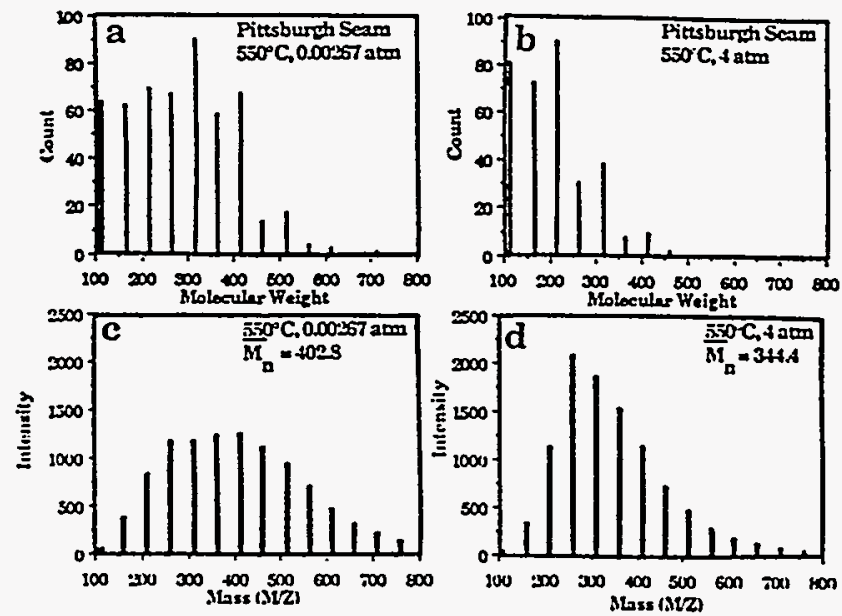

Figure 9. Comparison of predicted ( $a$ and $b$ ) and measured ( and d) tar molecular weight distribution for pyrolysis of a Pittsburgh Seam coal in a heat-grid apparatus at a heating rate of $500^{\circ} \mathrm{C} / \mathrm{s}$ to $550^{\circ} \mathrm{C}$. Parts a and $\mathrm{c}$ compare the prediction and the measurement at 0.00267 atm. Parts $b$ and $d$ compare the prediction and measurement at 4.0 atm. $\Delta P=0.2 \mathrm{~atm}$.

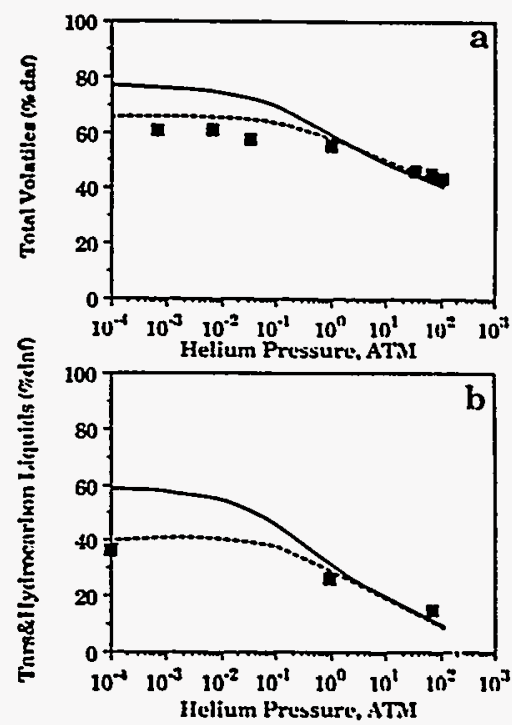

Figure 10. Comparison of measured and predicted volatile yield for a Pittsburgh Seam bituminous coal; (a) total volatiles, data of Anthony et al. ${ }^{22}$ (b) tars and hydrocarbon liquids, data of Suuberg et al. ${ }^{7}$ The solid line assumes $\Delta P=0$ atm; the dashed line assumes $\Delta P=0.2$ atm.

temperature of tar formation and not the heating rate per se which is important.

Consider first the effects of heating rate on the yields of a Pittsburgh Seam bituminous coal. Table III summarizes the results for three experiments $s^{16,87,88}$ in which the heating rate varied from 0.5 to $5000^{\circ} \mathrm{C} / \mathrm{s}$ and in which the final temperature reached is sufficiently high for tar

(87) Serio, M. A.; Solomon, P. R.; Carangelo, R. M. Prepr. Pap.-Am. Chem. Soc., Div. Fuel Chem. 1988, 33(2), 295.

(88) Solomon, P. R.; Hamblen, D. G.; Serio, M. A.; Smoot, L. D.; Brewster, S. "Measurement and Modeling of Advanced Coal Conversion"; First Annual Report for U.S. METC Contract No. DE-AC21-86MC23075, 1987. 

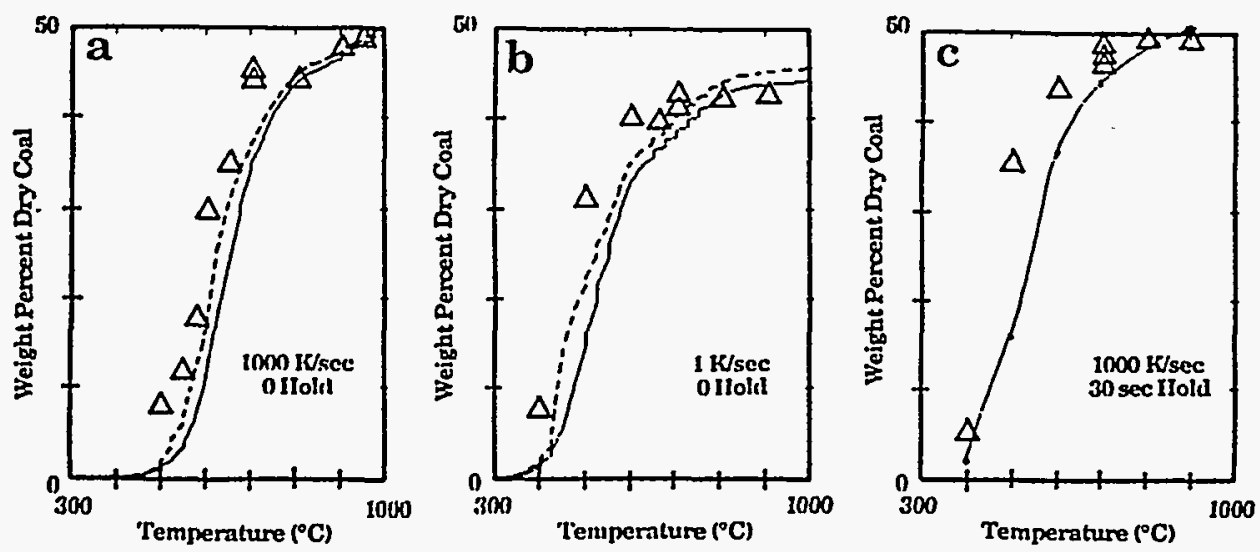

Figure 11. Comparison of FG-DVC model predictions with the data of Gibbins-Matham and Kandiyoti ${ }^{83}$ (symbols) for Pittsburgh Seam coal: (a) $1000 \mathrm{~K} / \mathrm{s}$, zero hold; (b) $1 \mathrm{~K} / \mathrm{s}$, zero hold; (c) $1000 \mathrm{~K} / \mathrm{s}, 30$-s hold. $P=1.18$ atm. Transport is by eq $4(\Delta P=0$ ) and no external-transport limitation. The dashed line assumes no transport limitations for molecules whose vapor pressure exceeds $P_{0}$ $+\Delta P$.

formation to be completed during the heating period. As can be seen, the predicted and measured volatile yields increased by about $10 \%$ from low to high heating rates. As can also be seen, the increase in yield results from the increase in tar plus aliphatic gases. Examination of the rates in the model shows that the major contribution to the variation in yield is the internal-transport rate relative to the bond-breaking rate. At low temperatures, internal transport severely limits the evolution of the heavier molecules, resulting in smaller tar molecules and inefficient use of the donatable hydrogens.

A set of data showing the effect of heating rate on yield for the Argonne Pittsburgh Seam coal was recently reported by Gibbins-Matham and Kandiyoti. ${ }^{84}$ Data were obtained in a wire-grid apparatus at 1 and $1000^{\circ} \mathrm{C} / \mathrm{s}$ with no holding time and at $1000^{\circ} \mathrm{C} / \mathrm{s}$ with a $30-\mathrm{s}$ hold. These data (triangles) are compared to predictions of the model in Figure 11. For all three cases, the theory predicts the correct pyrolysis final yields, the correct yield variation with heating rate, and the correct temperature shift with heating rate.

The predicted yields, however, occur at temperatures from 20 to $80^{\circ} \mathrm{C}$ higher than the comparable experimental yields. At this time, the reason for the discrepancy is not clear. One possible reason is the assumptions used for the internal transport limitations. Calculations were made assuming that molecules for which $P_{j} \geq P_{0}+\Delta P$ evolve as they are produced, while only heavier molecules evolve as described in eq 4. The predicted curves (dashed lines in Figure 11) are $20-40^{\circ} \mathrm{C}$ lower than in the original calculation. Alternatively, the vapor pressure may not be accurately described by the expression of Suuberg et al. ${ }^{32}$ $\mathrm{Oh}^{38}$ compared a number of correlations for the tar vapor pressure. At $1000^{\circ} \mathrm{C}$, the expression of Suuberg et al. ${ }^{32}$ gave vapor pressures from 1-2 orders of magnitude lower than other published expression. 89,90 Calculations using the expression for aliphatic molecules of Maiorella ${ }^{89}$ gave predictions at about $40^{\circ} \mathrm{C}$ lower temperatures, in better agreement with the data of Gibbons-Matham and Kandiyoti. The simulation, however, required a lower value of $W_{B}(0.060)$ to compensate for the higher volatility. Predictions using the same assumptions failed to match those of Fong et al. ${ }^{16}$ in Figure 6 with regard to the temperature of evolution and the amount of extract produced. Possible refinements of the internal-transport model are

(B9) Maiorella, B. L. B.S. Thesis, Department of Chemical Engineering, MIT, Cambridge, MA, 1975 .

(90) Grey, J. A.; Brady, A. J.; Cunningham, J. R.; Freeman, J. R.; Wilson, G. M. Ind. Eng. Chem. Process Des. Dev. 1983, 22, 410.
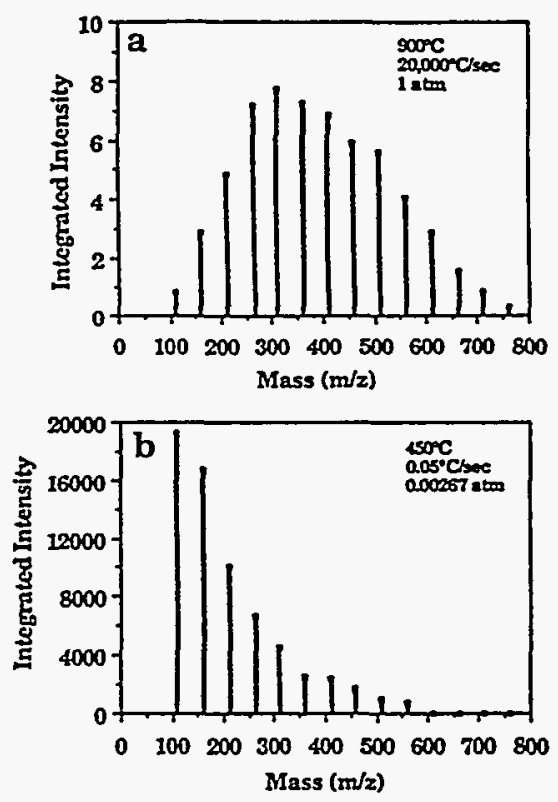

Figure 12. Comparison of FIMS spectra of tars of Beulah Zap lignite formed at (a) high heating rate $\left(20000^{\circ} \mathrm{C} / \mathrm{s}\right)$ and (b) low heating rate $\left(0.05^{\circ} \mathrm{C} / \mathrm{s}\right)$.

being considered.

Another possible explanation for the discrepancy is the accuracy of the reported pyrolysis temperature, which has been notoriously variable among investigators. Other Pittsburgh Seam coal data (not shown) for Niksa et al..$^{40}$ under the same conditions as Figure $11 \mathrm{c}\left(1000^{\circ} \mathrm{C} / \mathrm{s}, 30-\mathrm{s}\right.$ hold) and from $\mathrm{Oh}^{38}$ and Suuberg et al. ${ }^{7}$ for the same conditions as Figure 11a $\left(1000^{\circ} \mathrm{C} / \mathrm{s}\right.$, zero hold) show substantial yariations in temperature compared to the results of Gibbons-Matham and Kandiyoti. ${ }^{84}$ The theoretical predictions would lie within the scatter of the several data sets. Work is in progress to resolve this question.

Low-rank coals also exhibit heating-rates effects. It has been found that Beulah lignite chars soften and exhibit bubble formation at high heating rates $\left(\sim 20000^{\circ} \mathrm{C} / \mathrm{s}\right) .^{13}$ Under these conditions, molecular weight distribution of tars of Beulah lignite look like that of a bituminous coal..$^{3031}$ The infrared spectrum of the tar is also closer in appearance to that of the parent coal. ${ }^{31}$ The mass spectra of the tars formed at high heating rate $\left(20000^{\circ} \mathrm{C} / \mathrm{s}\right)$ and low heating rate $\left(0.05^{\circ} \mathrm{C} / \mathrm{s}\right)$ are shown in parts $\mathrm{a}$ and $\mathrm{b}$ of Figure 12, respectively. The low values of intensity between 100 and 200 mass units in Figure $12 \mathrm{~b}$ are believed 

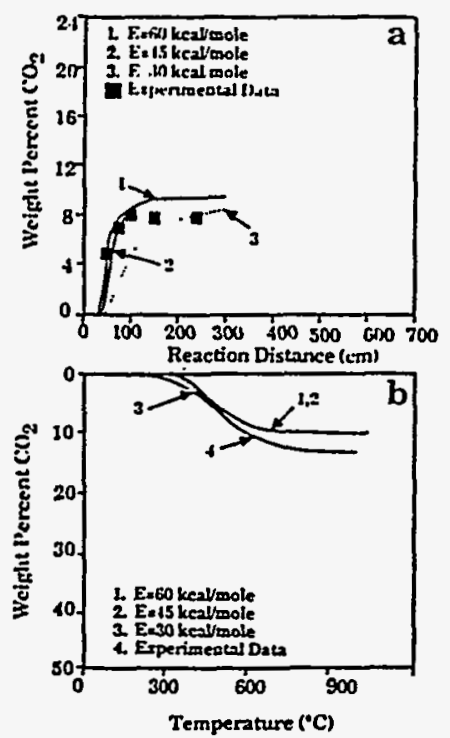

Figure 13. Comparison of $\mathrm{CO}_{2}$ evolution data from North Dakota lignite for low-heating-rate $\left(0.5^{\circ} \mathrm{C} / \mathrm{s}\right)$ and high-heating-rate $(20000$ - $\mathrm{C} / \mathrm{s}$ ) experiments with model predictions for different values of activation energy for $\mathrm{CO}_{2}$ (extra loose) in the FG-DVC Model: (a) heated-tube-reactor experiments; ${ }^{6}$ (b) TG-FTIR experiments. ${ }^{6}$

to be due to loss of these components due to their high volatility. The molecular weight distribution of the tars is very se. ssitive to the heating rate. The effect is attributed to the higher rate of depolymerization reactions relative $t$. cross-linking reactions at high temperatures, as discussed in the sensitivity section.

The FG-DVC model, assuming the internal-masstransport limitations, was used to simulate the low heating rate $\left(0.05^{\circ} \mathrm{C} / \mathrm{s}\right)$ and high heating rate $\left(20000^{\circ} \mathrm{C} / \mathrm{s}\right)$ pyrolysis of Beulah lignite. The activation energy for $\mathrm{CO}_{2}$ (extra louse) in the FG subroutine was reduced from 60 to $45 \mathrm{kcal} / \mathrm{mol}$ in order to make it lower than the activation energy for bond breaking ( $55 \mathrm{kcal} / \mathrm{mol}$ ). This was done since measurements of the rate of cross-linking at high heating rates suggested that the relative rate of bondbreaking and cross-linking reactions associated with $\mathrm{CO}_{2}$ evolution is increased with increasing temperature. ${ }^{91}$ This change in the activation energy makes only a slight change in the $\mathrm{CO}_{2}$ evolution profiles for high-heating-rate $(20000$ $\left.{ }^{\circ} \mathrm{C} / \mathrm{s}\right)$ and low-heating rate $\left(0.5^{\circ} \mathrm{C} / \mathrm{s}\right)$ predictions. The $\mathrm{CO}_{2}$ gas evolution profiles are compared to the data in Figure $13 \mathrm{a}, \mathrm{b}$ for high-heating-rate $\left(20000^{\circ} \mathrm{C} / \mathrm{s}\right)$ and lowheating-rate $\left(0.5^{\circ} \mathrm{C} / \mathrm{s}\right)$ experiments with Beulah lignite using activation energies of 60,45 , and $30 \mathrm{kcal} / \mathrm{mol}$. When the activation energy for $\mathrm{CO}_{2}$ (extra loose) evolution was reduced to $45 \mathrm{kcal} / \mathrm{mol}$, acceptable fits to the gas evoluation data were still obtained. However, at $30 \mathrm{kcal} / \mathrm{mol}$, the high-heating-rate $\mathrm{CO}_{2}$ evolution profile was quite different and did not agree with the experimental data.

The model, with internal-mass-transport limitations included, was used to simulate the tar molecular weight distributions with $\triangle P=0$ atm for Beulah lignite for high heating rate $\left(20000^{\circ} \mathrm{C} / \mathrm{s}\right)$ in Figure $14 \mathrm{a}, \mathrm{b}$. The simulations were done for both the original activation energy ( 60 $\mathrm{kcal} / \mathrm{mol}$ ) and altered activation energy $(45 \mathrm{kcal} / \mathrm{mol}$ ) for $\mathrm{CO}_{2}$ (extra loose) evolution. The tar molecular weight distributions (for $\triangle P=0 \mathrm{~atm}$ ) at high heating rates (Figure $14 a, b)$ show the observed high values of the tar molecular weight at high heating rate (Figure 12a). The lower activation energy case (Figure 14a) exhibits more high molecular weight molecules and gives a higher tar yield (10\%)

(91) Deshpande, G. V.: Solomon, P. R.; Serio, M. A. Prepr. Pap.-Am. Chem. Soc., Div. Fuel Chem. 1988, 33(2), 310.
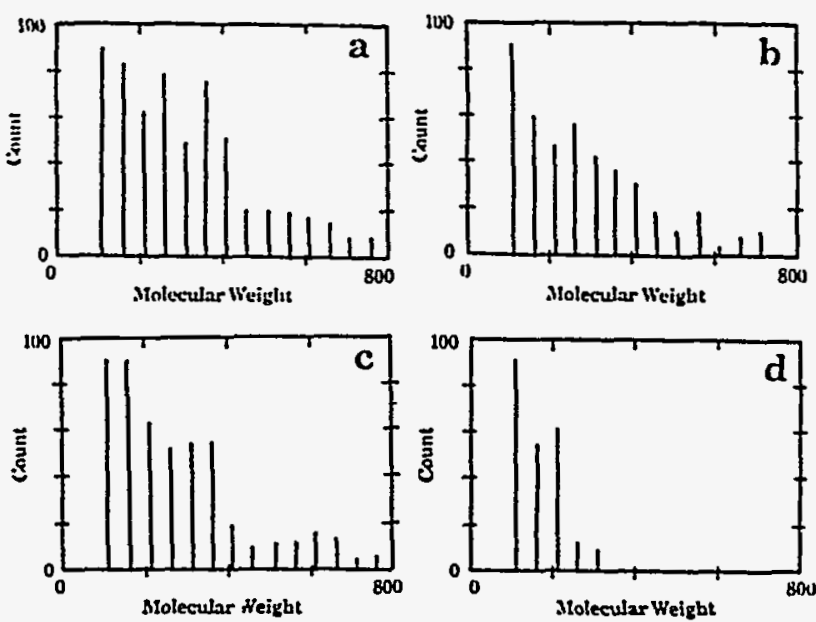

Figure 14. Comparison of predicted molecular weight distribution of tars of Beulah lignite for $(a$ and $b$ ) high heating rate $\left(20000^{\circ} \mathrm{C} / \mathrm{s}\right)$ and $\left(\mathrm{c}\right.$ and d) low heating rate $\left(0.05^{\circ} \mathrm{C} / \mathrm{s}\right)$. In parts $\mathrm{a}$, $\mathrm{c}$, and d the $\mathrm{CO}_{2}$ activation energy is $45 \mathrm{kcal} / \mathrm{mol}$ and in part $\mathrm{b}$ it is $60 \mathrm{kcal} / \mathrm{mol}$. In parts a-c $\Delta P=0 \mathrm{~atm}$, and in part $\mathrm{d} \Delta P$ $=10 \mathrm{~atm}$.
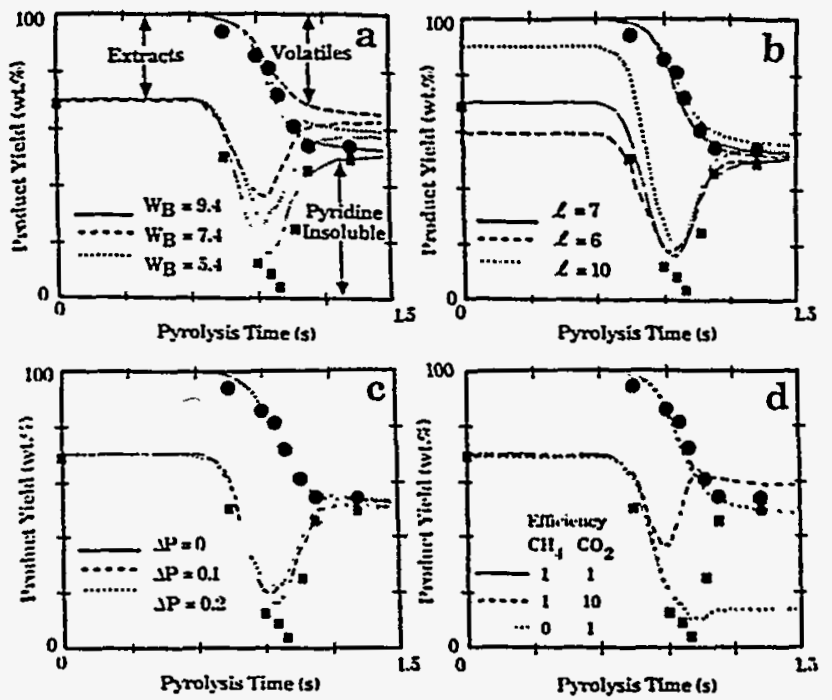

Figure 15. Effect on product yields of (a) fraction of labile bridges, $W_{\mathrm{B}}$, (b) oligomer length, $l$, (c) internal pressure difference, $\Delta P$, and (d) cross-linking efficiency. Data were taken from Fong et al. ${ }^{16}$ for Pittsburgh Seam bituminous coal (1018 K at $640 \mathrm{~K} / \mathrm{s}$, $P=0.85 \mathrm{~atm}) ; \Delta P=0 \mathrm{~atm}$.

than the high activation energy case (8\%) (Figure 14b). The low-heating-rate $\left(0.05^{\circ} \mathrm{C} / \mathrm{s}\right)$ case $(\Delta P=0)$ (Figure $\left.14 \mathrm{c}\right)$ exhibits lower molecular weights consistent with Figure 12b. At high heating rates, where cross-linking reactions are curbed and the lignite melts, $\Delta P$ is likely to be low. At low heating rate, due to the higher extent of cross-linking before tar evolution, the coal is less fluid, and hence $\Delta P$ (which is related to viscosity of the solid/liquid mixture) is likely to be higher. A simulation for the slow-heatingrate case with $\triangle P=10 \mathrm{~atm}$ is shown in Figure 14d. The measured molecular weight distribution in Figure $12 \mathrm{~b}$ appears to be intermediate between the $\Delta P=0$ and $\Delta P$ $=10 \mathrm{~atm}$ cases.

Sensitivity Analysis. This section considers the sensitivity of the FG-DVC model to variations in the DVC parameters. The FG parameter sensitivities have been considered elsewhere. ${ }^{52}$

(a) Variations in $W_{B}$. The number of labile bridges is the most important parameter in determining tar yield. The value of $W_{B}$ for the Pittsburgh Seam coal was reduced from its value of 9.4 to 7.4 and 5.4. The results in Figure 15a were calculated for the case considered in Figure 6d. 

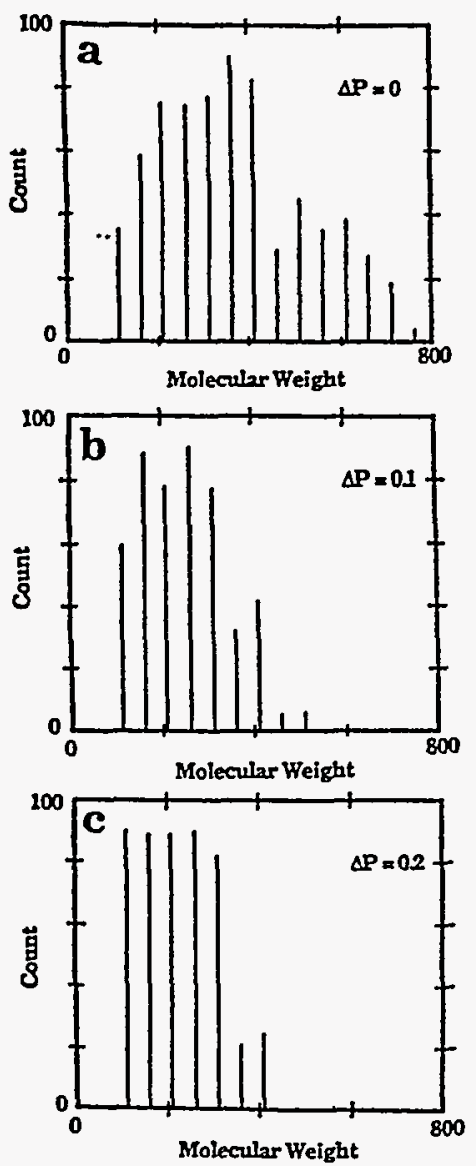

Figure 16. Effect of $\triangle P$ on tar molecular weight distribution for Pittsburgh Seam bituminous coal heated to $723 \mathrm{~K}$ at $0.05 \mathrm{~K} / \mathrm{s}$. $P=0.00267$ atm.

The reduction in $W_{\mathrm{B}}$ reduces the tar yield, the total volatile yield, and the extract yield. Higher values of $W_{B}$ could not be considered because the molecule already contained the maximum number of labile bridges. This is a limitation in the model as it is currently formulated since all the donatable hydrogens are assumed to be in bridges.

(b) Variations in $l$. The parameter $l$ affects mainly the extract yield in the raw coal. Figure $15 \mathrm{~b}$ demonstrates variations in $l$ from 6 to 10 around the base value of 7 . The initial extract yield varies substantially while there is only a minor effect on the tar yield, total volatile yield, and extract yield at elevated temperature.

(c) Variations in $\Delta P$. The effect of variations in $\Delta P$ on the overall yield are considered in Figure 10. There is no effect at 1 atm pressure and above but a strong effect at lower ambient pressures. Figure $15 \mathrm{c}$ confirms that $\Delta P$ has little effect on the tar yield or the total volatile yield for pyrolysis at 1 atm pressure. Only the extract yield is slightly affected.

Figure 16 illustrates the effect on the molecular weight distribution for three values of $\Delta P$ for pyrolysis in vacuum $\left(P_{0}=0\right)$. The yield of higher molecular weight tars present for $\Delta P=0$ is lower for $\Delta P=0.1$ atm and eliminated for $\Delta P=0.2$ atm. The total tar yields are $39 \%, 21 \%$, and $17 \%$ for $\Delta P=0,0.1$, and 0.2 atm respectively. The tar molecular weight distribution for $\Delta P=0$ atm gives the best match to Figure $9 c$, but $\Delta P=0.1-0.2$ atm provides the best match to the yield.

The variation of $\Delta P$ in the tar molecular weight distribution for lignite is discussed with reference to Figure 13.

(d) Variations in $m\left(\mathrm{CO}_{2}\right)$ and $m\left(\mathrm{CH}_{4}\right)$. Variations in $m\left(\mathrm{CO}_{2}\right)$ were considered for the lignite in the discussion accompanying Figure 7 . Variations in both $m\left(\mathrm{CO}_{2}\right)$ and
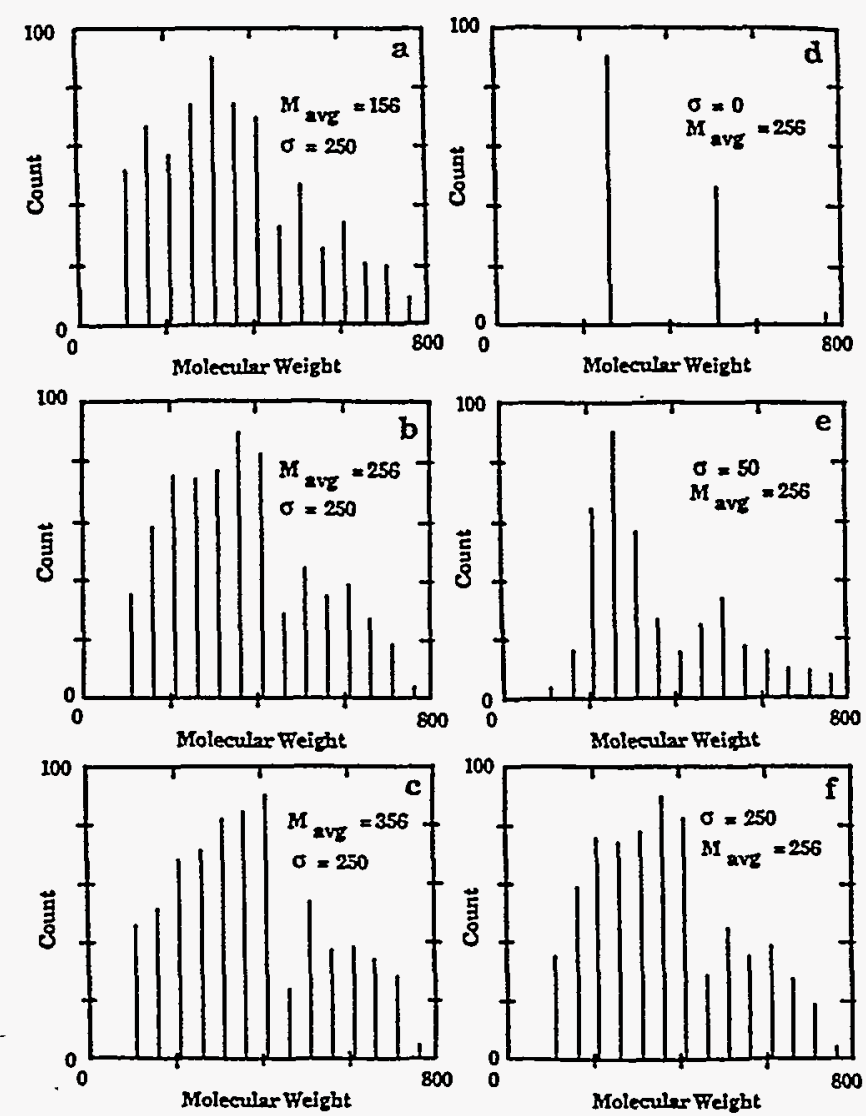

Figure 17. (a-c) Effect of $M_{\mathrm{av}}$ on the shape of the tar spectrum and $(d-f)$ effect of $\sigma$ on the shape of the tar spectrum. The heating rate is $0.05^{\circ} \mathrm{C} / 8$ to $450^{\circ} \mathrm{C}, P=0.00267 \mathrm{~atm}$, and $\Delta P=0 \mathrm{~atm}$.

$m\left(\mathrm{CH}_{4}\right)$ are considered in Figure 15d. These have a major effect on the yields. Increasing $m\left(\mathrm{CO}_{2}\right)$ from 1 to 10 reduces the extract and volatile yields while reducing $m\left(\mathrm{CH}_{4}\right)$ from 1 to 0 prevents the repolymerization of the extract.

(e) Variations in $M_{c}$. Variations in the $M_{c}$ values were made. These chiefly affect the extract yield, requiring an adjustment in $l$. They have little effect on the subsequent cross-linking in the coal. The reason for this can be seen in Table I. The initial value of $M_{c}$ consistent with the literature required only 0.09 and 0.18 cross-link/monomer for the bituminous coal and lignite, respectively. The total number of cross-links added during pyrolysis are 0.49 and 0.89 , respectively. The added number of cross-links is thus much larger than that in the raw coal and, consequently, dominates the char's behavior.

(f) Variations in $M_{\text {av }}$ and $\sigma$. Figure $17 \mathrm{a}-\mathrm{c}$ illustrates the effects of variations in $M_{\mathrm{av}}$. Varying $M_{\mathrm{av}}$ changes the shape of the tar spectrum, but not drastically. The shape is still dominated by the transport properties (e.g., see Figure 16). The effect on the tar yield is also modest, giving values of $45 \%, 44 \%$, and $42 \%$ for $M_{\mathrm{av}}$ values of 156 , 256 , and 356 , respectively.

A similar lack of sensitivity of the molecular weight distribution to $M_{\mathrm{av}}$ was exhibited for the lignite for both high-heating-rate $\left(\sim 20000^{\circ} \mathrm{C} / \mathrm{s}\right)$ and low-heating-rate $\left(0.05^{\circ} \mathrm{C} / \mathrm{s}\right)$ cases (not shown).

The effect of variations in $\sigma$ is illustrated in Figure 17d-f. $\sigma=250$ fills in the spectrum in a more realistic fashion and is more aesthetically pleasing than the two smaller values of $\sigma$. The effect on the total tar yield is minor with yields of $41 \%, 46 \%$, and $45 \%$ for $\sigma=0,50$, and 2:(1), respectively.

(g) Variations in $W_{\mathrm{N}}$. This parameter, which is taken from the FG model, controls the split between tar, char, and gas. 
Table IV. Summary of Sensitivity Analysis ${ }^{\circ}$

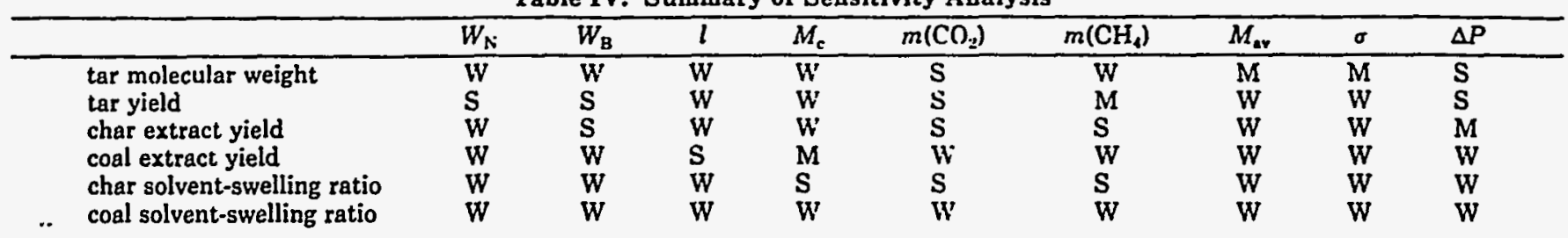

-Key: $W=$ weak or none; $M=$ moderate; $S=$ strong.

(h) Vaporization Law. The results are sensitive to the choice of the tar vapor pressure correlation. Higher vapor pressures result in faster tar evolution and higher yields as discussed in reference to Figure 11.

A summary of the sensitivity analysis is presented in Table IV. The concentration of labile bridges $W_{B}$ and the $\mathrm{CO}_{2}$ cross-linking parameter $m\left(\mathrm{CO}_{2}\right)$ are the most important parameters in determining yields.

\section{Conclusions}

A general FG-DVC model for coal devolatilization, which combines a functional group model for gas evolution and a statistical model for tar formation, has been presented. The tar formation model includes depolymerization, cross-linking, external transport and internal transport. The cross-linking is related to the evolutions of $\mathrm{CO}_{2}$ and $\mathrm{CH}_{4}$, with one cross-link formed per molecule evolved. The predictions of the tar formation model are made by using Monte Carlo calculation methods. Predictions take between $10 \mathrm{~s}$ and $10 \mathrm{~min}$ (depending on coal rank, experimental conditions, and accuracy required) on a Sun $3 / 260$ computer.

The FG-DVC model predictions compare favorably with a variety of data for the devolatilization of Pittsburgh Seam coal and North Dakota (Beulah) lignite, including volatile yields, extract yields, cross-link densities, and tar molecular weight distributions. The variations with pressure, devolatilization temperature, rank, and heating rate were accurately predicted. Comparison of the model with several sets of data employing alternative assumptions on transport suggests assuming that the particle is well mixed (i.e. the surface concentration of tar molecules is the same as the bulk) overpredicts the transport rate. For 50- $\mu \mathrm{m}$ particles, assuming that the internal-transport limitation dominates (i.e. neglecting the external transport) provides a good fit to the data. This is consistent with assuming (a) that the internal- and external-transport mechanisms act in series or (b) that they act in parallel but liquid-phase diffusion of $\operatorname{tar}$ molecules to the surface is very small and so the external transport term can be neglected.

The rank dependence of tar formation, extract yields, cross-linking, and viscosity appears to be explained by the rank dependence of $\mathrm{CO}_{2}$ yields. The high $\mathrm{CO}_{2}$ yields in low-rank coals produce rapid cross-linking at low temperatures and hence low tar yields, low extract yields, loss of solvent-swelling properties, and high viscosities. The relative importance of cross-linking compared to bond breaking is, however, sensitive to heating rate, and this effect is predicted by the FG-DVC model. The predicted cross-linking associated with methane evolution appears to match the observed cross-linking in high-rank coals (which evolve little $\mathrm{CO}_{2}$ ).

The model has eight coal structure parameters that must be determined for each coal from selected laboratory experiments. Once determined, these remain fixed for all experiments. The model also contains one adjustable parameter, $\Delta P$, the internal pressure difference that drives the volatiles out of the particle. A sensitivity analysis shows that the volatile yield is most sensitive to the fraction of labile bridges, $W_{B}$, the cross-linking parameters $m\left(\mathrm{CO}_{2}\right)$ and $m\left(\mathrm{CH}_{4}\right)$, and, in some cases (low-rank coals, low pressure), to $\triangle P$. The monomer molecular weight distribution parameters, $M_{\mathrm{sv}}$ and $\sigma$, have only a weak effect on yields and tar molecular weight distributions. The initial molecular weight between cross-links, $M_{c}$, and the initial oligomer length, $l$, affect the coal's solvent-swelling ratio and extract yield but have little effect on the subsequent pyrolysis behavior.

The model currently has several deficiencies. There is no model for estimating liquid-phase diffusion of tar molecules, which may be important for very small particles. The calculation of the average molecular weight between cross-links neglects the effect of labile bridge rupture. The assumption that all the donatable hydrogen is in bridges may be restrictive for some high hydrogen coals. The model presented here has neglected polymethylene in coal and the effect of other types of weak bonds besides ethylene bridges. There are some discrepancies between the predictions and reported temperatures of pyrolysis experiments. It is unclear at this time whether this is due to errors in the reported temperatures or in the transport predictions. Many of these deficiencies require only minor modifications to the model and are currently being addressed.

Acknowledgment. This work was supported under DOE Contracts DE-AC21-85MC22050, DE-AC2184MC21004, DW-AC21-86MC23075, and DE-FG2285PC80910. We express our thanks to Professor Eric Suuberg for many helpful discussions on transport properties and to Dr. Zhen Zhong Yu for assistance with the model calculations. 


\section{APPENDIX G}

A CHARACTERIZATION METHOD AND MODEL FOR PREDICTING COAL CONVERSION BEHAVIOUR 


\title{
A characterization method and model for predicting coal conversion behaviour
}

\author{
Peter R. Solomon, David G. Hamblen, Michael A. Serio, Zhen-Zhong Yu \\ and Sylvie Charpenay \\ Advanced Fuel Research Inc., 87 Church Street, East Hartford, CT 06108, USA \\ (Received 15 April 1991: revised 1 April 1992)
}

\begin{abstract}
This paper considers the development of a predictive macromolecular network decomposition model for coal conversion which is based on a variety of modern analytical techniques for coal characterization. Six concepts which are the foundation of the functional group-depolymerization-vaporization-cross-linking (FG-DVC) model are considered: (1) The decomposition of functional group sources in the coal yields the light gas species in thermal decomposition. The amount and evolution kinetics can be measured by t.g.-FT-i.r., the functional group changes by FT-i.r. and n.m.r. (2) The decomposition of a macromolecular network yields tar and metaplast. The amount and kinetics of the tar evolution can be measured by t.g.-FT-i.r. and the molecular weight by f.i.m.s. The kinetics of metaplast formation and destruction can he determined by solvent extraction, by Gieseler plastometer measurements and by proton magnetic resonance thermal analysis (p.m.r.t.a.). (3) The molecular weight distribution of the metaplast depends on the network coordination number (average number of attachments on aromatic ring clusters). The coordination number can be determined by solvent swelling and n.m.r. (4) The network decomposition is controlled by bridge breaking. The number of bridges broken is limited by the available donatable hydrogen. (5) The network solidification is controlled by cross-linking. The changing cross-link density can be measurcd by solvent swelling and n.m.r. Cruss-linking appears to occur with evolution of both $\mathrm{CO}_{2}$ (before bridge breaking) and $\mathrm{CH}_{4}$ (after bridge breaking). Thus low-rank coals (which evolve much $\mathrm{CO}_{2}$ ) cross-link before bridge breaking and are thus thermosetting. High-volatile bituminous coals (which form little $\mathrm{CO}_{2}$ ) undergo significant bridge breaking before cross-linking and become highly fiuid. Weathering. which increases the $\mathrm{CO}_{2}$ yield, causes increased cross-linking and lowers fluidity. (6) The evolution of tar is controlled by mass transport in which the tar molecules evaporate into the light gas or tar species and are carried out of the coal at rates proportional to their vapour pressure and the volume of light species. High pressures reduce the volume of light species and hence reduce the yield of heavy molecules with low vapour pressures. These changes can be studied with fi.m.s. The paper describes how the coal kinetic and composition parameters are obtained by t.g.-FT-i.r., solvent swelling, solvent extraction and Gieseler plastometer data. The model is compared with a variety of experimental data in which heating rate, temperature and pressure are all varied. There is good agreement with theory for most of the data available from the authors laboratory and in the literature.
\end{abstract}

(Keywords: characterization; coal; conversion)

The question addressed by this paper is : : an coal science be predictive? More specifically, is it possible to accurately predict the way a coal behaves in a coal conversion process, given coal characteristics which can be measured in the laboratory. For example, Figure $I$ illustrates the behaviour of coal in combustion. The left-hand side shows a picture of a coal burning in a reactor after injection into the centre of a hot air stream. The processes that occur are illustrated on the right-hand side: the heating of the coal, softening, devolatilization, swelling. the ignition of the volatiles, the formation of soot, the burning of the volatiles, the ignition of the char, the combustion of the char, and finally the fragmentation of the char which determines the ultimate distribution of the ash particles. Can one qualitatively predict pyrolysis yields, swelling, soot formation, char reactivity, etc.?

As a second example. consider coal in a liquefaction process. The important step is the fragmentation of the coal macromolecule into small pieces. As shown in Figure
2 , that fragmentation takes place very quickly for a bituminous coal. The coal dissolves into the solvent, and the subsequent reactions between the solvent and the coal are all liquid-liquid phase interactions, which can occur very rapidly. In a lignitc, this fragmentation process is prevented by low-temperature cross-linking. Thus for a lignite there is no quick solubilization of the coal, and most of the reaction takes place between the solvent and a solid cross-linked residue. Can one predict macromolecular fragmentation and cross-linking?

The research conducted during the last 10 years suggests that many of the steps discussed above can be accurately predicted. The concept used in the athors' laboratory is to employ a set of characterization procedures to determine the kinetic and composition parameters for a general predictive model. Five experiments define these parameters. The most important is t.g.-FT-i.r., thermogravimetry (t.g.) with analysis of the evolved product by Fourier transform infrared (FT i.r.) spectroscop! " This allows determination of 


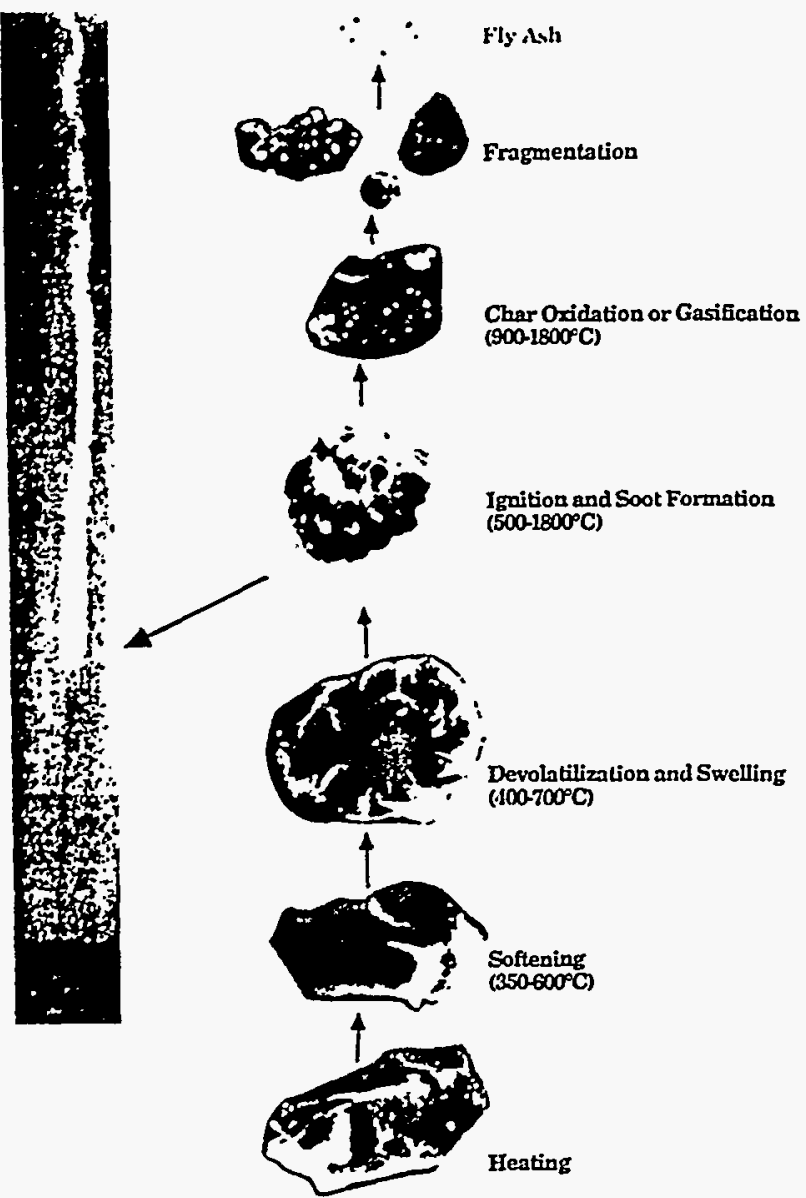

Figure 1 Processes in coal combustion

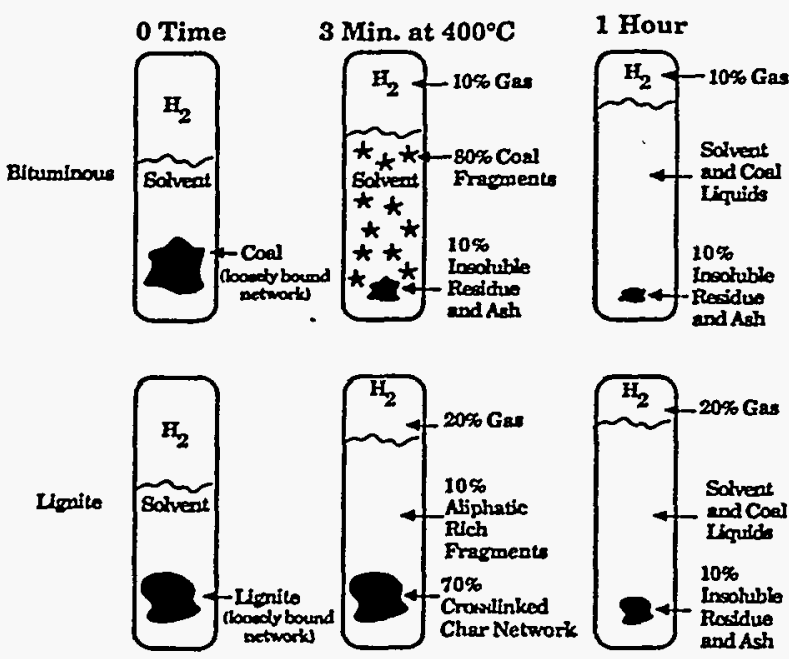

Figure 2 Processes in coal liquefaction

the amount of volatiles, their composition, the kinetics of their evolution, the reactivity of the char, and also the moisture and ash contents of the coal. Also measured are the solvent swelling ratio ${ }^{2,3}$, the extract yield, and the fluidity in a Gieseler plastometer ${ }^{4}$, to which are added nuclear magnetic resonance (n.m.r. $)^{5}$ and field ionization mass spectrometry (f.i.m.s.) data ${ }^{6}$. These experiments determine the matromolecular network parameters for the model.

The model is called FG-DVC 7.8 . The letters FG stand for functional group, and DVC for depolymerization, vaporization and cross-linking. The FG model considers certain functional groups in the coal which lead to the formation of the light gas species ${ }^{9-12}$. At the same time, the DVC model describes the overall depolymerization of the macromolecular network which combines bridge breaking and cross-linking to produce fraginents of the coal macromolecule ${ }^{13-15}$. These fragments are then subjected to transport behaviour, specifically the vaporization of the lightest fragments to form tar. The fragmentation process provides a second mechanism for the removal of functional groups from the coal. The model. whose parameters are determined in the laboratory at moderate temperatures and atmospheric pressure, can then be used to extrapolate from the laboratory conditions to predict pyrolysis and combustion in high-temperature reactions, or liquefaction at high pressure. Recently, extrapolation of the kinetics and reactions to low-temperature geological transformations in coal beds has been explored ${ }^{16}$.

The model for coal thermal decomposition has six basic concepts:

- functional groups (decompose to produce light gases)

- macromolecular network (decomposes to produce tar and metaplast )

- network coordination number (determines fragment molecular weight distribution)

- bridge breaking (limited by hydrogen availability)

- cross-linking (related to gas evolution)

- mass transport of tar (evaporation of network fragments into light gases and tars).

The first concept is that light gases are formed by the decomposition of certain functional groups in the coal. For example, methyl groups can detach and pick up a hydrogen to form methane, carboxyl groups can lead to the formation of $\mathrm{CO}_{2}$, etc. ${ }^{9-12.17-20}$. The second concept is that coal consists of a macromolecular network ${ }^{2.3 .7 .13-15.21-36}$. This network is made up of fused aromatic ring clusters ( which are described by their molecular weight ) linked by bridges, some of which are relatively weak. There are some unattached parts of the network which can be extracted. Sometimes there is also a second polymethylene component ${ }^{37-41}$. When heated, this network decomposes to produce smaller fragments. The lightest of the fragments evaporate to produce $\operatorname{tar}^{7.42}$, and the heavier fragments form the metaplast. These heavier molecules are the primary liquid fragments in liquefaction or the fragments that make coal fluid ${ }^{8.43}$.

The third concept is that one of the most important propertics of the network is its coordination number. The coordination number describes the geometry of the network by specifying how many possible attachments there are per aromatic ring cluster ${ }^{31-36}$. For example, a linear polymer chain has a coordination number of 2 , because each fused aromatic ring has two possible attachments to link it in the chain. On the other hand, a 'fishnet' has a coordination number of 4 , because there are four possible attachments at each ring cluster. The coordination number controls the molecular weight distribution of the network fragments at a given extent of decomposition. The extent of decomposition is specified by the probability that the possible attachments 
exist. For example, for $20 \%$ of broken bridges, a linear chain is totally fragmented, while a 'fishnet' will have some holes but is almost totally' connected. In describing the network, a cross-link site is defined to occur at a ring iuster where there are more than two possible ittachments. The coordination number is thus related to the density of cross-link sites. With no possible cross-links between chains, the coordination number is 2 . With increasing density of cross-link sites, the coordination number increases.

The other important property of the network is the fraction of possible attachments that actually exist. During thermal decomposition, this fraction is determined by the rates of bridge breaking and cross-linking 7.15.44-47. The factors which control how many of the bridges can break are the rate constant and the amount of hydrogen that can be donated from the coal to stabilize the free radicals that form when the bridges break ${ }^{10}$.

A competitive process with the bridge breaking is the retrogressive process of cross-linking. Cross-linking reactions appear to be related to the evolution of certain gases $^{7,15,44,47}$. Specifically, for low-rank coals, crosslinking at low temperature (before bridge breaking) seems to be related to the evolution of carbon dioxide (or possibly water). For coals of all ranks, a higher-temperature cross-linking event (following bridge breaking) seems to be related to the evolution of methane. At high temperatures, the evolution of hydrogen is also related to cross-linking in the form of aromatic ring condensation reactions.

The final concept is that the tar evolution is controlled by mass transport. Bridge breaking and cross-linking produce fragments with a molecular weight distribution. The lightest fragments can leave the coal melt by evaporation into the light gas and tar species ${ }^{7.42}$. The heavier fragments remain, forming the metaplast, which controls the coal's fluidity.

The remainder of the paper describes how these concepts' are incorporated into a practical predictive model. The next section considers the FG-DVC model in detail. It discusses each of the six concepts and the evidence for each assumption. The following two sections consider the experiments used to obtain the model parameters and compare predictions of the model with a variety of experimental data. A summary and conclusions complete the paper.

\section{COAL PYROLYSIS MODEL}

\section{Functional group decomposition model}

Figure 3 illustrates the phenomena in coal thermal decomposition considered in the functional group model. The diagrams are not meant to describe the exact structure of coal or the exact chemistry of pyrolysis, but rather to illustrate the kinds of structure considered and the classes of phenomena that can occur. The important processes are the decomposition or detachment of the individual functional groups to form the light gases and the competitive decomposition of the macromolecular network to form fragments, the lightest of which can evaporate as tar.

Figure $3 a$ shows a representative part of a Pittsburgh Seam coal macromolecule. The structure is based on measurements of the aromatic ring cluster size, the functional group composition and the elemental composition ${ }^{48}$. The molcculc consists of several fused

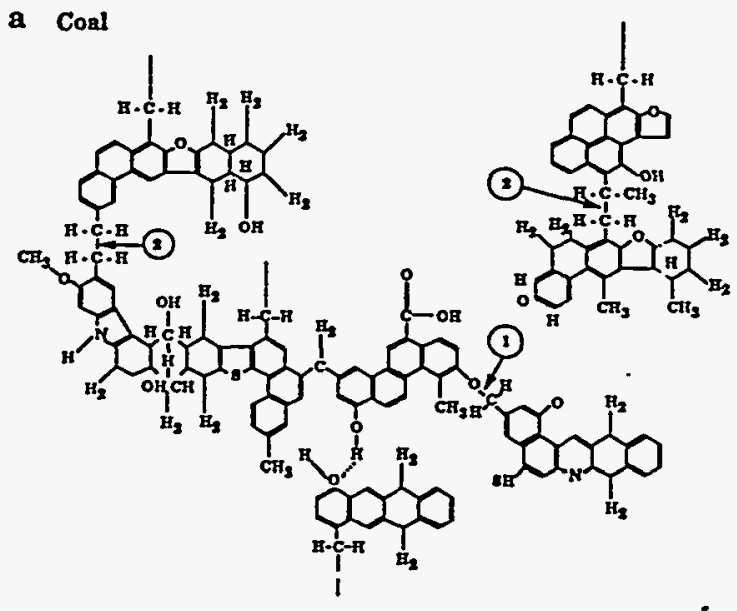

b Primary Pyrolyais

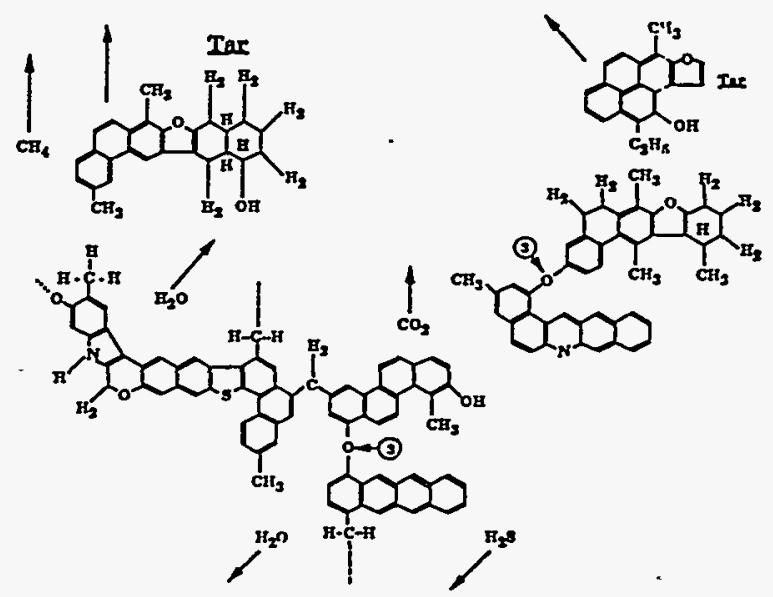

Figure 3 Hypothetical mondel of Pittsburgh seam coal: (a) original coal; (b) during pyrolysis. From ref. 12

aromatic ring clusters linked by labile bridges. The ring clusters have various functional groups attached to them. When the coal is heated, two things happen to the functional groups. The first is that certain functional groups can detach to form light gases. The second is that fragmentation of the network, and removal of light fragments as tar, can cause the same type of functional group to be removed as part of the tar. So there are two parallel processes for the volatilization of the functional groups.

The way the coal behaves during pyrolysis is illustrated in Figure $3 b$. The carbon-carbon aliphatic bridge in the upper left-hand corner of the molecule (labelled 2 in Figure $3 a$ ) has broken and picked up hydrogen to form two methyl groups. The hydrogen is assumed to come from hydroaromatic or aliphatic portions of the coal. For example, the hydroaromatic ring in the lower right-hand portion of the coal molecule of Figure $3 a$ has lost two hydrogens to become an aromatic ring in Figure $3 b$. This process creates a fragment which is light enough to evolve as tar. There is also independent detachment of functional groups to form light gases. The carboxyl group in the middle of Figure $3 a$ is shown as carbon dioxide evolving in Figure $3 b$. Methyl groups have detached to form methane, there has been a condensation of hydroxyl groups to form water and an ether link (labelied 3). mercaptans detach to form $\mathrm{H}_{2} \mathrm{~S}$, etc. 
The evidence for this description is as follows. (1) For bituminous coals and low-rank coals healed rapidly, the tar is strikingly similar in elemental and functional group composition to the parent coal ${ }^{9.10 .48-50}$. The tar appears to consist of representative fragments of the parent coal macromolecule. In contradiction to this result, however, is the observation that tar produced from low-rank coals at low heating rates appears to be significantly different in composition from the coal $^{51}$ and is probably dominated by polymethylenes. (2) There is a correlation between the decrease in the functional group sources in the char and the evolution of specific gases $s^{9-12}$. (3) There is a systematic variation in functional group composition with rank, and this variation is correlated with the evolved gas composition.

\section{Macromoleculur ne'work decomposition model}

The concept of a macromolecular network decomposition model is illustrated in Figure 4, which recently appeared in a paper by Grant and co-workers ${ }^{36}$. The figure represents aromatic ring clusters with four possible attachments to their neighbours, arranged in a 'fishnet' type of network (a network with a coordination number of 4). Figure $4 a$ illustrates what happens when $20 \%$ of the possible attachments are broken. As can be seen, only three fragments are created, shown by the clusters with boxes around them. The breaking of $20 \%$ of the bridges produces very little fragmentation of the network. On the other hand, consider (Figure $4 b$ ) what happens when $45 \%$ of the bridges are broken. Now there is a much higher concentration of fragments and the fragments have a molecular weight distribution from monomers up to heptamers (consisting of seven fused ring clusters linked together). The lightest of these fragments - monomers, dimers, and trimers - can evaporate into the light gas species and are removed from the coal particles as tar.

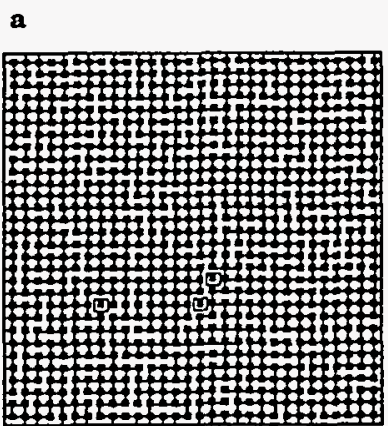

b

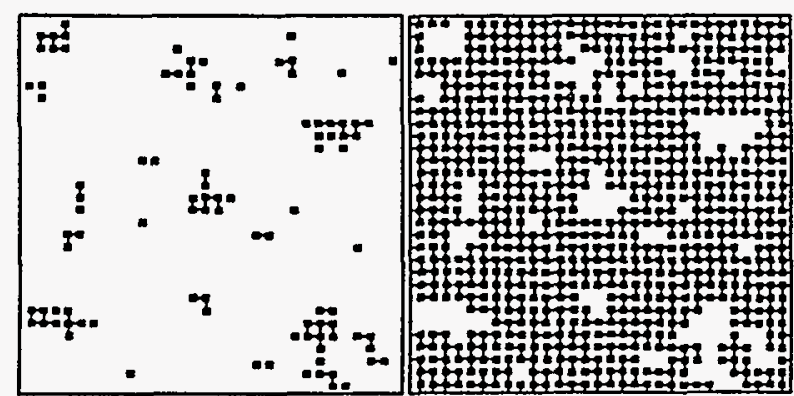

Figure 4 Macromolecular network decompusition model using Monte Carlo calculations for coordination number of $4:$ (a) $20 \%$ broken bridges; (b) $45 \%$ broken bridges (from ref. 36 )
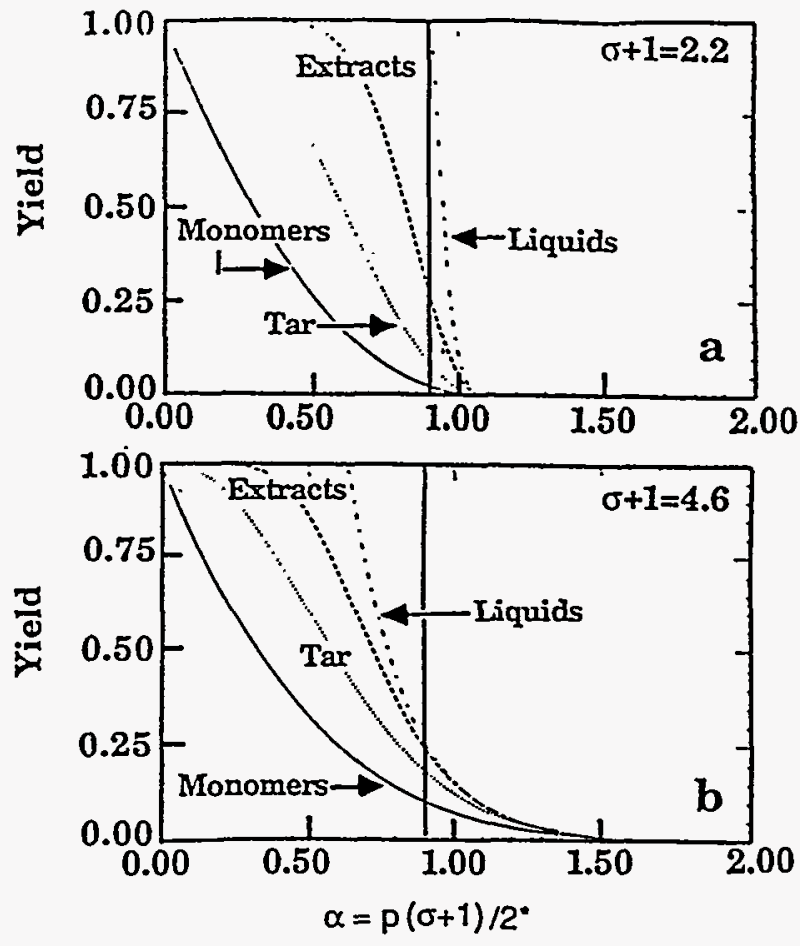

Figure 5 Percolation theory predictions for pyrolysis products for coordination numbers of 2.2 and 4.6

The heavier fragments make up the metaplast. The lightest of these can be extracted using a solvent, while others are too heavy to be extracted. The presence of a sufficiently large fraction of these fragments is what makes these materials fluid.

\section{Network courdination number}

The importance of the network coordination number is illustrated in Figures 4 and 5. In Figure $4 a$ with $20 \%$ of the bridges broken in a 'fishnet', only a small number of fragments are produced, and they are all monomers. On the other hand, if $20 \%$ of the bridges in the linear chain are broken, $100 \%$ of the material becomes fragments and there will be many dimers, trimers, etc. Thus the molecular weight distribution of the fragments depends very strongly on the coordination number.

In Figure 4, the molecular distribution was computed using Monte Carlo calculations in which a representative network is set up in computer memory and the fragment molecular weight distribution is calculated after the broken bridges are randomly distributed. Alternatively, a technique called percolation theory allows a closedform analytical solution of the molecular weight distribution as a function of the number of actual attachments per ring cluster.

Figure 5 shows percolation theory calculations for networks with two different coordination numbers: (a) 2.2 and (b) 4.6. The variable $\sigma$ is one less than the coordination number. The figure shows the calculated yield distributions of: (1) monomers; (2) up to trimers (i.e. monomer, dimer, trimer) representative of what might be evolved as tar for a ring cluster size of 300 daltons; (3) $n$-mers up to 10 , representative of extractable material; and (4) all $n$-mers, representative of liquids. These are plotted as a function of $\alpha$, which is the average number of bridges per fused aromatic ring, and is equal 
to the probability $p$ that a bridge is occupicd multiplied by the coordination number of the network divided by $2: \alpha=p(\sigma+1), 2$. As can be seen, there is a very different distribution of fragments, depending on the coordination number. For example, at $\alpha=0.9$, the network with coordination number 4.6 has most of the fragments in the tar. with only a small number of n-mers between 3 and 10 and almost no $n$-mers above 10 . On the other hand, for a network with a coordination number of 2.2 at $x=0.9$, there is a smaller number of monomers and a somewhat smaller concentration of tar, but a much higher concentration of $n$-mers up to 10 , and a $100 \%$ yield of all n-mers. In other words, for 0.9 bridges per cluster, most of the molecules have decomposed to produce fragments of one size or another.

The DVC model was originally implemented using a Monte Carlo solution method, which allows an arbitrary network geometry. Percolation theory, however, offers significant benefits in computational speed and reproducibility, at the cost of restricting the network geometries.

As will be seen below, in the Monte Carlo version of the model, the starting network is represented by linear chains of monomers (6-12 aromatic clusters) with some cross-linking which ties the chains together. Thus the starting network has a coordination number between 2 (straight chains) and $\geqslant 3$ (fully cross-linked). As pyrolysis proceeds, the linear chain bridges are broken, and cross-links are formed. Thus the coordination number, or degree of branching, increases with extent of pyrolysis. The conventional percolation theory models of coal decomposition do not model this feature. With conventional percolation theory, one can make any identification of the various chemical bonds with the percolation lattice bonds, so that the probability of a bond being occupied tracks the chemistry, but the occupied bonds must be randomly distributed within the lattice. The structure cannot be converted from 'chain-like' to 'fishnet-like'.

The DVC model predicts, and experiments confirm, that there are different kinds of bonds (bridges and cross-links), which have different coordination numbers, and independent probabilities of being broken. To take advantage of the benefits of percolation theory, percolation theory on a Bethe lattice (one with no loops) has been extended to use two independent sub-networks, as illustrated in Figure $6^{32}$. In the figure, double lines represent one of the bond types, while single lines represent the other. As can be seen by comparing Figures $6 a$ and $6 h$, this lattice has the desired feature of modelling a transition from chain-like structures (a) to fishnet structures (b). The mathematics of this ' $2-\sigma^{\prime}$ ' percolation theory follows closely that of the standard theory ${ }^{32}$. The results obtained from the ' $2-\sigma$ ' percolation theory agree well with those obtained from the original Monte Carlo calculations, as will be discussed under Comparison of theory and experiment.

\section{Bridge breaking and hydrogen utilization}

There are two questions with respect to bridge breaking: what is the bridge breaking rate, and how many bridges break? Pyrolysis rates have been reviewed by a number of authors ${ }^{10.52-54}$. One of the problems over the last two decades is the very wire variation in the reported rates for either weight loss or tar evolution in pyrolysis.
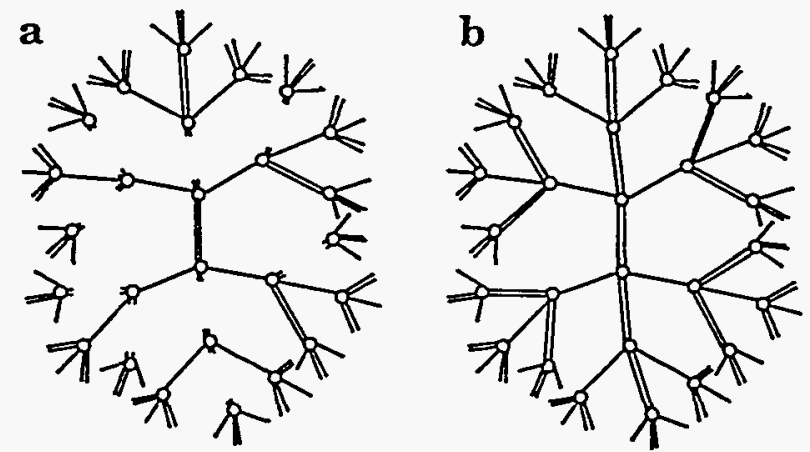

Figure 6 Bethe lattice for two- $\sigma$ model with $\sigma_{1}=\sigma_{2}=1$ (shown as single bonds): (a) with most double bonds representing the crosslinks not yet formed to represent the starting coal, the lattice is like a one- $\sigma$ model with $a=1$. linear chains: (b) fully linked case $(p=q=1)$ is like a one- $\sigma$ model with $\sigma=3$
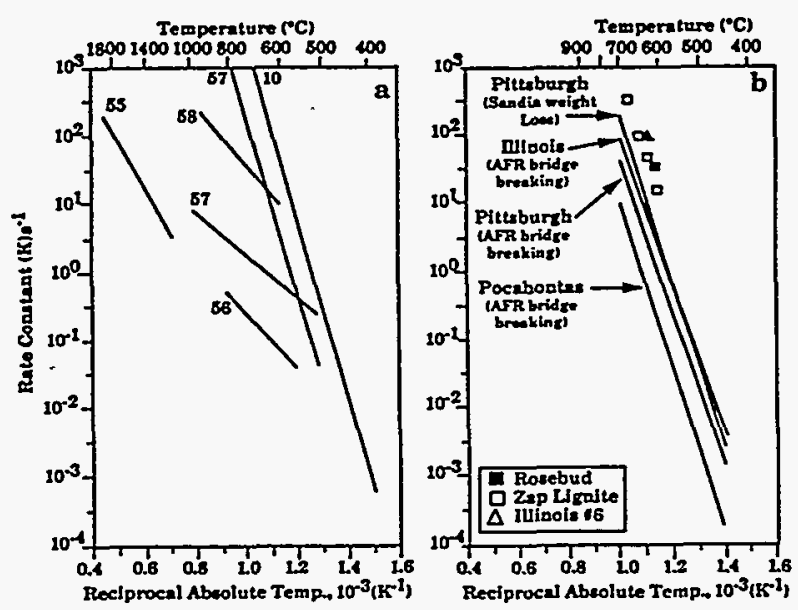

Figure 7 Kinetic rate constants for weight loss or bridge breaking: (a) extremes of published rates from experiments in which particle temperatures were not directly measured; (b) more recent rates from experiments in which particle temperatures were measured

Figure $7 a$ presents several of the extremes in rates reported before 1985 for high-heating-rate experiments ${ }^{10.55-58}$. At $800^{\circ} \mathrm{C}$ there is a variation of almost four orders of magnitude in the reported rate constants. An analysis of the data shows that this sort of variation in kinetics cannot be attributed to variations in cual type, because investigators who tested more than one coal type found that the variation in kinetic rates among coals is typically within a factor of 10 . So there has to be another explanation for such a wide variety of reported rites. The answer appears to lie in the estimation of the coal particle temperature ${ }^{53.54 .59-62}$. Almost none of the exp riments involved direct measurement of the particle temperature. For entrained-fiow reactor experiments, the temperature is usually calculated, and the calculation depends critically on the rate of mixing of the preheated gases with the coal stream. An error by a factor of 2 in the heating rate can lead to errors of hundreds of degrees celsius in the estimate of particle temperature during pyrolysis. For heated-grid experiments, temperature is measured with a thermocouple and it is inferred that the thermocouple temperature is the same as the coal particle temperature. Recent reviews of experiments for Pittsburgh Seam coal heated at $1000 \mathrm{~K} \mathrm{~s}^{-1}$ show a wide variation 
in pyrolysis temperatures. suggesting that this is not a good assumption ${ }^{100.63}$.

Since 1985, several experiments have been performed in which coal particle temperatures were measured during pyrolysis ${ }^{12,53,61,62,64}$. Careful experiments have also been performed at several low heating rates where the thermocouple temperature is a good measure of the coal particle temperature ${ }^{65,66}$. As can be seen from Figure $7 b$, the data are much more tightly grouped. There is a systematic variation with coal rank and the kinetic rate constants appear to have an activation energy between 190 and $230 \mathrm{~kJ} \mathrm{~mol}^{-1}$. This is the magnitude expected for the kind of labile bridges depicted in Figure $3^{14.67}$.

Besides the kinetic rate for bridge breaking, one needs to know the number of bridges that can be broken. The number depends on the amount of hydrogen that is available to stabilize the free radicals formed when bridges break. To understand why the hydrogen utilization controls the amount of tar and its hydrogen concentration, consider the following example. Assume that every time a bridge is broken, two hydrogens are used to stabilize the free radicals, and that two radicals are assumed to be stabilized per tar molecule. If the tar is made up of large fragments, the utilization of hydrogen per unit weight of tar is very efficient. On the other hand, if the tar consists of small molecules, the utilization of hydrogen is much less efficient. A sample calculation was made for $0.3 \mathrm{wt} \%$ hydrogen in the coal available for donation to the tar. For an average molecular weight of $100 \mathrm{u}$ (atomic mass units, or daltons), $15 \mathrm{wt} \%$ tar is produced. The amount of additional hydrogen in the tar is $2 \mathrm{wt} \%$. On the other hand, at an average molecular weight of $300 u$ the yield is up to $45 \mathrm{wt} \%$, while the additional hydrogen per unit mass is only $0.7 \mathrm{wt} \%$. The average molecular weight of the tar is affected by cross-linking, pressure, heating rate and bed geometry.

\section{Cross-linking}

During pyrolysis, another important process occurs besides bridge breaking. This is cross-linking, in which new bonds are formed between the fused aromatic ring clusters. One way of measuring the cross-link density is through solvent swelling ${ }^{2,3}$, in which a solvent (e.g. pyridine) is used to swell the char or coal ${ }^{7,15,44-47}$. To understand how solvent swelling indicates the cross-link density, consider the analogy of an air mattress, which is stitched in long rows along its length. When the mattress is inflated there are several connected small tubes instead of one big round tube. The small tubes have a smaller volume than one large tube and the volume can be used to infer the limiting circumference of the tubes. In a similar manner, the addition of the solvent to a coal indicates the circumference of linked molecules that make a loop to limit the swelling. Since it is cross-links (more than two attachments per cluster) that allow loops to be formed, the amount of swelling indicates the average molecular weight between cross-links.

Figure 8 shows the solvent swelling ratio as a function of the char temperature for coals of several ranks. Chars were produced by heating to the indicated temperatures at $30 \mathrm{~K} \mathrm{~min}^{-1}$ in an inert atmosphere and then cooling. These chars were subjected to solvent swelling experiments to determine the ratio of the volume of swollen material to the unswollen volume. Coals have solvent swelling ratios as high as 2.7. As char is formed,

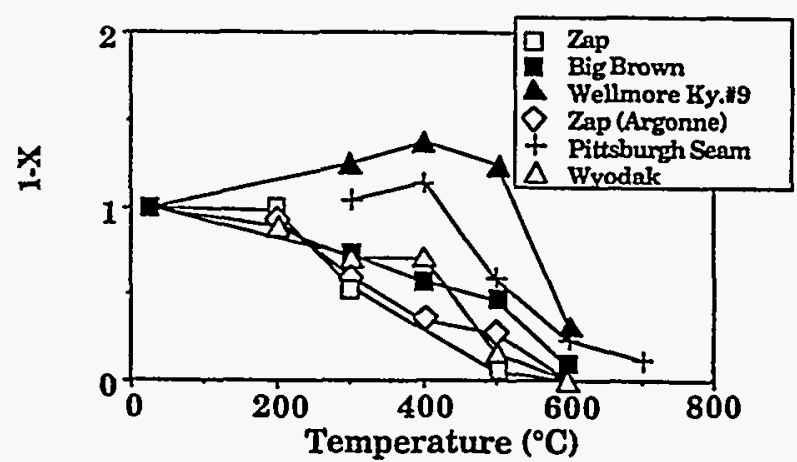

Figure 8 Solvent swelling ratios for coals of different rank at a series of final pyrolysis temperatures; heating rate $30 \mathrm{~K} \mathrm{~min}^{-1}$. From ref. 47

new cross-links reduce the swelling ratio to unity. The solvent swelling ratio in Figure 8 is normalized: the parameter $X$ is the difference in solvent swelling ratio between the coal and the char, divided by the maximum difference that can be achieved. This normalization allows different coals with different initial solvent swelling ratios to be compared conveniently. If $X=0$, the material swells the same as coal; if $X=1$, it is fully cross-linked.

There is a wide variation in behaviour, depending on rank. This rank-dependence of the cross-linking behaviour was first noted by Suuberg et al. ${ }^{44}$, who tested a lignite and a bituminous coal and found the same sort of difference as shown here. For the lowest-rank coal, Zap lignite, at temperatures as low as $200^{\circ} \mathrm{C}$ the char starts to undergo cross-linking, losing most of its solvent swelling properties by $400^{\circ} \mathrm{C}$. However, it is between 400 and $500^{\circ} \mathrm{C}$ that most of the weight loss occurs. Thus for a low-rank coal, the cross-linking occurs well in advance of bridge breaking.

For higher-rank coals, the cross-linking event is delayed relative to bridge breaking. For a highly softening bituminous coal (e.g. Pittsburgh Seam, or Kentucky No. 9) the materiai swells even more as it is heated into the region of pyrolysis, and loses its solvent swelling properties only after most of the weight loss has occurred. There is thus a very strong rank-dependence of cross-linking behaviour. Low-rank coals cross-link early, before bridge breaking, while high-rank coals undergo cross-linking after most of the bridge breaking has taken place.

The results of solvent swelling experiments are not unambiguous, because the solvent swelling ratio depends on two things: the cross-link density and the solvent interaction parameter. This parameter can change with the functional group composition of the coal. Since the functional group composition will change as the coal pyrolyses, the change in solvent swelling ratio could be due to the change in functional group composition, not to cross-linking. However, estimation of the likely change in solvent swelling ratio with functional group composition indicates that the drastic change from 2.7 typical of coal to 1 for char is not likely to be due to the small changes in functional group composition during low-temperature pyrolysis ${ }^{47}$.

Another way of investigating the cross-link density is by nuclear magnetic resonance (n.m.r.). Results of work in collaboration with the University of $\mathrm{Utah}^{68}$ are shown 
in Figure 9. The n.m.r. experiments used crosspolarization with magic angle spinning and dipolar dephasing (CP-MAS-DD) $)^{5}$. Dipolar dephasing allows determination of the functional-group form of the carbon atoms studied. When all the different kin.ss of bonds are considered, it is possible to determine an average molecular weight for the ring clusters and also the average number of attachments per ring cluster. In Figure 9, the average number of attachments is compared with the solvent swelling data for Pittsburgh Seam bituminous coal and a North Dakota lignite. The average number of attachments determined by n.m.r. is normalized to determine an index that can be compared with $1-X$. Figure $9 a$ presents the results for the lignite. Although there are differences in cross-link density determined by n.m.r. and by solvent swelling ratio, both techniques show that the material starts to cross-link at a reasonably low temperature, $573-673 \mathrm{~K}$, and is almost completely cross-linked by $773 \mathrm{~K}$. In contrast, for the Pittsburgh Seam coal shown in Figure $9 b$, the char has not undergone any cross-linking by $673 \mathrm{~K}$ and is not fully cross-linked even at $873 \mathrm{~K}$.

To develop an understanding of the chemistry of cross-linking, an attempt was made to determine whether the addition of cross-links could be correlated with any other observation, specifically the evolution of gases. In
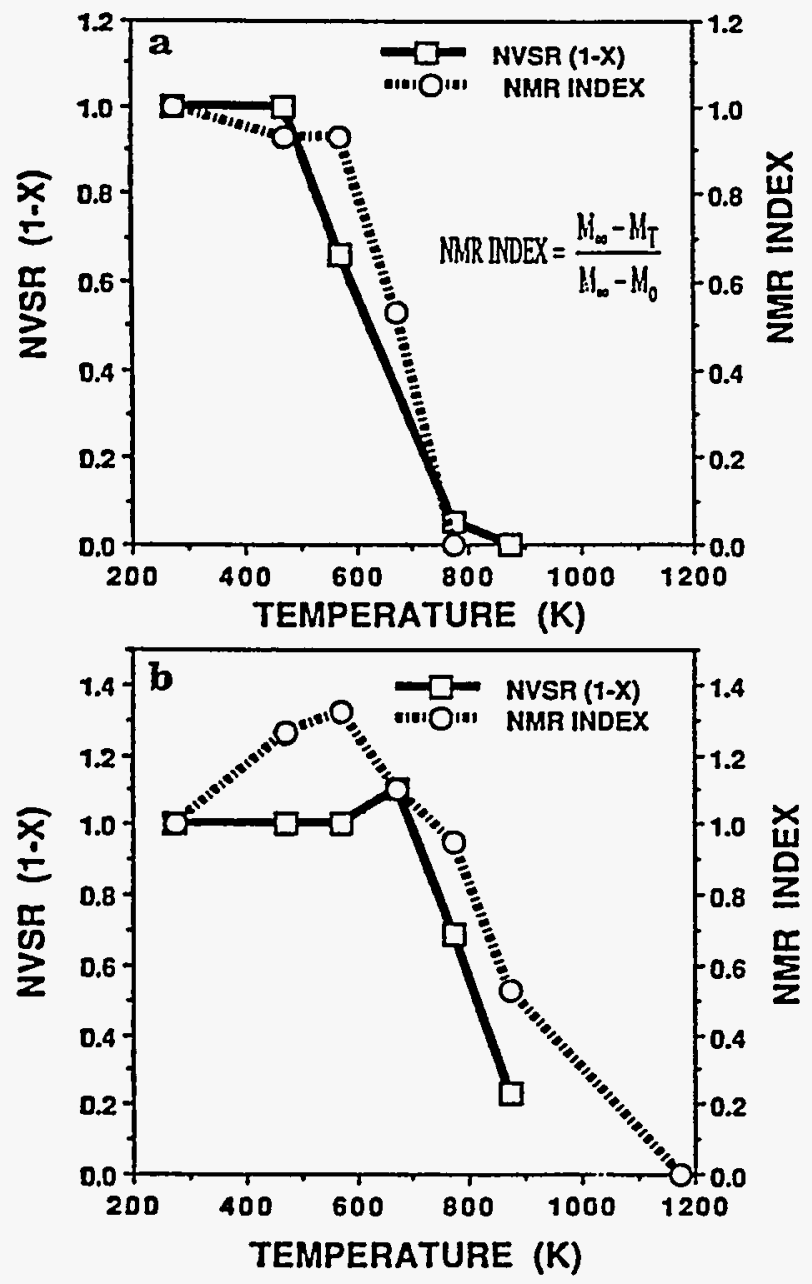

Figure 9 Comparison of n.m.r. and swelling data for (a) Zap lignite. (b) Pittshurgh No. 8 coal
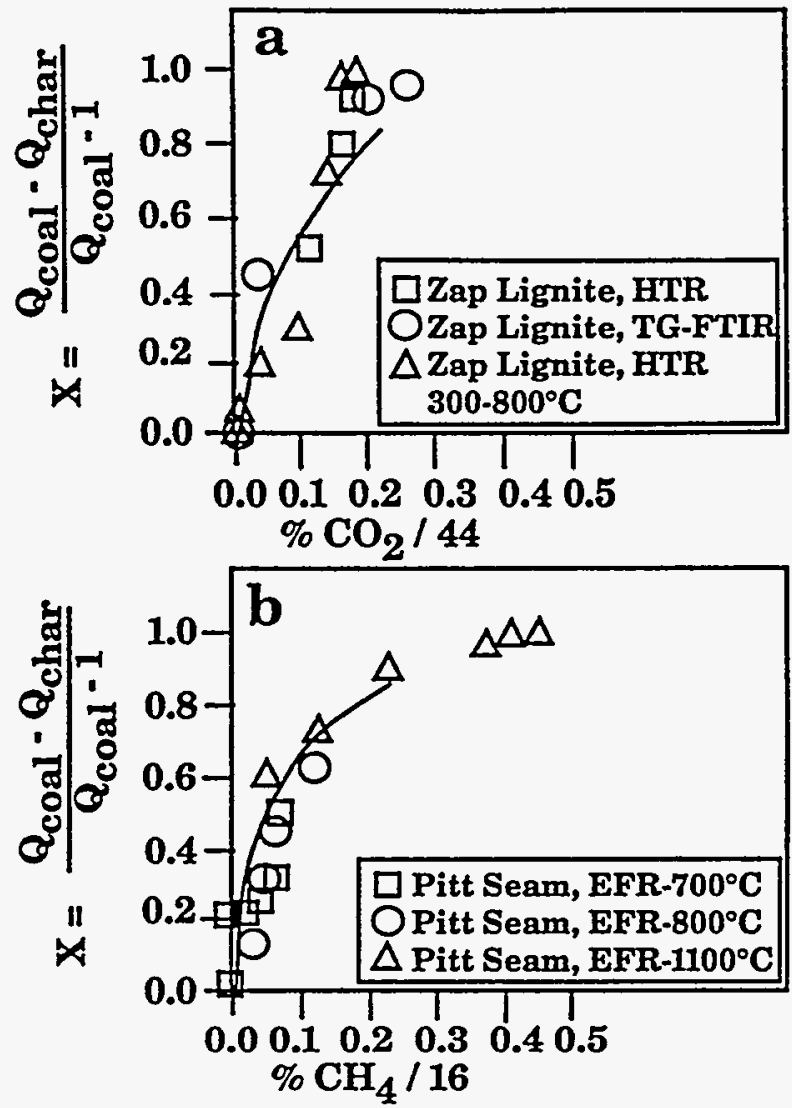

Figure 10 Measured and calculated normalized volumetric swelling ratios for coal and chars: (a) Zap lignite vs. $\mathrm{CO}_{2}$ yield; (b) Pittsburgh Seam bituminous coal vs. $\mathrm{CH}_{\alpha}$ yield. Chars prepared in an entrained-flow reactor (EFR). a heated-tube reactor (HTR), and a thermogravimetric analyser with evolved product analysis by FT-i.r. (t.g.-FT-i.r.) as described in ref. 7

their initial work, Suuberg et al. ${ }^{44}$ noted that the one gas species which correlated with the early creation of cross-linking in lignite was $\mathrm{CO}_{2}$. Figure 10 shows the results obtained in the authors' laboratory. Figure 10a presents the parameter $X$ as a function of $\mathrm{CO}_{2}$ yield divided by 44 so that it is on a molecular basis. For a wide variety of experiments (some at high and some at low heating rates) there is a very reasonable correlation between the loss of swelling and the appearance of $\mathrm{CO}_{2}$ in pyrolysis. For all the low-rank coals studied, there appears to be a good correlation between the appearance of cross-links and the appearance of $\mathrm{CO}_{2}$. The line shown in Figure 10a is from the FG-DVC model, where one cross-link is assumed for each molecule of $\mathrm{CO}_{2}$ evolved.

For a higher-rank coal, which does not produce significant yields of $\mathrm{CO}_{2}$, a different correlation is observed. Figure $10 b$ compares the normalized solvent swelling ratio for a Pittsburgh Seam coal with the evolution of methane divided by 16. There is a good correlation between these two parameters for chars produced at different temperatures at high heating rates. The line in Figure 10b is from the FG-DVC model, where it is assumed that one cross-link is formed for each methane molecule evolved.

The correlation of the loss of swelling with other parameters was examined. but the correlation for $\mathrm{CO}_{2}$ and methane was found to be the best. There was a 
correlation for low-rank coals between the furmation of cross-links and walter cvolution. hut not yuitc as good as for $\mathrm{CO}_{2}$. There was no good correlation for high-rank coals between cross-linking and tar evolution. Crosslinking correlated with hydrogen evolution is also expected at higher temperatures, as ring condensation by hydrogen elimination occurs. However, this phenomenon has not been studied.

Three sets of results illustrating the phenomena of bridge breaking and cross-linking are presented in Figure 11. Figure $11 a$ shows the proton magnetic resonance thermal analysis (p.m.r.t.a.) results obtained at CSIRO ${ }^{69-71}$. This technique, which measures the relaxation time for protons, can distinguish between protons attached to mobile molecules (which are free to rotate) and those attached to a rigid lattice. The higher the concentration of mobile protons. the lower the value of the parameter $M_{2 \mathrm{~T}}$. The data, obtained at $4 \mathrm{~K} \mathrm{~s}^{-1}$, were provided by Dr R. Sakurovs (personal communication). The decrease in $M_{2 T}$ at low temperatures appears to be associated with melting, the sharp drop above $400^{\circ} \mathrm{C}$ is due to bridge breaking, and the sharp increase above $440^{\circ} \mathrm{C}$ is due to cross-linking.

Figure $11 b$ shows fiuidity data measured with a Gieseler plastometer for the same coal at a similar heating rate $\left(3 \mathrm{~K} \mathrm{~s}^{-1}\right)^{4}$. While the fluidity below $400^{\circ} \mathrm{C}$ is probably due to melting, above $400^{\circ} \mathrm{C}$ bridge breaking becomes important, and above $440^{\circ} \mathrm{C}$ cross-linking resolidifies the network.

Figure $11 \mathrm{C}$ presents data ${ }^{72}$ on the extract yield from chars produced at a high heating rate, $640 \mathrm{~K} \mathrm{~s}^{-1}$. The maximum extract yield occurs at a much higher temperature than for the other two experiments, owing to the high heating rate. The increase in extract yield is due to bridge breaking, and the decrease to cross-linking.

\section{Transport}

The above discussion shows how bridge breaking and cross-linking can fragment the macromolecular network and allow small pieces to be formed. The evolution of tar is controlled by the formation of these small fragments and their transport out of the metaplast. In the FG-DVC model, a very simple transport process has been assumed. The assumption is that the fragments reach their equilibrium vapour pressure in the light gas species and are removed from the metaplast by convective transport in the light gas species?. In a highly fluid coal, the expulsion of the light gases occurs by bubble transport. In a low-rank thermosetting coal, the transport of the light gas species is through the pores. In either case, the degree to which the tar molecules are transported depends on the volume of light species that evolve and the vapour pressure of the molecule. The low-molecularweight species that have high vapour pressure are therefore easily transported, whereas heavy species are not. The result is that the tar from a bituminous coal pyrolysed at $\leqslant 1$ atm consists of molecules up to $\sim 800 u$. As the pressure is increased, the volume of the light gases is reduced and those marginal heavy products that were previously transported at $1 \mathrm{~atm}$ can no longer be transported. Thus, as the pressure is increased, the average molecular weight of the tar is reduced. The amount of tar is also reduced, because of the reduced efficiency of hydrogen utilization. As the pressure is decreased below $1 \mathrm{~atm}$, the molecular weights and tar amounts increase. There is a minimum pressure $\Delta P$, which is helieved to be the internal pressure within the particles. $\Delta P$ is in adjustable parameter, depending on the coal and heating rate, and has a minimum value of 0.2 for fluid coals.

For low-rank coals, low-temperature cross-linking increases the effective coordination number of the
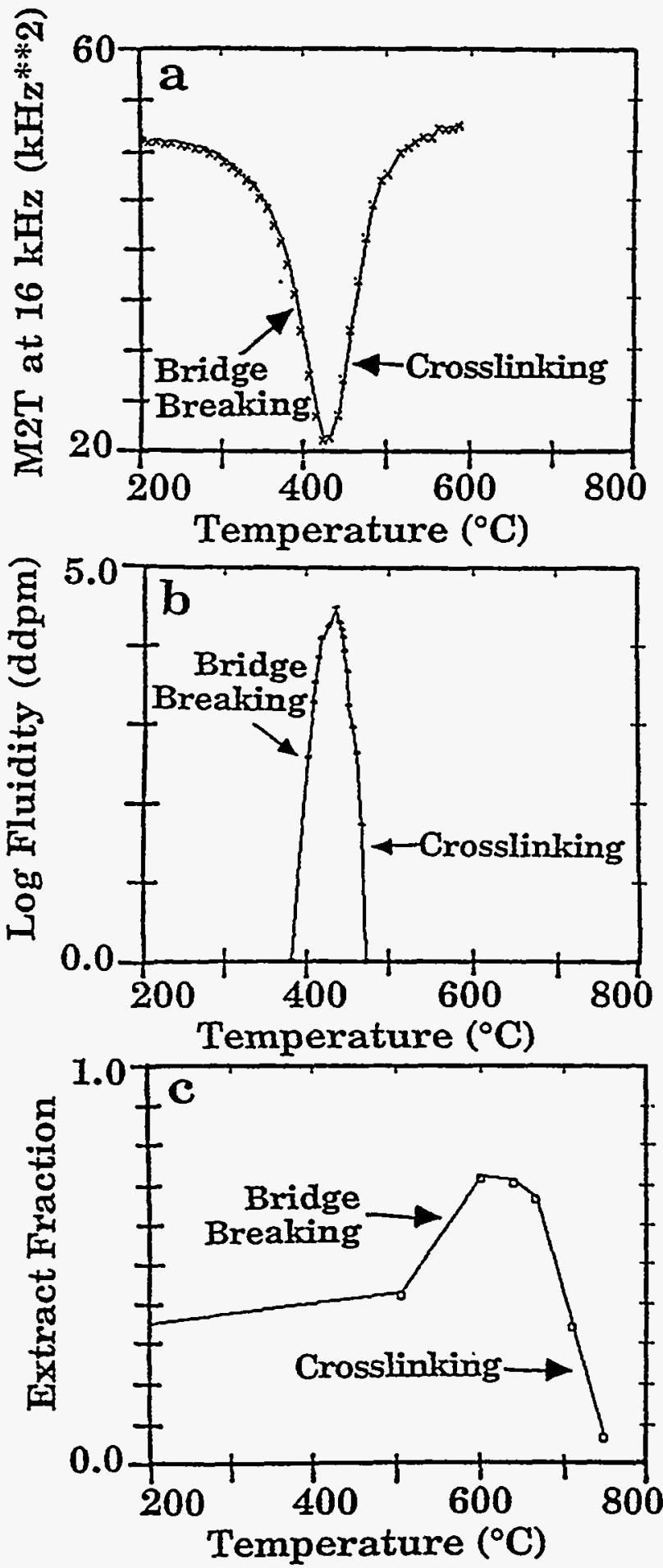

Figure 11 Results illustrating bridge breaking and cross-linking for Pittsburgh Seam coal: (a) p.m.r.t.a. at $4 \mathrm{~K} \mathrm{~s}^{-1}$ (R. Sakurovs, personal communication): (b) Gieseler fuidity at $3 \mathrm{~K} \mathrm{~s}^{-1}$ (data from Commercial Testing and Engineering Co.): (c) extract yield at $640 \mathrm{Ks}^{-1}$ (ref. 72 ) 
nctwork, and only small molecules are produced. The yiclds are low, and pressure has little influence on the iteld or molecular weight distribution.

\section{$\because$ inmmary of the FG-DVC model}

Figure 12 summarizes the FG-DVC model. In Figure $12 a$, an assumed macromolecular network is presented. In the Monte Carlo version of the model, each piece of this network is actually described in the computer memory. The description of the network contains the molecular weights of the aromatic ring clusters (shown as the numbers in the circles) and the cross-linking density (shown by the vertical double line). The potential numbers of labile bridges (related to the donatable hydrogen) are indicated by the single horizontal lines, while the unbreakable bridges are indicated by double horizontal lincs. The starting molccule is constructed from linear chains of a certain length $l$ (typically between 6 and 12 aromatic ring clusters) connected by the appropriate number of cross-links, which together with the monomer molecular weight defines the molecular weight between cross-links $M_{\mathrm{c}}$. When this is done, a certain number of the chains may be unattached to the rest of the macromolecular network. These are the guest molccules whose molecular weight is $<3000 \mathrm{u}$ and would he pyridine-soluble. The length of the chains $l$ is adjusted to obtain the proper amount of pyridine-solubles. The number of cross-links is chosen to obtain a coordination number that yields the right ratio of tar to heavier fragments (e.g. extracts and liquids) in the metaplast.

a. Starting Molecule
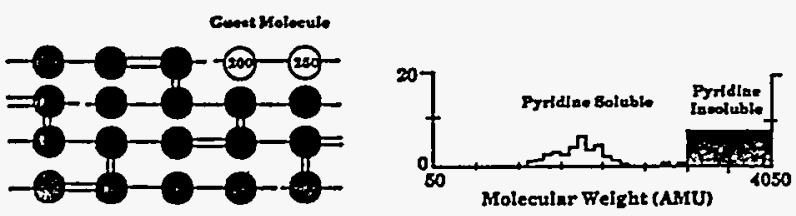

b. During Tar Formation

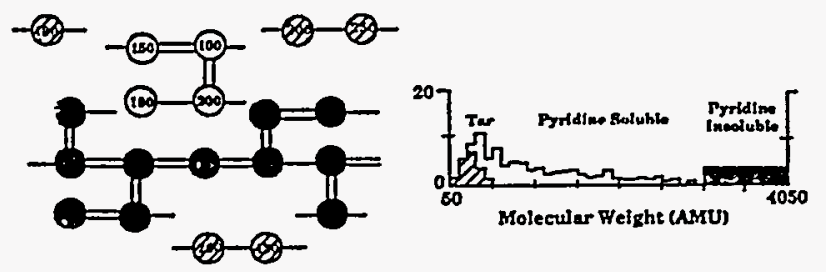

c. Char Formed

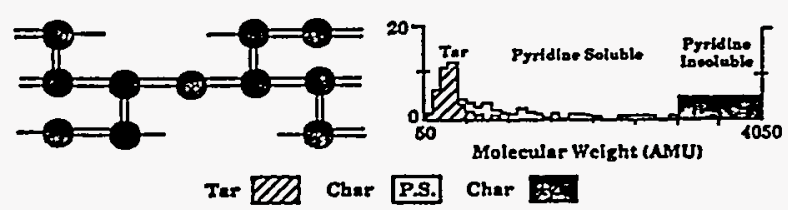

Figure 12 Representation of coal molecule in the DVC simulation and corresponding molecular weight distribution (from ref. 7). Circles represent monomers (ring clusters and peripheral groups): numbers denute the molecular weight of the monomer ineluding attached bridges. Single-line bridges are breakable and can donate hydrogen: double-line bridges are unbreakable and do not donate hydrogen. Histograms show molecular weight distribution of the coal. tar and char: pyridine-soluble and -insoluble fractions of coal and char are dilferentialed : the area under the histogram corresponds to the weight per cent of the oligomers
The number of labile bridges (amount of donatable hydrogen. $\mathrm{H}_{\mathrm{al}}$ ) is chosen to obtain the proper tar yield in the t.g.-FT-i.r. experiments. Thus the model has three parameters, $l, M_{c}$ and $H_{21}$. two of which are independently adjustable to obtain the correct tar, extract yield and viscosity.

Figure $12 b$ considers what happens during pyrolysis. As the temperature increases. some of the weak bridges (single horizontal lines) can break according to the bridge breaking rate. The hydrogen limitation is accomplished by requiring that for each bridge that is broken. another one of the labile bridges becomes an unbreakable bridge as its hydrogen is used to stabilize the free radicals generated by the broken bridges. Thus for each broken bridge, two of the labile bridges are consumed. The broken bridges and new unbreakable bridges (horizontal double lines) are distributed randomly.

In the model. the cross-linking is assumed to correlate with $\mathrm{CO}_{2}$ and $\mathrm{CH}_{4}$ evolution. The evolution of these species is determined from the functional-group part of the model: one cross-link is inserted randomly for each $\mathrm{CO}_{2}$ and each methane molecule evolved. If bridge breaking predominates over cross-linking, the macromolecular network is broken up into smaller fragments. On the right-hand side of Figure $12 b$, the molecular weight distribution that results from the bridge breaking and cross-linking events is shown. Molecules of $<3000 \mathrm{u}$ are greater in number, the lightest molecules escape as tar, and the rest of the network is described as pyridine-insoluble.

Figure $12 c$ shows the network at the conclusion of the pyrolysis process. When all the labile bridges are consumed, the decomposition of the network is complete. All of the network is completely connected by unbreakable bridges, and is highly cross-linked. All the previously loose fragments have been incorporated into the network by cross-linking or have escaped as tar.

\section{ANALYSIS OF COMPOSITION AND KINETIC PARAMETERS}

In this section, the laboratory characterization to determine the model parameters is considered. Analysis by t.g.-FT-i.r. is used to determine kinetic rales and functional group compositions. Solvent swelling, solvent extraction. and fluidity measurements in a Gieseler plastometer are used to obtain information on the molecular weight distribution of the metaplast and hence determine the network parameters. N.m.r. and f.i.m.s. are used to determine the molecular weight of the ring clusters.

\section{T.g. $-F T-i . r$.}

Apparatus and procedure. The instrument used, a Bomem TG/plus, comprises a DuPont 951 t.g.a., a hardware interface (including a furnace power supply). an Infrared Analysis 16-pass gas cell with transfer optics, a Michelson MB series FT-i.r. spectrometer (resolution $4 \mathrm{~cm}^{-1}$. detector $\mathrm{MCT}$ ), and a PC-AT compatible computer. The cell is connected without restrictions to the sample area and helium sweep gas is used to bring evolved products from the t.g.a. directly into the gas cell. The infrared spectra quantitatively track the composition of the volatiles as a function of time.

The most difficult volatiles in analyse are the tars which condense :It room lemperature. In the TG.'plus, the rapid 
cooling from the high-thermil-conductivity helium sweep gas causes these products to lorm an acrosol tine enough to follow the gas through the analysis cell. The aerosol is also sufficiently fine as to cuuse little scattering of the infrared beam and it thus appears as though the tar were in the gas phase.

As an example of the analysis procedure, the pyrolysis and oxidation of a bituminous coal is described. More detail can be found in ref. 1 . Figure $13 a$ illustrates the weight loss from this sample and the temperature history. A $25 \mathrm{mg}$ sample of Pittsburgh Seam coal, loaded in the sample basket of the DuPont 951, is heated at $30 \mathrm{~K} \mathrm{~min}^{-1}$ in the He sweep gas, first to $150^{\circ} \mathrm{C}$ to dry, then to $900^{\circ} \mathrm{C}$ for pyrolysis. After cooling, a small flow of $\mathrm{O}_{2}$ is added to the furnace at $57 \mathrm{~min}$ and the temperature is ramped to $900^{\circ} \mathrm{C}$ for oxidation.

During this procedure, infrared spectra are obtained once every $40 \mathrm{~s}$. As discussed previously ${ }^{1}$, the spectra show absorption bands for $\mathrm{CO}, \mathrm{CO}_{2}, \mathrm{CH}_{4}, \mathrm{H}_{2} \mathrm{O}, \mathrm{SO}_{2}$, $\mathrm{COS}, \mathrm{C}_{2} \mathrm{H}_{4}, \mathrm{HCl}$ and $\mathrm{NH}_{3}$. Unfortunately, $\mathrm{H}_{2}$ evolution is not directly determined by this technique, but can be determined by a second experiment in which the volatiles are oxidized. The spectra above $400^{\circ} \mathrm{C}$ also show aliphatic, aromitic, hydroxyl, carbonyl and ether bands from tar. The evolutions of gases derived from the i.r. absorbance spectra are obtained by a quantitative analysis program using a database of calibration spectra for ${ }_{z}$ different compounds. The routine decides which regions of each calibration spectrum to use for best quantitation with least interference. A correlation between the sample spectrum and the reference spectrum is performed to determine gas amounts. A database of integration windows is also available for tracking functional group absorption. Tar quantitation is
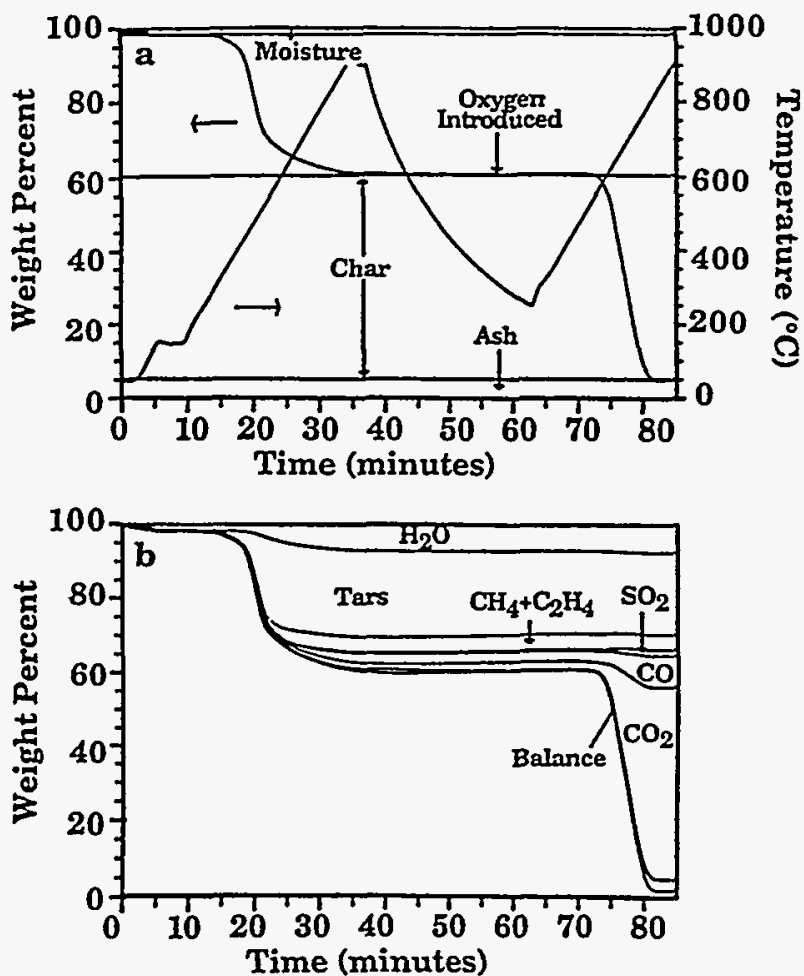

Figure 13 Thermogram and quantitative breakdown of evolved gases for Pittsburgh Seam coal
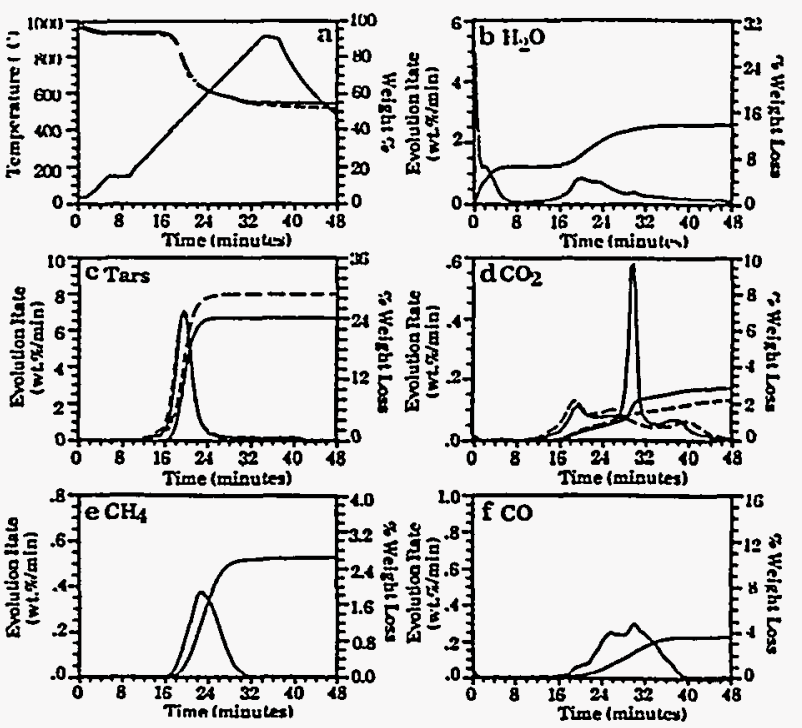

Figure 14 T.g.-FT-i.r. analysis of raw and demineralized Illinois No. 6 coal during pyrolysis: (a) weight loss ( - ), sum of evolved products (-- ) and temperature profile; (b) $\mathrm{H}_{2} \mathrm{O}$ evolution rate and integral; (c) tar evolution rate and integral for raw coal $(-)$ and demineralized coal (-- ); (d) $\mathrm{CO}_{2}$ evolution rate and integral for raw coal $(-)$ and demineralized coal (-- ) ; (e) $\mathrm{CH}_{4}$ evolution rate and integral; (f) $\mathrm{CO}$ evolution rate and integral; all weight losses are on the as-reccived basis

discussed in ref. 1. The routine is fast enough for the product anaiysis to be performed and displayed every $40 \mathrm{~s}$ during the actual experiment.

Figure $13 b$ illustrates the integral of the evolution curves to obtain cumulative evolved product amounts. Because the data are quantitative, the sum of these curves matches the weight loss as determined by the t.g.a. Discrepancies occur because of components such as $\mathrm{H}_{2}$ which cannot be seen by i.r.

Determination of FG-DVC model parameters. The kinetic and composition purameters for the FG-DVC model are obtained from the TG/plus pyrolysis cycle. The pyrolysis cycle for Illinois No. 6 coal (Argonne premium sample) is presented in Figure 14. Figure $14 a$ shows the weight loss and temperature profile; the dashed line is the sum of species ( $\mathrm{tar}, \mathrm{CH}_{4}, \mathrm{H}_{2} \mathrm{O}, \mathrm{CO}_{2}, \mathrm{CO}$, $\mathrm{SO}_{2}, \mathrm{NH}_{3}, \mathrm{C}_{2} \mathrm{H}_{4}$ and $\mathrm{COS}$ ). The sum of species is within a few per cent of the weight loss.

The water evolution (Figure 14b) consists of a low-temperature moisture peak followed by a pyrolysis peak. To fit the wide pyrolysis peak by the FG submodel, three sources are used for $\mathrm{H}_{2} \mathrm{O}$. Each source cvolves according to

$$
d \mathrm{~W}_{i} / \mathrm{d} t=k_{i} W_{i} \text { (char) }
$$

where $W_{i}$ is the gas species and $W_{i}$ (char) is the amount of the functional group source remaining in the char. The rate constant $k_{i}$ is given by an Arrhenius expression of the form

$$
k_{i}=A_{i} \exp \left(\left(-E_{i} \pm \sigma_{i}\right) / R T\right)
$$

where $A_{i}$ is the pre-exponential factor, $E_{i}$ the activation energy and $\sigma_{i}$ the distribution in activation energies. Two sources are used for $\mathrm{CH}_{4}$ and three for $\mathrm{CO}$ and $\mathrm{CO}_{2} . \mathrm{A}$ single source is used for $\mathrm{H}_{2}$, but the kinetic rates have not been finalized. Note the elimination of the calcite 
$\mathrm{CO}_{2}$ evolution peak (Figure $14 d$ ) and the increase in tar (Figure 14c) for the demineralized coal.

To obtain the model parameters, the model is fitted to the TG/plus data at three heating rates $(3,30$ and $\left.100 \mathrm{~K} \mathrm{~min}^{-1}\right)$. When there are multiple sources for a given species and the sources have overlapping peaks, the determination of parameters is not unique and some rules must be assumed. Based on chemical arguments, $A_{i}$ is restricted between $10^{12}$ and $10^{15} \mathrm{~s}^{-1}$. Also, the pre-exponential factor for a given species pool is assumed rank-invariant. This assumption is based on the observed rank variation of the evolution curves. With increasing coal rank, the leading edges and the early peaks (extra-loose or loose pools) shift to higher temperatures. while the trailing edges (tight or extra-tight pools) remain at the same temperature. An example of this is shown for water for five coals in Figure $15 a^{16}$. Ignoring the moisture release at low temperature, the figure shows a systematic increase with rank of the temperature $T_{\max }$ of the peak evolution rate. An interesting speculation is that the shift in the evolution curve with rank is due to natural pyrolysis over geological time. With increasing ageing temperature and time, the maturation process gradually evolves the loosely bound functional groups, while leaving the tightly bonded groups intact. Thus there is an increase in $T_{\max }$ with rank.

The shift can be simulated by pyrolysing a species described by a distribution of activation energies (equation (2)) up to different bed temperatures in order to represent natural ageing. An example is shown in Figure $15 b$. Starting with the evolution profile for Zap lignite, the coal is assumed to pyrolyse at $10 \mathrm{~K} \mathrm{Ma}^{-1}$ up to temperatures of $60,120,150$ and $180^{\circ} \mathrm{C}$. The

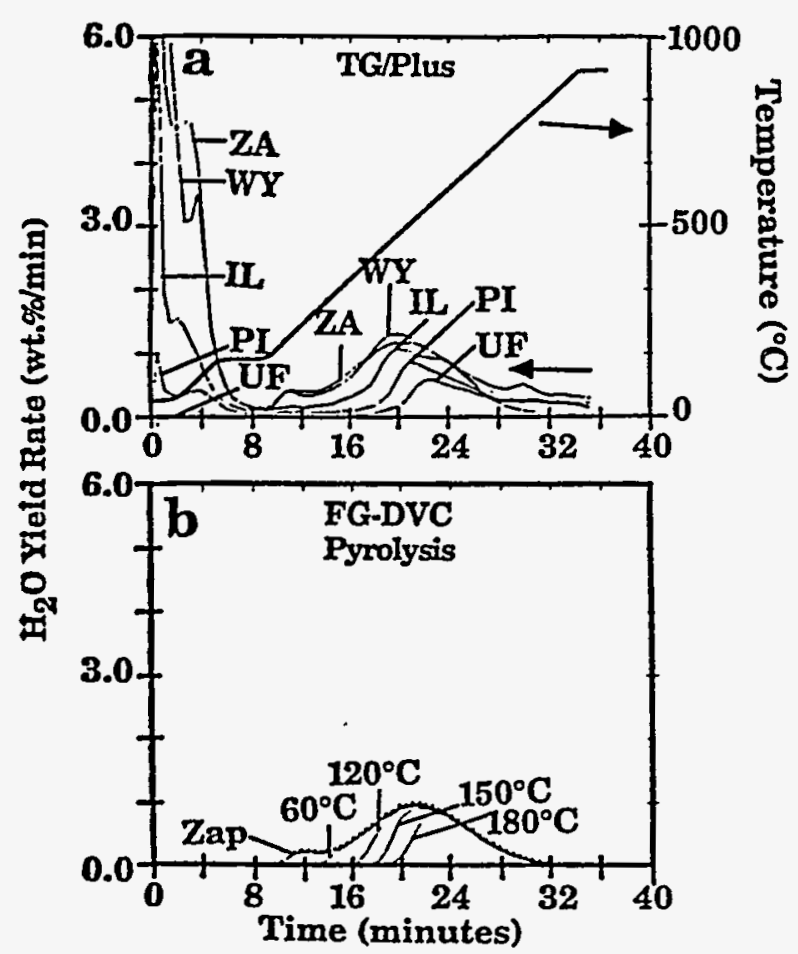

Figure 15 Evolution of $\mathrm{H}_{2} \mathrm{O}$ from five Argonne premium coals: (a) pyrolysed at $30 \mathrm{~K} \mathrm{~min}^{-1}:$ (b) predicted by $F G-D V C$ for slow pyrolysis (geological ageing) at different temperatures. ZA. Beulah Zap lignite; WY, Wyodak Anderson subbituminous: IL, Illinois No. 6 hvb: PI, Pittsburgh Ni. 8 hvb; UF. Upper Freeport mvb
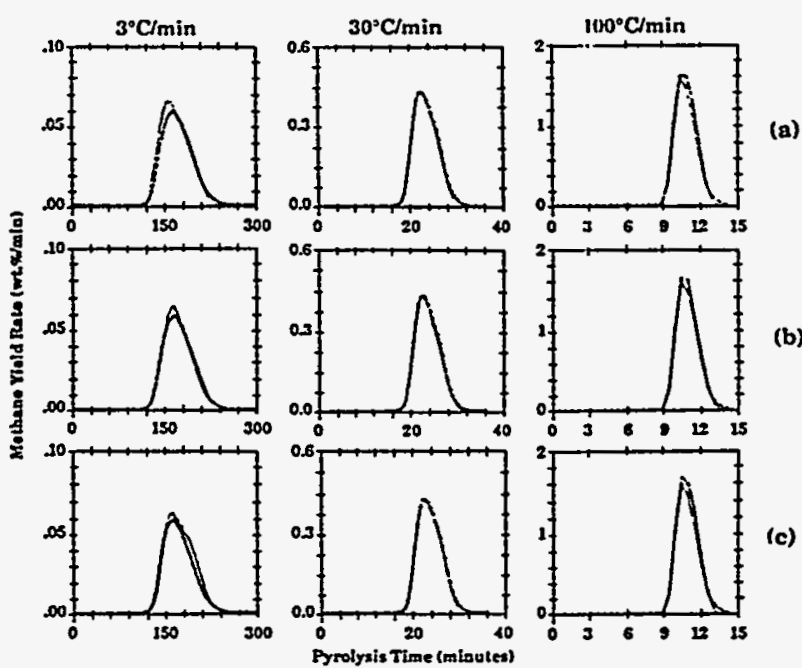

Figure 16 Kinetic analysis for $\mathrm{CH}_{4}$ evolution at three heating rates for Pittsburgh Seam coal: comparison of theory $(-)$ and experiment (-D.). $E_{\mathrm{CI} / 4}\left(\mathrm{~kJ} \mathrm{~mol}^{-1}\right)$ : (a) 193 (L), 226 (T): (b) 234 (L). 268 (T): (c) 276 (L). 310 (T)

resulting geologically aged sample is simulated using the TG/plus temperature profile and the predicted results are plotted. The curves for geological ageing at 120,150 and $180^{\circ} \mathrm{C}$ are similar to the actual TG/plus evolution curves shown in Figure 15a for Illinois No. 6, Pittsburgh No. 8 and Upper Freeport coals respectively. Thus, in the FG-DVC model, the pre-exponential factor is assumed to be constant as rank increases, and the activation energy of the pool is increased with increasing coal rank to fit the data. It is found that the activation energy of tight pools generally changes with rank much less than does that of the loose pools.

The amounts of the functional group pools and kinetic parameters are obtained by comparing the data from the t.g.-FT-i.r. with the model at heating rates of 3,30 and $100 \mathrm{~K} \mathrm{~min}^{-1}$. The resolution of the hydrocarbon evolution into paraffins, olefins, ethane, ethylene, propane and propylene and the determination of $\mathrm{H}_{2}$ evolution is done in other experiments if required. Figure 16 compares the theory and experiment for methane evolution from the Pittsburgh Seam coal at three heating rates and for three sets of $E_{i}, A_{i}$ and $\sigma_{i}$ values, where $E_{i}$ was specified and $A_{i}$ and $\sigma_{i}$ were adjusted to obtain the kinetic rate that fits the data at $30 \mathrm{~K} \mathrm{~min}^{-1}$. The kinetic parameters are then derived from this analysis, the kinetic rate being known within a factor of $\sim 2$. The agreement between the theory and experiment in Figure 16 is best for $E_{\mathrm{CH} 4(\mathrm{~L})}=234$ and $E_{\mathrm{CH} 4(\mathrm{~T})}=268 \mathrm{~kJ} \mathrm{~mol}^{-1}$. In the determination of kinetic parameters, uncertainties arise from the fact that each gas evolution is represented by several pools, which gives more degrees of freedom to the choice of parameters. Based on such analysis it is determined that $E$ can be specified to within $\pm 20 \mathrm{~kJ}$ mol $^{-1}$. Once $E$ is fixed, then $A$ is determined to within a factor of 2 , and $\sigma$ to $\pm 1.3 \mathrm{~kJ} \mathrm{~mol}^{-1}$. On the other hand, if $A$ is chosen based on chemical arguments, then $E$ is determined by the data to within $\pm 2 \mathrm{~kJ} \mathrm{~mol}^{-1}$.

These curve-fitting procedures have been applied to the eight Argonne premium coals according to the rules cited above (i.e. frequency factor between $10^{12}$ and $10^{15} \mathrm{~s}^{-1}$ and constant for a given gas species pool, independent of coal rank ). Results for $E, A$ and $\sigma$ for bridge breaking and the evolution of methane (two 
Table I Kinetie ditta for major functional group pouls in Argeune premium coals

Valus of $l: R(K)$ (upper tigure) and $\sigma K(K)$ (lower tigure)

\begin{tabular}{|c|c|c|c|c|c|c|c|c|}
\hline $\begin{array}{l}\text { Group-poola : } \\
A\left(\mathrm{~s}^{-1}\right)\end{array}$ & $\begin{array}{l}\text { Beulah } \\
\text { Zipp } \\
\text { lignite }\end{array}$ & $\begin{array}{l}\text { Wyodiak } \\
\text { Anderson } \\
\text { subbit. }\end{array}$ & $\begin{array}{l}\text { Illinois } \\
\text { No. } 6 \\
\text { hvb }\end{array}$ & $\begin{array}{l}\text { Blind } \\
\text { Canyun } \\
\text { hvb }\end{array}$ & $\begin{array}{l}\text { Lewiston } \\
\text { Stockton } \\
\text { hvb }\end{array}$ & $\begin{array}{l}\text { Pittsburgh } \\
\text { No. } 8 \\
\text { hvb }\end{array}$ & $\begin{array}{l}\text { Upper } \\
\text { Freeport } \\
\text { mvb }\end{array}$ & $\begin{array}{l}\text { Pocahontas } \\
\text { No. } 3 \\
\text { lvb }\end{array}$ \\
\hline $\mathrm{CO}_{2}-\mathrm{XL}$ & 18000 & 18000 & 20500 & 21000 & 21250 & 21500 & 22000 & 23000 \\
\hline $5.0 \times 10^{12}$ & 1500 & 1500 & 3000 & 40000 & 3500 & 3600 & 2000 & 2500 \\
\hline $\mathrm{CO}_{2}-\mathrm{L}$ & 23500 & 24000 & 24750 & 25000 & 26000 & 26500 & 27000 & 28000 \\
\hline $5.0 \times 10^{12}$ & 2000 & 2500 & 1750 & 1250 & 3000 & 3000 & 3000 & 2500 \\
\hline $\mathrm{CO}_{2}-\mathrm{T}$ & 31000 & 32000 & 32000 & 32000 & 32000 & 32000 & 32000 & 33500 \\
\hline $7.5 \times 10^{12}$ & 3000 & 2800 & 2750 & 5000 & 3200 & 2500 & 2500 & 2700 \\
\hline CO-L & 24500 & 24750 & 25000 & 25000 & 25500 & 26000 & 28000 & 29000 \\
\hline $5.0 \times 10^{12}$ & 3000 & 2500 & 1000 & 1250 & 1100 & 1250 & 750 & 1250 \\
\hline $\mathrm{CO}-\mathrm{T}$ & 30000 & 30250 & 30500 & 30500 & 30500 & 30750 & 31500 & 32000 \\
\hline $5.0 \times 100^{12}$ & $30 x(x)$ & $3(10 x)$ & 2000 & $2(x)(x)$ & $16(x)$ & $19(x)$ & $I A(X)$ & 1500 \\
\hline $\mathrm{CO}-\mathrm{xT}$ & $39(x \times)$ & 39) 750 & 40000 & 40000 & 400000 & 40000 & 40000 & 40000 \\
\hline $2.0 \times 10^{14}$ & $25(x)$ & $25(x)$ & $30 \times 0$ & 2500 & 3000 & 2800 & 2250 & 3200 \\
\hline $\mathrm{CH}_{4}-\mathrm{L}$ & 28000 & 28000 & 28000 & 28000 & 28000 & 28000 & 28750 & 29500 \\
\hline $3.0 \times 10^{13}$ & 2500 & 2250 & 1800 & 1500 & 1200 & 1300 & 800 & 750 \\
\hline $\mathrm{CH}_{2}-\mathrm{T}$ & $320 \times 0$ & 32000 & 32000 & 32000 & 32000 & 32000 & 32000 & 33000 \\
\hline $6.0 \times 10^{13}$ & 2200 & 2000 & 2200 & 2200 & 2200 & 2200 & 2000 & 1700 \\
\hline $\mathrm{H}_{2} \mathrm{O}-\mathrm{XL}$ & 16500 & 17000 & 18000 & 0 & 0 & 0 & 0 & 0 \\
\hline $5.0 \times 10^{12}$ & 1500 & 1500 & 1800 & 0 & 0 & 0 & 0 & 0 \\
\hline $\mathrm{H}_{2} \mathrm{O}-\mathrm{L}$ & 23000 & 24250 & 25000 & 250000 & 25500 & 26000 & 27500 & 28000 \\
\hline $5.0 \times 10^{12}$ & 2700 & 2500 & 1500 & 1250 & 1250 & 1250 & 1250 & 1250 \\
\hline $\mathrm{H}_{2} \mathrm{O}-\mathrm{T}$ & 31000 & 31000 & 32000 & 32000 & 32000 & 32000 & 34000 & 35000 \\
\hline $2.0 \times 10^{14}$ & 3500 & 3500 & 2800 & 2500 & 2500 & 2500 & 4000 & 3000 \\
\hline Tar-BB & 26000 & 26000 & 26000 & 27000 & 27250 & 27500 & 28250 & 29000 \\
\hline $1.0 \times 10^{14}$ & 1000 & 1000 & 750 & 1250 & 1000 & 1250 & 1250 & 750 \\
\hline
\end{tabular}

aB, bridge breaking: L, loose; $T$, tight: $X L$, extra-loose; XT, extra-tight

$R$ is the gas constant

pools), $\mathrm{CO}$ (three pools), $\mathrm{CO}_{2}$ (three pools) and $\mathrm{H}_{2} \mathrm{O}$ (three pools) are presented in Tuble 1. These Arrhenius parameters do not represent fundamental kinetics, but have been found to give good predictions at the three heating rates investigated. In addition, as shown in the next section, these parameters appear to give reasonable predictions at high heating rates. The values for $\mathrm{H}_{2}$ are not yet finalized and are not presented. There is a systematic increase in activation energy with increasing rank. The variation in activation energy is maximum for the loose pool and decreases as the activation energy increases. The amounts for these pools are presented in Figure 17, which shows a systematic variation with rank. Oxygenated species - $\mathrm{CO}, \mathrm{CO}_{2}$ and $\mathrm{H}_{2} \mathrm{O}$ - decrease with increasing rank. Methane increases with increasing rank.

Solvent swelling, extraction and n.m.r.

Solvent swelling and extraction data for the Argonne premium coals are presented in Table 2. As discussed above, the extract yield is used to determine the length of the chains (Monte Carlo) or the starting bond probabilities (percolation) used in the model. There appears to be some problem in this approach for the highest-rank coals (Pocahontas and Upper Freeport). The swelling and extract yields for these coals in pyridine appear to be limited by weak cross-linking (other than hydrogen bonding) forces which are not eliminated by pyridine.

The solvent swelling ratio has been used to determine the cross-link density ${ }^{2.3 .24-29}$. The various theories and
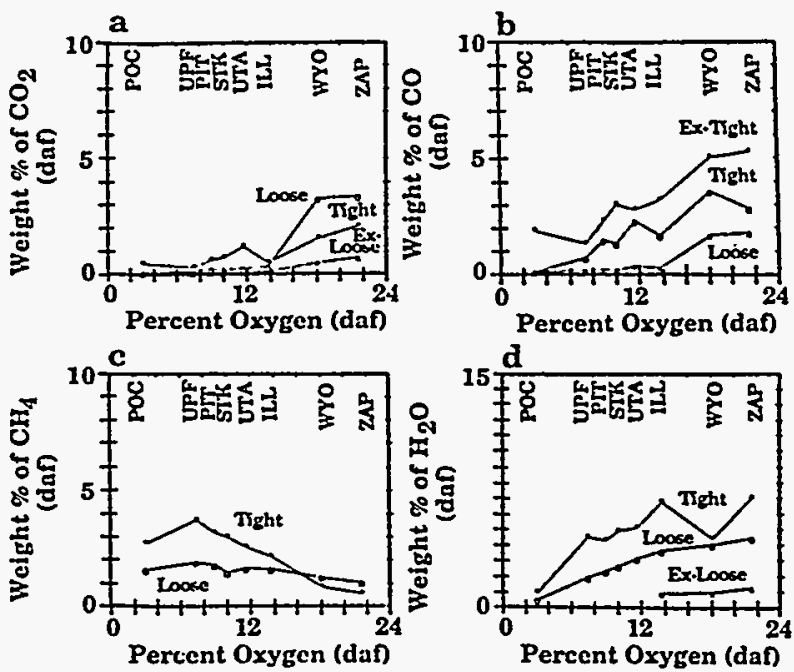

Figure 17 Amuunts of four gases released for functional group pools of the cight Argonne premium coals, as a function of the oxygen content of the coal. POC. Pocahontas No. 3: UPF, Upper Freeport; PIT, Pittsburgh No. 8; STK, Lewiston-Stockton; UTA, Blind Canyon; ILL, Illinois No. 6; WYO, Wyodak Anderson; ZAP. Beulah Zap

values for the solvent interaction parameter ${ }^{24-29}$ suggest that there are between four and eight ring clusters between cross-links, indicating a value of $\sigma+1$ between 2.13 and $2.25^{32}$. N.m.r. results of Solum et al. ${ }^{5}$ for the number of bridges and loops suggest a value of $\sigma+1$ of between 2 and 3 , so a value in the neighbourhood of 2.5 
Table 2 Fxtract yiclds and volunetric swelling ratio (VSR) for Irgonne premium coals

\begin{tabular}{lcl}
\hline & $\begin{array}{c}\text { Pyridine extract } \\
\text { ( wt\% daf) }\end{array}$ & $\begin{array}{l}\text { VSR } \\
\text { (vacuum-dried) }\end{array}$ \\
Bculah Zap & 5.4 & 2.7 \\
Wyodak Anderson & 10.7 & 2.7 \\
Lewiston-Stockton & 18.3 & 2.3 \\
Blind Canyon & 22.5 & 2.7 \\
Pittshurgh No. 8 & 27.7 & 2.3 \\
Illinois No. 6 & 35.7 & 2.5 \\
Pocahontas No. 3 & 1.0 & 1.1 \\
Upper Freeport & 10.4 & 1.3 \\
\hline
\end{tabular}
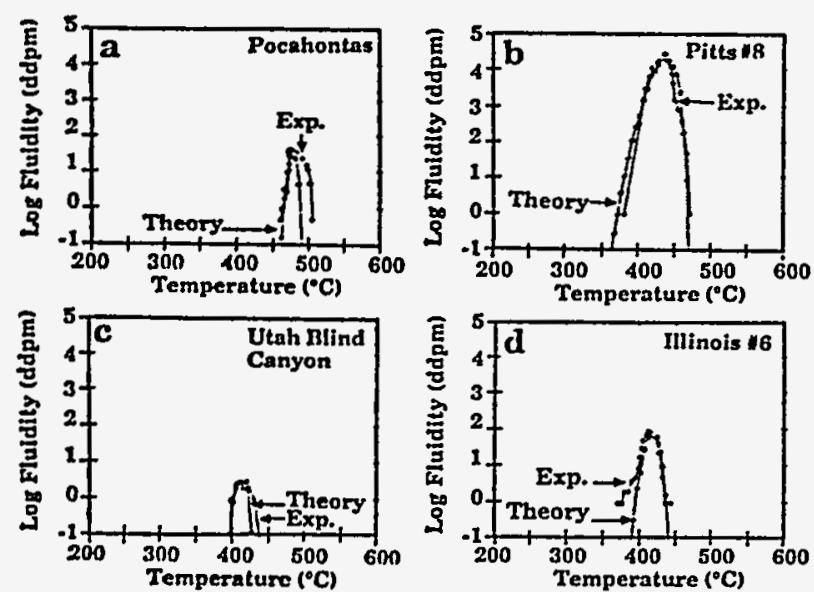

Figure 18 Measured and predicted Gieseler fluiditics of four Argonne premium coals. from ref. 77

seems reasonable. However, the uncertainty in the coordination number determined by n.m.r. is too large for use in the model. The cross-link density is instead considered as an adjustable parameter used to fit the fluidity data.

\section{Gieseler fuidity}

As discussed above, the cross-link density controls the effective coordination number of the network and hence the molecular weight distribution and amount of the fragments. For bituminous coals, it is the initial cross-link density that is important, since few new cross-links are formed before pyrolysis. A recent theory for fiuidity was developed based on the liquid fraction in the coal computed by the FG-DVC model ${ }^{8.43}$. Measurements of the tar and the fluidity thus provide a constraint on the molecular weight distribution of the fragments and hence on the cross-link density. Figure 18 presents a comparison of theory and experiment for four of the Argonne coals with the kinetic parameters fitted from t.g.-FT-i.r. data. The fitting procedure for fluidity and tar determines a unique combination of the cross-link density and donatable hydrogen.

\section{Monomer molecular weight distribution}

The molecular weight distribution of the monomers is chosen based on the ring cluster size determined by n.m.r. ${ }^{5}$ and the results of the model checked with f.i.m.s. data ${ }^{6}$.

\section{COMPARISON OF THEORY AND EXPERIMENT}

\section{Volatiles evolution}

A good test of the validity of using the t.g.-FT-i.r. method over a range of low heating rates to obtain kinetic parameters is the ability to use the kinetic parameters to extrapolate to high heating rate conditions. Figure $19 a$ presents results for Illinois No. 6 coal using the complete FG-DVC model and the most recent kinetic and composition parameters derived from the TG/plus ${ }^{65}$. The data were obtained in the heated-tube reactor, where FT-i.r. emission and transmission measurements of coal particle temperatures determined the heating rate to be $>2 \times 10^{4} \mathrm{~K} \mathrm{~s}^{-1}$. The predicted rates of cvolution for each species are in good agreement with the observed ratcs except for water, for which moisture sometimes creates measurcment errors.

The sensitivity of the predictions to the assumed kinetic rates has been considered by running cases with rates at a factor of 10 lower and higher. Such a change produces a $\pm 65 \mathrm{~K}$ shift in the curves or a variation of $\pm 5 \mathrm{~cm}$ in the position on the $x$-axis. The results thus suggest a knowledge of the kinetic rates at these high heating rates to within a factor of 10 . On the other hand, the accuracy of the temperature measurements is estimated to be $\pm 25 \mathrm{~K}$.

\section{Char}

A number of char characteristics can be measured and compared with the model. These include fluidity (already discussed in the previous section), functional group composition, cross-link density, p.m.r.t.a., and extract yield. These are discussed below.

Functional group composition. The functional group composition can be determined by FT-i.r. ${ }^{73-76}$ or CP-MAS n.m.r. with dipolar dephasing ${ }^{5}$. Chars were prepared by heating Pittsburgh Seam coal to temperatures of $200,300,400,500$ and $600^{\circ} \mathrm{C}$ at $30 \mathrm{~K} \mathrm{~s}^{-1}$ and were
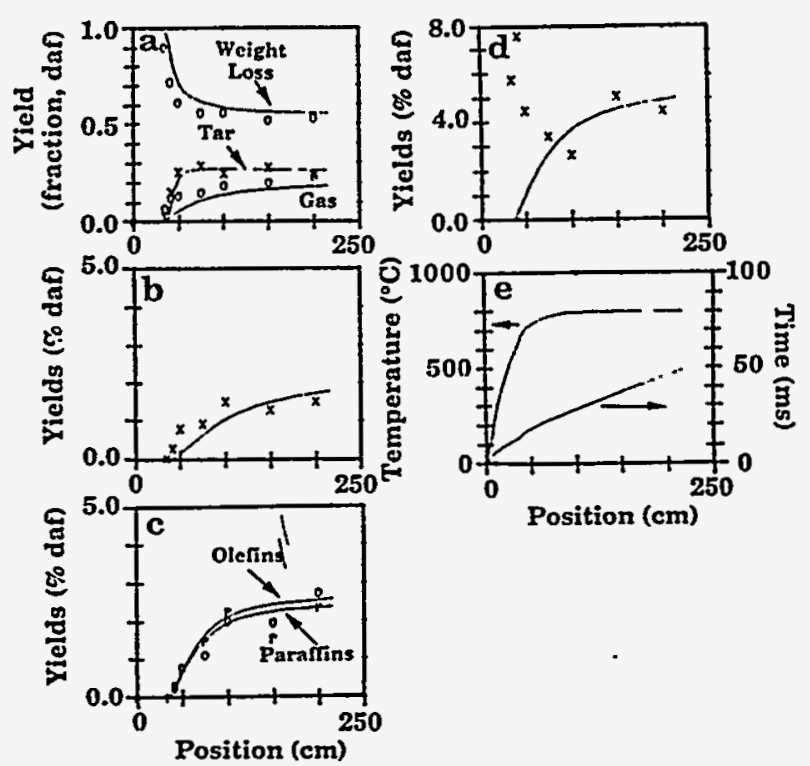

Figure 19 Predictions of the FG-DVC mudel for Illinois No. 6 conl pyrolysed in the heitted-tube reactor: (a) wivght loss, tar and gas; (b) methane: (c) olclin and paraltins: / d I water:(c) time and temperature as a function of pumition. Data points are frum ref. 5.3 
characterized"s. Figure 20 compares the theory with n.m.r. and FT i.r. measurements. The fractions of aliphatic and aromatic carbons are compared in Figure 20a, and aliphatic, aromatic, methyl and hydroxyl hydrogens in Figure 20b. Figures $20 b$ and 20d compare the n.m.r. and FT-i.r. measurements for the same quantitics (except that methyl and aliphatic hydrogen are lumped together in the latter). The tar (Figure 21a) and methane (Figurc $21 \mathrm{~b}$ ) yield rates are presented for comparison. Although there are some discrepancies between the theory and experiment in the magnitudes of the quantities (the FT-i.r. quantities are the less well predicted), the theory matches the data for the temperatures at which variations occur to within $\pm 20 \mathrm{~K}$ or the kinetic rate to within a factor of 2 . The experimental uncertainty in the temperature is $\pm 10 \mathrm{~K}$. The model predictions are thus in good agreement with the data.

Cross-link density. The application of the volumetric swelling ratio to obtain the changing cross-link density in the char was discussed in the section dealing with the FG-DVC model. Comparison with theory was discussed in ref. 7. Figure 2Ic compares the theory and experiment on the cross-link density as determined by the solvent swelling ratio for the set of chars of Figures 20 and $21 a, b$. The agreement is good except for the increase in $1-X$, which is not predicted. Figure 22 compares the theory and experiment for two coals. The theory predicts the early cross-linking in Zap lignite (related to $\mathrm{CO}_{2}$ evolution), not seen for the bituminous coal. The agreement between theory and experiment is good, except that as in Figure 21c the increase in $1-X$ for the Pittsburgh Seam coal is not predicted.

The n.m.r. data also provide a direct measurement of the number of attachments per cluster ${ }^{5}$ (see Figure 9). Figure 23 presents data ${ }^{68}$ for the total number of attachments (which includes peripheral groups, bridges and loops), and only bridges and loops (B \& L) as a function of final temperature for Pittsburgh Seam coal
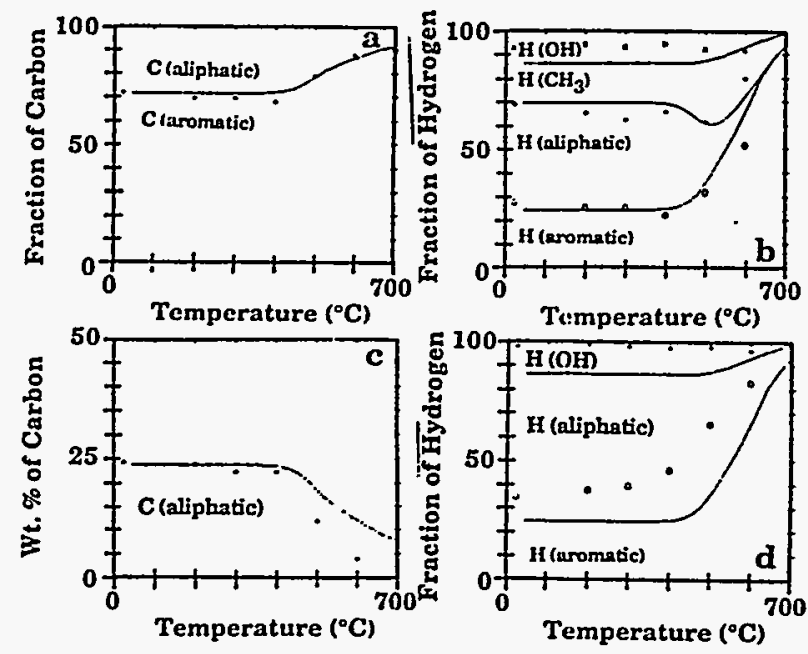

Figure 20 Comparison of measured (points) and predicted (-) functional group composition of char from Pittsburgh Scam coal pyrolysed at $30 \mathrm{~K} \mathrm{~min}^{-1}$ to the indicated temperatures: (a) carbon distribution by n.m.r.; (b) hydrogen distribution by n.m.r.; (c) aliphatic carbon by FT-i.r.; (d) hydrogen distribution by FT-i.r. Data from ref. 68
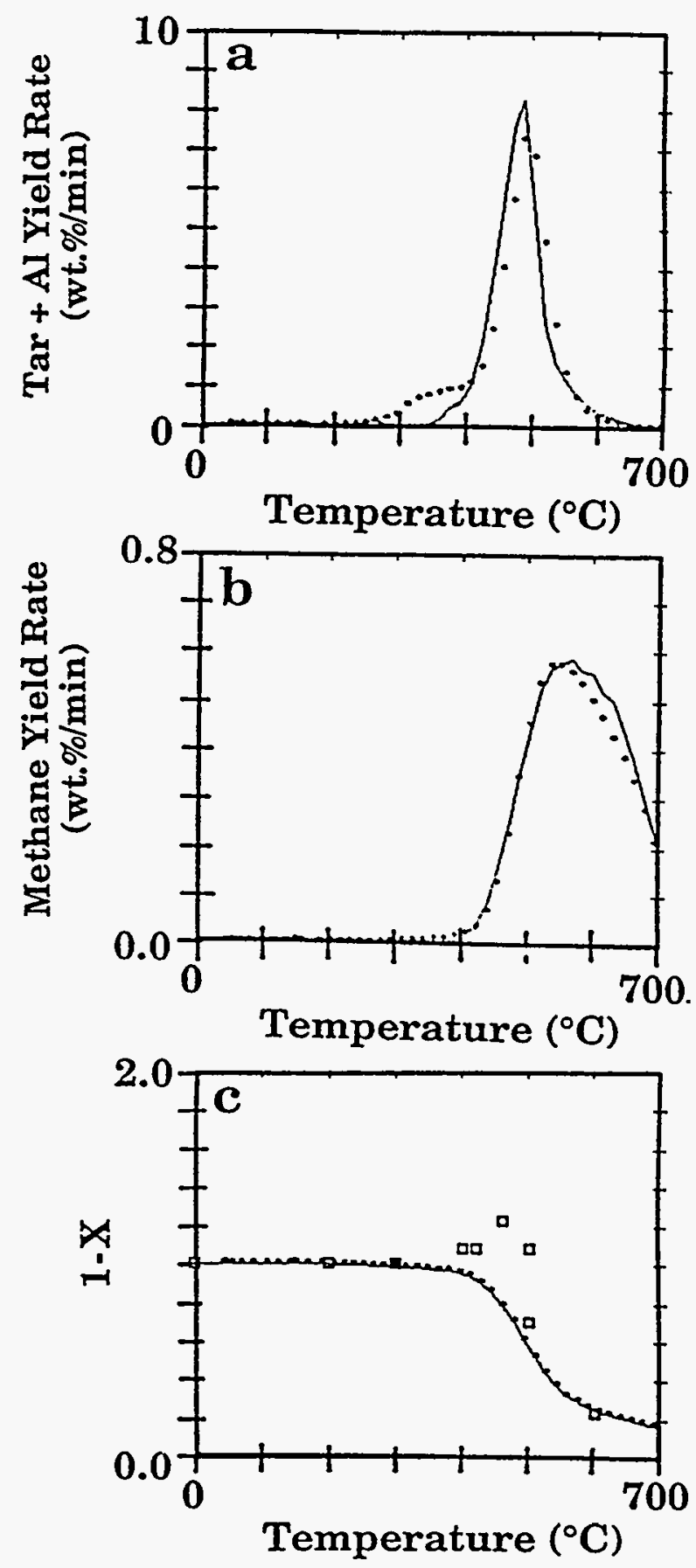

Figure 21 Comparison of measured (points) and predicted (-), pyrolysis behaviour of Pittsburgh Stam coal heated at $30 \mathrm{~K} \mathrm{~min}^{-1} 10$ the indicated temperatures: (a) tar yield rate; (b) methane yield rate: (c) solvent swelling ratio

heated at $30 \mathrm{~K} \mathrm{~min}^{-1}$ to the indicated temperature. These are the same chars as in Figures 20 and 21. The FG-DVC model predicts the number of B \& L. This quantity $(\sim 2$ for coal) is the coordination number, suggesting that coal is quite chain-like. There is little change in $B \& L$ up to $400^{\circ} \mathrm{C}$, but an increase at $500^{\circ} \mathrm{C}$ and above, where cross-linking related to methane evolution is believed to occur.

Notice that the total number of attachments changes very little. This would be reasonable if the methane 


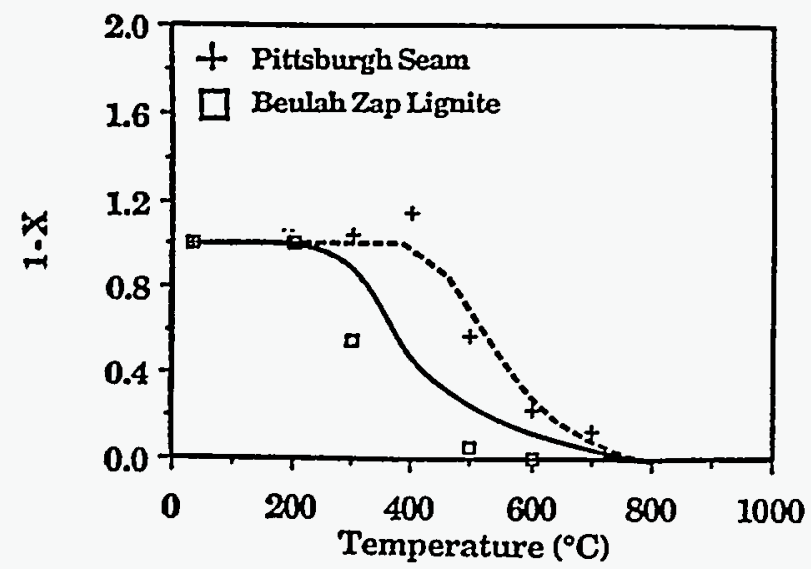

Figure 22 Comparison of measured (points) and predicted (..... $---)$ normalized volumetric swelling ratio as a function of pyrolysis temperature for a lignite and a bituninous coal

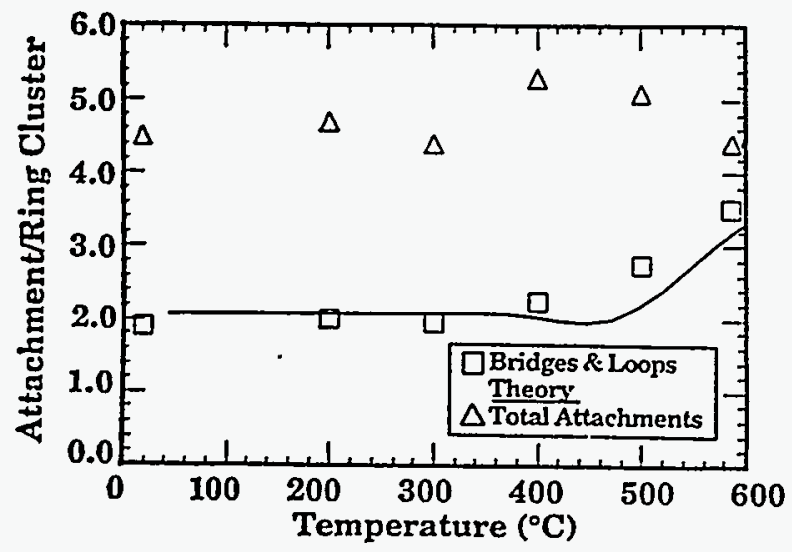

Figure 23 Comparison of theory with n.m.r. data for attachments to ring clusters (data from University of Utah)

peripheral groups were replaced by bridges in substitution reactions. This is believed to be the reason for the correlation between methane evolution and cross-linking.

P.m.r.t.a. Proton magnetic resonance thermal :unalysis p.m.r.t.a.) is used at CSIRO as an alternative to fluidity measurements. The measurement of proton mobility can distinguish protons on molecules free to rotate from protons on a rigid lattice. The molecule's ability to rotate depends on its freedom from the network (i.e. it must be unattached or attached at only one place) and on the mobility of free molecules to rotate (which depends on the temperature). From the measured $M_{2 \mathrm{~T}}$ values, a 'mobile' liquid fraction can be defined by the expression (R. Sakurovs, personal communication):

$$
\text { Liquid fraction }=\frac{M_{2 T}(\text { room temperature })-M_{2 T}(T)}{. M_{2 T}(\text { room temperature })}
$$

At sufficiently high temperature, when the free molecules have sufficient energy to rotate, this quantity should be equal to the FG-DVC liquid fraction. Figures $24 a$ and $24 d$ compare the measured and predicted liquid fraction using both the Monte Carlo and percolation models. As expected, the theory and experiment do not agree at low temperature where the material is solid and the molecules are not free to rotate. However, there is goud agreement on the softening temperature, peak fluidity temperature and solidification temperature, which are predicted to within $\pm 20 \mathrm{~K}$ (a factor of 2 in rate). The liquid amounts in the two theories are defined differently and so the absolute amounts do not agree. Also shown in Figures 24h, c. $c$ and $f$ are comparisons of both theories and experiment for the fluidity and tar evolution curves for the same coal at a similar heating rate. The agreement between the data and both theorics is good.

Extract yields. Figure 25 compares the FG-DVC predictions with the data of Fong et $a l^{72}$ on total volatiles yield and extract yield as a function of temperature in pyrolysis at $0.85 \mathrm{~atm}$. The experiment was performed in a heated-grid apparatus at heating rates of $470 \mathrm{~K} \mathrm{~s}^{-1}$ to $814 \mathrm{~K}$ (Figure $25 \mathrm{u}$ ) and $640 \mathrm{~K} \mathrm{~s}^{-1}$ to $1018 \mathrm{~K}$ (Figure $25 b$ ), with variable holding times and rapid cooldown. The predictions in Figure 25 are in reasonable agreement with the data. The temperatures at which the extract yield increases owing to bridge breaking and decreases owing to cross-linking are predicted to within $\pm 30 \mathrm{~K}$. This is well within the accuracy of the experiment. The predicted extract yields are not as high as the measured yields. However. such high yields of extracts have not been duplicated by others. and it is possible that the extracted fraction also contains some colloidal material.

Weathering. Oxidation of Pittsburgh Seam coal was performed at $80^{\circ} \mathrm{C}$ for 10.20 and $62 \mathrm{~d}$. In the model, the loss of fluidity with increasing oxygen concentration is related to the increase in $\mathrm{CO}_{2}$ evolution and hence to increases in low-temperature cross-linking. To determine the $\mathrm{CO}_{2}$ evolution, measurements were made in the t.g.-FT-i.r. ${ }^{77}$. Figure $26 a$ shows that the lowtemperature $\mathrm{CO}_{2}$ evolution significantly increased after $10 \mathrm{~d}$ of oxidation, becoming comparable with that for Illinois No. 6 coal. After $20 \mathrm{~d}$, the early $\mathrm{CO}_{2}$ evolution
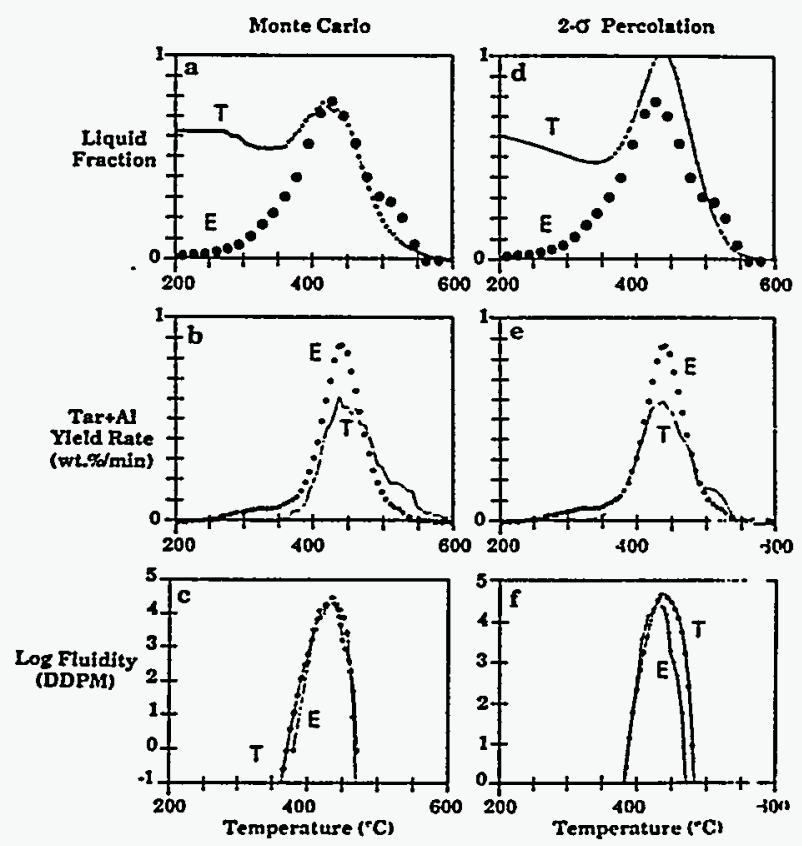

Figure 24 Comparison of Monte Carlo and 2- $\sigma$ percolation theory for liquid fraction, tar yicld rate and Giescler fuidity for Pittsburgh No. 8 bituminous coil pyrolysed at $3 \mathrm{~K} \mathrm{~min}^{-1}$. T. theory: E, experimentill ditti 

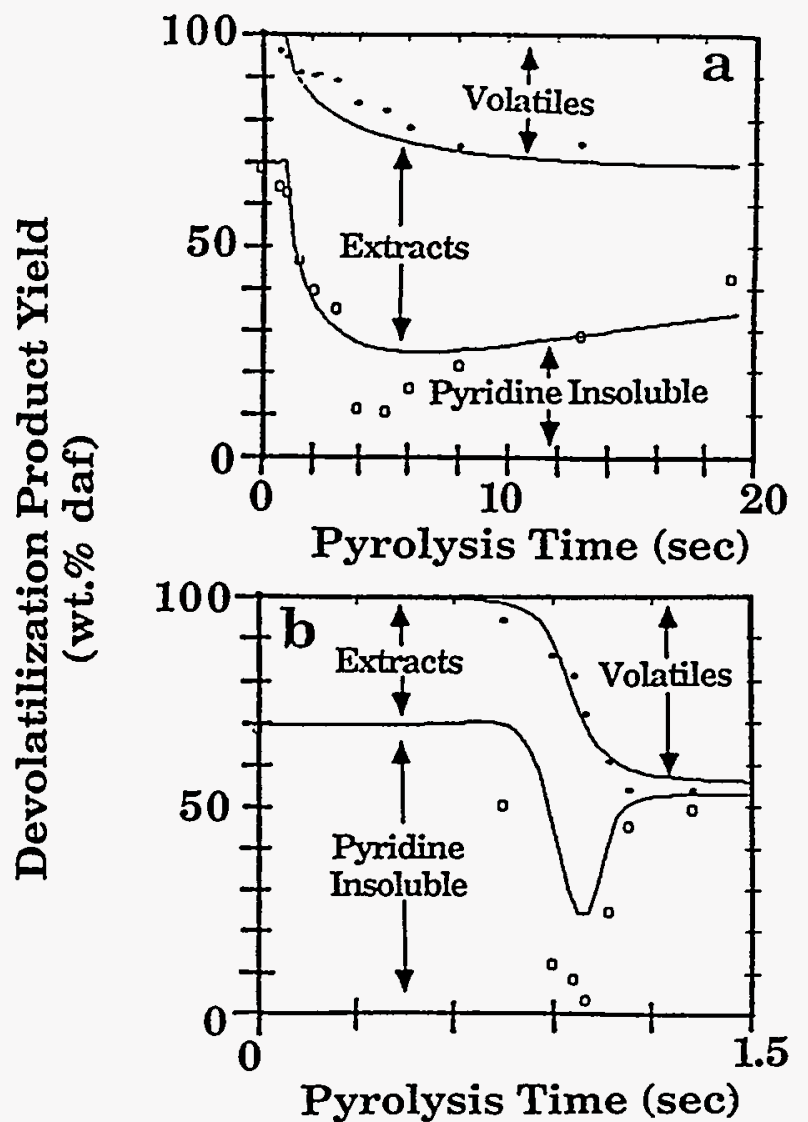

Figure 25 Comparison of FG-DVC model predictions (-) with the data of ref. 72 (points) for Pittsburgh Seam coal: (a) $813 \mathrm{~K}$, $470 \mathrm{~K} \mathrm{~s}^{-1}$; (b) $1018 \mathrm{~K}, 640 \mathrm{~K} \mathrm{~s}^{-1} ; P=0.85 \mathrm{~atm}$. The predictions assume transport by equation (4) $(\Delta P=0 \mathrm{~atm})$ and no external transport

was greater than that for Utah bituminous coal. After 3 months at $110^{\circ} \mathrm{C}$ the $\mathrm{CO}_{2}$ evolution was comparable with that of a lignite. When these increased $\mathrm{CO}_{2}$ yields were incorporated in the simulation for the oxidized Pittsburgh Seam coal's fluidity, the maximum fluidity was reduced. The predicted maximum fuidity is compared in Figure $26 b$ with the measurements of $\mathrm{Wu}$ et $a .^{78}$ for comparable coal and oxidation treatment. The agreement is quite reasonable.

Predicted molecular weight distribution. The dominant factors determining the char's properties are the starting cross-link density and low-temperature cross-linking behaviour. To illustrate the differences between plastic and non-plastic coals, Figure 27 compares the predicted molecular weight distribution of macromolecular network fragments in the char and tar for Zap lignite and Pittsburgh Seam bituminous coal. The bituminous coal (Figure 27a) exhibits substantial fragmentation of tar precursors with molecular weights of 300 to 900 daltons (11-mers $1-3$ ), extracts or pyridine solubles with molecular weights of 1200 to 3000 daltons (n-mers 4-10), and liquids with molecular weights of 3300 to 30000 daltons ( $n$-mers $11-100$ ). On the other hand, the initial cross-link density in the lignite, and the subsequent increase due to $\mathrm{CO}_{2}$-related cross-linking, allow only minimal fragmentation producing only small molecules (Figure 27b). (Note the factor of 10 scale difference between the bituminous coal and the lignite.) These predictions of the model are related to the extract yields, p.m.r.t.a. analysis. fluidity and tar yields. Bituminous coals have high extract yields, high fluidity and high tar yields; lignites have low values of these quantities. The chief difference is the cross-link density.

Tar

Molecular nceight distribution. The tar is evolved from the lightest fractions of the metaplast and depends on the metaplast distribution and the transport. For the bituminous coul, the upper molecular weight is limited only by the vapour pressure of the large molecules. For
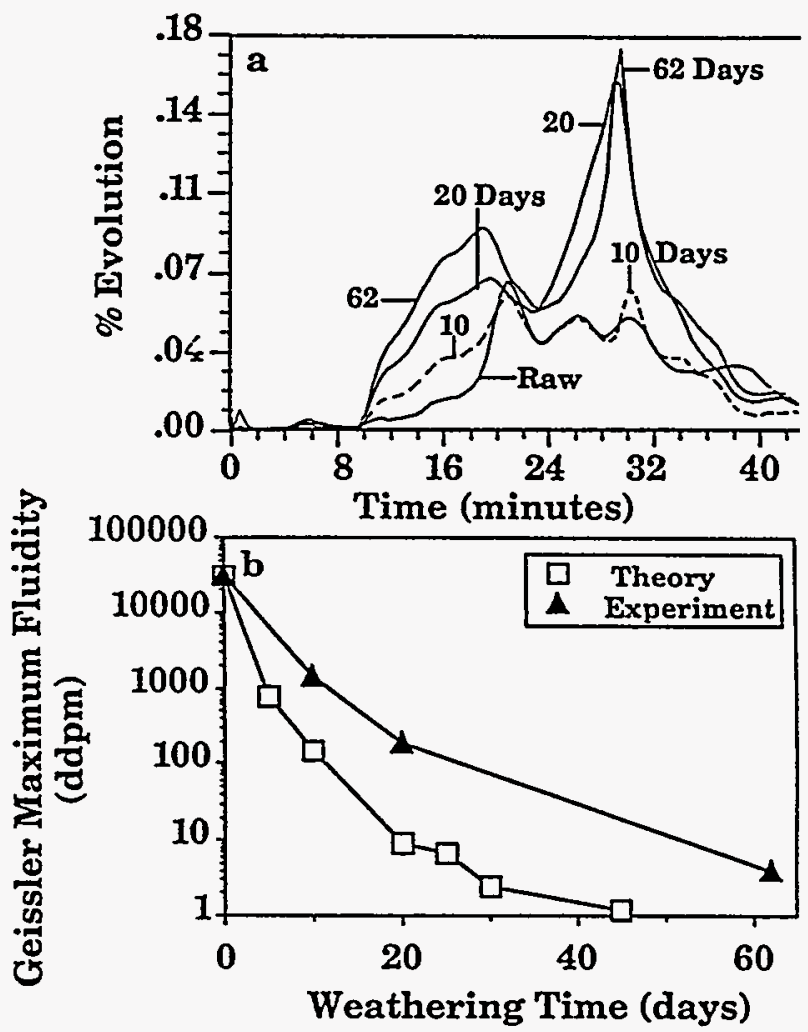

Figure 26 Reduction in Gieseler fluidity due to whathering of Pittshurgh Seam coal by oxidation at $80^{\circ} \mathrm{C}$ for the number of day: shown: (a) $\mathrm{CO}_{2}$ evolution of raw and weathered coal; (b) prediclial and measured fluidity (measurements from ref. 78)
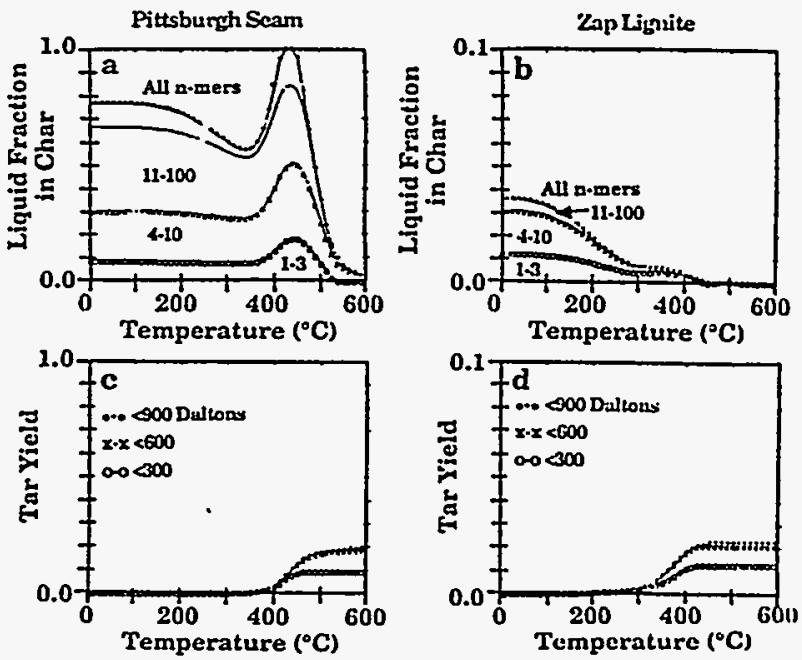

Figure 27 Predicted molecular weight distribution of macromolecular network fragments in char and tar for a bituminous coal and a lignite 
the lignite, the metaplast distribution limıs the amount and molecular weight distribution. Figtures $28 \mathrm{c}$ and $28 \mathrm{~d}$ show measurements for the Beulah Zap lignite and the Pittsburgh Seam bituminous coal pyrolysed in the f.i.m.s. apparatus, in which the tar is ionized immediately upon evolution and is directly transported to the mass spectrometer, so thë spectra are a good indicator of the tar molecular weight distribution. The data have been summed over $50 \mathrm{u}$ intervals. Whereas the Pittsburgh bituminous coal shows a peak intensity at $\sim 400 \mathrm{u}$ the lignite peak is at $100 \mathrm{u}$. The predicted average tar molecular weight distributions are in good agreement with T.i.m.s. data, as shown in Figures $28 a$ and $28 b$. In the FG-DVC model, the same monomer distribution is used for both the lignite and bituminous coal. Thus the greater fall in amplitude with increasing molecular.weight for the lignite than for the bituminous coal must be due to early cross-linking and transport effects in the lignite.

Pressure effects. The predicted effect of pressure on the tar molecular weight distribution is illustrated in Figures $29 a$ and $29 b$. Pressure enters the model through the transport assumption. The internal transport rate is inversely proportional to the ambient pressure. The reduced transport rate reduces the evolution rate of the heavier molecules. Therefore the average molecular weight and vaporization 'cutoff' decrease with increasing

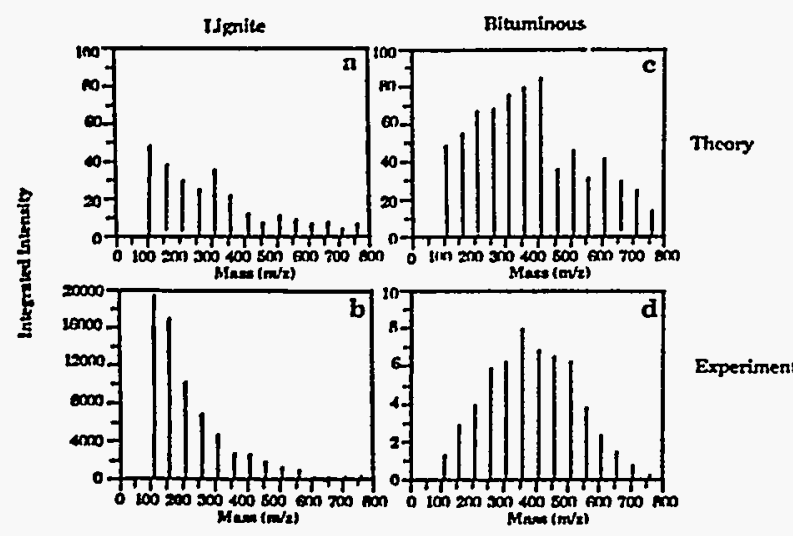

Figure 28 Comparison of measured and predicted tar molccular weight distributions for Zap lignite and Pittsburgh Seam bituminous coal pyrolysed in an f.i.m.s. apparatus. Intensities are summed over $50 \mathrm{u}$ intervals

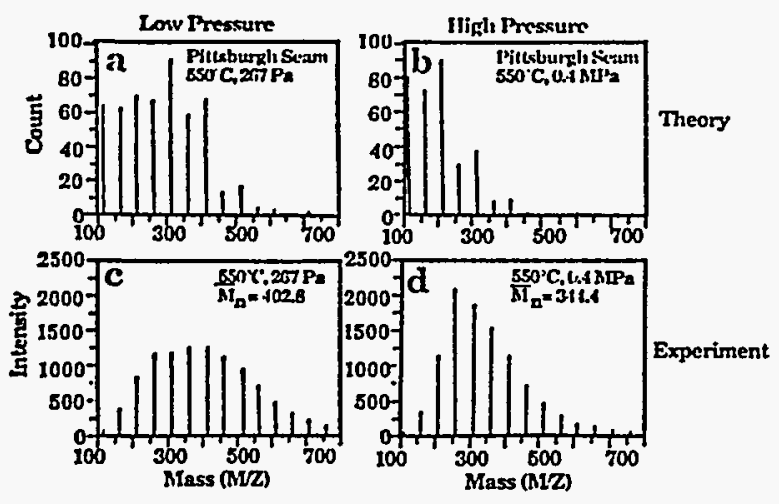

Figure 29 Comparison of predicted (a, b) and measured (c, d) tar molecular weight distributions for Pittsburgh Seam coal pyrolysed in a heated-grid apparatus at $500 \mathrm{~K} \mathrm{~s}^{-1}: \Delta P=0.2 \mathrm{~atm}$

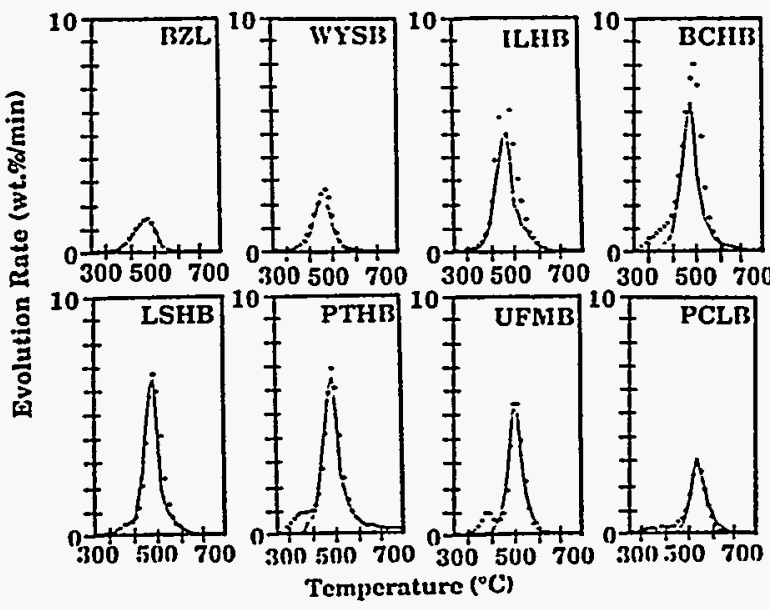

Figure 30 Comparison of measured (points) and predicted (-) t.g.-FT-i.r. analysis of tar evolution for the eight Argonne premium coals. BZL. Beulah Zap lignite: WYSB, Wyodak Anderson subbituminous: ILHB. Illinois No. 6 hvb: BCHB, Blind Canyon hvb; LSHB. Lewiston-Stockton hvb: PTHB, Pittsburgh No. 8 hvb:UFMB. Upper Freeport mub; PCLB. Pocahontas No. 3 lvb

pressure. The amount of tar also decreases because of the inefficient use of the available hydrogen in stabilizing small tar molecules. The predicted trends are in reasonable agreement with the observed tar molecular weight distributions shown in Figures $29 c$ and $29 d$. The spectra are for previously formed tars analysed by f.i.m.s. ${ }^{6}$. The low intensities between 100 and $200 \mathrm{u}$ are believed to be due to loss of these components as a result of their higher volatility.

Yield. The evolution rates measured with t.g.-FT-i.r. are compared in Figure 30 with the predicted values as a function of temperature for the eight Argonne premium coals. These are the data used in establishing the kinetic rates, so the agreement between the theory and experiments is within the $\pm 10 \mathrm{~K}$ accuracy of the experiment. However, the low-temperature evolution of guest molecules (see peaks and shoulders between 300 and $400^{\circ} \mathrm{C}$ for Blind Canyon, Lewiston Stockton, Pittsburgh and Upper Freeport) is not well predicted in the standard model. Improvements to predict this early peak have been published elsewhere.

\section{Netirork parameters}

As discussed under 'Summary of the FG-DVC model', there are thrce network parameters $l, M_{\mathrm{c}}$ and $\mathrm{H}_{21}$ which are adjusted to fit the laboratory characterization data (t.g.-FT-i.r. tar yield, extract yield and Gieseler fluidity). Whether or not these parameters have any physical or chemical meaning or whether they are only adjustable curve-fitting parameters remains to be seen. To address this question, it is useful to examine their dependence on rank. Figure 31 shows the adjustable network parameters as functions of the oxygen content of the coal. The oligomer length $l$ and the molecular weight between crosslinks $M_{\mathrm{c}}$ increase smoothly with rank. The concentration of available hydrogen $\mathrm{H}_{\mathrm{al}}$ for ring stabilization has a maximum for the high-volatile bituminous coals.

Figure $32 a$ compares the predicted extract yield required to obtain the correct Gieseler fluidity and the 

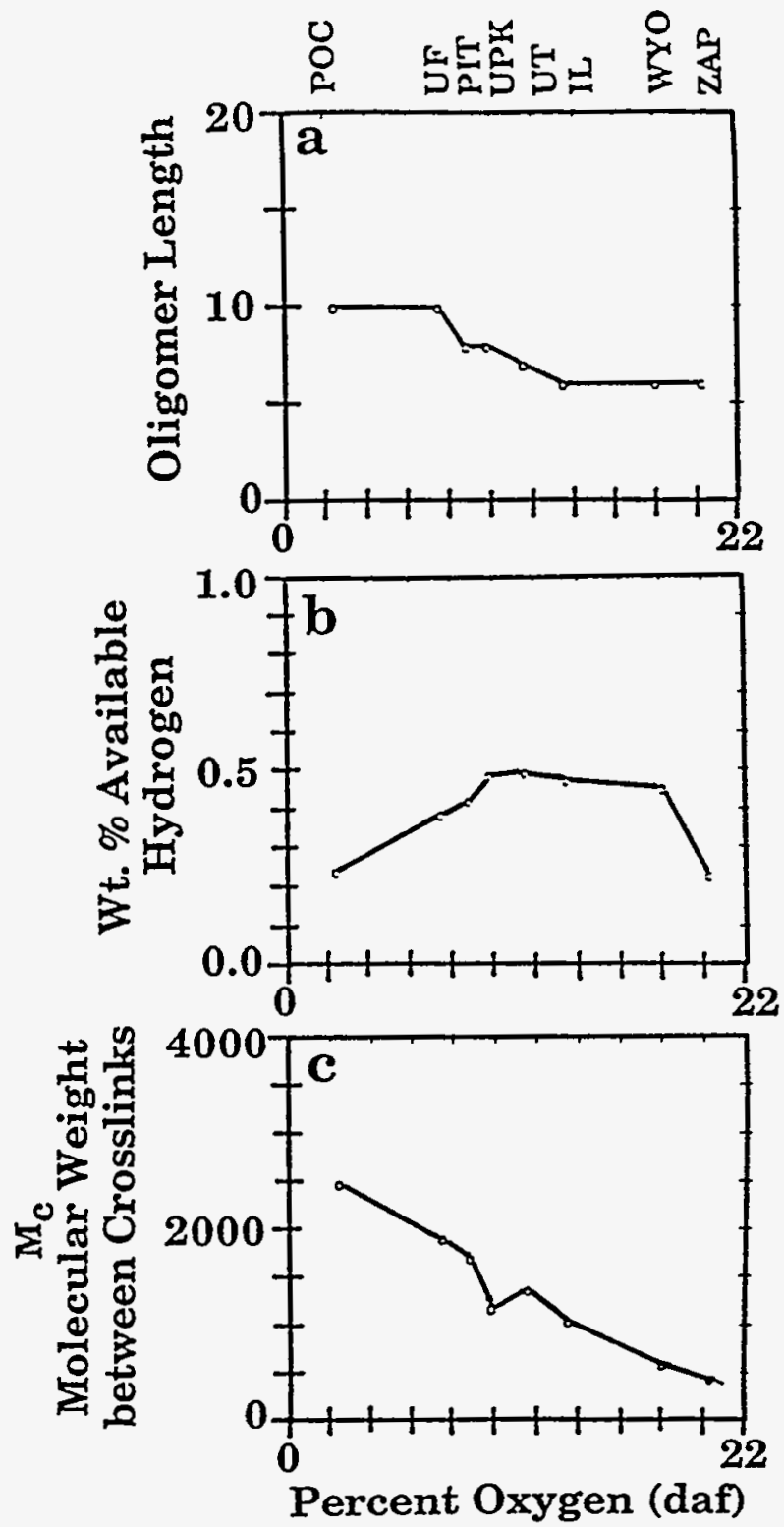

Figure 31 'Network parameters for Monte Carlo versiun of FG-DVC model for the eight Argonne premium coals, as a funclion of the oxygen content of the coal. The order of the coals is the same as in Figure 17

measured extract yield. The model requires a higher extract yield for Upper Frecport and Pocahontas coals than the measured yield. The problem is that there may be an additional kind of weak cross-link for high-rank coals, which affects the solvent extraction experiments at low temperature but not the Gieseler fluidity. Such weak cross-linking could be the aromatic-aromatic interactions suggested by Larsen ${ }^{79}$. When the Upper Freeport coal is heated to $300^{\circ} \mathrm{C}$ and then cooled, the solvent swelling ratio increases from 1.32 to 2.13 and the extract yield from 10.4 to $21 \mathrm{wt} \%$, suggesting that this treatment may loosen some of these weak bonds irreversibly.

Figure 32h compares the predicted and estimated (from solvent swelling experiments) molecular weight between cross-links. The cross-link density for the bituminous coals is within the range of measured values. However, the model requires a high molecular weight between

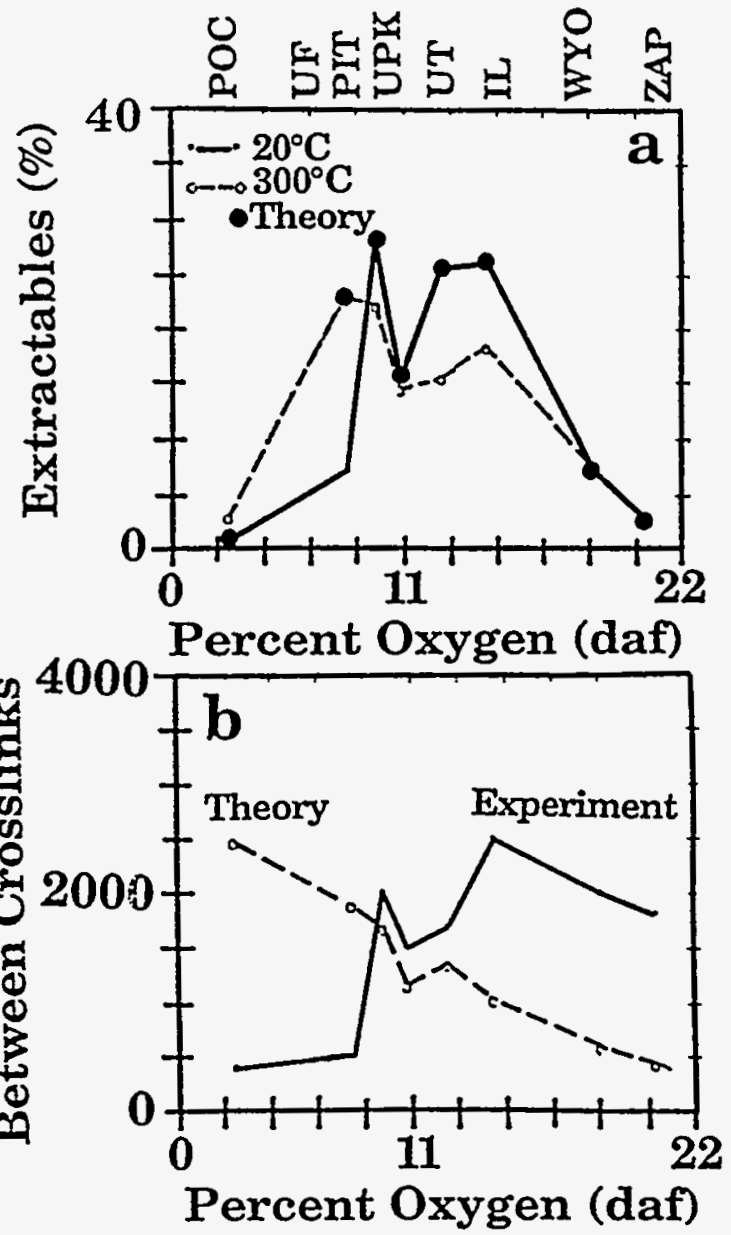

Figure 32 Comparison of experimental and theoretical parameters for the eight Argonne premium coals, as a function of the oxygen content of the coal. The order of the coals is the same as in Figure 17

crosslinks for Pocahontas and Upper Freeport, whereas the solvent swelling ratios would indicate a low $M_{\mathrm{c}}$. This discrepancy probably has the same cause as discussed above for the solvent extract. The predicted $M_{\mathrm{e}}$ values for the low-rank coals are much lower than those derived from solvent swelling experiments. There are two possible explanations. First, the theories for deriving $M_{\mathrm{s}}$ from solvent swelling are highly controversial. Second, the predicted values are not well-defined, because of the lack of Gieseler fluidity data and the presence of polymethylenes in the extracts of low-rank couls which are unrelated to unattached macromolecular network fragments corsidered in the model. These discrepancies cannot be resolved without further study.

\section{SUMMARY AND CONCLUSIONS}

The paper poses the question: can coal science be predictive? The answer is 'yes' for coal thermal decomposition in particles small enough to be isothermal. It is possible to construct a model based on reasonable assumptions to predict almost all of the observed behaviour. The model has only one parameter (the internal pressure in the transport submodel) which is adjusted for the process conditions. All other model parameters of the coal are fixed for each coal, and are 
based on simple laboratory experiments. The model has composition and kinetic parameters to describe the evolution of each individual gas species. These can be determined in t.g.-FT-i.r. experiments and exhibit a systematic variation with rank. There are three network parameters in the Monte Carlo version of the model: the chain length $l$, the molecular weight between cross-links $M_{c}$, and the available donatable hydrogen $\mathrm{H}_{21}$. A similar set of network parameters is used in the percolation theory. They are adjusted to fit the t.g.-FT-i.r. tar yields, extract yiclds and fluidity. These also exhibit a systematic variation with rank.

The paper explores the six concepts that are the foundation of the FG-DVC model:

1. The thermal decomposition of functional group sources in the coal yields the light gas species. The amount and evolution kinetics can be measured by t.g.-FT-i.r., and the functional group changes by FT-i.r. and n.m.r. (CP-MAS-DD). There is good agreement between the model and the results of these measurements on Pittsburgh Scam coal heated at $30 \mathrm{~K} \mathrm{~min}^{-1}$ and for gas evolution for a lignite and Illinois No. 6 bituminous coal at $36, \mathrm{~K} \mathrm{~min}^{-1}$ and $2 \times 10^{4} \mathrm{~K} \mathrm{~s}^{-1}$.

2. The decomposition of a macromolecular network yields tar and metaplast. The amount and kinetics of the tar evolution can be measured by t.g.-FT-i.r. and the molecular weight by f.i.m.s. The kinetics of metaplast formation and destruction can be measured by solvent extraction, Gieseler plastometry and proton magnetic resonance thermal analysis (p.m.r.t.a.). Reasonable agreement has been demonstrated for the solvent extract of Pittsburgh Seam coal pyrolysed at $30 \mathrm{~K} \mathrm{~min}^{-1}$ and $640 \mathrm{~K} \mathrm{~s}^{-1}$. Good agreement is shown for four of the Argonne premium coal samples for Gieseler fluidity, and for one coal by p.m.t.r.a.

3. The molecular weight distribution of the metaplast depends on the network coordination number. The coordination number can be determined by solvent swelling and n.m.r. (CP-MAS-DD).

4. The network decomposition is controlled by bridge breaking, and the amount of bridge breaking is limited by the available donatable hydrogen $H_{21}$.

5. The network solidification is controlled by cross-linking. The changing cross-link density can be measured by solvent swelling and n.m.r. (CP-MAS-DD). Crosslinking appears to occur with evolution of both $\mathrm{CO}_{2}$ (before bridge breaking) and $\mathrm{CH}_{4}$ (after bridge breaking). Thus low-rank coals (which form much $\mathrm{CO}_{2}$ ) cross-link before bridge breaking and are thus thermosetting. High-volatile bituminous coals (which form little $\mathrm{CO}_{2}$ ) undergo significant bridge breaking before cross-linking and become highly fiuid. Weathering, which increases the $\mathrm{CO}_{2}$ yield, causes increased cross-linking and lowers fluidity. There is good agreement between the predicted and measured cross-link densities and fluidities in the FG-DVC model in which cross-links are correlated with $\mathrm{CO}_{2}$ and $\mathrm{CH}_{4}$ gas evolution.

6. The evolution of tar is controlled by mass transport in which the tar molecules evaporate into the light gas species and are carried out of the coal at rates proportional to their vapour pressure and the volume of light gases. High pressure reduces the volume of light gases and hence reduces the yield of heavy molecules with low vapour pressure. These changes can be studied with f.i.m.s. The changes in tar yield and molecular weight distribution with pressure have been accurately predicted using the vapour pressure law of Suuberg and co-workers.

Although the experimental results and the model are consistent with the suggested processes, the chemical reactions for bridge breaking, cross-linking and functional group decomposition are not defined in detail. Also there are only sparse data to validate the transport assumption, and the internal pressure in the particle is an adjustable parameter of the model.

\section{ACKNOWLEDGEMENTS}

The authors wish to acknowledge the following organizations for their support over the last 10 years: US Department of Energy, Morgantown Energy Technology Center; US Department of Energy, Pittsburgh Energy Technology Center; National Science Foundation; and Gas Research Institute. The authors also wish to acknowledge the extensive contribution from many co-authors who have collaborated in this work: Robert Carangelo, James Markham, Philip Best, Erik Kroo, Yan Ping Zhang, Rosemary Bassilakis, Marie DiTaranto, Girish Deshpande, Po-Liang Chien. H. H. King, Tom Squires and Kevin Squire, who worked at Advanced Fuel Research, Inc.; Meredith Colket from United Technologies Research Center; Douglas Smoot and Scott Brewster from Brigham Young University: Eric Suuberg from Brown University; Ronald Pugmire, David Grant and Mark Solum from the University of Utah; Donald McMillen and Ripudaman Malhotra from SRI International: Rashid Khan from Texaco; Tom Fletcher from Sandia National Laboratory; Leo Lynch and Richard Sakurovs from CSIRO; Bernie Gerstein from Iowa State University: Jean Whelan from Woods Hole Oceanographic Institute; Harold Schobert and Caroline Burgess at Penn State University; Garry Vail, Fred Baudais, Michel Baillargeon and Daniel Gravel from Bomem, Inc.; and Dennis Gerson from IBM Instruments.

\section{REFERENCES}

1 Solomon. P. R., Serio. M. A.. Carangelo, R. M., Bassilakis, R., Gravel. D., Baillargeon. M., Baudais, F. and Vail. G. Energy Fuels 1990, 4. 319

2 Green, T. K., Kovac. J. and Larsen, 3. W. Fud 1984, 63. 935

3 Green, T. K.. Kovac. J. and Larsen. J. W. In 'Coal Structure' (ed. R. A. Meyers). Academic Press. Ncw York, 1982. p. 199

4 Gieseler plastometer data were supplied by Mr George Engelke of Commercial Testing and Engineering Company

5 Solum, M. A., Pugmire. R. J. and Grant, D. M. Energy Fuels 1989. 3. 187

6 St John, G. A.. Butrill, S. E. Jr and Anbar, M. Am. Chem. Soc. Symp. Ser. 1978, 71, 223

7 Solomon. P. R. Hamblen. D. G., Carangelo. R. M., Serio. M. A. and Deshpande. G. V. Energy Fuels 1988, 2.405

8 Solomon. P. R., Serio, M. A., Hamblen, D. G., Yu, Z. Z. and Charpenay, S. Am. Chem. Snc. Div. Fuel Chem. Preprints 1990, Charpenay, S. Ain. Chem. Soc. Dir. Fuel Chem. Preprints 1990. 35(2), 479

9 Solomon. P. R. Am. Chem. Suc. Adr. Chem. Ser. 1981, 192. 95

10 Solomon. P. R. and Hamblen. D. G. In Chemistry of Coal Conversion (ed. R. H. Schlosberg). PIenum Press, New York, 1985. pp. 121-251

11 Solomon. P. R.. Hamblen. D. G.. Carangelo. R. M. and Krause, J. L. In Ninetecnth Symp. IInt.) Combustion. Combustion Institute. Pittshurgh. 1982. p. 1131 
12 Strio, M. A.. Hamblen. D. (j.. Alurhham. J. R. and Sulomenn. P. R. Einerul Fu'ls 1987,1 , 13,

13 Solumon. P. R. and King. H. H. Fect 198. 6.3. 1302

It Squire, K. R.. Carangelo. R. M., DiTarantu. M. B. and Solomon. P. R. Fuel 1986. 65. 833

15 Solomon, P. R., Hamblen. D. G.. Carangelo, R.M., Serio, M. A. and Deshpande, G. V. Comibusi. Flame 1988. 71, 137

16 Solomon. P. R., Serio. M. A.. Carangelo. R. M.. Bassilakis. R., Yu, Z.Z., Charpenay, S. and Whelan, J. J. Antul. Appl. Pyrul. 1991. 19,1

17 Suuberg. E. M., Peters. W. A. and Howard.J. B. In 'Seventeenth Symp. (Int.) Combustion', Combustion Institutc. Pittsburgh, 1979. p. 117

18 Gavalas, G. R., Cheong, P. H. and Jain, R. Ind. Eng. Chem. Fundam. 1981, 20, 113

19 Gavalas, G. R., Cheong, P. H. and Jain, R. Ind. Eng. Chem. Fundam. 1981, 20, 122

20) Xu, W. C. and Tomita, A. Fuel 1987, 66, 627

21 Van Krevelen. D. W. 'Coal', Elsevicr, Amsterdam, 1961

22 Solomon, P. R., Squire, K. R. and Carangelo. R. M. In Int. Conf. Coal Science, Pergamon. Sydncy. 1985, p. 945

Brenner, D. Fuel 1985, 64, 167

Lucht, L. M. and Peppas, N. A. Fiuel 1987, 66, 803

Lucht, L. M., Larsen, J. M. and Peppas, N. A. Energy Fuels $1987,1,56$

26 Larsen, J. W. Am. Chem. Soc. Div. Fitul Chem. Preprints 1985, $30(4), 444$

27 Green, T., Kovac, J., Brenner, D. and Larsen. J. In 'Coal Structure' (ed. R. A. Meyers), Academic Press, New York, 1982, p. 199

28 Hall, P. J., Marsh, H. and Thomas, K. M. Fuel 1988, 67, 863

29 Sanada, Y. and Honda, H. Fuel 1966, 45, 295

30 Suuberg, E. M.. Yoshi, O. and Deevo, S. Am. Chem. Sic. Div. Fut Chem. Priprints 1988. 33(1). 387

.31 Gavalas, G. R. In 'Coal Pyrolysis', Elsevier, Amsterdam. 1982, p. 51

32 Solomon, P. R., Hamblen, D. G., Yu, Z. Z. and Serio, M. A. Fuel 1990, 69, 754

33 Niksa, S. and Kerstein, A. R. Combust. Flame 1986, 66, 95

34 Niksa, S. Combust. Flame 1986, 66, 111

35 Niksa, S. and Kerstein, A. R. Fuel 1987, 66, 1389

36 Grant, D. M., Pugmire, R. J., Fletcher, T. H. and Kerstein, A. R. Energy Fuels 1989, 3, 175

Nelson, P. F. Fuel 1987, 66, 1264

Calkins, W. H., Hagaman, E. and Zeldes, H. Fuel 1984,63, 1113

Calkins, W. H. and Tyler, R. J. Fuel 1984, 63, 1119

Calkins, W. H. Fuel 1985, 64, 1125

Calkins, W. H., Hovsepian, B. K., Drykacz, G. R., Bloomquist, C. A. A. and Ruscic, L. Fuel 1984, 63, 1226

Niksa, S. AIChE J. 1988, 34, 790

43 Solomon, P. R., Best, P. E., Yu, Z. Z. and Deshpande, G. V. Am. Chen. Soc. Div. Fuel Chem. Preprints 1989, 34(3). 895 Suuberg. E. M., Lee, D. and Larsen, J. W. Fuel 1985, 64, 1668 Suuberg, E. M., Unger, P. E. and Larsen, J. W. Energy" Fuels $1987,1,305$

Bockrath, B. C., Illig, E. G. and Eassell-Bridger, W. D. Energy Fuels 1987, 1, 227

47 Solomon, P. R., Serio, M. A., Deshpande, G. V. and Kroo, E. Energy Fuel: 1990, 4, 42 ts Sulumor: 1'. R. Am Chem. Sur. Symm. Sir. 1981, 169,61

t4 Brown. J. K.. Drydun. I. G. C., Dunewin. D. H., Joy, W. K. and Pankhurst, K. S. J. Inst. Fuet 1958, 31, 259

50) Orning. A. A. and Greifer. B. Fuel 1956. 35, 318

51 Freihaut, J. D., Proscia, W. M. and Seery, D. J. Energy Fuels $1989,3,692$

52 Anthony, D. B. and Howard, J. B. AIChE J. 1976, 22, 625

53 Sulomon, P. R.. Serio, M. A., Carangelo, R. M. and Markliam, J. R. Fuel 1986, 65, 182

54 Solumon, P. R. and Serio, M. A. In Fundamentals of the Physical-Chemistry of Pulverized Coal Combustion' (ed. J. Lahaye and G. Prado). Martinus NijholT, 1987, p. 126

55 Kobayashi. H. Howard, J. B. and Sarofim, A. F. In Sixteenth Symp. (Int.) Combustion. Cumbustion Institute. Pittsburgh. 1977, p. 411

56 Solomon, P. R. and Colket. M. B. In Seventeenth Symp. (Int.) Combustion, Combustion Institute, Pittsburgh, 1979, p. 131

57 Anthony, D. B.. Howard, J. B., Hottel. H.C. and Meissner, H. P. In Fifteenth Symp. (Int.) Combustion, Combustion Institute. Pjttsburgh, 1974, p. 1303

58 Badzioch, S. and Hawksley. P. G. Ind. Eny. Chem. Process Des. Der. 1970, 9, 521

59 Solomon, P. R., Fletcher, T.H. and Pugmire, R.J. Fuclin press

60 Solomon, P. R.. Sirio, M. A. and Suuberg, E. M. Prog. Energy' Combust. Sci. 1992, 18. 133

61 Fletcher, T. H. Combust. Flame 1989, 78, 223

62 Fletcher, T. H. Combist. Sci. Technol. 1989, 63, 89

63 Freihaut, J. D. and Proscia. W. M. Energy Fuels 1989, 3, 625

64 Solomon, P. R., Serio. M. A. and Markham, J. R. In Proc. Int. Conf. Coal Science, Tokyo, 1989. p. 575

65 Serio M. A.. Solomon, P. R. Charpenay, S, Yu, Z Z and Bassilakis, R. Am. Chem. Suc. Div. Fuel Chem. Preprints 1990. $35(3), 808$

66 Burnham, A.K., Oh, M.S., Crawford, R. W. and Samoun. A. M. Energy Fuels 1989, 3, 42

67 Stein, S. E., Robauch, D. A., Alfieri, A. D. and Miller, R. E. J. Am. Chem. Soc. 1982, 104, 6567

68 Solomon, P. R., Charpenay, S., Yu. Z. Z., Serio, M. A., Kroo, E., Solum, M. S. and Pugmire, R. J. In Proc. Int. Conf. Coal Science, Newcastle upon Tyne, 1991, p. 484

69 Lynch, L. J., Sakurovs, R., Webster, D. S. and Redlich, P. J. Fuel 1988, 67, 1036

70 Lynch, L. J., Webster, D. S., Sakurovs, R., Barton, W. A. and Maher, T. P. Fuel $1988,67,579$

71 Barton, W. A. and Lynch, L. J. Energy Fuels 1989, 3, 402

72 Fong. W.S., Peters. W.A. and Howard. J. B. Fuel 1986, 65, 25!

73 Sobkowwiak, M., Riesser, B., Given, R. and Painter, P. Fuel $1984,63,1245$

74 Riesser, B., Starsinic, M., Squires, E., Davis, A. and Painter. P. Fuel 1984, 63, 1253

75 Solomon, P. R. and Carangelo, R. M. Fuel 1988, 67, 949

76 Solomon, P. R. and Carangelo, R. M. Fuel 1982, 61, 663

77 Solomon, P. R., Best, P. E., Yu, Z.Z. and Charpenay, S. Emıryy Fuels 1992, 6, 143

78 Wu, M. M., Robbins, G. A., Winschel, R. A. and Burke, F. P. Energy Futls 1988, 2, 150

79 Larsen. J. W. Am. Chem. Soc. Dir. Fuel Chem. Preprints 1988. $33(1), 400$ 


\section{APPENDIX H}

\section{FBED-1 SUBROUTINES}

Subroutine

Name

ADJSTY0

ASHCP

ASHH

AVGATW

BALANC

CALC

\section{Description}

Adjusts the functional group composition of the selected standard coal to match the ultimate composition of the feed coal.

Initializes the coefficients for the polynomial expression for the ash heat capacity based on either Kopp-Neumann rule or Kirov's expression.

Computes the total enthalpy of ash.

Computes the average atomic weight based on the ultimate composition.

Computes the overall mass, energy, and elemental balance for the gasifier.

This routine is the part of the CREE equilibrium subroutines library. It constructs the Newton-Raphson derivative matrix 
for equilibrium solution and solves it using pivotal Gaussian reduction.

COALIN Reads the input data for the devolatilization submodel.

CONVY

Converts the dependent variables to the quantities of interest, such as total gas enthalpy to gas temperature, total solid enthalpy to solid temperature etc.

CNVYUP Counterpart of CONVY during the upward integration pass.

CPUTMM Tracks the total CPU time since the start of the execution.

CREE Calculates chemical equilibrium composition for the gas phase.

CREE0 Part of the CREE equilibrium subroutines library. Reads and initializes the elemental and species input data and computes various parameters used in the chemical equilibrium calculations.

DDZONE Computes the ultimate yields and composition of gas, tar and char in the drying and devolatilization zone of the zerodimensional submodel.

DELTAP

Calculates the pressure drop based on Ergun's equation for packed beds.

DEVOLO Computes the devolatilization rates for the zero-dimensional submodel.

DEVOL1 Computes the devolatilization rates for the one-dimensional portion of the model. 
DLSODES

DRYING

ECHOIN

ELRATE

EQZONE

EQEXIT

EXITT

F

F0

FUP

FBEDOD
This is the Lawrence Livermore Solver for the Ordinary Differential Equations. It includes the sparse solver as well as the regular version. The auxiliary routines needed for LSODE or LSODES reside in the file dlsodes. $f$.

Computes the rate of evaporation of moisture and the heat of vaporization.

Echos the input data and writes the derived quantities to the output file fblout.

Converts the volumetric evolution rates to elemental basis.

Computes the composition and temperature of the streams, leaving the equilibrium zone of the zero-dimensional submodel.

Computes the temperature of the drying and devolatilization zone, assuming full chemical and thermal equilibrium.

Computes the temperature of the drying and devolatilization zone, keeping the devolatilized gases out of equilibrium.

Constructs the derivatives of the dependent variables for the downward integration pass.

Constructs the derivatives for the dependent variables for the zero-dimensional portion of the model.

Constructs the derivatives of the dependent variables for the upward integration pass.

Provides the initial estimates for the effluent gas and tar streams to be used as initial guess to convert the split boundary value problem to an initial value problem. 
FBED1D

FGDVC

FGSET

FREEBD

GASIN

GTEMP

HSCHAR

HCPS

HDEVOL

HFORM
The main program to predict detailed axial profiles for fixedbed gasification and combustion.

Computes the devolatilization rates based on the FG-DVC submodel developed by AFR. This subroutine calls RTS, which computes the kinetic rate of devolatilization and PERCVAP, which computes the tar evolution from the char. These and other FG-DVC auxiliary routines are lumped in the file fgdvc. $f$.

Computes the devolatilization rates based on the FG-SET submodel.

Calculates the heat exchange between the gas and the solid phase in the free board zone.

Computes the composition and the temperature of the blast gas entering the gasifier.

Calculates the temperature of the gas phase given the enthalpy and composition.

Computes the sensible enthalpy of char based on Merrick's correlation.

Part of the CREE equilibrium subroutines library. Calculates the heat capacity, enthalpy and entropy of the gaseous species, as well as of the gas mixture.

Calculates the heat of devolatilization associated with tar and gas evolution from the char matrix.

Calculates the heat of formation of char or coal. 
THTAR

HTCOEF

HVH2O

IVALUO

IVALU1

IVIUP

MTCOEF

OMEGAD

OMEGAM

OXGAS

PDPND

PTEMP

RTSFXG
Computes the total enthalpy of tar.

Calculates the heat transfer coefficients for solid-to-gas, solid-to-wall, and gas-to-wall.

Computes the heat of vaporization of moisture at the solid temperature.

Initializes the dependent variables for the zero-dimensional portion of the model.

Initializes the dependent variables for the downward integration pass of the one-dimensional model.

Initializes the dependent variables for the upward integration pass of the one-dimensional model.

Calculates the mass transfer coefficients.

Computes the collision integral for diffusivity.

Computes the collision integral for viscosity.

Calculates the oxidation and gasification rates and associated heat exchange effects.

Computes the particle diameter and particle number density.

Calculates the solid temperature given the solid composition and enthalpy.

Calculates the kinetic rates for devolatilization, assuming that Gaussian remains fixed in shape. 
READIN

SET

SPECE

T2GEQZ

TCRACK

TRANSP

ULTCOM

WRTOD
Reads the input data for the fixed-bed model and computes basis parameters for later use.

Computes the potential tar forming fraction based on Ko's correlation.

Part of the CREE equilibrium subroutine library. This subroutine calls CALC to compute the corrections to chemical species and temperature.

Calculates the gas phase decomposition of tar to yield gas in equilibrium zone of the the zero-dimensional portion.

Calculates the gas phase decomposition of tar to yield gas.

Calculates the transport properties of the gaseous species and the gas mixture.

Computes the ultimate composition given the functional group composition of char or tar.

Prints the results of the zero-dimensional portion of the code to the output file fblout. 


\section{APPENDIX I}

\section{CODE VARIABLES}

A partial listing of variables used in FBED- 1 is included in this appendix. Variables used within the LSODE routines are not included. Most of the FBED-1 subroutines contain a list of code variables at the beginning. Units are included where appropriate. Also, uppercase letters are used to show how the code variable name was chosen (e.g., diach DIAmeter of reactor CHamber, $\mathrm{m}$ ).

Variables

$a(1)$

$a(1, J)$

absth2

abstol

acpash

adfco 2

adfaev

adfh2

adfhoo

adfhgw

adfhsg

adfhow

adfo2

alanda(1)

alpha

apd(1)
Description and Units

In of the pre-exponential factor for 1th functional group and bond-breaking kinetics.

composition of 1 th element in $\mathrm{Jth}$ functional group

amount of ABstactable $\mathrm{H2}$

ABSolute TOLerance used in LSODE

coefflclent A for heat CaPacity of ASH, ( $J / \mathrm{kg} \mathrm{K}$ )

ADJustable Factor for $\mathrm{CO}$ gasification reaction

ADJustable pactor for DEVolatilization rates

ADJustable Factor for 132 gasification reaction

ADJustable Pactor for H2O gasification reaction

ADJustable Pactor for Heat transfer between Gas and Fall

ADjustable Factor for Heat transfer between solld and Gas

ADJustable Factor for Heat transfer between solld and WaIl

ADJustable Factor for oxidation reaction

IAMbDA (Carbon stolchlometrle coefflclent for the oxidation

reaction) at 1th output point

coefficient ALPHA in Ro's correlation for $x 0$

Particle Diameter at the 1th output point, m 
Code Variables

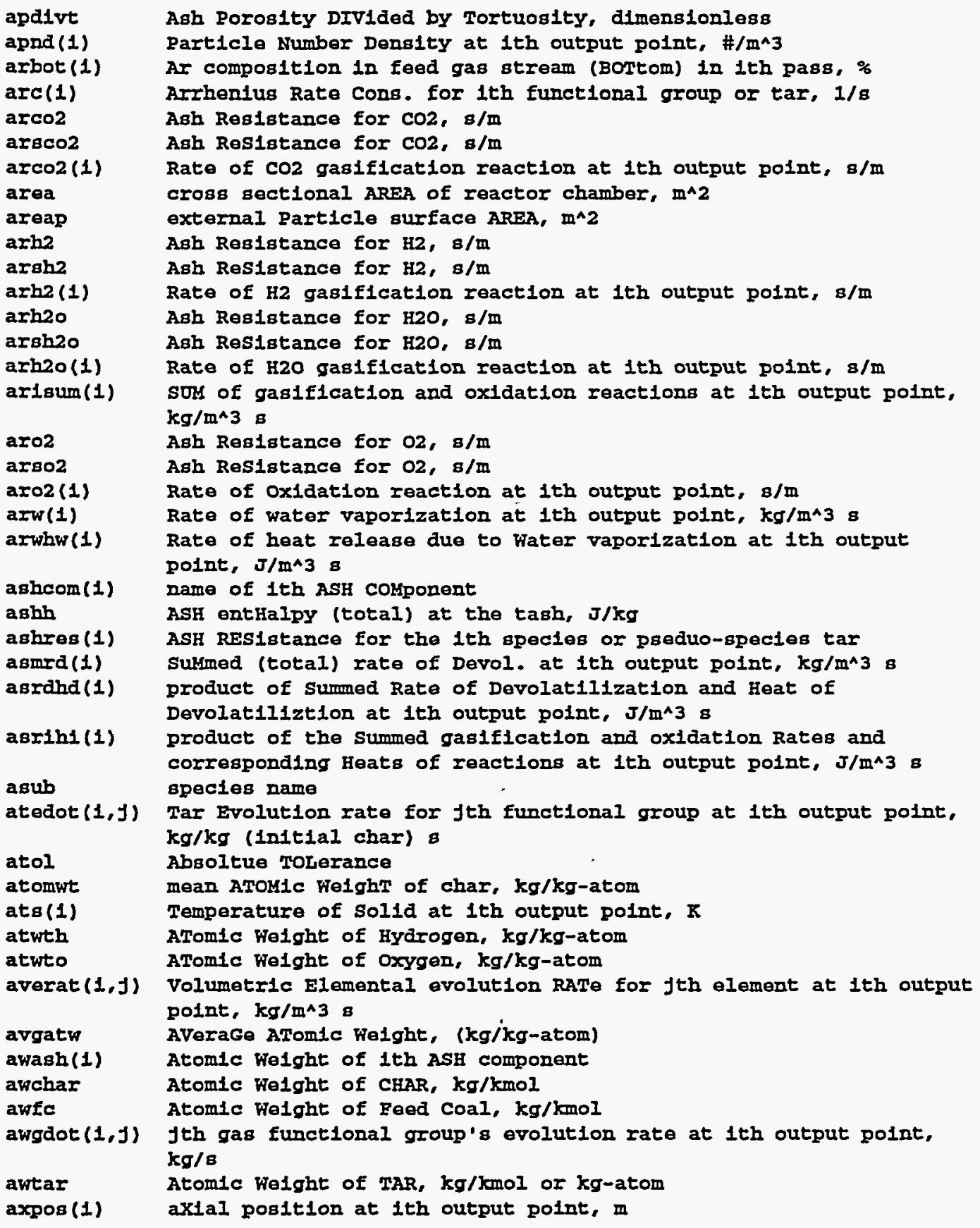


azrihl(1) product of $z 1$ and Heat of reaction for the oxidation and gasification reactions at ith output point, $J / \mathrm{m}^{\wedge} 3 \mathrm{~s}$

$b$ (1) Activation energy divided by $R$ for lth functional group and bondbreaking kinetics, $R$

bo(1) atom number for reactant mixture, $\mathrm{kmol} 1 / \mathrm{kg}$ mixture

bas1s

bcpash

beta

specifies whether ash composition 18 on mass or molar basis

coefficlent B for heat CaPaclty of ASH, (J/kg $K^{\wedge} 2$ )

effective length between centers of nelgbboring solid particles divided by equivalent diameter of the particles

beta

bfo(1)

bf1(1)

boutod

brnbot (1)

brnout

cepash

cfrac

coefficlent BETA in $\mathrm{XO} / \mathrm{s}$ correlation for $\times 0$

atom number for reed stream $0, \mathrm{kmol}$ (atom 1)/ $\mathrm{kg}$

atom number for peed stream 1, kmol (atom 1)/kg

Burn ODT for 0-dimensionl submodel

predicted BuRN ODT (at Bortom) in 1th pass

BuRN OUT for 1-dimensional model

coofficlent $C$ for hoat Capacity of ASH, ( $\mathrm{J} \mathrm{K} / \mathrm{kg}$ )

cg

cgco2

cgh2

cgh20

cgo2

ch4top (1)

chlgh

chrtot

chvol

$\operatorname{co} 2$ bot (1)

co2crc

instantaneous FRACtion of organic matter in daf coal

molar concentration of Gas, $\mathrm{kmol} / \mathrm{m}^{\wedge} 3$

molar $\mathrm{CO}$ Concentration in $\mathrm{Gas}, \mathrm{kmol}(\mathrm{CO} 2) / \mathrm{m}^{\wedge} 3$

molar H2 concentration in Gas, $\mathrm{kmol}(\mathrm{h} 2) / \mathrm{m}^{\wedge} 3$

molar h2o Concentration in Gas, $\mathrm{kmol}(\mathrm{b} 20) / \mathrm{m}^{\wedge} 3$

molar 02 concentration in Gas, $\mathrm{kmol}(02) / \mathrm{m}^{\wedge} 3$

CH4 composition in exit gas stream (at TOP) in ith pass, \% reactor chamber Lencth, m

TOTal instantaneous chaR functional groups

reactor chamber Volume, m^3

CO2 composition in feed gas stream (BoTtom) in 1th pass, \%

Chemical Rate constant for $\mathrm{CO} 2$ gasification, m/s

co2top (1)

$\operatorname{cotop}(1)$

$\operatorname{cp}(1)$

cpa (1)

$\mathrm{cpb}(1)$

$\operatorname{cpc}(1)$

cpg

cph201

cplash

cplig(1)

cpsout

cpsum

crco2

crh2

crh2o

cro2

CO2 composition in exit gas stream (at TOP) in ith pass, \%

co composition in exit gas stream (at TOP) in ith pass, \%

non-dimensional heat Capacity of spectes 1

heat capacity cofflcient A for 1th ash component, ( $/ \mathrm{kg} \mathrm{K}$ )

heat Capacity cofficient B for 1th ash component, (J/kg $k^{\wedge} 2$ )

heat Capacity cofficient $C$ for ith ash component, ( $\mathrm{J} / \mathrm{kg}$ )

heat Capacity of gas mixture, (J/kg K)

heat CaPacity of Liquid $\mathrm{H2O}, \mathrm{J} / \mathrm{kg} \mathrm{K}$

heat Capacity of Ifquid ASH, (J/Kg K)

heat Capacity of 1th IIQuid Ast component

heat CaPacity of ooTgoing Solids from the eq. zone, J/kg $K$

non-dimensional heat Capacity of gaseous mixture

Chemical Resistance for $\mathrm{CO}, \mathrm{s} / \mathrm{m}$

Chemical Resistance for $\mathrm{H} 2, \mathrm{~B} / \mathrm{m}$

Chemical Resistance for H2O, $8 / m$

Chemical Resistance for $02,8 / \mathrm{m}$

dafcrg

datafl

dateld

delab

inftlal DAP Charge of feed coal, $\mathrm{kg} / \mathrm{m}^{\wedge} 3$

name of selected (or user specified) DATA FiLe

DATE IDentification stamp

Binary polar contribution parameter for species $a$ and $b$

deltap

particle over pressure, FG-DVC parameter, atm 
Code Variables

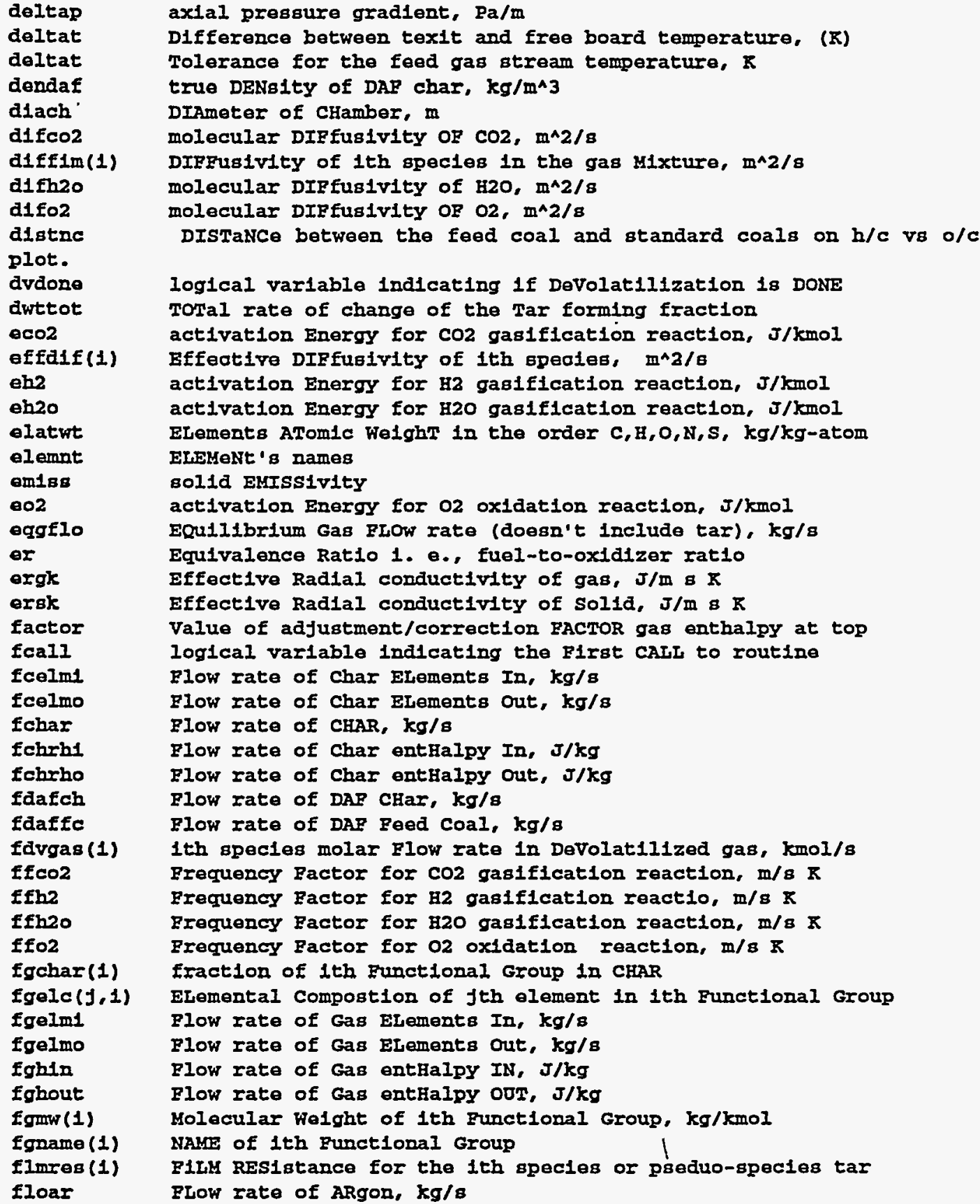




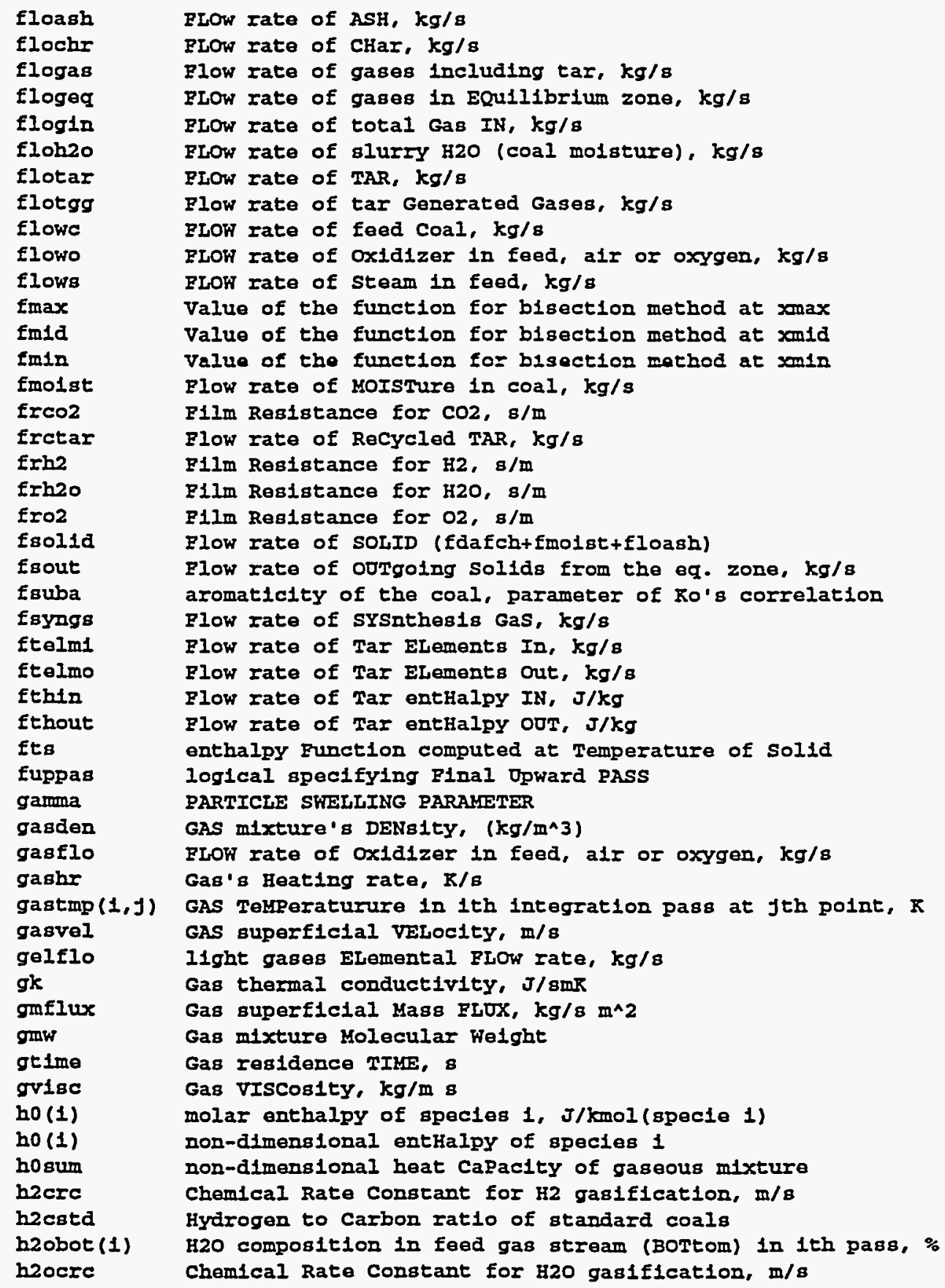


Code Variables

\begin{tabular}{|c|c|}
\hline $\begin{array}{l}\text { fash } \\
\text { fchar } \\
\text { ffc } \\
\text { fh2o } \\
\text { fh2ol } \\
\text { g1n } \\
\text { gtop } \\
\text { h2o } \\
\text { ho } \\
\text { lh2o } \\
\text { mash } \\
\text { max } \\
\text { molot } \\
\text { Ictar }\end{array}$ & 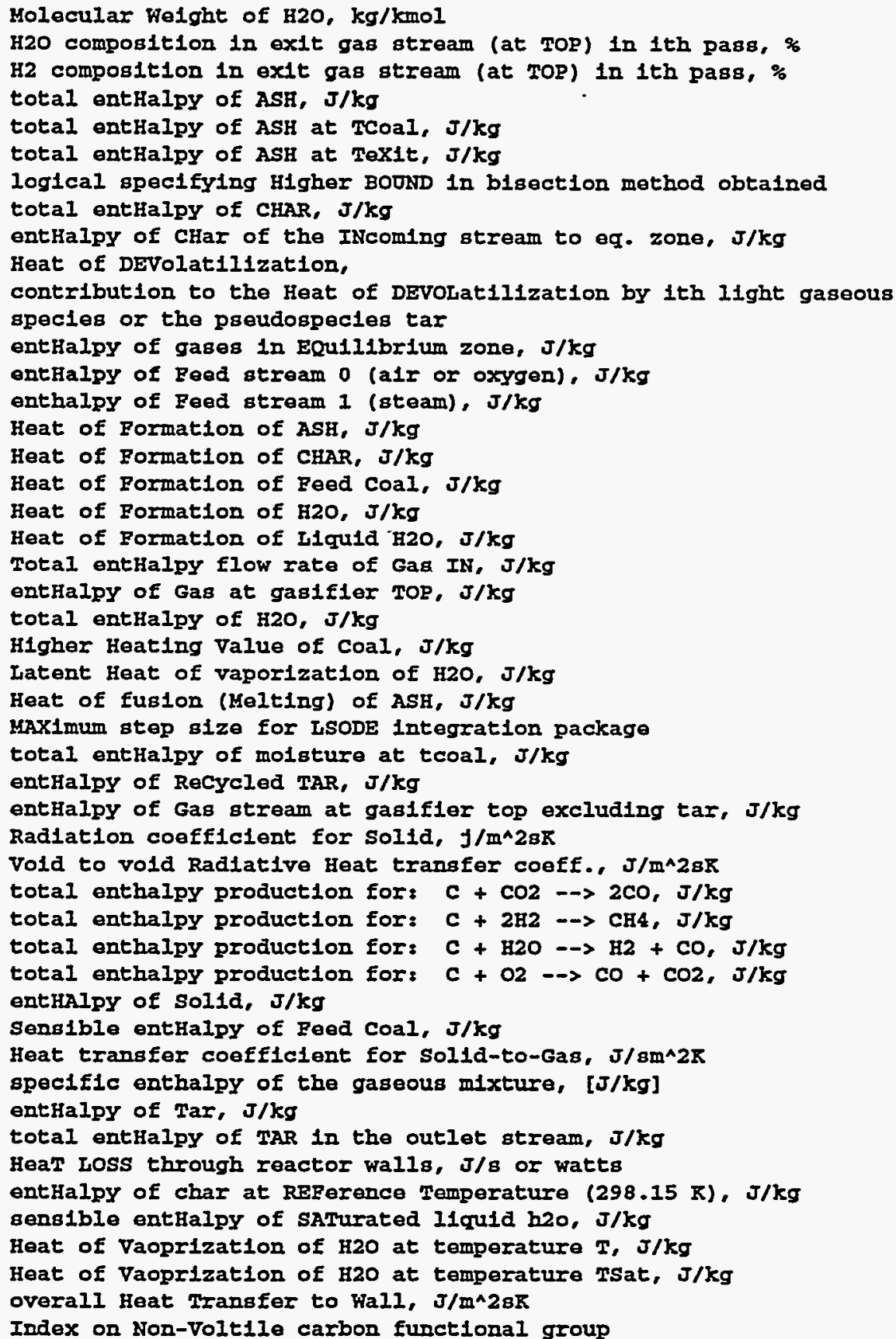 \\
\hline
\end{tabular}




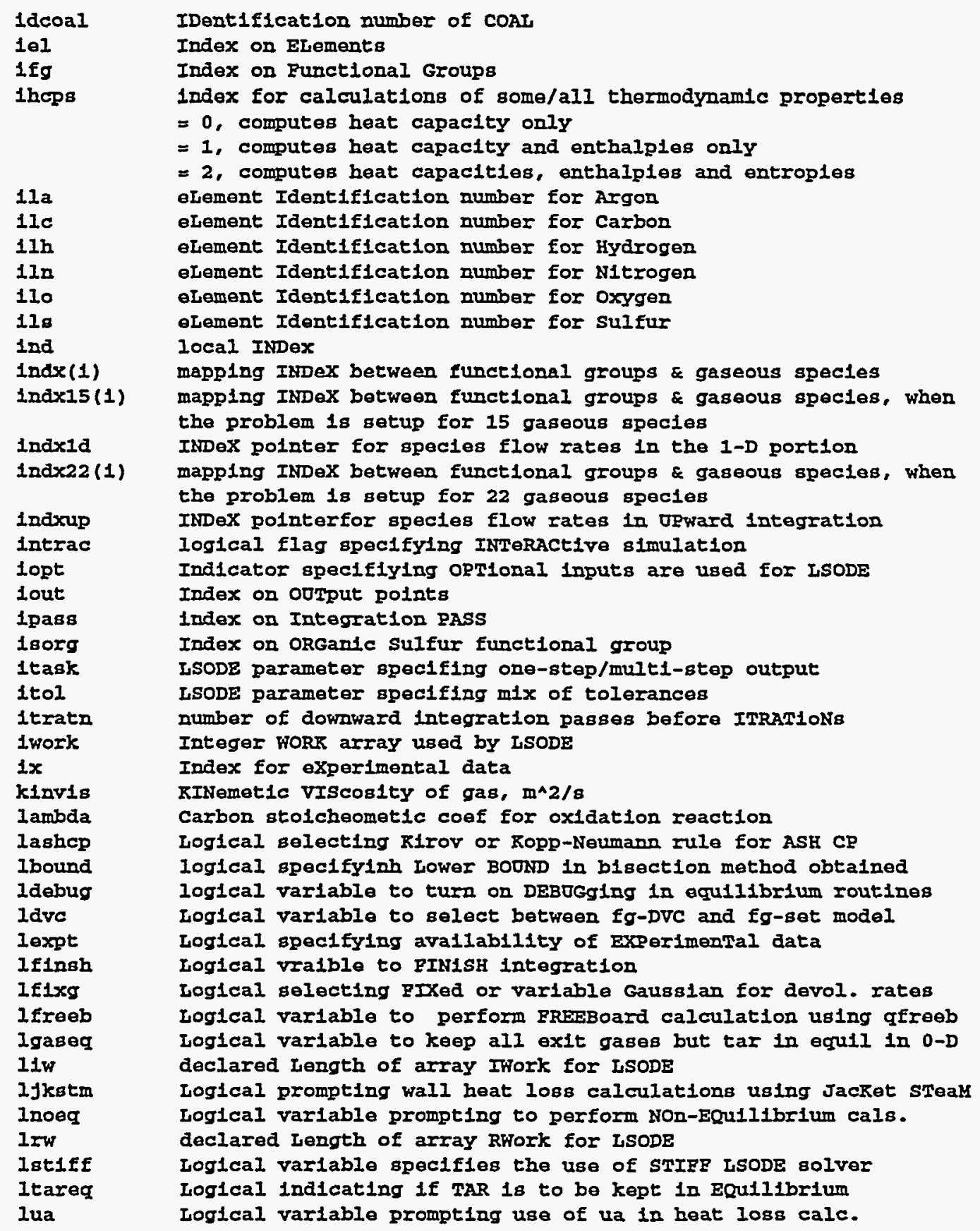


Code Variables

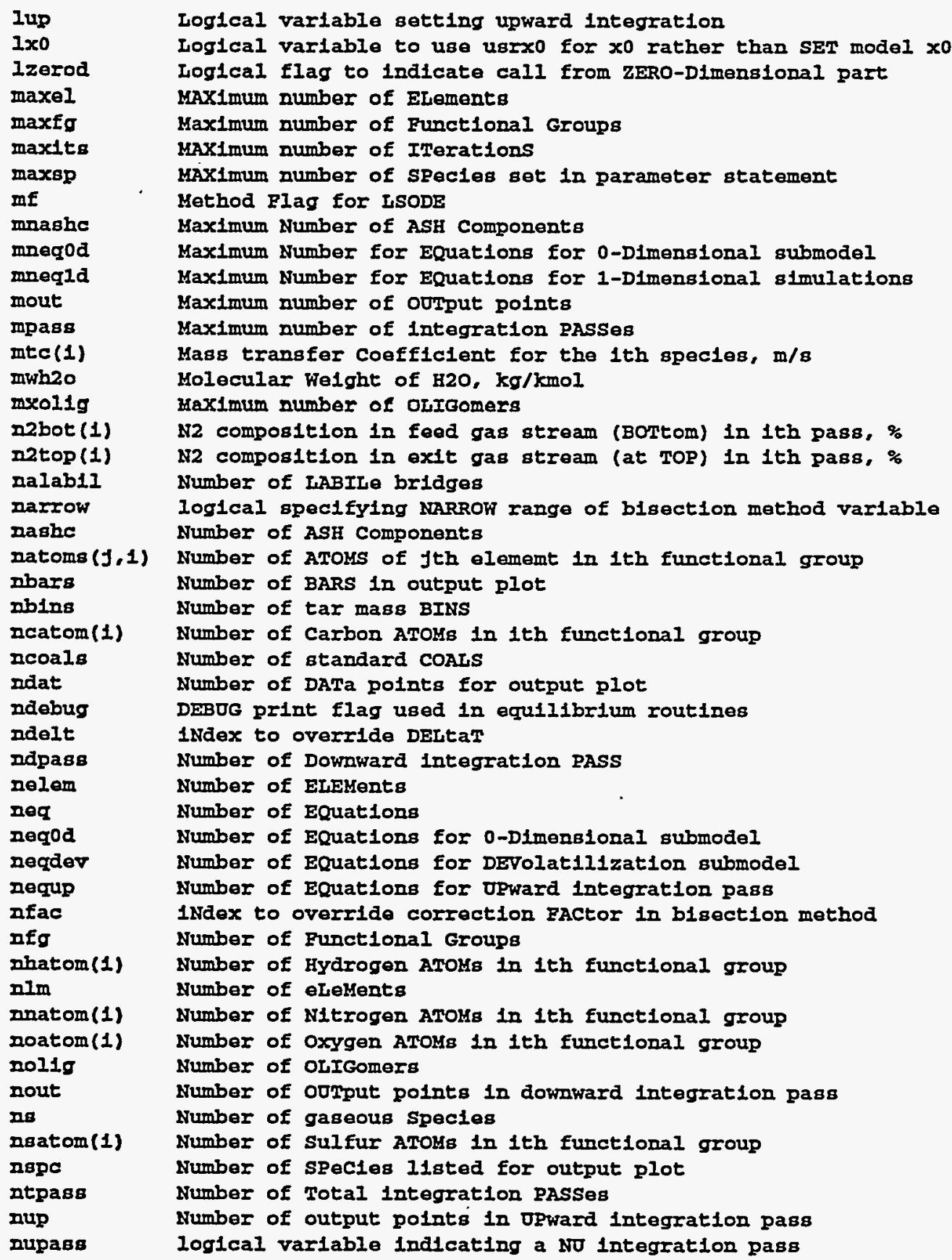


Appendix I

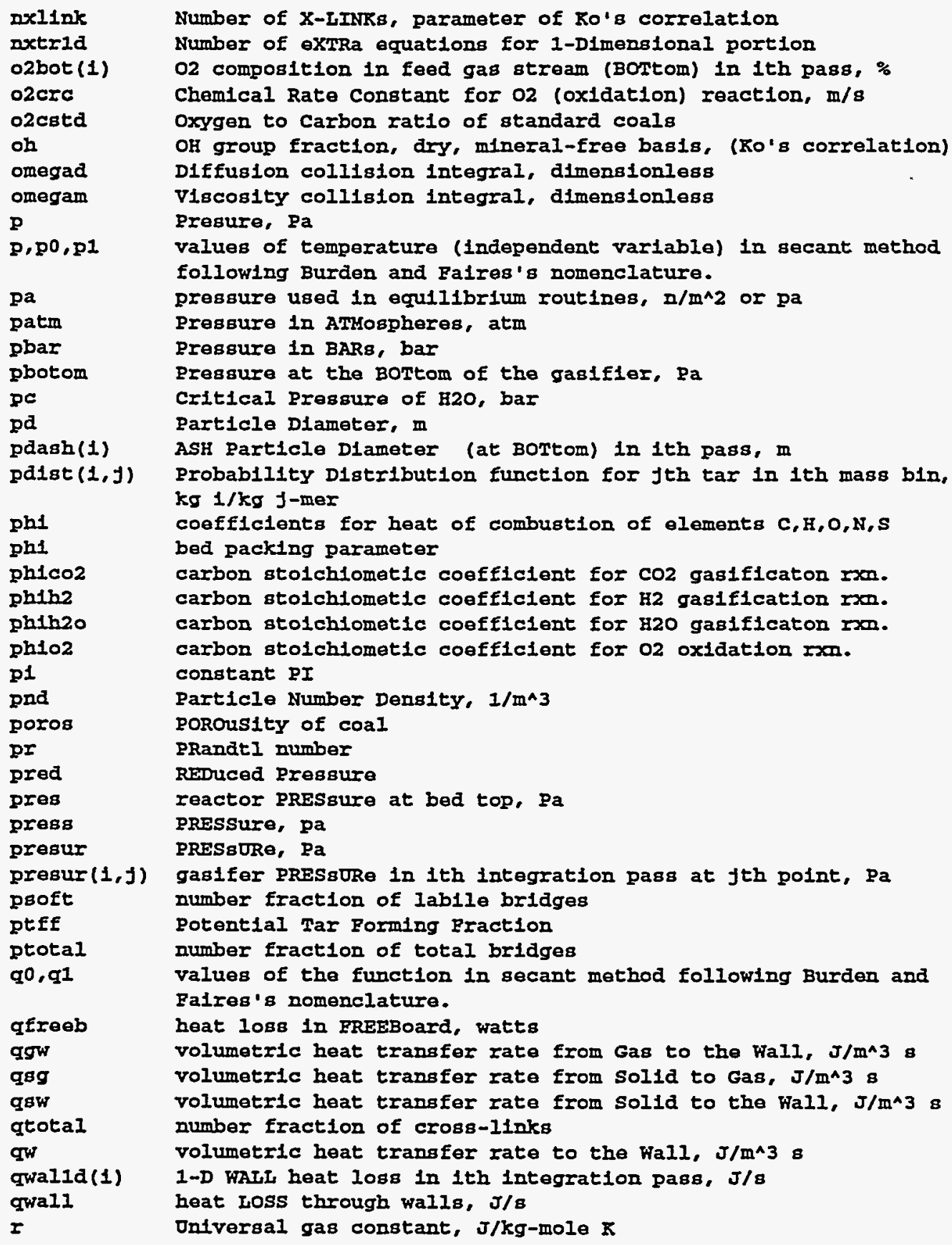


Code Variables

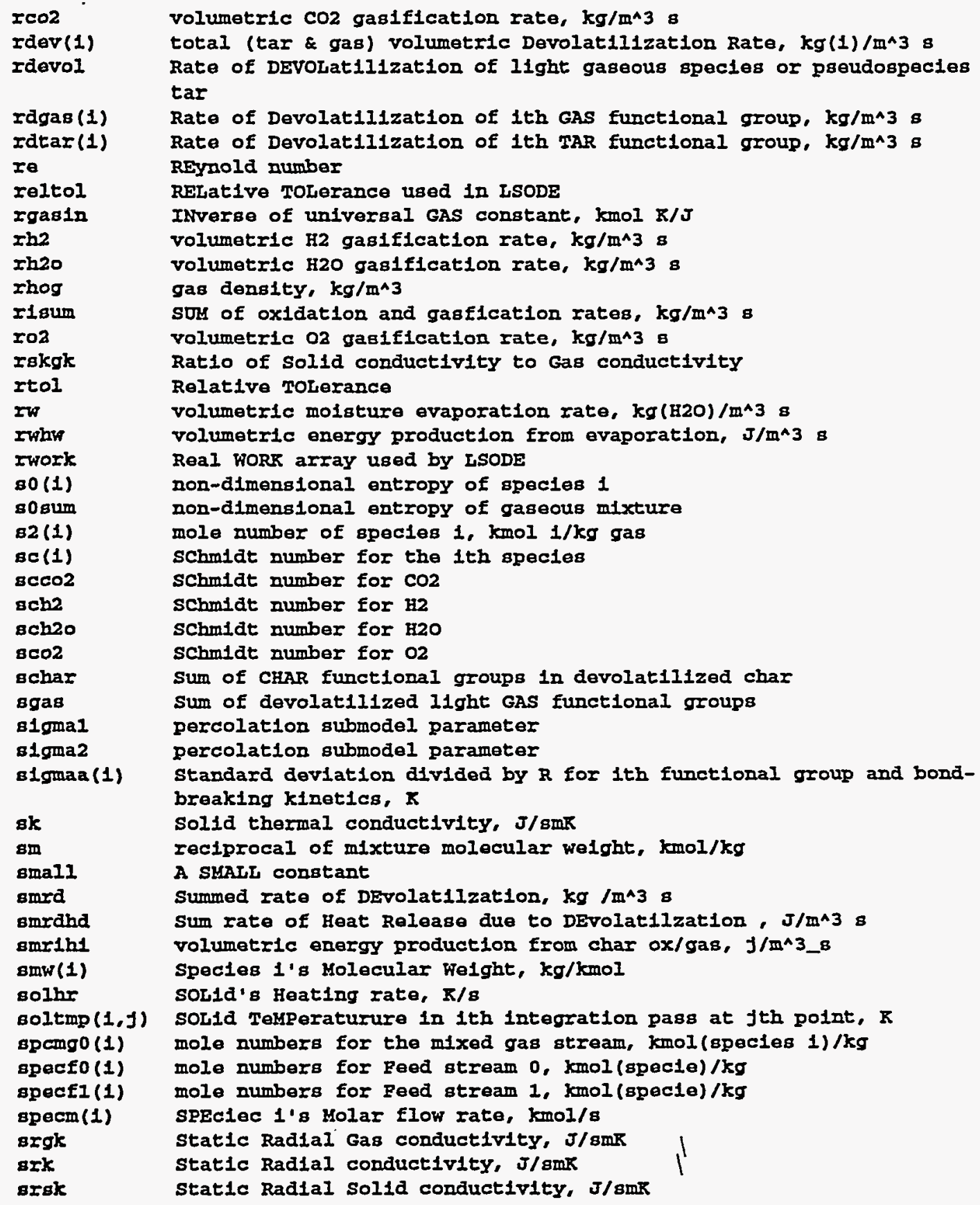




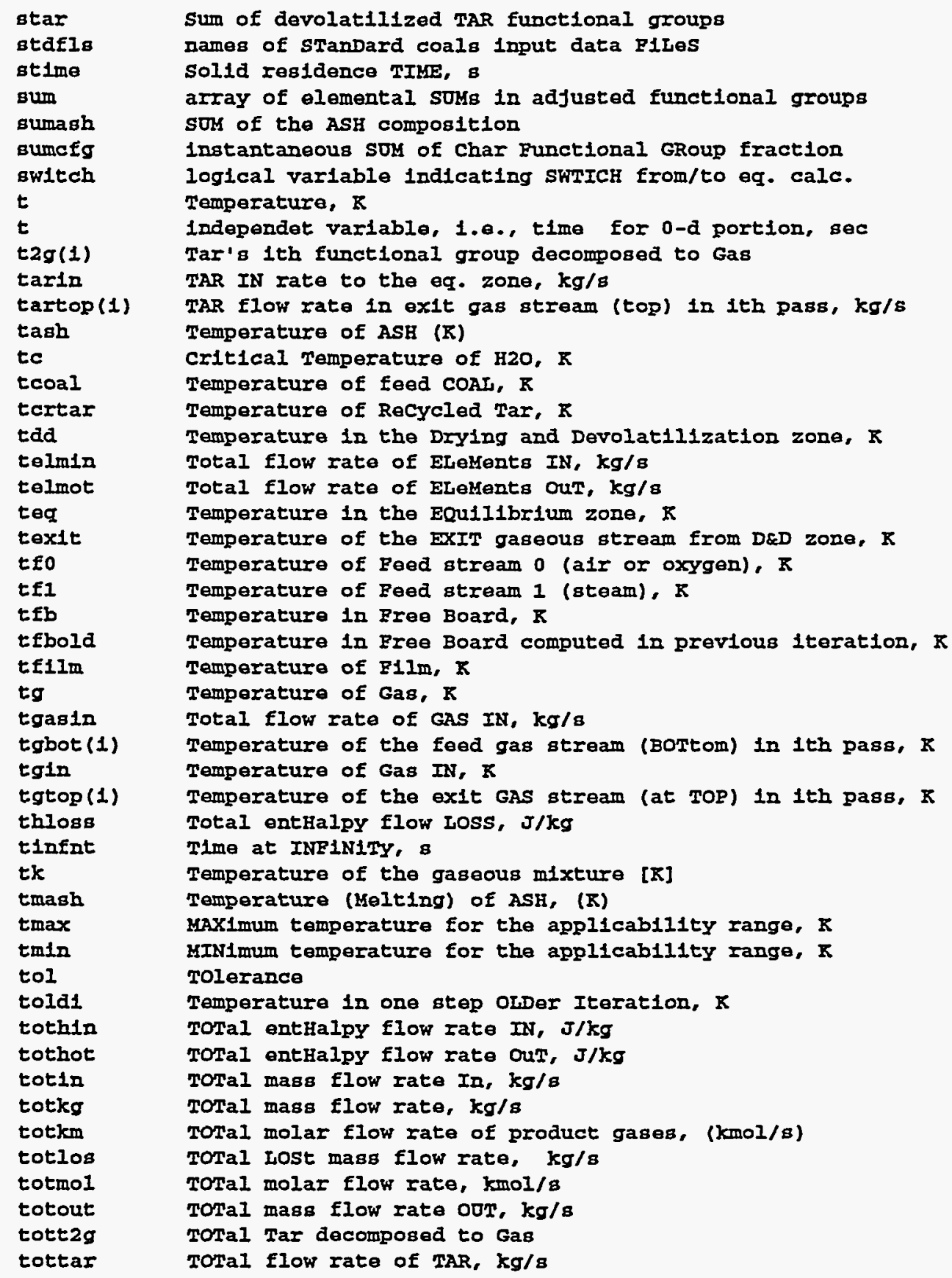


Code Variables

\begin{tabular}{|c|c|}
\hline 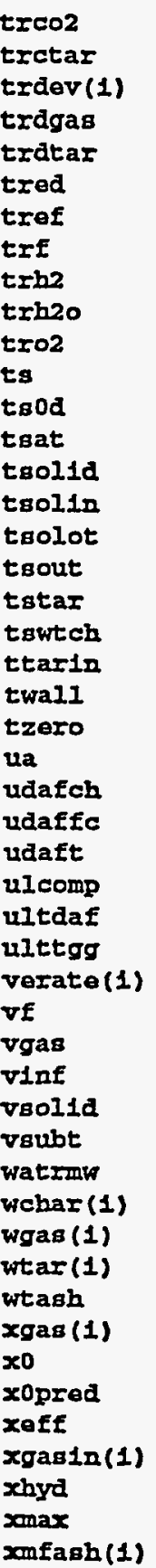 & 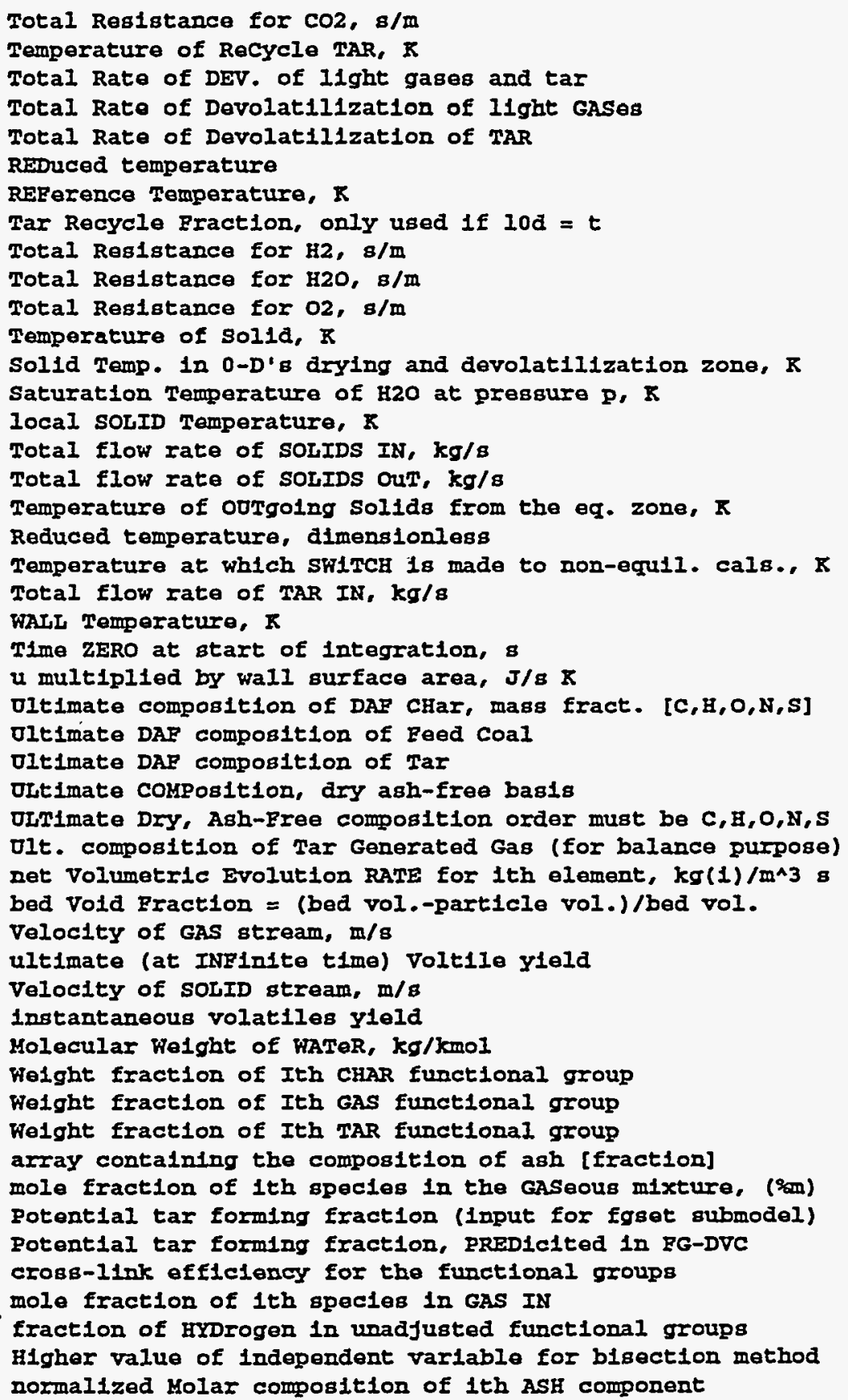 \\
\hline
\end{tabular}


Appendix I

\begin{tabular}{|c|c|}
\hline $\begin{array}{l}\text { xoid } \\
\text { xoin } \\
\text { xotrgn } \\
\text { xoxygn } \\
\text { xsurf } \\
\text { xtar } \\
\text { yo } \\
\text { yo } \\
\text { yot } \\
\text { yoneq(1) } \\
\text { yoav } \\
\text { yup } \\
\text { z } \\
\text { zbotom } \\
\text { zeta } \\
\text { zi } \\
\text { zirini }\end{array}$ & 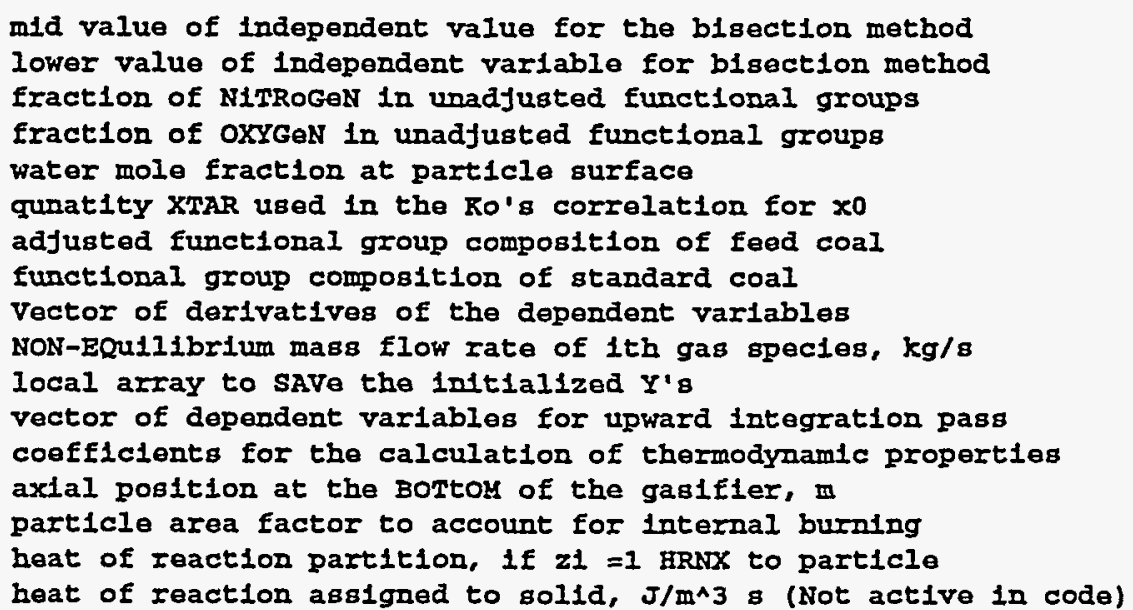 \\
\hline
\end{tabular}




\section{APPENDIX J}

\section{FBED-1 GRAPHICS}

FBED-1 code produces output in standard ASCII files. These files can be used in conjunction with any popular graphics package to plot the quantities of interest. In addition, two FORTRAN programs written for the DISSPLA graphics package are also distributed with the FBED-1 code. This Appendix includes the input files to setup these plots, and the procedure to compile, execute and print the plots. The reader is referred to the DISSPLA user's manual (Computer Associates, 1989) for the description of the subroutines.

The programs PLOT1 and PLOT2 generate the plots shown in Figures 6.1 and 6.2. The corresponding input files to set up these plots are PLOT1.DIM and PLOT2.DIM, as shown in Tables J.1 and J.2, respectively. These files contain the information regarding the names of the files containing the FBED-1 output data, as well as sizes and the locations of the subplots. All input data in these files are explained as well. The output of these programs is PLOT1.POP and PLOT2.POP respectively. These output files are in a device independent format and can be viewed or plotted on various output devices using a script program called POPOUT. The programs needed to run this script file are also distributed with the FBED-1 code. However, it is pointed out that the DISSPLA graphics package in our Research Center (ACERC, BYU) is available on a SUN computer, and some 
FBED-1 Graphics

Table J.1 Input file plotl.dim for the program plot 1

\begin{tabular}{|c|c|}
\hline 'ccons.out' & DATEILE1 \\
\hline 'pd1a.out' & DATRILE2 \\
\hline 'compos.out' & DATEILE3 \\
\hline 'tandp.out' & DATFILE4 \\
\hline 8.50 .11 .0 & XPAGE, YPAGE \\
\hline $2.75,2.75$ & XSIZE,YSIZE \\
\hline $0.0,0.5,2.25$ & $X S, X I, X F$ \\
\hline 1.50 .2 .00 & XORIGN (1), YORIGN (1) \\
\hline $0.0,0.10,0.495$ & $Y S(1), Y I(1), Y F(1)$ \\
\hline 4.30 .2 .00 & XORIGN (2), YORIGN (2) \\
\hline $0.0,0.5,2.95$ & $Y S(2), Y I(2), Y E(2)$ \\
\hline $1.50,4.80$ & XORIGN (3), YORIGN (3) \\
\hline $0.0,10.0,69.9$ & $Y S(3), Y I(3), Y F(3)$ \\
\hline $4.30,4.80$ & XORIGN (4), YORIGN (4) \\
\hline $0.0,0.2,0.895$ & $Y S(4), Y I(4), Y F(4)$ \\
\hline $1.50,7.60$ & XORIGN (5), YORIGN (5) \\
\hline $0.0,500.0,2500.0$ & $Y S(5), Y I(5), Y R(5)$ \\
\hline $4.30,7.60$ & $\operatorname{XORIGN}(6), \operatorname{YORIGN}(6)$ \\
\hline $102.0,1.0,104.0$ & $Y S(6), Y I(6), Y F(6)$ \\
\hline $0.0,0.5,2.7$ & YS2, YI2, YF2 \\
\hline $1.225,0.15$ & $X$ AND $Y$ COORDINATES FOR DRYING RATE CURVE LABEL \\
\hline $1,1.75,0.15,2.0,0$ & 2867 COORDINATES FOR THE ARROW FOR DRYING RATE CURVE \\
\hline $0.70,0.40$ & $X$ AND $Y$ COORDINATES FOR DEVOLATILIZATIONOLATILIZATION RATE \\
\hline $0.125,0.16$ & $X$ AND Y COORDINATES FOR OXIDATION RATE CURVE LABEL \\
\hline $0.975,0.05$ & $X$ AND Y COORDINATES FOR CO2 GASIFICATION RATE CURVE LABEL \\
\hline $1.1 .20,0.045,1.00$ & 01729 COORDINATES FOR THE ARROW EOR CO2 GASIFICATION CURVE \\
\hline $0.70,0.11$ & $X$ AND $Y$ COORDINATES FOR FOR H2O GASIFICATION CURVE LABEL \\
\hline $0,1.00,0.20$ & $X$ AND Y COORDINATES FOR FOR H2 GASIFICATION CORVE LABEL \\
\hline $1.00,0.55$ & $X$ AND Y COORDINATES FOR BURNOUT PRORILE ILABEL \\
\hline $0.80,2.10$ & $X$ AND $Y$ COORDINATES FOR PARTICLE DIAMETER PROEILE LABEL \\
\hline $0.725,1.15$ & $X$ AND $\bar{Y}$ COORDINATES FOR ONREACTED CORE DIAMETER PROFILE LAB \\
\hline $1.0,55.0$ & $X$ AND $\Psi$ COORDINATES FOR N2 COMPOSITION PROFILE LABEL \\
\hline $0.725,22.5$ & $X$ AND $Y$ COORDINATES FOR H2O COMPOSITION PRORILE LABEL \\
\hline $0.20,17.5$ & $X$ AND $Y$ COORDINATES FOR O2 COMPOSITION PROFILE ILABEL \\
\hline $0.40,3.5$ & $X$ AND Y COORDINATES FOR CO2 COMPOSITION PRORILE LABEL \\
\hline $1.60,14.5$ & $X$ AND Y COORDINATES ROR H2 COMPOSITION PRORILE ILABEL \\
\hline $1.7,25.5$ & $X$ AND Y COORDINATES FOR CO COMPOSITION PROFILE IABEL \\
\hline 1.85 .2 .50 & $X$ AND Y COORDINATES IOR CH4 COMPOSITION PROFILE IABEL \\
\hline $1.0,0.70$ & $X$ AND Y COORDINATES FOR AR COMPOSITION PROEILE LABEL \\
\hline $0.65,0.365$ & $X$ AND $Y$ COORDINATES FOR OH COMPOSITION PROEILE LAABEL \\
\hline $0.45,0.175$ & $X$ AND $Y$ COORDINATES FOR NO COMPOSITION PRORILE ILABEL \\
\hline $1.775,0.285$ & $X$ AND Y COORDINATES BOR H2S COMPOSITION PRORILE IIABEL \\
\hline $0.8,0.20$ & $X$ AND Y COORDIMATES FOR SO2 COMPOSITION PROFILE IABEL \\
\hline $1.80,0.145$ & $X$ AND Y COORDINATES FOR HCN COMPOSITION PROFILE ILABEI \\
\hline $0.90,2050.0$ & $X$ AND Y COORDINATES ROR GAS TEMPERATURE CURVE IABEL \\
\hline $1.25,1300.0$ & $X$ AND Y COORDINATES FOR SOLID TEMPERATURE CORVE ILABEL \\
\hline
\end{tabular}


Table J.2 Input data file plot2.dim for the program plot2

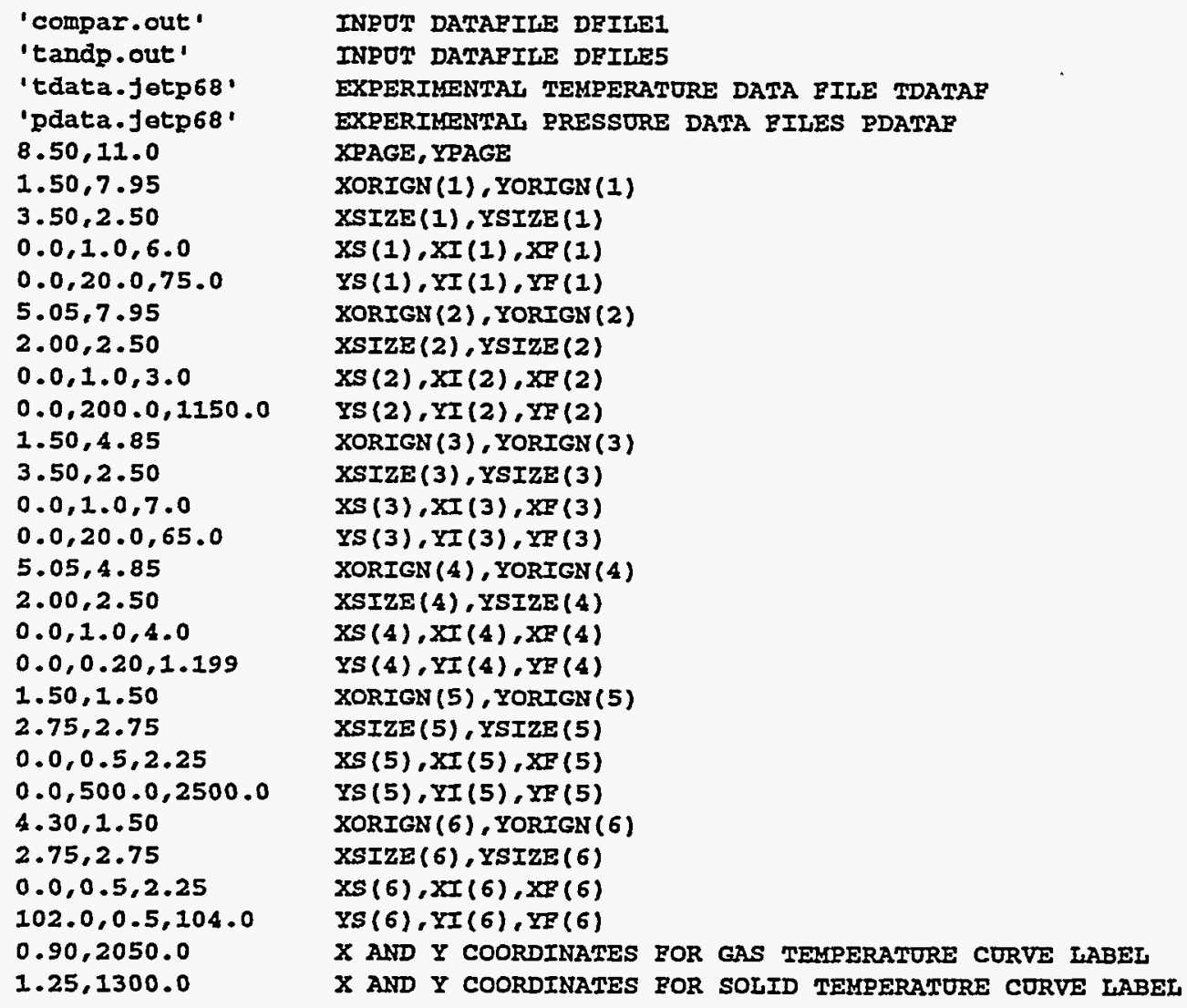

changes might be necessary to run this script file on a different platform. The user should consult with the system's manager for help, if needed. 


\section{Compilation, Execution and Viewing/Printing of the Program PLOT1}

In our center, the DISSPLA graphics package is installed on a computer named hodg. The plotting routines reside in the subdirectory jetp68. The symbol " $>$ " is the system prompt. The procedure to compile and execute the program is as follows:

Step 1: Compile the program plotl.

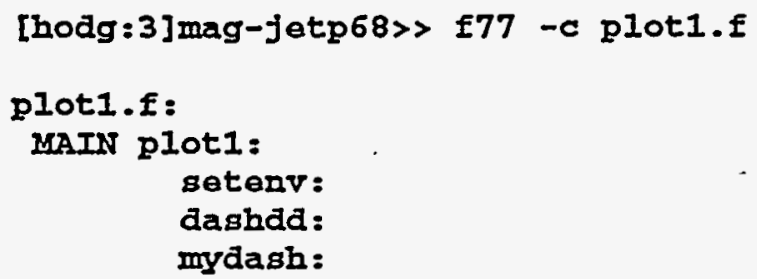

Step 2: Link and generate the executable code plot1.

[hodg:4]mag-jetp68>> dis771ink -o plot1 plot1.0

Step 3: Execute plotl to generate the device independent file plotl.pop

[hodg:5]mag-jetp68>> plot1

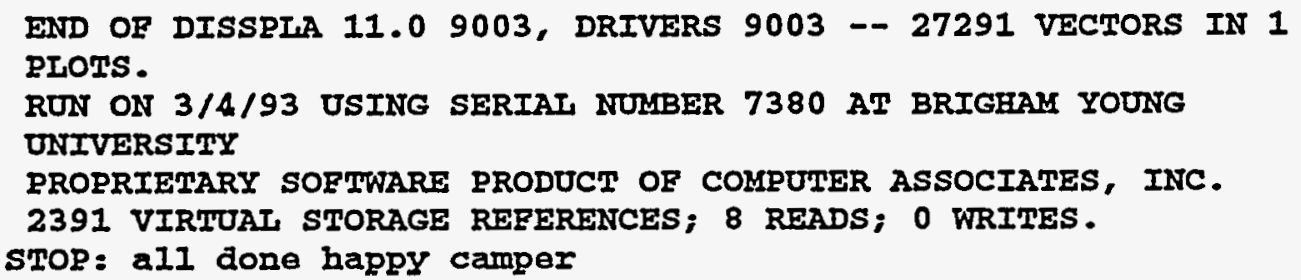

Step 4: Run the script file to view the plotl.pop on a $X$-Windows environment.

[hodg: 6] mag-jetp68>> popout

Choose your output device

x) $X$ window

h) IIP Iaserjet III Si 


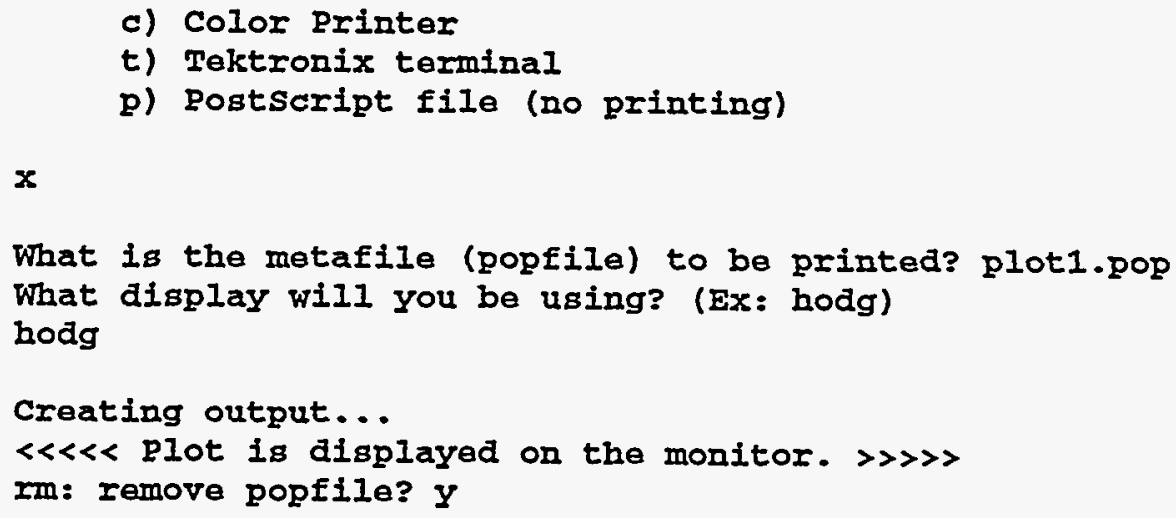

Step 5: run the script file to generate the postscript file plotl.ps. [hodg: 7] mag-jetp68>> popout

Step 6: Print the file plotl.ps using the appropriate print command.

Other output devices supported by this script file are an HP Laserjet III Si printer, a color printer, and Tektronix terminal.

The second program plot 2 can also be executed in a similar manner, by replacing plot1 with plot 2 in the above commands. 


\section{USER'S FORUM}

If you have any comments concerning your experiences using FBED-1, please foward them to:

Dr. Andrew M. Eaton

ACERC Software Specialist

75 Crabtree Building

Brigham Young University

Provo, UT 84602

Phone: (801) 378-5008

E-mail:ame@homer.et.byu.edu

You are also invited to submit your comments concerning the clarity, accuracy, and usefulness of this manual. Constructive critical comments are most welcome and will help us continue our efforts to generate quality user documentation. Please list the page number for questions and comments. Use other side if necessary.

From:

Name Title

Company Date

Address and Phone No. 
August 20, 1993

\section{EBED-1 Code Installation Instructions}

We have executed the FBED-1 code on our Sun 4/310 and Silicon Graphics workstations and made sample runs. The code runs properly. We have copied all the files, including the sample input and the sample output files on the enclosed tapes. The files can be recovered as follows:

\section{$\%$ mkdir FBED1}

$\%$ cd FBED1

$\% \operatorname{tar}-\mathrm{xvf} / \mathrm{dev} / \mathrm{rst8}$ (please use the appropriate device name for the magnetic tape drive on your workstation)

Next the sample output files, fb1out, outa - outj, balanc.out, cconv.out, compar.out, compos.out and pdia.out, should be moved to a subdirectory outfiles as follows:

$\%$ mkdir outfiles

$\% \mathrm{mv} *$ out* outfiles

The sample case is for Wellman-Galusha gasifier fired with Jetson bituminous coal and may be run while in FBED1 directory simply by typing:

$\%$ fbed1

The new output files, fb1out, outa - outj, balanc.out, cconv.out, compar.out, compos.out and pdia.out, will be generated and may be compared with our results in the subdirectory outfiles. We also recommend that you recompile the code and rerun the sample case and again compare the results with the earlier ones. The code may be recompiled by first removing all object (with extension 0 ) files, and then by typing:

\% make -f makefbed

After successful compilation, linking and loading, the sample case can be run as before by typing:

$\%$ fbed1

The tape also includes two plotting programs for the DISSPLA package. The executable files on the tape are for SUN computer and can be run following the procedure outlined in Appendix $\mathbf{J}$ of the FBED-1 User's Manual. Please make the appropriate changes, if needed, for running these programs on a Silicon Graphics machine.

If you need any further assistance, please call Dr. Predrag T. Radulovic at (801) 3783097 or Dr. M. Usman Ghani at (801) 378-6074. 